\title{
An N-Fluorinated Imide for Practical Catalytic Imidations
}

Yuno Oe, ${ }^{\dagger, \|}$ Ryuhei Yoshida, ${ }^{\dagger, \|}$ Airi Tanaka, ${ }^{\dagger, \|}$ Akiya Adachi, ${ }^{\ddagger}$ Yuichiro Ishibashi,§ Takashi Okazoe,, ${ }^{\ddagger} \S$ Kohsuke Aikawa, ${ }^{*, \ddagger}$ and Takuya Hashimoto ${ }^{*, \dagger}$

${ }^{\dagger}$ Chiba lodine Resource Innovation Center and Department of Chemistry, Graduate School of Science, Chiba University, 1-33, Yayoi, Inage, Chiba 263-8522, Japan

‡Department of Chemistry and Biotechnology, Graduate School of Engineering, The University of Tokyo, 2-11-16 Yayoi, Bunkyo-ku, Tokyo 113-8656, Japan

§Yokohama Technical Center, AGC Inc., 1-1 Suehiro-cho, Tsurumi-ku, Yokohama 230-0045, Japan "Equal contribution 


\section{Table of Contents}

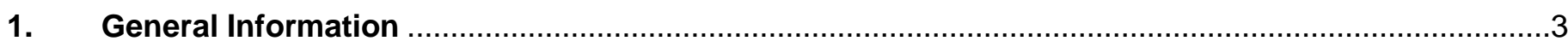

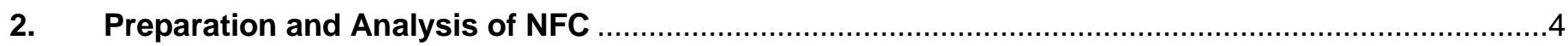

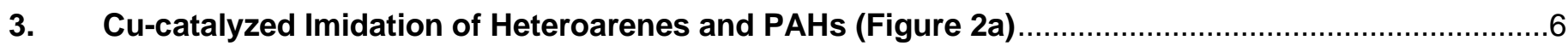

4. Computed Energy Profile for the Imidyl Radical Addition to Benzene (Figure 2b) ........................20

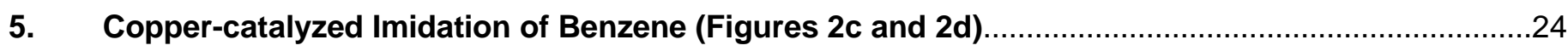

6. Cu-catalyzed Imidation of Benzene Derivatives (Figure 2e) ....................................................25

7. Copper-Catalyzed Imidocyanation of Alkenes with NFC (Figure 3) ...........................................32

8. Deprotection of Imidated Products to Amines (Figure 4) …...................................................... 44

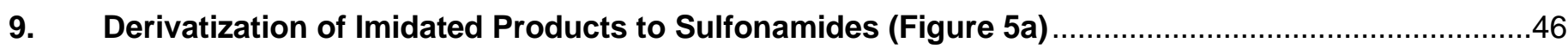

10. Derivatization of Imidated Products to Sulfamides (Figure 5b) …...........................................50

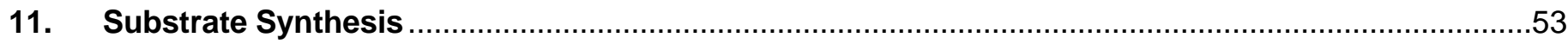

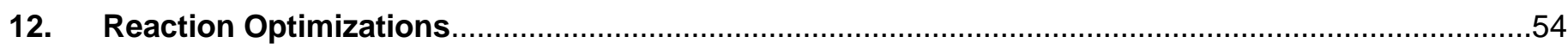

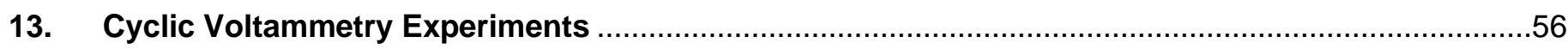

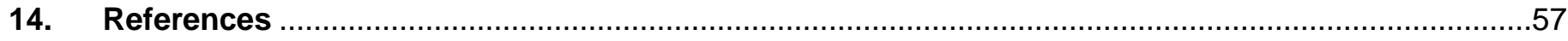

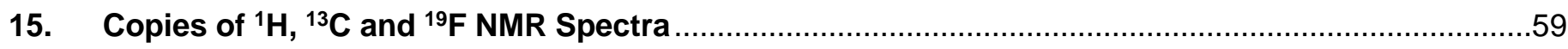




\section{General Information}

${ }^{1}$ H NMR spectra were measured on JEOL JNM-ECS400 spectrometer or JEOL JNM-ECA500 spectrometer. Data were reported as follows: chemical shifts in ppm from tetramethylsilane as an internal standard, integration, multiplicity ( $\mathrm{s}=$ singlet, $\mathrm{d}=$ doublet, $\mathrm{t}=$ triplet, $\mathrm{q}=$ quartet, $\mathrm{dd}=$ double-doublet, $\mathrm{m}=$ multiplet and $\mathrm{br}=\mathrm{broad}$ ), coupling constants $(\mathrm{Hz}) .{ }^{13} \mathrm{C}$ NMR spectra were measured on JEOL JNM-ECS400 spectrometer or JEOL JNMECA500 spectrometer with complete proton decoupling. Chemical shifts were reported in ppm from the residual solvent as an internal standard. ${ }^{19} \mathrm{~F}$ NMR spectra were measured on JEOL JNM-ECS400 spectrometer or JEOL JNM-ECA500 spectrometer, using hexafluorobenzene as internal standard (-163.0 ppm).

High-resolution mass spectra (HRMS) were performed on a Thermo Exactive Plus (ESI) or JEOL JMS-T100LP spectrometer in the electron spray ionization time-of-flight (ESI-TOF) mode.

Cyclic voltammograms were collected with a BAS ALS600E.

Thermogravimetric and differential thermal analysis (TG/DTA) measurements were performed on a Seiko EXSTAR 6000 TG/DTA 6200 analyzer.

UV-Vis measurements were performed on a Shimadzu UV-1800 using $2 \mathrm{~mm}$ quarts cell.

Commercially obtained reagents were used without further purification. The products were purified by Merck precoated preparative thin layer chromatography (PLC) plate (silica gel $60 \mathrm{GF} 254,0.25 \mathrm{~mm}$ ) or a Biotage Isolera purification system using Wakogel ${ }^{8}(60 \mathrm{~N}$, neutral, 38-100 $\mu \mathrm{m})$ or Wakogel $(\mathrm{C}-300,45-75 \mu \mathrm{m})$. 


\section{Preparation and Analysis of NFC}

\section{lithium (fluorosulfonyl)((neopentyloxy)carbonyl)amide (1)}

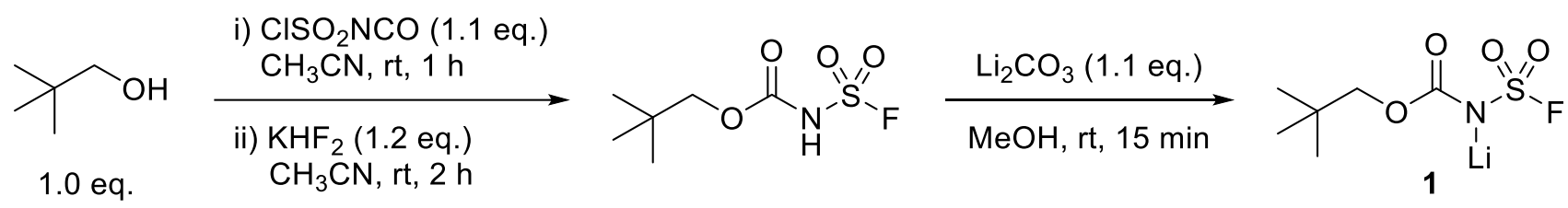

To an argon-purged flask, acetonitrile $(50 \mathrm{~mL})$, chlorosulfonyl isocyanate $(110 \mathrm{mmol}, 9.77 \mathrm{~mL}, 98.0 \%)$ and neopentyl alcohol (100 mmol, $8.82 \mathrm{~g}$ ) were added at $0{ }^{\circ} \mathrm{C}$. After stirring for $1 \mathrm{~h}$ at room temperature, potassium hydrogenfluoride $(120 \mathrm{mmol}, 9.37 \mathrm{~g})$ was added to the flask at room temperature. After stirring for $2 \mathrm{~h}$ at the same temperature, the reaction was quenched with water $(150 \mathrm{~mL})$ and extracted with hexane/EtOAc (1:1) $(100 \mathrm{~mL} \times$ 3). The combined organic layers were washed with brine, dried over anhydrous $\mathrm{Na}_{2} \mathrm{SO}_{4}$ and evaporated under reduced pressure. To the residue, $\mathrm{MeOH}(100 \mathrm{~mL})$ and lithium carbonate $(110 \mathrm{mmol}, 8.13 \mathrm{~g})$ were added. The mixture was stirred for $15 \mathrm{~min}$ at room temperature and the solvent was removed under reduced pressure. The residue was diluted with EtOAc, filtered through Celite pad and concentrated. The residue was washed with $\mathrm{CH}_{2} \mathrm{Cl}_{2}$ to give the title compound as a white solid in $80 \%$ yield $(79.9 \mathrm{mmol}, 17.5 \mathrm{~g})$ for 2 steps.

${ }^{1} \mathrm{H}$ NMR $\left(396 \mathrm{MHz},\left(\mathrm{CD}_{3}\right)_{2} \mathrm{SO}\right) \delta 3.50(\mathrm{~s}, 2 \mathrm{H}), 0.86(\mathrm{~s}, 9 \mathrm{H})$.

${ }^{13} \mathrm{C}$ NMR $\left(126 \mathrm{MHz},\left(\mathrm{CD}_{3}\right)_{2} \mathrm{SO}\right) \delta 157.9,73.4,31.2,26.4$.

${ }^{19} \mathrm{~F}$ NMR $\left(471 \mathrm{MHz},\left(\mathrm{CD}_{3}\right)_{2} \mathrm{SO}\right) \delta 48.92(\mathrm{~s}, 1 \mathrm{~F})$.

HRMS (ESI) exact mass calculated for $\mathrm{C}_{6} \mathrm{H}_{11} \mathrm{O}_{4} \mathrm{NFS}$ : m/z 212.0398 ([M-Li]'), found: 212.0397 ([M-Li]').

\section{neopentyl $\mathrm{N}$-fluoro- $\mathrm{N}$-(fluorosulfonyl)carbamate (2)}

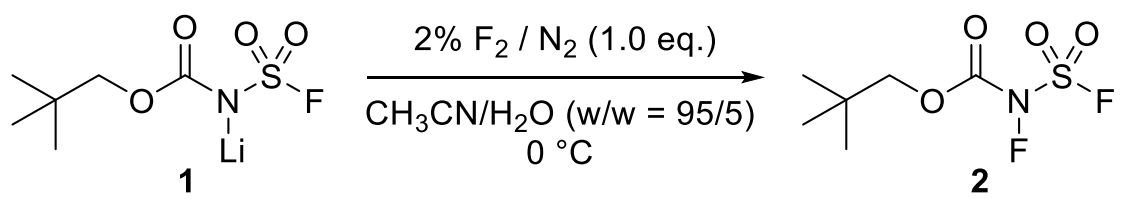

The solution of $1(6.00 \mathrm{~g}, 27.38 \mathrm{mmol})$ in a mixture of acetonitrile $(142.5 \mathrm{~g})$ and $\mathrm{H}_{2} \mathrm{O}(7.5 \mathrm{~g})$ was stirred in a 250 $\mathrm{mL}$ PFA vessel and cooled to $0{ }^{\circ} \mathrm{C}$. A gaseous mixture of $4 \% \mathrm{~F}_{2}$ in $\mathrm{N}_{2}$ (volume percent) was introduced at a rate of $100 \mathrm{~mL} / \mathrm{min}$ to the solution for $162.3 \mathrm{~min}\left(F_{2}: 1.0\right.$ eq.). $\mathrm{N}_{2}$ gas was introduced to remove the residual $F_{2}$ gas for $30 \mathrm{~min}$. The yield of the compound was determined by ${ }^{19} \mathrm{~F}$ NMR analysis using benzotrifluoride as an internal standard ( ${ }^{19} \mathrm{~F}$ NMR yield $\left.89 \%\right)$. The insoluble solid was filtrated, and the filtrate was evaporated under reduced pressure $(50 \mathrm{mmHg})$. The resulting crude product was extracted with $\mathrm{n}$-hexane $(30 \mathrm{~mL}, 3$ times). The collected organic phase was concentrated under reduced pressure $(50 \mathrm{mmHg})$ at ambient temperature and gave the product $2(4.85 \mathrm{~g}, 77 \%$ yield) as a colorless liquid.

${ }^{1} \mathrm{H}$ NMR $\left(500 \mathrm{MHz}, \mathrm{CDCl}_{3}\right) \delta 4.16(\mathrm{~s}, 2 \mathrm{H}), 1.01$ (s, 9H).

${ }^{13} \mathrm{C}$ NMR $\left(100 \mathrm{MHz}, \mathrm{CDCl}_{3}\right) \delta 148.5\left(\mathrm{~d}, \mathrm{~J}_{\mathrm{C}-\mathrm{F}}=8.7 \mathrm{~Hz}\right), 80.6,31.9,26.0$.

${ }^{19} \mathrm{~F} \mathrm{NMR}\left(470 \mathrm{MHz}, \mathrm{CDCl}_{3}\right) \delta 44.1$ (s, 1F), -46.3 (s, 1F).

HRMS (ESI-TOF) exact mass calculated for $\mathrm{C}_{6} \mathrm{H}_{11} \mathrm{~F}_{2} \mathrm{NNaO}_{4} \mathrm{~S}[\mathrm{M}+\mathrm{Na}]^{+}: \mathrm{m} / z$ 231.0377, found: 231.0348 .

Thermogravimetric and Differential Thermal Analysis (TG/DTA) measurements were performed at a heating rate of $10^{\circ} \mathrm{C} / \mathrm{min}$. Reagent 2 shows a vaporization at $120-125^{\circ} \mathrm{C}$ without decomposition. 


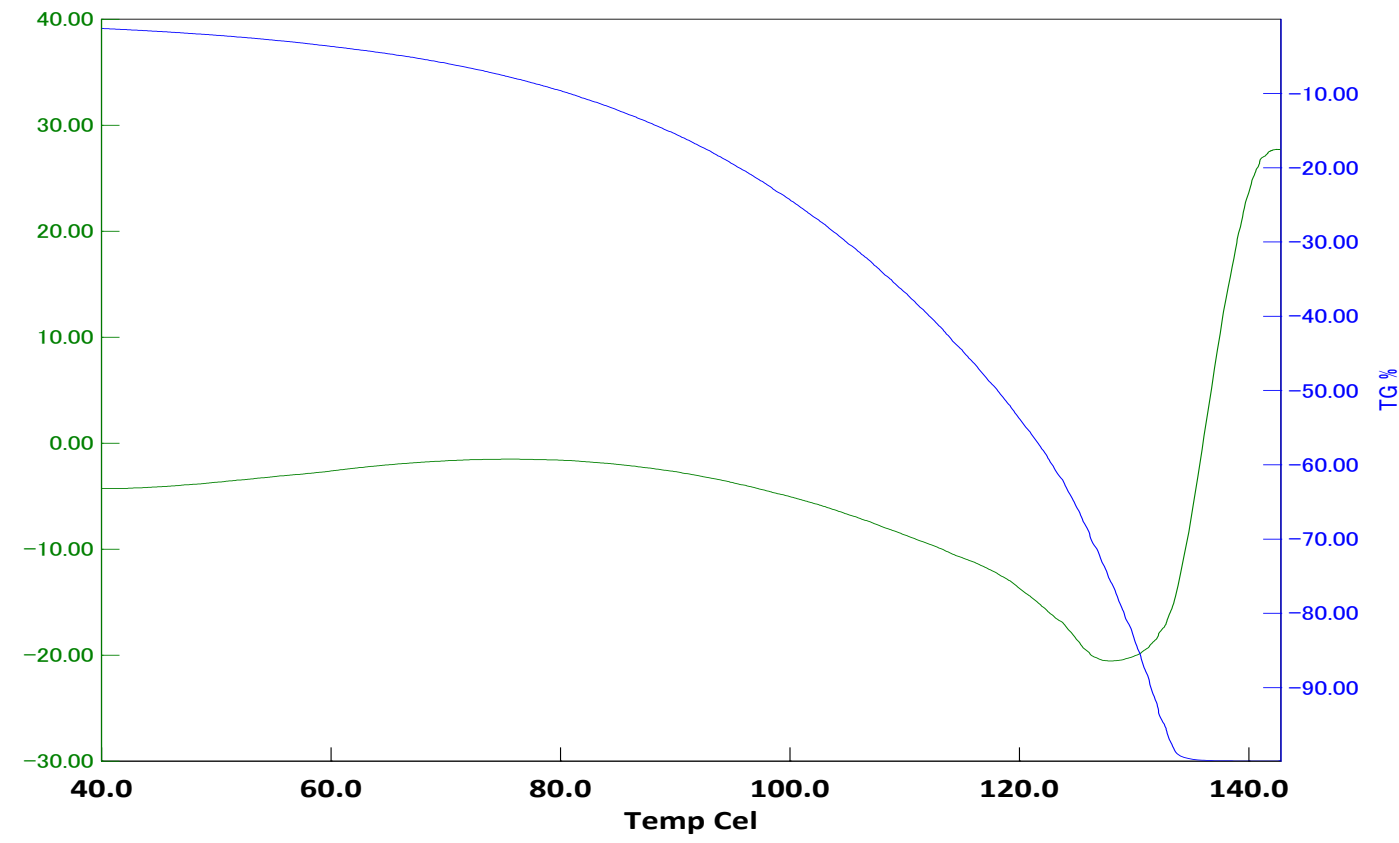


3. Cu-catalyzed Imidation of Heteroarenes and PAHs (Figure 2a)

\section{General Procedure}

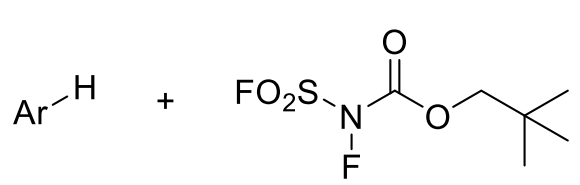

1.0 eq.

$1.4 \mathrm{eq}$

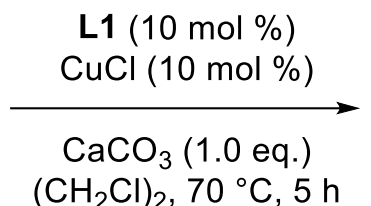

$\left(\mathrm{CH}_{2} \mathrm{Cl}\right)_{2}, 70^{\circ} \mathrm{C}, 5 \mathrm{~h}$

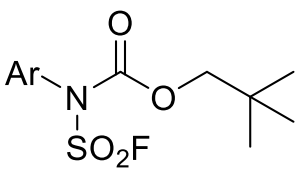

$\mathrm{SO}_{2} \mathrm{~F}$

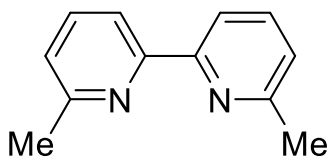

L1

To an argon-purged Schlenk tube, calcium carbonate $(200 \mu \mathrm{mol}, 20.0 \mathrm{mg})$, copper $(\mathrm{I})$ chloride $(20.0 \mu \mathrm{mol}, 2.1 \mathrm{mg}$,

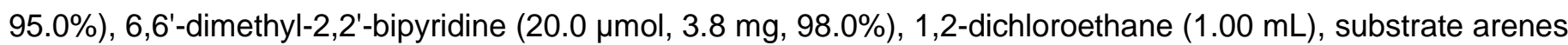
(200 $\mu \mathrm{mol}$ ) and neopentyl $N$-fluoro- $N$-(fluorosulfonyl)carbamate (NFC) $(280 \mu \mathrm{mol}, 64.7 \mathrm{mg},>98 \%)$ were added. After stirring for $5 \mathrm{~h}$ at $70^{\circ} \mathrm{C}$, the mixture was cooled to room temperature. The crude solution was filtered through a pad of $\mathrm{Al}_{2} \mathrm{O}_{3}$ eluting with EtOAc and concentrated under vacuum. The residue was purified by column chromatography on silica gel.

neopentyl (fluorosulfonyl)(thiophen-2-yl)carbamate (3)<smiles>CC(C)(C)COC(=O)N(c1ccccc1)c1cccs1</smiles>

Prepared according to the general procedure with thiophene (200 $\mu \mathrm{mol}, 17.3 \mathrm{mg}, 97.0 \%)$. The crude material was purified by column chromatography (EtOAc/hexane 3-26\%) to give the title compound as a pale yellowish oil in $49 \%$ yield $(97.9 \mu \mathrm{mol}, 28.9 \mathrm{mg})$.

${ }^{1}$ H NMR $\left(392 \mathrm{MHz}, \mathrm{CDCl}_{3}\right) \delta 7.40(\mathrm{dd}, J=5.6,1.3 \mathrm{~Hz}, 1 \mathrm{H}), 7.12(\mathrm{dd}, J=3.8,1.6 \mathrm{~Hz}, 1 \mathrm{H}), 7.01(\mathrm{dd}, J=5.6,3.8$ $\mathrm{Hz}, 1 \mathrm{H}), 3.97$ (s, 2H), $0.89(\mathrm{~s}, 9 \mathrm{H})$.

${ }^{13} \mathrm{C}$ NMR $\left(99 \mathrm{MHz}, \mathrm{CDCl}_{3}\right) \delta 150.2,134.6,129.1,127.9,125.9,78.8,31.5,26.0$.

${ }^{19} \mathrm{~F}$ NMR $\left(369 \mathrm{MHz}, \mathrm{CDCl}_{3}\right) \delta 49.84(\mathrm{~s}, 1 \mathrm{~F})$.

HRMS (ESI) exact mass calculated for $\mathrm{C}_{10} \mathrm{H}_{14} \mathrm{O}_{4} \mathrm{NFS}_{2} \mathrm{Na}: \mathrm{m} / z 318.0240\left([\mathrm{M}+\mathrm{Na}]^{+}\right)$, found: $\mathrm{m} / z 318.0237\left([\mathrm{M}+\mathrm{Na}]^{+}\right)$.

neopentyl (fluorosulfonyl)(5-phenylthiophen-2-yl)carbamate (4)

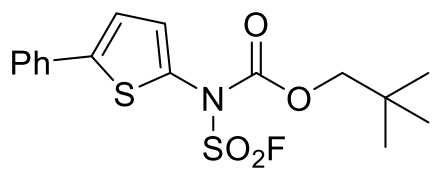

Prepared according to the general procedure with neopentyl fluoro(fluorosulfonyl)carbamate (200 $\mu \mathrm{mol}, 33.7 \mathrm{mg})$. The crude material was purified by column chromatography (EtOAc/hexane 4-34\%) to give the title compound as a white solid in $71 \%$ yield ( $142 \mu \mathrm{mol}, 52.7 \mathrm{mg})$.

${ }^{1} \mathrm{H}$ NMR $\left(392 \mathrm{MHz}, \mathrm{CDCl}_{3}\right)$ ठ 7.56-7.58 (m, 2H), 7.32-7.42 (m, 3H), 7.18 (d, J=4.0 Hz, $\left.1 \mathrm{H}\right), 7.10(\mathrm{~d}, J=4.0 \mathrm{~Hz}$, $1 \mathrm{H}), 4.00(\mathrm{~s}, 2 \mathrm{H}), 0.91(\mathrm{~s}, 9 \mathrm{H})$.

${ }^{13} \mathrm{C}$ NMR $\left(99 \mathrm{MHz}, \mathrm{CDCl}_{3}\right) \delta 150.2,146.8,133.2,133.0,130.2,129.1,128.6,126.0,121.4,78.9,31.5,26.0$.

${ }^{19} \mathrm{~F}$ NMR $\left(369 \mathrm{MHz}, \mathrm{CDCl}_{3}\right) \delta 50.10$ (s, 1F).

HRMS (ESI) exact mass calculated for $\mathrm{C}_{16} \mathrm{H}_{18} \mathrm{O}_{4} \mathrm{NFS}{ }_{2} \mathrm{Na}: \mathrm{m} / z 394.0553\left([\mathrm{M}+\mathrm{Na}]^{+}\right)$, found: $\mathrm{m} / z 394.0556\left([\mathrm{M}+\mathrm{Na}]^{+}\right)$.

methyl 5-((fluorosulfonyl)((neopentyloxy)carbonyl)amino)thiophene-2-carboxylate (5) 


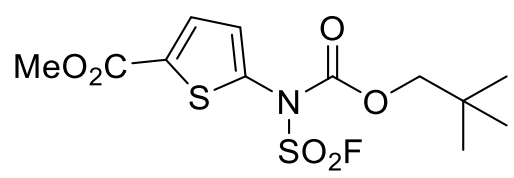

Prepared according to the general procedure with methyl thiophene-2-carboxylate (200 $\mu \mathrm{mol}, 28.4 \mathrm{mg}) .^{1}$ The crude material was purified by column chromatography (EtOAc/hexane 4-34\%) to give the title compound as a yellowish solid in $48 \%$ yield $(96.2 \mu \mathrm{mol}, 34.0 \mathrm{mg})$.

${ }^{1} \mathrm{H}$ NMR $\left(396 \mathrm{MHz}, \mathrm{CDCl}_{3}\right) \delta 7.70(\mathrm{~d}, J=3.9 \mathrm{~Hz}, 1 \mathrm{H}), 7.13(\mathrm{~d}, J=4.1 \mathrm{~Hz}, 1 \mathrm{H}), 4.00(\mathrm{~s}, 2 \mathrm{H}), 3.91(\mathrm{~s}, 3 \mathrm{H}), 0.90(\mathrm{~s}$, $9 \mathrm{H})$.

${ }^{13} \mathrm{C}$ NMR $\left(99 \mathrm{MHz}, \mathrm{CDCl}_{3}\right) \delta 161.5,149.6,139.7,135.1,131.7,129.8,79.2,52.6,31.5,26.0$.

${ }^{19} \mathrm{~F}$ NMR $\left(369 \mathrm{MHz}, \mathrm{CDCl}_{3}\right) \delta 50.76(\mathrm{~s}, 1 \mathrm{~F})$.

HRMS (ESI) exact mass calculated for $\mathrm{C}_{12} \mathrm{H}_{16} \mathrm{O}_{6} \mathrm{NFS}_{2} \mathrm{Na}: \mathrm{m} / z 376.0295\left([\mathrm{M}+\mathrm{Na}]^{+}\right)$, found: $\mathrm{m} / z 376.0294\left([\mathrm{M}+\mathrm{Na}]^{+}\right)$.

neopentyl (5-bromothiophen-2-yl)(fluorosulfonyl)carbamate (6)

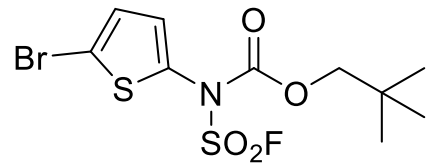

Prepared according to the general procedure with 2-bromothiophene (200 $\mu \mathrm{mol}, 33.3 \mathrm{mg}, 98.0 \%)$. The crude material was purified by column chromatography (EtOAc/hexane $5 \%$ ) to give the title compound as a pale yellowish oil in $86 \%$ yield $(173 \mu \mathrm{mol}, 64.7 \mathrm{mg})$.

${ }^{1} \mathrm{H}$ NMR $\left(392 \mathrm{MHz}, \mathrm{CDCl}_{3}\right) \delta 7.02(\mathrm{~d}, J=4.0 \mathrm{~Hz}, 1 \mathrm{H}), 6.91(\mathrm{~d}, J=4.0 \mathrm{~Hz}, 1 \mathrm{H}), 3.99(\mathrm{~s}, 2 \mathrm{H}), 0.92(\mathrm{~s}, 9 \mathrm{H})$.

${ }^{13} \mathrm{C}$ NMR $\left(99 \mathrm{MHz}, \mathrm{CDCl}_{3}\right) \delta 149.8,134.6,129.9,128.8,114.9,79.1,31.5,26.0$.

${ }^{19} \mathrm{~F}$ NMR $\left(369 \mathrm{MHz}, \mathrm{CDCl}_{3}\right) \delta 50.53(\mathrm{~s}, 1 \mathrm{~F})$.

HRMS (ESI) exact mass calculated for $\mathrm{C}_{10} \mathrm{H}_{13} \mathrm{O}_{4} \mathrm{NFS}{ }_{2} \mathrm{BrNa}: \mathrm{m} / z 395.9346\left([\mathrm{M}+\mathrm{Na}]^{+}\right)$, found: $\mathrm{m} / z 395.9342$ $\left([\mathrm{M}+\mathrm{Na}]^{+}\right)$.

neopentyl (3-acetylthiophen-2-yl)(fluorosulfonyl)carbamate (7) and neopentyl (4-acetylthiophen-2-yl)(fluorosulfonyl)carbamate (7')

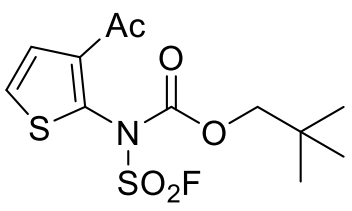

7

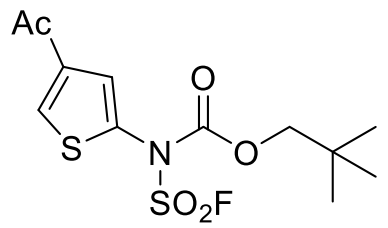

$7^{\prime}$

Prepared according to the general procedure with 3-acetylthiophene (200 $\mu \mathrm{mol}, 25.7 \mathrm{mg}, 98.0 \%)$. The crude material was purified by column chromatography (EtOAc/hexane 3-26\%) to give a mixture of the title compounds as a yellowish solid in $68 \%$ yield $\left(136 \mu \mathrm{mol}, 45.8 \mathrm{mg}, \mathbf{7 : 7 ^ { \prime }}=61: 39\right)$.

${ }^{1} \mathrm{H}$ NMR $\left(500 \mathrm{MHz}, \mathrm{CDCl}_{3}\right) \delta 8.08(\mathrm{~d}, J=1.7 \mathrm{~Hz}, 0.6 \mathrm{H}), 7.56(\mathrm{~d}, J=1.7 \mathrm{~Hz}, 0.6 \mathrm{H}), 7.41(\mathrm{~s}, 0.8 \mathrm{H}), 4.01(\mathrm{~s}, 1.2 \mathrm{H})$, $3.97(\mathrm{br}, 0.8 \mathrm{H}), 2.53(\mathrm{~s}, 1.2 \mathrm{H}), 2.52(\mathrm{~s}, 1.8 \mathrm{H}), 0.92(\mathrm{~s}, 5.4 \mathrm{H}), 0.89(\mathrm{~s}, 3.6 \mathrm{H})$.

${ }^{13} \mathrm{C}$ NMR $\left(126 \mathrm{MHz}, \mathrm{CDCl}_{3}\right) \delta 191.7,191.0,149.8,149.6,140.7,138.5,136.4,135.4,133.5,128.8,127.1,126.2$, 79.2, 78.8, 31.5, 31.4, 29.3, 26.9, 26.0.

${ }^{19} \mathrm{~F}$ NMR $\left(471 \mathrm{MHz}, \mathrm{CDCl}_{3}\right) \delta 51.95$ (s, 0.4F), 50.80 (s, 0.6F).

HRMS (ESI) exact mass calculated for $\mathrm{C}_{12} \mathrm{H}_{16} \mathrm{O}_{5} \mathrm{NFS}{ }_{2} \mathrm{Na}: \mathrm{m} / \mathrm{z} 360.0346\left([\mathrm{M}+\mathrm{Na}]^{+}\right)$, found: $\mathrm{m} / \mathrm{z} 360.0340$ $\left([\mathrm{M}+\mathrm{Na}]^{+}\right)$. 


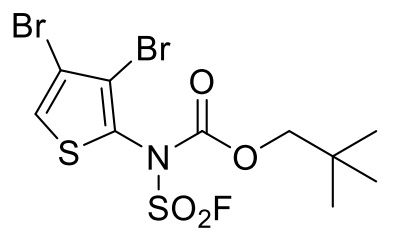

Prepared according to the general procedure with 3,4-dibromothiophene (200 $\mu \mathrm{mol}, 49.4 \mathrm{mg}, 98.0 \%)$. The crude material was purified by column chromatography (DCM/hexane 4-34\%) to give the title compound as a pale yellowish oil in $73 \%$ yield $(147 \mu \mathrm{mol}, 66.4 \mathrm{mg})$.

${ }^{1} \mathrm{H}$ NMR $\left(500 \mathrm{MHz}, \mathrm{CDCl}_{3}\right) \delta 7.51(\mathrm{~s}, 1 \mathrm{H}), 4.02(\mathrm{~s}, 2 \mathrm{H}), 0.91(\mathrm{~s}, 9 \mathrm{H})$.

${ }^{13} \mathrm{C}$ NMR $\left(126 \mathrm{MHz}, \mathrm{CDCl}_{3}\right) \delta 148.9,130.9,124.9,118.8,113.0,79.2,31.5,26.0$.

${ }^{19} \mathrm{~F}$ NMR $\left(471 \mathrm{MHz}, \mathrm{CDCl}_{3}\right) \delta 54.25$ (s, 1F).

HRMS (ESI) exact mass calculated for $\mathrm{C}_{10} \mathrm{H}_{12} \mathrm{O}_{4} \mathrm{NFS}_{2} \mathrm{Br}_{2} \mathrm{Na}: \mathrm{m} / z 473.8451$ ([M+Na] $]^{+}$), found: $\mathrm{m} / \mathrm{z} 473.8451$ $\left([\mathrm{M}+\mathrm{Na}]^{+}\right)$.

neopentyl (2,5-dibromothiophen-3-yl)(fluorosulfonyl)carbamate (9)<smiles>CC(C)(C)COC(=O)N(c1cc(Br)sc1Br)S(=O)(=O)F</smiles>

Prepared according to the general procedure with 2,5-dibromothiophene (200 $\mu \mathrm{mol}, 49.2 \mathrm{mg}, 98.4 \%){ }^{2}$ The crude material was purified by column chromatography (1st DCM/hexane $5-40 \%$, 2nd acetone/hexane $5 \%$ ) to give the title compound as a pale yellowish oil in $21 \%$ yield $(41.0 \mu \mathrm{mol}, 18.6 \mathrm{mg})$.

${ }^{1} \mathrm{H}$ NMR $\left(500 \mathrm{MHz}, \mathrm{CDCl}_{3}\right) \delta 6.99(\mathrm{~s}, 1 \mathrm{H}), 4.00(\mathrm{~s}, 2 \mathrm{H}), 0.92(\mathrm{~s}, 9 \mathrm{H})$.

${ }^{13} \mathrm{C}$ NMR $\left(126 \mathrm{MHz}, \mathrm{CDCl}_{3}\right) \delta 149.1,131.6,128.9,114.6,111.8,78.9,31.5,26.0$.

${ }^{19} \mathrm{~F}$ NMR $\left(471 \mathrm{MHz}, \mathrm{CDCl}_{3}\right) \delta 53.59$ (s, 1F).

HRMS (ESI) exact mass calculated for $\mathrm{C}_{10} \mathrm{H}_{12} \mathrm{O}_{4} \mathrm{NFS}_{2} \mathrm{Br}_{2} \mathrm{Na}: \mathrm{m} / z 473.8451$ ([M+Na $]^{+}$), found: $\mathrm{m} / z 473.8454$ $\left([\mathrm{M}+\mathrm{Na}]^{+}\right)$.

neopentyl (fluorosulfonyl)(5-phenylfuran-2-yl)carbamate (10)<smiles>CC(C)(C)COC(=O)N(c1ccc(-c2ccccc2)o1)[S+](=O)[O-]</smiles>

Prepared according to the general procedure with 2-phenylfuran $(200 \mu \mathrm{mol}, 28.8 \mathrm{mg}) .^{3}$ The crude material was purified by column chromatography (DCM/hexane 5-40\%) to give the title compound as a white solid in $61 \%$ yield (122 $\mu \mathrm{mol}, 43.5 \mathrm{mg})$.

${ }^{1} \mathrm{H}$ NMR $\left(500 \mathrm{MHz}, \mathrm{CDCl}_{3}\right)$ ठ $7.66(\mathrm{~m}, 2 \mathrm{H}), 7.39-7.42(\mathrm{~m}, 2 \mathrm{H}), 7.31-7.34(\mathrm{~m}, 1 \mathrm{H}), 6.71(\mathrm{~d}, J=3.4 \mathrm{~Hz}, 1 \mathrm{H}), 6.58(\mathrm{~d}$, $J=3.7 \mathrm{~Hz}, 1 \mathrm{H}), 4.01(\mathrm{~s}, 2 \mathrm{H}), 0.90(\mathrm{~s}, 9 \mathrm{H})$.

${ }^{13} \mathrm{C}$ NMR $\left(126 \mathrm{MHz}, \mathrm{CDCl}_{3}\right) \delta 153.9,149.6,137.8,129.4,128.8,128.5,124.2,111.8,106.4,78.9,31.5,26.0$.

${ }^{19} \mathrm{~F}$ NMR $\left(471 \mathrm{MHz}, \mathrm{CDCl}_{3}\right) \delta 50.92(\mathrm{~s}, 1 \mathrm{~F})$.

HRMS (ESI) exact mass calculated for $\mathrm{C}_{16} \mathrm{H}_{18} \mathrm{O}{ }_{5} \mathrm{NFSNa}: \mathrm{m} / z 378.0782\left([\mathrm{M}+\mathrm{Na}]^{+}\right)$, found: $m / z 378.0774\left([\mathrm{M}+\mathrm{Na}]^{+}\right)$. 


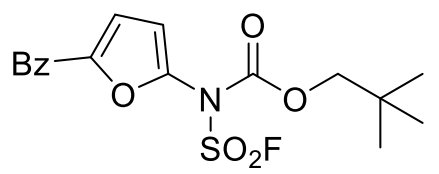

Prepared according to the general procedure with 2-benzoylfuran $(200 \mu \mathrm{mol}, 34.4 \mathrm{mg})$. The crude material was purified by column chromatography (1st EtOAc/hexane 2-20\%, 2nd DCM/hexane 12-100\%) to give the title compound as a pale yellowish solid in $62 \%$ yield $(124 \mu \mathrm{mol}, 47.4 \mathrm{mg})$.

${ }^{1} \mathrm{H}$ NMR $\left(500 \mathrm{MHz}, \mathrm{CDCl}_{3}\right) \delta 7.96(\mathrm{~m}, 2 \mathrm{H}), 7.60-7.65(\mathrm{~m}, 1 \mathrm{H}), 7.50-7.54(\mathrm{~m}, 2 \mathrm{H}), 7.29(\mathrm{~d}, J=3.4 \mathrm{~Hz}, 1 \mathrm{H}), 6.72(\mathrm{~d}$, $J=3.7 \mathrm{~Hz}, 1 \mathrm{H}), 4.03(\mathrm{~s}, 2 \mathrm{H}), 0.92(\mathrm{~s}, 9 \mathrm{H})$.

${ }^{13} \mathrm{C}$ NMR $\left(126 \mathrm{MHz}, \mathrm{CDCl}_{3}\right) \delta 182.0,150.9,148.8,141.8,136.2,133.2,129.4,128.6,120.6,111.9,79.3,31.5$, 25.9.

${ }^{19} \mathrm{~F}$ NMR $\left(471 \mathrm{MHz}, \mathrm{CDCl}_{3}\right) \delta 52.24(\mathrm{~s}, 1 \mathrm{~F})$.

HRMS (ESI) exact mass calculated for $\mathrm{C}_{17} \mathrm{H}_{18} \mathrm{O}_{6} \mathrm{NFSNa:} \mathrm{m} / z \quad 406.0731\left([\mathrm{M}+\mathrm{Na}]^{+}\right)$, found: $\mathrm{m} / z 406.0726$ $\left([\mathrm{M}+\mathrm{Na}]^{+}\right)$.

tert-butyl 2-((fluorosulfonyl)((neopentyloxy)carbonyl)amino)-1H-pyrrole-1-carboxylate (12)

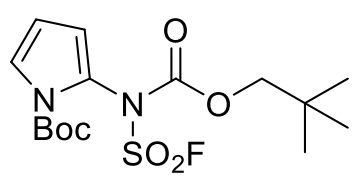

Prepared according to the general procedure with tert-butyl $1 \mathrm{H}$-pyrrole-1-carboxylate $(200 \mu \mathrm{mol}, 33.4 \mathrm{mg}) .^{4}$ The crude material was purified by column chromatography (DCM/hexane $12-100 \%)$ to give the title compound as a white solid in $54 \%$ yield (108 $\mu \mathrm{mol}, 40.9 \mathrm{mg}$ ).

${ }^{1} \mathrm{H}$ NMR $\left(500 \mathrm{MHz}, \mathrm{CDCl}_{3}\right) \delta 7.33(\mathrm{dd}, J=3.6,1.9 \mathrm{~Hz}, 1 \mathrm{H}), 6.40(\mathrm{dd}, J=3.7,2.0 \mathrm{~Hz}, 1 \mathrm{H}), 6.22(\mathrm{dd}, J=3.6,3.6$ $\mathrm{Hz}, 1 \mathrm{H}$ ), 3.95 (d, $J=10.0 \mathrm{~Hz}, 1 \mathrm{H}), 3.92(\mathrm{~d}, J=10.0 \mathrm{~Hz}, 1 \mathrm{H}), 1.58(\mathrm{~s}, 9 \mathrm{H}), 0.88(\mathrm{~s}, 9 \mathrm{H})$.

${ }^{13} \mathrm{C}$ NMR $\left(126 \mathrm{MHz}, \mathrm{CDCl}_{3}\right) \delta 150.0,147.5,122.6,121.1,115.0,109.6,85.7,78.3,31.3,27.8,26.0$.

${ }^{19} \mathrm{~F}$ NMR $\left(471 \mathrm{MHz}, \mathrm{CDCl}_{3}\right) \delta 50.96(\mathrm{~s}, 1 \mathrm{~F})$.

HRMS (ESI) exact mass calculated for $\mathrm{C}_{15} \mathrm{H}_{23} \mathrm{O}_{6} \mathrm{~N}_{2} \mathrm{FSNa}: \mathrm{m} / z 401.1153\left([\mathrm{M}+\mathrm{Na}]^{+}\right)$, found: $\mathrm{m} / z 401.1157\left([\mathrm{M}+\mathrm{Na}]^{+}\right)$.

neopentyl (fluorosulfonyl)(1-tosyl-1H-pyrrol-2-yl)carbamate (13)

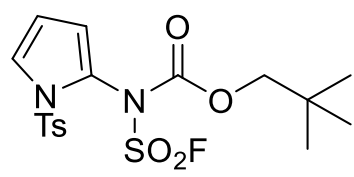

Prepared according to the general procedure with 1-tosyl-1 $\mathrm{H}$-pyrrole $(200 \mu \mathrm{mol}, 44.3 \mathrm{mg}) .^{5}$ The crude material was purified by column chromatography (EtOAc/hexane 3-20\%) to give the title compound as a colorless oil in $62 \%$ yield $(124 \mu \mathrm{mol}, 53.8 \mathrm{mg})$.

${ }^{1} \mathrm{H}$ NMR $\left(500 \mathrm{MHz}, \mathrm{CDCl}_{3}\right) \delta 7.79(\mathrm{~d}, J=8.3 \mathrm{~Hz}, 2 \mathrm{H}), 7.32-7.34(\mathrm{~m}, 3 \mathrm{H}), 6.40(\mathrm{dd}, J=3.7,1.7 \mathrm{~Hz}, 1 \mathrm{H}), 6.33$ (dd, $J=3.7,3.7 \mathrm{~Hz}, 1 \mathrm{H}), 3.96(\mathrm{~d}, J=10.3 \mathrm{~Hz}, 1 \mathrm{H}), 3.77(\mathrm{~d}, J=10.0 \mathrm{~Hz}, 1 \mathrm{H}), 2.43(\mathrm{~s}, 3 \mathrm{H}), 0.88(\mathrm{~s}, 9 \mathrm{H})$.

${ }^{13} \mathrm{C}$ NMR $\left(126 \mathrm{MHz}, \mathrm{CDCl}_{3}\right) \delta 149.8,145.8,135.0,130.0,127.7,123.2,121.6,115.1,110.8,78.6,31.3,26.0,21.7$. ${ }^{19} \mathrm{~F}$ NMR $\left(471 \mathrm{MHz}, \mathrm{CDCl}_{3}\right) \delta 51.39(\mathrm{~s}, 1 \mathrm{~F})$.

HRMS (ESI) exact mass calculated for $\mathrm{C}_{17} \mathrm{H}_{21} \mathrm{O}_{6} \mathrm{~N}_{2} \mathrm{FS}_{2} \mathrm{Na}: \mathrm{m} / z 455.0717\left([\mathrm{M}+\mathrm{Na}]^{+}\right)$, found: $\mathrm{m} / \mathrm{z} 455.0691$ $\left([\mathrm{M}+\mathrm{Na}]^{+}\right)$. 


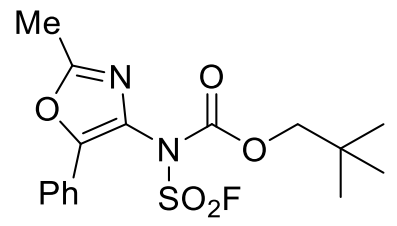

Prepared according to the general procedure with 2-methyl-5-phenyloxazole (200 $\mu \mathrm{mol}, 31.8 \mathrm{mg}) .^{6}$ The crude material was purified by column chromatography (DCM/hexane 17-100\%) to give the title compound as a pale yellowish oil in $79 \%$ yield $(157 \mu \mathrm{mol}, 58.3 \mathrm{mg})$.

${ }^{1} \mathrm{H}$ NMR $\left(500 \mathrm{MHz}, \mathrm{CDCl}_{3}\right)$ ठ 7.70-7.72 (m, 2H), 7.36-7.45 (m, 3H), $4.01(\mathrm{~s}, 2 \mathrm{H}), 2.53(\mathrm{~s}, 3 \mathrm{H}), 0.84(\mathrm{~s}, 9 \mathrm{H})$.

${ }^{13} \mathrm{C} \mathrm{NMR}\left(126 \mathrm{MHz}, \mathrm{CDCl}_{3}\right) \delta 160.8,148.9,136.9,132.0,129.2,128.9,128.8,126.3,79.2,31.5,25.8,14.1$.

${ }^{19} \mathrm{~F}$ NMR $\left(471 \mathrm{MHz}, \mathrm{CDCl}_{3}\right) \delta 52.99$ (s, 1F).

HRMS (ESI) exact mass calculated for $\mathrm{C}_{16} \mathrm{H}_{19} \mathrm{O}_{5} \mathrm{~N}_{2} \mathrm{FSNa}: \mathrm{m} / z 393.0891\left([\mathrm{M}+\mathrm{Na}]^{+}\right)$, found: $m / z 393.0893\left([\mathrm{M}+\mathrm{Na}]^{+}\right)$.

\section{neopentyl (3-bromobenzo[b]thiophen-2-yl)(fluorosulfonyl)carbamate (15)}

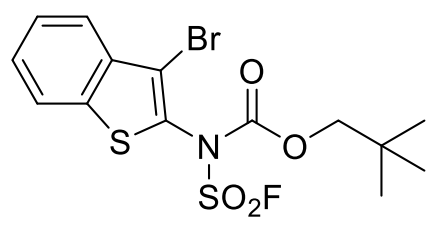

Prepared according to the general procedure with 3-bromobenzothiophene (200 $\mu \mathrm{mol}, 42.6 \mathrm{mg}) .{ }^{7}$ The crude material was purified by column chromatography (DCM/hexane $5-40 \%$ ) to give the title compound as a white solid in $63 \%$ yield $(126 \mu \mathrm{mol}, 53.6 \mathrm{mg})$.

${ }^{1} \mathrm{H}$ NMR (392 MHz, $\left.\mathrm{CDCl}_{3}\right)$ ס 7.84-7.90 (m, 1H), 7.79-7.84 (m, 1H), 7.50-7.54 (m, 2H), $4.03(\mathrm{~s}, 2 \mathrm{H}), 0.88(\mathrm{~s}, 9 \mathrm{H})$. ${ }^{13} \mathrm{C}$ NMR $\left(126 \mathrm{MHz}, \mathrm{CDCl}_{3}\right) \delta 149.1,137.4,135.4,130.2,127.6,125.9,124.5,122.8,113.0,79.1,31.5,26.0$. ${ }^{19} \mathrm{~F}$ NMR $\left(369 \mathrm{MHz}, \mathrm{CDCl}_{3}\right) \delta 54.28(\mathrm{~s}, 1 \mathrm{~F})$.

HRMS (ESI) exact mass calculated for $\mathrm{C}_{14} \mathrm{H}_{15} \mathrm{O}_{4} \mathrm{NFS}{ }_{2} \mathrm{BrNa}: \mathrm{m} / \mathrm{z} 445.9502\left([\mathrm{M}+\mathrm{Na}]^{+}\right)$, found: $\mathrm{m} / \mathrm{z} 445.9502$ $\left([\mathrm{M}+\mathrm{Na}]^{+}\right)$.

neopentyl (2-chlorobenzo[b]thiophen-3-yl)(fluorosulfonyl)carbamate (16)<smiles>CC(C)(C)COC(=O)N(c1c(Cl)sc2ccccc12)[S+](=O)[O-]</smiles>

Prepared according to the general procedure with 2-chlorobenzothiophene (200 $\mu \mathrm{mol}, 33.7 \mathrm{mg}){ }^{8}$ The crude material was purified by column chromatography (DCM/hexane 8-66\%) to give the title compound as a white solid in $71 \%$ yield ( $142 \mu \mathrm{mol}, 54.1 \mathrm{mg})$.

${ }^{1} \mathrm{H}$ NMR $\left(500 \mathrm{MHz}, \mathrm{CDCl}_{3}\right)$ ठ 7.75-7.73 (m, 1H), 7.61-7.59 (m, 1H), 7.47 (ddd, J = 7.8, 7.2, 1.2 Hz, 1H), 7.43 (ddd, 8.1, 7.2, 1.2 Hz, 1H), $4.01(\mathrm{~d}, \mathrm{~J}=10.3 \mathrm{~Hz}, 1 \mathrm{H}), 3.96(\mathrm{~d}, \mathrm{~J}=10.0 \mathrm{~Hz}, 1 \mathrm{H}), 0.81(\mathrm{~s}, 9 \mathrm{H})$

${ }^{13} \mathrm{C}$ NMR $\left(126 \mathrm{MHz}, \mathrm{CDCl}_{3}\right) \delta 149.4,134.8,133.7,126.1,124.1,122.5,120.0,78.7,31.4,25.9$.

${ }^{19} \mathrm{~F}$ NMR $\left(471 \mathrm{MHz}, \mathrm{CDCl}_{3}\right) \delta 54.45(\mathrm{~s}, 1 \mathrm{~F})$.

HRMS (ESI) exact mass calculated for $\mathrm{C}_{14} \mathrm{H}_{15} \mathrm{O}_{4} \mathrm{NFS}{ }_{2} \mathrm{CINa}: \mathrm{m} / \mathrm{z} 402.0007\left([\mathrm{M}+\mathrm{Na}]^{+}\right)$, found: $\mathrm{m} / \mathrm{z} 402.0014$ $\left([\mathrm{M}+\mathrm{Na}]^{+}\right)$. 


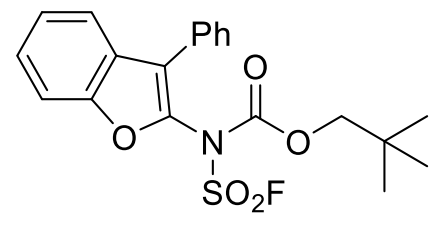

Prepared according to the general procedure with 3-phenylbenzofuran $(200 \mu \mathrm{mol}, 38.8 \mathrm{mg}) .^{9}$ The crude material was purified by column chromatography (1st DCM/hexane $4-34 \%$, 2nd DCM/hexane $5-40 \%$ ) to give the title compound as a colorless oil in $88 \%$ yield $(176 \mu \mathrm{mol}, 71.3 \mathrm{mg})$.

${ }^{1}$ H NMR $\left(500 \mathrm{MHz}, \mathrm{CDCl}_{3}\right) \delta 7.70(\mathrm{~m}, 1 \mathrm{H}), 7.42-7.55(\mathrm{~m}, 7 \mathrm{H}), 7.34(\mathrm{ddd}, J=8.0,7.7,1.1 \mathrm{~Hz}, 1 \mathrm{H}), 3.98(\mathrm{~s}, 2 \mathrm{H})$, $0.82(\mathrm{~s}, 9 \mathrm{H})$.

${ }^{13} \mathrm{C}$ NMR $\left(126 \mathrm{MHz}, \mathrm{CDCl}_{3}\right) \delta 152.6,149.4,136.9,129.1,128.7,128.4,127.0,126.8,123.8,121.3,120.6,111.8$, 79.0, 31.5, 25.8.

${ }^{19} \mathrm{~F}$ NMR $\left(471 \mathrm{MHz}, \mathrm{CDCl}_{3}\right) \delta 52.47(\mathrm{~s}, 1 \mathrm{~F})$.

HRMS (ESI) exact mass calculated for $\mathrm{C}_{20} \mathrm{H}_{20} \mathrm{O}_{5} \mathrm{NFSNa:} \mathrm{m} / \mathrm{z} 428.0938\left([\mathrm{M}+\mathrm{Na}]^{+}\right)$, found: $\mathrm{m} / \mathrm{z} 428.0938$ $\left([\mathrm{M}+\mathrm{Na}]^{+}\right)$.

neopentyl (fluorosulfonyl)(3-methylbenzofuran-2-yl)carbamate (18)

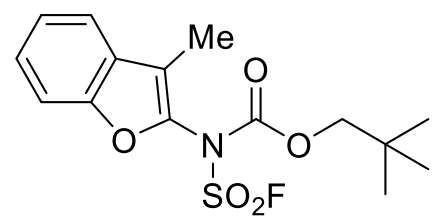

Prepared according to the general procedure with 3-methylbenzofuran (200 $\mu \mathrm{mol}, 27.0 \mathrm{mg}, 98.0 \%)$. The crude material was purified by column chromatography (EtOAc/hexane $5 \%$ ) to give the title compound as a yellowish oil in $66 \%$ yield ( $133 \mu \mathrm{mol}, 45.6 \mathrm{mg}$ ).

${ }^{1} \mathrm{H}$ NMR (392 MHz, $\mathrm{CDCl}_{3}$ ) $\delta 7.56$ (ddd, J = 7.7, 1.3, 0.7 Hz, 1H), 7.46 (ddd, J = 8.3, 1.1, 0.7 Hz, 1H), 7.39 (ddd, 8.5, 7.2, 1.3 Hz, 1H), 7.30 (ddd, J = 7.9, 6.9, $0.8 \mathrm{~Hz}, 1 \mathrm{H}$ ), 4.03 (s, 2H), 2.27 (s, 3H), $0.88(\mathrm{~s}, 9 \mathrm{H}$ ).

${ }^{13} \mathrm{C}$ NMR $\left(99 \mathrm{MHz}, \mathrm{CDCl}_{3}\right) \delta 152.5,149.3,137.6,128.2,126.4,123.2,120.4,115.4,111.6,78.9,31.5,25.9,7.6$. ${ }^{19} \mathrm{~F}$ NMR $\left(369 \mathrm{MHz}, \mathrm{CDCl}_{3}\right) \delta 52.35$ (s, 1F).

HRMS (ESI) exact mass calculated for $\mathrm{C}_{15} \mathrm{H}_{18} \mathrm{O}_{5} \mathrm{NFSNa:} \mathrm{m} / z 366.0782\left([\mathrm{M}+\mathrm{Na}]^{+}\right)$, found: $\mathrm{m} / z 366.0780\left([\mathrm{M}+\mathrm{Na}]^{+}\right)$.

ethyl 3-((fluorosulfonyl)((neopentyloxy)carbonyl)amino)benzofuran-2-carboxylate (19)<smiles>CCOC(=O)c1oc2ccccc2c1N(C(=O)OCC(C)(C)C)S(=O)(=O)[O-]</smiles>

Prepared according to the general procedure with ethyl benzofuran-2-carboxylate $(200 \mu \mathrm{mol}, 38.0 \mathrm{mg}) .{ }^{10}$ The crude material was purified by column chromatography (EtOAc/hexane $5 \%$ ) to give the title compound as a white solid in $27 \%$ yield $(54.3 \mu \mathrm{mol}, 21.8 \mathrm{mg})$.

${ }^{1}$ H NMR $\left(500 \mathrm{MHz}, \mathrm{CDCl}_{3}\right)$ ठ 7.63-7.66 (m, 2H), 7.55 (ddd, J = 8.6, 7.2, 1.2 Hz, 1H), 7.44 (ddd, 7.7, 7.2, 0.9 Hz, $1 \mathrm{H}), 4.44-4.51(\mathrm{~m}, 2 \mathrm{H}), 4.01(\mathrm{~s}, 2 \mathrm{H}), 1.42(\mathrm{t}, J=7.2 \mathrm{~Hz}, 3 \mathrm{H}), 0.87(\mathrm{~s}, 9 \mathrm{H})$.

${ }^{13} \mathrm{C}$ NMR $\left(126 \mathrm{MHz}, \mathrm{CDCl}_{3}\right) \delta 157.7,153.7,149.4,141.6,128.9,125.2,124.2,122.1,119.7,113.1,78.8,62.4$, $31.5,25.9,14.1$. 
${ }^{19} \mathrm{~F}$ NMR $\left(471 \mathrm{MHz}, \mathrm{CDCl}_{3}\right) \delta 52.92(\mathrm{~s}, 1 \mathrm{~F})$.

HRMS (ESI) exact mass calculated for $\mathrm{C}_{17} \mathrm{H}_{20} \mathrm{O}{ }_{7} \mathrm{NFSNa}: \mathrm{m} / \mathrm{z} 424.0837\left([\mathrm{M}+\mathrm{Na}]^{+}\right)$, found: $\mathrm{m} / \mathrm{z} 424.0836\left([\mathrm{M}+\mathrm{Na}]^{+}\right)$.

tert-butyl 3-acetyl-2-((fluorosulfonyl)((neopentyloxy)carbonyl)amino)-1 $H$-indole-1-carboxylate (20)

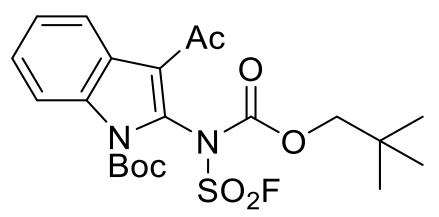

Prepared according to the general procedure with tert-butyl 3-acetyl-1H-indole-1-carboxylate (200 $\mu$ mol, 51.9 $\mathrm{mg}$ ). ${ }^{11}$ The crude material was purified by column chromatography (1st EtOAc/hexane 3-26\%, 2nd DCM/hexane $12-100 \%)$ to give the title compound as a pale yellowish solid in $51 \%$ yield $(101 \mu \mathrm{mol}, 47.7 \mathrm{mg}$ ).

${ }^{1} \mathrm{H}$ NMR $\left(500 \mathrm{MHz}, \mathrm{CDCl}_{3}\right) \delta 8.21(\mathrm{~d}, J=8.6 \mathrm{~Hz}, 1 \mathrm{H}), 8.10(\mathrm{~d}, J=8.0 \mathrm{~Hz}, 1 \mathrm{H}), 7.49$ (ddd, 8.6, 7.5, $\left.1.5 \mathrm{~Hz}, 1 \mathrm{H}\right)$, 7.42 (ddd, 8.0, 7.2, $0.9 \mathrm{~Hz}, 1 \mathrm{H}$ ), 4.06 (d, J=10.0 Hz, 1H), 4.03 (d, J=10.0 Hz, 1H), 2.70 (s, 3H), 1.68 (s, 9H).

${ }^{13} \mathrm{C}$ NMR $\left(126 \mathrm{MHz}, \mathrm{CDCl}_{3}\right) \delta 193.3,149.3,148.1,134.4,128.8,127.0,124.7,124.0,121.8,119.1,115.9,87.2$, 78.8, 31.4, 31.1, 27.9, 26.0.

${ }^{19} \mathrm{~F}$ NMR $\left(471 \mathrm{MHz}, \mathrm{CDCl}_{3}\right) \delta 54.17(\mathrm{~s}, 1 \mathrm{~F})$.

HRMS (ESI) exact mass calculated for $\mathrm{C}_{21} \mathrm{H}_{27} \mathrm{O}_{7} \mathrm{~N}_{2} \mathrm{FSNa}: \mathrm{m} / z$ 493.1415 ([M+Na] $]^{+}$, found: $m / z 493.1419\left([\mathrm{M}+\mathrm{Na}]^{+}\right)$.

1-(tert-butyl) 2-ethyl 3-((fluorosulfonyl)((neopentyloxy)carbonyl)amino)-1H-indole-1,2-dicarboxylate (21)

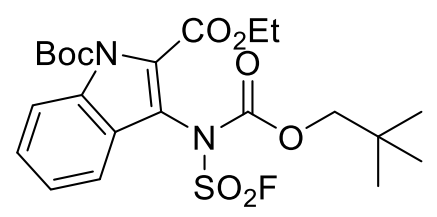

Prepared according to the general procedure with 1-(tert-butyl) 2-ethyl $1 \mathrm{H}$-indole-1,2-dicarboxylate $(200 \mu \mathrm{mol}$, $57.9 \mathrm{mg}) .{ }^{12}$ The crude material was purified by column chromatography (EtOAc/hexane 4-34\%) to give the title compound as a pale yellowish oil in $75 \%$ yield $(151 \mu \mathrm{mol}, 75.5 \mathrm{mg})$.

${ }^{1} \mathrm{H}$ NMR $\left(392 \mathrm{MHz}, \mathrm{CDCl}_{3}\right) \delta 8.14(\mathrm{~d}, J=8.3 \mathrm{~Hz}, 1 \mathrm{H}), 7.55(\mathrm{~d}, J=7.9 \mathrm{~Hz}, 1 \mathrm{H}), 7.47-7.51(\mathrm{~m}, 1 \mathrm{H}), 7.35-7.39(\mathrm{~m}$, $1 \mathrm{H}), 4.34-4.49(\mathrm{~m}, 2 \mathrm{H}), 3.97$ (s, 2H), $1.63(\mathrm{~s}, 9 \mathrm{H}), 1.39$ (t, J= $7.2 \mathrm{~Hz}, 3 \mathrm{H}), 0.83(\mathrm{~s}, 9 \mathrm{H})$.

${ }^{13} \mathrm{C}$ NMR $\left(126 \mathrm{MHz}, \mathrm{CDCl}_{3}\right) \delta 159.4,150.0,148.3,135.3,128.4,127.8,124.4,124.3,119.2,118.3,115.2,85.9$, 78.6, 62.3, 31.4, 27.7, 25.9, 14.0 .

${ }^{19} \mathrm{~F}$ NMR $\left(369 \mathrm{MHz}, \mathrm{CDCl}_{3}\right) \delta 52.65(\mathrm{~s}, 1 \mathrm{~F})$.

HRMS (ESI) exact mass calculated for $\mathrm{C}_{22} \mathrm{H}_{29} \mathrm{O}_{8} \mathrm{~N}_{2} \mathrm{FSNa}: m / z 523.1521\left([\mathrm{M}+\mathrm{Na}]^{+}\right)$, found: $m / z 523.1523\left([\mathrm{M}+\mathrm{Na}]^{+}\right)$.

neopentyl (10-bromoanthracen-9-yl)(fluorosulfonyl)carbamate (22)<smiles>CC(C)(C)COC(=O)N(c1c2ccccc2c(Br)c2ccccc12)[S+](=O)[O-]</smiles> 
Prepared according to the general procedure with 9-bromoanthracene (200 $\mu \mathrm{mol}, 52.5 \mathrm{mg}, 98.0 \%)$. The crude material was purified by column chromatography (DCM/hexane 6-50\%) to give the title compound as a yellow solid in $81 \%$ yield ( $162 \mu \mathrm{mol}, 75.7 \mathrm{mg})$.

${ }^{1} \mathrm{H}$ NMR $\left(392 \mathrm{MHz}, \mathrm{CDCl}_{3}\right)$ ठ 8.63-8.66 (m, 2H), 8.10-8.07 (m, 2H), 7.64-7.72 (m, 4H), $3.94(\mathrm{~s}, 2 \mathrm{H}), 0.65(\mathrm{~s}, 9 \mathrm{H})$. ${ }^{13} \mathrm{C}$ NMR $\left(99 \mathrm{MHz}, \mathrm{CDCl}_{3}\right) \delta 150.7,130.8,130.1,128.9,128.8,127.6,127.1,126.6,121.8,78.5,31.4,25.8$.

${ }^{19} \mathrm{~F}$ NMR $\left(369 \mathrm{MHz}, \mathrm{CDCl}_{3}\right) \delta 53.36(\mathrm{~s}, 1 \mathrm{~F})$.

HRMS (ESI) exact mass calculated for $\mathrm{C}_{20} \mathrm{H}_{19} \mathrm{O}_{4} \mathrm{NFSBrNa:} \mathrm{m} / \mathrm{z} 490.0094\left([\mathrm{M}+\mathrm{Na}]^{+}\right)$, found: $\mathrm{m} / \mathrm{z} 490.0098$ $\left([\mathrm{M}+\mathrm{Na}]^{+}\right)$.

neopentyl (fluorosulfonyl)(pyren-1-yl)carbamate (23)<smiles>CC(C)(C)COC(=O)N(c1ccccc1)c1ccc2ccc3cccc4ccc1c2c34</smiles>

Prepared according to the general procedure with pyrene $(200 \mu \mathrm{mol}, 41.7 \mathrm{mg}, 97.0 \%)$. The crude material was purified by column chromatography (DCM/hexane 6-50\%) to give the title compound as a white solid in $65 \%$ yield (129 $\mu \mathrm{mol}, 53.5 \mathrm{mg})$.

${ }^{1} \mathbf{H}$ NMR $\left(392 \mathrm{MHz}, \mathrm{CDCl}_{3}\right)$ ठ 8.20-8.25 (m, 4H), $8.14(\mathrm{~d}, J=9.2 \mathrm{~Hz}, 2 \mathrm{H}), 8.03-8.08(\mathrm{~m}, 2 \mathrm{H}), 8.00(\mathrm{~d}, J=8.1 \mathrm{~Hz}$, $1 \mathrm{H}), 3.99(\mathrm{~d}, J=10.3 \mathrm{~Hz}, 1 \mathrm{H}), 3.96(\mathrm{~d}, J=10.3 \mathrm{~Hz}, 1 \mathrm{H}), 0.71(\mathrm{~s}, 9 \mathrm{H})$.

${ }^{13} \mathrm{C}$ NMR $\left(99 \mathrm{MHz}, \mathrm{CDCl}_{3}\right) \delta 151.1,132.7,130.9,130.5,130.2,129.2,129.1,128.1,126.9,126.7,126.6,126.39$, $126.38,125.3,125.1,124.1,120.3,78.4,31.4,25.9$.

${ }^{19} \mathrm{~F}$ NMR $\left(369 \mathrm{MHz}, \mathrm{CDCl}_{3}\right) \delta 52.05$ (s, 1F).

HRMS (ESI) exact mass calculated for $\mathrm{C}_{22} \mathrm{H}_{20} \mathrm{O}_{4} \mathrm{NFSNa}: \mathrm{m} / z 436.0989\left([\mathrm{M}+\mathrm{Na}]^{+}\right)$, found: $\mathrm{m} / z 436.0995\left([\mathrm{M}+\mathrm{Na}]^{+}\right)$.

neopentyl (fluorosulfonyl)(naphthalen-1-yl)carbamate (24)<smiles>CC(C)(C)COC(=O)N(c1ccccc1)c1cccc2ccccc12</smiles>

Prepared according to the general procedure with naphthalene ( $200 \mu \mathrm{mol}, 25.9 \mathrm{mg}, 99.0 \%)$. The crude material was purified by column chromatography (DCM/hexane 5-40\%) to give the title compound as a pale yellowish solid in $27 \%$ yield $(53.3 \mu \mathrm{mol}, 18.1 \mathrm{mg})$.

${ }^{1} \mathrm{H}$ NMR $\left(392 \mathrm{MHz}, \mathrm{CDCl}_{3}\right) \delta 8.00(\mathrm{dd}, J=7.2,2.2 \mathrm{~Hz}, 1 \mathrm{H}), 7.93(\mathrm{dd}, J=9.5,9.5 \mathrm{~Hz}, 2 \mathrm{H}), 7.52-7.66(\mathrm{~m}, 4 \mathrm{H}), 3.92$ (s, 2H), $0.71(\mathrm{~s}, 9 \mathrm{H})$.

${ }^{13} \mathrm{C}$ NMR $\left(126 \mathrm{MHz}, \mathrm{CDCl}_{3}\right) \delta 150.7,134.5,131.7,131.1,130.6,128.6,128.2,127.3,127.0,125.3,121.4,78.3$, 31.4, 25.8 .

${ }^{19} \mathrm{~F}$ NMR $\left(369 \mathrm{MHz}, \mathrm{CDCl}_{3}\right) \delta 51.87(\mathrm{~s}, 1 \mathrm{~F})$.

HRMS (ESI) exact mass calculated for $\mathrm{C}_{16} \mathrm{H}_{18} \mathrm{O}{ }_{4} \mathrm{NFSNa}: \mathrm{m} / \mathrm{z} 362.0833\left([\mathrm{M}+\mathrm{Na}]^{+}\right)$, found: $\mathrm{m} / \mathrm{z} 362.0801\left([\mathrm{M}+\mathrm{Na}]^{+}\right)$. 


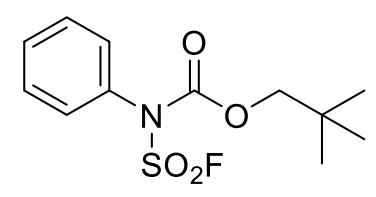

Prepared according to the general procedure with benzene $(200 \mu \mathrm{mol}, 15.6 \mathrm{mg})$. The yield $(6 \%)$ was determined by ${ }^{1} \mathrm{H}$ NMR of the crude mixture using mesitylene as an internal standard. Characterization of the product was performed in the next section.

neopentyl (3,5-dimethylbenzyl)(fluorosulfonyl)carbamate (26)<smiles>Cc1cc(C)cc(CN(C(=O)OCC(C)(C)C)S(=O)(=O)[O-])c1</smiles>

Prepared according to the general procedure with mesitylene (200 $\mu \mathrm{mol}, 24.5 \mathrm{mg}, 98.0 \%)$. The crude material was purified by column chromatography (EtOAc/hexane 3-20\%) to give the title compound as a colorless oil in $61 \%$ yield (122 $\mu \mathrm{mol}, 40.5 \mathrm{mg})$.

${ }^{1}$ H NMR (396 MHz, CDCl $) \delta 6.97(\mathrm{~s}, 2 \mathrm{H}), 6.96(\mathrm{~s}, 1 \mathrm{H}), 4.95\left(\mathrm{~d}, J_{\mathrm{H}-\mathrm{F}}=2.0 \mathrm{~Hz}, 2 \mathrm{H}\right), 4.00(\mathrm{~s}, 2 \mathrm{H}), 2.31$ (d, $J=0.5$ $\mathrm{Hz}, 6 \mathrm{H}), 0.96$ (s, 9H).

${ }^{13} \mathrm{C}$ NMR $\left(126 \mathrm{MHz}, \mathrm{CDCl}_{3}\right) \delta 151.0,138.4,134.8,130.1,125.7,78.5,53.0,31.5,26.1,21.2$.

${ }^{19} \mathrm{~F}$ NMR $\left(471 \mathrm{MHz}, \mathrm{CDCl}_{3}\right) \delta 54.80(\mathrm{~s}, 1 \mathrm{~F})$.

HRMS (ESI) exact mass calculated for $\mathrm{C}_{15} \mathrm{H}_{22} \mathrm{O}{ }_{4} \mathrm{NFSNa}: \mathrm{m} / \mathrm{z} 354.1146\left([\mathrm{M}+\mathrm{Na}]^{+}\right)$, found: $m / z 354.1132\left([\mathrm{M}+\mathrm{Na}]^{+}\right)$.

Table S1. Additional substrate scope of Cu-catalyzed imidation of heteroarenes and PAHs

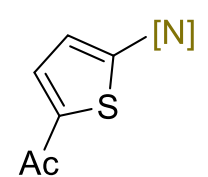

$55 \%(S 1)$

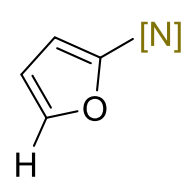

$27 \%(S 2)$

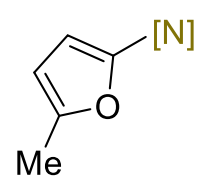

$38 \%$ (S3)

$[\mathrm{N}]: \mathrm{ArCH}_{2}[\mathrm{~N}]=84: 16$

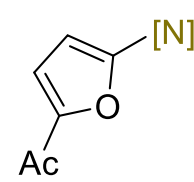

$38 \%$ (S4)

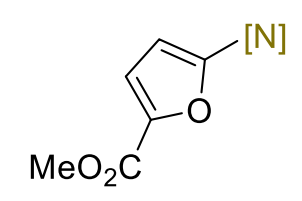

$62 \%$ (S5)<smiles>N#Cc1ccc(Br)o1</smiles>

$57 \%(\mathbf{S 6})$<smiles>N#Cc1ccc(C#N)n1C(=O)OCc1ccccc1</smiles>

$67 \%(\mathrm{~S} 7)$<smiles>N#Cc1oc2ccccc2c1Br</smiles>

$52 \%(\mathbf{S 1 0})$<smiles>COC(=O)c1cc(Br)c(C#N)n1C(=O)OCc1ccccc1</smiles>

$69 \%$ (S8)<smiles>CC(C)(C)n1c(C#N)c(Cl)c2ccccc21</smiles>

$84 \%$ (S11)<smiles>N#Cc1nc(-c2ccccc2)oc1-c1ccccc1</smiles>

$81 \%$ (S9)<smiles>N#[N+]c1ccc2c3cccc4cccc(c5cccc1c52)c43</smiles>

$57 \%$ (S12) 


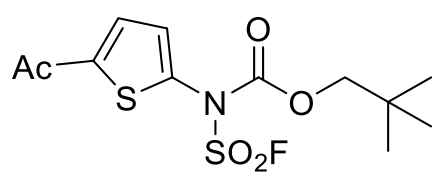

Prepared according to the general procedure with 1-(thiophen-2-yl)ethan-1-one (200 $\mu \mathrm{mol}, 25.7 \mathrm{mg}, 98.0 \%)$. The crude material was purified by column chromatography (EtOAc/hexane 4-34\%) to give the title compound as a yellowish oil in $55 \%$ yield $(110 \mu \mathrm{mol}, 37.0 \mathrm{mg})$.

${ }^{1} \mathrm{H}$ NMR $\left(392 \mathrm{MHz}, \mathrm{CDCl}_{3}\right) \delta 7.58(\mathrm{~d}, J=4.0 \mathrm{~Hz}, 1 \mathrm{H}), 7.16(\mathrm{~d}, J=4.0 \mathrm{~Hz}, 1 \mathrm{H}), 4.01(\mathrm{~s}, 2 \mathrm{H}), 2.57(\mathrm{~s}, 3 \mathrm{H}), 0.91(\mathrm{~s}$, $9 \mathrm{H})$.

${ }^{13} \mathrm{C}$ NMR $\left(99 \mathrm{MHz}, \mathrm{CDCl}_{3}\right) \delta 190.2,149.5,145.8,141.2,130.4,130.2,79.2,31.5,26.4,26.0$.

${ }^{19} \mathrm{~F}$ NMR $\left(369 \mathrm{MHz}, \mathrm{CDCl}_{3}\right) \delta 50.95(\mathrm{~s}, 1 \mathrm{~F})$.

HRMS (ESI) exact mass calculated for $\mathrm{C}_{12} \mathrm{H}_{16} \mathrm{O}_{5} \mathrm{NFS}{ }_{2} \mathrm{Na}: \mathrm{m} / z 360.0346\left([\mathrm{M}+\mathrm{Na}]^{+}\right)$, found: $\mathrm{m} / z 360.0331\left([\mathrm{M}+\mathrm{Na}]^{+}\right)$.

neopentyl (fluorosulfonyl)(furan-2-yl)carbamate (S2)

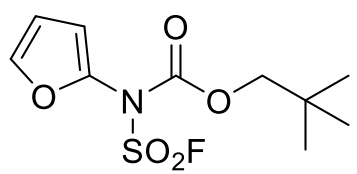

Prepared according to the general procedure with furan $(200 \mu \mathrm{mol}, 13.8 \mathrm{mg}, 99.0 \%)$. The crude material was purified by column chromatography (EtOAc/hexane 4-34\%) to give the title compound as a pale yellowish oil in $27 \%$ yield $(54.1 \mu \mathrm{mol}, 15.1 \mathrm{mg})$.

${ }^{1} \mathrm{H}$ NMR $\left(500 \mathrm{MHz}, \mathrm{CDCl}_{3}\right) \delta 7.42(\mathrm{dd}, J=2.0,0.9 \mathrm{~Hz}, 1 \mathrm{H}), 6.49-6.52(\mathrm{~m}, 2 \mathrm{H}), 3.99(\mathrm{~s}, 2 \mathrm{H}), 0.90(\mathrm{~s}, 9 \mathrm{H})$.

${ }^{13} \mathrm{C} \mathrm{NMR}\left(126 \mathrm{MHz}, \mathrm{CDCl}_{3}\right) \delta 149.5,142.6,138.9,111.9,109.8,78.8,31.5,25.9$.

${ }^{19} \mathrm{~F}$ NMR $\left(471 \mathrm{MHz}, \mathrm{CDCl}_{3}\right) \delta 50.81$ (s, 1F).

HRMS (ESI) exact mass calculated for $\mathrm{C}_{10} \mathrm{H}_{14} \mathrm{O}_{5} \mathrm{NFSNa}: \mathrm{m} / z 302.0469\left([\mathrm{M}+\mathrm{Na}]^{+}\right)$, found: $\mathrm{m} / z 302.0469\left([\mathrm{M}+\mathrm{Na}]^{+}\right)$.

neopentyl (fluorosulfonyl)(5-methylfuran-2-yl)carbamate (S3) and neopentyl (fluorosulfonyl)(furan-2-ylmethyl)carbamate (S3')<smiles>Cc1ccc(N(C(=O)OCC(C)(C)C)S(=O)(=O)[O-])o1</smiles>

S3<smiles>CC(C)(C)COC(=O)N(Cc1ccco1)S(=O)(=O)OF</smiles>

S3"

Prepared according to the general procedure with 2-methylfuran ( $200 \mu \mathrm{mol}, 16.4 \mathrm{mg})$. The crude material was purified by column chromatography (DCM/hexane 6-50\%) to give the title compound as a pale yellowish oil in $38 \%$ yield $\left(75.7 \mu \mathrm{mol}, 22.2 \mathrm{mg}, \mathbf{S 3}^{\prime} \mathbf{S 3}^{\prime}=84: 16\right)$.

${ }^{1} \mathrm{H}$ NMR $\left(500 \mathrm{MHz}, \mathrm{CDCl}_{3}\right) \delta 6.38(\mathrm{~d}, J=3.2 \mathrm{~Hz}, 1 \mathrm{H}), 6.08-6.09(\mathrm{~m}, 1 \mathrm{H}), 3.98(\mathrm{~s}, 2 \mathrm{H}), 2.31(\mathrm{~s}, 3 \mathrm{H}), 0.91(\mathrm{~s}, 9 \mathrm{H})$. $\delta 7.41$ (dd, J = 2.0, $0.9 \mathrm{~Hz}, 0.2 \mathrm{H}$ ), $6.42(\mathrm{dd}, \mathrm{J}=3.2,0.6 \mathrm{~Hz}, 0.2 \mathrm{H}), 6.38(\mathrm{~d}, \mathrm{~J}=3.2 \mathrm{~Hz}, 0.8 \mathrm{H}), 6.35$ (dd, J = 3.2, 1.7 $\mathrm{Hz}, 0.2 \mathrm{H}), 6.09-6.08(\mathrm{~m}, 1 \mathrm{H}), 5.02\left(\mathrm{~d}, J_{\mathrm{H}-\mathrm{F}}=1.7 \mathrm{~Hz}, 0.2 \mathrm{H}\right), 4.02(\mathrm{~s}, 0.3 \mathrm{H}), 3.98(\mathrm{~s}, 1.7 \mathrm{H}), 2.31(\mathrm{~s}, 2.5 \mathrm{H}), 0.98(\mathrm{~s}$, $1 \mathrm{H}), 0.91(\mathrm{~s}, 7.6 \mathrm{H})$

${ }^{13} \mathrm{C}$ NMR $\left(126 \mathrm{MHz}, \mathrm{CDCl}_{3}\right.$, S3) $\delta 152.7,149.7,136.7,110.7,107.9,78.7,31.5,25.9,13.7$.

${ }^{19} \mathrm{~F}$ NMR $\left(471 \mathrm{MHz}, \mathrm{CDCl}_{3}\right) \delta 54.41$ (s, $\left.0.2 \mathrm{~F}\right), 50.48$ (s, 0.8F).

HRMS (ESI) exact mass calculated for $\mathrm{C}_{11} \mathrm{H}_{16} \mathrm{O}_{5} \mathrm{NFSNa}: \mathrm{m} / z 316.0625\left([\mathrm{M}+\mathrm{Na}]^{+}\right)$, found: $\mathrm{m} / z 316.0624\left([\mathrm{M}+\mathrm{Na}]^{+}\right)$. 


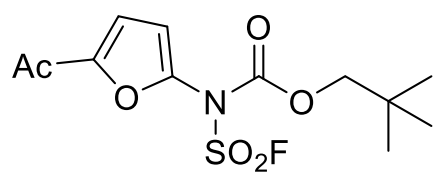

Prepared according to the general procedure with 1-(furan-2-yl)ethan-1-one (200 $\mu \mathrm{mol}, 22.2 \mathrm{mg}, 99.0 \%)$. The crude material was purified by column chromatography (EtOAc/hexane $8-66 \%$ ) to give the title compound as a yellowish oil in $38 \%$ yield $(76.9 \mu \mathrm{mol}, 24.7 \mathrm{mg})$.

${ }^{1} \mathrm{H}$ NMR $\left(500 \mathrm{MHz}, \mathrm{CDCl}_{3}\right) \delta 7.22(\mathrm{~d}, J=3.7 \mathrm{~Hz}, 1 \mathrm{H}), 6.66(\mathrm{~d}, J=3.7 \mathrm{~Hz}, 1 \mathrm{H}), 4.02(\mathrm{~s}, 2 \mathrm{H}), 2.50(\mathrm{~s}, 3 \mathrm{H}), 0.92(\mathrm{~s}$, $9 \mathrm{H})$.

${ }^{13} \mathrm{C}$ NMR $\left(126 \mathrm{MHz}, \mathrm{CDCl}_{3}\right) \delta 186.4,151.4,148.8,141.2,117.5,112.1,79.3,31.5,26.0,25.9$.

${ }^{19} \mathrm{~F}$ NMR $\left(471 \mathrm{MHz}, \mathrm{CDCl}_{3}\right) \delta 52.13(\mathrm{~s}, 1 \mathrm{~F})$.

HRMS (ESI) exact mass calculated for $\mathrm{C}_{12} \mathrm{H}_{16} \mathrm{O}_{6} \mathrm{NFSNa}: \mathrm{m} / z 344.0575\left([\mathrm{M}+\mathrm{Na}]^{+}\right)$, found: $\mathrm{m} / \mathrm{z} 344.0574\left([\mathrm{M}+\mathrm{Na}]^{+}\right)$.

methyl 5-((fluorosulfonyl)((neopentyloxy)carbonyl)amino)furan-2-carboxylate (S5)

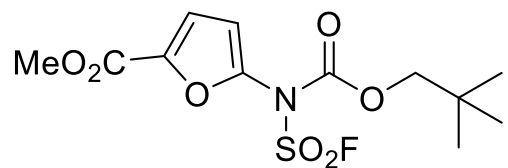

Prepared according to the general procedure with methyl furan-2-carboxylate ( $200 \mu \mathrm{mol}, 25.5 \mathrm{mg}, 99.0 \%)$. The crude material was purified by column chromatography (DCM/hexane $8-66 \%$ ) to give the title compound as a yellowish oil in $62 \%$ yield $(124 \mu \mathrm{mol}, 41.9 \mathrm{mg})$.

${ }^{1} \mathrm{H}$ NMR $\left(500 \mathrm{MHz}, \mathrm{CDCl}_{3}\right) \delta 7.23(\mathrm{~d}, J=3.4 \mathrm{~Hz}, 1 \mathrm{H}), 6.64(\mathrm{~d}, J=3.7 \mathrm{~Hz}, 1 \mathrm{H}), 4.01(\mathrm{~s}, 2 \mathrm{H}), 3.92(\mathrm{~s}, 3 \mathrm{H}), 0.91$ (s, $9 \mathrm{H})$.

${ }^{13} \mathrm{C}$ NMR $\left(126 \mathrm{MHz}, \mathrm{CDCl}_{3}\right) \delta 158.1,148.8,143.8,141.3,118.9,111.8,79.2,52.4,31.5,25.9$.

${ }^{19} \mathrm{~F}$ NMR $\left(471 \mathrm{MHz}, \mathrm{CDCl}_{3}\right) \delta 52.10(\mathrm{~s}, 1 \mathrm{~F})$.

HRMS (ESI) exact mass calculated for $\mathrm{C}_{12} \mathrm{H}_{16} \mathrm{O}{ }_{7} \mathrm{NFSNa}: \mathrm{m} / z 360.0524\left([\mathrm{M}+\mathrm{Na}]^{+}\right)$, found: $\mathrm{m} / z 360.0524\left([\mathrm{M}+\mathrm{Na}]^{+}\right)$.

neopentyl (5-bromofuran-2-yl)(fluorosulfonyl)carbamate (S6)<smiles>CC(C)(C)COC(=O)N(c1ccc(F)o1)c1ccc(Br)o1</smiles>

Prepared according to the general procedure with 2-bromofuran ( $200 \mu \mathrm{mol}, 29.4 \mathrm{mg})$. The crude material was purified by column chromatography (1st DCM/hexane 5-40\%, 2nd toluene/hexane 4-34\%, 3rd acetone/hexane $3 \%)$ to give the title compound as a colorless oil in $57 \%$ yield $(113 \mu \mathrm{mol}, 40.6 \mathrm{mg})$.

${ }^{1}$ H NMR (392 MHz, $\left.\mathrm{CDCl}_{3}\right) \delta 6.49(\mathrm{~d}, J=3.4 \mathrm{~Hz}, 1 \mathrm{H}), 6.45(\mathrm{~d}, J=3.3 \mathrm{~Hz}, 1 \mathrm{H}), 4.00(\mathrm{~s}, 2 \mathrm{H}), 0.92(\mathrm{~s}, 9 \mathrm{H})$.

${ }^{13} \mathrm{C}$ NMR $\left(99 \mathrm{MHz}, \mathrm{CDCl}_{3}\right) \delta 149.1,139.6,122.8,113.8,112.8,79.1,31.5,25.9$.

${ }^{19} \mathrm{~F}$ NMR $\left(369 \mathrm{MHz}, \mathrm{CDCl}_{3}\right) \delta 51.20$ (s, 1F).

HRMS (ESI) exact mass calculated for $\mathrm{C}_{10} \mathrm{H}_{13} \mathrm{O}_{5} \mathrm{NFSBrNa:} \mathrm{m} / z 379.9574\left([\mathrm{M}+\mathrm{Na}]^{+}\right)$, found: $\mathrm{m} / z 379.9574$ $\left([\mathrm{M}+\mathrm{Na}]^{+}\right)$.

tert-butyl 2-acetyl-5-((fluorosulfonyl)((neopentyloxy)carbonyl)amino)-1H-pyrrole-1-carboxylate (S7)<smiles></smiles> 
Prepared according to the general procedure with tert-butyl 2-acetyl-1 $H$-pyrrole-1-carboxylate $(200 \mu$ mol, 41.8 $\mathrm{mg}) .{ }^{13}$ The crude material was purified by column chromatography (1st EtOAc/hexane 2-20\%, 2nd DCM/hexane $12-100 \%)$ to give the title compound as a pale yellowish solid in $67 \%$ yield ( $134 \mu \mathrm{mol}, 56.5 \mathrm{mg}$ ).

${ }^{1} \mathrm{H}$ NMR $\left(500 \mathrm{MHz}, \mathrm{CDCl}_{3}\right) \delta 6.84(\mathrm{~d}, J=4.0 \mathrm{~Hz}, 1 \mathrm{H}), 6.37(\mathrm{~d}, J=4.0 \mathrm{~Hz}, 1 \mathrm{H}), 4.02(\mathrm{~d}, J=10.3 \mathrm{~Hz}, 1 \mathrm{H}), 3.94(\mathrm{~d}$, $J=10.0 \mathrm{~Hz}, 1 \mathrm{H}), 2.46(\mathrm{~s}, 3 \mathrm{H}), 1.56(\mathrm{~s}, 9 \mathrm{H}), 0.89(\mathrm{~s}, 9 \mathrm{H})$.

${ }^{13} \mathrm{C}$ NMR $\left(126 \mathrm{MHz}, \mathrm{CDCl}_{3}\right) \delta 187.6,149.5,147.2,133.7,126.6,117.2,111.4,86.7,78.8,31.4,27.1,26.8,25.9$. ${ }^{19} \mathrm{~F}$ NMR $\left(471 \mathrm{MHz}, \mathrm{CDCl}_{3}\right) \delta 51.87(\mathrm{~s}, 1 \mathrm{~F})$.

HRMS (ESI) exact mass calculated for $\mathrm{C}_{17} \mathrm{H}_{25} \mathrm{O}_{7} \mathrm{~N}_{2} \mathrm{FSNa}: \mathrm{m} / z 443.1259\left([\mathrm{M}+\mathrm{Na}]^{+}\right)$, found: $\mathrm{m} / z 443.1252\left([\mathrm{M}+\mathrm{Na}]^{+}\right)$.

1-(tert-butyl) 2-methyl 4-bromo-5-((fluorosulfonyl)((neopentyloxy)carbonyl)amino)-1H-pyrrole-1,2dicarboxylate (S8)

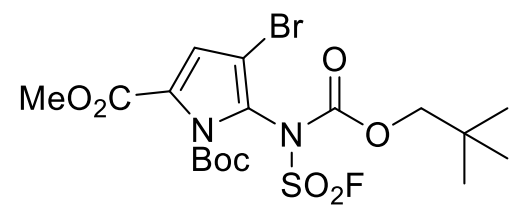

Prepared according to the general procedure with 1-(tert-butyl) 2-methyl 4-bromo-1H-pyrrole-1,2-dicarboxylate $(200 \mu \mathrm{mol}, 60.8 \mathrm{mg}){ }^{5}$ The crude material was purified by column chromatography (1st EtOAc/hexane 2-16\%, 2nd EtOAc/hexane $5 \%$ ) to give the title compound as a pale yellowish oil in $69 \%$ yield (138 $\mu \mathrm{mol}, 71.0 \mathrm{mg}$ ).

${ }^{1} \mathrm{H}$ NMR $\left(500 \mathrm{MHz}, \mathrm{CDCl}_{3}\right) \delta 6.88(\mathrm{~s}, 1 \mathrm{H}), 4.05(\mathrm{~d}, J=10.0 \mathrm{~Hz}, 1 \mathrm{H}), 3.94(\mathrm{~d}, J=10.3 \mathrm{~Hz}, 1 \mathrm{H}), 3.87(\mathrm{~s}, 3 \mathrm{H}), 1.56$ (s, 9H), $0.90(\mathrm{~s}, 9 \mathrm{H})$.

${ }^{13} \mathrm{C}$ NMR $\left(126 \mathrm{MHz}, \mathrm{CDCl}_{3}\right) \delta 159.4,148.9,146.0,125.6,123.9,118.8,102.0,87.6,78.9,52.4,31.5,27.2,25.9$.

${ }^{19} \mathrm{~F}$ NMR $\left(471 \mathrm{MHz}, \mathrm{CDCl}_{3}\right) \delta 54.78(\mathrm{~s}, 1 \mathrm{~F})$.

HRMS (ESI) exact mass calculated for $\mathrm{C}_{17} \mathrm{H}_{24} \mathrm{O}_{8} \mathrm{~N}_{2} \mathrm{FSBrNa}: \mathrm{m} / z 537.0313\left([\mathrm{M}+\mathrm{Na}]^{+}\right)$, found: $\mathrm{m} / z 537.0313$ $\left([\mathrm{M}+\mathrm{Na}]^{+}\right)$.

neopentyl (2,5-diphenyloxazol-4-yl)(fluorosulfonyl)carbamate (S9)

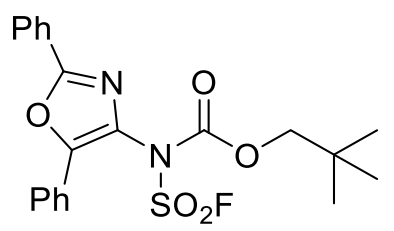

Prepared according to the general procedure with 2,5-diphenyloxazole $(200 \mu \mathrm{mol}, 44.3 \mathrm{mg}){ }^{6}$ The crude material was purified by column chromatography (DCM/hexane $8-66 \%$ ) to give the title compound as a yellowish oil in $81 \%$ yield (161 $\mu \mathrm{mol}, 69.8 \mathrm{mg})$.

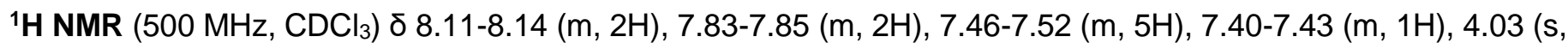
$2 \mathrm{H}), 0.82(\mathrm{~s}, 9 \mathrm{H})$.

${ }^{13} \mathrm{C}$ NMR $\left(126 \mathrm{MHz}, \mathrm{CDCl}_{3}\right) \delta 160.3,148.9,138.1,132.1,131.4,129.4,129.0,128.9,128.8,126.82,126.75,126.7$, 126.5, 126.3, 79.3, 31.5, 25.8.

${ }^{19} \mathrm{~F}$ NMR $\left(471 \mathrm{MHz}, \mathrm{CDCl}_{3}\right) \delta 53.18(\mathrm{~s}, 1 \mathrm{~F})$.

HRMS (ESI) exact mass calculated for $\mathrm{C}_{21} \mathrm{H}_{21} \mathrm{O}_{5} \mathrm{~N}_{2} \mathrm{FSNa}: \mathrm{m} / z$ 455.1047 ([M+Na] $\left.]^{+}\right)$, found: $\mathrm{m} / z 455.1018\left([\mathrm{M}+\mathrm{Na}]^{+}\right)$. 


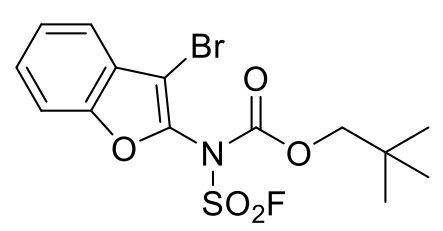

Prepared according to the general procedure with 3-bromobenzofuran $(200 \mu \mathrm{mol}, 39.4 \mathrm{mg}) \cdot{ }^{14}$ The crude material was purified by column chromatography (DCM/hexane 4-34\%) to give the title compound as a yellowish oil in $52 \%$ yield (103 $\mu \mathrm{mol}, 42.1 \mathrm{mg})$.

${ }^{1} \mathrm{H}$ NMR $\left(396 \mathrm{MHz}, \mathrm{CDCl}_{3}\right) \delta 7.60(\mathrm{ddd}, J=7.9,1.3,0.7 \mathrm{~Hz}, 1 \mathrm{H}), 7.46-7.53(\mathrm{~m}, 2 \mathrm{H}), 7.37-7.41(\mathrm{~m}, 1 \mathrm{H}), 4.05(\mathrm{~s}$, $2 \mathrm{H}), 0.89(\mathrm{~s}, 9 \mathrm{H})$.

${ }^{13} \mathrm{C}$ NMR $\left(126 \mathrm{MHz}, \mathrm{CDCl}_{3}\right) \delta 152.2,148.5,139.0,127.7,126.9,124.3,120.8,112.1,99.2,79.2,31.6,25.9$.

${ }^{19} \mathrm{~F}$ NMR $\left(471 \mathrm{MHz}, \mathrm{CDCl}_{3}\right) \delta 53.86(\mathrm{~s}, 1 \mathrm{~F})$.

HRMS (ESI) exact mass calculated for $\mathrm{C}_{14} \mathrm{H}_{15} \mathrm{O}_{5} \mathrm{NFSBrNa:} \mathrm{m} / z$ $429.9731\left([\mathrm{M}+\mathrm{Na}]^{+}\right)$, found: $\mathrm{m} / \mathrm{z} 429.9725$ $\left([\mathrm{M}+\mathrm{Na}]^{+}\right)$.

tert-butyl 3-chloro-2-((fluorosulfonyl)((neopentyloxy)carbonyl)amino)-1H-indole-1-carboxylate (S11)

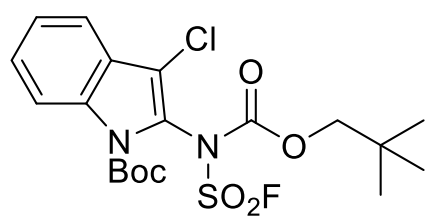

Prepared according to the general procedure with tert-butyl 3-chloro-1H-indole-1-carboxylate (200 $\mu$ mol, 50.3 $\mathrm{mg}) .{ }^{15}$ The crude material was purified by column chromatography (DCM/hexane $8-66 \%$ ) to give the title compound as a colorless solid in $84 \%$ yield $(169 \mu \mathrm{mol}, 78.1 \mathrm{mg})$.

${ }^{1} \mathrm{H}$ NMR $\left(500 \mathrm{MHz}, \mathrm{CDCl}_{3}\right) \delta 8.23(\mathrm{~d}, J=8.6 \mathrm{~Hz}, 1 \mathrm{H}), 7.65(\mathrm{~d}, J=8.0 \mathrm{~Hz}, 1 \mathrm{H}), 7.48(\mathrm{ddd}, J=8.5,7.5,1.5 \mathrm{~Hz}, 1 \mathrm{H})$, 7.37 (ddd, J = 8.0, 7.2, $0.6 \mathrm{~Hz}, 1 \mathrm{H}$ ), $4.04(\mathrm{~d}, \mathrm{~J}=10.0 \mathrm{~Hz}, 1 \mathrm{H}$ ), $3.98(\mathrm{~d}, \mathrm{~J}=10.0 \mathrm{~Hz}, 1 \mathrm{H}), 1.65(\mathrm{~s}, 9 \mathrm{H}), 0.88(\mathrm{~s}, 9 \mathrm{H})$. ${ }^{13} \mathrm{C}$ NMR $\left(126 \mathrm{MHz}, \mathrm{CDCl}_{3}\right) \delta 149.2,148.2,133.8,127.7,124.3,124.0,122.8,119.3,116.1,115.1,86.3,78.7$, 31.5, 28.0, 25.9.

${ }^{19} \mathrm{~F}$ NMR $\left(471 \mathrm{MHz}, \mathrm{CDCl}_{3}\right) \delta 54.95(\mathrm{~s}, 1 \mathrm{~F})$.

HRMS (ESI) exact mass calculated for $\mathrm{C}_{19} \mathrm{H}_{24} \mathrm{O}_{6} \mathrm{~N}_{2} \mathrm{FSCINa}: \mathrm{m} / z 485.0920\left([\mathrm{M}+\mathrm{Na}]^{+}\right)$, found: $\mathrm{m} / z 485.0922$ $\left([\mathrm{M}+\mathrm{Na}]^{+}\right)$.

\section{neopentyl (fluorosulfonyl)(perylen-3-yl)carbamate (S12)}<smiles></smiles>

Prepared according to the general procedure with perylene (200 $\mu \mathrm{mol}, 51.5 \mathrm{mg}, 98.0 \%)$. The crude material was purified by column chromatography (DCM/hexane 6-50\%) to give the title compound as a yellowish solid in $57 \%$ yield (113 $\mu \mathrm{mol}, 52.4 \mathrm{mg})$.

${ }^{1} \mathrm{H}$ NMR $\left(392 \mathrm{MHz}, \mathrm{CDCl}_{3}\right) \delta 8.22(\mathrm{~d}, J=7.0 \mathrm{~Hz}, 1 \mathrm{H}), 8.19-8.16(\mathrm{~m}, 3 \mathrm{H}), 7.72-7.68(\mathrm{~m}, 3 \mathrm{H}), 7.61(\mathrm{dd}, J=8.0,8.0$ $\mathrm{Hz}, 1 \mathrm{H}), 7.53(\mathrm{~d}, J=7.9 \mathrm{~Hz}, 1 \mathrm{H}), 7.47(\mathrm{dd}, J=7.9,7.9 \mathrm{~Hz}, 2 \mathrm{H}), 3.96(\mathrm{~s}, 2 \mathrm{H}), 0.75(\mathrm{~s}, 9 \mathrm{H})$. 
${ }^{13} \mathrm{C}$ NMR $\left(99 \mathrm{MHz}, \mathrm{CDCl}_{3}\right) \delta 150.7,134.4,134.2,132.1,132.0,130.6,130.3,129.84,129.76,129.2,128.6,128.5$, 128.2, 127.9, 126.7, 126.6, 121.6, 121.2, 120.94, 120.86, 119.5, 78.4, 31.4, 25.9.

${ }^{19} \mathrm{~F}$ NMR $\left(369 \mathrm{MHz}, \mathrm{CDCl}_{3}\right) \delta 52.26$ (s, 1F).

HRMS (ESI) exact mass calculated for $\mathrm{C}_{26} \mathrm{H}_{22} \mathrm{O}_{4} \mathrm{NFSNa:} \mathrm{m} / z 486.1146\left([\mathrm{M}+\mathrm{Na}]^{+}\right)$, found: $m / z 486.1150\left([\mathrm{M}+\mathrm{Na}]^{+}\right)$. 


\section{Computed Energy Profile for the Imidyl Radical Addition to Benzene (Figure 2b)}

All calculations were performed with Gaussian 16, Revision A.03. All geometries and harmonic vibrational frequencies were calculated at the UB3LYP-D3/6-311G+(d,p)/IEFPCM(dichloroethane) level of theory. Stationary points, minima, and transition states on the potential energy surface were identified by vibrational analysis. It was confirmed that the transition state had only one imaginary frequency. Energy diagrams, wherein all Gibbs free energies at $298.15 \mathrm{~K}$ and 1 bar were estimated using Gaussian16, are illustrated.

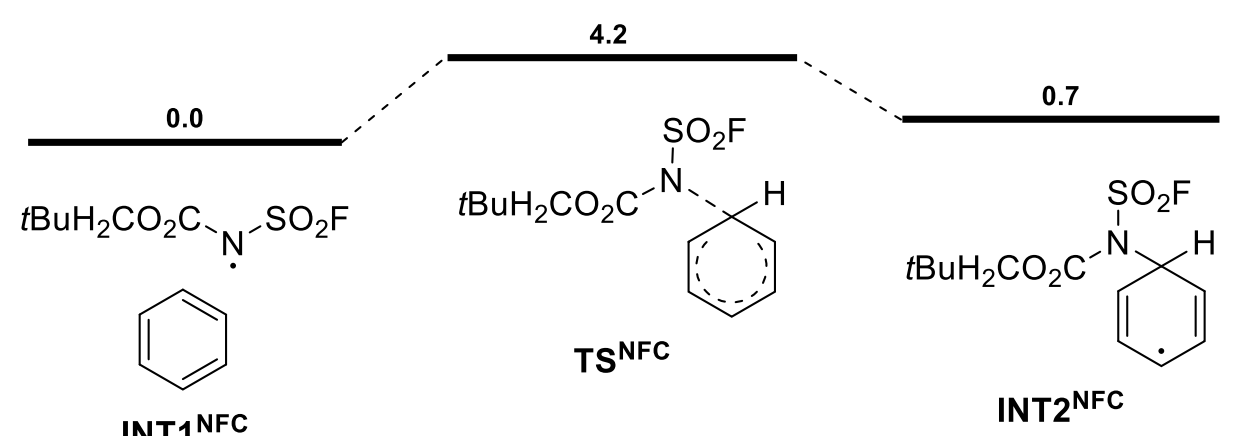

Figure S1. Addition of the imidyl radical generated from NFC to benzene
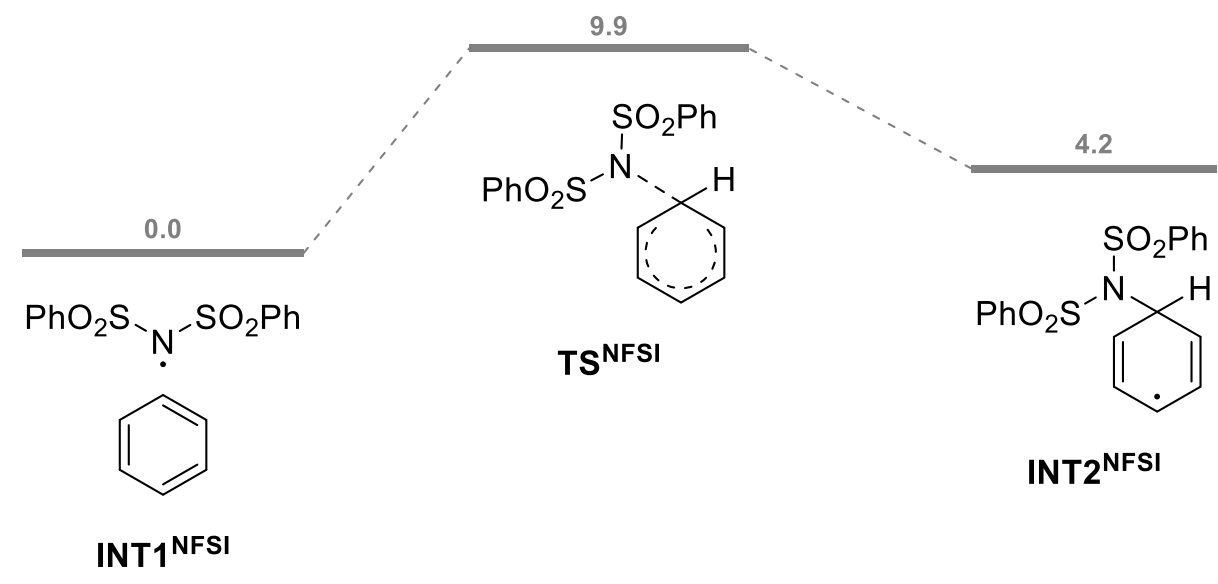

INT2 ${ }^{\text {NFSI }}$

Figure S2. Addition of the imidyl radical generated from NFSI to benzene

Table S2. Calculated Gibbs free energies

\begin{tabular}{|c|c|c|c|c|c|}
\hline & $\mathrm{G}_{\text {corr }}$ (Hartree) & EE (Hartree) & $\mathrm{EE}+\mathrm{G}_{\text {corr }}($ Hartree $)$ & $\Delta G(\mathrm{kcal} / \mathrm{mol})$ & imaginary frequency $\left(\mathrm{cm}^{-1}\right)$ \\
\hline benzene & 0.072658 & -232.318833 & -232.246175 & 0.0 & \\
\hline INT1 NFC & 0.134507 & -1089.081066 & -1088.946559 & 0.0 & \\
\hline $\mathrm{TS}^{\mathrm{NFC}}$ & 0.230768 & -1321.416877 & -1321.186109 & 4.2 & -268.74 \\
\hline INT2 ${ }^{\text {NFC }}$ & 0.231584 & -1321.423132 & -1321.191548 & 0.7 & \\
\hline INT1 $1^{\text {NFSI }}$ & 0.153274 & -1615.433772 & -1615.280498 & 0.0 & \\
\hline $\mathrm{TS}^{\mathrm{NFSI}}$ & 0.252022 & -1847.762994 & -1847.510973 & 9.9 & -264.44 \\
\hline INT2 ${ }^{\text {NFSI }}$ & 0.252068 & -1847.772008 & -1847.519940 & 4.2 & \\
\hline
\end{tabular}


benzene

\begin{tabular}{|l|l|l|l|}
\hline C & -0.49492 & -1.30493 & -0.000001 \\
\hline C & -1.37758 & -0.2238 & 0.000029 \\
\hline C & -0.88265 & 1.081062 & -0.000022 \\
\hline C & 0.494972 & 1.30491 & 0.000004 \\
\hline C & 1.377572 & 0.223851 & 0.00002 \\
\hline C & 0.882605 & -1.0811 & -0.000024 \\
\hline H & -0.87951 & -2.31871 & -0.000003 \\
\hline H & -2.44782 & -0.39772 & 0.00002 \\
\hline$H$ & -1.5683 & 1.921019 & -0.000024 \\
\hline$H$ & 0.879434 & 2.318739 & -0.000006 \\
\hline$H$ & 2.447834 & 0.39764 & 0.000017 \\
\hline$H$ & 1.568366 & -1.92097 & -0.000036 \\
\hline
\end{tabular}

\section{INT1 $^{\text {NFC }}$}

\begin{tabular}{|c|c|c|c|}
\hline$S$ & 2.403307 & -0.40664 & -0.1301 \\
\hline $\mathrm{O}$ & 2.594452 & 0.056843 & -1.48056 \\
\hline $\mathrm{O}$ & 1.915651 & -1.7273 & 0.183679 \\
\hline$F$ & 3.834145 & -0.27329 & 0.598356 \\
\hline C & 0.397441 & 1.226378 & 0.272714 \\
\hline $\mathrm{O}$ & 0.447393 & 2.335751 & -0.20141 \\
\hline $\mathrm{O}$ & -0.62191 & 0.427221 & 0.413543 \\
\hline C & -1.93816 & 0.895868 & -0.06768 \\
\hline $\mathrm{H}$ & -2.25314 & 1.702832 & 0.596332 \\
\hline $\mathrm{H}$ & -1.80088 & 1.283519 & -1.07792 \\
\hline $\mathrm{N}$ & 1.598692 & 0.700967 & 0.826852 \\
\hline C & -2.90312 & -0.28745 & -0.03595 \\
\hline C & -3.05057 & -0.813 & 1.402242 \\
\hline $\mathrm{H}$ & -3.76779 & -1.63749 & 1.428568 \\
\hline $\mathrm{H}$ & -2.0973 & -1.18097 & 1.788389 \\
\hline $\mathrm{H}$ & -3.41251 & -0.0277 & 2.07276 \\
\hline C & -4.25828 & 0.240426 & -0.54376 \\
\hline $\mathrm{H}$ & -4.1804 & 0.622819 & -1.56577 \\
\hline $\mathrm{H}$ & -4.99316 & -0.56852 & -0.54281 \\
\hline $\mathrm{H}$ & -4.63986 & 1.042246 & 0.095299 \\
\hline C & -2.39378 & -1.40431 & -0.96366 \\
\hline $\mathrm{H}$ & -2.28331 & -1.0425 & -1.99045 \\
\hline $\mathrm{H}$ & -1.42651 & -1.78603 & -0.6288 \\
\hline $\mathrm{H}$ & -3.10212 & -2.23671 & -0.9727 \\
\hline
\end{tabular}

TS $^{\mathrm{NFC}}$

\begin{tabular}{|c|c|c|c|}
\hline C & -2.7066 & 2.40091 & -1.10215 \\
\hline C & -1.46298 & 1.832686 & -1.19665 \\
\hline $\mathrm{H}$ & -3.20101 & 2.775941 & -1.99019 \\
\hline $\mathrm{H}$ & -0.9533 & 1.745222 & -2.1477 \\
\hline C & -3.3476 & 2.509919 & 0.149467 \\
\hline C & -2.70837 & 2.064174 & 1.325615 \\
\hline $\mathrm{H}$ & -4.33792 & 2.944629 & 0.209316 \\
\hline $\mathrm{H}$ & -3.20391 & 2.183479 & 2.281636 \\
\hline C & -0.80172 & 1.319203 & -0.01393 \\
\hline $\mathrm{H}$ & 0.278802 & 1.388477 & -0.01136 \\
\hline C & -1.45867 & 1.503735 & 1.265101 \\
\hline $\mathrm{H}$ & -0.94449 & 1.175668 & 2.159779 \\
\hline$N$ & -0.6513 & -0.57145 & -0.26481 \\
\hline$S$ & -2.004 & -1.48125 & -0.23678 \\
\hline C & 0.582633 & -1.18643 & 0.003027 \\
\hline $\mathrm{O}$ & -3.14061 & -0.61806 & -0.45318 \\
\hline $\mathrm{O}$ & -1.87861 & -2.73512 & -0.94528 \\
\hline$F$ & -2.1077 & -1.90968 & 1.358356 \\
\hline $\mathrm{O}$ & 0.73796 & -2.33884 & 0.339428 \\
\hline $\mathrm{O}$ & 1.565924 & -0.3012 & -0.17576 \\
\hline C & 2.921761 & -0.76555 & 0.088247 \\
\hline $\mathrm{H}$ & 2.943688 & -1.22 & 1.08086 \\
\hline $\mathrm{H}$ & 3.167378 & -1.52785 & -0.65471 \\
\hline C & 3.872137 & 0.429867 & -0.00121 \\
\hline C & 5.291742 & -0.10976 & 0.253598 \\
\hline $\mathrm{H}$ & 6.01678 & 0.706765 & 0.202387 \\
\hline $\mathrm{H}$ & 5.372019 & -0.56919 & 1.243438 \\
\hline $\mathrm{H}$ & 5.573433 & -0.85709 & -0.49422 \\
\hline C & 3.513469 & 1.472227 & 1.072268 \\
\hline $\mathrm{H}$ & 2.508665 & 1.872208 & 0.918104 \\
\hline $\mathrm{H}$ & 3.555725 & 1.03469 & 2.074383 \\
\hline $\mathrm{H}$ & 4.217019 & 2.308478 & 1.038039 \\
\hline C & 3.798948 & 1.062435 & -1.40177 \\
\hline $\mathrm{H}$ & 4.50436 & 1.894441 & -1.47833 \\
\hline $\mathrm{H}$ & 4.052269 & 0.331407 & -2.1757 \\
\hline $\mathrm{H}$ & 2.797203 & 1.445208 & -1.61011 \\
\hline
\end{tabular}

\section{INT2 $^{\text {NFC }}$}

\begin{tabular}{|l|l|l|l|}
\hline C & -2.5264 & 2.478321 & -1.15285 \\
\hline
\end{tabular}




\begin{tabular}{|c|c|c|c|}
\hline C & -1.46651 & 1.624207 & -1.22687 \\
\hline $\mathrm{H}$ & -2.92653 & 2.909784 & -2.06383 \\
\hline $\mathrm{H}$ & -1.00899 & 1.365929 & -2.1745 \\
\hline C & -3.12308 & 2.807834 & 0.088422 \\
\hline C & -2.61794 & 2.247416 & 1.28702 \\
\hline $\mathrm{H}$ & -3.97256 & 3.478276 & 0.120255 \\
\hline $\mathrm{H}$ & -3.08756 & 2.503453 & 2.230504 \\
\hline C & -0.86623 & 1.020464 & 0.003344 \\
\hline $\mathrm{H}$ & 0.182579 & 1.325398 & 0.066237 \\
\hline C & -1.5576 & 1.390552 & 1.279123 \\
\hline $\mathrm{H}$ & -1.16732 & 0.962335 & 2.194974 \\
\hline$N$ & -0.69641 & -0.50454 & -0.13484 \\
\hline$S$ & -2.0531 & -1.47417 & -0.20771 \\
\hline C & 0.560144 & -1.14865 & 0.019815 \\
\hline $\mathrm{O}$ & -3.20677 & -0.6223 & -0.32302 \\
\hline $\mathrm{O}$ & -1.85812 & -2.62163 & -1.05415 \\
\hline$F$ & -2.09413 & -2.0587 & 1.321657 \\
\hline $\mathrm{O}$ & 0.686039 & -2.32887 & 0.232877 \\
\hline $\mathrm{O}$ & 1.543014 & -0.26779 & -0.10752 \\
\hline C & 2.904074 & -0.77046 & 0.072003 \\
\hline $\mathrm{H}$ & 2.954815 & -1.27446 & 1.039095 \\
\hline $\mathrm{H}$ & 3.099312 & -1.49666 & -0.71966 \\
\hline C & 3.866266 & 0.415484 & 0.001323 \\
\hline C & 3.753813 & 1.107656 & -1.36819 \\
\hline $\mathrm{H}$ & 2.75095 & 1.511425 & -1.52553 \\
\hline $\mathrm{H}$ & 4.466905 & 1.933908 & -1.43306 \\
\hline $\mathrm{H}$ & 3.971654 & 0.407764 & -2.18069 \\
\hline C & 3.555682 & 1.414797 & 1.129248 \\
\hline $\mathrm{H}$ & 4.266665 & 2.244924 & 1.104985 \\
\hline $\mathrm{H}$ & 2.5499 & 1.828787 & 1.025879 \\
\hline $\mathrm{H}$ & 3.628104 & 0.935166 & 2.11009 \\
\hline C & 5.285561 & -0.1533 & 0.184519 \\
\hline $\mathrm{H}$ & 6.019488 & 0.655581 & 0.141052 \\
\hline $\mathrm{H}$ & 5.392982 & -0.65332 & 1.151792 \\
\hline $\mathrm{H}$ & 5.531129 & -0.87288 & -0.60225 \\
\hline
\end{tabular}

\begin{tabular}{|c|c|c|c|}
\hline $\mathrm{O}$ & -1.19248 & 1.246715 & -1.9451 \\
\hline C & -2.64798 & -0.13552 & -0.18871 \\
\hline C & -2.89934 & -1.10687 & -1.15746 \\
\hline C & -3.3327 & -0.0902 & 1.025336 \\
\hline C & -3.87769 & -2.06089 & -0.89585 \\
\hline $\mathrm{H}$ & -2.35137 & -1.10779 & -2.09081 \\
\hline C & -4.30693 & -1.05423 & 1.269135 \\
\hline $\mathrm{H}$ & -3.11312 & 0.681554 & 1.751513 \\
\hline C & -4.57614 & -2.03472 & 0.313233 \\
\hline $\mathrm{H}$ & -4.0958 & -2.8213 & -1.63553 \\
\hline $\mathrm{H}$ & -4.85551 & -1.03691 & 2.202749 \\
\hline $\mathrm{H}$ & -5.33598 & -2.78182 & 0.509799 \\
\hline$S$ & 1.388645 & 1.082722 & 0.502228 \\
\hline O & 1.192468 & 1.246702 & 1.945105 \\
\hline $\mathrm{O}$ & 1.610316 & 2.247792 & -0.35056 \\
\hline C & 2.647982 & -0.13552 & 0.188714 \\
\hline C & 2.899338 & -1.10688 & 1.15745 \\
\hline C & 3.332705 & -0.09019 & -1.02533 \\
\hline C & 3.877692 & -2.0609 & 0.895843 \\
\hline $\mathrm{H}$ & 2.351363 & -1.1078 & 2.090798 \\
\hline C & 4.306942 & -1.05422 & -1.26913 \\
\hline $\mathrm{H}$ & 3.113129 & 0.681567 & -1.75151 \\
\hline C & 4.576145 & -2.03472 & -0.31324 \\
\hline $\mathrm{H}$ & 4.095797 & -2.82131 & 1.635517 \\
\hline $\mathrm{H}$ & 4.855523 & -1.03689 & -2.20274 \\
\hline $\mathrm{H}$ & 5.335984 & -2.78181 & -0.5098 \\
\hline
\end{tabular}

TS $^{\mathrm{NFSI}}$

\begin{tabular}{|l|l|l|l|}
\hline C & -0.79778 & 3.671835 & -0.52801 \\
\hline C & 0.030742 & 2.931878 & 0.276518 \\
\hline H & -0.38448 & 4.425612 & -1.18729 \\
\hline H & 1.10217 & 3.085101 & 0.270904 \\
\hline C & -2.19044 & 3.459913 & -0.49921 \\
\hline C & -2.75399 & 2.506705 & 0.373631 \\
\hline H & -2.83523 & 4.037549 & -1.15052 \\
\hline H & -3.82908 & 2.376604 & 0.402706 \\
\hline C & -0.51277 & 1.905771 & 1.138119 \\
\hline H & 0.029573 & 1.700894 & 2.053121 \\
\hline C & -1.94973 & 1.75575 & 1.193017 \\
\hline H & -2.36673 & 1.026206 & 1.875225 \\
\hline
\end{tabular}

\section{INT1 ${ }^{\text {NFSI }}$}

\begin{tabular}{|l|l|l|l|}
\hline$N$ & 0 & 0.194646 & $-6 \mathrm{E}-06$ \\
\hline $\mathrm{S}$ & -1.38865 & 1.082725 & -0.50223 \\
\hline $\mathrm{O}$ & -1.61032 & 2.24779 & 0.350567 \\
\hline
\end{tabular}




\begin{tabular}{|c|c|c|c|}
\hline$N$ & 0.150388 & 0.241089 & 0.396408 \\
\hline$S$ & 1.326483 & -0.59687 & 1.263734 \\
\hline O & 1.499842 & 0.185454 & 2.494943 \\
\hline O & 0.984279 & -2.01799 & 1.381986 \\
\hline$S$ & -0.53659 & -0.34887 & -1.01623 \\
\hline O & -0.82739 & 0.805845 & -1.86599 \\
\hline $\mathrm{O}$ & 0.321746 & -1.41775 & -1.54106 \\
\hline C & -2.11102 & -1.06487 & -0.5192 \\
\hline C & -2.12158 & -2.11187 & 0.401167 \\
\hline C & -3.28166 & -0.5664 & -1.08419 \\
\hline$C$ & -3.34562 & -2.66623 & 0.765046 \\
\hline $\mathrm{H}$ & -1.19599 & -2.47592 & 0.828422 \\
\hline C & -4.49918 & -1.13485 & -0.71287 \\
\hline $\mathrm{H}$ & -3.23718 & 0.253096 & -1.78849 \\
\hline C & -4.53146 & -2.17899 & 0.210685 \\
\hline $\mathrm{H}$ & -3.37281 & -3.47811 & 1.482091 \\
\hline $\mathrm{H}$ & -5.41932 & -0.75712 & -1.14259 \\
\hline $\mathrm{H}$ & -5.48078 & -2.61472 & 0.499891 \\
\hline$C$ & 2.839888 & -0.45706 & 0.307114 \\
\hline C & 3.202368 & -1.49241 & -0.54944 \\
\hline C & 3.6118 & 0.695036 & 0.447114 \\
\hline$C$ & 4.376521 & -1.36443 & -1.28822 \\
\hline $\mathrm{H}$ & 2.573385 & -2.36667 & -0.63877 \\
\hline C & 4.780292 & 0.810167 & -0.30158 \\
\hline $\mathrm{H}$ & 3.31257 & 1.475187 & 1.135165 \\
\hline C & 5.160692 & -0.21672 & -1.16734 \\
\hline $\mathrm{H}$ & 4.676043 & -2.16141 & -1.95824 \\
\hline $\mathrm{H}$ & 5.394738 & 1.697066 & -0.20315 \\
\hline $\mathrm{H}$ & 6.071856 & -0.12285 & -1.74662 \\
\hline
\end{tabular}

\begin{tabular}{|c|c|c|c|}
\hline $\mathrm{H}$ & -0.95003 & 4.66504 & 1.058413 \\
\hline C & 0.678669 & 1.612005 & 0.698468 \\
\hline $\mathrm{H}$ & 1.283197 & 1.311842 & 1.566537 \\
\hline $\mathrm{C}$ & -0.28477 & 2.657695 & 1.169826 \\
\hline $\mathrm{H}$ & -0.99208 & 2.358632 & 1.933899 \\
\hline $\mathrm{N}$ & -0.04092 & 0.309115 & 0.368035 \\
\hline$S$ & 0.58447 & -1.11604 & 1.093204 \\
\hline 0 & 0.725674 & -0.80984 & 2.515828 \\
\hline 0 & -0.27245 & -2.21314 & 0.655992 \\
\hline$S$ & -1.00809 & 0.203303 & -1.06901 \\
\hline $\mathrm{O}$ & -1.19835 & 1.592328 & -1.47465 \\
\hline $\mathrm{O}$ & -0.41594 & -0.75099 & -2.00437 \\
\hline C & -2.56714 & -0.44128 & -0.47332 \\
\hline$C$ & -2.91227 & -1.76003 & -0.74896 \\
\hline$C$ & -3.40474 & 0.419186 & 0.234255 \\
\hline$C$ & -4.14446 & -2.2296 & -0.29975 \\
\hline $\mathrm{H}$ & -2.22773 & -2.39884 & -1.28918 \\
\hline$C$ & -4.62896 & -0.06752 & 0.682597 \\
\hline $\mathrm{H}$ & -3.10828 & 1.443232 & 0.423869 \\
\hline$C$ & -4.9969 & -1.38797 & 0.415187 \\
\hline $\mathrm{H}$ & -4.43387 & -3.25317 & -0.50461 \\
\hline $\mathrm{H}$ & -5.2953 & 0.583956 & 1.234869 \\
\hline $\mathrm{H}$ & -5.95218 & -1.76107 & 0.765494 \\
\hline C & 2.222586 & -1.30585 & 0.396883 \\
\hline$C$ & 2.348699 & -1.85784 & -0.87774 \\
\hline $\mathrm{C}$ & 3.322719 & -0.86422 & 1.130063 \\
\hline$C$ & 3.621546 & -1.96013 & -1.43089 \\
\hline $\mathrm{H}$ & 1.472333 & -2.18056 & -1.42253 \\
\hline$C$ & 4.589272 & -0.97377 & 0.559729 \\
\hline $\mathrm{H}$ & 3.190171 & -0.45224 & 2.121794 \\
\hline $\mathrm{C}$ & 4.736728 & -1.51712 & -0.71634 \\
\hline $\mathrm{H}$ & 3.741951 & -2.38556 & -2.41981 \\
\hline $\mathrm{H}$ & 5.456723 & -0.63685 & 1.114063 \\
\hline $\mathrm{H}$ & 5.723998 & -1.59839 & -1.1557 \\
\hline
\end{tabular}

INT2 $^{\text {NFSI }}$

\begin{tabular}{|l|l|l|l|}
\hline C & 1.587082 & 3.354079 & -0.84169 \\
\hline C & 1.601059 & 2.067022 & -0.39286 \\
\hline H & 2.276756 & 3.651924 & -1.62419 \\
\hline H & 2.289179 & 1.333436 & -0.79546 \\
\hline C & 0.678759 & 4.307934 & -0.32126 \\
\hline C & -0.24831 & 3.929115 & 0.680631 \\
\hline H & 0.680467 & 5.321516 & -0.70216 \\
\hline
\end{tabular}




\section{Copper-catalyzed Imidation of Benzene (Figures $2 c$ and 2d) \\ Photoinduced, Cu-Catalyzed Imidation of Benzene (Figure 2c)}

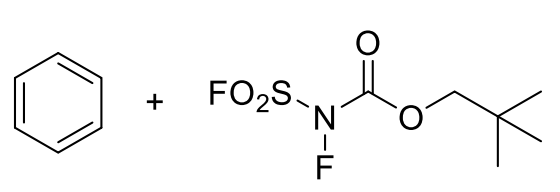

1.0 eq. $1.4 \mathrm{eq}$.

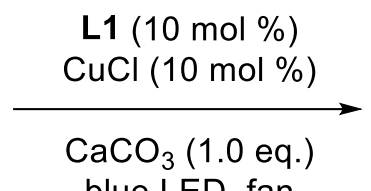

$\left(\mathrm{CH}_{2} \mathrm{Cl}\right)_{2}, 30-40^{\circ} \mathrm{C}, 5 \mathrm{~h}$<smiles>CC(C)(C)COC(=O)N(c1ccccc1)S(=O)(=O)[O-]</smiles>

$46 \%$<smiles>Cc1cccc(-c2cccc(C)n2)n1</smiles>

L1

To an argon-purged Schlenk tube, calcium carbonate $(100 \mu \mathrm{mol}, 10.0 \mathrm{mg})$, copper $(\mathrm{I})$ chloride $(10.0 \mu \mathrm{mol}, 1.0 \mathrm{mg}$,

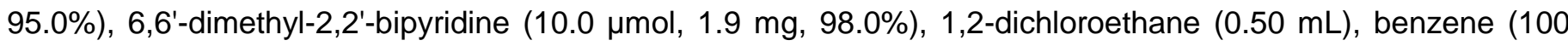
$\mu \mathrm{mol}, 7.9 \mathrm{mg}, 99.0 \%$ ) and neopentyl $\mathrm{N}$-fluoro- $N$-(fluorosulfonyl)carbamate (NFC) (140 $\mu \mathrm{mol}, 48.2 \mathrm{mg},>96 \%$ ) were added. After stirring for $5 \mathrm{~h}$ under blue LED irradiation (Kessil A160WE Controllable LED Aquarium Light, $2 \mathrm{~cm}$ from the light source) maintaining the temperature to $30-40{ }^{\circ} \mathrm{C}$ by a fan, the crude solution was filtered through a pad of $\mathrm{SiO}_{2}$ eluting with dichloromethane and concentrated under vacuum. The yield was determined by ${ }^{1} \mathrm{H}$ NMR using 1,2-dichloroethane as an internal standard.

\section{Cationic Cu-Catalyzed Imidation of Benzene (Figure 2d)}<smiles>CC(C)(C)COC(=O)N(F)S(=O)(=O)O[Na]</smiles>

1.0 eq. 1.4 eq.

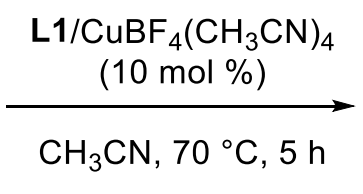<smiles>CC(C)(C)COC(=O)N(c1ccccc1)c1ccccc1</smiles>

$68 \%$

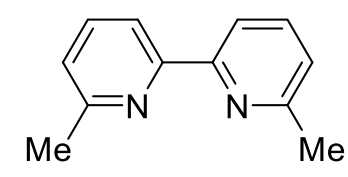

L1

To an argon-purged Schlenk tube, tetrakis(acetonitrile)copper(l) tetrafluoroborate (10.0 $\mu \mathrm{mol}, 3.2 \mathrm{mg}, 98.0 \%)$, 6,6'-dimethyl-2,2'-bipyridine (10.0 $\mu \mathrm{mol}, 1.9 \mathrm{mg}, 98.0 \%), 1,2$-dichloroethane $(0.50 \mathrm{~mL})$, benzene (100 $\mu \mathrm{mol}, 7.9$ mg, 99.0\%) and neopentyl $N$-fluoro- $N$-(fluorosulfonyl)carbamate (NFC) $(140 \mu \mathrm{mol}, 48.2 \mathrm{mg},>96 \%)$ were added. After stirring for $5 \mathrm{~h}$ at $70^{\circ} \mathrm{C}$, the mixture was cooled to room temperature. The crude solution was filtered through a pad of $\mathrm{SiO}_{2}$ eluting with dichloromethane and concentrated under vacuum. The yield was determined by ${ }^{1} \mathrm{H}$ NMR using 1,2-dichloroethane as an internal standard. 


\section{Cu-catalyzed Imidation of Benzene Derivatives (Figure 2e)}

\section{General Procedure}

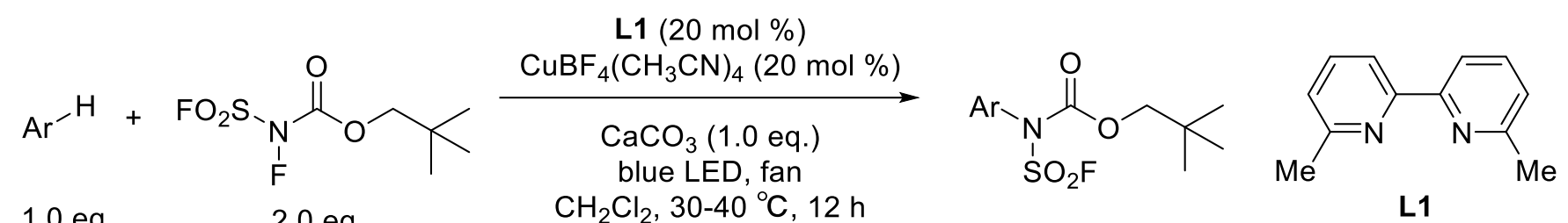

1.0 eq.

2.0 eq.

$\mathrm{CH}_{2} \mathrm{Cl}_{2}, 30-40{ }^{\circ} \mathrm{C}, 12 \mathrm{~h}$

To an argon-purged Schlenk tube, calcium carbonate $(200 \mu \mathrm{mol}, 20.0 \mathrm{mg})$, tetrakis(acetonitrile)copper(I) tetrafluoroborate $(40.0 \mu \mathrm{mol}, 12.8 \mathrm{mg}, 98.0 \%), 6,6$ '-dimethyl-2,2'-bipyridine $(40.0 \mu \mathrm{mol}, 7.5 \mathrm{mg}, 98.0 \%)$, dichloromethane $(1.00 \mathrm{~mL})$ and substrate arenes $(200 \mu \mathrm{mol})$ were added and stirred for $10 \mathrm{~min}$. Then, neopentyl $\mathrm{N}$-fluoro- $\mathrm{N}$-(fluorosulfonyl)carbamate (NFC) $(400 \mu \mathrm{mol}, 96.3 \mathrm{mg},>96 \%)$ were added. After stirring for $12 \mathrm{~h}$ under blue LED irradiation (Kessil A160WE Controllable LED Aquarium Light, $2 \mathrm{~cm}$ from the light source) maintaining the temperature to $30-40{ }^{\circ} \mathrm{C}$ by a fan, the crude solution was filtered through a pad of $\mathrm{SiO}_{2}$ eluting with dichloromethane and concentrated under vacuum. The residue was purified by column chromatography on silica gel.

\section{Reaction Setup}

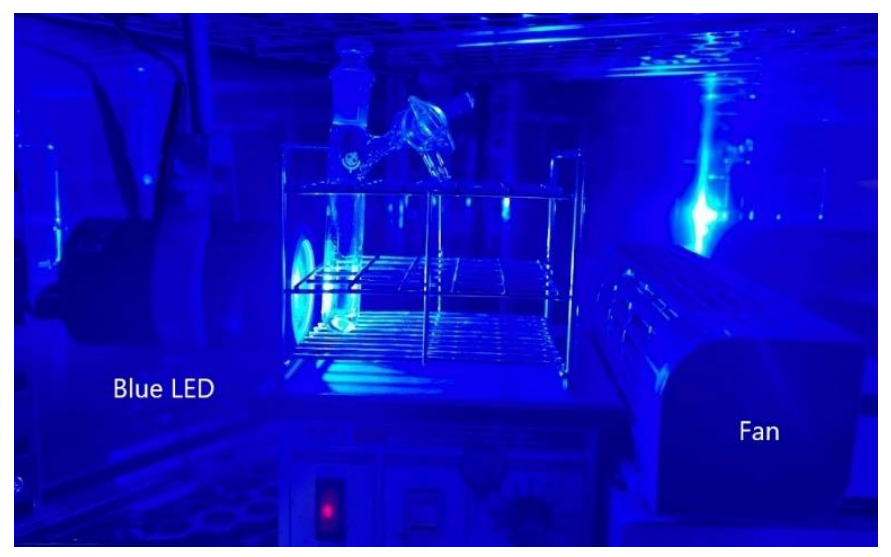

neopentyl (fluorosulfonyl)(phenyl)carbamate (25)<smiles>CC(C)(C)COC(=O)N(c1ccccc1)c1ccccc1</smiles>

Prepared according to the general procedure with benzene (200 $\mu \mathrm{mol}, 15.8 \mathrm{mg}, 99.0 \%)$. The crude material was purified by column chromatography on silica gel (DCM/hexane $20-80 \%)$ to give the title compound as a pale greenish oil in $91 \%$ yield $(182 \mu \mathrm{mol}, 52.7 \mathrm{mg})$.

${ }^{1}$ H NMR (392 MHz, CDCl$)$ ס 7.49-7.47 (m, 3H), 7.37-7.34 (m, 2H), 3.94 (s, 2H), $0.85(\mathrm{~s}, 9 \mathrm{H})$.

${ }^{13} \mathrm{C}$ NMR $\left(126 \mathrm{MHz}, \mathrm{CDCl}_{3}\right) \delta 150.7,135.5,130.4,129.9,128.9,78.4,31.5,26.1$.

${ }^{19} \mathrm{~F}$ NMR $\left(471 \mathrm{MHz}, \mathrm{CDCl}_{3}\right) \delta 51.58(\mathrm{~s}, 1 \mathrm{~F})$.

HRMS (ESI) exact mass calculated for $\mathrm{C}_{12} \mathrm{H}_{16} \mathrm{O}_{4} \mathrm{NFSNa}: \mathrm{m} / \mathrm{z} 312.0676\left([\mathrm{M}+\mathrm{Na}]^{+}\right)$, found: $\mathrm{m} / \mathrm{z} 312.0678\left([\mathrm{M}+\mathrm{Na}]^{+}\right)$.

neopentyl (fluorophenyl)(fluorosulfonyl)carbamate (27) 


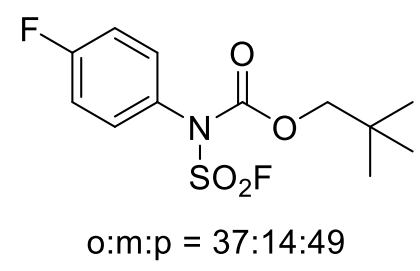

Prepared according to the general procedure with fluorobenzene $(200 \mu \mathrm{mol}, 19.2 \mathrm{mg})$. The crude material was purified by column chromatography on silica gel (DCM/hexane $20-80 \%$ ) to give the title compound as a colorless oil in $81 \%$ yield $(161 \mu \mathrm{mol}, 49.5 \mathrm{mg})$.

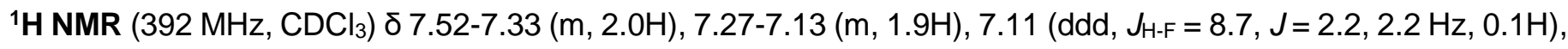
$3.98(\mathrm{~s}, 0.7 \mathrm{H}), 3.97(\mathrm{~s}, 0.3 \mathrm{H}), 3.96(\mathrm{~s}, 1.0 \mathrm{H}), 0.88(\mathrm{~s}, 1.3 \mathrm{H}), 0.87(\mathrm{~s}, 4.4 \mathrm{H}), 0.87(\mathrm{~s}, 3.3 \mathrm{H})$.

${ }^{13} \mathrm{C}$ NMR $\left(99 \mathrm{MHz}, \mathrm{CDCl}_{3},\left\{{ }^{1} \mathrm{H},{ }^{19} \mathrm{~F}(-116.0 \mathrm{ppm})\right\}\right) \delta 163.41,163.35,163.3,158.4,150.6,150.3,150.0,136.3$, $132.5,131.3,131.0,130.9,125.1$, 124.9, 123.3, 117.8, 117.0, 116.9, 116.7, 78.70, 78.66, 78.6, 31.6, 26.2, 26.1, 26.0 .

${ }^{19} \mathrm{~F} \mathrm{NMR}\left(369 \mathrm{MHz}, \mathrm{CDCl}_{3}\right) \delta 51.93(\mathrm{~s}, 0.14 \mathrm{~F}), 51.75\left(\mathrm{~d}, \mathrm{~J}_{\mathrm{F}-\mathrm{F}}=5.7 \mathrm{~Hz}, 0.37 \mathrm{~F}\right), 51.55$ (s, 0.49F), -110.66 (m, 0.49F), $-111.19(\mathrm{~m}, 0.14 \mathrm{~F}),-121.23(\mathrm{~m}, 0.37 \mathrm{~F})$.

HRMS (ESI) exact mass calculated for $\mathrm{C}_{12} \mathrm{H}_{15} \mathrm{O}_{4} \mathrm{NF}_{2} \mathrm{SNa}: \mathrm{m} / z 330.0582\left([\mathrm{M}+\mathrm{Na}]^{+}\right)$, found: $\mathrm{m} / z 330.0591\left([\mathrm{M}+\mathrm{Na}]^{+}\right)$.

neopentyl (chlorophenyl)(fluorosulfonyl)carbamate (28)

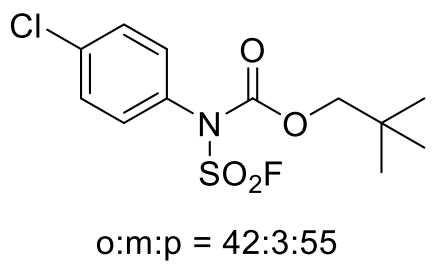

Prepared according to the general procedure with chlorobenzene ( $200 \mu \mathrm{mol}, 23.0 \mathrm{mg}, 98.0 \%)$. The crude material was purified by column chromatography on silica gel (DCM/hexane $20-80 \%$ ) to give the title compound as a colorless oil in $46 \%$ yield $(92.7 \mu \mathrm{mol}, 30.0 \mathrm{mg})$.

${ }^{1} \mathbf{H}$ NMR $\left(500 \mathrm{MHz}, \mathrm{CDCl}_{3}\right)$ ठ 7.56-7.54 (m, 0.4H), 7.49-7.43 (m, 2.0H), 7.41-7.37 (m, 0.5H), $7.30(\mathrm{~d}, J=8.9 \mathrm{~Hz}$, $1.1 \mathrm{H}), 3.97(\mathrm{~s}, 0.9 \mathrm{H}), 3.97(\mathrm{~s}, 1.1 \mathrm{H}), 0.88(\mathrm{~s}, 5.2 \mathrm{H}), 0.85(\mathrm{~s}, 3.8 \mathrm{H})$.

${ }^{13} \mathrm{C}$ NMR (126 MHz, $\mathrm{CDCl}_{3}$, o- and p-isomers) $\delta 150.4,149.8,136.6,134.1,133.8,133.2,131.8,131.0,130.7$, 130.3, 130.2, 128.2, 78.7, 78.6, 31.6, 31.5, 26.1, 26.0.

${ }^{19} \mathrm{~F} \mathrm{NMR} \mathrm{(471} \mathrm{MHz,} \mathrm{CDCl} 3$ ) $\delta 53.32$ (s, 0.42F), 52.12 (s, 0.03F), 51.88 (s, 0.55F).

HRMS (ESI) exact mass calculated for $\mathrm{C}_{12} \mathrm{H}_{15} \mathrm{O}_{4} \mathrm{NFSCINa:} \mathrm{m} / z 346.0287\left([\mathrm{M}+\mathrm{Na}]^{+}\right)$, found: $\mathrm{m} / \mathrm{z} 346.0288$ $\left([\mathrm{M}+\mathrm{Na}]^{+}\right)$.

neopentyl (bromophenyl)(fluorosulfonyl)carbamate (29)

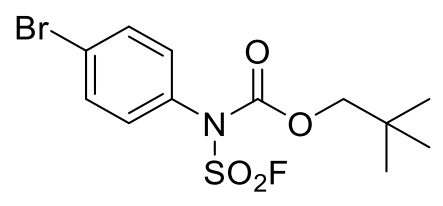

$0: m: p=44: 6: 50$ 
Prepared according to the general procedure with bromobenzene ( $200 \mu \mathrm{mol}, 31.7 \mathrm{mg}, 99.0 \%)$. The crude material was purified by column chromatography on silica gel (DCM/hexane $20-80 \%)$ to give the title compound as a colorless oil in $61 \%$ yield (122 $\mu \mathrm{mol}, 45.0 \mathrm{mg})$.

${ }^{1} \mathrm{H}$ NMR $\left(500 \mathrm{MHz}, \mathrm{CDCl}_{3}\right) \delta 7.72(\mathrm{~d}, J=7.7 \mathrm{~Hz}, 0.4 \mathrm{H}), 7.64-7.61(\mathrm{~m}, 1.1 \mathrm{H}), 7.54(\mathrm{dd}, J=2.0,2.0 \mathrm{~Hz}, 0.1 \mathrm{H}), 7.45-$ $7.43(\mathrm{~m}, 0.9 \mathrm{H}), 7.38-7.34(\mathrm{~m}, 0.5 \mathrm{H}), 7.33-7.31(\mathrm{~m}, 0.1 \mathrm{H}), 7.23(\mathrm{~d}, J=8.6 \mathrm{~Hz}, 1.0 \mathrm{H}), 3.97(\mathrm{~s}, 2.0 \mathrm{H}), 0.88(\mathrm{~s}, 5.0 \mathrm{H})$, $0.85(\mathrm{~s}, 4.0 \mathrm{H})$.

${ }^{13} \mathrm{C}$ NMR (126 MHz, $\mathrm{CDCl}_{3}$, o- and p-isomers) $\delta 150.3,149.7,134.8,134.4,134.0,133.2,131.9,131.0,130.5$, $128.9,124.8,124.3,78.7,78.6,31.6,31.5,26.11,26.07$.

${ }^{19} \mathrm{~F}$ NMR (471 MHz, CDCl 3$) \delta 53.90$ (s, 0.44F), 52.17 (s, 0.06F), 51.94 (s, 0.50F).

HRMS (ESI) exact mass calculated for $\mathrm{C}_{12} \mathrm{H}_{15} \mathrm{O}_{4} \mathrm{NFSBrNa:} \mathrm{m} / \mathrm{z} 389.9781\left([\mathrm{M}+\mathrm{Na}]^{+}\right)$, found: $\mathrm{m} / \mathrm{z} 389.9782$ $\left([\mathrm{M}+\mathrm{Na}]^{+}\right)$.

((fluorosulfonyl)((neopentyloxy)carbonyl)amino)phenyl acetate (30)

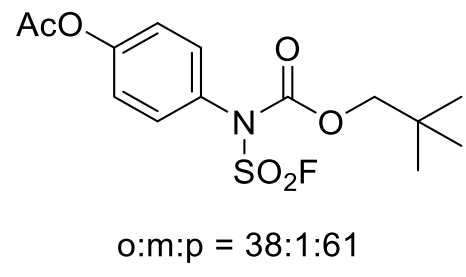

Prepared according to the general procedure with phenyl acetate ( $200 \mu \mathrm{mol}, 27.8 \mathrm{mg}, 98.0 \%)$. The crude material was purified by column chromatography on silica gel (DCM/hexane $35-95 \%)$ to give the title compound as a pale yellowish solid in $80 \%$ yield $(159 \mu \mathrm{mol}, 55.3 \mathrm{mg}$ ).

${ }^{1} \mathrm{H}$ NMR (392 MHz, $\mathrm{CDCl}_{3}$, o- and p-isomers) $\delta 7.51$ (ddd, $J=7.9,7.9,1.3 \mathrm{~Hz}, 0.4 \mathrm{H}$ ), 7.43 (dd, $J=8.0,1.5 \mathrm{~Hz}$, $0.4 \mathrm{H}), 7.37-7.31(\mathrm{~m}, 2.0 \mathrm{H}), 7.23(\mathrm{~d}, J=8.8 \mathrm{~Hz}, 1.2 \mathrm{H}), 3.95(\mathrm{~s}, 2.0 \mathrm{H}), 2.31(\mathrm{~s}, 1.8 \mathrm{H}), 2.30(\mathrm{~s}, 1.2 \mathrm{H}), 0.87(\mathrm{~s}, 5.6 \mathrm{H})$, $0.85(\mathrm{~s}, 3.4 \mathrm{H})$.

${ }^{13} \mathrm{C}$ NMR (126 MHz, $\mathrm{CDCl}_{3}$, o- and p-isomers) $\delta 168.7,167.8,151.9,150.5,150.1,147.3,132.6,131.6,130.6$, 130.0, 127.5, 126.7, 123.9, 123.0, 78.6, 78.5, 31.6, 31.5, 26.1, 26.0, 21.2, 20.7.

${ }^{19} \mathrm{~F}$ NMR $\left(471 \mathrm{MHz}, \mathrm{CDCl}_{3}\right) \delta 51.84(\mathrm{~s}, 0.01 \mathrm{~F}), 51.80(\mathrm{~s}, 0.38 \mathrm{~F}), 51.62(\mathrm{~s}, 0.61 \mathrm{~F})$.

HRMS (ESI) exact mass calculated for $\mathrm{C}_{14} \mathrm{H}_{18} \mathrm{O}_{6} \mathrm{NFSNa}: \mathrm{m} / \mathrm{z} 370.0731\left([\mathrm{M}+\mathrm{Na}]^{+}\right)$, found: $\mathrm{m} / \mathrm{z} 370.0727\left([\mathrm{M}+\mathrm{Na}]^{+}\right)$.

neopentyl ((1,3-dioxoisoindolin-2-yl)phenyl)(fluorosulfonyl)carbamate (31)

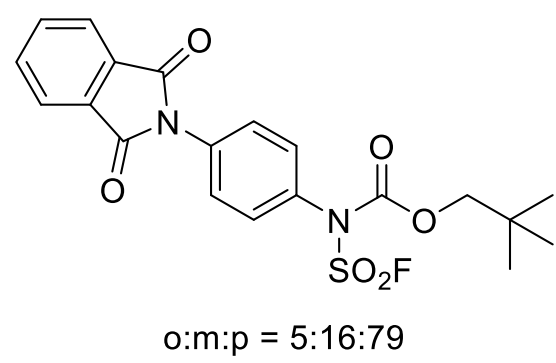

Prepared according to the general procedure with 2-phenylisoindoline-1,3-dione ( $200 \mu \mathrm{mol}, 44.6 \mathrm{mg}) .{ }^{16} \mathrm{The}$ crude material was purified by column chromatography on silica gel (TBME/hexane 15-40\%) to give the title compound as a white solid in $72 \%$ yield ( $144 \mu \mathrm{mol}, 62.6 \mathrm{mg})$.

${ }^{1} \mathrm{H}$ NMR $\left(392 \mathrm{MHz}, \mathrm{CDCl}_{3}\right)$ ठ 7.97-7.94 (m, 2.0H), 7.84-7.80 (m, 2.0H), 7.68-7.58 (m, 2.2H), 7.51-7.47 (m, 1.8H), 3.99 (s, 2.0H), 0.90-0.87 (m, 9.0H). 
${ }^{13} \mathrm{C}$ NMR $\left(126 \mathrm{MHz}, \mathrm{CDCl}_{3}, \mathrm{~m}\right.$ - and p-isomers) $\delta 166.8,166.4,150.5,149.8,134.9,134.8,134.2,133.7,132.6$, $131.5,131.3,130.3,130.2,130.0,129.6,127.3,124.1,78.7,78.6,31.6,31.5,29.8,26.1$.

${ }^{19} \mathrm{~F}$ NMR $\left(471 \mathrm{MHz}, \mathrm{CDCl}_{3}\right) \delta 57.35$ (t, JF-H $\left.=5.4 \mathrm{~Hz}, 0.05 \mathrm{~F}\right), 53.67$ (s, 0.16F), 51.96 (s, 0.79F).

HRMS (ESI) exact mass calculated for $\mathrm{C}_{20} \mathrm{H}_{19} \mathrm{O}_{6} \mathrm{~N}_{2} \mathrm{FSNa}: \mathrm{m} / z 457.0840\left([\mathrm{M}+\mathrm{Na}]^{+}\right)$, found: $\mathrm{m} / z 457.0842\left([\mathrm{M}+\mathrm{Na}]^{+}\right)$.

neopentyl (fluorosulfonyl)(tolyl)carbamate (32)

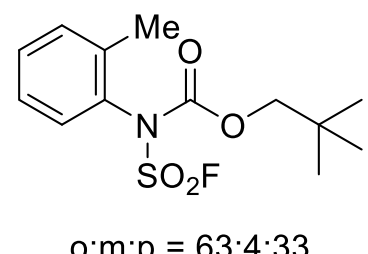

Prepared according to the general procedure with toluene (200 $\mu \mathrm{mol}, 18.5 \mathrm{mg}, 99.5 \%)$. The crude material was purified by column chromatography on silica gel (DCM/hexane $20-80 \%$ ) to give the title compound as a colorless oil in $94 \%$ yield ( $189 \mu \mathrm{mol}, 57.3 \mathrm{mg})$.

${ }^{1} \mathrm{H}$ NMR (392 MHz, CDCl$)$ ס 7.60-7.05 (m, 4.0H), 3.99-3.91 (m, 2.0H), 2.40-2.39 (m, 1.1H), $2.34(\mathrm{~s}, 1.9 \mathrm{H}), 0.87$ (s, 3.3H), $0.84(\mathrm{~s}, 5.7 \mathrm{H})$.

${ }^{13} \mathrm{C}$ NMR (126 MHz, $\mathrm{CDCl}_{3}$, o- and p-isomers) $\delta 150.9,150.5,140.7,137.3,134.7,132.8,131.6,130.7,130.5$, 129.0, 128.6, 127.5, 78.40, 78.38, 31.6, 31.5, 26.1, 26.0, 21.4, 17.6 .

${ }^{19} \mathrm{~F} \mathrm{NMR}\left(471 \mathrm{MHz}, \mathrm{CDCl}_{3}\right) \delta 52.46$ (s, 0.63F), 51.54 (s, 0.04F), 51.35 (s, 0.33F).

HRMS (ESI) exact mass calculated for $\mathrm{C}_{13} \mathrm{H}_{18} \mathrm{O}_{4} \mathrm{NFSNa}: \mathrm{m} / z 326.0833\left([\mathrm{M}+\mathrm{Na}]^{+}\right)$, found: $\mathrm{m} / z 326.0828\left([\mathrm{M}+\mathrm{Na}]^{+}\right)$.

neopentyl (2,4-dibromophenyl)(fluorosulfonyl)carbamate (33) and neopentyl (2,6-dibromophenyl)(fluorosulfonyl)carbamate (33')<smiles></smiles>

33

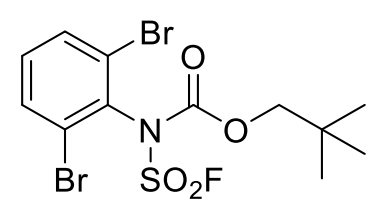

33'

Prepared according to the general procedure with 1,3-dibromobenzene (200 $\mu \mathrm{mol}, 48.1 \mathrm{mg}, 98.0 \%)$. The crude material was purified by column chromatography on silica gel (DCM/hexane $20-80 \%)$ to give the title compound as a pale yellowish oil in $58 \%$ yield $\left(116 \mu \mathrm{mol}, 51.9 \mathrm{mg}, 33: 33^{\prime}=83: 17\right)$.

${ }^{1} \mathrm{H}$ NMR $\left(392 \mathrm{MHz}, \mathrm{CDCl}_{3}\right) \delta 7.89(\mathrm{~d}, J=2.0 \mathrm{~Hz}, 0.8 \mathrm{H}), 7.68(\mathrm{~d}, J=8.3 \mathrm{~Hz}, 0.3 \mathrm{H}), 7.57(\mathrm{dd}, J=8.4,2.1 \mathrm{~Hz}, 0.8 \mathrm{H})$, $7.30(\mathrm{~d}, J=8.5 \mathrm{~Hz}, 0.8 \mathrm{H}), 7.22(\mathrm{t}, J=8.2 \mathrm{~Hz}, 0.2 \mathrm{H}), 4.00(\mathrm{~s}, 0.3 \mathrm{H}), 3.99(\mathrm{~d}, J=1.3 \mathrm{~Hz}, 1.7 \mathrm{H}), 0.89(\mathrm{~s}, 7.5 \mathrm{H}), 0.87$ $(\mathrm{s}, 1.5 \mathrm{H})$.

${ }^{13} \mathrm{C}$ NMR $\left(126 \mathrm{MHz}, \mathrm{CDCl}_{3}\right) \delta 149.4,148.9,136.5,134.4,133.9,133.0,132.5,132.3,131.9,125.9,125.3,125.2$, 124.9, 78.9, 78.7, 31.6, 26.11, 26.05.

${ }^{19} \mathrm{~F} \mathrm{NMR}\left(471 \mathrm{MHz}, \mathrm{CDCl}_{3}\right) \delta 56.86$ (s, 0.17F), 54.19 (s, 0.83F).

HRMS (ESI) exact mass calculated for $\mathrm{C}_{12} \mathrm{H}_{14} \mathrm{O}_{4} \mathrm{NFSBr}{ }_{2} \mathrm{Na}: \mathrm{m} / z 467.8887\left([\mathrm{M}+\mathrm{Na}]^{+}\right)$, found: $\mathrm{m} / z 467.8892$ $\left([\mathrm{M}+\mathrm{Na}]^{+}\right)$. 


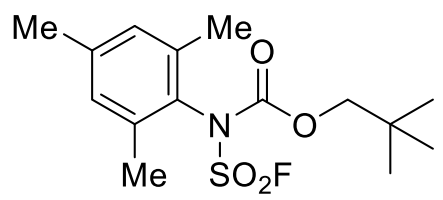

Prepared according to the general procedure with mesitylene ( $200 \mu \mathrm{mol}, 24.5 \mathrm{mg}, 98.0 \%)$ and NFC (280 $\mu \mathrm{mol}$, $67.4 \mathrm{mg},>96 \%)$. The crude material was purified by column chromatography on silica gel (DCM/hexane $20-80 \%)$ to give the title compound as a pale greenish oil in $48 \%$ yield $(96.9 \mu \mathrm{mol}, 32.1 \mathrm{mg})$.

${ }^{1} \mathrm{H}$ NMR $\left(392 \mathrm{MHz}, \mathrm{CDCl}_{3}\right) \delta 6.96(\mathrm{~s}, 2 \mathrm{H}), 3.97$ (s, 2H), 2.30 (s, 3H), 2.26 (s, 6H), $0.86(\mathrm{~s}, 9 \mathrm{H})$.

${ }^{13} \mathrm{C} \mathrm{NMR}\left(126 \mathrm{MHz}, \mathrm{CDCl}_{3}\right) \delta 150.5,140.4,136.9,131.5,129.9,78.3,31.6,26.1,21.2,17.9$.

${ }^{19} \mathrm{~F}$ NMR $\left(471 \mathrm{MHz}, \mathrm{CDCl}_{3}\right) \delta 53.95$ (s, 1F).

HRMS (ESI) exact mass calculated for $\mathrm{C}_{15} \mathrm{H}_{22} \mathrm{O}_{4} \mathrm{NFSNa}: \mathrm{m} / z 354.1146\left([\mathrm{M}+\mathrm{Na}]^{+}\right)$, found: $\mathrm{m} / z 354.1141\left([\mathrm{M}+\mathrm{Na}]^{+}\right)$.

neopentyl (2,4-dichloro-3-methylphenyl)(fluorosulfonyl)carbamate (35)

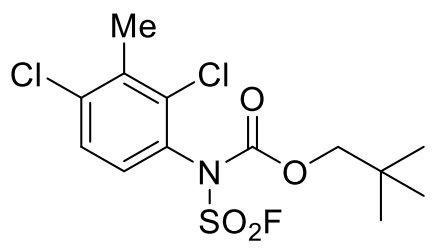

Prepared according to the general procedure with 1,3-dichloro-2-methylbenzene ( $200 \mu \mathrm{mol}, 32.5 \mathrm{mg}, 99.0 \%$ ). The crude material was purified by column chromatography on silica gel (DCM/hexane $20-80 \%)$ to give the title compound as a pale yellowish solid in $80 \%$ yield $(161 \mu \mathrm{mol}, 59.8 \mathrm{mg})$ as a single regioisomer.

${ }^{1} \mathrm{H}$ NMR $\left(500 \mathrm{MHz}, \mathrm{CDCl}_{3}\right) \delta 7.41(\mathrm{~d}, J=8.6 \mathrm{~Hz}, 1 \mathrm{H}), 7.24(\mathrm{~d}, J=8.6 \mathrm{~Hz}, 1 \mathrm{H}), 4.00(\mathrm{~d}, J 10.0 \mathrm{~Hz}, 1 \mathrm{H}), 3.98(\mathrm{~d}, J$ $10.3 \mathrm{~Hz}, 1 \mathrm{H}), 2.54(\mathrm{~s}, 3 \mathrm{H}), 0.89(\mathrm{~s}, 9 \mathrm{H})$.

${ }^{13} \mathrm{C}$ NMR $\left(126 \mathrm{MHz}, \mathrm{CDCl}_{3}\right) \delta$ 149.7, 137.8, 136.9, 135.5, 132.0, 128.5, 78.8, 31.6, 26.1, 18.3.

${ }^{19} \mathrm{~F}$ NMR $\left(471 \mathrm{MHz}, \mathrm{CDCl}_{3}\right) \delta 53.67(\mathrm{~s}, 1 \mathrm{~F})$.

HRMS (ESI) exact mass calculated for $\mathrm{C}_{13} \mathrm{H}_{16} \mathrm{O}_{4} \mathrm{NFSCl}{ }_{2} \mathrm{Na}: \mathrm{m} / z 394.0053\left([\mathrm{M}+\mathrm{Na}]^{+}\right)$, found: $\mathrm{m} / z 394.0049$ $\left([\mathrm{M}+\mathrm{Na}]^{+}\right)$.

neopentyl (3-bromo-2,4-dimethylphenyl)(fluorosulfonyl)carbamate (36) and neopentyl (4-bromo-3,5-dimethylphenyl)(fluorosulfonyl)carbamate (36')<smiles>Cc1ccc(N(C(=O)OCC(C)(C)C)S(=O)(=O)O)c(C)c1Br</smiles>

36<smiles>Cc1cc(N(C(=O)OCC(C)(C)C)S(=O)(=O)OF)cc(C)c1Br</smiles>

36'

Prepared according to the general procedure with 2-bromo-1,3-dimethylbenzene ( $200 \mu \mathrm{mol}, 37.0 \mathrm{mg})$. The crude material was purified by column chromatography on silica gel (DCM/hexane $20-80 \%)$ to give the title compound as a pale greenish oil in $75 \%$ yield $\left(149 \mu \mathrm{mol}, 59.1 \mathrm{mg}, \mathbf{3 6 : 3 6}{ }^{\prime}=91: 9\right)$.

${ }^{1} \mathrm{H}$ NMR $\left(392 \mathrm{MHz}, \mathrm{CDCl}_{3}\right) \delta 7.20(\mathrm{~d}, J=8.1 \mathrm{~Hz}, 0.9 \mathrm{H}), 7.16(\mathrm{~d}, J=8.1 \mathrm{~Hz}, 0.9 \mathrm{H}), 7.08(\mathrm{~s}, 0.2 \mathrm{H}), 4.01-3.93(\mathrm{~m}$, 2.0H), $2.46(\mathrm{~s}, 2.7 \mathrm{H}), 2.44(\mathrm{~s}, 0.5 \mathrm{H}), 2.42(\mathrm{~s}, 2.7 \mathrm{H}), 0.91(\mathrm{~s}, 0.8 \mathrm{H}), 0.88(\mathrm{~s}, 8.2 \mathrm{H})$.

${ }^{13} \mathrm{C}$ NMR $\left(126 \mathrm{MHz}, \mathrm{CDCl}_{3}\right) \delta 150.6,150.4,141.6,140.4,137.9,136.4,133.6,132.7,129.9,128.8,128.2,127.5$, 78.7, 78.6, 31.63, 31.60, 26.13, 26.09, 24.4, 24.0, 19.1. 
${ }^{19} \mathrm{~F}$ NMR $\left(471 \mathrm{MHz}, \mathrm{CDCl}_{3}\right) \delta 52.38$ (s, 0.91F), 51.87 (s, 0.09F).

HRMS (ESI) exact mass calculated for $\mathrm{C}_{14} \mathrm{H}_{19} \mathrm{O}_{4} \mathrm{NFSBrNa:} \mathrm{m} / z 418.0094\left([\mathrm{M}+\mathrm{Na}]^{+}\right)$, found: $\mathrm{m} / \mathrm{z} 418.0092$ $\left([\mathrm{M}+\mathrm{Na}]^{+}\right)$.

4-((fluorosulfonyl)((neopentyloxy)carbonyl)amino)-5-methyl-1,3-phenylene diacetate (37) and 2-((fluorosulfonyl)((neopentyloxy)carbonyl)amino)-5-methyl-1,3-phenylene diacetate (37')<smiles>[C]c1cc(OC(C)=O)cc(OC(C)=O)c1N(C(=O)OCC(C)(C)C)S(=O)(=O)OF</smiles>

37<smiles>CC(=O)Oc1cc(C)cc(OC(=O)O)c1N(C(=O)OCC(C)(C)C)S(=O)(=O)O</smiles>

37'

Prepared according to the general procedure with 5-methyl-1,3-phenylene diacetate (200 $\mu \mathrm{mol}, 41.6 \mathrm{mg}) .{ }^{17}$ The crude material was purified by column chromatography on silica gel (TBME/hexane 10-50\%) to give the title compound as a pale yellowish oil in 93\% yield (186 $\left.\mu \mathrm{mol}, 78.2 \mathrm{mg}, 37: 37^{\prime}=82: 18\right)$.

${ }^{1} \mathrm{H}$ NMR $\left(500 \mathrm{MHz}, \mathrm{CDCl}_{3}\right) \delta 7.07$ (d, $\left.J=2.6 \mathrm{~Hz}, 0.8 \mathrm{H}\right), 7.06$ (s, 0.4H), 7.00 (dd, $\left.J=2.6,0.6 \mathrm{~Hz}, 0.8 \mathrm{H}\right), 3.98-3.93$ $(\mathrm{m}, 2.0 \mathrm{H}), 2.41(\mathrm{~s}, 0.5 \mathrm{H}), 2.35(\mathrm{~s}, 2.5 \mathrm{H}), 2.29(\mathrm{~s}, 1.1 \mathrm{H}), 2.28(\mathrm{~s}, 4.9 \mathrm{H}), 0.87(\mathrm{~s}, 1.6 \mathrm{H}), 0.86(\mathrm{~s}, 7.4 \mathrm{H})$.

${ }^{13} \mathrm{C}$ NMR $\left(126 \mathrm{MHz}, \mathrm{CDCl}_{3}\right) \delta 168.5,167.7,167.5,151.6,149.9,149.7,147.9,147.8,142.5,140.0,124.1,121.4$, $121.1,117.9,114.8,78.6,31.6,31.5,26.0,21.8,21.2,20.7,17.9$.

${ }^{19} \mathrm{~F}$ NMR $\left(471 \mathrm{MHz}, \mathrm{CDCl}_{3}\right) \delta 52.71$ (s, 0.82F), 52.22 (s, 0.18F).

HRMS (ESI) exact mass calculated for $\mathrm{C}_{17} \mathrm{H}_{22} \mathrm{O}_{8} \mathrm{NFSNa}: \mathrm{m} / z 442.0942\left([\mathrm{M}+\mathrm{Na}]^{+}\right)$, found: $\mathrm{m} / z 442.0938\left([\mathrm{M}+\mathrm{Na}]^{+}\right)$.

\section{Measurement of UV-Vis Absorption Spectra of $\mathrm{CuBF}_{4} / \mathrm{L} 1$ complex}

To an argon-purged Schlenk tube, tetrakis(acetonitrile)copper(I) tetrafluoroborate (10.0 $\mu \mathrm{mol}, 3.2 \mathrm{mg}, 98.0 \%)$, 6,6'-dimethyl-2,2'-bipyridine ( $10.0 \mu \mathrm{mol}, 1.9 \mathrm{mg}, 98.0 \%)$ and dichloromethane $(0.50 \mathrm{~mL})$ were added (Figure S3). To this orange solution, neopentyl $N$-fluoro- $N$-(fluorosulfonyl)carbamate (NFC) (50 $\mu \mathrm{mol}, 48.2 \mathrm{mg}$ ) was added and stirred for $30 \mathrm{~min}$ (Figure S4). The supernatant was taken from the heterogeneous mixture, and subjected to the UV-Vis measurement.

\begin{tabular}{|c|c|c|}
\hline & \\
\hline
\end{tabular}




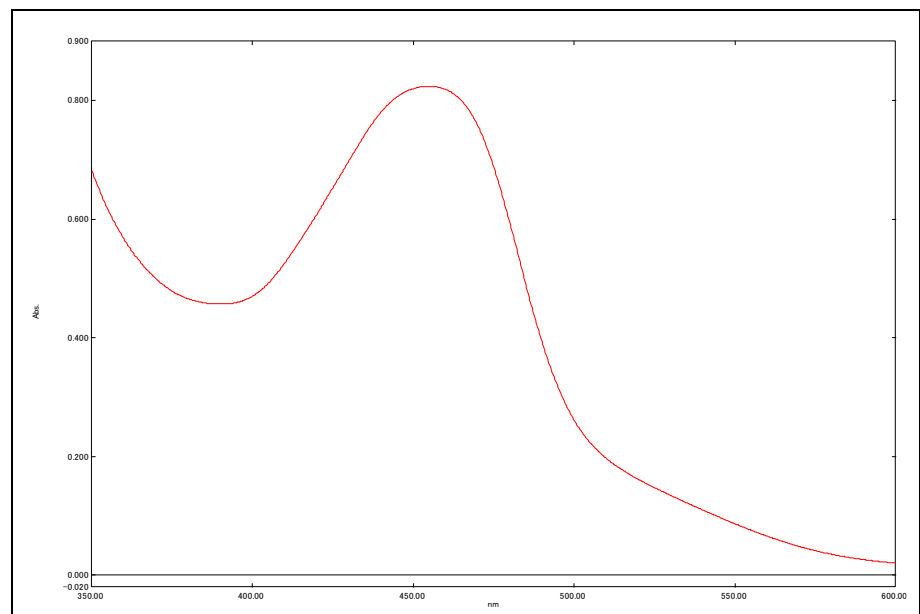

Figure S5. UV-Vis of $\mathrm{CuBF}_{4} / \mathrm{L} 1$ (4 times dilution)

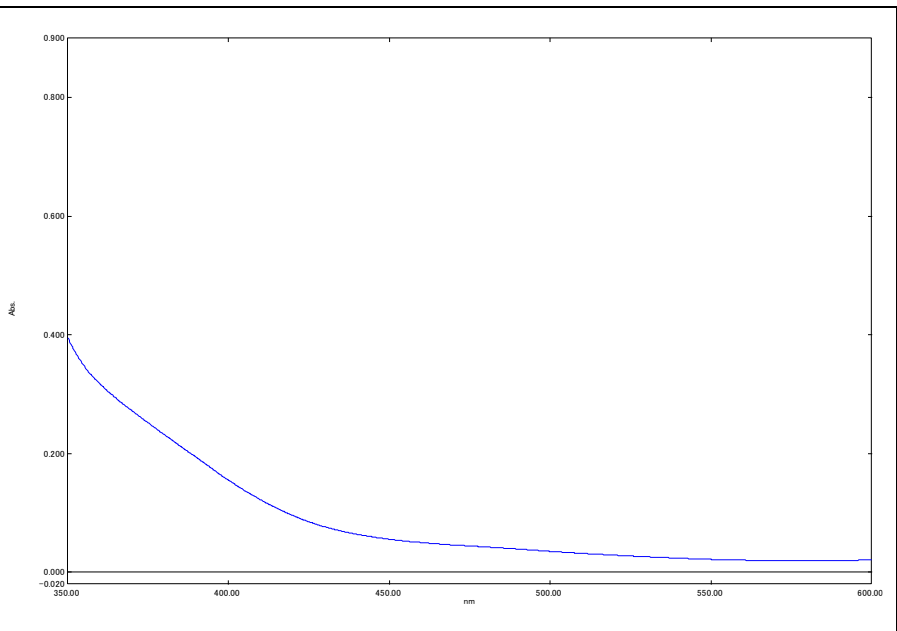

Figure S6. UV-Vis of $\mathrm{CuBF}_{4} / \mathrm{L1} / \mathrm{NFC}$ 


\section{Copper-Catalyzed Imidocyanation of Alkenes with NFC (Figure 3)}

\section{General Procedure A}

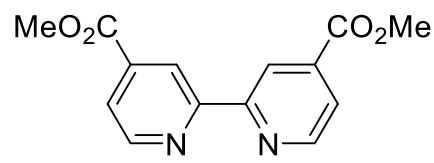

L2 $(10 \mathrm{~mol} \%)$

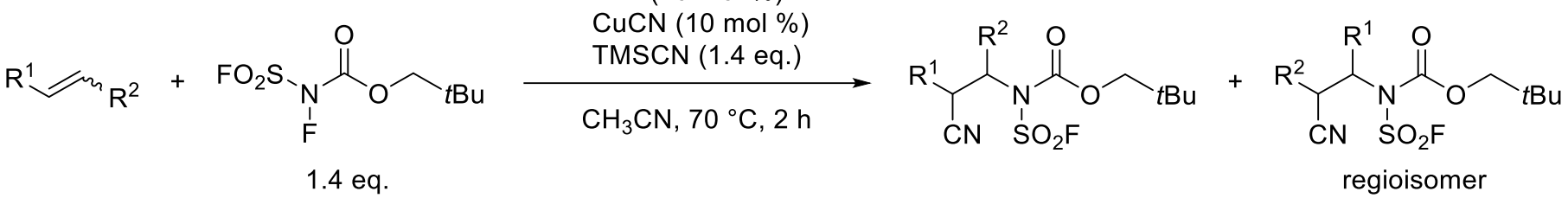

To an argon-purged Schlenk tube, CuCN $(20.0 \mu \mathrm{mol}, 1.8 \mathrm{mg})$, L2 $(20.0 \mu \mathrm{mol}, 5.5 \mathrm{mg})$, and acetonitrile $(1.00 \mathrm{~mL})$ were added at room temperature. After stirring for $10 \mathrm{~min}$ at the same temperature, alkene $(200 \mu \mathrm{mol})$, TMSCN $(280 \mu \mathrm{mol}, 38.9 \mu \mathrm{L}, 90 \%)$ and NFC (280 $\mu \mathrm{mol})$ were added at the same temperature. The solution was degassed and heated to $70^{\circ} \mathrm{C}$. After stirring for $2 \mathrm{~h}$, the solution was cooled to room temperature, diluted with DCM, filtered through a pad of silica gel and concentrated under vacuum. The residue was purified by flash column chromatography on silica gel.

\section{General Procedure B}

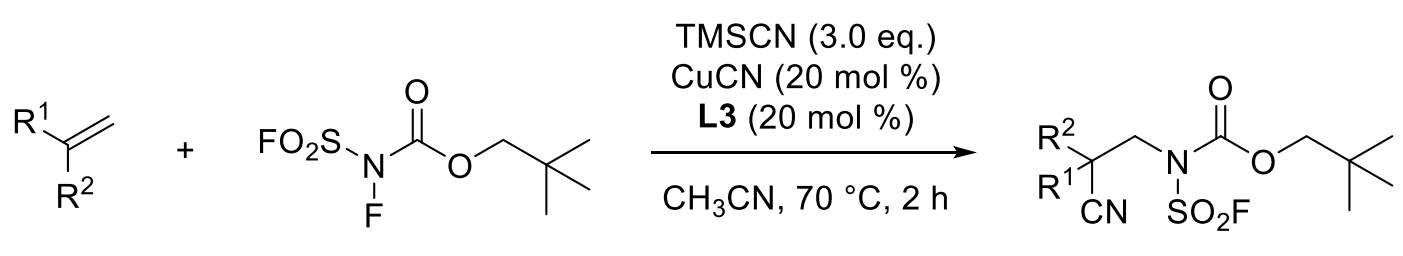

$1.4 \mathrm{eq}$.<smiles>O=C1C(=O)c2cccnc2-c2ncccc21</smiles>

L3

To an argon-purged Schlenk tube, CuCN $(40.0 \mu \mathrm{mol}, 3.6 \mathrm{mg})$, L3 $(40.0 \mu \mathrm{mol}, 8.4 \mathrm{mg})$, and acetonitrile $(1.00 \mathrm{~mL})$ were added at room temperature. After stirring for $10 \mathrm{~min}$ at the same temperature, alkene $(200 \mu \mathrm{mol})$, TMSCN $(600 \mu \mathrm{mol}, 83.4 \mu \mathrm{L}, 90 \%)$ and NFC $(280 \mu \mathrm{mol})$ were added at the same temperature. The solution was degassed and heated to $70^{\circ} \mathrm{C}$. After stirring for $2 \mathrm{~h}$, the solution was cooled to room temperature, diluted with DCM, filtered through a pad of silica gel and concentrated under vacuum. The residue was purified by flash column chromatography on silica gel.

\section{General Procedure C}

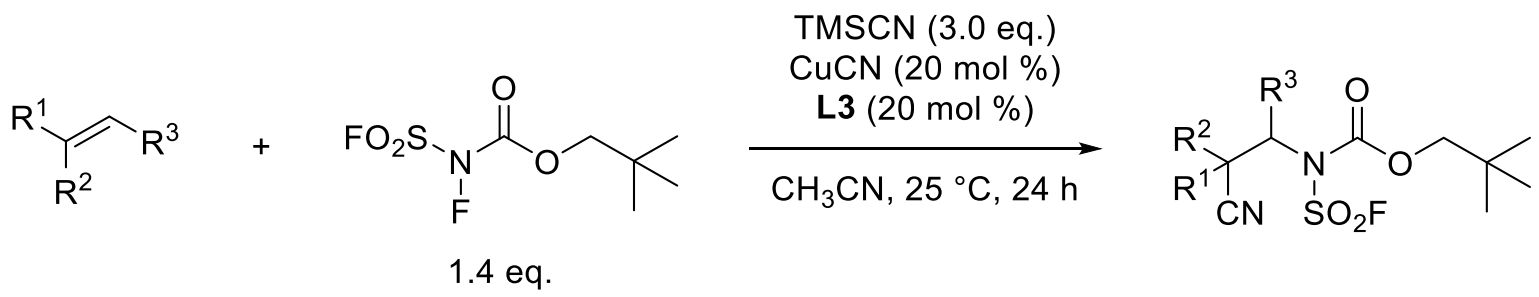

To an argon-purged Schlenk tube, CuCN $(40.0 \mu \mathrm{mol}, 3.6 \mathrm{mg})$, L3 $(40.0 \mu \mathrm{mol}, 8.4 \mathrm{mg})$, and acetonitrile $(1.00 \mathrm{~mL})$ were added at room temperature. After stirring for $10 \mathrm{~min}$ at the same temperature, alkene (200 $\mu \mathrm{mol})$, TMSCN $(600 \mu \mathrm{mol}, 83.4 \mu \mathrm{L}, 90 \%)$ and NFC (280 $\mu \mathrm{mol})$ were added at the same temperature. The solution was degassed and heated to $25^{\circ} \mathrm{C}$. After stirring for $24 \mathrm{~h}$, the solution was cooled to room temperature, diluted with DCM, filtered through a pad of silica gel and concentrated under vacuum. The residue was purified by flash column chromatography on silica gel. 


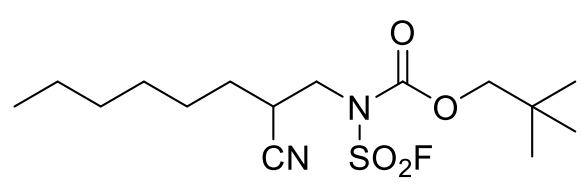

(38)

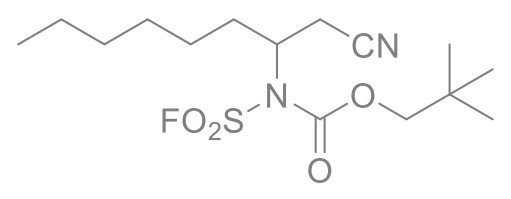

(38')

Prepared according to general procedure A with 1-octene $(200 \mu \mathrm{mol}, 31.4 \mu \mathrm{L})$ and NFC $(280 \mu \mathrm{mol}, 64.7 \mathrm{mg})$. The crude material was purified by flash column chromatography on silica gel (EtOAc/hexane 4-34\%) to give the title compound as a yellowish oil in $77 \%$ yield $(154 \mu \mathrm{mol}, 54.0 \mathrm{mg}, \mathrm{rr} 90: 10)$.

${ }^{1} \mathrm{H}$ NMR (392 MHz, CDCl $) \delta 4.18$ (ddd, $\left.J=14.6,9.3, J_{\mathrm{H}-\mathrm{F}}=1.9 \mathrm{~Hz}, 1 \mathrm{H}\right), 4.07(\mathrm{~d}, J=10.1 \mathrm{~Hz}, 1 \mathrm{H}), 4.03(\mathrm{~d}, J=$ $10.1 \mathrm{~Hz}, 1 \mathrm{H}), 3.91\left(\mathrm{ddd}, J=14.6,6.3, J_{\mathrm{H}-\mathrm{F}}=1.9 \mathrm{~Hz}, 1 \mathrm{H}\right), 3.06-3.15(\mathrm{~m}, 1 \mathrm{H}), 1.53-1.69(\mathrm{~m}, 3 \mathrm{H}), 1.41-1.52(\mathrm{~m}, 1 \mathrm{H})$, 1.27-1.38 (m, 6H), $1.01(\mathrm{~s}, 9 \mathrm{H}), 0.89(\mathrm{t}, J=7.0 \mathrm{~Hz}, 3 \mathrm{H})$.

${ }^{13} \mathrm{C}$ NMR $\left(99 \mathrm{MHz}, \mathrm{CDCl}_{3}\right) \delta 150.5,118.8,79.1,50.0,31.7,31.5,31.3,29.3,28.5,26.7,26.0,22.4,14.0$.

${ }^{19} \mathrm{~F}$ NMR $\left(369 \mathrm{MHz}, \mathrm{CDCl}_{3}\right) \delta 55.11$ (s, 1F).

HRMS (ESI) exact mass calculated for $\mathrm{C}_{15} \mathrm{H}_{27} \mathrm{O}_{4} \mathrm{~N}_{2} \mathrm{FSNa}: \mathrm{m} / z 373.1568\left([\mathrm{M}+\mathrm{Na}]^{+}\right)$, found: $\mathrm{m} / z 373.1558\left([\mathrm{M}+\mathrm{Na}]^{+}\right)$.

Scale up experiment To an argon-purged round bottom flask, CuCN (200 $\mu \mathrm{mol}, 17.9 \mathrm{mg})$, L2 (200 $\mu \mathrm{mol}, 54.5$ $\mathrm{mg})$, and acetonitrile $(10.0 \mathrm{~mL}$ ) were added at room temperature. After stirring for $10 \mathrm{~min}$ at the same temperature, 1-octene $(2.00 \mathrm{mmol}, 314 \mu \mathrm{L})$, TMSCN (2.80 mmol, $389 \mu \mathrm{L}, 90 \%)$ and NFC (2.80 mmol, $694 \mathrm{mg}, 93.3 \%)$ were added at the same temperature. The solution was degassed and heated to $70^{\circ} \mathrm{C}$. After stirring for $2 \mathrm{~h}$, the solution was cooled to room temperature, diluted with $\mathrm{CH}_{2} \mathrm{Cl}_{2}$, filtered through silica gel and concentrated under vacuum. The residue was purified by flash column chromatography on silica gel (1st DCM/hexane 12-100\%, 2nd EtOAc/hexane 2-20\%) to give the title compound (38) as a yellowish oil in $74 \%$ yield $(1.47 \mathrm{mmol}, 515 \mathrm{mg})$ and $\left.\mathbf{( 3 8}^{\prime}\right)$ as a yellowish oil in $9 \%$ yield $(186 \mu \mathrm{mol}, 65.1 \mathrm{mg})$.

\section{neopentyl (1-cyanooctan-2-yl)(fluorosulfonyl)carbamate (38')}

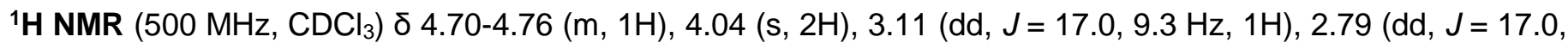
$6.4 \mathrm{~Hz}, 1 \mathrm{H}), 2.03-2.10(\mathrm{~m}, 1 \mathrm{H}), 1.70-1.77(\mathrm{~m}, 1 \mathrm{H}), 1.27-1.40(\mathrm{~m}, 8 \mathrm{H}), 1.01(\mathrm{~s}, 9 \mathrm{H}), 0.88(\mathrm{t}, J=7.0 \mathrm{~Hz}, 3 \mathrm{H})$.

${ }^{13} \mathrm{C} \mathrm{NMR}\left(126 \mathrm{MHz}, \mathrm{CDCl}_{3}\right) \delta 150.4,116.2,78.8,59.4,32.1,31.6,31.4,28.5,26.0,25.9,22.4,22.1,13.9$.

${ }^{19} \mathrm{~F}$ NMR $\left(471 \mathrm{MHz}, \mathrm{CDCl}_{3}\right) \delta 56.47(\mathrm{br}, 1 \mathrm{~F})$.

\section{neopentyl (2-cyano-5-oxohexyl)(fluorosulfonyl)carbamate (39)}

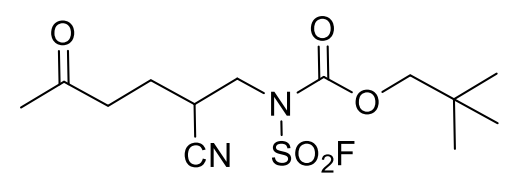

Prepared according to general procedure A with hex-5-en-2-one $(200 \mu \mathrm{mol}, 23.2 \mu \mathrm{L})$ and NFC (280 $\mu \mathrm{mol}, 64.7$ $\mathrm{mg}$ ). After stirring for $2 \mathrm{~h}$, the solution was cooled to room temperature, diluted with EtOAc, filtered through a pad of silica gel and concentrated under vacuum. The crude material was purified by flash column chromatography on silica gel (EtOAc/hexane 8-66\%) to give the title compound as a yellowish solid in 79\% yield (158 $\mu \mathrm{mol}, 53.0 \mathrm{mg}$, rr 89:11). 
${ }^{1} \mathbf{H}$ NMR $\left(392 \mathrm{MHz}, \mathrm{CDCl}_{3}\right) \delta 4.17$ (ddd, $\left.J=14.6,9.0, J_{\mathrm{H}-\mathrm{F}}=1.8 \mathrm{~Hz}, 1 \mathrm{H}\right), 4.07(\mathrm{~d}, J=10.1 \mathrm{~Hz}, 1 \mathrm{H}), 4.03(\mathrm{~d}, J=$ $10.1 \mathrm{~Hz}, 1 \mathrm{H}), 3.95\left(\mathrm{ddd}, J=14.7,6.5, J_{\mathrm{H}-\mathrm{F}}=1.7 \mathrm{~Hz}, 1 \mathrm{H}\right), 3.15-3.28(\mathrm{~m}, 1 \mathrm{H}), 2.65-2.80(\mathrm{~m}, 2 \mathrm{H}), 2.20(\mathrm{~s}, 3 \mathrm{H}), 1.95-$ $2.05(\mathrm{~m}, 1 \mathrm{H}), 1.75-1.84(\mathrm{~m}, 1 \mathrm{H}), 1.01(\mathrm{~s}, 9 \mathrm{H})$.

${ }^{13} \mathrm{C}$ NMR $\left(99 \mathrm{MHz}, \mathrm{CDCl}_{3}\right) \delta 206.0,150.4,137.1,118.3,79.1,49.8,39.7,31.5,30.8,29.9,26.0,22.9$.

${ }^{19} \mathrm{~F}$ NMR $\left(369 \mathrm{MHz}, \mathrm{CDCl}_{3}\right) \delta 55.06$ (s, 1F).

HRMS (ESI) exact mass calculated for $\mathrm{C}_{13} \mathrm{H}_{21} \mathrm{O}_{5} \mathrm{~N}_{2} \mathrm{FSNa}: \mathrm{m} / z 359.1047\left([\mathrm{M}+\mathrm{Na}]^{+}\right)$, found: $m / z 359.1041\left([\mathrm{M}+\mathrm{Na}]^{+}\right)$.

\section{methyl 5-cyano-6-((fluorosulfonyl)((neopentyloxy)carbonyl)amino)hexanoate (40)}<smiles>COC(=O)CCCC(C#N)CN(C(=O)O)C(=O)OCC(C)(C)C</smiles>

Prepared according to general procedure A with methyl hex-5-enoate $(200 \mu \mathrm{mol}, 29 \mu \mathrm{L}, 98 \%)$ and NFC (280 $\mu \mathrm{mol}$, $66.1 \mathrm{mg}, 98 \%$ ). After stirring for $2 \mathrm{~h}$, the solution was cooled to room temperature, diluted with EtOAc, filtered through a pad of silica gel and concentrated under vacuum. The crude material was purified by flash column chromatography on silica gel (EtOAc/hexane 8-66\%) to give the title compound as a yellowish oil in $88 \%$ yield (175 $\mu \mathrm{mol}, 64.1 \mathrm{mg}, \mathrm{rr} 89: 11)$.

${ }^{1} \mathbf{H}$ NMR $\left(500 \mathrm{MHz}, \mathrm{CDCl}_{3}\right) \delta 4.19$ (ddd, $\left.J=14.6,9.3, J_{\mathrm{H}-\mathrm{F}}=1.5 \mathrm{~Hz}, 1 \mathrm{H}\right), 4.07(\mathrm{~d}, J=10.0 \mathrm{~Hz}, 1 \mathrm{H}), 4.04(\mathrm{~d}, J=$ $10.0 \mathrm{~Hz}, 1 \mathrm{H}$ ), 3.93 (ddd, $\left.J=14.6,6.3, J_{\mathrm{H}-\mathrm{F}}=1.4 \mathrm{~Hz}, 1 \mathrm{H}\right), 3.69(\mathrm{~s}, 3 \mathrm{H}), 3.11-3.17(\mathrm{~m}, 1 \mathrm{H}), 2.40(\mathrm{t}, J=7.0 \mathrm{~Hz}, 2 \mathrm{H})$, 1.90-1.98 (m, 1H), 1.76-1.85 (m, 1H), 1.67-1.73 (m, 2H), $1.01(\mathrm{~s}, 9 \mathrm{H})$.

${ }^{13} \mathrm{C}$ NMR $\left(126 \mathrm{MHz}, \mathrm{CDCl}_{3}\right) \delta 172.8,150.4,118.3,79.1,51.7,49.8,32.9,31.5,31.4,28.5,26.0,22.0$.

${ }^{19} \mathrm{~F}$ NMR $\left(471 \mathrm{MHz}, \mathrm{CDCl}_{3}\right) \delta 55.13(\mathrm{~s}, 1 \mathrm{~F})$.

HRMS (ESI) exact mass calculated for $\mathrm{C}_{14} \mathrm{H}_{23} \mathrm{O}_{6} \mathrm{~N}_{2} \mathrm{FSNa}: \mathrm{m} / z 389.1153\left([\mathrm{M}+\mathrm{Na}]^{+}\right)$, found: $m / z 389.1147\left([\mathrm{M}+\mathrm{Na}]^{+}\right)$.

\section{neopentyl (2-cyano-4-(1,3-dioxoisoindolin-2-yl)butyl)(fluorosulfonyl)carbamate (41)}

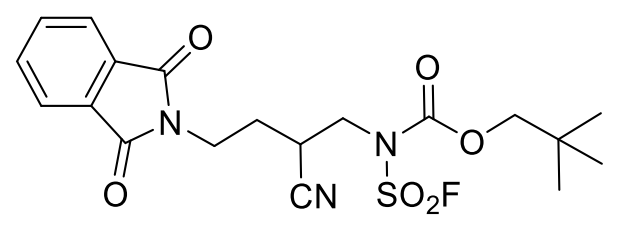

Prepared according to general procedure A with 2-(but-3-en-1-yl)isoindoline-1,3-dione (200 $\mu \mathrm{mol}, 40.2 \mathrm{mg})^{18}$ and NFC $(280 \mu \mathrm{mol}, 66.1 \mathrm{mg}, 98 \%)$. After stirring for $2 \mathrm{~h}$, the solution was cooled to room temperature, diluted with EtOAc, filtered through a pad of silica gel and concentrated under vacuum. The crude material was purified by flash column chromatography on silica gel (EtOAc/hexane 8-66\%) to give the title compound as a white solid in 89\% yield (178 $\mu \mathrm{mol}, 78.3 \mathrm{mg}$, rr 89:11).

${ }^{1} \mathbf{H}$ NMR $\left(392 \mathrm{MHz}, \mathrm{CDCl}_{3}\right) \delta$ 7.83-7.88 (m, 2H), 7.72-7.77 (m, 2H), 4.23 (ddd, $\left.J=14.7,9.1, J_{\mathrm{H}-\mathrm{F}}=1.3 \mathrm{~Hz}, 1 \mathrm{H}\right)$, 3.98-4.07 (m, 3H), 3.87-3.96 (m, 2H), 3.15-3.25 (m, 1H), 1.98-2.17 (m, 2H), $0.99(\mathrm{~s}, 9 \mathrm{H})$.

${ }^{13} \mathrm{C}$ NMR $\left(99 \mathrm{MHz}, \mathrm{CDCl}_{3}\right) \delta 168.0,150.3,134.2,131.7,123.4,117.9,79.1,49.5,35.0,31.4,29.4,28.2,26.0$.

${ }^{19} \mathrm{~F}$ NMR $\left(369 \mathrm{MHz}, \mathrm{CDCl}_{3}\right) \delta 55.13(\mathrm{~s}, 1 \mathrm{~F})$.

HRMS (ESI) exact mass calculated for $\mathrm{C}_{19} \mathrm{H}_{22} \mathrm{O}_{6} \mathrm{~N}_{3} \mathrm{FSNa}: \mathrm{m} / z 462.1106\left([\mathrm{M}+\mathrm{Na}]^{+}\right)$, found: $m / z 462.1107\left([\mathrm{M}+\mathrm{Na}]^{+}\right)$. 
<smiles></smiles>

Prepared according to general procedure A with 4-bromobut-1-ene $(200 \mu \mathrm{mol}, 20.9 \mu \mathrm{L})$ and NFC $(280 \mu \mathrm{mol}, 66.1$ $\mathrm{mg}, 98 \%$ ). The crude material was purified by flash column chromatography on silica gel (EtOAc/hexane $4-34 \%$ ) to give the title compound as a yellowish oil in $83 \%$ yield $(166 \mu \mathrm{mol}, 61.8 \mathrm{mg}, \mathrm{rr} 88: 12)$.

${ }^{1} \mathrm{H}$ NMR $\left(500 \mathrm{MHz}, \mathrm{CDCl}_{3}\right) \delta 4.23$ (ddd, $\left.J=14.7,8.7, J_{\mathrm{H}-\mathrm{F}}=1.6 \mathrm{~Hz}, 1 \mathrm{H}\right), 4.08(\mathrm{~d}, J=10.0 \mathrm{~Hz}, 1 \mathrm{H}), 4.05(\mathrm{~d}, J=$ $10.0 \mathrm{~Hz}, 1 \mathrm{H}), 4.00$ (ddd, $\left.J=14.6,6.6, J_{\mathrm{H}-\mathrm{F}}=1.7 \mathrm{~Hz}, 1 \mathrm{H}\right), 3.61-3.65(\mathrm{~m}, 1 \mathrm{H}), 3.44-3.55(\mathrm{~m}, 2 \mathrm{H}), 2.25(\mathrm{qd}, J=9.9$, $4.9 \mathrm{~Hz}, 1 \mathrm{H}), 2.07-2.14(\mathrm{~m}, 1 \mathrm{H}), 1.01(\mathrm{~s}, 9 \mathrm{H})$.

${ }^{13} \mathrm{C}$ NMR $\left(126 \mathrm{MHz}, \mathrm{CDCl}_{3}\right) \delta 150.4,117.5,79.3,49.4,32.2,31.5,30.6,28.3,26.0$.

${ }^{19} \mathrm{~F}$ NMR $\left(471 \mathrm{MHz}, \mathrm{CDCl}_{3}\right) \delta 55.06(\mathrm{~s}, 1 \mathrm{~F})$.

HRMS (ESI) exact mass calculated for $\mathrm{C}_{11} \mathrm{H}_{18} \mathrm{O}_{4} \mathrm{~N}_{2} \mathrm{FSBrNa}: \mathrm{m} / z 395.0047\left([\mathrm{M}+\mathrm{Na}]^{+}\right)$, found: $\mathrm{m} / \mathbf{z} 395.0033$ $\left([\mathrm{M}+\mathrm{Na}]^{+}\right)$.

neopentyl (2-cyano-4-phenylbutyl)(fluorosulfonyl)carbamate (43)<smiles></smiles>

Prepared according to general procedure A with but-3-en-1-ylbenzene $(200 \mu \mathrm{mol}, 30.0 \mu \mathrm{L})$ and NFC $(280 \mu \mathrm{mol}$, $64.7 \mathrm{mg}$ ). The crude material was purified by flash column chromatography on silica gel (EtOAc/hexane 4-34\%) to give the title compound as a yellowish solid in 39\% yield $(78.8 \mu \mathrm{mol}, 29.2 \mathrm{mg}$, $\mathrm{rr} 91: 9)$.

${ }^{1} \mathrm{H}$ NMR $\left(392 \mathrm{MHz}, \mathrm{CDCl}_{3}\right) \delta$ 7.15-7.34 (m, $\left.5 \mathrm{H}\right), 4.18$ (ddd, $\left.J=14.6,9.0, J_{\mathrm{H}-\mathrm{F}}=1.8 \mathrm{~Hz}, 1 \mathrm{H}\right), 4.04(\mathrm{~d}, J=10.1 \mathrm{~Hz}$, $1 \mathrm{H}), 4.01(\mathrm{~d}, J=10.3 \mathrm{~Hz}, 1 \mathrm{H}), 3.93\left(\mathrm{ddd}, J=14.8,6.5, J_{\mathrm{H}-\mathrm{F}}=1.7 \mathrm{~Hz}, 1 \mathrm{H}\right), 3.04-3.12(\mathrm{~m}, 1 \mathrm{H}), 2.92-2.99(\mathrm{~m}, 1 \mathrm{H})$, 2.73-2.81 (m, 1H), 1.86-2.02 (m, 2H), $0.98(\mathrm{~s}, 9 \mathrm{H})$.

${ }^{13} \mathrm{C}$ NMR (99 MHz, $\left.\mathrm{CDCl}_{3}\right) \delta 150.4,139.2,128.8,128.3,126.7,118.4,79.1,49.9,32.7,31.5,31.1,31.0,26.0$.

${ }^{19} \mathrm{~F}$ NMR $\left(369 \mathrm{MHz}, \mathrm{CDCl}_{3}\right) \delta 55.04$ (s, 1F).

HRMS (ESI) exact mass calculated for $\mathrm{C}_{17} \mathrm{H}_{23} \mathrm{O}_{4} \mathrm{~N}_{2} \mathrm{FSNa}: \mathrm{m} / z 393.1255\left([\mathrm{M}+\mathrm{Na}]^{+}\right)$, found: $\mathrm{m} / z 393.1253\left([\mathrm{M}+\mathrm{Na}]^{+}\right)$.

\section{neopentyl (2-cyano-5-hydroxypentyl)(fluorosulfonyl)carbamate (44)}<smiles>CC(C)(C)COC(=O)N(CC(C#N)CCCO)S(=O)(=O)[O-]</smiles>

Prepared according to general procedure A with pent-4-en-1-ol (200 $\mu \mathrm{mol}, 20.7 \mu \mathrm{L})$ and NFC (280 $\mu \mathrm{mol}, 64.7 \mathrm{mg})$. After stirring for $2 \mathrm{~h}$, the solution was cooled to room temperature, diluted with EtOAc, filtered through a pad of silica gel and concentrated under vacuum. The crude material was purified by flash column chromatography on silica gel (EtOAc/hexane 12-100\%) to give the title compound as a yellowish solid in 66\% yield (132 $\mu$ mol, 42.8 mg, rr 89:11).

${ }^{1} \mathrm{H}$ NMR $\left(392 \mathrm{MHz}, \mathrm{CDCl}_{3}\right) \delta 4.20\left(\mathrm{ddd}, J=14.6,9.4, J_{\mathrm{H}-\mathrm{F}}=1.9 \mathrm{~Hz}, 1 \mathrm{H}\right), 4.07(\mathrm{~d}, J=10.1 \mathrm{~Hz}, 1 \mathrm{H}), 4.03(\mathrm{~d}, J=$ $10.1 \mathrm{~Hz}, 1 \mathrm{H}), 3.94\left(\mathrm{ddd}, J=14.6,6.3, J_{\mathrm{H}-\mathrm{F}}=1.8 \mathrm{~Hz}, 1 \mathrm{H}\right), 3.69-3.73(\mathrm{~m}, 2 \mathrm{H}), 3.16-3.24(\mathrm{~m}, 1 \mathrm{H}), 1.72-1.93(\mathrm{~m}, 5 \mathrm{H})$, $1.01(\mathrm{~s}, 9 \mathrm{H})$.

${ }^{13} \mathrm{C}$ NMR $\left(99 \mathrm{MHz}, \mathrm{CDCl}_{3}\right) \delta 150.5,118.7,79.2,61.4,50.0,31.5,31.5,29.4,26.0,26.0$.

${ }^{19} \mathrm{~F}$ NMR $\left(369 \mathrm{MHz}, \mathrm{CDCl}_{3}\right) \delta 55.06$ (s, 1F). 
HRMS (ESI) exact mass calculated for $\mathrm{C}_{12} \mathrm{H}_{21} \mathrm{O}_{5} \mathrm{~N}_{2} \mathrm{FSNa}: \mathrm{m} / z 347.1047\left([\mathrm{M}+\mathrm{Na}]^{+}\right)$, found: $\mathrm{m} / z 347.1037\left([\mathrm{M}+\mathrm{Na}]^{+}\right)$.

4-cyano-5-((fluorosulfonyl)((neopentyloxy)carbonyl)amino)pentanoic acid (45)<smiles></smiles>

Prepared according to general procedure A with pent-4-enoic acid $(200 \mu \mathrm{mol}, 20.8 \mu \mathrm{L})$ and NFC $(280 \mu \mathrm{mol}, 66.1$ $\mathrm{mg}, 98 \%)$. After stirring for $2 \mathrm{~h}$, the solution was cooled to room temperature, diluted with EtOAc, filtered through a pad of silica gel and concentrated under vacuum. The crude material was purified by flash column chromatography on silica gel (1st EtOAc/hexane 25-50\% with $\mathrm{AcOH} 1 \%$, 2nd acetone/hexane $20 \%$ with $\mathrm{AcOH}$ $1 \%)$ to give the title compound as a yellowish solid in 69\% yield (137 $\mu \mathrm{mol}, 46.5 \mathrm{mg}, \mathrm{rr} 85: 15)$.

H NMR (396 MHz, CDCl $)$ ס $9.60(\mathrm{br}, 1 \mathrm{H}), 4.20$ (ddd, $\left.J=14.6,8.7, J_{\mathrm{H}-\mathrm{F}}=1.6 \mathrm{~Hz}, 1 \mathrm{H}\right), 4.07(\mathrm{~d}, J=10.2 \mathrm{~Hz}, 1 \mathrm{H})$, $4.04(\mathrm{~d}, J=10.2 \mathrm{~Hz}, 1 \mathrm{H}), 3.98$ (ddd, $\left.J=14.7,6.6, J_{\mathrm{H}-\mathrm{F}}=1.4 \mathrm{~Hz}, 1 \mathrm{H}\right), 3.25-3.33(\mathrm{~m}, 1 \mathrm{H}), 2.58-2.76(\mathrm{~m}, 2 \mathrm{H}), 1.87-$ $2.10(\mathrm{~m}, 2 \mathrm{H}), 1.01(\mathrm{~s}, 9 \mathrm{H})$.

${ }^{13} \mathrm{C}$ NMR $\left(99 \mathrm{MHz}, \mathrm{CDCl}_{3}\right) \delta 177.4,150.4,117.9,79.3,49.6,31.5,30.9,30.6,26.0,24.1$.

${ }^{19} \mathrm{~F}$ NMR $\left(369 \mathrm{MHz}, \mathrm{CDCl}_{3}\right) \delta 55.04$ (s, 1F).

HRMS (ESI) exact mass calculated for $\mathrm{C}_{12} \mathrm{H}_{19} \mathrm{O}_{6} \mathrm{~N}_{2} \mathrm{FSNa}: \mathrm{m} / z 361.0840\left([\mathrm{M}+\mathrm{Na}]^{+}\right)$, found: $\mathrm{m} / z 361.0838\left([\mathrm{M}+\mathrm{Na}]^{+}\right)$.

neopentyl (2-cyano-2-cyclohexylethyl)(fluorosulfonyl)carbamate (46)

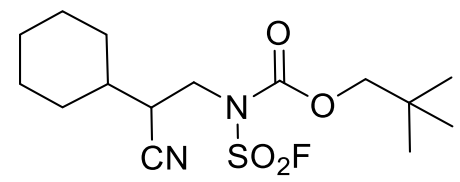

Prepared according to general procedure A with vinylcyclohexane $(200 \mu \mathrm{mol}, 27.4 \mu \mathrm{L})$ and NFC $(280 \mu \mathrm{mol}, 71.9$ $\mathrm{mg}, 90 \%)$. The crude material was purified by flash column chromatography on silica gel (EtOAc/hexane 4-34\%) to give the title compound as a white solid in $73 \%$ yield $(145 \mu \mathrm{mol}, 50.5 \mathrm{mg}, \mathrm{rr} 91: 9)$.

${ }^{1} \mathrm{H}$ NMR $\left(392 \mathrm{MHz}, \mathrm{CDCl}_{3}\right) \delta 4.23\left(\mathrm{ddd}, J=14.6,10.2, J_{\mathrm{H}-\mathrm{F}}=2.1 \mathrm{~Hz}, 1 \mathrm{H}\right), 4.07(\mathrm{~d}, J=10.1 \mathrm{~Hz}, 1 \mathrm{H}), 4.03(\mathrm{~d}, J=$ $10.1 \mathrm{~Hz}, 1 \mathrm{H}), 3.93\left(\mathrm{ddd}, J=14.5,5.8, J_{\mathrm{H}-\mathrm{F}}=1.6 \mathrm{~Hz}, 1 \mathrm{H}\right), 2.99-3.04(\mathrm{~m}, 1 \mathrm{H}), 1.57-1.90(\mathrm{~m}, 7 \mathrm{H}), 1.21-1.34(\mathrm{~m}, 5 \mathrm{H})$, $1.01(\mathrm{~s}, 9 \mathrm{H})$.

${ }^{13} \mathrm{C}$ NMR $\left(99 \mathrm{MHz}, \mathrm{CDCl}_{3}\right) \delta 150.4,117.9,79.0,48.6,38.0,37.4,31.5,31.2,29.0,26.0,25.8,25.6,25.5$.

${ }^{19} \mathrm{~F}$ NMR $\left(369 \mathrm{MHz}, \mathrm{CDCl}_{3}\right) \delta 55.38$ (s, 1F).

HRMS (ESI) exact mass calculated for $\mathrm{C}_{15} \mathrm{H}_{25} \mathrm{O}_{4} \mathrm{~N}_{2} \mathrm{FSNa}: \mathrm{m} / z 371.1411\left([\mathrm{M}+\mathrm{Na}]^{+}\right)$, found: $\mathrm{m} / z 371.1416\left([\mathrm{M}+\mathrm{Na}]^{+}\right)$.

neopentyl (2-cyano-3-hydroxy-3-methylbutyl)(fluorosulfonyl)carbamate (47)<smiles>CC(C)(C)COC(=O)N(CC(C#N)C(C)(C)O)S(=O)(=O)[O-]</smiles>

Prepared according to general procedure A with 2-methylbut-3-en-2-ol (200 $\mu \mathrm{mol}, 21.6 \mu \mathrm{L}, 97 \%)$ and NFC (280 $\mu \mathrm{mol}, 66.1 \mathrm{mg}, 98 \%)$. After stirring for $2 \mathrm{~h}$, the solution was cooled to room temperature, diluted with EtOAc, filtered through a pad of silica gel and concentrated under vacuum. The crude material was diluted with THF $(1.0 \mathrm{~mL})$, and $\mathrm{AcOH}(1.0 \mathrm{mmol}, 57.2 \mu \mathrm{L})$ and TBAF ( $1 \mathrm{M} \mathrm{THF}$ solution, $0.2 \mathrm{~mL})$ were added to this solution at $0{ }^{\circ} \mathrm{C}$ to cleave the TMS group from the alcohol. After stirring for $3 \mathrm{~h}$ at the same temperature, the reaction was quenched with 1 $\mathrm{N} \mathrm{HCl}$ aq. $(10 \mathrm{~mL})$, extracted with EtOAc $(10 \mathrm{~mL} \times 3)$ and concentrated under vacuum. The residue was diluted 
with EtOAc, filtered through a pad of silica gel and concentrated under vacuum. The crude material was purified by flash column chromatography on silica gel (1st acetone/hexane 8-66\%, 2nd EtOAc/hexane 8-66\%) to give the title compound as a yellowish oil in $30 \%$ yield $(59.8 \mu \mathrm{mol}, 19.4 \mathrm{mg}, \mathrm{rr} 97: 3)$.

${ }^{1} \mathrm{H}$ NMR $\left(392 \mathrm{MHz}, \mathrm{CDCl}_{3}\right) \delta 4.38$ (ddd, $\left.J=14.7,11.0, J_{\mathrm{H}-\mathrm{F}}=2.1 \mathrm{~Hz}, 1 \mathrm{H}\right), 4.12\left(\mathrm{ddd}, J=14.7,4.9, J_{\mathrm{H}-\mathrm{F}}=1.8 \mathrm{~Hz}\right.$, $1 \mathrm{H}), 4.08(\mathrm{~d}, J=10.1 \mathrm{~Hz}, 1 \mathrm{H}), 4.03(\mathrm{~d}, J=10.1 \mathrm{~Hz}, 1 \mathrm{H}), 3.18(\mathrm{dd}, J=11.0,4.9 \mathrm{~Hz}, 1 \mathrm{H}), 1.97(\mathrm{br}, 1 \mathrm{H}), 1.47(\mathrm{~s}$, $3 \mathrm{H}), 1.44(\mathrm{~s}, 3 \mathrm{H}), 1.01(\mathrm{~s}, 9 \mathrm{H})$.

${ }^{13} \mathrm{C}$ NMR $\left(99 \mathrm{MHz}, \mathrm{CDCl}_{3}\right) \delta 150.5,117.6,79.1,70.9,47.1,43.7,31.5,28.1,27.3,26.1$.

${ }^{19} \mathrm{~F}$ NMR $\left(369 \mathrm{MHz}, \mathrm{CDCl}_{3}\right) \delta 55.55(\mathrm{~s}, 1 \mathrm{~F})$.

HRMS (ESI) exact mass calculated for $\mathrm{C}_{12} \mathrm{H}_{21} \mathrm{O}_{5} \mathrm{~N}_{2} \mathrm{FSNa}: \mathrm{m} / z 347.1047\left([\mathrm{M}+\mathrm{Na}]^{+}\right)$, found: $\mathrm{m} / z 347.1046\left([\mathrm{M}+\mathrm{Na}]^{+}\right)$.

neopentyl rel-(4R,5S)-(5-cyanooctan-4-yl)(fluorosulfonyl)carbamate (48) and neopentyl rel-(4S,5S)-(5cyanooctan-4-yl)(fluorosulfonyl)carbamate (48')

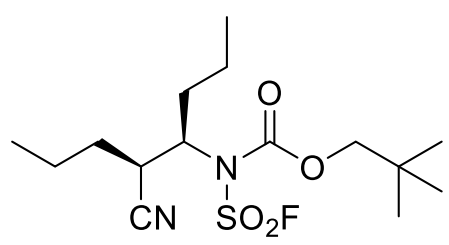

48

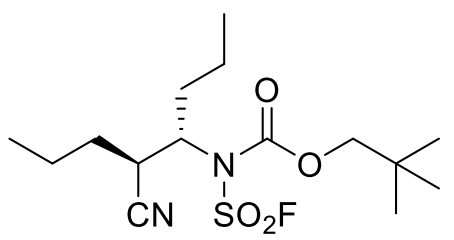

48'

Prepared according to general procedure A with (E)-oct-4-ene (200 $\mu \mathrm{mol}, 31.7 \mu \mathrm{L}, 99 \%)$ and NFC (280 $\mu \mathrm{mol}, 66.1$ $\mathrm{mg}, 98 \%)$. The crude material was purified by flash column chromatography on silica gel (1st DCM/hexane 12 $100 \%$, 2nd EtOAc/hexane 4-34\%) to give the title compound as a yellowish oil in $67 \%$ yield (134 $\mu \mathrm{mol}, 46.9 \mathrm{mg}$, 48:48' $=69: 31)$.

${ }^{1} \mathrm{H}$ NMR $\left(500 \mathrm{MHz}, \mathrm{CDCl}_{3}\right) \delta 4.50(\mathrm{br}, 1 \mathrm{H}), 4.04-4.08(\mathrm{~m}, 2 \mathrm{H}), 3.38(\mathrm{br}, 1 \mathrm{H}), 1.91-2.17(\mathrm{~m}, 2 \mathrm{H}), 1.32-1.73(\mathrm{~m}, 6 \mathrm{H})$, 0.96-1.01 (m, 15H).

${ }^{13} \mathrm{C}$ NMR $\left(99 \mathrm{MHz}, \mathrm{CDCl}_{3}\right) \delta 150.2,119.6,118.8,78.8,63.7,36.0,35.3,33.0,31.9,31.8,31.6,30.9,26.03,26.00$, $20.3,19.9,19.34,19.30,13.51,13.48,13.4,13.3$.

${ }^{19} \mathrm{~F}$ NMR (471 MHz, $\left.\mathrm{CDCl}_{3}\right) \delta 61.29$ (br 0.3F), 52.63 (br, 0.7F).

HRMS (ESI) exact mass calculated for $\mathrm{C}_{15} \mathrm{H}_{27} \mathrm{O}_{4} \mathrm{~N}_{2} \mathrm{FSNa}: \mathrm{m} / z 373.1568\left([\mathrm{M}+\mathrm{Na}]^{+}\right)$, found: $\mathrm{m} / z 373.1563\left([\mathrm{M}+\mathrm{Na}]^{+}\right)$. CIF File CCDC 2084401 contains the supplementary crystallographic data of 48 after derivatization to $\mathrm{N}$-(4nitrobenzoyl)amide.

Reaction of (Z)-oct-4-ene Prepared according to general procedure A with (Z)-oct-4-ene (200 $\mu \mathrm{mol}, 33 \mu \mathrm{L}, 95 \%$ ) and NFC (280 $\mu \mathrm{mol}, 64.7 \mathrm{mg},>99 \%)$. The crude material was purified by flash column chromatography on silica gel (1st DCM/hexane 12-100\%, 2nd EtOAc/hexane 4-34\%) to give the title compound as a yellowish oil in $65 \%$ yield (130 $\mu \mathrm{mol}, 45.4 \mathrm{mg}$, dr 68:32).

neopentyl trans-(2-cyanocyclohexyl)(fluorosulfonyl)carbamate (49)<smiles>CC(C)(C)COC(=O)N([O-])[C@H]1CCCC[C@H]1C#N</smiles>

Prepared according to general procedure A with cyclohexene (200 $\mu \mathrm{mol}, 20.9 \mu \mathrm{L}, 97 \%)$ and NFC (280 $\mu \mathrm{mol}, 66.1$ $\mathrm{mg}, 98 \%)$. The crude material was purified by flash column chromatography on silica gel (EtOAc/hexane 4-34\%) to give the title compound as a yellowish oil in 73\% yield (147 $\mu \mathrm{mol}, 47.0 \mathrm{mg}$, dr 97:3). 


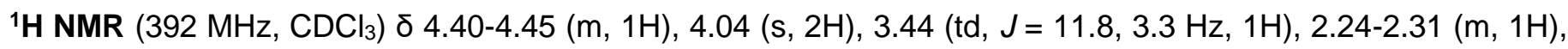
$2.11(\mathrm{dtd}, J=12.6,12.6,3.8 \mathrm{~Hz}, 1 \mathrm{H}), 1.97-2.02(\mathrm{~m}, 1 \mathrm{H}), 1.87-1.94(\mathrm{~m}, 1 \mathrm{H}), 1.76-1.83(\mathrm{~m}, 1 \mathrm{H}), 1.67(\mathrm{dtd}, J=13.0$, 13.0, 3.6 Hz, $1 \mathrm{H}), 1.41$ (dtt, $J=13.2,13.2,3.7 \mathrm{~Hz}, 1 \mathrm{H}), 1.24(\mathrm{dtt}, J=13.3,13.3,3.6 \mathrm{~Hz}, 1 \mathrm{H}), 1.01(\mathrm{~s}, 9 \mathrm{H})$

${ }^{13} \mathrm{C}$ NMR $\left(99 \mathrm{MHz}, \mathrm{CDCl}_{3}\right) \delta 150.5,119.1,78.8,63.0,32.1,31.5,30.4,29.4,26.0,25.1,23.7$.

${ }^{19} \mathrm{~F}$ NMR $\left(369 \mathrm{MHz}, \mathrm{CDCl}_{3}\right) \delta 55.50(\mathrm{~s}, 1 \mathrm{~F})$.

HRMS (ESI) exact mass calculated for $\mathrm{C}_{13} \mathrm{H}_{21} \mathrm{O}_{4} \mathrm{~N}_{2} \mathrm{FSNa}: \mathrm{m} / z 343.1098\left([\mathrm{M}+\mathrm{Na}]^{+}\right)$, found: $m / z 343.1103\left([\mathrm{M}+\mathrm{Na}]^{+}\right)$.

neopentyl (rel-(1S,2R,3R,4R)-3-cyanobicyclo[2.2.1]heptan-2-yl)(fluorosulfonyl)carbamate (50)

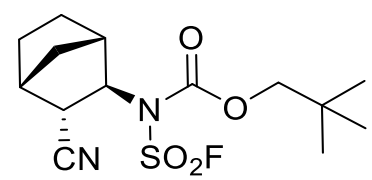

Prepared according to general procedure A with bicyclo[2.2.1]hept-2-ene (200 $\mu \mathrm{mol}, 19.0 \mathrm{mg}, 99 \%)$ and NFC (280 $\mu \mathrm{mol}, 66.1 \mathrm{mg}, 98 \%)$. The crude material was purified by flash column chromatography on silica gel (EtOAc/hexane 4-34\%) to give the title compound as a colorless oil in $82 \%$ yield ( $160 \mu \mathrm{mol}, 55.3 \mathrm{mg}, 98 \%$, $\mathrm{dr}$ 82:13:5).

${ }^{1} \mathrm{H}$ NMR $\left(392 \mathrm{MHz}, \mathrm{CDCl}_{3}\right) \delta 4.37(\mathrm{~d}, J=6.7 \mathrm{~Hz}, 1 \mathrm{H}), 4.02(\mathrm{~d}, J=10.3 \mathrm{~Hz}, 1 \mathrm{H}), 3.98(\mathrm{~d}, J=10.1 \mathrm{~Hz}, 1 \mathrm{H}), 3.50-$ $3.53(\mathrm{~m}, 1 \mathrm{H}), 2.68(\mathrm{~s}, 1 \mathrm{H}), 2.54(\mathrm{~s}, 1 \mathrm{H}), 2.21-2.26(\mathrm{~m}, 1 \mathrm{H}), 1.63-1.82(\mathrm{~m}, 3 \mathrm{H}), 1.45-1.59(\mathrm{~m}, 1 \mathrm{H}), 1.36-1.39(\mathrm{~m}$, $1 \mathrm{H}), 1.00(\mathrm{~s}, 9 \mathrm{H})$

${ }^{13} \mathrm{C}$ NMR $\left(99 \mathrm{MHz}, \mathrm{CDCl}_{3}\right) \delta 150.7,119.8,79.0,68.8,42.8,40.8,38.1,36.8,31.4,30.1,26.0,23.3$.

${ }^{19} \mathrm{~F}$ NMR $\left(369 \mathrm{MHz}, \mathrm{CDCl}_{3}\right) \delta 55.55(\mathrm{br}, 1 \mathrm{~F})$.

HRMS (ESI) exact mass calculated for $\mathrm{C}_{14} \mathrm{H}_{21} \mathrm{O}_{4} \mathrm{NFS}{ }_{2} \mathrm{Na}: \mathrm{m} / z 355.1098\left([\mathrm{M}+\mathrm{Na}]^{+}\right)$, found: $m / z 355.1092\left([\mathrm{M}+\mathrm{Na}]^{+}\right)$. CIF File CCDC 2084402 contains the supplementary crystallographic data of $\mathbf{5 0}$ after derivatization to $\mathrm{N}$-benzyl sulfamide.

neopentyl (2-cyano-2-methyldecyl)(fluorosulfonyl)carbamate (51)

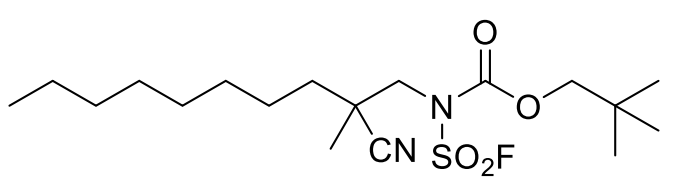

Prepared according to general procedure B with 2-methyldec-1-ene $(200 \mu \mathrm{mol}, 30.9 \mathrm{mg})$ and NFC (280 $\mu \mathrm{mol}$, $66.1 \mathrm{mg}, 98 \%)$. The crude material was purified by flash column chromatography on silica gel (DCM/hexane 12$100 \%)$ to give the title compound as a yellowish oil in $57 \%$ yield $(113 \mu \mathrm{mol}, 44.5 \mathrm{mg})$.

${ }^{1} \mathrm{H}$ NMR $\left(392 \mathrm{MHz}, \mathrm{CDCl}_{3}\right) \delta 4.16\left(\mathrm{dd}, J=15.1, J_{\mathrm{H}-\mathrm{F}}=2.1 \mathrm{~Hz}, 1 \mathrm{H}\right), 4.08(\mathrm{~d}, J=10.1 \mathrm{~Hz}, 1 \mathrm{H}), 4.05(\mathrm{~d}, J=10.1 \mathrm{~Hz}$, $1 \mathrm{H}), 3.96\left(\mathrm{dd}, J=15.0, J_{\mathrm{H}-\mathrm{F}}=1.6 \mathrm{~Hz}, 1 \mathrm{H}\right), 1.62-1.70(\mathrm{~m}, 1 \mathrm{H}), 1.47-1.53(\mathrm{~m}, 3 \mathrm{H}), 1.41(\mathrm{~s}, 3 \mathrm{H}), 1.28-1.32(\mathrm{~m}, 10 \mathrm{H})$, $1.01(\mathrm{~s}, 9 \mathrm{H}), 0.89(\mathrm{t}, J=7.0 \mathrm{~Hz}, 3 \mathrm{H})$.

${ }^{13} \mathrm{C}$ NMR $\left(99 \mathrm{MHz}, \mathrm{CDCl}_{3}\right) \delta 150.9,121.4,79.1,54.9,38.5,37.6,31.7,31.5,29.5,29.2,29.1,26.1,24.6,22.6$, 22.5, 14.1 .

${ }^{19} \mathrm{~F}$ NMR $\left(369 \mathrm{MHz}, \mathrm{CDCl}_{3}\right) \delta 54.88(\mathrm{~s}, 1 \mathrm{~F})$.

HRMS (ESI) exact mass calculated for $\mathrm{C}_{18} \mathrm{H}_{33} \mathrm{O}_{4} \mathrm{~N}_{2} \mathrm{FSNa}: \mathrm{m} / z 415.2037\left([\mathrm{M}+\mathrm{Na}]^{+}\right)$, found: $m / z 415.2033\left([\mathrm{M}+\mathrm{Na}]^{+}\right)$.

neopentyl (2-cyano-4-hydroxy-2-methylbutyl)(fluorosulfonyl)carbamate (52) 


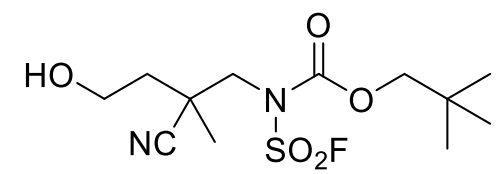

Prepared according to general procedure B with 3-methylbut-3-en-1-ol (200 $\mu \mathrm{mol}, 20.6 \mu \mathrm{L}, 98 \%)$ and NFC (280 $\mu \mathrm{mol}, 66.1 \mathrm{mg}, 98 \%)$. After stirring for $2 \mathrm{~h}$, the solution was cooled to room temperature, diluted with EtOAc, filtered through a pad of silica gel and concentrated under vacuum. THF (1.0 mL), AcOH (1.0 mmol, $57.2 \mu \mathrm{L})$ and TBAF ( $1 \mathrm{M}$ THF solution, $0.2 \mathrm{~mL}$ ) were added to the residue at $0{ }^{\circ} \mathrm{C}$. After stirring for $4 \mathrm{~h}$ at the same temperature, the reaction was quenched with $1 \mathrm{~N} \mathrm{HCl}$ aq. $(10 \mathrm{~mL})$, extracted with EtOAc $(10 \mathrm{~mL} \times 3)$ and concentrated under vacuum. The residue was diluted with EtOAc, filtered through a pad of silica gel and concentrated under vacuum. The crude material was purified by flash column chromatography on silica gel (EtOAc/hexane 12-100\%) to give the title compound as a yellowish solid in 50\% yield $(101 \mu \mathrm{mol}, 32.6 \mathrm{mg})$.

${ }^{1} \mathrm{H}$ NMR $\left(396 \mathrm{MHz}, \mathrm{CDCl}_{3}\right) \delta 4.38\left(\mathrm{dd}, J=15.2, J_{\mathrm{H}-\mathrm{F}}=2.3 \mathrm{~Hz}, 1 \mathrm{H}\right), 4.09(\mathrm{~d}, J=10.2 \mathrm{~Hz}, 1 \mathrm{H}), 4.05(\mathrm{~d}, J=10.2 \mathrm{~Hz}$, $1 \mathrm{H}), 3.91-4.03(\mathrm{~m}, 3 \mathrm{H}), 1.93-2.00(\mathrm{~m}, 1 \mathrm{H}), 1.82-1.89(\mathrm{~m}, 1 \mathrm{H}), 1.66(\mathrm{br}, 1 \mathrm{H}), 1.50(\mathrm{~s}, 3 \mathrm{H}), 1.01(\mathrm{~s}, 9 \mathrm{H})$.

${ }^{13} \mathrm{C} \mathrm{NMR}\left(99 \mathrm{MHz}, \mathrm{CDCl}_{3}\right) \delta 151.0,121.3,79.2,58.9,54.9,39.5,37.4,31.5,26.0,23.2$.

${ }^{19} \mathrm{~F}$ NMR $\left(369 \mathrm{MHz}, \mathrm{CDCl}_{3}\right) \delta 54.79(\mathrm{~s}, 1 \mathrm{~F})$.

HRMS (ESI) exact mass calculated for $\mathrm{C}_{12} \mathrm{H}_{21} \mathrm{O}_{5} \mathrm{~N}_{2} \mathrm{FSNa}: \mathrm{m} / z 347.1047\left([\mathrm{M}+\mathrm{Na}]^{+}\right)$, found: $\mathrm{m} / z 347.1042\left([\mathrm{M}+\mathrm{Na}]^{+}\right)$.

neopentyl ((1-cyanocyclohexyl)methyl)(fluorosulfonyl)carbamate (53)<smiles>CC(C)(C)COC(=O)N(CC1(C)CCCCC1)S(=O)(=O)OF</smiles>

Prepared according to general procedure B with methylenecyclohexane $(200 \mu \mathrm{mol}, 19.2 \mathrm{mg})$ and NFC $(280 \mu \mathrm{mol}$, $71.9 \mathrm{mg}, 90 \%)$. The crude material was purified by flash column chromatography on silica gel (EtOAc/hexane 4$34 \%)$ to give the title compound as a colorless solid in $44 \%$ yield $(88.5 \mu \mathrm{mol}, 29.6 \mathrm{mg})$.

${ }^{1} \mathrm{H}$ NMR $\left(396 \mathrm{MHz}, \mathrm{CDCl}_{3}\right) \delta 4.06(\mathrm{~s}, 2 \mathrm{H}), 4.05\left(\mathrm{~d}, \mathrm{~J}_{\mathrm{H}-\mathrm{F}}=2.0 \mathrm{~Hz}, 2 \mathrm{H}\right), 1.99-2.03(\mathrm{~m}, 2 \mathrm{H}), 1.76-1.83(\mathrm{~m}, 3 \mathrm{H}), 1.60-$ $1.71(\mathrm{~m}, 2 \mathrm{H}), 1.32-1.40(\mathrm{~m}, 2 \mathrm{H}), 1.12-1.25(\mathrm{~m}, 1 \mathrm{H}), 1.01(\mathrm{~s}, 9 \mathrm{H})$

${ }^{13} \mathrm{C} \mathrm{NMR}\left(99 \mathrm{MHz}, \mathrm{CDCl}_{3}\right) \delta 150.9,120.5,79.1,55.6,40.6,33.6,31.5,26.1,24.7,22.5$.

${ }^{19} \mathrm{~F}$ NMR $\left(369 \mathrm{MHz}, \mathrm{CDCl}_{3}\right) \delta 54.60(\mathrm{~s}, 1 \mathrm{~F})$.

HRMS (ESI) exact mass calculated for $\mathrm{C}_{14} \mathrm{H}_{23} \mathrm{O}_{4} \mathrm{~N}_{2} \mathrm{FSNa}: \mathrm{m} / z 357.1255\left([\mathrm{M}+\mathrm{Na}]^{+}\right)$, found: $m / z 357.1251\left([\mathrm{M}+\mathrm{Na}]^{+}\right)$.

\section{neopentyl ((4-cyano-1-tosylpiperidin-4-yl)methyl)(fluorosulfonyl)carbamate (54)}<smiles>CC(C)(C)COC(=O)N(CC1(C)CCN([As])CC1)S(=O)(=O)[O-]</smiles>

Prepared according to general procedure B with 4-methylene-1-tosylpiperidine ( $200 \mu \mathrm{mol}, 50.3 \mathrm{mg})$ and NFC (280 $\mu \mathrm{mol}, 66.1 \mathrm{mg}, 98 \%)$. After stirring for $2 \mathrm{~h}$, the solution was cooled to room temperature, diluted with EtOAc, filtered through a pad of silica gel and concentrated under vacuum. The crude material was purified by flash column chromatography on silica gel (EtOAc/hexane $8-50 \%$ ) to give the title compound as a white solid in $48 \%$ yield (96.4 $\mu \mathrm{mol}, 47.2 \mathrm{mg})$.

${ }^{1} \mathrm{H}$ NMR $\left(392 \mathrm{MHz}, \mathrm{CDCl}_{3}\right)$ ס 7.62-7.65 (m, 2H), $7.34(\mathrm{~d}, \mathrm{~J}=8.1 \mathrm{~Hz}, 2 \mathrm{H}), 4.08(\mathrm{~s}, 2 \mathrm{H}), 4.05(\mathrm{~s}, 2 \mathrm{H}), 3.86-3.91(\mathrm{~m}$, $2 \mathrm{H}), 2.52-2.59(\mathrm{~m}, 2 \mathrm{H}), 2.45(\mathrm{~s}, 3 \mathrm{H}), 2.00-2.03(\mathrm{~m}, 2 \mathrm{H}), 1.76-1.84(\mathrm{~m}, 2 \mathrm{H}), 0.99(\mathrm{~s}, 9 \mathrm{H})$ 
${ }^{13} \mathrm{C}$ NMR $\left(99 \mathrm{MHz}, \mathrm{CDCl}_{3}\right) \delta 150.7,144.2,132.6,129.9,127.4,118.7,79.4,54.5,42.8,38.9,32.4,31.5,26.0$, 21.5.

${ }^{19} \mathrm{~F}$ NMR $\left(369 \mathrm{MHz}, \mathrm{CDCl}_{3}\right) \delta 54.67(\mathrm{~s}, 1 \mathrm{~F})$.

HRMS (ESI) exact mass calculated for $\mathrm{C}_{20} \mathrm{H}_{28} \mathrm{O}_{6} \mathrm{~N}_{3} \mathrm{FS}_{2} \mathrm{Na}: \mathrm{m} / z 512.1296\left([\mathrm{M}+\mathrm{Na}]^{+}\right)$, found: $\mathrm{m} / z 512.1296$ $\left([\mathrm{M}+\mathrm{Na}]^{+}\right)$.

neopentyl (2-cyano-2-methylundecan-3-yl)(fluorosulfonyl)carbamate (55)

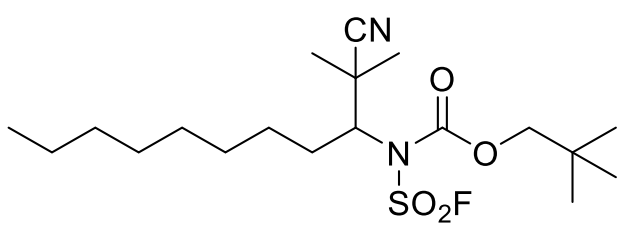

Prepared according to general procedure $\mathrm{C}$ with 2-methylundec-2-ene $(200 \mu \mathrm{mol}, 35.4 \mathrm{mg}, 95 \%)$ and NFC (280 $\mu \mathrm{mol}, 66.1 \mathrm{mg}, 98 \%)$. The crude material was purified by flash column chromatography on silica gel (1st EtOAc/hexane 4-34\%, 2nd DCM/hexane 12-100\%, 3rd acetone/hexane 4-34\%) to give the title compound as a yellowish oil in $38 \%$ yield $(76.5 \mu \mathrm{mol}, 31.1 \mathrm{mg})$.

${ }^{1} \mathrm{H}$ NMR $\left(396 \mathrm{MHz}, \mathrm{CDCl}_{3}\right) \delta 4.12(\mathrm{~d}, J=10.2 \mathrm{~Hz}, 1 \mathrm{H}), 4.04-4.08(\mathrm{~m}, 2 \mathrm{H}), 2.34-2.44(\mathrm{~m}, 1 \mathrm{H}), 1.70-1.81(\mathrm{~m}, 1 \mathrm{H})$, $1.53(\mathrm{~s}, 3 \mathrm{H}), 1.45(\mathrm{~s}, 3 \mathrm{H}), 1.27-1.42(\mathrm{~m}, 12 \mathrm{H}), 1.00(\mathrm{~s}, 9 \mathrm{H}), 0.88(\mathrm{t}, J=6.9 \mathrm{~Hz}, 3 \mathrm{H})$.

${ }^{13} \mathrm{C}$ NMR $\left(99 \mathrm{MHz}, \mathrm{CDCl}_{3}\right) \delta 149.9,122.5,78.5,69.1,37.0,31.7,31.6,29.2,29.0,28.8,28.4,28.3,26.3,26.0$, 24.3, 22.6, 14.0.

${ }^{19} \mathrm{~F}$ NMR $\left(369 \mathrm{MHz}, \mathrm{CDCl}_{3}\right) \delta 59.75$ (s, 0.2F), 51.91 (s, 0.8F).

HRMS (ESI) exact mass calculated for $\mathrm{C}_{19} \mathrm{H}_{35} \mathrm{O}_{4} \mathrm{~N}_{2} \mathrm{FSNa}: \mathrm{m} / z$ 429.2194 ([M+Na] $]^{+}$, found: $m / z 429.2191\left([\mathrm{M}+\mathrm{Na}]^{+}\right)$.

neopentyl (2-cyano-2-((R)-4-methyl-5-oxocyclohex-3-en-1-yl)propyl)(fluorosulfonyl)carbamate (56)

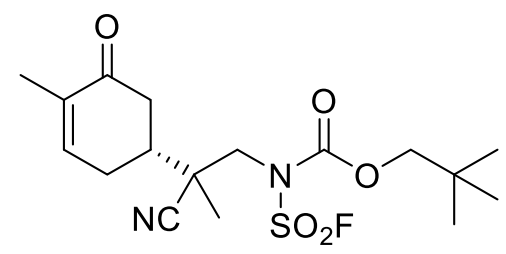

Prepared according to general procedure $C$ with $(R)$-2-methyl-5-(prop-1-en-2-yl)cyclohex-2-en-1-one (200 $\mu \mathrm{mol}$, $31.6 \mu \mathrm{L}, 99 \%)$ and NFC $(280 \mu \mathrm{mol}, 66.1 \mathrm{mg}, 98 \%)$. After stirring for $2 \mathrm{~h}$, the solution was cooled to room temperature, diluted with EtOAc, filtered through a pad of silica gel and concentrated under vacuum. The crude material was purified by flash column chromatography on silica gel (1st EtOAc/hexane 8-66\%, 2nd TBME/hexane $12-100 \%)$ to give the title compound as a yellowish solid in $29 \%$ yield $(57.9 \mu \mathrm{mol}, 22.5 \mathrm{mg}, \mathrm{dr} 56: 44)$.

${ }^{1} \mathbf{H}$ NMR $\left(392 \mathrm{MHz}, \mathrm{CDCl}_{3}\right)$ d 6.75-6.78 (m, 1H), 4.37-4.30 (m, 1H), $4.08(\mathrm{~m}, 2 \mathrm{H}), 3.95(\mathrm{~d}, J=15.0 \mathrm{~Hz}, 0.6 \mathrm{H}), 3.90$ (d, $J=15.0 \mathrm{~Hz}, 0.4 \mathrm{H}), 2.20-2.81(\mathrm{~m}, 5 \mathrm{H}), 1.80(\mathrm{~d}, J=1.1 \mathrm{~Hz}, 3 \mathrm{H}), 1.47(\mathrm{~s}, 1.2 \mathrm{H}), 1.44(\mathrm{~s}, 1.8 \mathrm{H}), 1.01(\mathrm{~s}, 9 \mathrm{H})$.

${ }^{13} \mathrm{C}$ NMR $\left(99 \mathrm{MHz}, \mathrm{CDCl}_{3}\right) \delta 196.8,196.7,150.92,150.86,142.8,142.6,136.0,119.8,119.7,79.4,53.0,52.8$, $41.7,41.2,40.8,40.0,39.0,31.6,28.0,27.5,26.0,20.3,19.8,15.50,15.47$.

${ }^{19} \mathrm{~F}$ NMR $\left(369 \mathrm{MHz}, \mathrm{CDCl}_{3}\right) \delta 55.15$ (s, 0.6F), 55.05 (s, 0.4F).

HRMS (ESI) exact mass calculated for $\mathrm{C}_{17} \mathrm{H}_{25} \mathrm{O}_{5} \mathrm{~N}_{2} \mathrm{FSNa}: \mathrm{m} / z 411.1360\left([\mathrm{M}+\mathrm{Na}]^{+}\right)$, found: $\mathrm{m} / z 411.1354\left([\mathrm{M}+\mathrm{Na}]^{+}\right)$. 


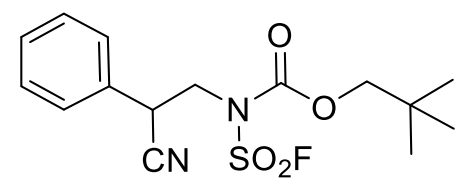

Prepared according to general procedure A with styrene $(200 \mu \mathrm{mol}, 23.0 \mu \mathrm{L})$ and NFC $(280 \mu \mathrm{mol}, 66.1 \mathrm{mg}, 98 \%)$. The crude material was purified by flash column chromatography on silica gel (EtOAc/hexane 4-34\%) to give the title compound as a yellowish solid in $79 \%$ yield $(157 \mu \mathrm{mol}, 54.0 \mathrm{mg}, \mathrm{rr}$ 99:1).

${ }^{1} \mathrm{H}$ NMR $\left(392 \mathrm{MHz}, \mathrm{CDCl}_{3}\right)$ ठ 7.37-7.46 (m, 5H), 4.32-4.40 (m, 2H), 4.06-4.15 (m, 1H), $4.04(\mathrm{~d}, J=10.1 \mathrm{~Hz}, 1 \mathrm{H})$, $4.00(\mathrm{~d}, J=10.1 \mathrm{~Hz}, 1 \mathrm{H}), 0.99(\mathrm{~s}, 9 \mathrm{H})$.

${ }^{13} \mathrm{C}$ NMR $\left(99 \mathrm{MHz}, \mathrm{CDCl}_{3}\right) \delta 150.3,131.0,129.5,129.3,127.8,117.6,79.1,52.2,37.1,31.5,26.0$.

${ }^{19} \mathrm{~F}$ NMR $\left(369 \mathrm{MHz}, \mathrm{CDCl}_{3}\right) \delta 55.43(\mathrm{~s}, 1 \mathrm{~F})$.

HRMS (ESI) exact mass calculated for $\mathrm{C}_{15} \mathrm{H}_{19} \mathrm{O}_{4} \mathrm{~N}_{2} \mathrm{FSNa}: \mathrm{m} / z 365.0942\left([\mathrm{M}+\mathrm{Na}]^{+}\right)$, found: $\mathrm{m} / z 365.0947\left([\mathrm{M}+\mathrm{Na}]^{+}\right)$.

neopentyl (2-(2-chlorophenyl)-2-cyanoethyl)(fluorosulfonyl)carbamate (58)

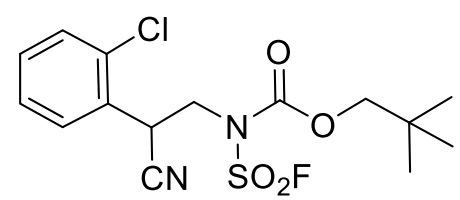

Prepared according to general procedure A with 1-chloro-2-vinylbenzene $(200 \mu \mathrm{mol}, 26.5 \mu \mathrm{L}, 95 \%)$ and NFC (280 $\mu \mathrm{mol}, 64.7 \mathrm{mg},>99 \%)$. The crude material was purified by flash column chromatography on silica gel (1st EtOAc/hexane $4-34 \%$, 2nd DCM/hexane $12-100 \%$ ) to give the title compound as a white solid in 93\% yield (185 umol, $69.8 \mathrm{mg}$, rr 99:1).

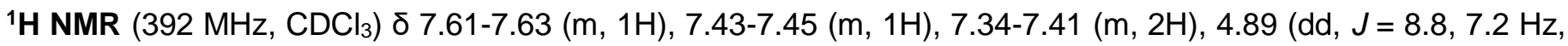
$1 \mathrm{H}$ ), 4.36 (ddd, $J=14.6,8.8, J_{\mathrm{H}-\mathrm{F}}=2.5 \mathrm{~Hz}, 1 \mathrm{H}$ ), 4.22 (ddd, $J=14.4,7.2, J_{\mathrm{H}-\mathrm{F}}=1.4 \mathrm{~Hz}, 1 \mathrm{H}$ ), $4.06(\mathrm{~d}, J=10.1 \mathrm{~Hz}$, $1 \mathrm{H}), 4.03(\mathrm{~d}, J=10.1 \mathrm{~Hz}, 1 \mathrm{H}), 1.00(\mathrm{~s}, 9 \mathrm{H})$.

${ }^{13} \mathrm{C}$ NMR $\left(99 \mathrm{MHz}, \mathrm{CDCl}_{3}\right) \delta 150.4,133.4,130.8,130.3,129.9,129.1,128.0,117.1,79.1,50.4,34.3,31.5,26.0$. ${ }^{19} \mathrm{~F}$ NMR $\left(369 \mathrm{MHz}, \mathrm{CDCl}_{3}\right) \delta 55.09$ (s, 1F).

HRMS (ESI) exact mass calculated for $\mathrm{C}_{15} \mathrm{H}_{18} \mathrm{O}_{4} \mathrm{~N}_{2} \mathrm{FSCINa:} \mathrm{m} / z 399.0552\left([\mathrm{M}+\mathrm{Na}]^{+}\right)$, found: $\mathrm{m} / z 399.0548$ $\left([\mathrm{M}+\mathrm{Na}]^{+}\right)$.

neopentyl (2-(3-chlorophenyl)-2-cyanoethyl)(fluorosulfonyl)carbamate (59)<smiles></smiles>

Prepared according to general procedure A with 1-chloro-3-vinylbenzene $(200 \mu \mathrm{mol}, 25.4 \mu \mathrm{L}, 95 \%)$ and NFC $(280$ $\mu \mathrm{mol}, 64.7 \mathrm{mg},>99 \%)$. The crude material was purified by flash column chromatography on silica gel (1st EtOAc/hexane $4-34 \%$, 2nd DCM/hexane 12-100\%) to give the title compound as a yellowish oil in $86 \%$ yield (172 $\mu \mathrm{mol}, 67.5 \mathrm{mg}, 96 \%$, rr 99:1).

${ }^{1} \mathrm{H}$ NMR $\left(392 \mathrm{MHz}, \mathrm{CDCl}_{3}\right)$ ठ 7.36-7.41 (m, 3H), 7.29-7.31 (m, 1H), 4.31-4.39 (m, 2H), 4.08-4.15 (m, $\left.1 \mathrm{H}\right), 4.06(\mathrm{~d}$, $J=10.1 \mathrm{~Hz}, 1 \mathrm{H}), 4.01(\mathrm{~d}, J=10.1 \mathrm{~Hz}, 1 \mathrm{H}), 1.00(\mathrm{~s}, 9 \mathrm{H})$.

${ }^{13} \mathrm{C}$ NMR $\left(99 \mathrm{MHz}, \mathrm{CDCl}_{3}\right) \delta 150.3,135.4,132.9,130.8,129.6,128.0,126.0,117.1,79.2,52.0,36.7,31.5,26.0$. ${ }^{19} \mathrm{~F}$ NMR $\left(369 \mathrm{MHz}, \mathrm{CDCl}_{3}\right) \delta 55.45$ (s, 1F). 
HRMS (ESI) exact mass calculated for $\mathrm{C}_{15} \mathrm{H}_{18} \mathrm{O}_{4} \mathrm{~N}_{2} \mathrm{FSCINa}: \mathrm{m} / z 399.0552\left([\mathrm{M}+\mathrm{Na}]^{+}\right)$, found: $\mathrm{m} / z 399.0551$ $\left([\mathrm{M}+\mathrm{Na}]^{+}\right)$.

neopentyl (2-cyano-2-(4-tolyl)ethyl)(fluorosulfonyl)carbamate (60)<smiles>Cc1ccc(C(C#N)CN(C(=O)O[Na])C(=O)OCC(C)(C)C)cc1</smiles>

Prepared according to general procedure A with 1-methyl-4-vinylbenzene ( $200 \mu \mathrm{mol}, 27.7 \mu \mathrm{L}, 95 \%)$ and NFC (280 $\mu \mathrm{mol}, 64.7 \mathrm{mg},>99 \%)$. The crude material was purified by flash column chromatography on silica gel (EtOAc/hexane 4-34\%) to give the title compound as a yellowish oil in $70 \%$ yield ( $141 \mu \mathrm{mol}, 50.1 \mathrm{mg}$ ).

${ }^{1} \mathrm{H}$ NMR (392 MHz, $\left.\mathrm{CDCl}_{3}\right)$ ס 7.21-7.28 (m, 4H), 4.28-4.37 (m, 2H), 3.99-4.12 (m, 3H), $2.36(\mathrm{~s}, 3 \mathrm{H}), 0.99(\mathrm{~s}, 9 \mathrm{H})$.

${ }^{13} \mathrm{C}$ NMR $\left(99 \mathrm{MHz}, \mathrm{CDCl}_{3}\right) \delta 150.3,139.3,130.1,128.0,127.7,117.8,79.0,52.2,36.7,31.5,26.0,21.1$.

${ }^{19} \mathrm{~F}$ NMR $\left(369 \mathrm{MHz}, \mathrm{CDCl}_{3}\right) \delta 55.43$ (s, 1F).

HRMS (ESI) exact mass calculated for $\mathrm{C}_{16} \mathrm{H}_{21} \mathrm{O}_{4} \mathrm{~N}_{2} \mathrm{FSNa}: \mathrm{m} / z 379.1098\left([\mathrm{M}+\mathrm{Na}]^{+}\right)$, found: $m / z 379.1095\left([\mathrm{M}+\mathrm{Na}]^{+}\right)$.

neopentyl (rel-(1S,2R)-1-cyano-1-phenylpropan-2-yl)(fluorosulfonyl)carbamate (61)<smiles>CC(C(C#N)c1ccccc1)N(C(=O)[O-])C(=O)OCC(C)(C)C</smiles>

Prepared according to general procedure A with ( $E$ )-prop-1-en-1-ylbenzene (200 $\mu \mathrm{mol}, 26.2 \mu \mathrm{L}, 99 \%)$ and NFC ( $280 \mu \mathrm{mol}, 64.7 \mathrm{mg},>99 \%)$. The crude material was purified by flash column chromatography on silica gel (1st EtOAc/hexane 4-34\%, 2nd TBME/hexane 4-34\%) to give the title compound as a white solid in $81 \%$ yield (161 $\mu \mathrm{mol}, 57.4 \mathrm{mg}$, dr 97:3).

${ }^{1} \mathrm{H}$ NMR $\left(392 \mathrm{MHz}, \mathrm{CDCl}_{3}\right) \delta 7.30-7.41(\mathrm{~m}, 5 \mathrm{H}), 4.93\left(\mathrm{dqd}, J=11.0,7.1, J_{\mathrm{H}-\mathrm{F}}=0.8 \mathrm{~Hz}, 1 \mathrm{H}\right), 4.53(\mathrm{~d}, J=11.0 \mathrm{~Hz}$, $1 \mathrm{H}), 3.94(\mathrm{~d}, J=10.3 \mathrm{~Hz}, 1 \mathrm{H}), 3.89(\mathrm{~d}, J=10.1 \mathrm{~Hz}, 1 \mathrm{H}), 1.84(\mathrm{~d}, J=7.0 \mathrm{~Hz}, 3 \mathrm{H}), 0.88(\mathrm{~s}, 9 \mathrm{H})$.

${ }^{13} \mathrm{C}$ NMR $\left(99 \mathrm{MHz}, \mathrm{CDCl}_{3}\right) \delta 150.1,132.1,129.3,128.1,119.0,78.6,61.3,40.9,31.4,25.9,17.6$.

${ }^{19} \mathrm{~F}$ NMR $\left(369 \mathrm{MHz}, \mathrm{CDCl}_{3}\right) \delta 55.80(\mathrm{~s}, 1 \mathrm{~F})$.

HRMS (ESI) exact mass calculated for $\mathrm{C}_{16} \mathrm{H}_{21} \mathrm{O}_{4} \mathrm{~N}_{2} \mathrm{FSNa}$ : m/z 379.1098 ([M+Na] $]^{+}$), found: $m / z 379.1099\left([\mathrm{M}+\mathrm{Na}]^{+}\right)$. CIF File CCDC 2084403 contains the supplementary crystallographic data of 61.

neopentyl (rel-(1S,2S)-1-cyano-3-hydroxy-1-phenylpropan-2-yl)(fluorosulfonyl)carbamate (62)<smiles></smiles>

Prepared according to general procedure A with (E)-3-phenylprop-2-en-1-ol (200 $\mu \mathrm{mol}, 25.7 \mu \mathrm{L})$ and NFC (280 $\mu \mathrm{mol}, 64.7 \mathrm{mg},>99 \%)$. After stirring for $2 \mathrm{~h}$, the solution was cooled to room temperature, diluted with EtOAc, filtered through a pad of silica gel and concentrated under vacuum. The crude material was purified by flash column chromatography on silica gel (1st EtOAc/hexane 8-66\%, 2nd acetone/hexane 8-66\%, 3rd EtOAc/DCM 5\%) to give the title compound as a yellowish oil in $49 \%$ yield $(97.9 \mu \mathrm{mol}, 37.6 \mathrm{mg}, 97 \%)$.

${ }^{1}$ H NMR (392 MHz, CDCl $)$ ס 7.40-7.49 (m, 5H), $6.62(\mathrm{~s}, 1 \mathrm{H}), 4.41-4.47(\mathrm{~m}, 2 \mathrm{H}), 4.15-4.21(\mathrm{~m}, 2 \mathrm{H}), 3.89(\mathrm{~d}, J=$ $10.1 \mathrm{~Hz}, 1 \mathrm{H}), 3.86(\mathrm{~d}, J=10.3 \mathrm{~Hz}, 1 \mathrm{H}), 0.96(\mathrm{~s}, 9 \mathrm{H})$. 
${ }^{13} \mathrm{C}$ NMR $\left(99 \mathrm{MHz}, \mathrm{CDCl}_{3}\right) \delta$ 155.3, 129.8, 129.7, 129.5, 127.8, 116.8, 78.5, 64.4, 57.4, 40.7, 31.5, 26.1.

${ }^{19} \mathrm{~F}$ NMR $\left(369 \mathrm{MHz}, \mathrm{CDCl}_{3}\right) \delta 53.57$ (s, 1F).

HRMS (ESI) exact mass calculated for $\mathrm{C}_{16} \mathrm{H}_{20} \mathrm{O}_{5} \mathrm{~N}_{2} \mathrm{FS}$ : $\mathrm{m} / \mathrm{z} 371.1082$ ([M-H]), found: $m / z 371.1094$ ([M-H]'). 


\section{Deprotection of Imidated Products to Amines (Figure 4)}

5-phenylthiophen-2-amine (63)

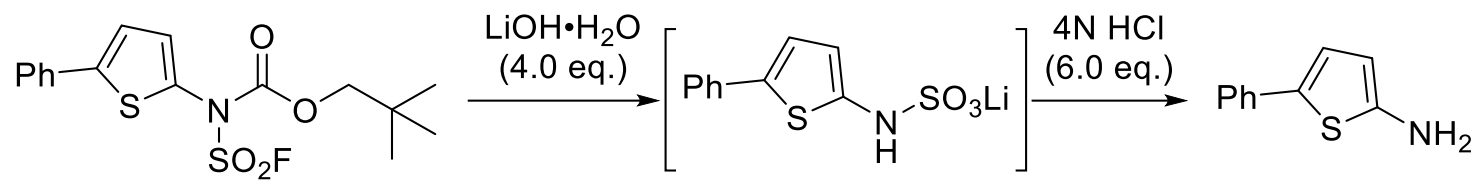

A test tube was charged with lithium hydroxide hydrate $(400 \mu \mathrm{mol}, 17.0 \mathrm{mg}, 99.0 \%)$, water $(150 \mu \mathrm{L}), 1,4$-dioxane $(150 \mu \mathrm{L})$ and neopentyl (fluorosulfonyl)(5-phenylthiophen-2-yl)carbamate (4) (100 $\mu \mathrm{mol}, 37.1 \mathrm{mg})$. After stirring for $1 \mathrm{~h}$ at room temperature, $4 \mathrm{~N} \mathrm{HCl}$ aq. $(150 \mu \mathrm{L})$ was added to the tube. After stirring for $0.5 \mathrm{~h}$ at $60^{\circ} \mathrm{C}$, the reaction was quenched with sat. $\mathrm{NaHCO}_{3}$ aq. The organic layer was extracted with $\mathrm{EtOAc}$, washed with brine, dried over anhydrous $\mathrm{Na}_{2} \mathrm{SO}_{4}$ and evaporated under reduced pressure. The residue was purified by column chromatography (EtOAc/hexane 2-20\%) to give the title compound as a white solid in $70 \%$ yield $(69.6 \mu \mathrm{mol}, 12.2 \mathrm{mg}$ ). The spectral data matched with those reported in the literature. ${ }^{19}$

\section{tert-butyl (2-cyanooctyl)carbamate (65)}<smiles>CC(C=O)CC(C#N)N(CC(=O)O)OS(=O)(=O)O</smiles>

38
i) $\mathrm{LiOH} \cdot \mathrm{H}_{2} \mathrm{O}$ (4.0 eq.) dioxane $/ \mathrm{H}_{2} \mathrm{O}, 25^{\circ} \mathrm{C}, 1 \mathrm{~h}$
ii) $\mathrm{HCl}$ (6.0 eq.) dioxane $/ \mathrm{H}_{2} \mathrm{O}, 60^{\circ} \mathrm{C}, 36 \mathrm{~h}$
iii) $\mathrm{Boc}_{2} \mathrm{O}$ (3.0 eq.), $\mathrm{NaOH}$ (9.0 eq.) dioxane $/ \mathrm{H}_{2} \mathrm{O}, 25^{\circ} \mathrm{C}, 1 \mathrm{~h}$

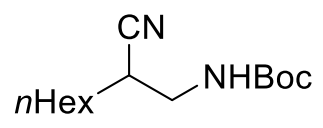

65

A test tube was charged with lithium hydroxide hydrate $(400 \mu \mathrm{mol}, 17.0 \mathrm{mg}, 99.0 \%)$, water (150 $\mu \mathrm{L}), 1,4$-dioxane $(150 \mu \mathrm{L})$ and neopentyl (2-cyanooctyl)(fluorosulfonyl)carbamate (38) $(100 \mu \mathrm{mol}, 35.0 \mathrm{mg})$. After stirring for $1 \mathrm{~h}$ at $25^{\circ} \mathrm{C}, 4 \mathrm{~N} \mathrm{HCl}$ aq. $(150 \mu \mathrm{L})$ was added to the tube. After stirring for $36 \mathrm{~h}$ at $60{ }^{\circ} \mathrm{C}, 4 \mathrm{M} \mathrm{NaOH}$ aq. $(225 \mu \mathrm{L})$ and $\mathrm{Boc}_{2} \mathrm{O}(300 \mu \mathrm{mol}, 69 \mu \mathrm{L})$ were added to the tube. After stirring for $1 \mathrm{~h}$ at $25^{\circ} \mathrm{C}$, the solution was quenched with sat. $\mathrm{NH}_{4} \mathrm{Cl}$ aq. The organic layer was extracted with $\mathrm{EtOAc}$, washed with brine, dried over anhydrous $\mathrm{Na}_{2} \mathrm{SO}_{4}$ and evaporated under reduced pressure. The crude material was purified by flash column chromatography on silica gel (EtOAc/hexane 8-66\%) to give the title compound as a yellowish oil in $80 \%$ yield $(80.2 \mu \mathrm{mol}, 20.4 \mathrm{mg})$.

${ }^{1} \mathrm{H}$ NMR $\left(392 \mathrm{MHz}, \mathrm{CDCl}_{3}\right.$, VT $\left.40{ }^{\circ} \mathrm{C}\right) \delta 4.95(\mathrm{~s}, 1 \mathrm{H}), 3.40-3.43(\mathrm{~m}, 1 \mathrm{H}), 3.16-3.23(\mathrm{~m}, 1 \mathrm{H}), 2.82(\mathrm{~s}, 1 \mathrm{H}), 1.48-1.62$ $(\mathrm{m}, 4 \mathrm{H}), 1.45(\mathrm{~s}, 9 \mathrm{H}), 1.24-1.39(\mathrm{~m}, 6 \mathrm{H}), 0.89(\mathrm{t}, J=6.7 \mathrm{~Hz}, 3 \mathrm{H})$.

${ }^{13} \mathrm{C}$ NMR $\left(99 \mathrm{MHz}, \mathrm{CDCl}_{3}\right.$, VT $\left.40{ }^{\circ} \mathrm{C}\right) \delta 155.6,121.0,80.1,42.3,33.2,31.4,29.5,28.7,28.3,26.9,22.4,13.9$.

HRMS (ESI) exact mass calculated for $\mathrm{C}_{14} \mathrm{H}_{26} \mathrm{O}_{2} \mathrm{~N}_{2} \mathrm{Na}: m / z 277.1886\left([\mathrm{M}+\mathrm{Na}]^{+}\right)$, found: $m / z 277.1884\left([\mathrm{M}+\mathrm{Na}]^{+}\right)$.

\section{$\mathrm{LiBH}_{4}$-Mediated Reduction of 36}<smiles>Cc1ccc(N(C(=O)OF)C(=O)OCC(C)(C)C)c(OC#N)c1Br</smiles>

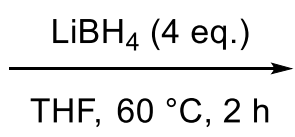

$36: 36^{\prime}=90: 10$<smiles>Cc1ccc(N)c(C)c1Br</smiles>

$83 \%\left(66: 66^{\prime}=91: 9\right)$

To an argon-purged Schlenk tube, $\mathrm{LiBH}_{4}(390 \mu \mathrm{mol}, 9.5 \mathrm{mg})$, THF $(0.5 \mathrm{~mL})$ and $36\left(88.3 \mu \mathrm{mol}, 35.0 \mathrm{mg}, 36: 36^{\prime}=\right.$ $90: 10)$ were added sequentially at room temperature. The reaction solution was warmed to $60^{\circ} \mathrm{C}$, stirred for 2 hours and then quenched with $\mathrm{NaHCO}_{3}$ aq. The organic layer was extracted with AcOEt, dried over $\mathrm{Na}_{2} \mathrm{SO}_{4}$ and evaporated under vacuum. The residue was purified by column chromatography on silica gel (DCM/hexane 50$100 \%)$ to give the title compound as a white solid in $83 \%$ yield $\left(88.3 \mu \mathrm{mol}, 14.6 \mathrm{mg}, 66: 66^{\prime}=91: 9\right)^{20,21}$. 


\section{Derivatization of Imidated Products to Sulfonamides (Figure 5a) \\ Derivatization of Imidated Arenes}

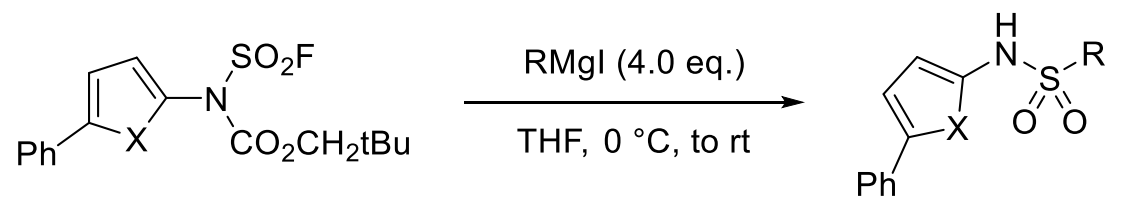

To an argon-purged Schlenk tube, freshly prepared alkyl or aryl magnesium iodide (400 $\mu \mathrm{mol}, \mathrm{Et}_{2} \mathrm{O}$ solution) and THF $(0.50 \mathrm{~mL})$ were added. After stirring for $15 \mathrm{~min}$ at $0{ }^{\circ} \mathrm{C}$, imidated product $(100 \mu \mathrm{mol})$ was added. After stirring for $2 \mathrm{~h}$ at $25^{\circ} \mathrm{C}$, the reaction mixture was treated with $1 \mathrm{~N} \mathrm{HCl}$ aq. The organic layer was extracted with EtOAc, washed with brine, dried over anhydrous $\mathrm{Na}_{2} \mathrm{SO}_{4}$ and evaporated under reduced pressure. The residue was purified by column chromatography on silica gel.

\section{Preparation of Grignard Reagents}

$$
\mathrm{R}-\mathrm{I}+\underset{1.5 \text { eq. }}{\mathrm{Mg}} \frac{\mathrm{I}_{2}(3 \mathrm{~mol} \%)}{\mathrm{Et}_{2} \mathrm{O}} \mathrm{R}-\mathrm{Mgl}
$$

To an argon-purged two-necked round bottom flask charged with magnesium turnings (1.5 eq.), $\mathrm{Et}_{2} \mathrm{O}$ (1.0 -3.0 M) and iodine ( $3 \mathrm{~mol} \%)$ were added and the mixture was sonicated for $20 \mathrm{~min}$. To this solution, aryl or alkyl iodide (1.0 eq.) was added dropwise over $0.5-1 \mathrm{~h}$ at room temperature and stirred for additional $1 \mathrm{~h}$. The solution was titrated following the literature procedure. ${ }^{22}$

\section{$N$-(5-phenylfuran-2-yl)benzenesulfonamide (67)}<smiles>O=S(=O)(Nc1ccc(-c2ccccc2)o1)c1ccccc1</smiles>

Prepared with neopentyl (fluorosulfonyl)(5-phenylfuran-2-yl)carbamate $\quad(\mathbf{1 0}) \quad(100 \mu \mathrm{mol}, \quad 35.5 \quad \mathrm{mg})$,

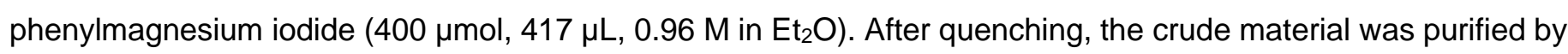
column chromatography (EtOAc/hexane $5-40 \%$ ) to give the title compound as a pale yellowish solid in $71 \%$ yield (71.2 $\mu \mathrm{mol}, 21.3 \mathrm{mg})$.

${ }^{1} \mathbf{H}$ NMR $\left(392 \mathrm{MHz}, \mathrm{CDCl}_{3}\right) \delta$ 7.85-7.88 (m, 2H), 7.59-7.63 (m, 1H), 7.49-7.53 (m, 2H), 7.40-7.43 (m, 2H), 7.30$7.34(\mathrm{~m}, 2 \mathrm{H}), 7.22-7.26(\mathrm{~m}, 1 \mathrm{H}), 6.64(\mathrm{br}, 1 \mathrm{H}), 6.55(\mathrm{~d}, J=3.6 \mathrm{~Hz}, 1 \mathrm{H}), 6.23(\mathrm{~d}, J=3.4 \mathrm{~Hz}, 1 \mathrm{H})$.

${ }^{13} \mathrm{C}$ NMR $\left(99 \mathrm{MHz}, \mathrm{CDCl}_{3}\right) \delta 151.4,141.4,139.4,133.3,130.0,129.0,128.6,127.6,127.4,123.5,106.9,106.2$. HRMS (ESI) exact mass calculated for $\mathrm{C}_{16} \mathrm{H}_{13} \mathrm{O}_{3} \mathrm{NSNa}: \mathrm{m} / z 322.0508\left([\mathrm{M}+\mathrm{Na}]^{+}\right)$, found: $\mathrm{m} / z 322.0509\left([\mathrm{M}+\mathrm{Na}]^{+}\right)$.

\section{$N$-(5-phenylfuran-2-yl)methanesulfonamide (68)}<smiles>CS(=O)(=O)Nc1ccc(-c2ccccc2)o1</smiles>

Prepared with neopentyl (fluorosulfonyl)(5-phenylfuran-2-yl)carbamate $\quad(\mathbf{1 0}) \quad(100 \mu \mathrm{mol}, \quad 35.5 \quad \mathrm{mg}$ ),

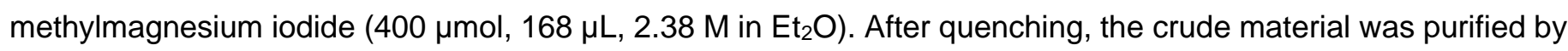


column chromatography (acetone/hexane 6-50\%) to give the title compound as a pale yellowish solid in $94 \%$ yield (94.0 $\mu \mathrm{mol}, 22.3 \mathrm{mg})$.

${ }^{1}$ H NMR $\left(396 \mathrm{MHz}, \mathrm{CDCl}_{3}\right)$ ס 7.60-7.62 (m, 2H), 7.37-7.40 (m, 2H), 7.27-7.31 (m, 1H), $6.76(\mathrm{br}, 1 \mathrm{H}), 6.63(\mathrm{~d}, J=$ $3.4 \mathrm{~Hz}, 1 \mathrm{H}), 6.35(\mathrm{~d}, J=3.4 \mathrm{~Hz}, 1 \mathrm{H}), 3.16(\mathrm{~s}, 3 \mathrm{H})$.

${ }^{13} \mathrm{C}$ NMR $\left(99 \mathrm{MHz}, \mathrm{CDCl}_{3}\right) \delta 151.7,141.2,130.0,128.7,127.9,123.6,107.4,106.4,40.9$.

HRMS (ESI) exact mass calculated for $\mathrm{C}_{11} \mathrm{H}_{11} \mathrm{O}_{3} \mathrm{NSNa}: \mathrm{m} / z 260.0352\left([\mathrm{M}+\mathrm{Na}]^{+}\right)$, found: $\mathrm{m} / z 260.0352\left([\mathrm{M}+\mathrm{Na}]^{+}\right)$.

\section{2-methyl-N-(5-phenylthiophen-2-yl)benzenesulfonamide (69)}

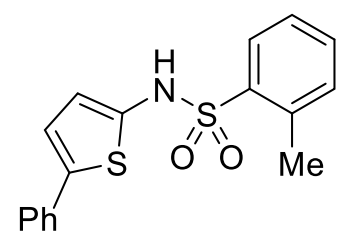

Prepared with neopentyl (fluorosulfonyl)(5-phenylthiophen-2-yl)carbamate (4) (100 $\mu \mathrm{mol}, 37.1 \mathrm{mg})$, 2tolylmagnesium iodide ( $400 \mu \mathrm{mol}, 548 \mu \mathrm{L}, 0.73 \mathrm{M}$ in $\mathrm{Et}_{2} \mathrm{O}$ ). After quenching, the crude material was purified by column chromatography (acetone/hexane $6-50 \%$ ) to give the title compound as a pale yellowish solid in $72 \%$ yield (72.2 $\mu \mathrm{mol}, 23.8 \mathrm{mg})$.

${ }^{1} \mathbf{H}$ NMR $\left(392 \mathrm{MHz}, \mathrm{CDCl}_{3}\right) \delta 7.91(\mathrm{dd}, J=7.9,1.3 \mathrm{~Hz}, 1 \mathrm{H}), 7.42-7.49(\mathrm{~m}, 3 \mathrm{H})$, 7.29-7.34 (m, 3H), 7.23-7.28 (m, $2 \mathrm{H}), 6.97(\mathrm{~d}, J=3.8 \mathrm{~Hz}, 1 \mathrm{H}), 6.84(\mathrm{br}, 1 \mathrm{H}), 6.64(\mathrm{~d}, J=4.0 \mathrm{~Hz}, 1 \mathrm{H}), 2.69(\mathrm{~s}, 3 \mathrm{H})$.

${ }^{13} \mathrm{C}$ NMR $\left(99 \mathrm{MHz}, \mathrm{CDCl}_{3}\right) \delta 142.1,137.5,136.3,135.5,133.7,133.4,132.5,130.5,128.9,127.8,126.3,125.5$, 125.0, 121.3, 20.5.

HRMS (ESI) exact mass calculated for $\mathrm{C}_{17} \mathrm{H}_{15} \mathrm{O}_{2} \mathrm{NS}_{2} \mathrm{Na}: \mathrm{m} / z 352.0436\left([\mathrm{M}+\mathrm{Na}]^{+}\right)$, found: $\mathrm{m} / \mathrm{z} 352.0436\left([\mathrm{M}+\mathrm{Na}]^{+}\right)$.

\section{4-chloro- $N$-(5-phenylthiophen-2-yl)benzenesulfonamide (70)}

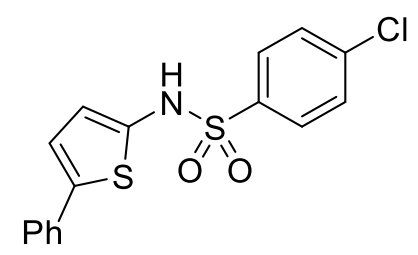

Prepared with neopentyl (fluorosulfonyl)(5-phenylthiophen-2-yl)carbamate (4) (100 $\mu \mathrm{mol}, 37.1 \mathrm{mg})$, (4chlorophenyl)magnesium iodide $\left(400 \mu \mathrm{mol}, 370 \mu \mathrm{L}, 1.08 \mathrm{M}\right.$ in $\left.\mathrm{Et}_{2} \mathrm{O}\right)$. After quenching, the crude material was purified by column chromatography (1st acetone/hexane $5-40 \%$, 2nd acetone/hexane $8-50 \%$ ) to give the title compound as a white solid in $70 \%$ yield $(70.3 \mu \mathrm{mol}, 24.6 \mathrm{mg})$.

${ }^{1} \mathbf{H}$ NMR $\left(392 \mathrm{MHz}, \mathrm{CDCl}_{3}\right) \delta 7.75(\mathrm{dt}, J=9.1,2.2 \mathrm{~Hz}, 2 \mathrm{H}), 7.45-7.50(\mathrm{~m}, 4 \mathrm{H}), 7.33-7.38(\mathrm{~m}, 2 \mathrm{H})$, 7.26-7.30 (m, $1 \mathrm{H}), 7.03(\mathrm{~d}, J=3.8 \mathrm{~Hz}, 1 \mathrm{H}), 6.69(\mathrm{~d}, J=3.8 \mathrm{~Hz}, 1 \mathrm{H}), 6.67(\mathrm{br}, 1 \mathrm{H})$.

${ }^{13} \mathrm{C}$ NMR $\left(99 \mathrm{MHz}, \mathrm{CDCl}_{3}\right) \delta 142.4,140.0,136.7,135.1,133.6,129.4,129.04,128.95,128.0,125.6,125.3,121.4$. HRMS (ESI) exact mass calculated for $\mathrm{C}_{16} \mathrm{H}_{12} \mathrm{O}_{2} \mathrm{NS}_{2} \mathrm{CINa}: \mathrm{m} / z 371.9890\left([\mathrm{M}+\mathrm{Na}]^{+}\right)$, found: $\mathrm{m} / z 371.9890$ $\left([\mathrm{M}+\mathrm{Na}]^{+}\right)$.

\section{$N$-(5-phenylthiophen-2-yl)cyclohexanesulfonamide (71)}<smiles>O=S(=O)(Nc1ccc(-c2ccccc2)s1)C1CCCCC1</smiles> 
Prepared with neopentyl (fluorosulfonyl)(5-phenylthiophen-2-yl)carbamate (4) (100 $\mu \mathrm{mol}, 37.1 \mathrm{mg}$ ), cyclohexylmagnesium iodide ( $400 \mu \mathrm{mol}, 588 \mu \mathrm{L}, 0.68 \mathrm{M}$ in $\mathrm{Et}_{2} \mathrm{O}$ ). After quenching, the crude material was purified by column chromatography (1st EtOAc/hexane 4-34\%, 2nd acetone/hexane 4-34\%) to give the title compound as a yellowish solid in $55 \%$ yield $(55.4 \mu \mathrm{mol}, 17.8 \mathrm{mg})$.

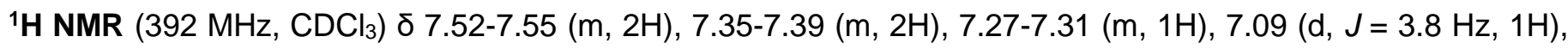
$6.87(\mathrm{~d}, J=3.8 \mathrm{~Hz}, 1 \mathrm{H}), 6.57(\mathrm{br}, 1 \mathrm{H}), 3.11(\mathrm{tt}, J=12.1,3.4 \mathrm{~Hz}, 1 \mathrm{H}), 1.90-2.24(\mathrm{~m}, 4 \mathrm{H}), 1.57-1.72(\mathrm{~m}, 3 \mathrm{H}), 1.15-$ $1.34(\mathrm{~m}, 3 \mathrm{H})$.

${ }^{13} \mathrm{C}$ NMR $\left(99 \mathrm{MHz}, \mathrm{CDCl}_{3}\right) \delta 141.4,136.4,133.8,129.0,127.8,125.5,124.0,121.5,59.9,26.4,25.0,25.0$.

HRMS (ESI) exact mass calculated for $\mathrm{C}_{16} \mathrm{H}_{19} \mathrm{O}_{2} \mathrm{NS}_{2} \mathrm{Na:} \mathrm{m} / \mathrm{z} 344.0749\left([\mathrm{M}+\mathrm{Na}]^{+}\right)$, found: $m / z 344.0748\left([\mathrm{M}+\mathrm{Na}]^{+}\right)$.

\section{Derivatization of Imidocyanated Alkenes}<smiles>N#CC(C=O)CN(C(=O)OF)S(=O)(=O)O</smiles>

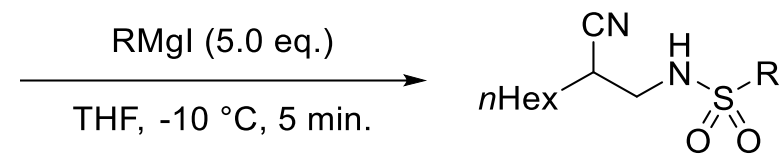

38

To an argon-purged Schlenk tube, freshly prepared alkyl or aryl magnesium iodide ( $500 \mu \mathrm{mol}, \mathrm{Et}_{2} \mathrm{O}$ solution) and THF $(0.50 \mathrm{~mL})$ were added. After stirring for $15 \mathrm{~min}$ at $-10{ }^{\circ} \mathrm{C}$, neopentyl (2-cyanooctyl)(fluorosulfonyl)carbamate (38) $(100 \mu \mathrm{mol}, 35.0 \mathrm{mg})$ was added. After stirring for $5 \mathrm{~min}$ at the same tempreture, the reaction mixture was treated with $1 \mathrm{~N} \mathrm{HCl}$ aq. The organic layer was extracted with EtOAc, washed with brine, dried over anhydrous $\mathrm{Na}_{2} \mathrm{SO}_{4}$ and evaporated under reduced pressure. The residue was purified by column chromatography on silica gel.

\section{N-(2-cyanooctyl)benzenesulfonamide (72)}<smiles>N#CC([Ge])CNS(=O)(=O)c1ccccc1</smiles>

Prepared with phenylmagnesium iodide $\left(500 \mu \mathrm{mol}, 410 \mu \mathrm{L}, 1.22 \mathrm{M}\right.$ in $\left.\mathrm{Et}_{2} \mathrm{O}\right)$. The crude material was purified by column chromatography (1st EtOAc/hexane 5-40\%, 2nd TBME/hexane 8-66\%) to give the title compound as a white solid in $61 \%$ yield $(60.5 \mu \mathrm{mol}, 17.8 \mathrm{mg})$.

${ }^{1} \mathrm{H}$ NMR $\left(500 \mathrm{MHz}, \mathrm{CDCl}_{3}\right) \delta 7.88(\mathrm{dt}, J=8.4,1.6 \mathrm{~Hz}, 2 \mathrm{H}), 7.60-7.64(\mathrm{~m}, 1 \mathrm{H}), 7.54-7.57(\mathrm{~m}, 2 \mathrm{H}), 5.17(\mathrm{t}, J=6.6$ $\mathrm{Hz}, 1 \mathrm{H}), 3.11-3.22(\mathrm{~m}, 2 \mathrm{H}), 2.73-2.79(\mathrm{~m}, 1 \mathrm{H}), 1.57(\mathrm{td}, J=14.8,7.0 \mathrm{~Hz}, 2 \mathrm{H}), 1.44-1.52(\mathrm{~m}, 1 \mathrm{H}), 1.35-1.40(\mathrm{~m}$, $1 \mathrm{H}), 1.21-1.33(\mathrm{~m}, 7 \mathrm{H}), 0.85-0.89(\mathrm{~m}, 3 \mathrm{H})$.

${ }^{13} \mathrm{C}$ NMR $\left(126 \mathrm{MHz}, \mathrm{CDCl}_{3}\right) \delta 139.6,133.1,129.4,126.9,120.2,44.3,33.1,31.4,29.3,28.6,26.7,22.4,14.0$.

HRMS (ESI) exact mass calculated for $\mathrm{C}_{15} \mathrm{H}_{22} \mathrm{O}_{2} \mathrm{~N}_{2} \mathrm{SNa}: \mathrm{m} / \mathrm{z} 317.1294\left([\mathrm{M}+\mathrm{Na}]^{+}\right)$, found: $m / z 317.1294\left([\mathrm{M}+\mathrm{Na}]^{+}\right)$.

$N$-(2-cyanooctyl)methanesulfonamide (73)<smiles>CS(=O)(=O)NCC(C#N)C#N</smiles>

73

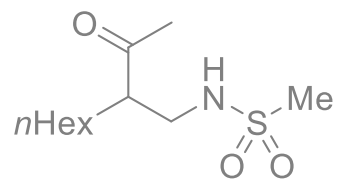

$73^{\prime}$ 
Prepared with methylmagnesium iodide ( $500 \mu \mathrm{mol}, 204 \mu \mathrm{L}, 2.45 \mathrm{M}$ in $\mathrm{Et}_{2} \mathrm{O}$ ). The crude material was purified by column chromatography (EtOAc/hexane 16-100\%) to give the title compound as a white solid in $68 \%$ yield. (68 $\mu \mathrm{mol}, 16.7 \mathrm{mg}, 73: 73^{\prime}=96: 4$, purity $\left.95 \%\right)$.

${ }^{1} \mathbf{H}$ NMR $\left(392 \mathrm{MHz}, \mathrm{CDCl}_{3}\right)$ ס 4.99-5.04 (m, 1H), 3.27-3.43 (m, 2H), 3.04 (s, 3H), 2.81-2.88 (m, 1H), 1.27-1.66 (m, $10 \mathrm{H}), 0.89$ (t, $J=6.8 \mathrm{~Hz}, 3 \mathrm{H})$.

${ }^{13} \mathrm{C}$ NMR $\left(126 \mathrm{MHz}, \mathrm{CDCl}_{3}\right) \delta 120.5,44.3,41.2,33.7,31.4,29.3,28.6,26.8,22.4,14.0$.

HRMS (ESI) exact mass calculated for $\mathrm{C}_{10} \mathrm{H}_{20} \mathrm{O}_{2} \mathrm{~N}_{2} \mathrm{SNa}: \mathrm{m} / \mathrm{z} 255.1138\left([\mathrm{M}+\mathrm{Na}]^{+}\right)$, found: $m / z 255.1141\left([\mathrm{M}+\mathrm{Na}]^{+}\right)$. 
10. Derivatization of Imidated Products to Sulfamides (Figure 5b)

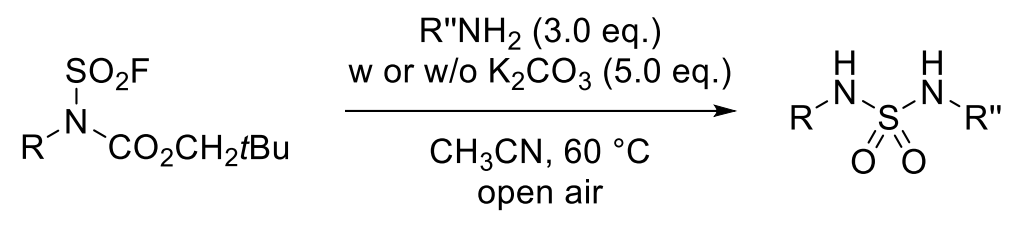

To a test tube, amine $(300 \mu \mathrm{mol})$, imidated product $(100 \mu \mathrm{mol})$ and acetonitrile were added under air. After stirring for the designated time at $60^{\circ} \mathrm{C}$, the reaction was quenched with $1 \mathrm{~N} \mathrm{HCl}$ aq. The organic layer was extracted with EtOAc, washed with brine, dried over anhydrous $\mathrm{Na}_{2} \mathrm{SO}_{4}$ and evaporated under reduced pressure. The residue was purified by flash column chromatography on silica gel.

\section{$N$-phenyl- $N$-(5-phenyl-2-furyl)sulfamide (74)}<smiles>O=S(=O)(Nc1ccccc1)Nc1ccc(-c2ccccc2)o1</smiles>

Prepared with neopentyl (fluorosulfonyl)(5-phenylfuran-2-yl)carbamate (10) $(100 \mu \mathrm{mol}, 35.5 \mathrm{mg})$, aniline (300 $\mu \mathrm{mol}$, $27.9 \mu \mathrm{L}, 98.0 \%)$ and potassium carbonate $(500 \mu \mathrm{mol}, 69.1 \mathrm{mg})$ in acetonitrile $(0.10 \mathrm{~mL})$ for $21 \mathrm{~h}$. The crude material was purified by column chromatography (silica gel pretreated with $1 \% \mathrm{AcOH}$ in $\mathrm{DCM}$, filtered and dried; EtOAc/hexane 5-40\%) to give the target compound as a pale yellowish solid in $18 \%$ yield (18 $\mu \mathrm{mol}, 5.6 \mathrm{mg}$ ). ${ }^{1} \mathrm{H}$ NMR $\left(392 \mathrm{MHz}, \mathrm{CDCl}_{3}\right)$ ס 7.48-7.51 (m, 2H), 7.34-7.39 (m, 4H), 7.20-7.30 (m, 4H), $6.62(\mathrm{br}, 1 \mathrm{H}), 6.58(\mathrm{~d}, J=$ $3.4 \mathrm{~Hz}, 1 \mathrm{H}), 6.58(\mathrm{br}, 1 \mathrm{H}), 6.24(\mathrm{~d}, J=3.4 \mathrm{~Hz}, 1 \mathrm{H})$.

${ }^{13} \mathrm{C}$ NMR $\left(99 \mathrm{MHz}, \mathrm{CDCl}_{3}\right) \delta 151.5,141.1,136.3,129.9,129.5,128.7,127.8,125.5,123.6,121.2,107.3,106.2$. HRMS (ESI) exact mass calculated for $\mathrm{C}_{16} \mathrm{H}_{14} \mathrm{O}_{3} \mathrm{~N}_{2} \mathrm{SNa}: \mathrm{m} / \mathrm{z} 337.0617\left([\mathrm{M}+\mathrm{Na}]^{+}\right)$, found: $\mathrm{m} / \mathrm{z} 337.0617\left([\mathrm{M}+\mathrm{Na}]^{+}\right)$.

\section{N-benzyl-N-(5-phenyl-2-furyl)sulfamide (75)}<smiles>O=S(=O)(NCc1ccccc1)Nc1ccc(-c2ccccc2)o1</smiles>

Prepared with neopentyl (fluorosulfonyl)(5-phenylfuran-2-yl)carbamate (10) (100 $\mu \mathrm{mol}, 35.5 \mathrm{mg}$ ), benzylamine (300 $\mu \mathrm{mol}, 33.1 \mu \mathrm{L}, 99.0 \%)$ and potassium carbonate $(500 \mu \mathrm{mol}, 69.1 \mathrm{mg})$ in acetonitrile $(0.10 \mathrm{~mL})$ for $2 \mathrm{~h}$. The crude material was purified by column chromatography (1st acetone/hexane 8-66\%, 2nd acetone/hexane 5-40\%) to give the target compound as a white solid in $72 \%$ yield $(71.6 \mu \mathrm{mol}, 23.5 \mathrm{mg})$.

${ }^{1} \mathrm{H}$ NMR $\left(396 \mathrm{MHz}, \mathrm{CDCl}_{3}\right)$ ס 7.60-7.57 (m, 2H), 7.42-7.27 (m, 8H), $6.62(\mathrm{~d}, \mathrm{~J}=3.4 \mathrm{~Hz}, 1 \mathrm{H}), 6.43(\mathrm{br}, 1 \mathrm{H}), 6.30$ (dd, J = 3.4, 1.1 Hz, 1H), $4.69(\mathrm{t}, \mathrm{J}=5.9 \mathrm{~Hz}, 1 \mathrm{H}), 4.38(\mathrm{~d}, \mathrm{~J}=5.9 \mathrm{~Hz}, 2 \mathrm{H})$

${ }^{13} \mathrm{C}$ NMR $\left(99 \mathrm{MHz}, \mathrm{CDCl}_{3}\right) \delta 151.3,142.0,135.9,130.0,128.8,128.7,128.2,128.1,127.8,123.6,106.5,106.4$, 47.7.

HRMS (ESI) exact mass calculated for $\mathrm{C}_{17} \mathrm{H}_{16} \mathrm{O}_{3} \mathrm{~N}_{2} \mathrm{SNa}: \mathrm{m} / \mathrm{z} 351.0774\left([\mathrm{M}+\mathrm{Na}]^{+}\right)$, found: $\mathrm{m} / \mathrm{z} 351.0779\left([\mathrm{M}+\mathrm{Na}]^{+}\right)$. 
<smiles>Cc1ccccc1NS(=O)(=O)Nc1ccc(-c2ccccc2)s1</smiles>

Prepared with neopentyl (fluorosulfonyl)(5-phenylthiophen-2-yl)carbamate (4) (100 $\mu \mathrm{mol}, 37.1 \mathrm{mg}$ ) and o-toluidine (300 $\mu \mathrm{mol}, 32.2 \mu \mathrm{L}, 99.0 \%)$ in acetonitrile $(0.050 \mathrm{~mL})$ for $2 \mathrm{~h}$. The crude material was purified by column chromatography (acetone/hexane 5-40\%) to give the target compound as a pale yellowish solid in $86 \%$ yield (85.9 $\mu \mathrm{mol}, 30.2 \mathrm{mg}, 98.0 \%)$.

${ }^{1} \mathbf{H}$ NMR $\left(392 \mathrm{MHz}, \mathrm{CDCl}_{3}\right) \delta 7.54(\mathrm{~d}, J=7.9 \mathrm{~Hz}, 1 \mathrm{H}), 7.45-7.49(\mathrm{~m}, 2 \mathrm{H}), 7.32-7.36(\mathrm{~m}, 2 \mathrm{H}), 7.09-7.29(\mathrm{~m}, 4 \mathrm{H})$, $7.02(\mathrm{~d}, J=3.8 \mathrm{~Hz}, 1 \mathrm{H}), 6.84(\mathrm{br}, 1 \mathrm{H}), 6.65(\mathrm{~d}, J=3.8 \mathrm{~Hz}, 1 \mathrm{H}), 6.50(\mathrm{br}, 1 \mathrm{H}), 2.12(\mathrm{~s}, 3 \mathrm{H})$.

${ }^{13} \mathrm{C}$ NMR $\left(99 \mathrm{MHz}, \mathrm{CDCl}_{3}\right) \delta 141.8,135.6,134.7,133.7,131.0,129.2,128.9,127.8,127.2,125.5,125.5,124.2$, 121.3, 120.7, 17.5 .

HRMS (ESI) exact mass calculated for $\mathrm{C}_{17} \mathrm{H}_{16} \mathrm{O}_{2} \mathrm{~N}_{2} \mathrm{~S}_{2} \mathrm{Na}: \mathrm{m} / z 367.0545\left([\mathrm{M}+\mathrm{Na}]^{+}\right)$, found: $\mathrm{m} / z 367.0545\left([\mathrm{M}+\mathrm{Na}]^{+}\right)$.

\section{N-t-butyl-N-(5-phenyl-2-thienyl)sulfamide (77)}<smiles>CC(C)(C)NS(=O)(=O)Nc1ccc(-c2ccccc2)s1</smiles>

Prepared with neopentyl (fluorosulfonyl)(5-phenylthiophen-2-yl)carbamate (4) (100 $\mu \mathrm{mol}, 37.1 \mathrm{mg}$ ) and tertbutylamine $(300 \mu \mathrm{mol}, 31.8 \mu \mathrm{L}, 99.0 \%)$ in acetonitrile $(0.050 \mathrm{~mL})$ for $2 \mathrm{~h}$. The crude material was purified by column chromatography (EtOAc/hexane 5-40\%) to give the target compound as a white solid in $55 \%$ yield $(54.8 \mu \mathrm{mol}$, $17.0 \mathrm{mg}$ ).

${ }^{1} \mathbf{H}$ NMR $\left(396 \mathrm{MHz}, \mathrm{CDCl}_{3}\right) \delta$ 7.51-7.54 (m, 2H), 7.33-7.38 (m, 2H), 7.25-7.29 (m, 1H), 7.07 (d, J = 4.0 Hz, 1H), $6.85(\mathrm{~d}, J=3.8 \mathrm{~Hz}, 1 \mathrm{H}), 6.54(\mathrm{br}, 1 \mathrm{H}), 4.57(\mathrm{br}, 1 \mathrm{H}), 1.39(\mathrm{~s}, 9 \mathrm{H})$.

${ }^{13} \mathrm{C}$ NMR (99 MHz, $\left.\mathrm{CDCl}_{3}\right) \delta$ 140.8, 137.2, 134.0, 128.9, 127.6, 125.5, 123.3, 121.4, 54.7, 29.7.

HRMS (ESI) exact mass calculated for $\mathrm{C}_{14} \mathrm{H}_{18} \mathrm{O}_{2} \mathrm{~N}_{2} \mathrm{~S}_{2} \mathrm{Na}: \mathrm{m} / \mathrm{z} 333.0702\left([\mathrm{M}+\mathrm{Na}]^{+}\right)$, found: $\mathrm{m} / \mathrm{z} 333.0696$ $\left([\mathrm{M}+\mathrm{Na}]^{+}\right)$.

\section{$N$-(2-hydroxyethyl)- $N$-(5-phenyl-2-thienyl)sulfamide (78)}<smiles>O=S(=O)(NCCO)Nc1ccc(-c2ccccc2)s1</smiles>

Prepared with neopentyl (fluorosulfonyl)(5-phenylthiophen-2-yl)carbamate (4) (100 $\mu \mathrm{mol}, 37.1 \mathrm{mg}$ ) and 2aminoethanol (300 $\mu \mathrm{mol}, 18.3 \mu \mathrm{L}, 99.0 \%)$ in acetonitrile $(0.050 \mathrm{~mL})$ for $2 \mathrm{~h}$. The crude material was purified by column chromatography (EtOAc/hexane $8-66 \%$ ) to give the target compound as a yellowish solid in $52 \%$ yield (52.3 $\mu \mathrm{mol}, 15.6 \mathrm{mg})$.

${ }^{1}$ H NMR $\left(392 \mathrm{MHz}, \mathrm{CD}_{3} \mathrm{CN}\right) \delta 7.83(\mathrm{br}, 1 \mathrm{H})$, 7.58-7.61 (m, 2H), 7.37-7.42 (m, 2H), 7.28-7.32 (m, 1H), 7.20 (d, $J=$ $3.8 \mathrm{~Hz}, 1 \mathrm{H}), 6.81(\mathrm{~d}, J=3.8 \mathrm{~Hz}, 1 \mathrm{H}), 5.65(\mathrm{t}, J=5.6 \mathrm{~Hz}, 1 \mathrm{H}), 3.58(\mathrm{t}, J=5.6 \mathrm{~Hz}, 2 \mathrm{H}), 3.11-3.16(\mathrm{~m}, 2 \mathrm{H}), 2.99(\mathrm{br}$, $1 \mathrm{H})$.

${ }^{13} \mathrm{C}$ NMR $\left(99 \mathrm{MHz}, \mathrm{CD}_{3} \mathrm{CN}\right) \delta 140.3,139.5,134.9,130.0,128.5,126.1,122.8,122.7,61.4,46.4$. 


\section{$N$-(2-cyanooctyl)-N-(phenyl)sulfamide (79)}<smiles>[14CH3]C(C#N)CNS(=O)(=O)Nc1ccccc1</smiles>

Prepared with neopentyl (2-cyanooctyl)(fluorosulfonyl)carbamate (38) $(100 \mu \mathrm{mol}, 35.0 \mathrm{mg})$ and aniline (300 $\mu \mathrm{mol}$, $27.7 \mu \mathrm{L})$ in acetonitrile $(0.050 \mathrm{~mL})$ for $48 \mathrm{~h}$. The crude material was purified by column chromatography (EtOAc/hexane 8-66\%) to give the title compound as a pale brown solid in $89 \%$ yield ( $88.9 \mu \mathrm{mol}, 27.5 \mathrm{mg}$ ).

${ }^{1} \mathrm{H}$ NMR $\left(392 \mathrm{MHz}, \mathrm{CDCl}_{3}\right)$ ס 7.33-7.39 (m, 2H), 7.16-7.21 (m, 3H), $6.66(\mathrm{~s}, 1 \mathrm{H}), 5.04(\mathrm{t}, J=6.7 \mathrm{~Hz}, 1 \mathrm{H}), 3.19-3.31$ $(\mathrm{m}, 2 \mathrm{H}), 2.69-2.76(\mathrm{~m}, 1 \mathrm{H}), 1.25-1.55(\mathrm{~m}, 10 \mathrm{H}), 0.88(\mathrm{t}, J=6.9 \mathrm{~Hz}, 3 \mathrm{H})$.

${ }^{13} \mathrm{C}$ NMR $\left(99 \mathrm{MHz}, \mathrm{CDCl}_{3}\right) \delta$ 136.6, 129.6, 125.1, 120.5, 120.3, 44.5, 33.0, 31.4, 29.2, 28.6, 26.7, 22.4, 14.0.

HRMS (ESI) exact mass calculated for $\mathrm{C}_{15} \mathrm{H}_{23} \mathrm{O}_{2} \mathrm{~N}_{3} \mathrm{SNa}: \mathrm{m} / z 332.1403\left([\mathrm{M}+\mathrm{Na}]^{+}\right)$, found: $m / z 332.1403\left([\mathrm{M}+\mathrm{Na}]^{+}\right)$.

\section{$N$-(2-cyanooctyl)- $N$-(propyl)sulfamide (80)}<smiles>CC(C)CNS(=O)(=O)NC(C)C</smiles>

Prepared with neopentyl (2-cyanooctyl)(fluorosulfonyl)carbamate (38) $(100 \mu \mathrm{mol}, 35.0 \mathrm{mg})$ and propylamine (300 $\mu \mathrm{mol}, 25.2 \mu \mathrm{L})$ in acetonitrile $(0.050 \mathrm{~mL})$ for $6 \mathrm{~h}$ at $40{ }^{\circ} \mathrm{C}$. The crude material was purified by column chromatography (EtOAc/hexane 8-66\%) to give the title compound as a white solid in 70\% yield (69.7 $\mu$ mol, 19.2 $\mathrm{mg})$.

${ }^{1} \mathrm{H}$ NMR $\left(392 \mathrm{MHz}, \mathrm{CDCl}_{3}\right)$ ठ 4.98-5.02 $(\mathrm{m}, 1 \mathrm{H}), 4.48-4.51(\mathrm{~m}, 1 \mathrm{H}), 3.18-3.32(\mathrm{~m}, 2 \mathrm{H}), 3.06(\mathrm{dd}, J=13.4,7.1 \mathrm{~Hz}$, $2 \mathrm{H}), 2.87-2.94(\mathrm{~m}, 1 \mathrm{H}), 1.26-1.64(\mathrm{~m}, 12 \mathrm{H}), 0.96(\mathrm{t}, J=7.4 \mathrm{~Hz}, 3 \mathrm{H}), 0.89(\mathrm{t}, J=6.8 \mathrm{~Hz}, 3 \mathrm{H})$.

${ }^{13} \mathrm{C}$ NMR $\left(99 \mathrm{MHz}, \mathrm{CDCl}_{3}\right) \delta 120.7,45.0,44.5,32.9,31.4,29.5,28.7,26.8,22.8,22.5,14.0,11.2$.

HRMS (ESI) exact mass calculated for $\mathrm{C}_{12} \mathrm{H}_{25} \mathrm{O}_{2} \mathrm{~N}_{3} \mathrm{SNa}: \mathrm{m} / \mathrm{z} 298.1560\left([\mathrm{M}+\mathrm{Na}]^{+}\right)$, found: $m / z 298.1556\left([\mathrm{M}+\mathrm{Na}]^{+}\right)$.

\section{Derivatization of 10 to 74 using $n$-BuLi}<smiles>CCCCOC(=O)N(c1ccccc1)c1ccc(-c2ccccc2)o1</smiles>

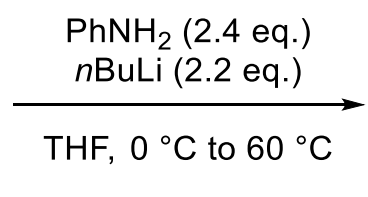<smiles>O=S(=O)(Nc1ccccc1)Nc1ccc(-c2ccccc2)o1</smiles>

To an argon-purged Schlenk tube, aniline (240 $\mu \mathrm{mol}, 22.3 \mu \mathrm{L}, 98.0 \%)$, THF (1.0 mL), $n$-BuLi $(220 \mu \mathrm{mol}, 78.6 \mu \mathrm{L}$, $2.80 \mathrm{M}$ ) were added at $0{ }^{\circ} \mathrm{C}$. After stirring for $15 \mathrm{~min}$ at the same temperature, neopentyl (fluorosulfonyl)(5phenylfuran-2-yl)carbamate (10) $(100 \mu \mathrm{mol}, 35.5 \mathrm{mg})$ was added. After stirring for $2 \mathrm{~h}$ at $60^{\circ} \mathrm{C}$, the reaction was quenched with $1 \mathrm{M}$ citric acid aq. $(1.5 \mathrm{~mL})$. The organic layer was extracted with EtOAc, washed with brine, dried over anhydrous $\mathrm{Na}_{2} \mathrm{SO}_{4}$ and evaporated under reduced pressure. The residue was purified by column chromatography on silica gel to give the target compound as a pale yellowish solid in $63 \%$ yield (62.7 $\mu$ mol, 19.7 $\mathrm{mg}$ ). 


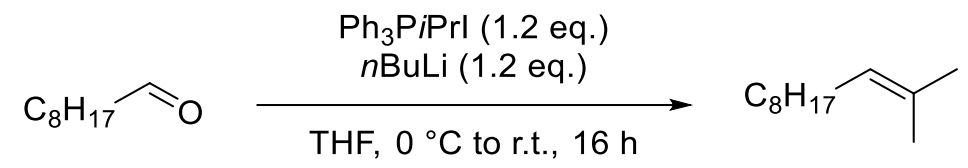

To an argon purged flask, THF (3.0 mL), isopropyltriphenylphosphonium iodide (1.20 mmol, $529 \mathrm{mg}, 98 \%)$ and $n$ BuLi $(2.6 \mathrm{M}, 0.464 \mathrm{~mL})$ were added at $0{ }^{\circ} \mathrm{C}$. After stirring for $30 \mathrm{~min}$ at the same temperature, nonanal $(1.00 \mathrm{mmol}$, $0.172 \mathrm{~mL}$ ) was added at the same temperature. The solution was warmed to room temperature. After stirring for $16 \mathrm{~h}$, the reaction was quenched with sat. $\mathrm{NH}_{4} \mathrm{Cl}$ aq. $(30 \mathrm{~mL})$, extracted with hexane $(30 \mathrm{~mL} \times 3)$. The combined organic layers were washed with brine, dried over anhydrous $\mathrm{Na}_{2} \mathrm{SO}_{4}$ and concentrated under vacuum. The residue was diluted with hexane, filtered through a pad of silica gel and concentrated under vacuum o give the title compound as a colorless oil in $74 \%$ yield $(740 \mu \mathrm{mol}, 131 \mathrm{mg}, 95 \%)$. The spectral data matched with those reported in the literature. ${ }^{23}$

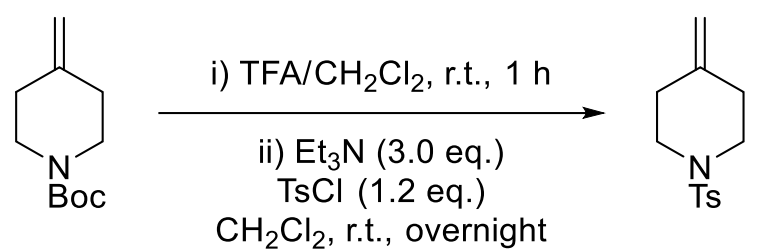

A flask was charged with tert-butyl 4-methylenepiperidine-1-carboxylate $(0.49 \mathrm{mmol}, 97 \mathrm{mg}){ }^{24} \mathrm{DCM}(2 \mathrm{~mL})$ and 2,2,2-trifluoroacetic acid ( $2 \mathrm{~mL})$. After stirred for $1 \mathrm{~h}$ at room temperature, the mixture was concentrated under vacuum. The flask was purged argon and added DCM $(2 \mathrm{~mL})$, triethylamine $(1.47 \mathrm{mmol}, 0.205 \mathrm{~mL})$ and 4methylbenzenesulfonyl chloride $(0.588 \mathrm{mmol}, 112 \mathrm{mg})$. After stirring overnight, the reaction was quenched with 1 $\mathrm{N} \mathrm{HCl}$ aq. $(20 \mathrm{~mL})$, extracted with DCM. The combined organic layers were washed with sat. $\mathrm{NaHCO}_{3}$ aq. and water, dried over anhydrous $\mathrm{Na}_{2} \mathrm{SO}_{4}$ and concentrated under vacuum. The crude material was purified by flash column chromatography on silica gel (EtOAc/hexane 6-50\%) to give the title compound as a white solid in $85 \%$ yield $(0.417 \mathrm{mmol}, 105 \mathrm{mg})$. The spectral data matched with those reported in the literature. ${ }^{25}$ 
12. Reaction Optimizations

Cu-catalyzed Imidation of Heteroarenes and PAHs (Figure 2a)
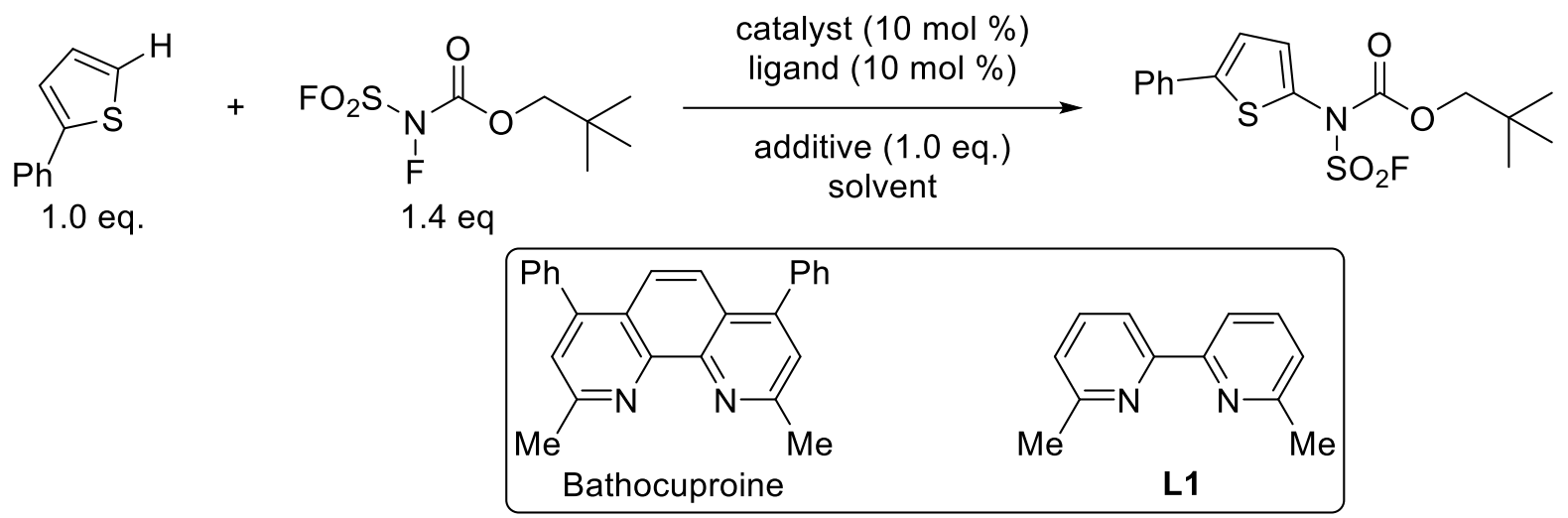

\begin{tabular}{|c|c|c|c|c|c|c|c|}
\hline entry & catalyst & ligand & additive & solvent & temp. $\left({ }^{\circ} \mathrm{C}\right)$ & time $(\mathrm{h})$ & NMR yield $(\%)$ \\
\hline 1 & $\mathrm{CuBr}$ & Bathocuproine & $\mathrm{CaCO}_{3}$ & $\mathrm{CH}_{3} \mathrm{CN}$ & rt & 25 & 28 \\
\hline 2 & $\mathrm{CuCN}$ & Bathocuproine & $\mathrm{CaCO}_{3}$ & $\mathrm{CH}_{3} \mathrm{CN}$ & rt & 22 & 30 \\
\hline 3 & $\mathrm{CuCl}$ & Bathocuproine & $\mathrm{CaCO}_{3}$ & $\mathrm{CH}_{3} \mathrm{CN}$ & rt & 22 & 38 \\
\hline 4 & $\mathrm{CuCl}$ & Bathocuproine & $\mathrm{CaCO}_{3}$ & $\mathrm{CH}_{3} \mathrm{CN}$ & 40 & 23 & 56 \\
\hline 5 & $\mathrm{CuCl}$ & Bathocuproine & $\mathrm{CaCO}_{3}$ & $\mathrm{CH}_{3} \mathrm{CN}$ & 40 & 6 & 43 \\
\hline 6 & $\mathrm{CuCl}$ & $\mathbf{L 1}$ & $\mathrm{CaCO}_{3}$ & $\mathrm{CH}_{3} \mathrm{CN}$ & 40 & 6 & 49 \\
\hline 7 & $\mathrm{CuCl}$ & $\mathbf{L 1}$ & $\mathrm{CaCO}_{3}$ & $\left(\mathrm{CH}_{2} \mathrm{Cl}\right)_{2}$ & 40 & 7 & 67 \\
\hline 8 & $\mathrm{CuCl}$ & $\mathbf{L 1}$ & $\mathrm{CaCO}_{3}$ & $\left(\mathrm{CH}_{2} \mathrm{Cl}\right)_{2}$ & 70 & 5 & 75 \\
\hline 9 & $\mathrm{CuCl}$ & $\mathbf{L 1}$ & $\mathbf{w} / 0$ & $\left(\mathrm{CH}_{2} \mathrm{Cl}\right)_{2}$ & 70 & 5 & 68 \\
\hline
\end{tabular}

\section{Cu-catalyzed Imidation of Benzene Derivatives (Figure 2e)}

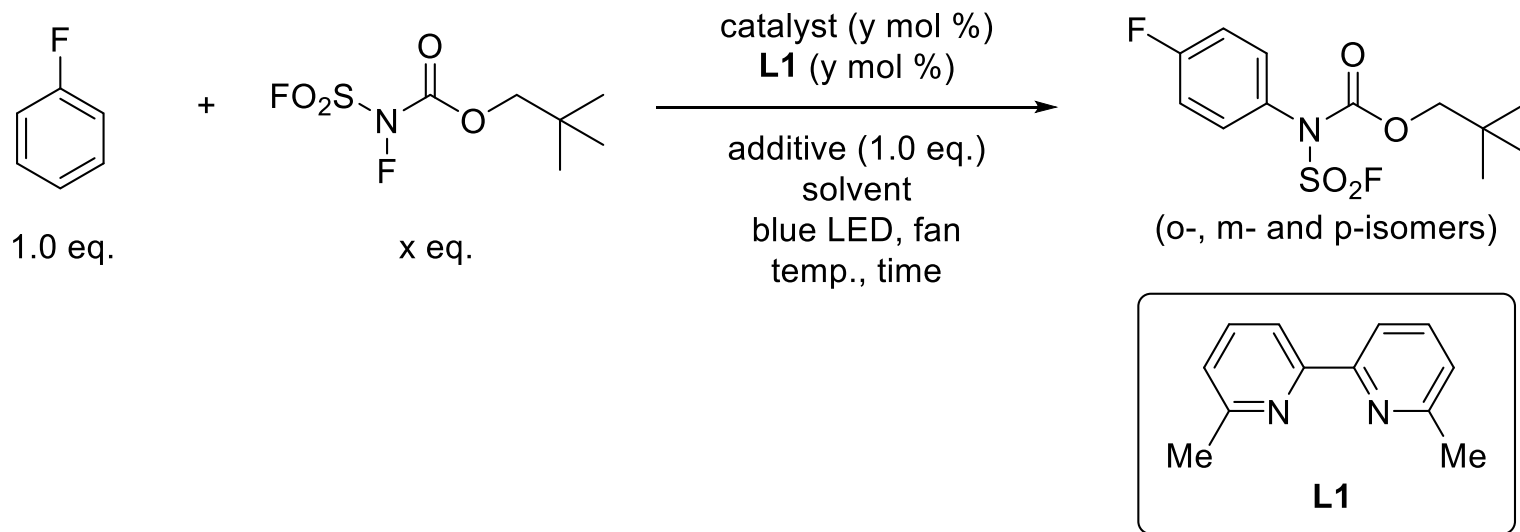

\begin{tabular}{|c|c|c|c|c|c|c|c|c|c|c|}
\hline entry & $\begin{array}{c}\mathrm{x} \\
\text { (eq.) }\end{array}$ & $\begin{array}{c}\mathrm{y} \\
(\mathrm{mol} \%)\end{array}$ & catalyst & additive & solvent & $\begin{array}{c}\text { blue } \\
\text { LED }\end{array}$ & & temp. $\left({ }^{\circ} \mathrm{C}\right)$ & $\begin{array}{c}\text { time } \\
(\mathrm{h})\end{array}$ & $\begin{array}{c}\text { NMR } \\
\text { yield }(\%)\end{array}$ \\
\hline 1 & 1.4 & 10 & $\mathrm{CuCl}$ & $\mathrm{CaCO}_{3}$ & $\left(\mathrm{CH}_{2} \mathrm{Cl}\right)_{2}$ & on & $\mathrm{Ar}$ & $30 \sim 40$ & 5 & 15 \\
\hline 2 & 1.4 & 10 & $\mathrm{CuBF}_{4}\left(\mathrm{CH}_{3} \mathrm{CN}\right)_{4}$ & none & $\mathrm{CH}_{3} \mathrm{CN}$ & off & $\mathrm{Ar}$ & 70 & 5 & 33 \\
\hline 3 & 1.4 & 10 & $\mathrm{CuBF}_{4}\left(\mathrm{CH}_{3} \mathrm{CN}\right)_{4}$ & none & $\left(\mathrm{CH}_{2} \mathrm{Cl}\right)_{2}$ & on & $\mathrm{Ar}$ & $30 \sim 40$ & 5 & 46 \\
\hline 4 & 1.4 & 10 & $\mathrm{CuBF}_{4}\left(\mathrm{CH}_{3} \mathrm{CN}\right)_{4}$ & none & $\mathrm{CH}_{2} \mathrm{Cl}_{2}$ & on & $\mathrm{Ar}$ & $30 \sim 40$ & 5 & 39 \\
\hline 5 & 1.4 & 10 & $\mathrm{CuBF}_{4}\left(\mathrm{CH}_{3} \mathrm{CN}\right)_{4}$ & none & $\mathrm{CH}_{2} \mathrm{Cl}_{2}$ & on & $\mathrm{Ar}$ & $30 \sim 40$ & 12 & 40 \\
\hline 6 & 2.0 & 20 & $\mathrm{CuBF}_{4}\left(\mathrm{CH}_{3} \mathrm{CN}\right)_{4}$ & none & $\mathrm{CH}_{2} \mathrm{Cl}_{2}$ & on & $\mathrm{Ar}$ & $30 \sim 40$ & 14 & 63 \\
\hline 7 & 2.0 & 20 & $\mathrm{CuBF}_{4}\left(\mathrm{CH}_{3} \mathrm{CN}\right)_{4}$ & $\mathrm{CaCO}_{3}$ & $\mathrm{CH}_{2} \mathrm{Cl}_{2}$ & on & $\mathrm{Ar}$ & $30 \sim 40$ & 12 & 94 \\
\hline 8 & 2.0 & 20 & $\mathrm{CuBF}_{4}\left(\mathrm{CH}_{3} \mathrm{CN}\right)_{4}$ & $\mathrm{CaCO}_{3}$ & $\mathrm{CH}_{2} \mathrm{Cl}_{2}$ & on & air & $30 \sim 40$ & 12 & 77 \\
\hline
\end{tabular}


Cu-catalyzed Imidocyanation of Terminal and Vicinally Disubstituted Alkenes (Figure 3)

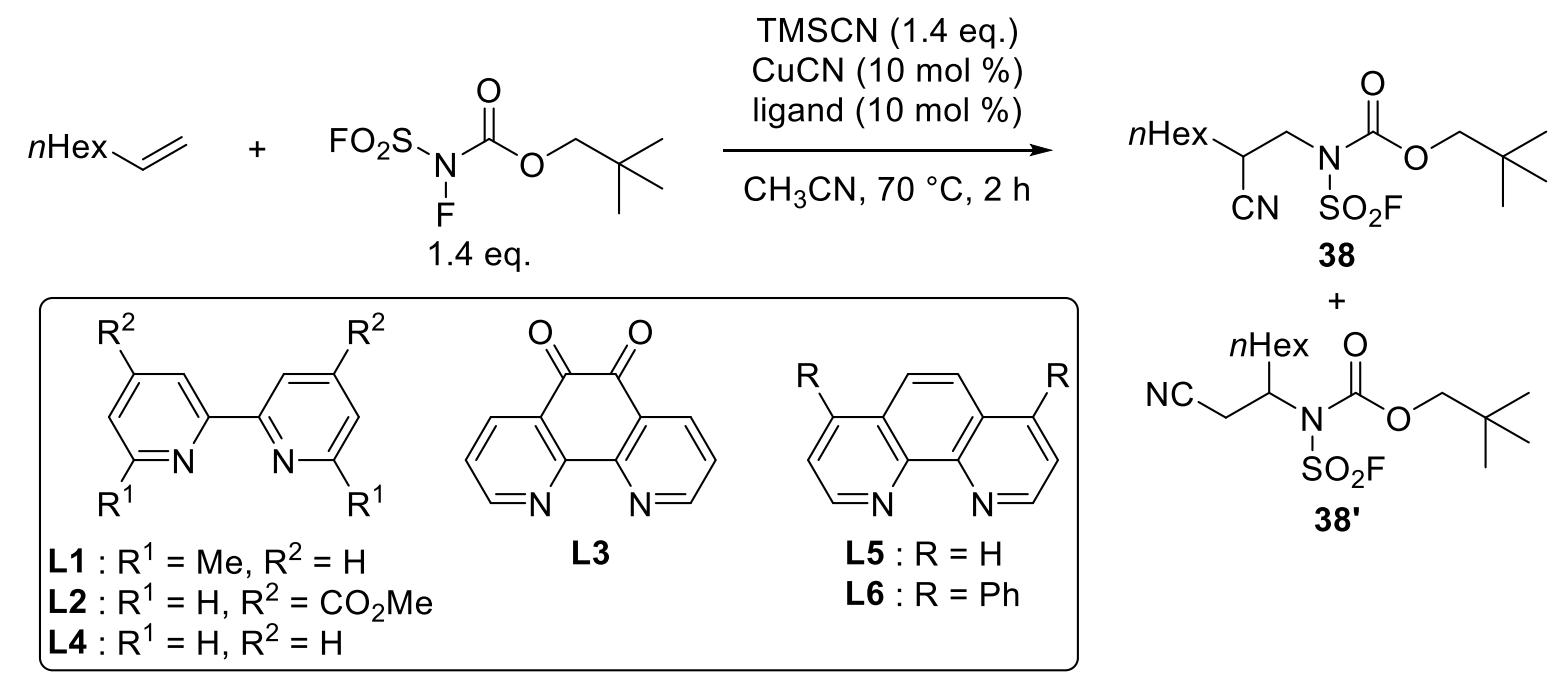

\begin{tabular}{|c|c|c|c|}
\hline entry & ligand & NMR yield (38+38') (\%) & rr \\
\hline 1 & L1 & 22 & $91: 9$ \\
\hline 2 & L2 & 82 & $89: 11$ \\
\hline 3 & L3 & 71 & $87: 13$ \\
\hline 4 & L4 & 75 & $89: 11$ \\
\hline 5 & L5 & 71 & $89: 11$ \\
\hline 6 & L6 & 78 & $88: 12$ \\
\hline
\end{tabular}

Cu-catalyzed Imidocyanation of Geminally Disubstituted Alkenes (Figure 3)

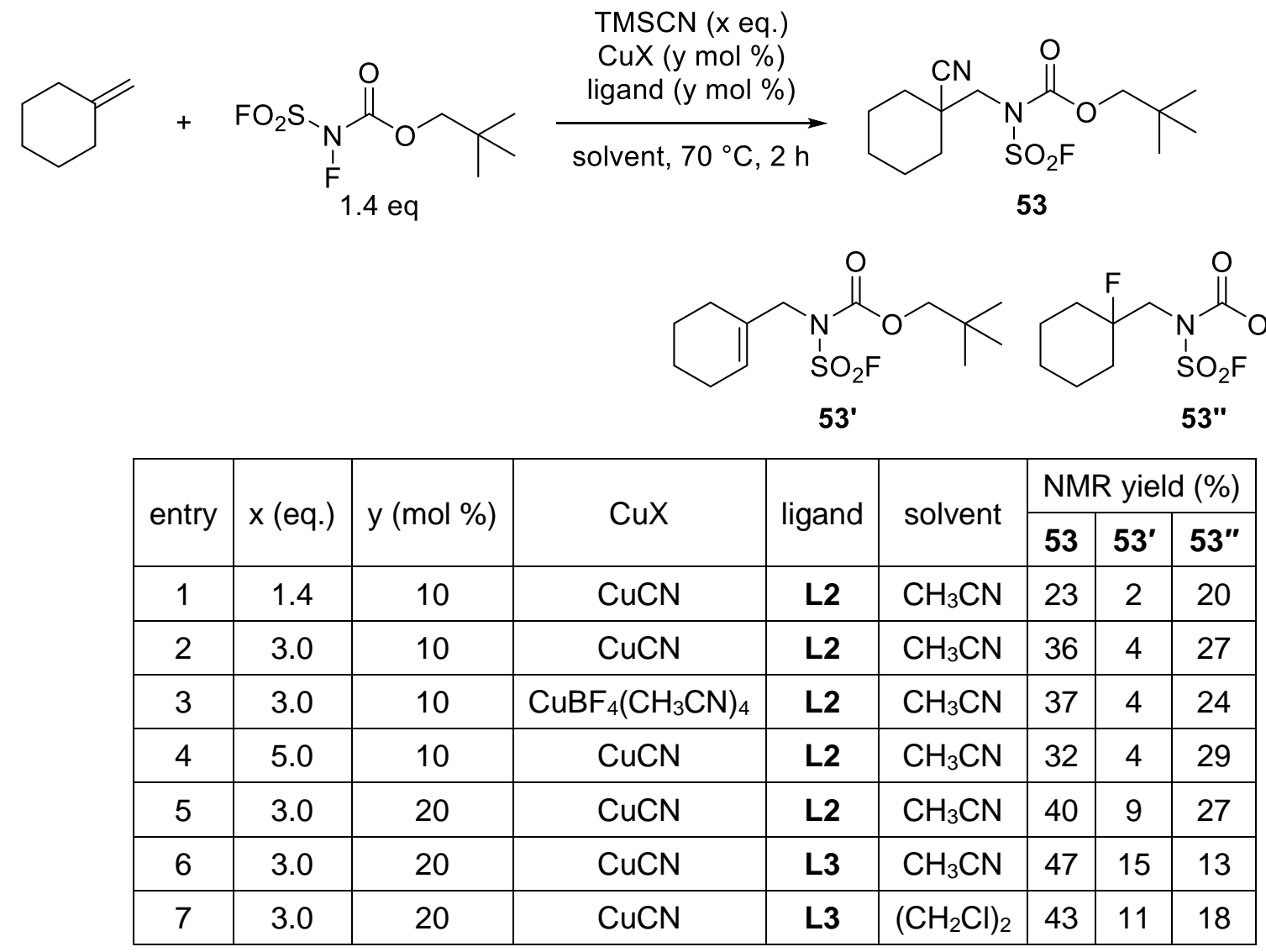




\section{Cyclic Voltammetry Experiments}

$1 \mathrm{mM}$ samples were prepared in $0.1 \mathrm{M}$ tetra- $n$-butylammonium hexafluorophosphate solution of dry, degassed acetonitrile $(10 \mathrm{~mL})$. Measurements employed a glassy carbon working electrode, platinum wire counter electrode, $0.01 \mathrm{M}$ silver-silver nitrate reference electrode, and a scan rate of $100 \mathrm{mV} / \mathrm{s}$ at $23^{\circ} \mathrm{C}$. $\mathrm{E}_{\text {calc }}$ were estimated according to the literature procedure. ${ }^{26}$

$$
\mathrm{FO}_{2} \mathrm{~S}_{\Theta} \mathrm{N}^{-\mathrm{CO}_{2} \mathrm{CH}_{2}{ }^{t} \mathrm{Bu}}
$$

$$
E_{p / 2}=+1.80 \vee v s \mathrm{Ag} / \mathrm{Ag}+
$$

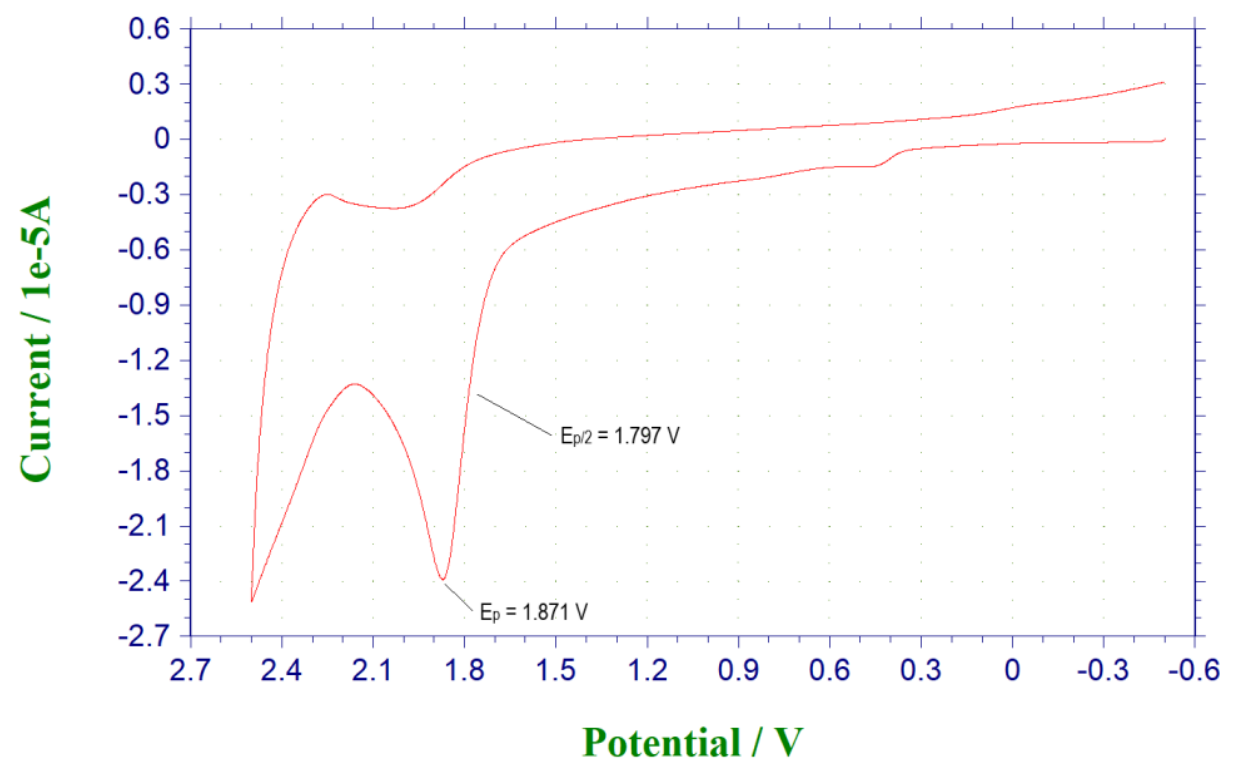

$$
\begin{gathered}
\mathrm{PhO}_{2} \mathrm{~S}_{-} \mathrm{N}^{-\mathrm{SO}_{2} \mathrm{Ph}} \\
\Theta \\
\mathrm{E}_{\mathrm{p} / 2}=+1.61 \vee \text { vs } \mathrm{Ag} / \mathrm{Ag}+
\end{gathered}
$$

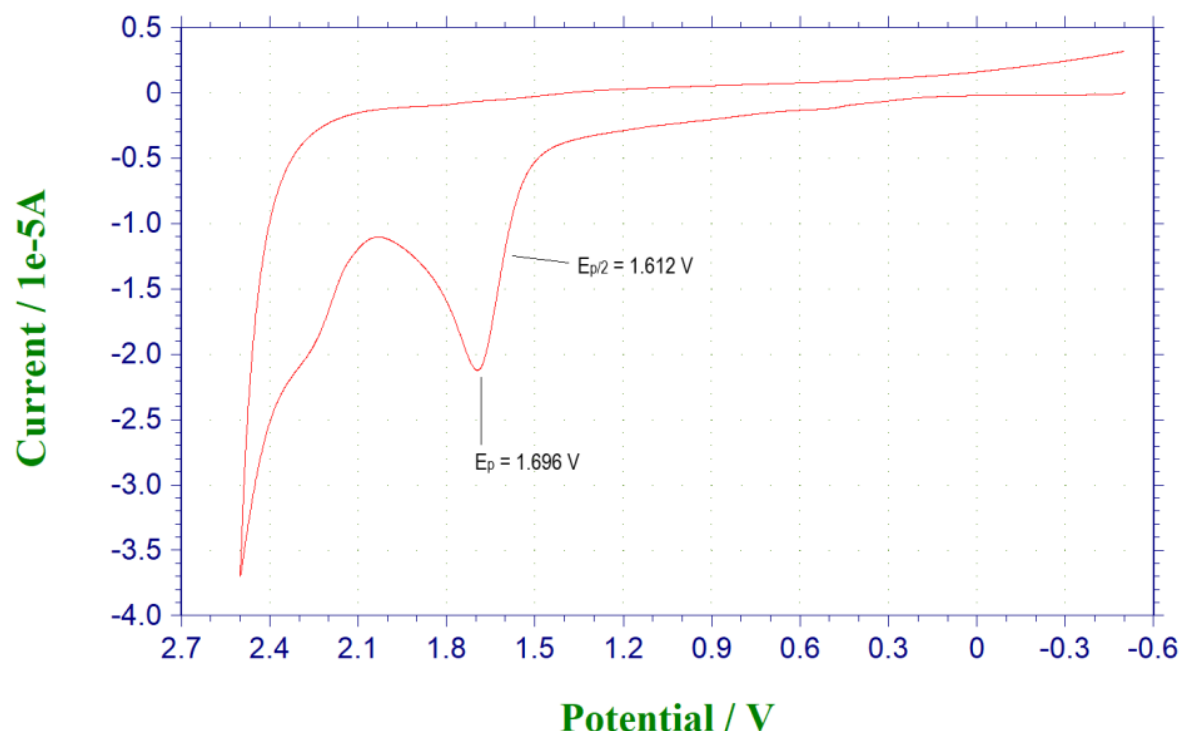




\section{References}

(1) Ma, L.; Yuan, L.; Xu, C.; Li, G.; Tao, M.; Zhang, W. An Efficient Synthesis of 2-Aminothiophenes via the Gewald Reaction Catalyzed by an N-Methylpiperazine-Functionalized Polyacrylonitrile Fiber. Synthesis 2013, 45, 45-52.

(2) Ozdemir, M.; Choi, D.; Kwon, G.; Zorlu, Y.; Cosut, B.; Kim, H.; Facchetti, A.; Kim, C.; Usta, H. Solution-Processable BODIPY-Based Small Molecules for Semiconducting Microfibers in Organic Thin-Film Transistors. ACS Applied Materials \& Interfaces 2016, 8, 14077-14087.

(3) Paul, S.; Das, K. K.; Manna, S.; Panda, S. Transition-Metal-Free Synthesis of Heterobiaryls through 1,2-Migration of Boronate Complex. Chem. Eur. J. 2020, 26, 1922-1927.

(4) Duan, Y.-N.; Cui, L.-Q.; Zuo, L.-H.; Zhang, C. Recyclable Hypervalent-lodine-Mediated Dehydrogenative $\alpha, \beta^{\prime}$-Bifunctionalization of $\beta$-Keto Esters Under Metal-Free Conditions. Chem. Eur. J. 2015, 21, $13052-13057$.

(5) Fu, J.; Wurzer, N.; Lehner, V.; Reiser, O.; Davies, H. M. L. Rh(II)-Catalyzed Monocyclopropanation of Pyrroles and Its Application to the Synthesis Pharmaceutically Relevant Compounds. Org. Lett. 2019, 21, 6102-6106.

(6) Cai, Z.-J.; Liu, C.-X.; Gu, Q.; Zheng, C.; You, S.-L. Pdll-Catalyzed Regio- and Enantioselective Oxidative $\mathrm{C}-\mathrm{H} / \mathrm{C}-\mathrm{H}$ Cross-Coupling Reaction between Ferrocenes and Azoles. Angew. Chem. Int. Ed. 2019, 58, 2149-2153.

(7) Li, Z.; Zhang, J.; Zhang, W.; Guo, L.; Huang, J.; Yu, G.; Wong, M. S. Effect of fluorine substitution on naphtho[2,1-b:3,4-b']bis[1]-benzothiophene-derived semiconductors for transistor application. Org. Electron. 2016, 32, 47-53.

(8) Liégault, B.; Petrov, I.; Gorelsky, S. I.; Fagnou, K. Modulating Reactivity and Diverting Selectivity in Palladium-Catalyzed Heteroaromatic Direct Arylation Through the Use of a Chloride Activating/Blocking Group. J. Org. Chem. 2010, 75, 1047-1060.

(9) Friedfeld, M. R.; Shevlin, M.; Margulieux, G. W.; Campeau, L.-C.; Chirik, P. J. Cobalt-Catalyzed Enantioselective Hydrogenation of Minimally Functionalized Alkenes: Isotopic Labeling Provides Insight into the Origin of Stereoselectivity and Alkene Insertion Preferences. J. Am. Chem. Soc. 2016, 138, 3314-3324.

(10) Fang, C.; Li, M.; Hu, X.; Mo, W.; Hu, B.; Sun, N.; Jin, L.; Shen, Z. A Mild TEMPO-Catalyzed Aerobic Oxidative Conversion of Aldehydes into Nitriles. Adv. Synth. Catal. 2016, 358, 1157-1163.

(11) Carmen de la Fuente, M.; Domínguez, D. Normal electron demand Diels-Alder cycloaddition of indoles to 2,3-dimethyl-1,3-butadiene. Tetrahedron 2011, 67, 3997-4001.

(12) Inahashi, N.; Matsumiya, A.; Sato, T. Efficient N-tert-Butoxycarbonylation of Indoles with Di-tertbutyl Dicarbonate Catalyzed by Cesium Fluoride. Synlett 2008, 2008, 294-296.

(13) Bayindir, S.; Saracoglu, N. A facile one-pot method to synthesise 2-alkylated indole and 2,2'bis(indolyl)methane derivatives using ketones as electrophiles and their anion sensing ability. RSC Advances 2016, 6, 72959-72967.

(14) Saxena, P.; Maida, N.; Kapur, M. Dioxazolones as masked ester surrogates in the $\mathrm{Pd}(\mathrm{ii})-$ catalyzed direct C-H arylation of 6,5-fused heterocycles. Chem. Commun. 2019, 55, 11187-11190.

(15) Samanta, R. C.; Yamamoto, H. Selective Halogenation Using an Aniline Catalyst. Chem. Eur. J. 2015, 21, 11976-11979.

(16) Bai, Y.; Shi, L.; Zheng, L.; Ning, S.; Che, X.; Zhang, Z.; Xiang, J. Electroselective and Controlled Reduction of Cyclic Imides to Hydroxylactams and Lactams. Org. Lett. 2021, 23, 2298-2302. 
(17) Yang, Z.-D.; Song, Z.-W.; Ren, J.; Yang, M.-J.; Li, S. Improved Thin-layer Chromatography Bioautographic Assay for the Detection of Actylcholinesterase Inhibitors in Plants. Phytochem. Anal 2011, 22, 509515.

(18) Xiong, T.; Li, Y.; Mao, L.; Zhang, Q.; Zhang, Q. Palladium-catalyzed allylic C-H amination of alkenes with $\mathrm{N}$-fluorodibenzenesulfonimide: water plays an important role. Chem. Commun. 2012, 48, 2246-2248.

(19) Yue, H.; Guo, L.; Liao, H.-H.; Cai, Y.; Zhu, C.; Rueping, M. Catalytic Ester and Amide to Amine Interconversion: Nickel-Catalyzed Decarbonylative Amination of Esters and Amides by $\mathrm{C}-\mathrm{O}$ and $\mathrm{C}-\mathrm{C}$ Bond Activation. Angew. Chem. Int. Ed. 2017, 56, 4282-4285.

(20) Meltzer, P. C.; Staskun, B. Double migration of methyl and bromine accompanying indeno[1,2,3de]quinolinone formation. Tetrahedron 1977, 33, 2965-2967.

(21) Zysman-Colman, E.-C.; Arias, K.; Siegel, J. S. S. S. Synthesis of arylbromides from arenes and $\mathrm{N}$-bromosuccinimide (NBS) in acetonitrile - A convenient method for aromatic bromination. Canadian Journal of Chemistry 2009, 87, 440-447.

(22) Krasovskiy, A.; Knochel, P. Convenient Titration Method for Organometallic Zinc, Magnesium, and Lanthanide- Reagents. Synthesis 2006, 2006, 0890-0891.

(23) Cahiez, G.; Duplais, C.; Moyeux, A. Iron-Catalyzed Alkylation of Alkenyl Grignard Reagents. Org. Lett. 2007, 9, 3253-3254.

(24) McManus, J. B.; Onuska, N. P. R.; Nicewicz, D. A. Generation and Alkylation of $\alpha$-Carbamyl Radicals via Organic Photoredox Catalysis. J. Am. Chem. Soc. 2018, 140, 9056-9060.

(25) Furman, B.; Dziedzic, M. An efficient route to 4-(substituted benzyl)piperidines. Tetrahedron Lett. 2003, 44, 8249-8252.

(26) Roth, H. G.; Romero, N. A.; Nicewicz, D. A. Experimental and Calculated Electrochemical Potentials of Common Organic Molecules for Applications to Single-Electron Redox Chemistry. Synlett 2016, 27, 714-723. 
15. Copies of ${ }^{1} \mathrm{H},{ }^{13} \mathrm{C}$ and ${ }^{19} \mathrm{~F}$ NMR Spectra 
lithium (fluorosulfonyl)((neopentyloxy)carbonyl)amide (1)<smiles>CC(C)(C)COC(=O)NS(=O)(=O)O</smiles>
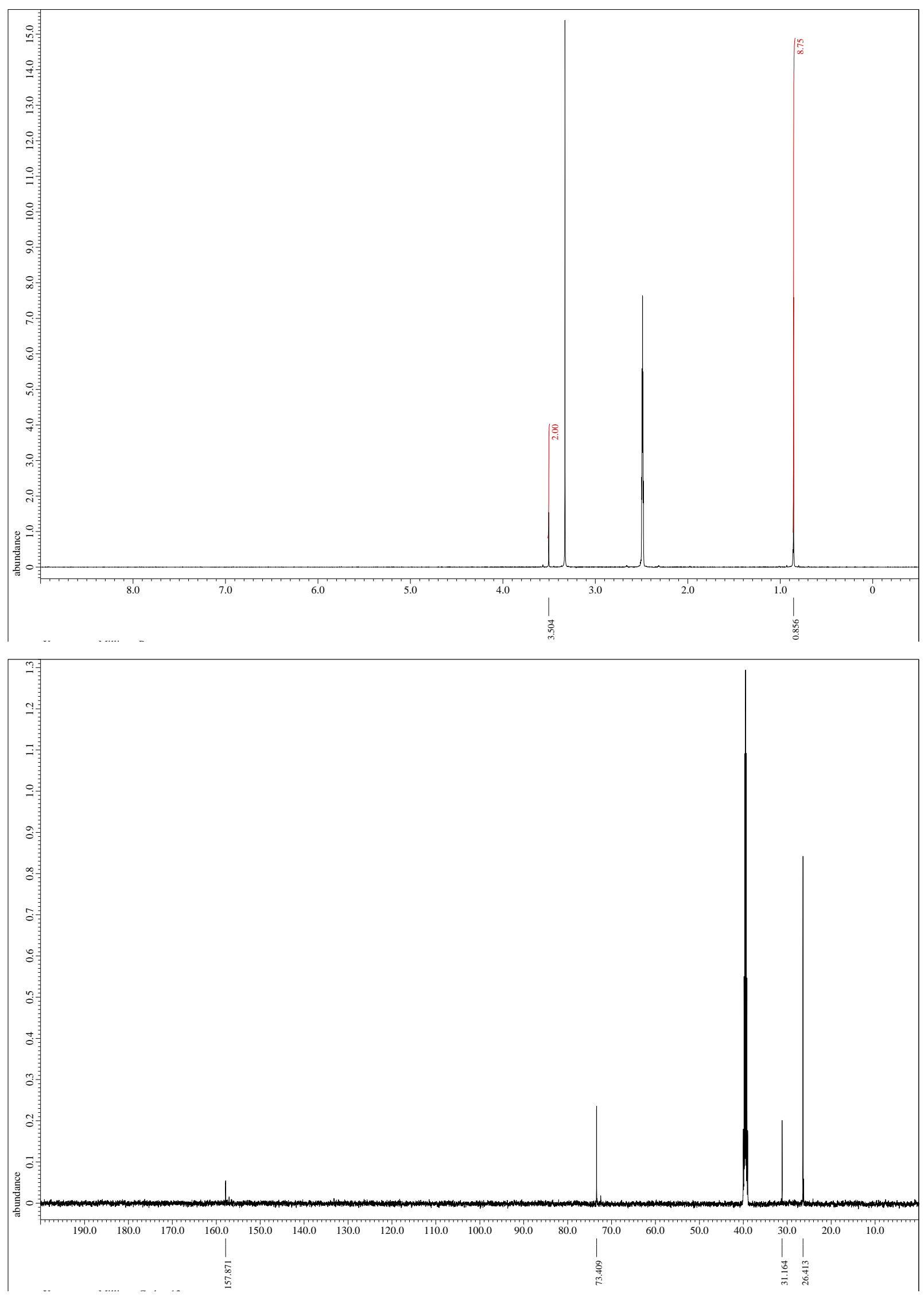


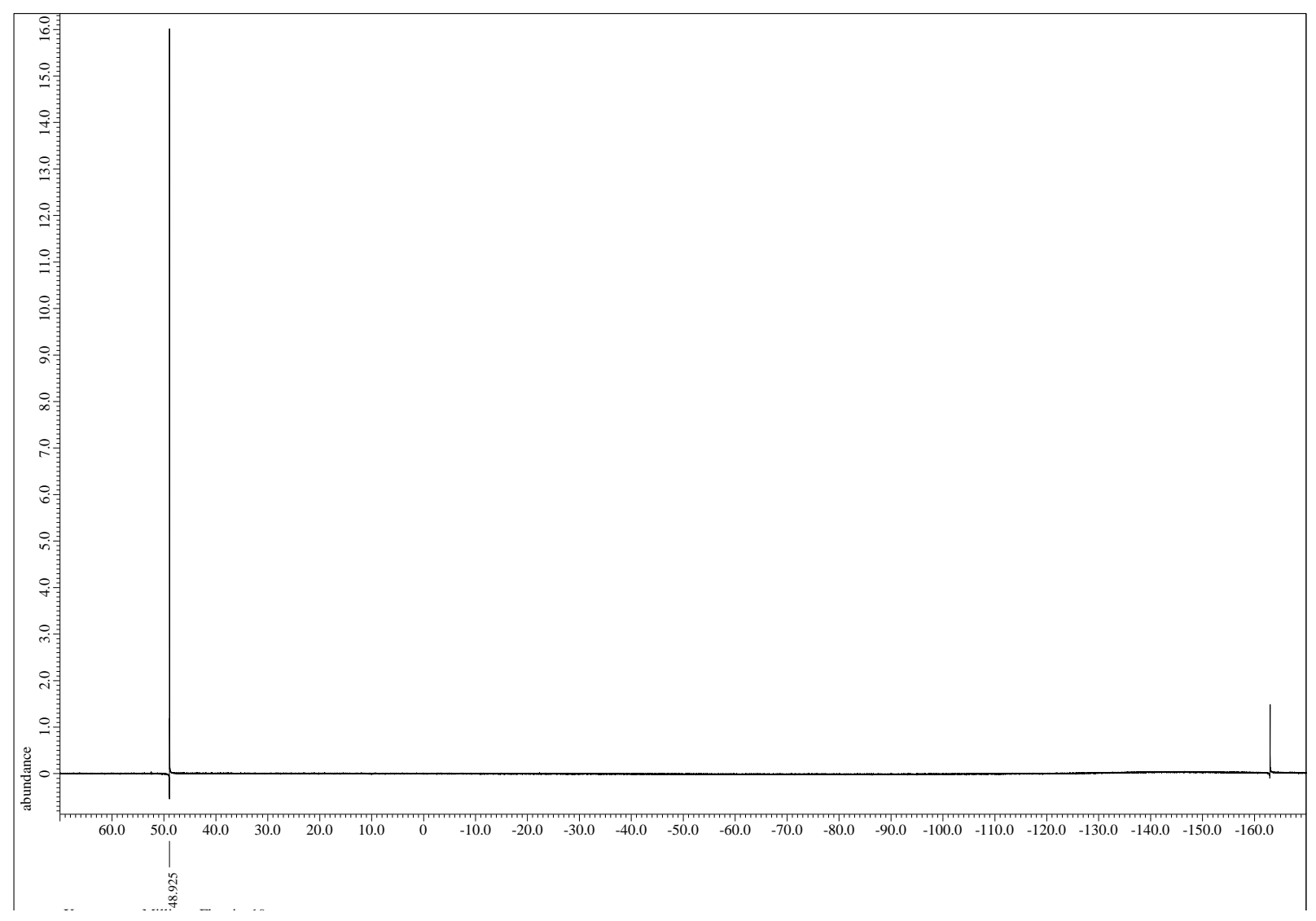



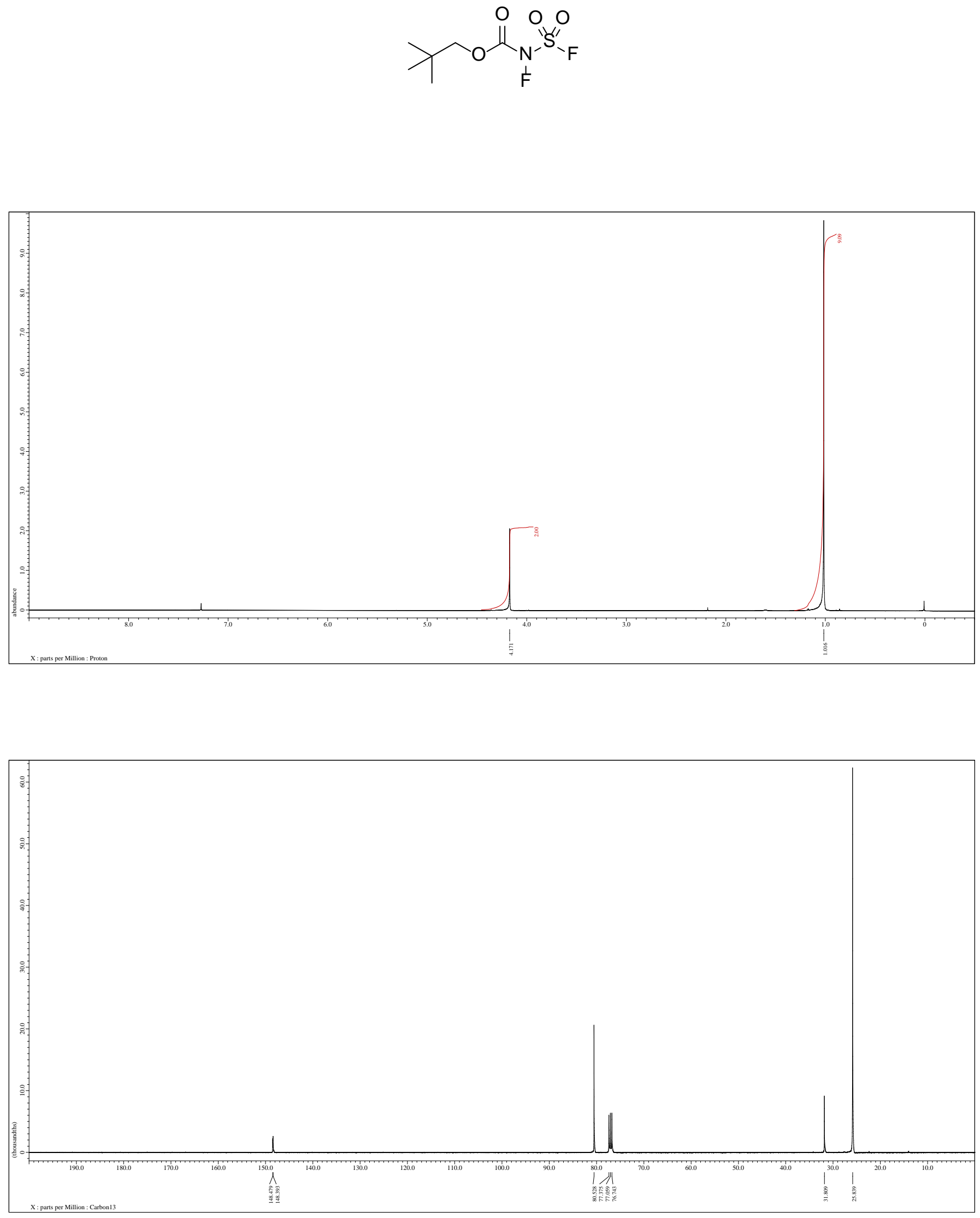


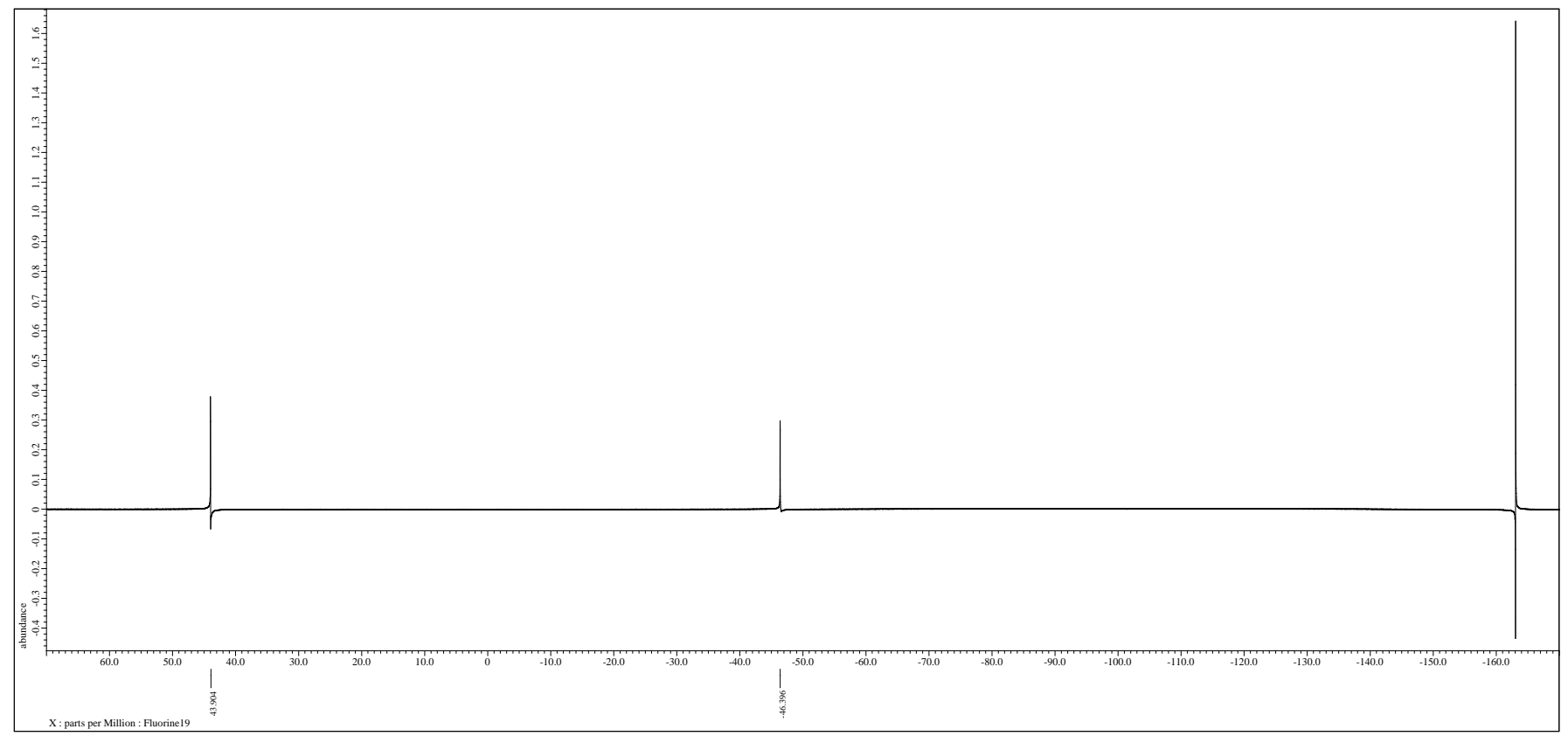


neopentyl (fluorosulfonyl)(thiophen-2-yl)carbamate (3)
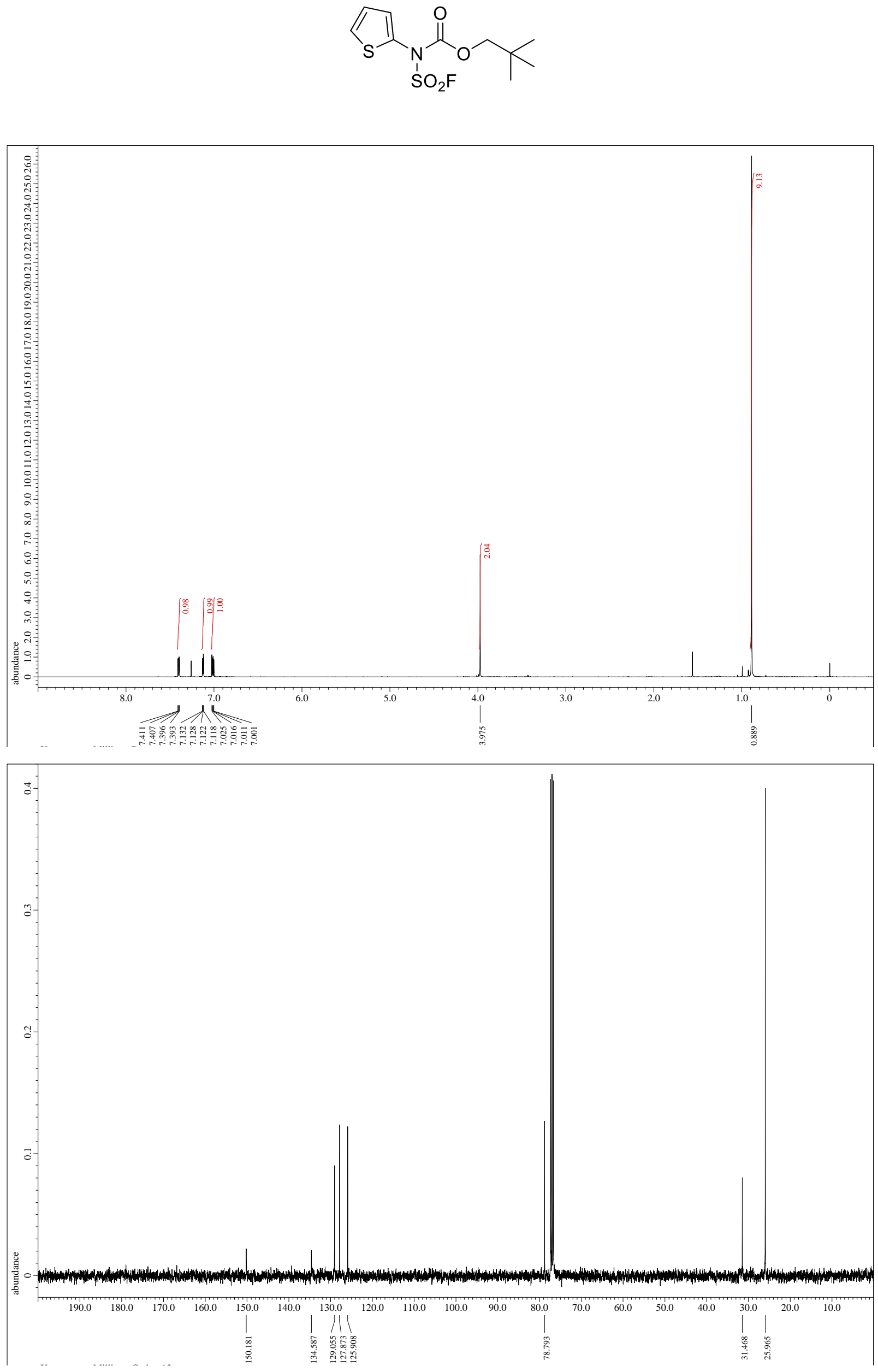


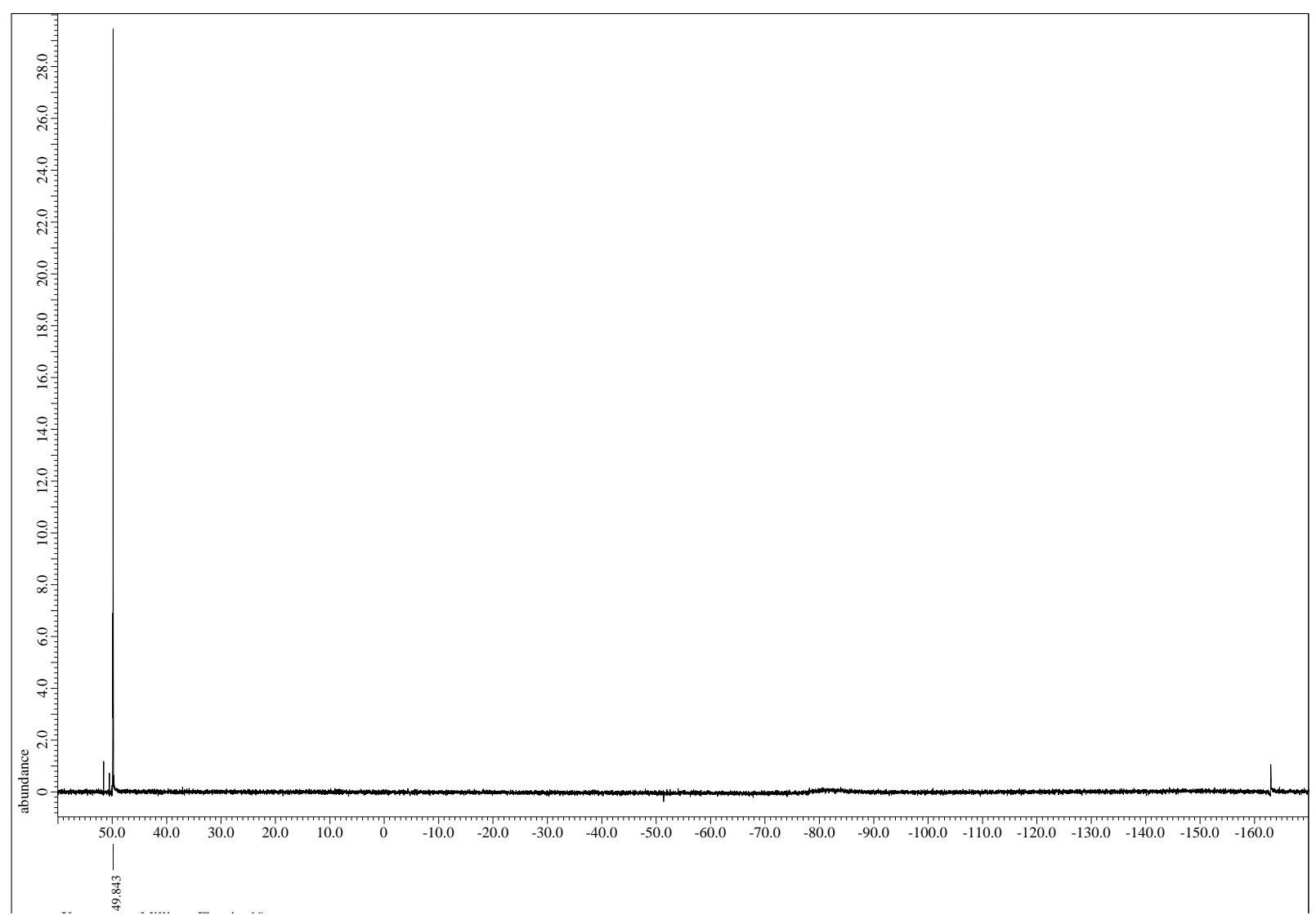


neopentyl (fluorosulfonyl)(5-phenylthiophen-2-yl)carbamate (4)
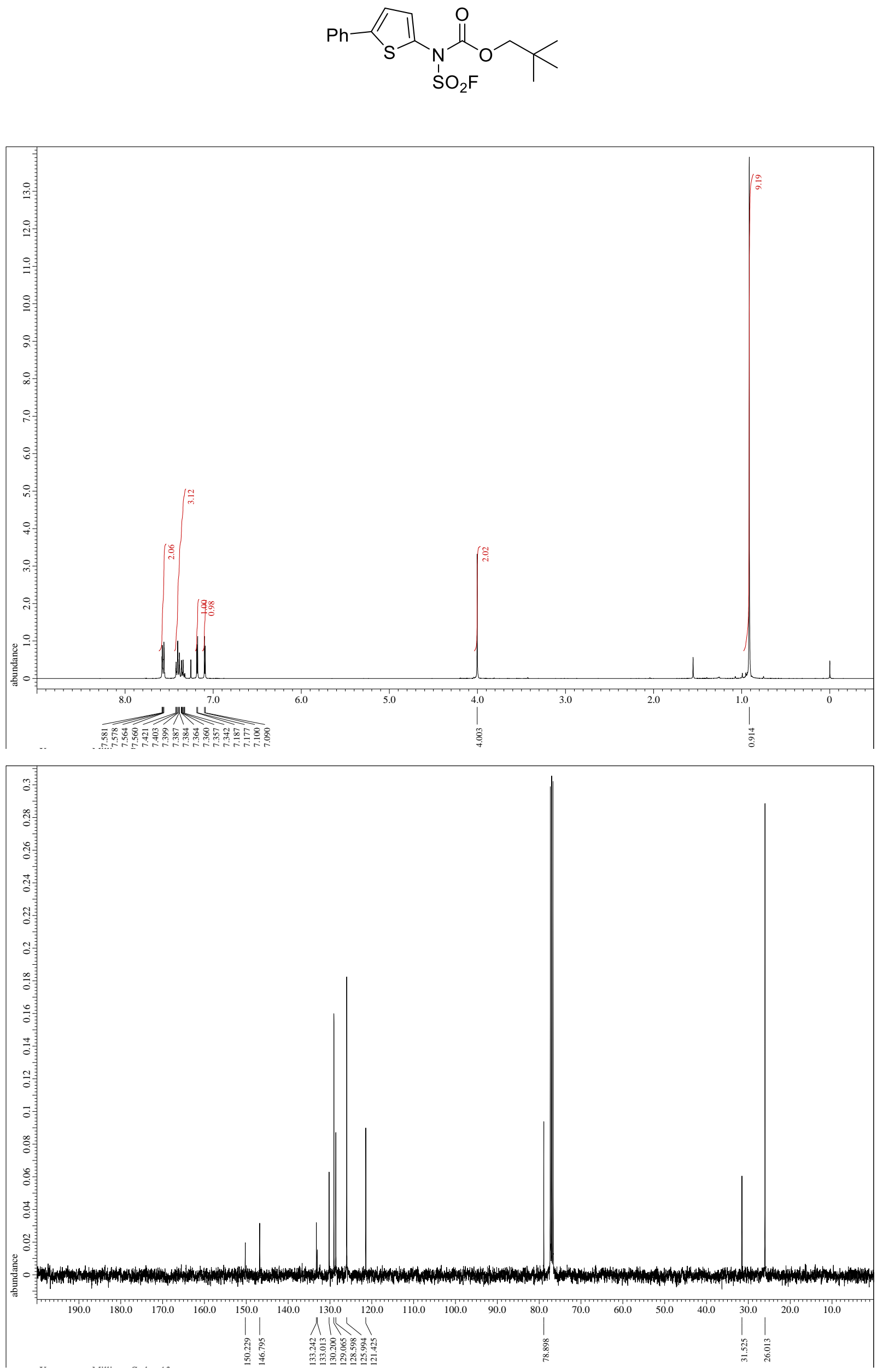


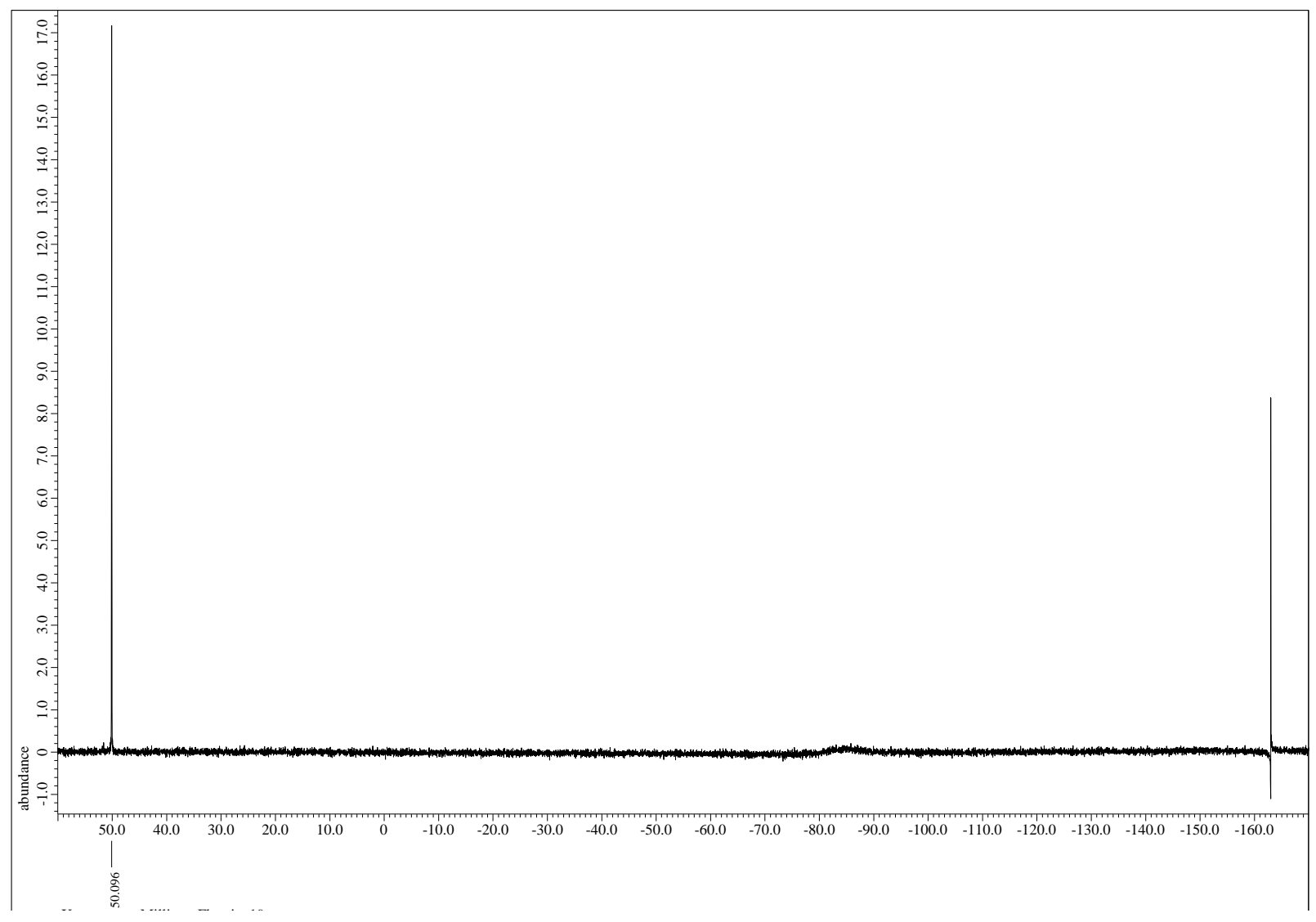


methyl 5-((fluorosulfonyl)((neopentyloxy)carbonyl)amino)thiophene-2-carboxylate (5)
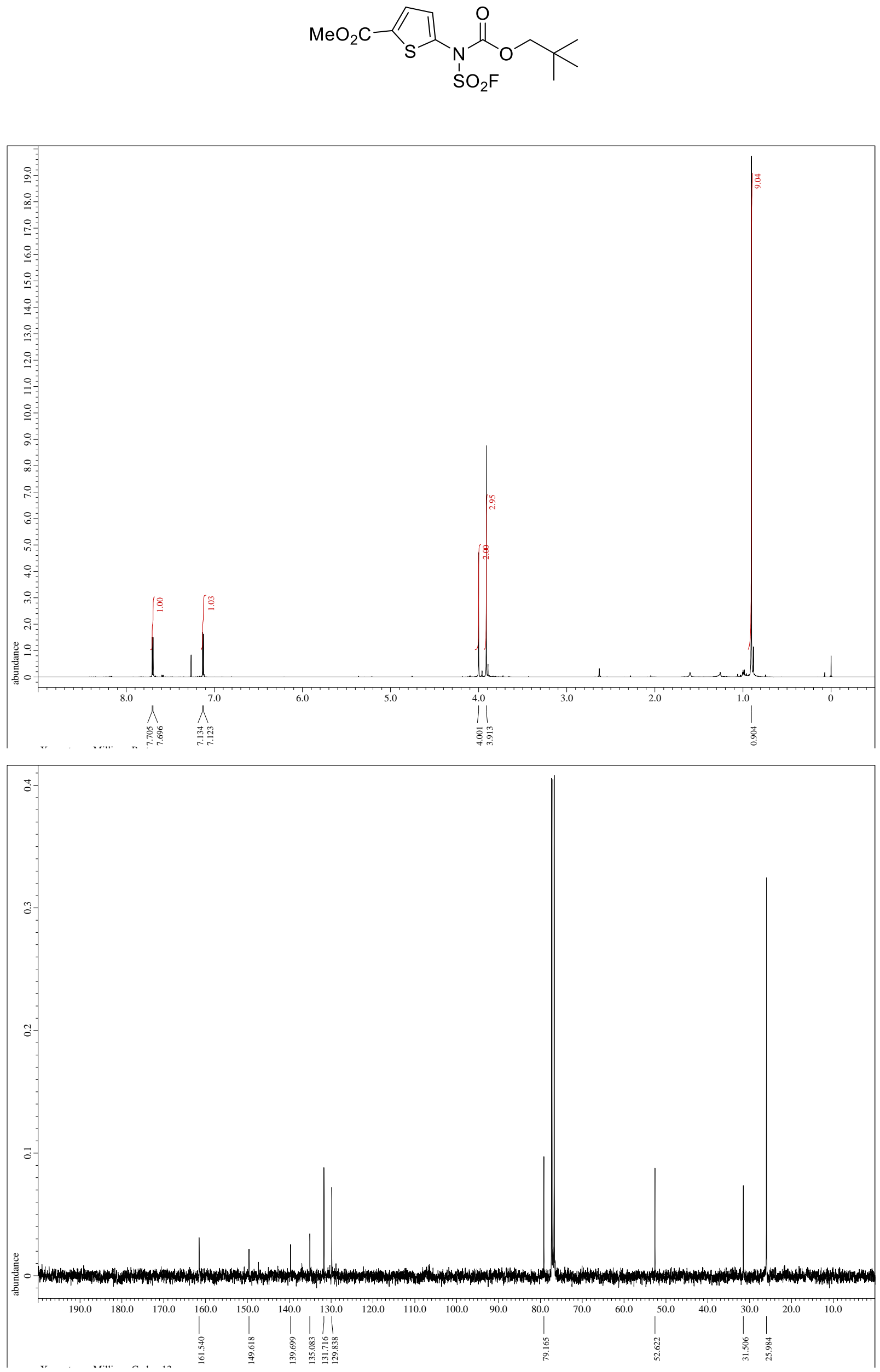


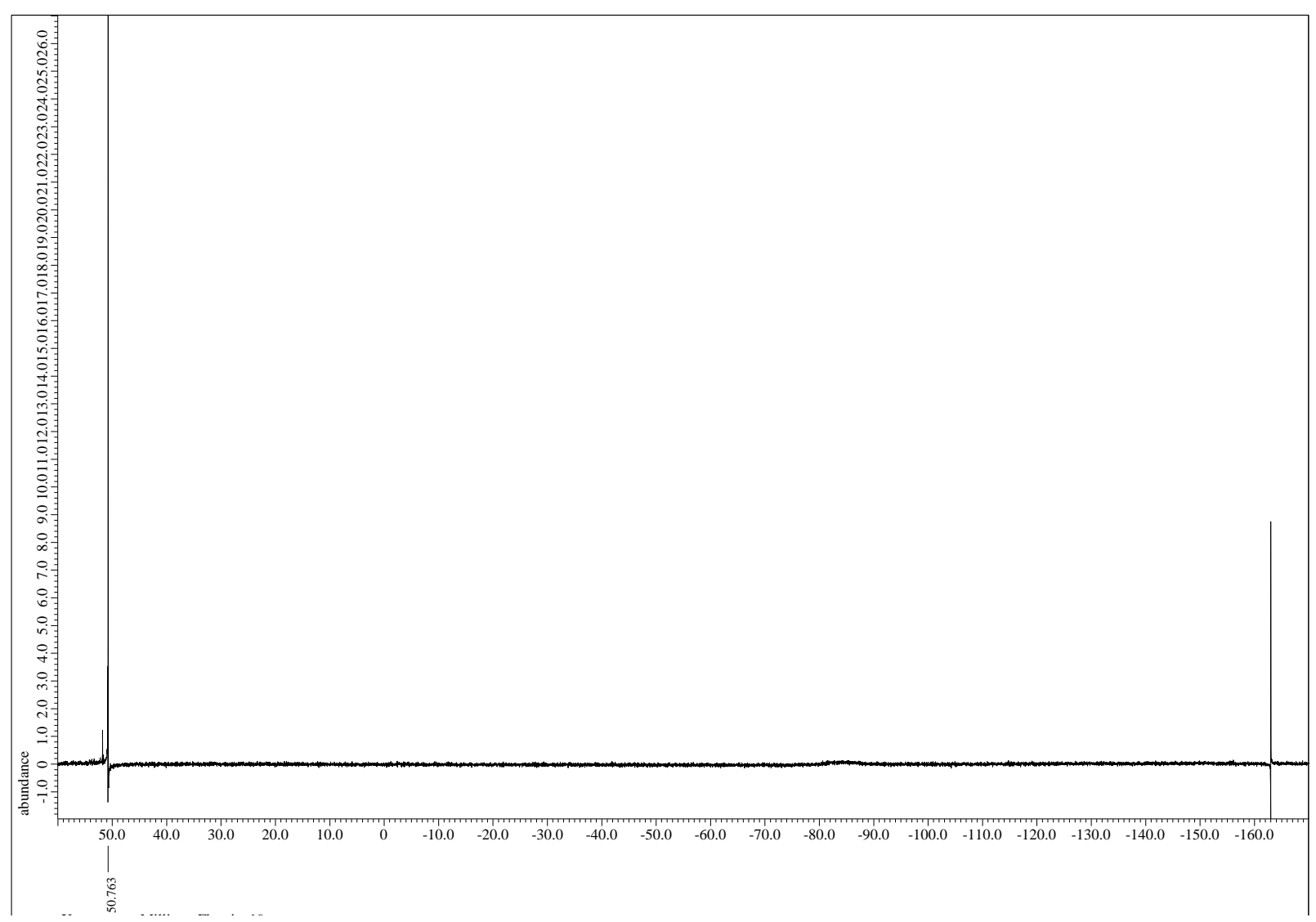


neopentyl (5-bromothiophen-2-yl)(fluorosulfonyl)carbamate (6)
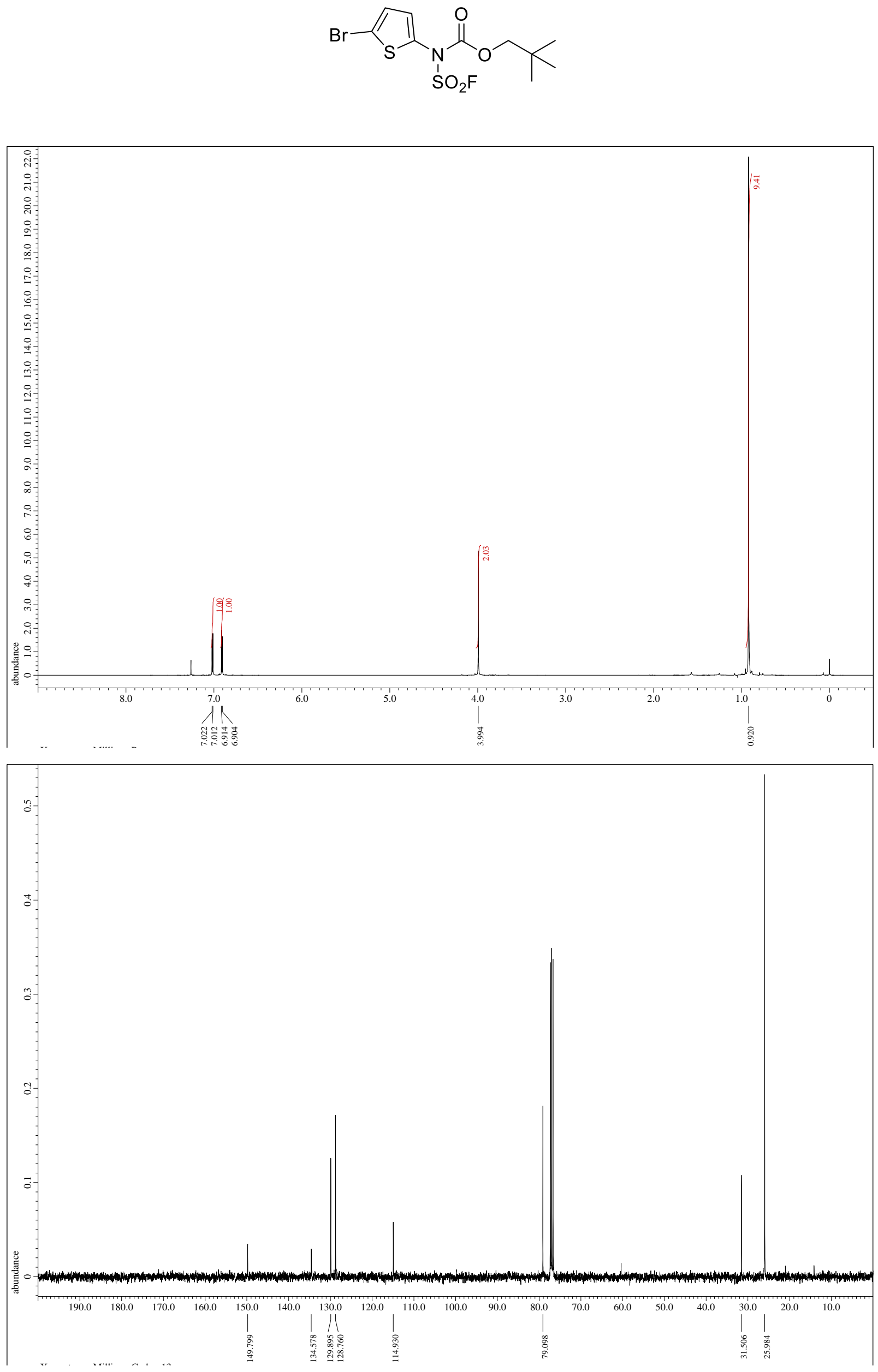


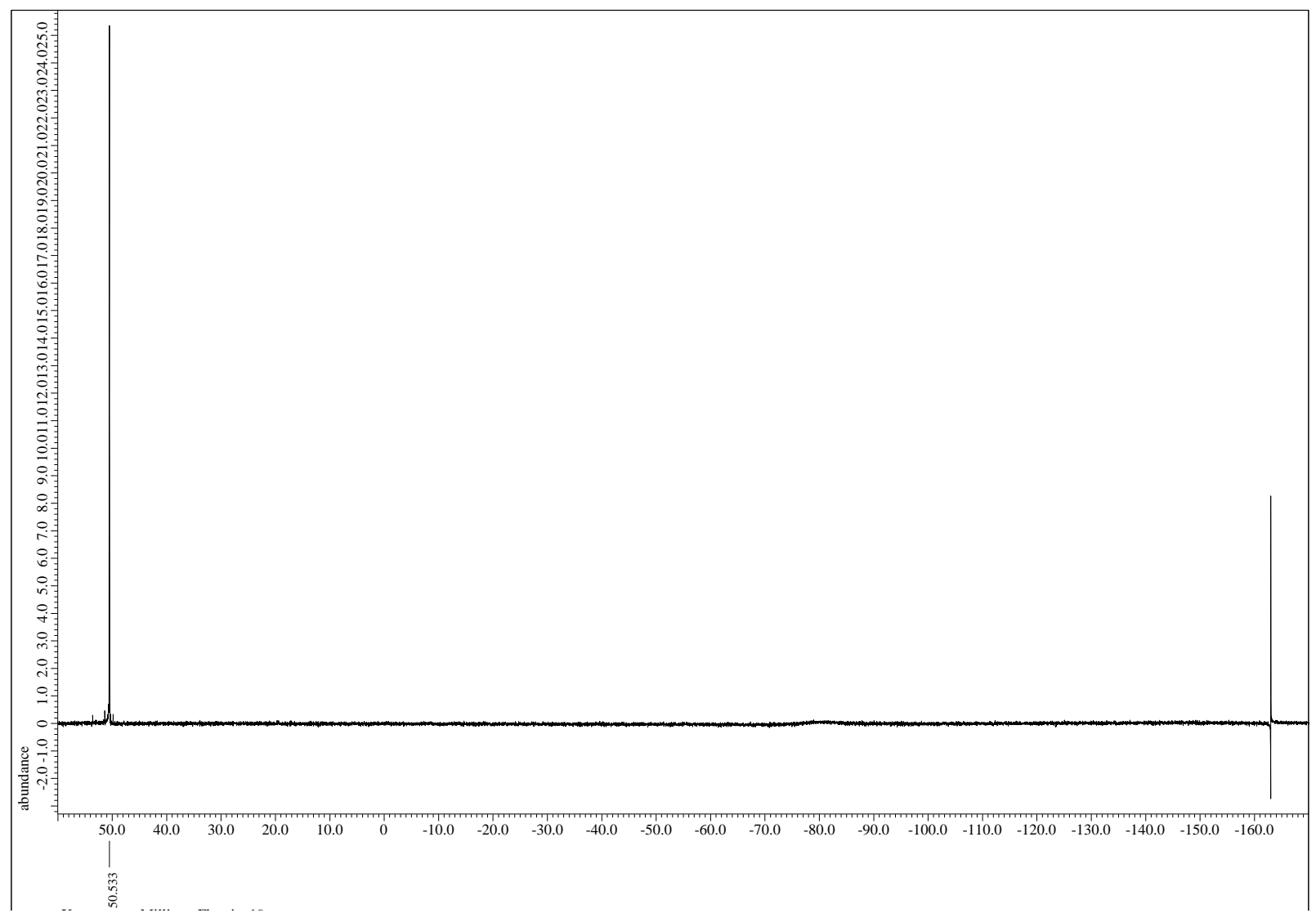


neopentyl (3-acetylthiophen-2-yl)(fluorosulfonyl)carbamate (7) and

neopentyl (4-acetylthiophen-2-yl)(fluorosulfonyl)carbamate $\left(7^{\prime}\right)$
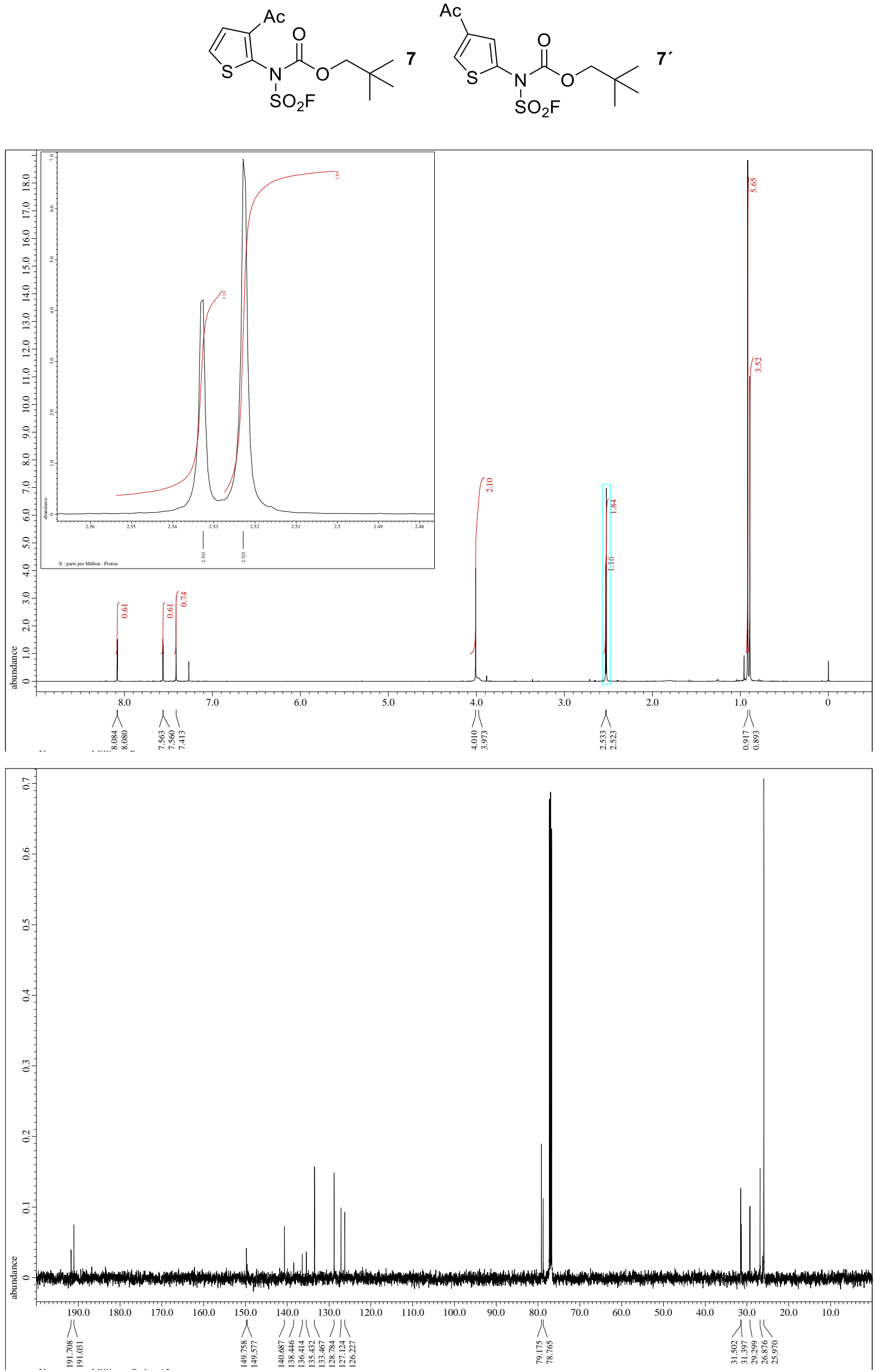


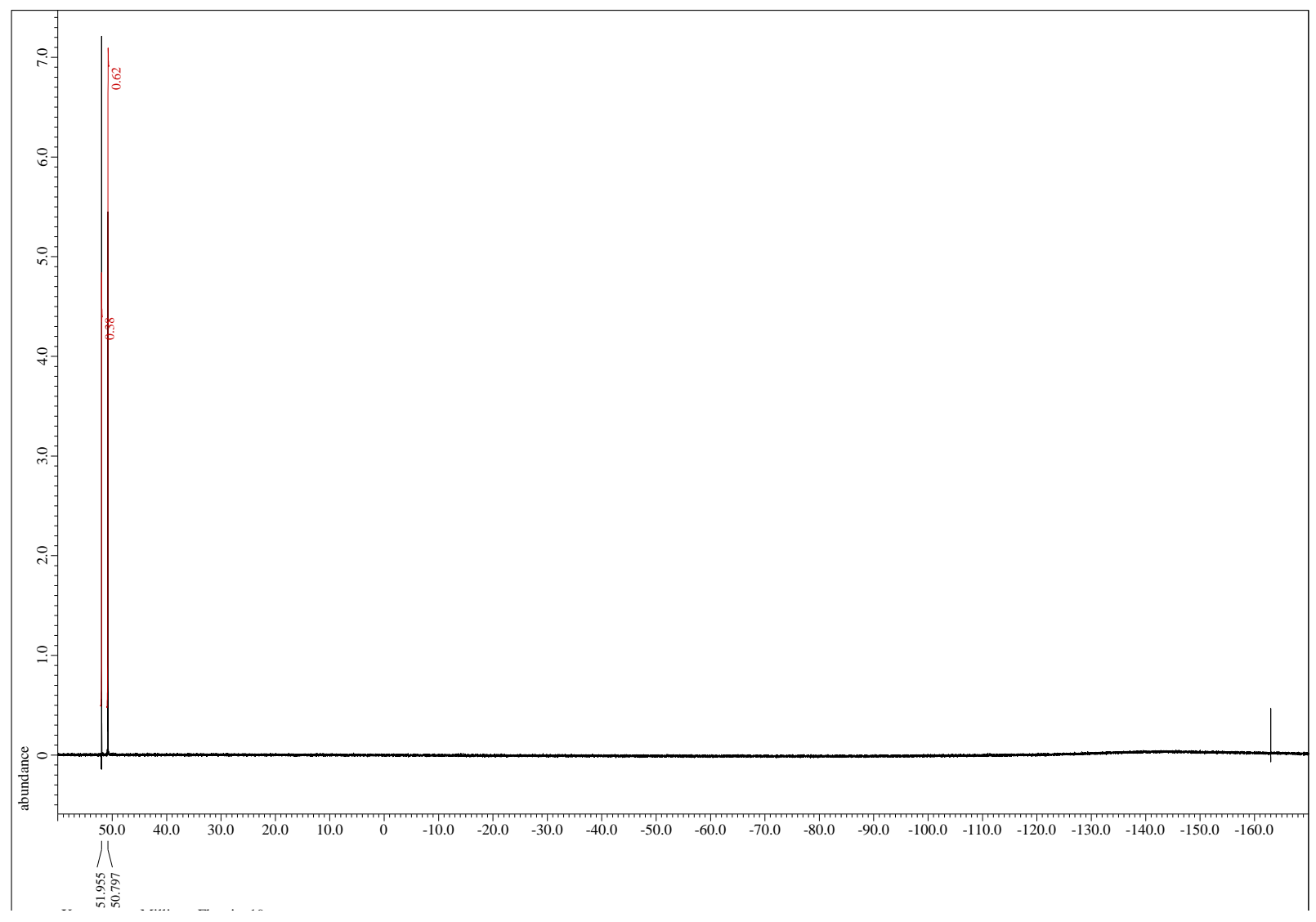


neopentyl (3,4-dibromothiophen-2-yl)(fluorosulfonyl)carbamate (8)
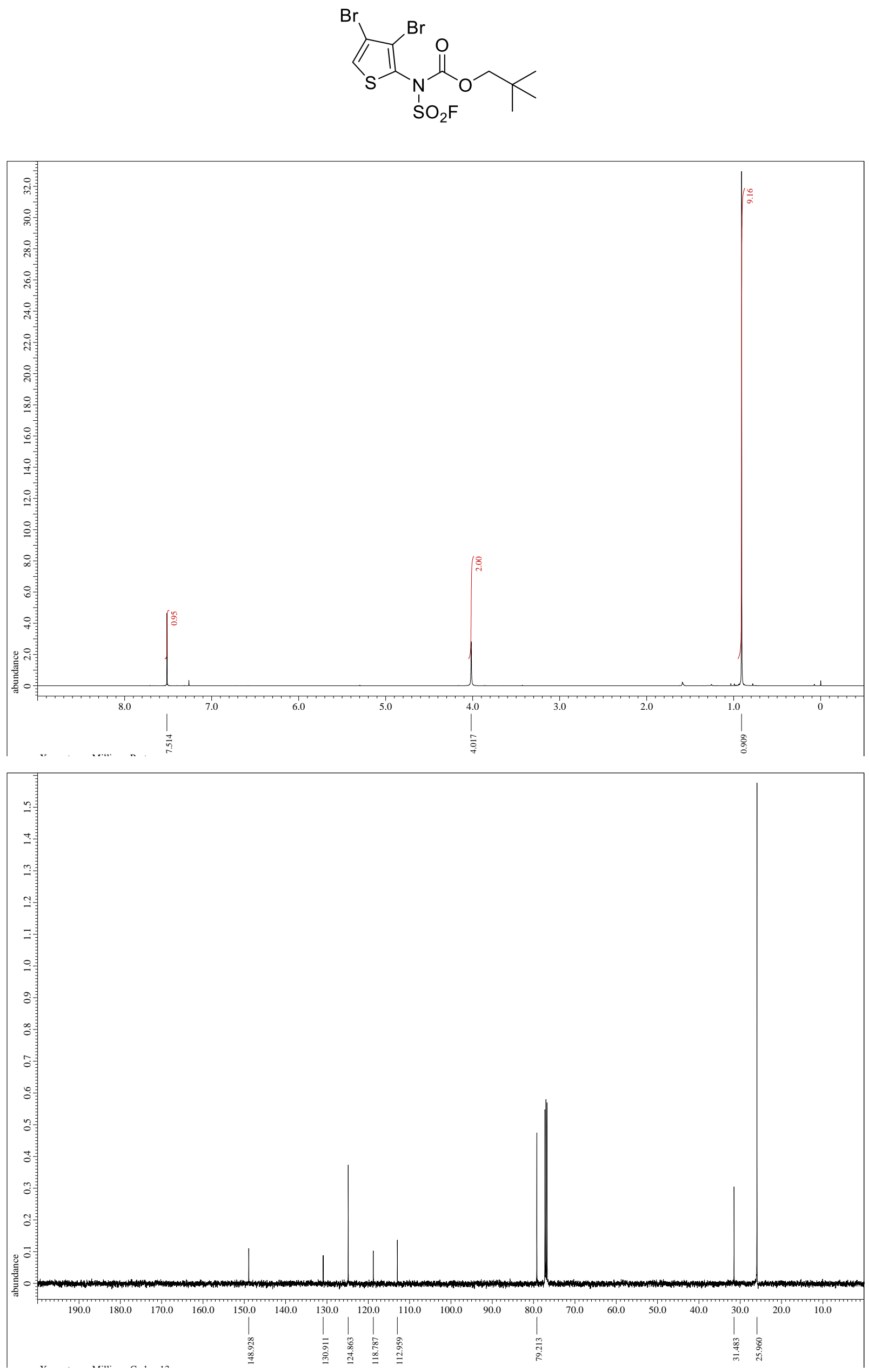


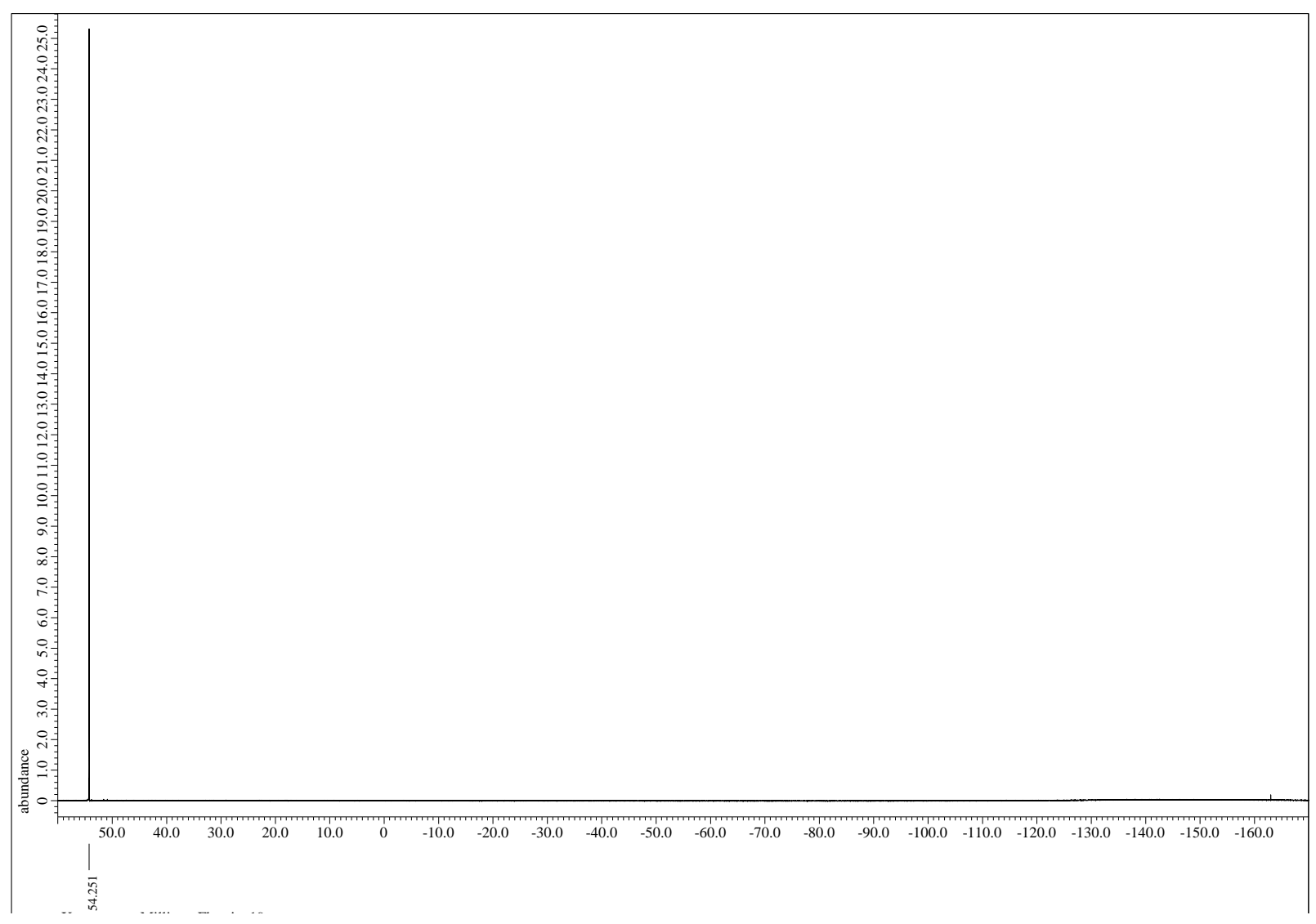


neopentyl (2,5-dibromothiophen-3-yl)(fluorosulfonyl)carbamate (9)
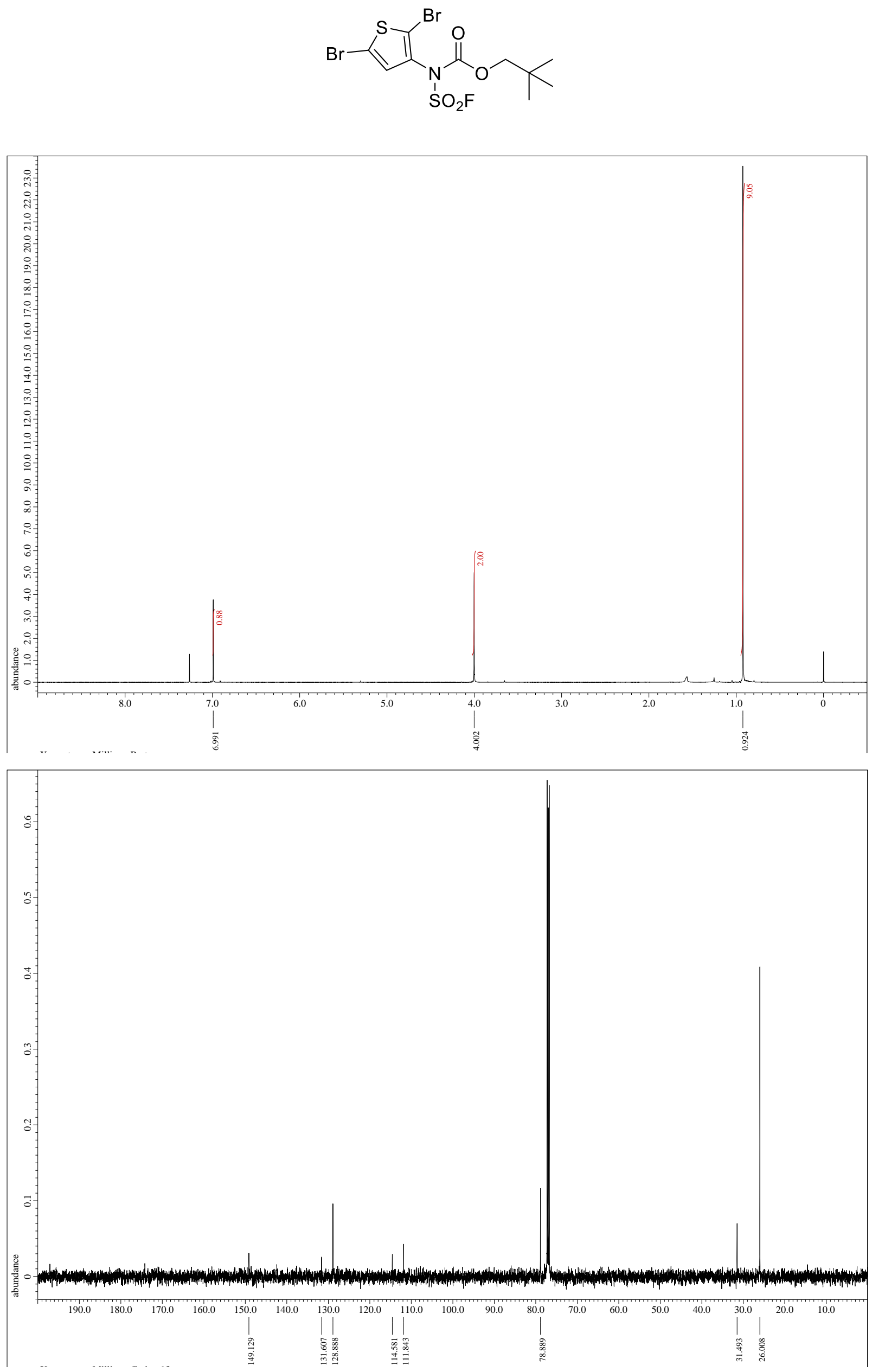


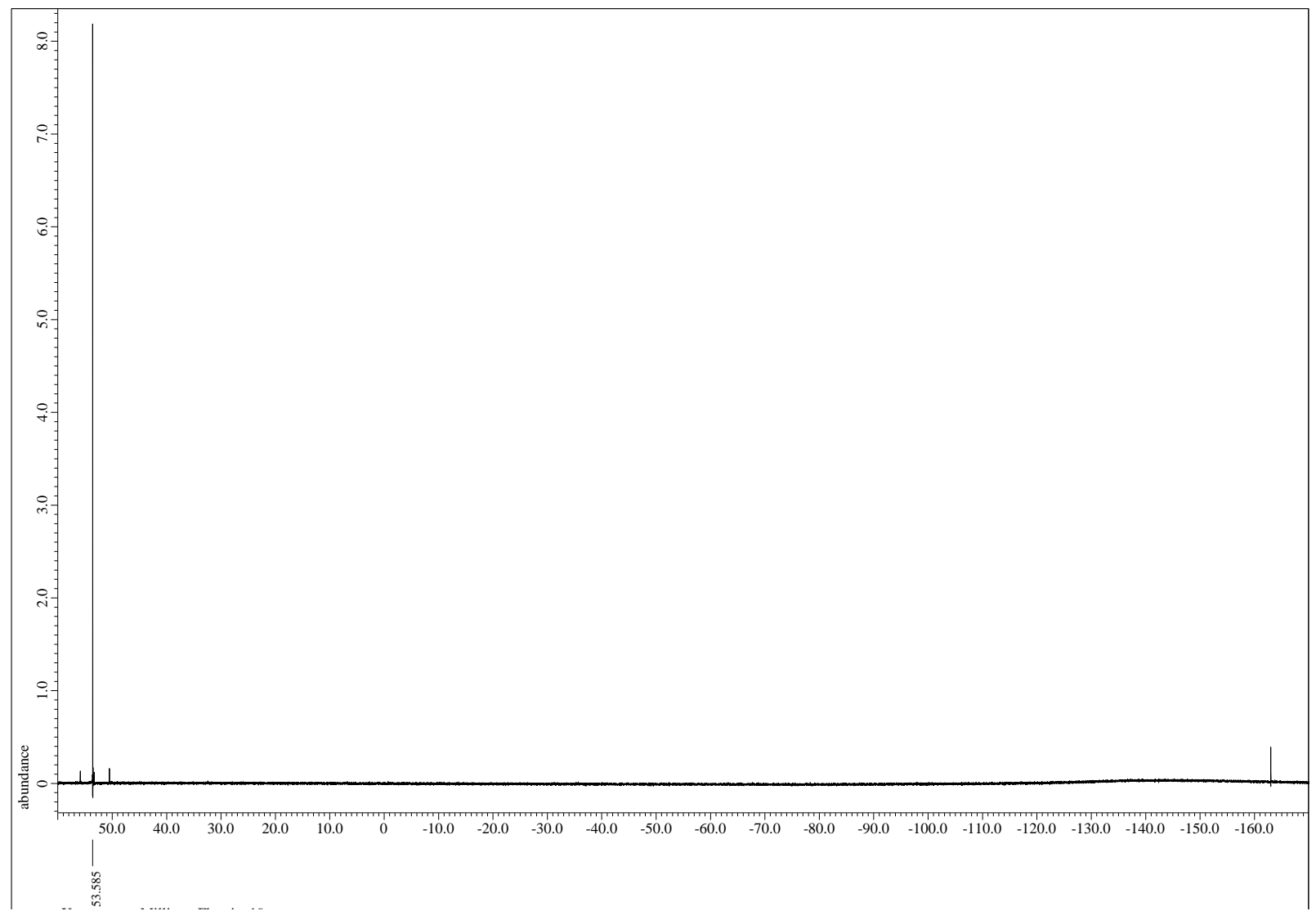


neopentyl (fluorosulfonyl)(5-phenylfuran-2-yl)carbamate (10)
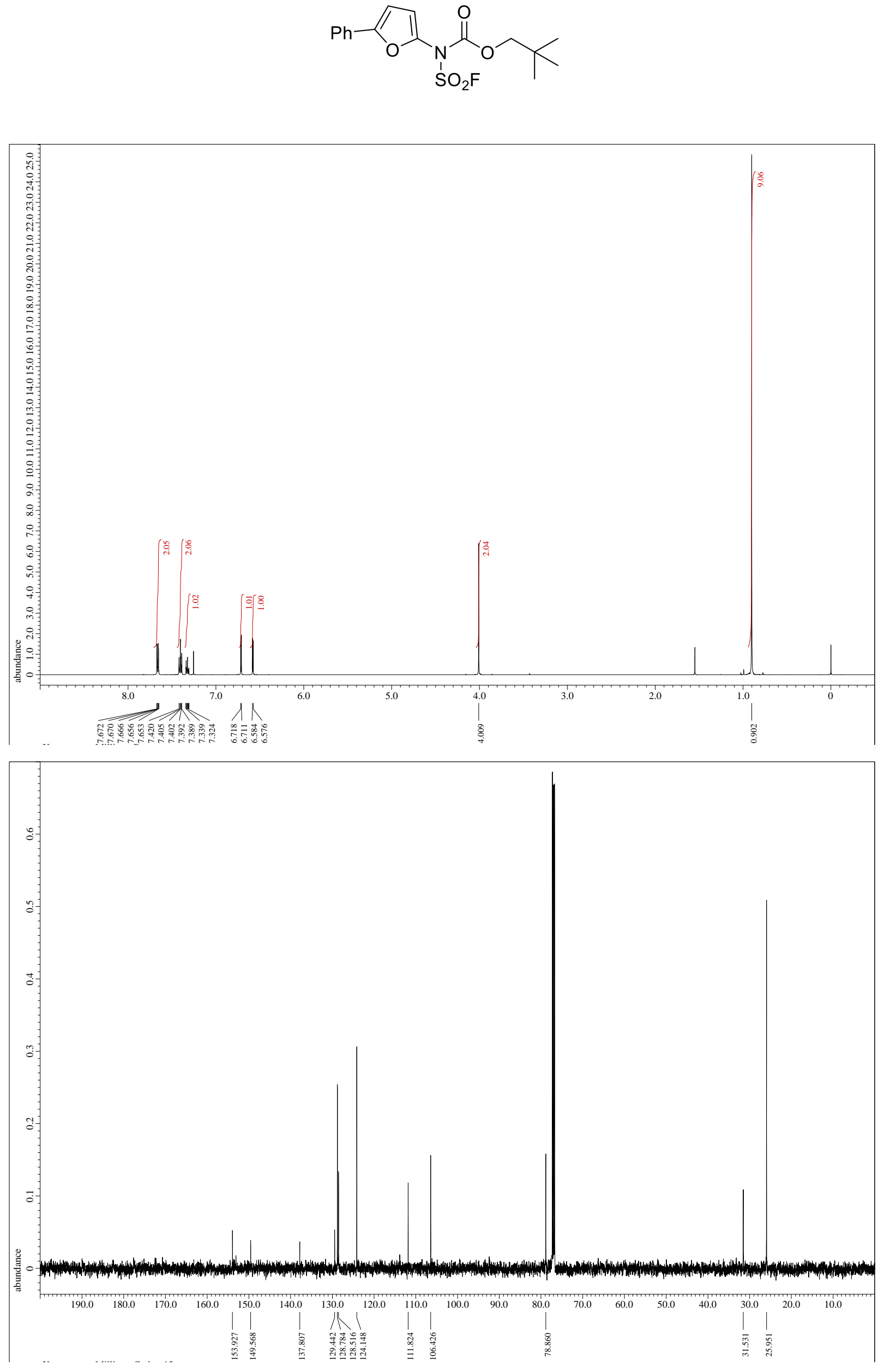


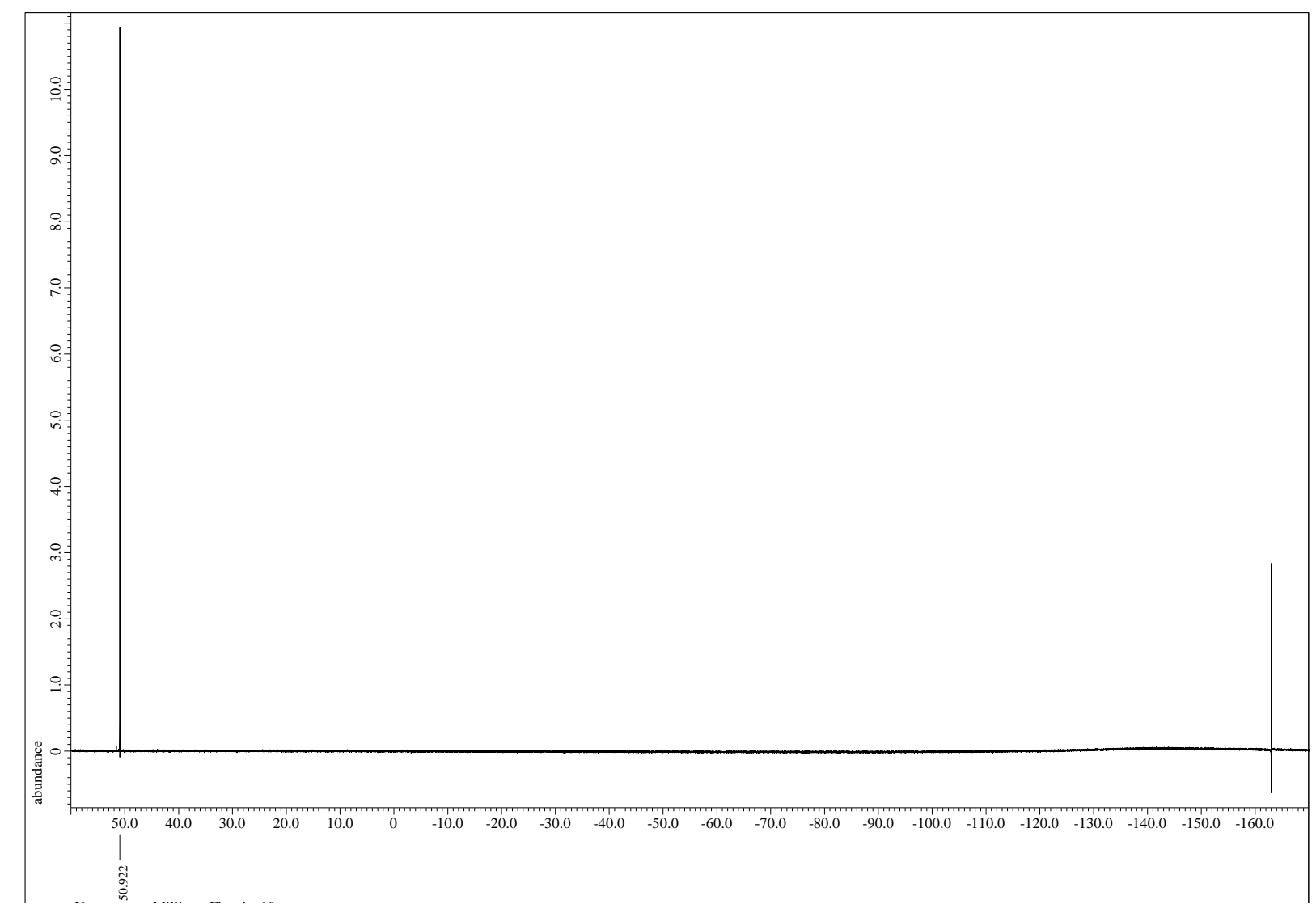


neopentyl (5-benzoylfuran-2-yl)(fluorosulfonyl)carbamate (11)
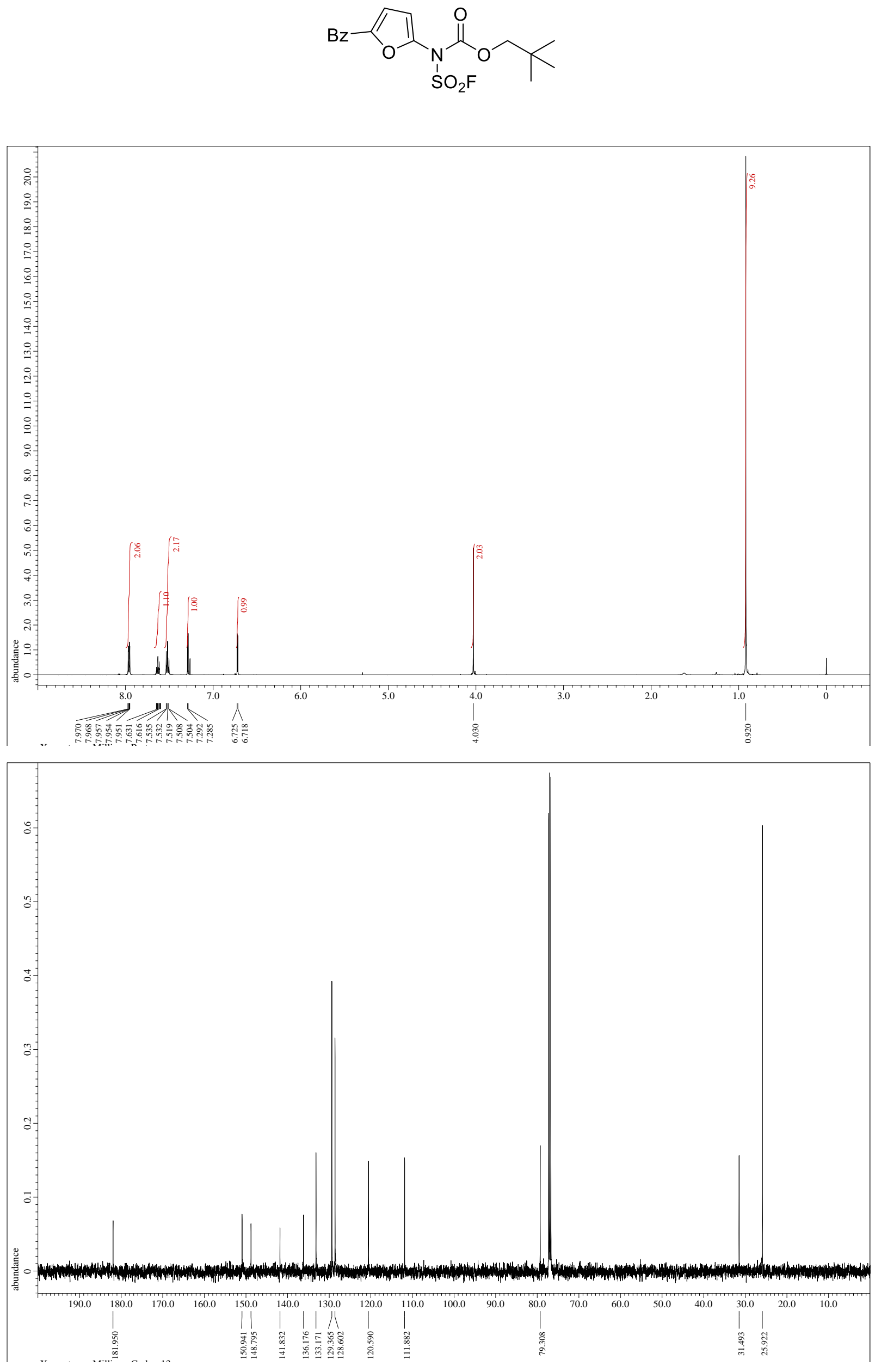


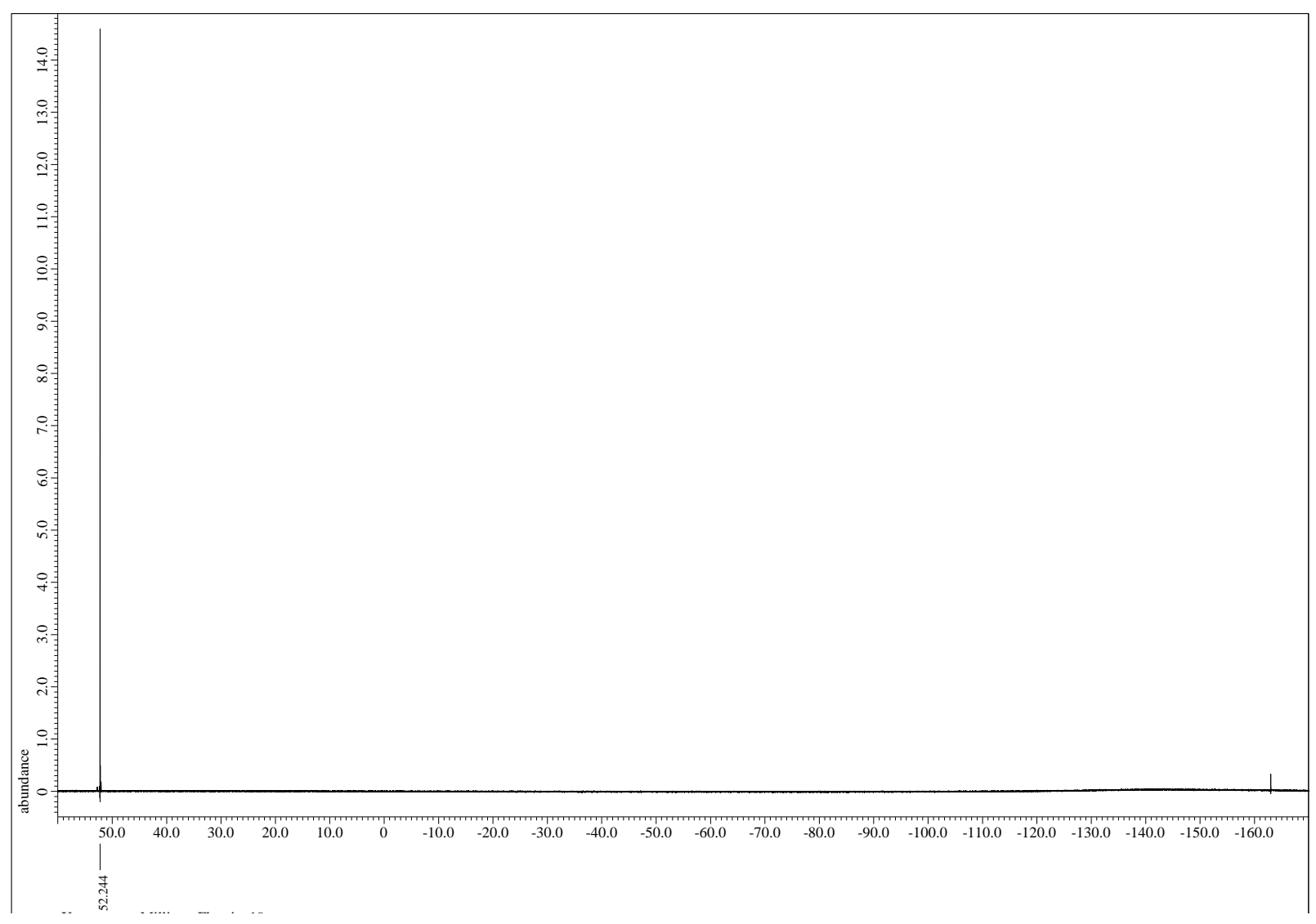



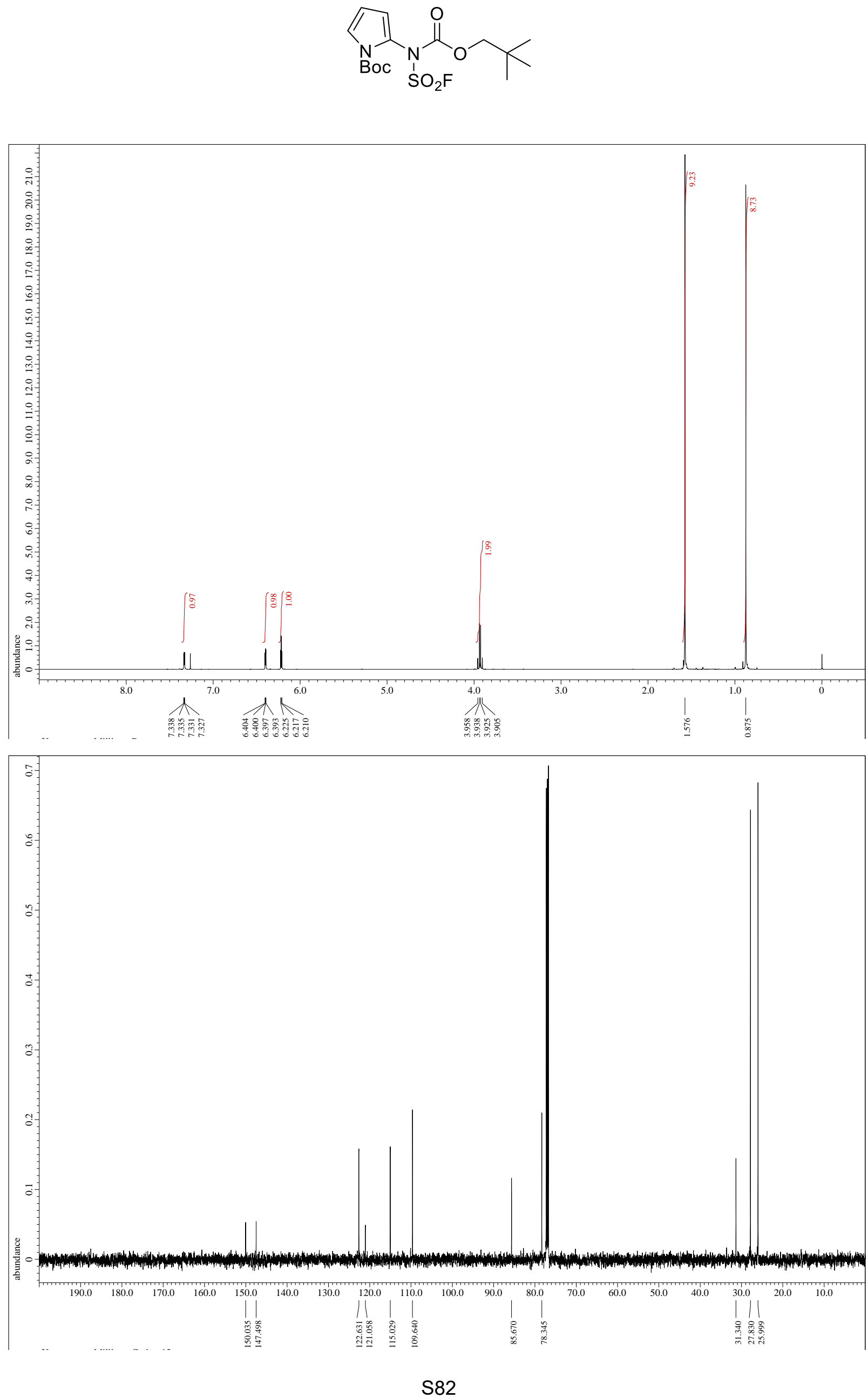


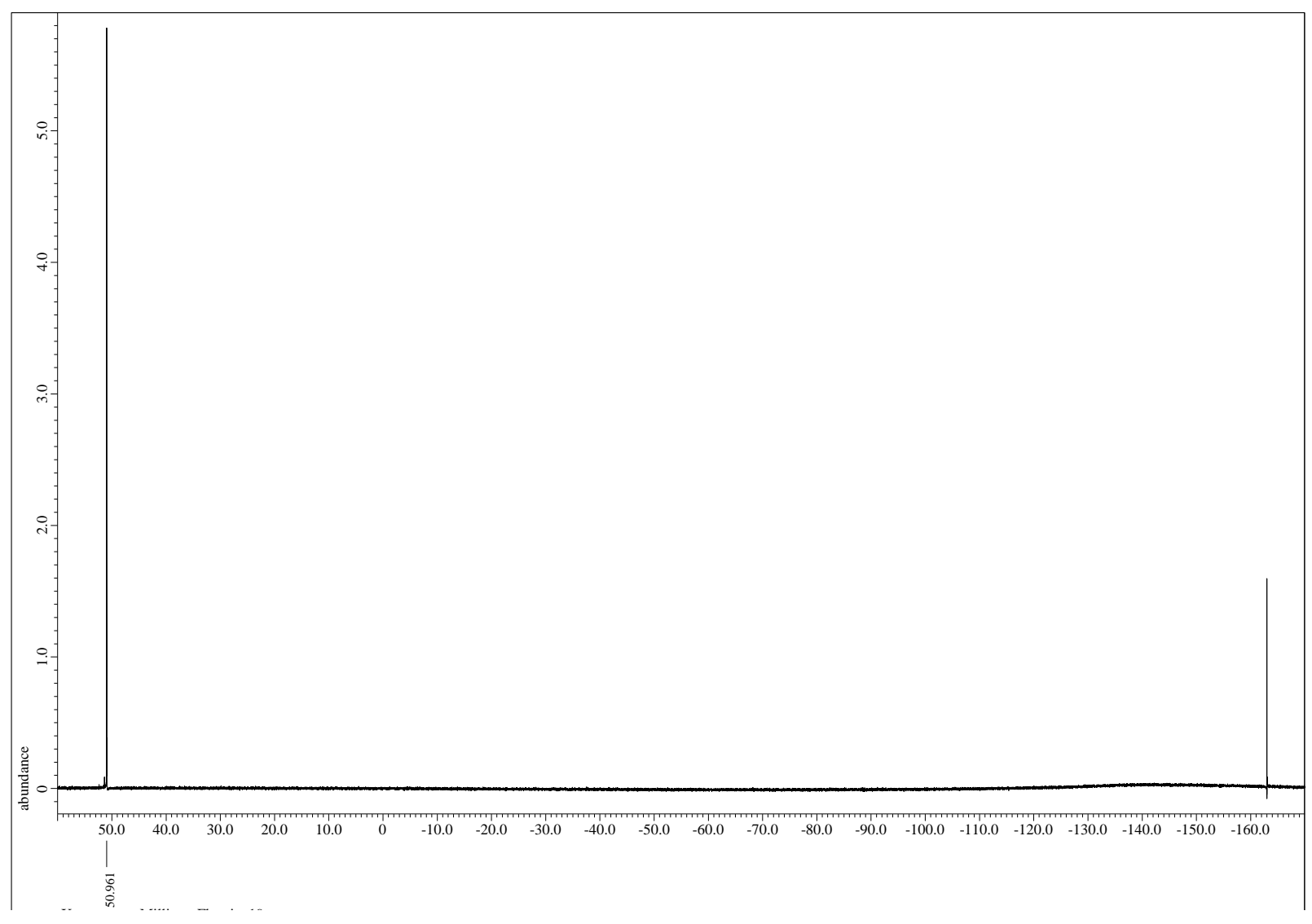



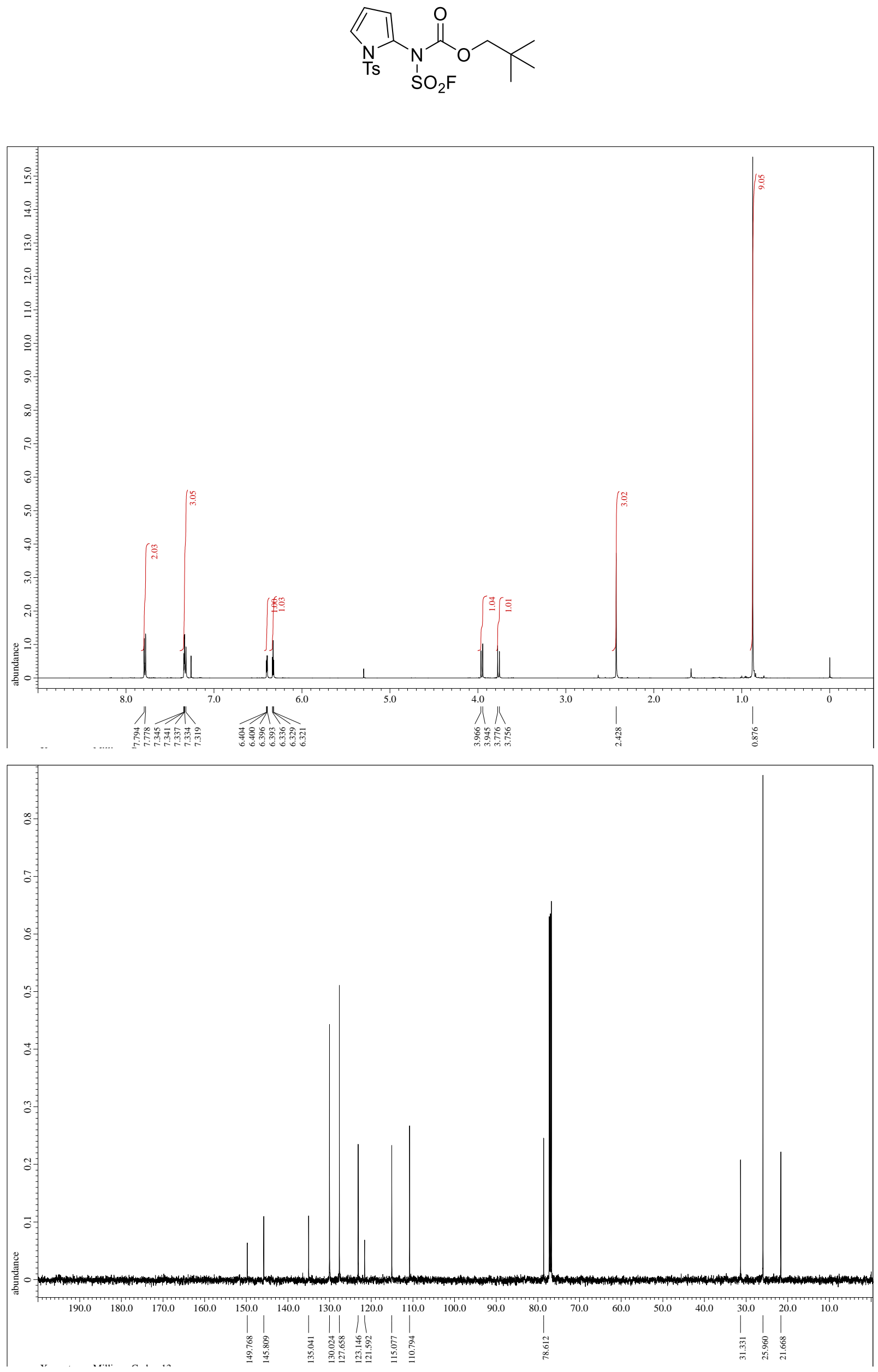


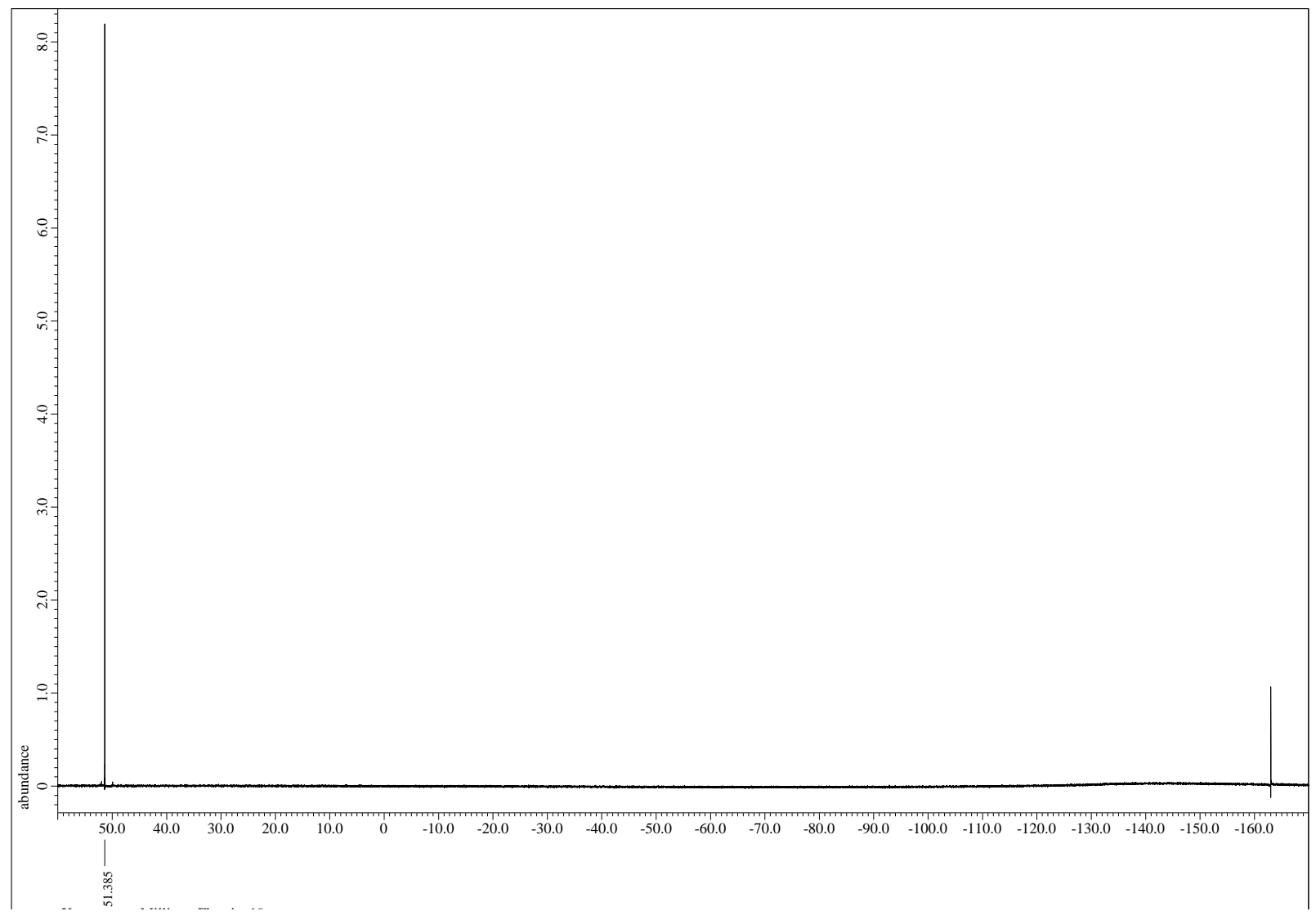


neopentyl (fluorosulfonyl)(2-methyl-5-phenyloxazol-4-yl)carbamate (14)
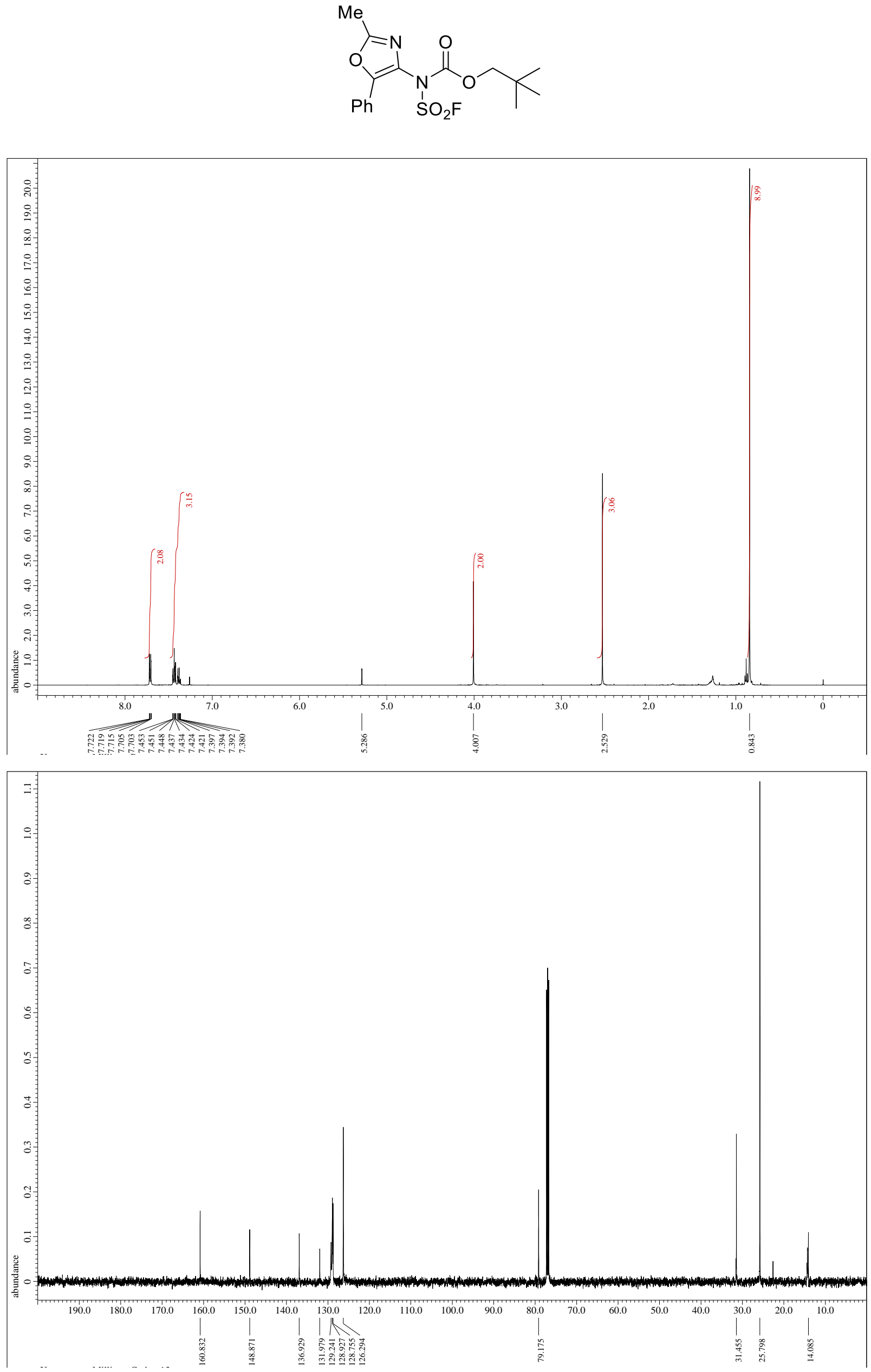


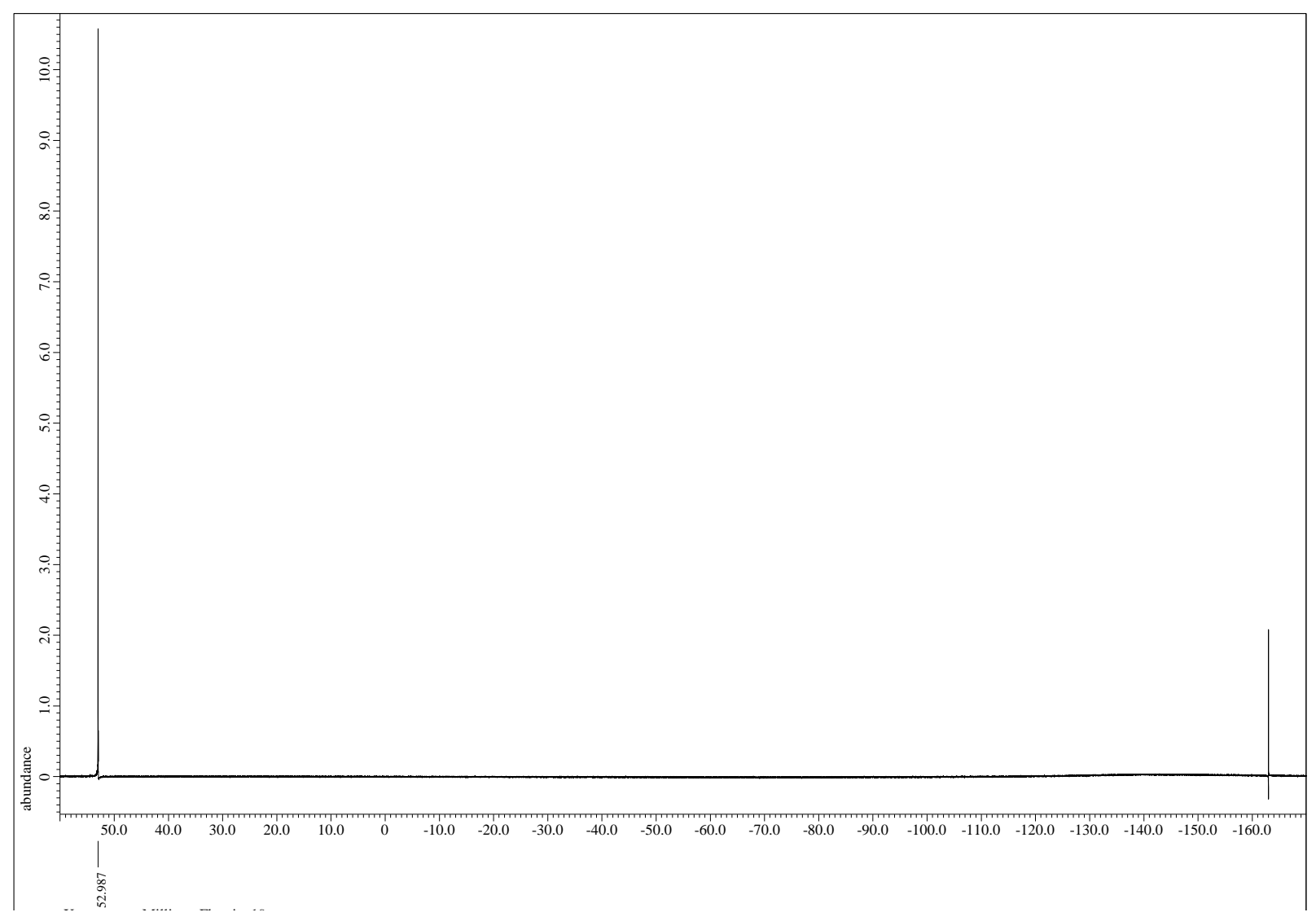



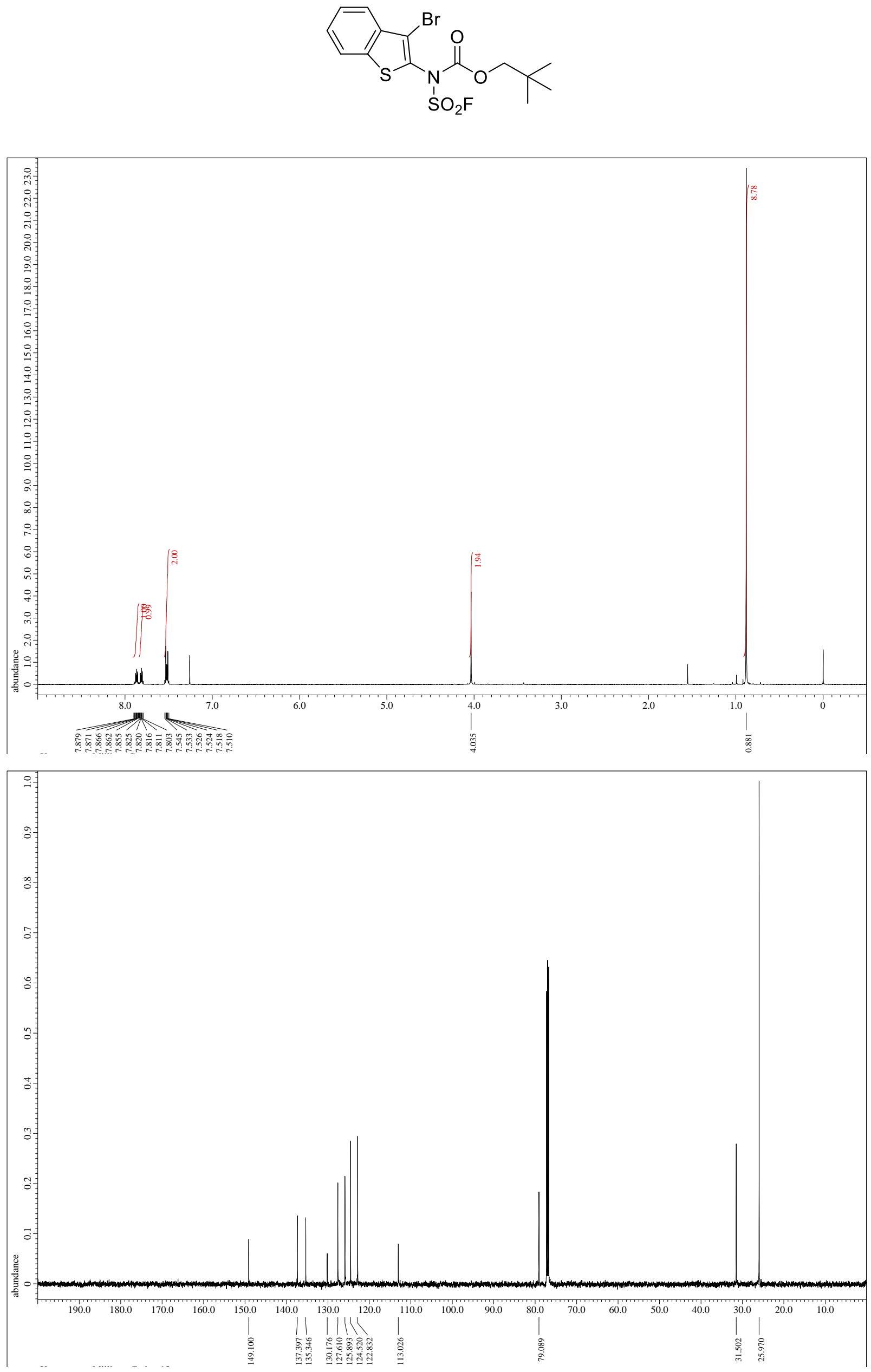


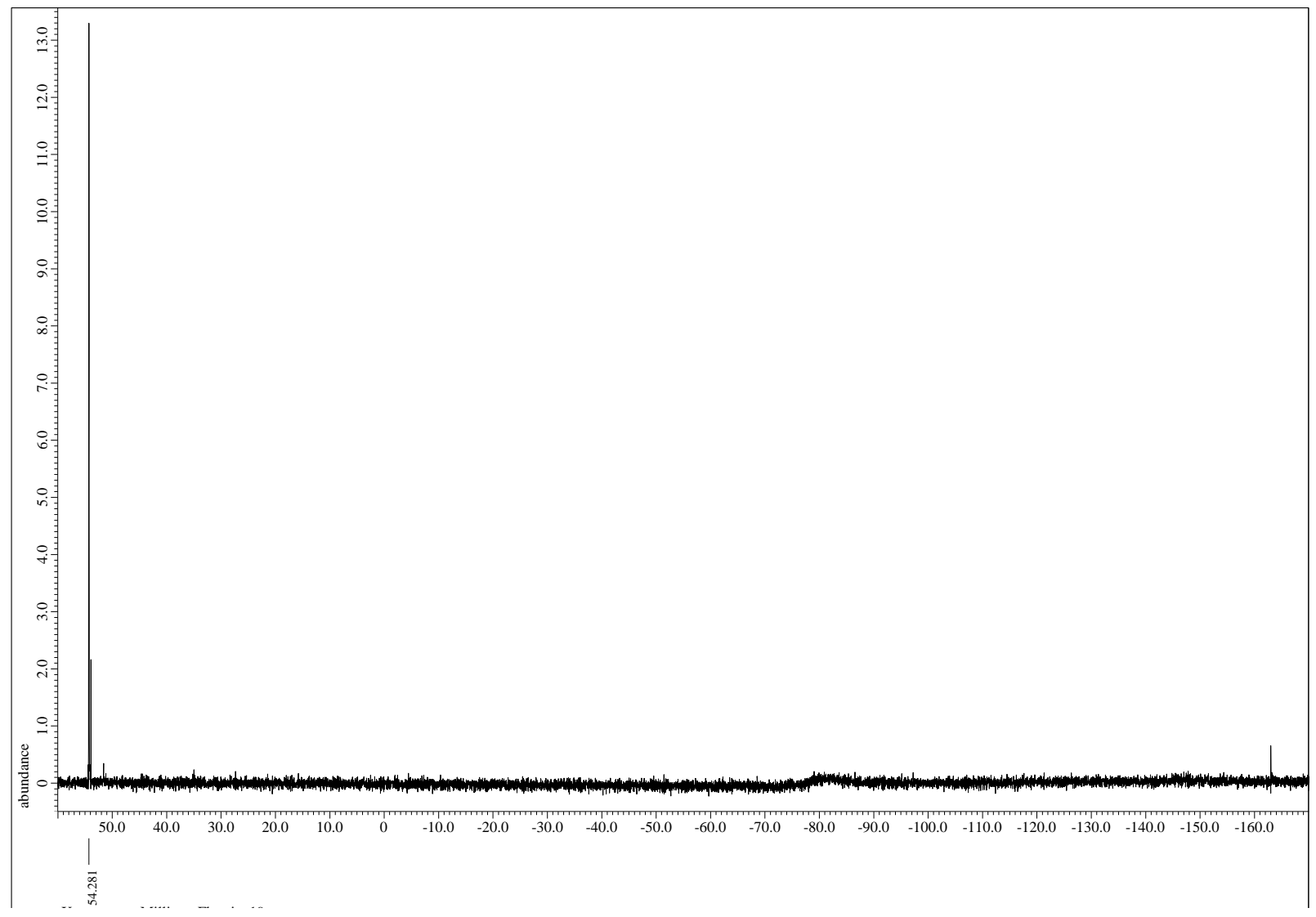



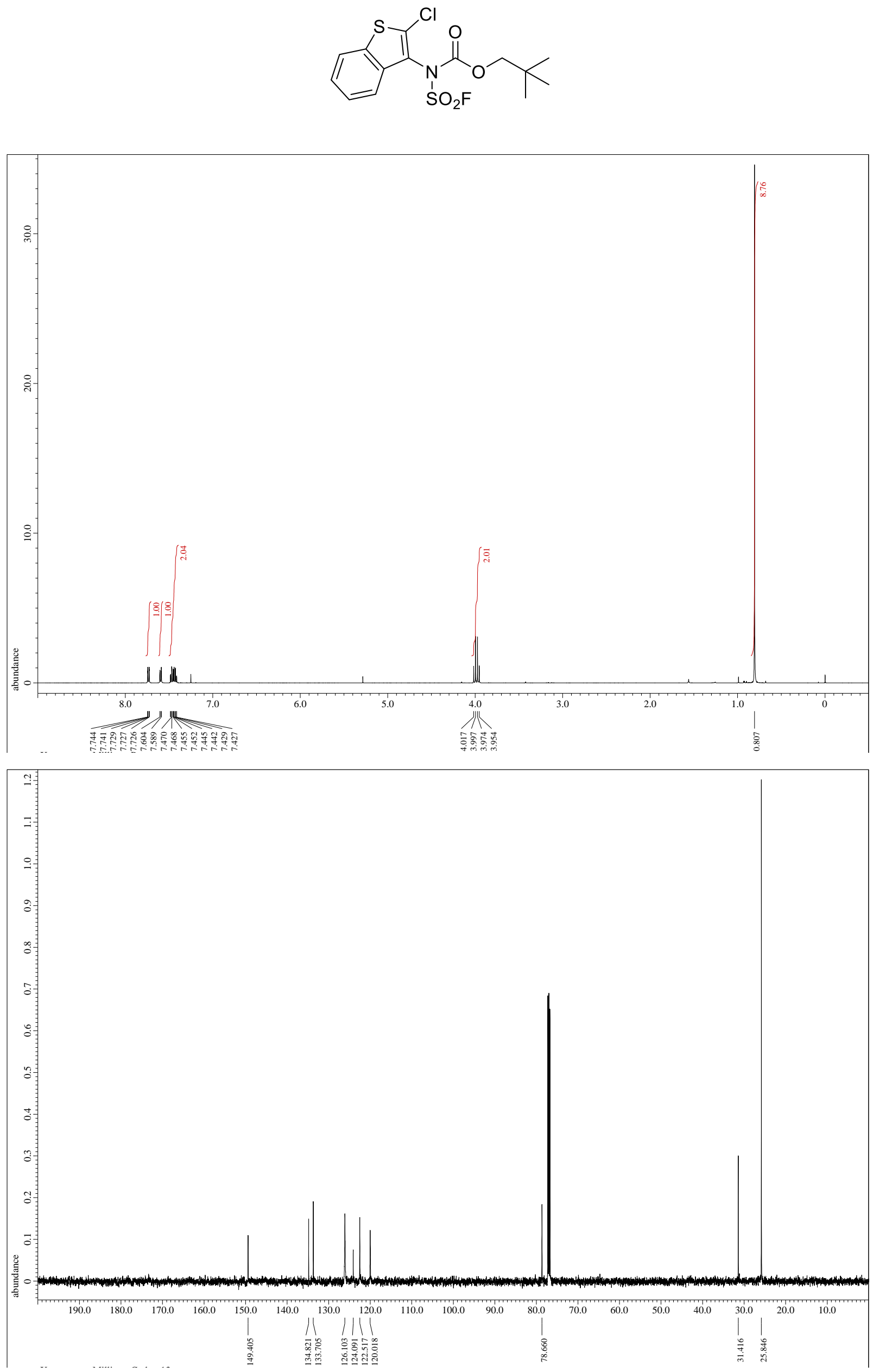


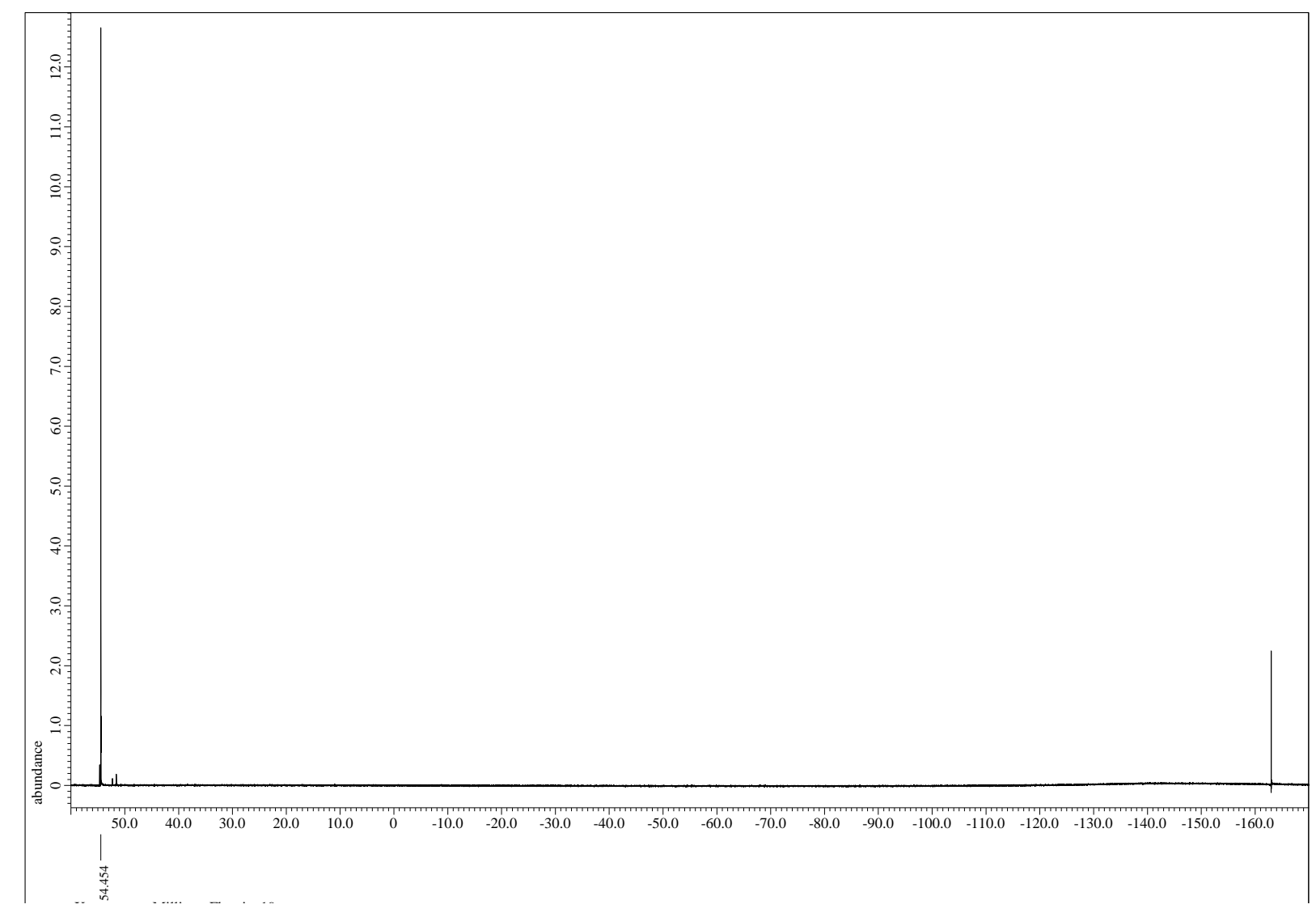


neopentyl (fluorosulfonyl)(3-phenylbenzofuran-2-yl)carbamate (17)
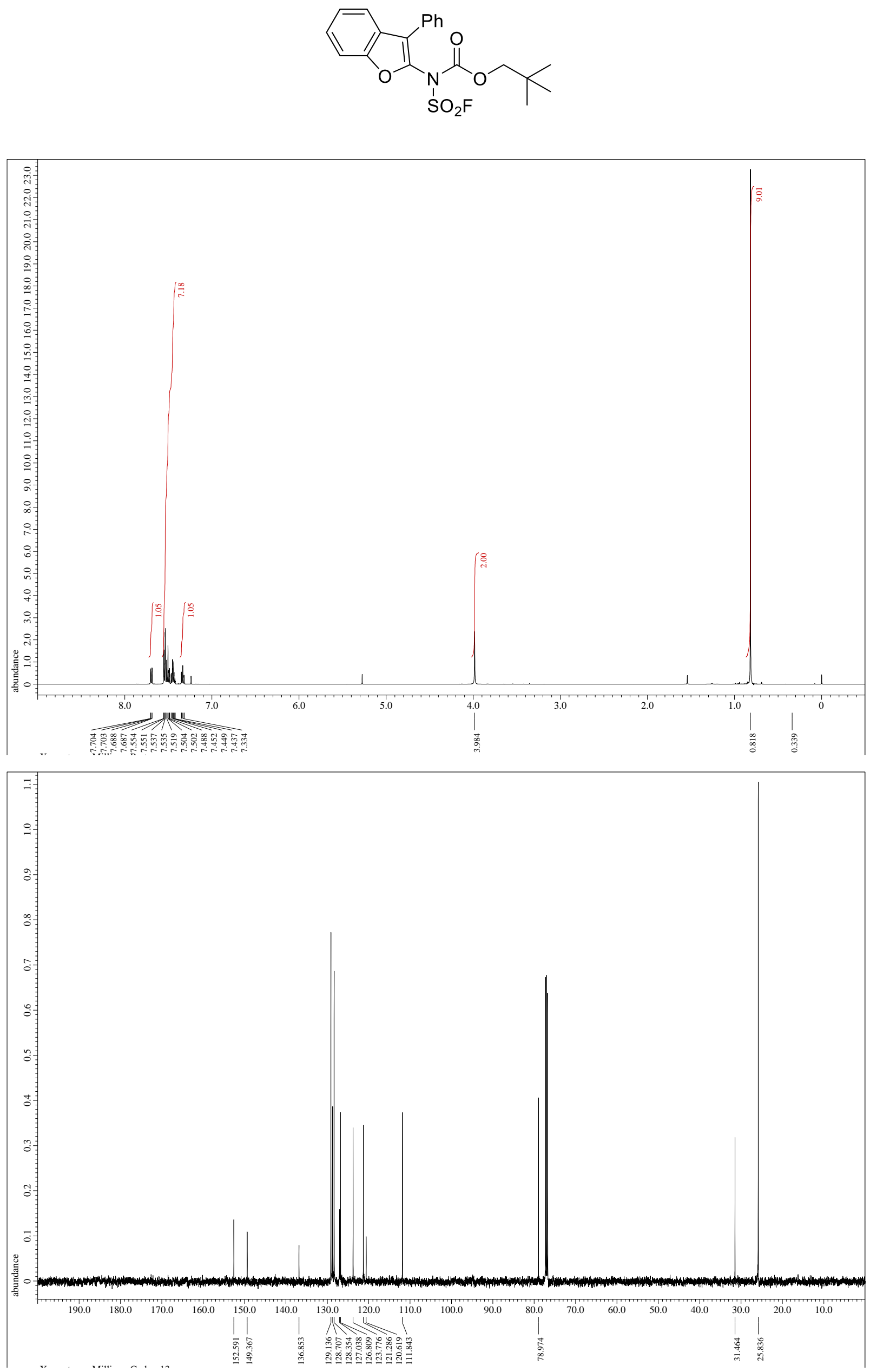


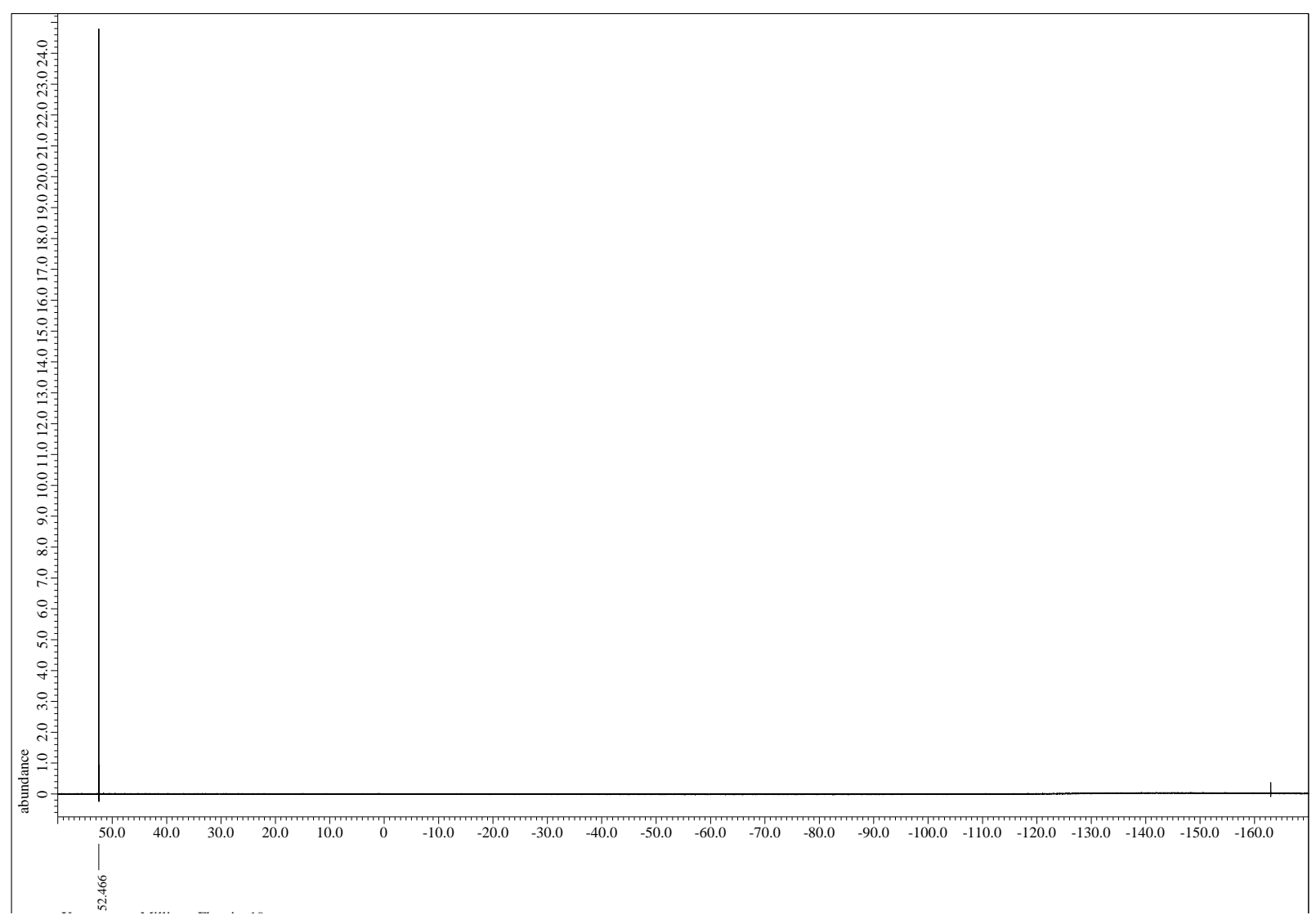


neopentyl (fluorosulfonyl)(3-methylbenzofuran-2-yl)carbamate (18)
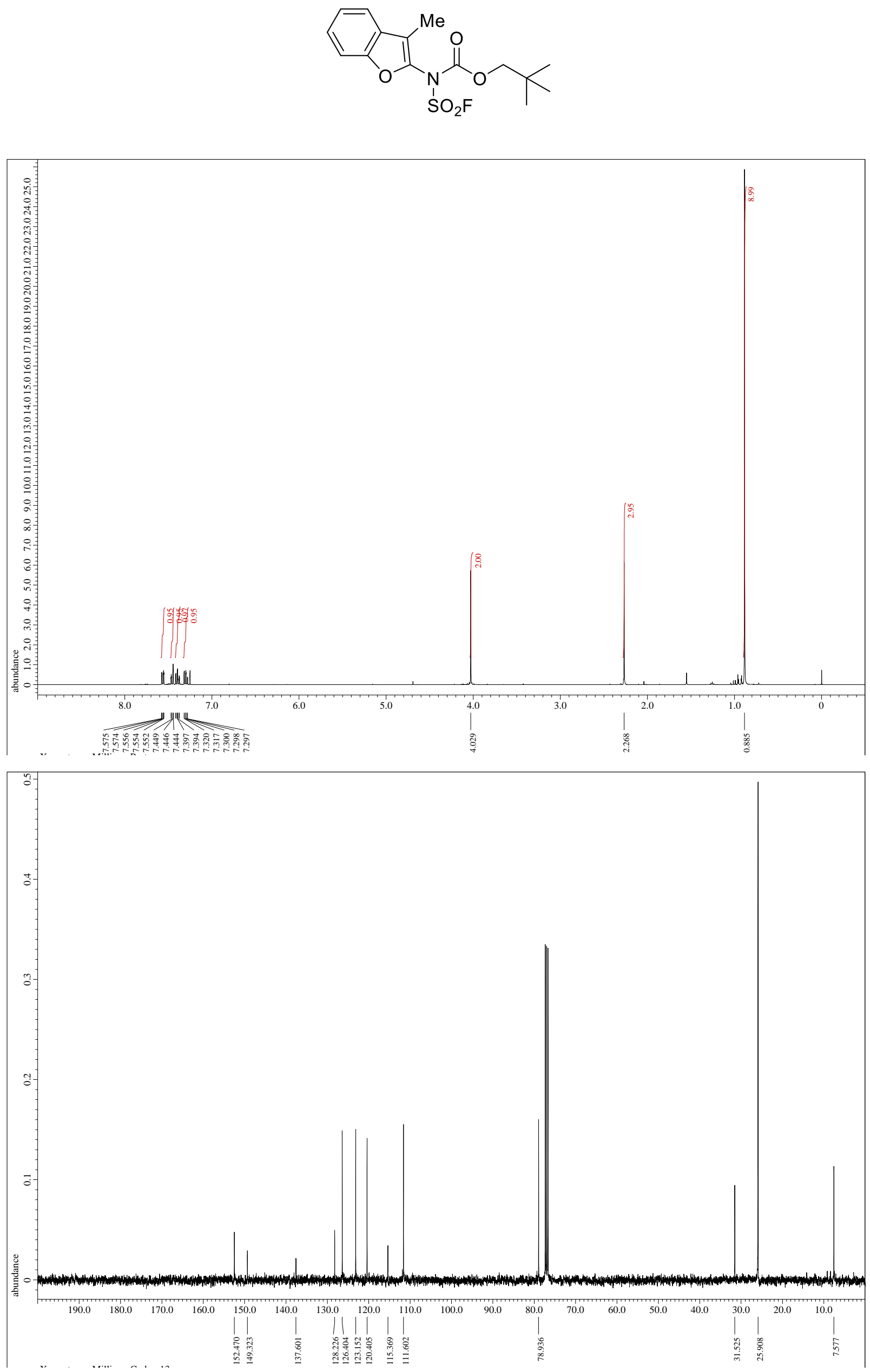


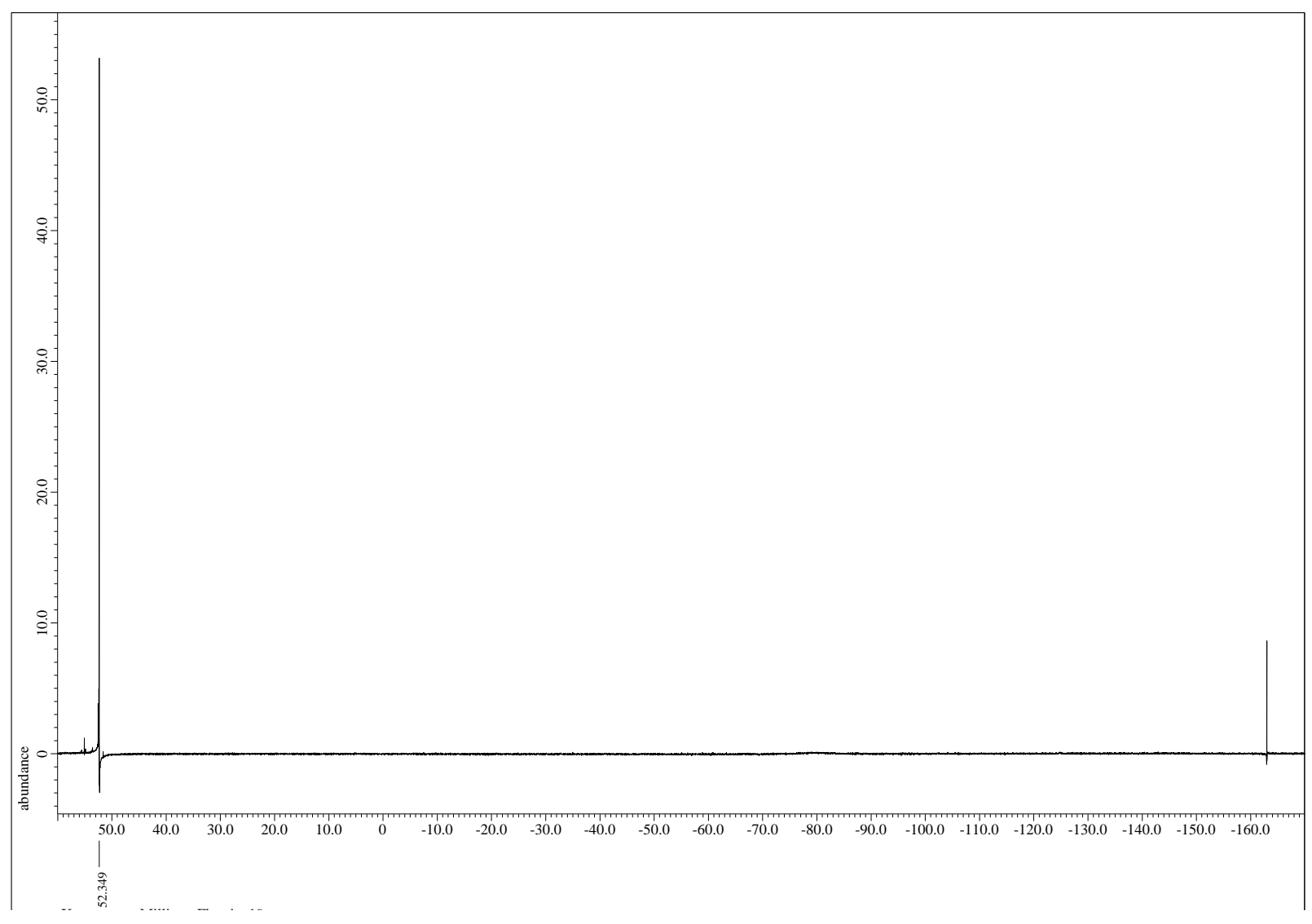



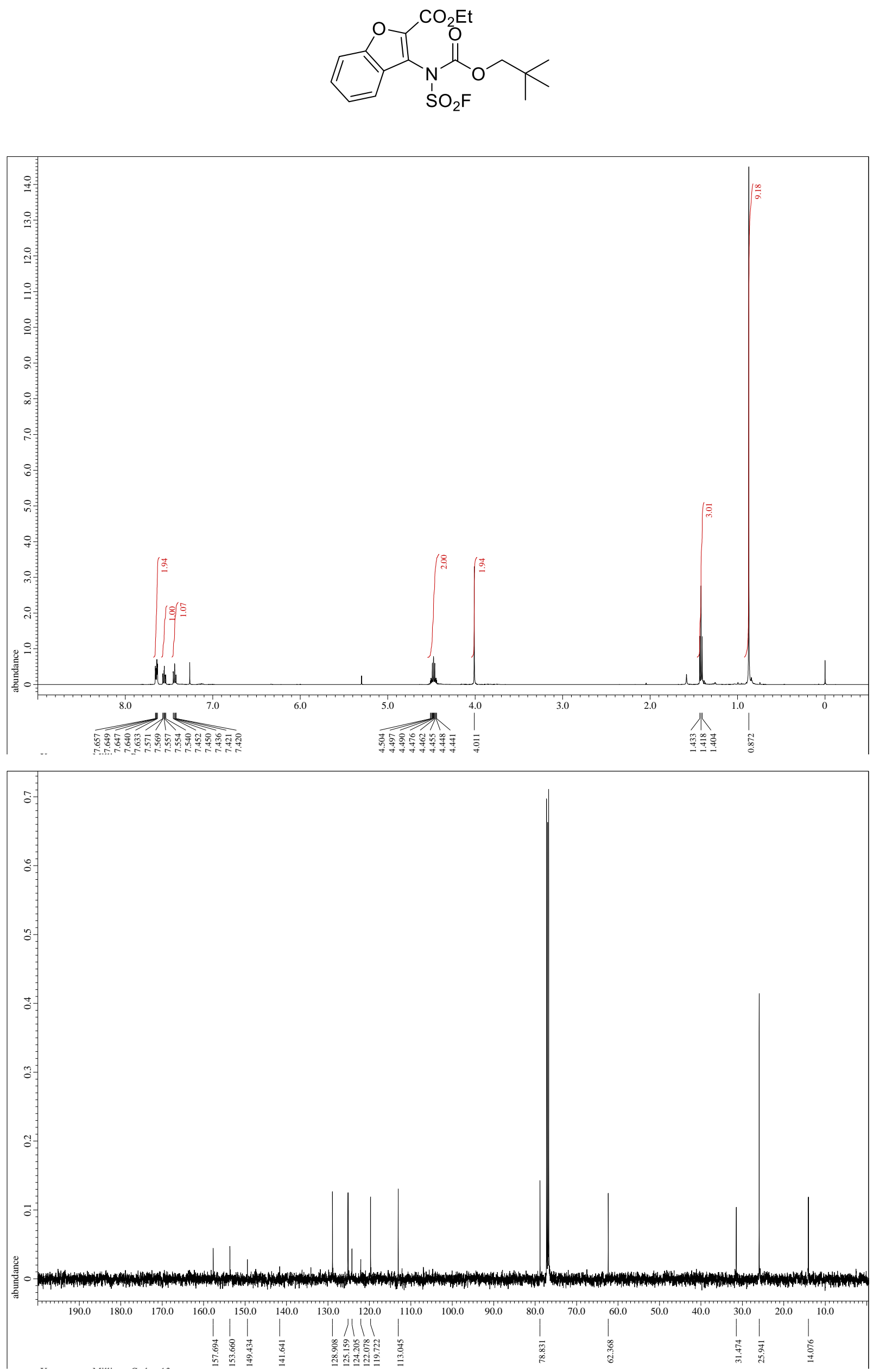


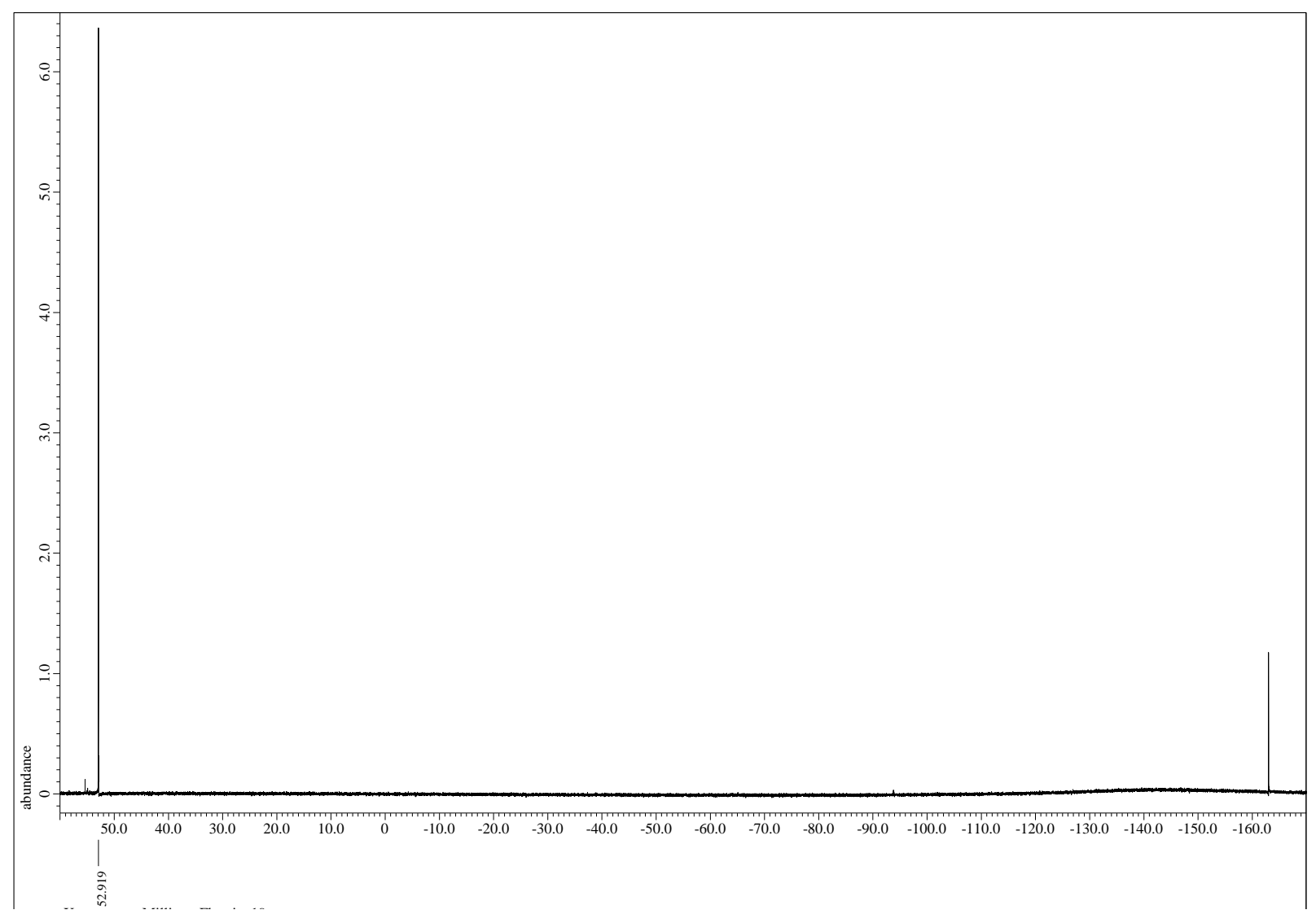



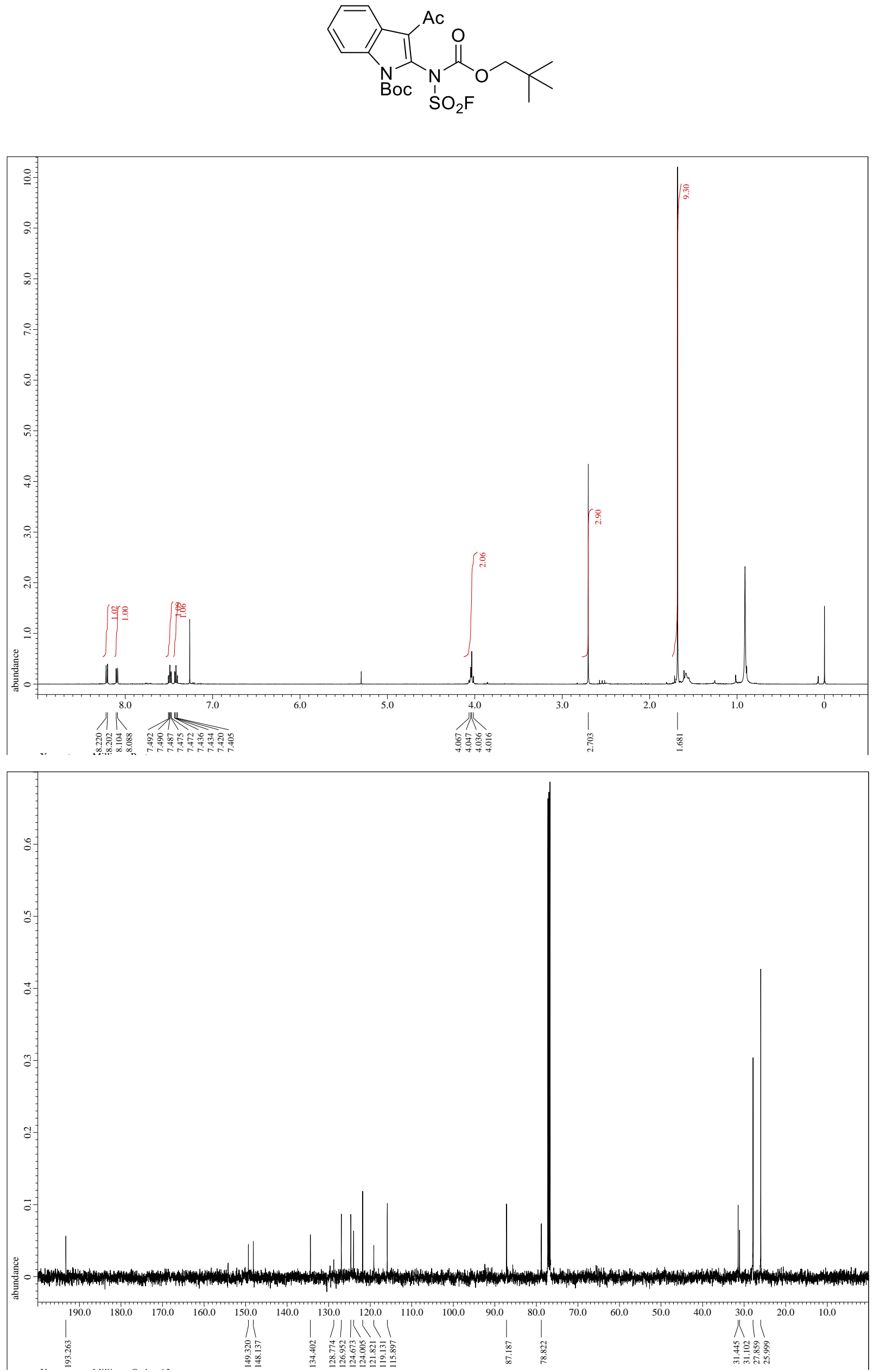


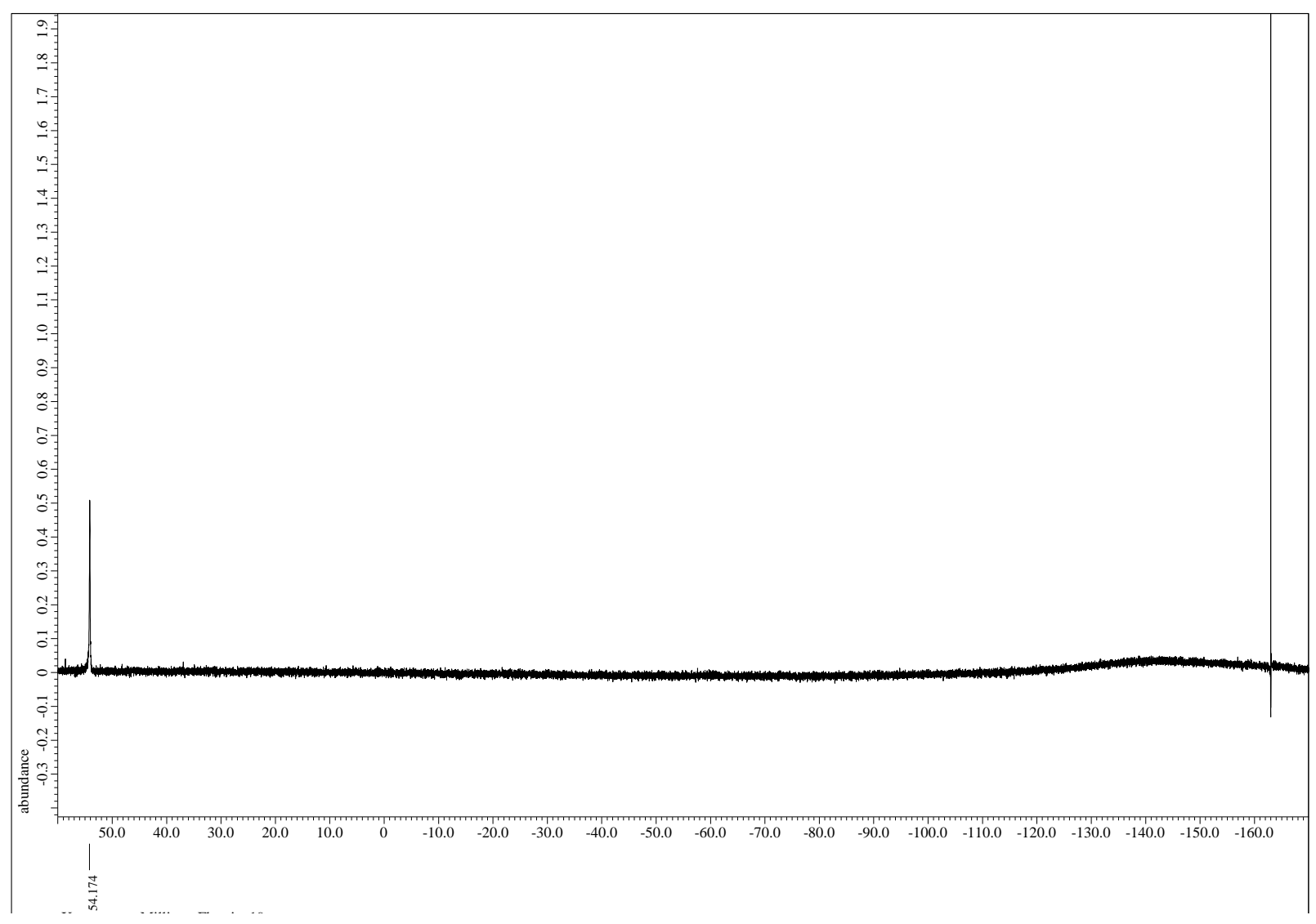



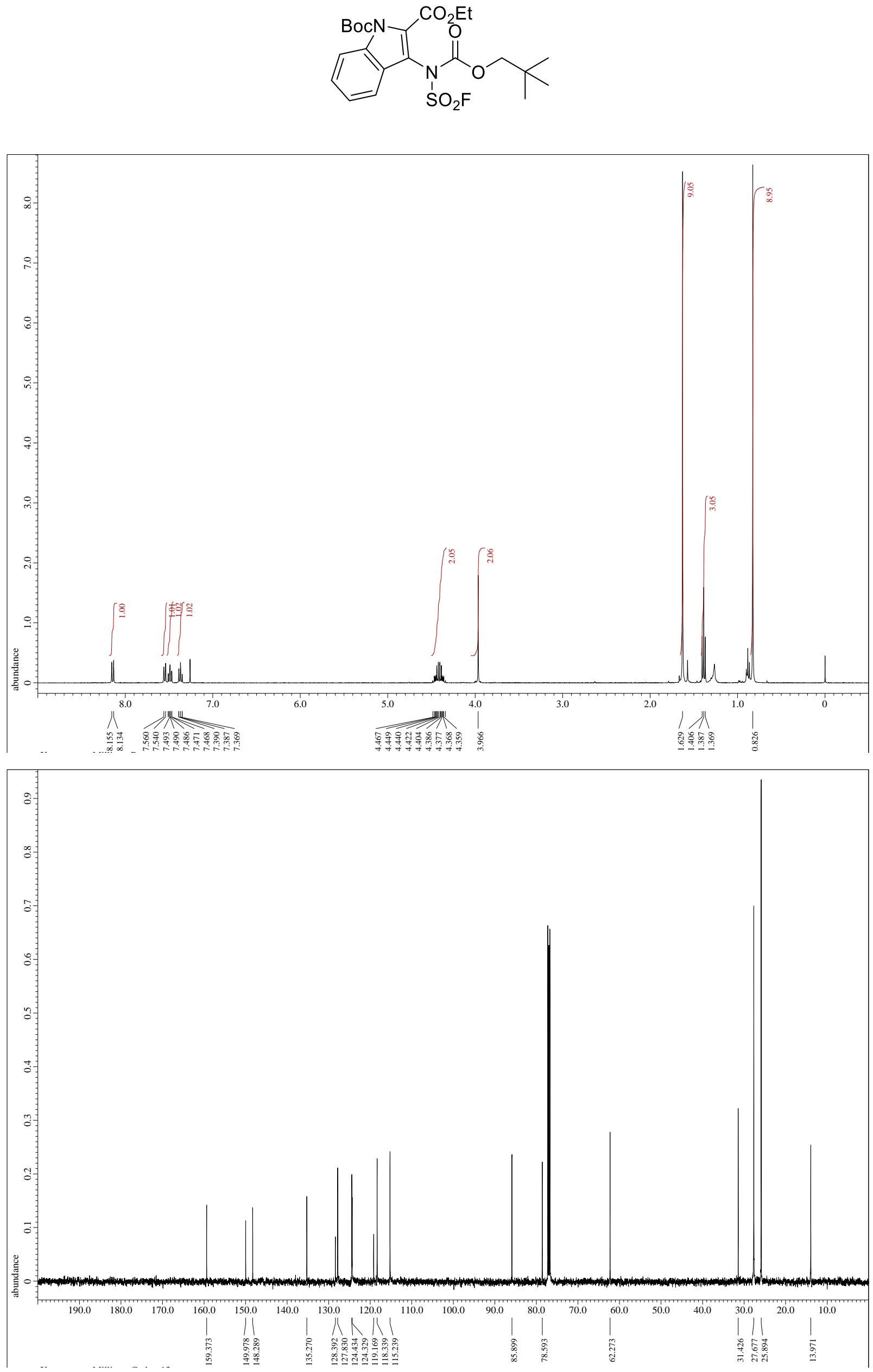


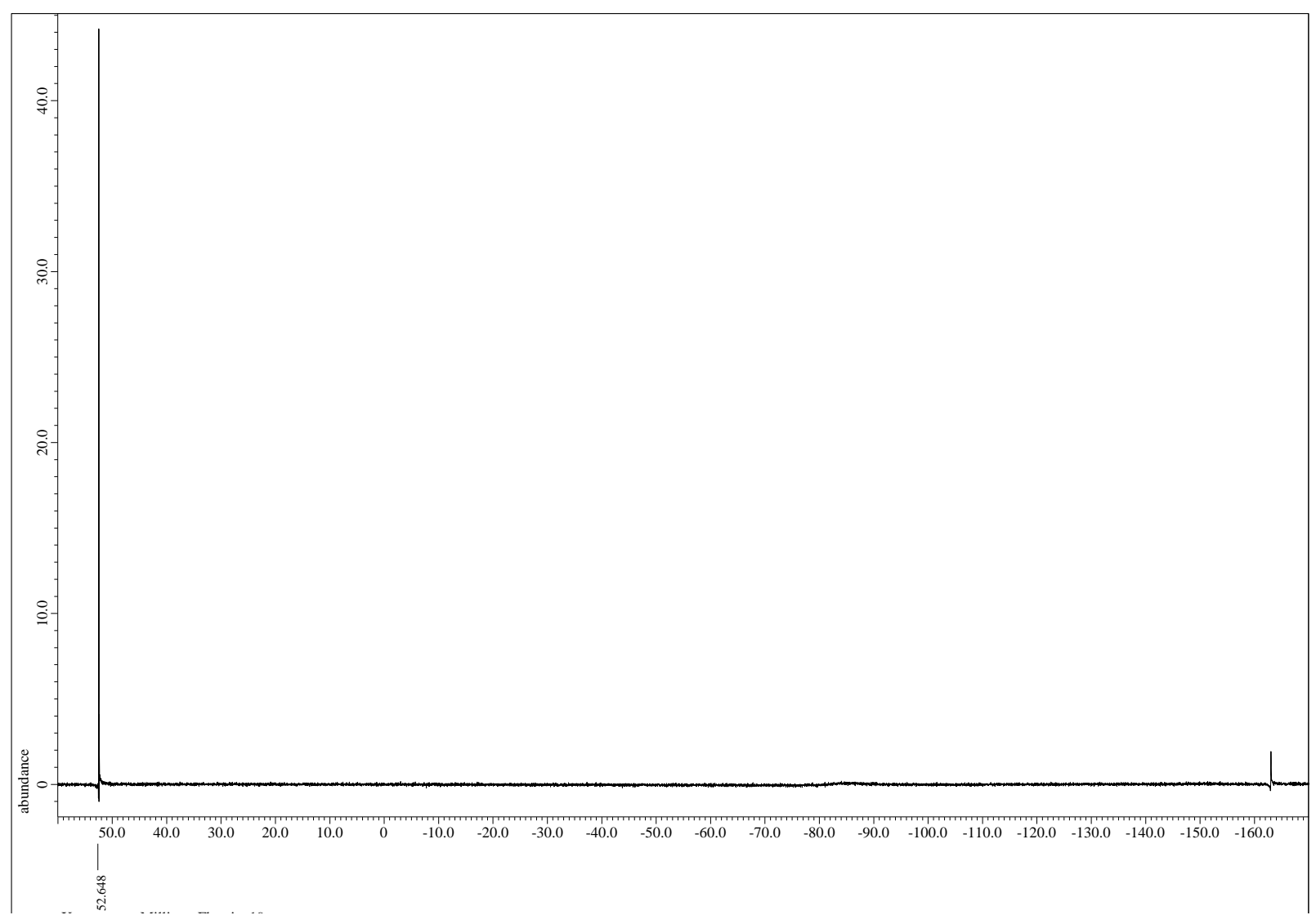


neopentyl (10-bromoanthracen-9-yl)(fluorosulfonyl)carbamate (22)
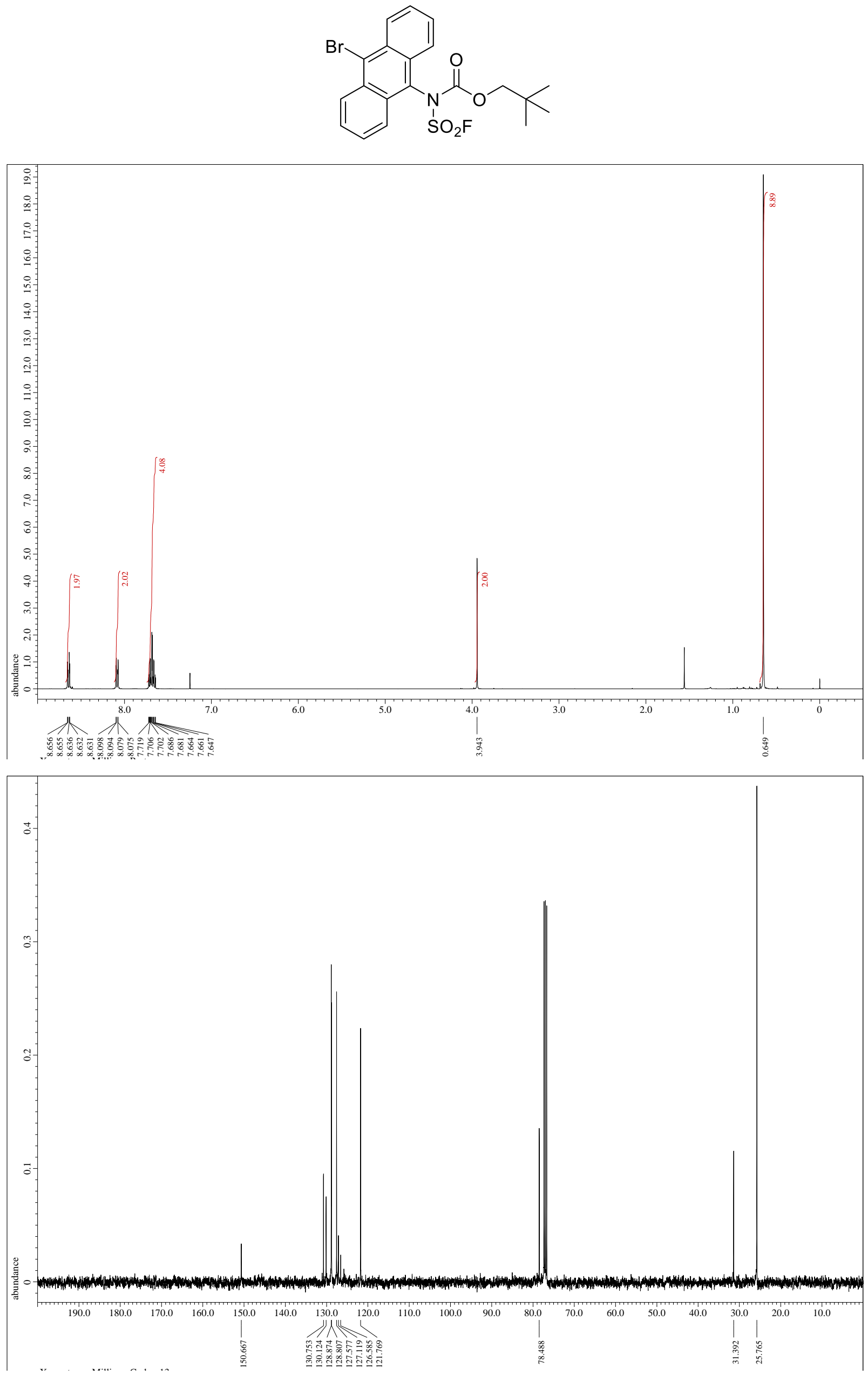


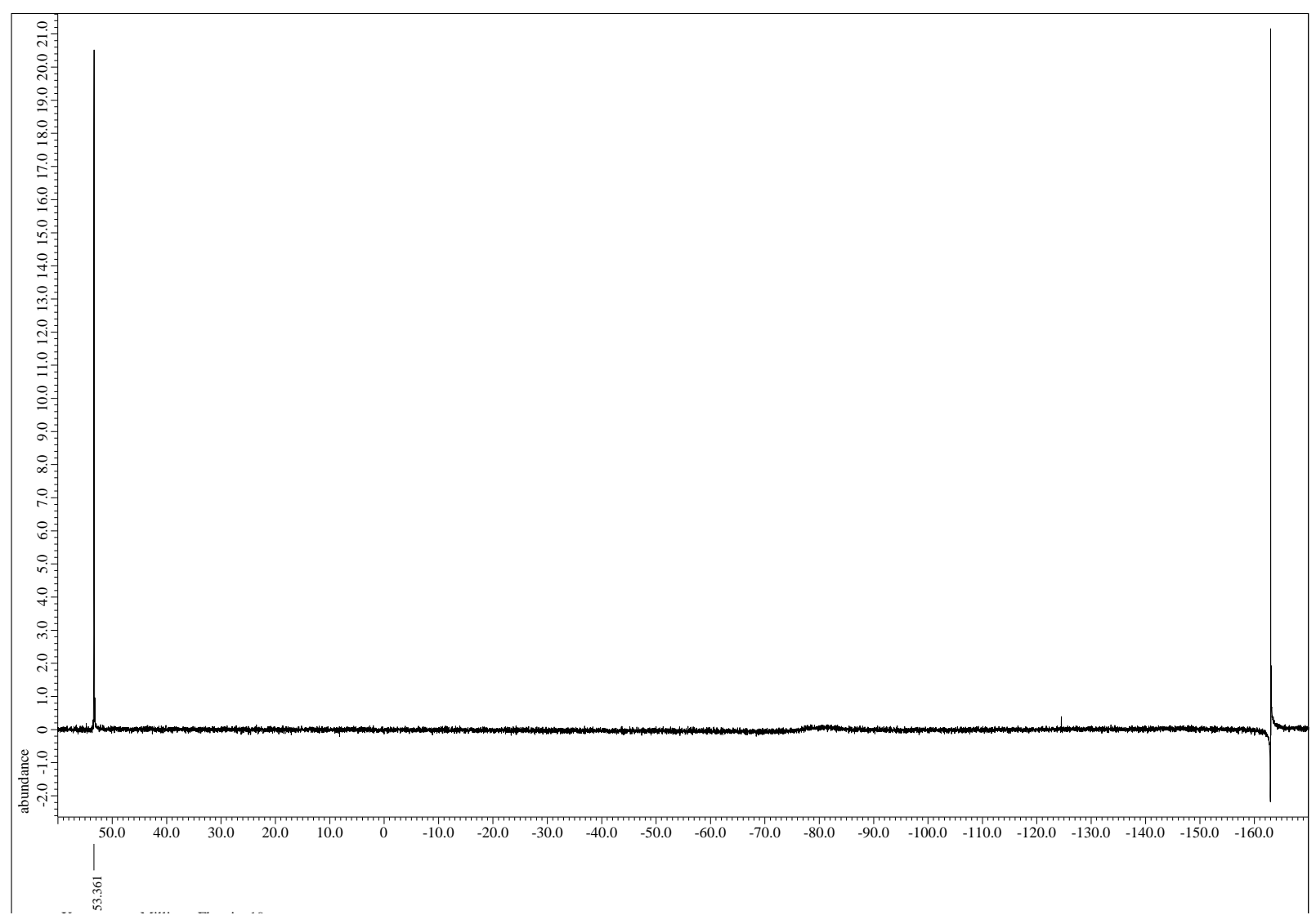


<smiles></smiles>
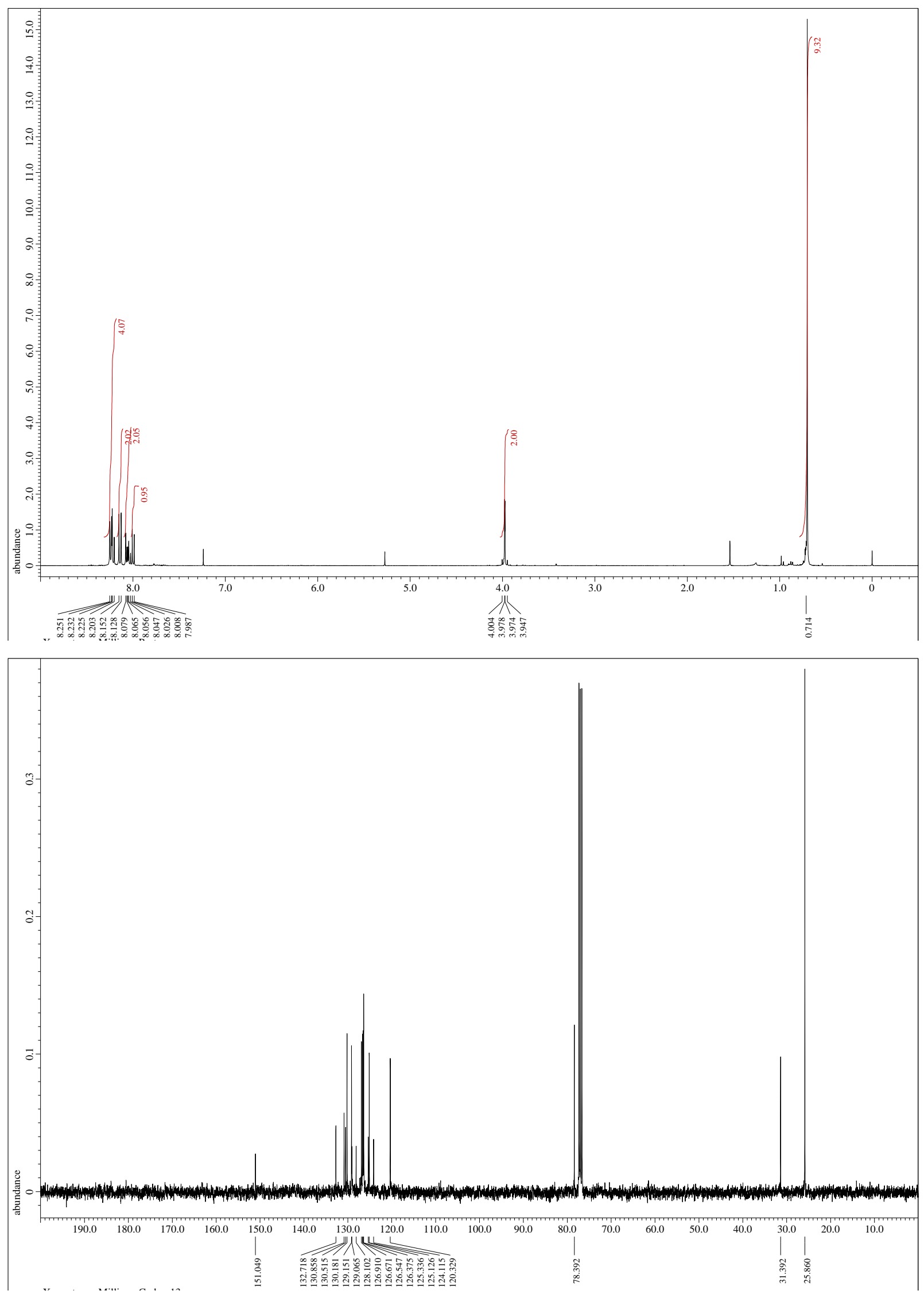


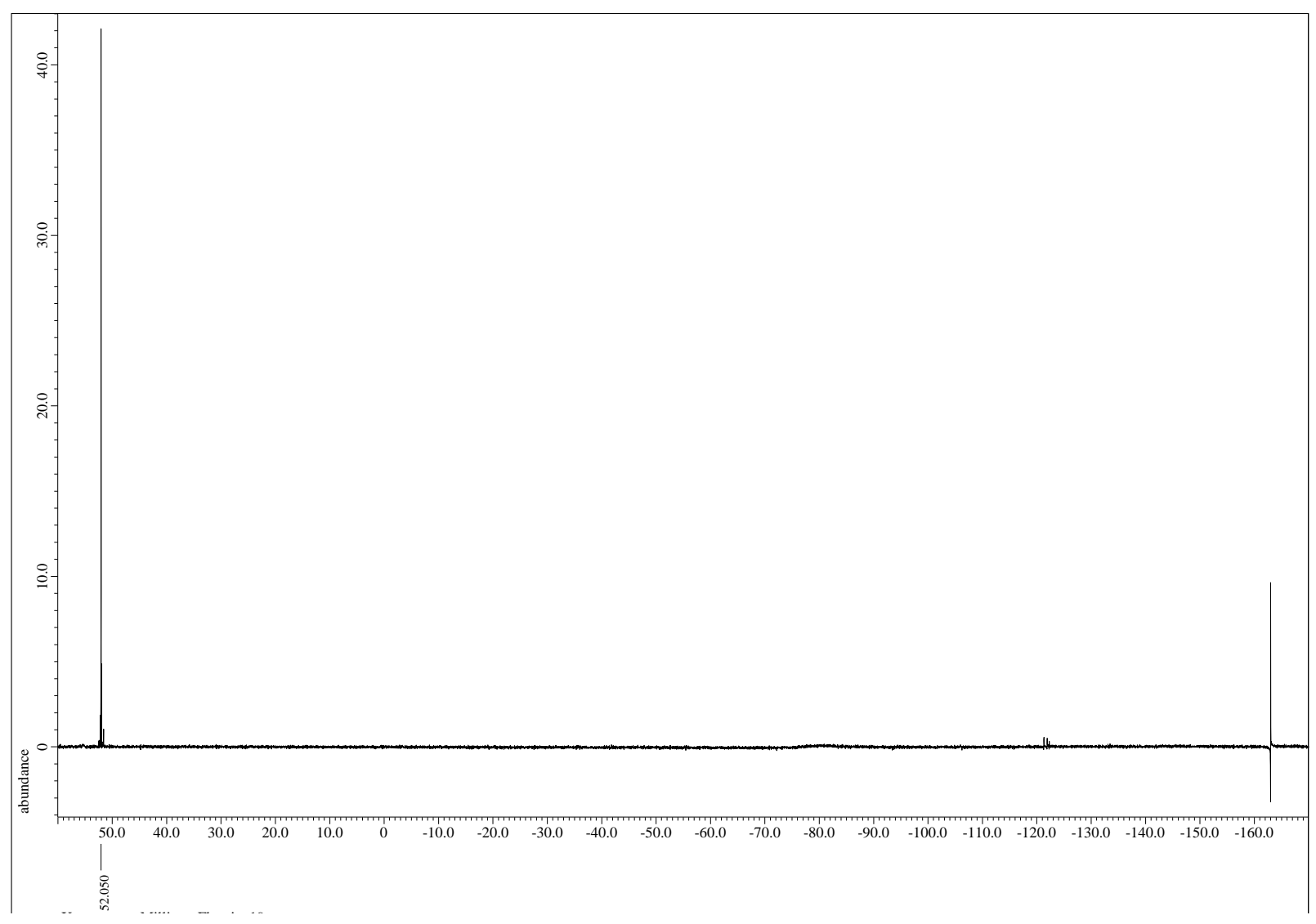



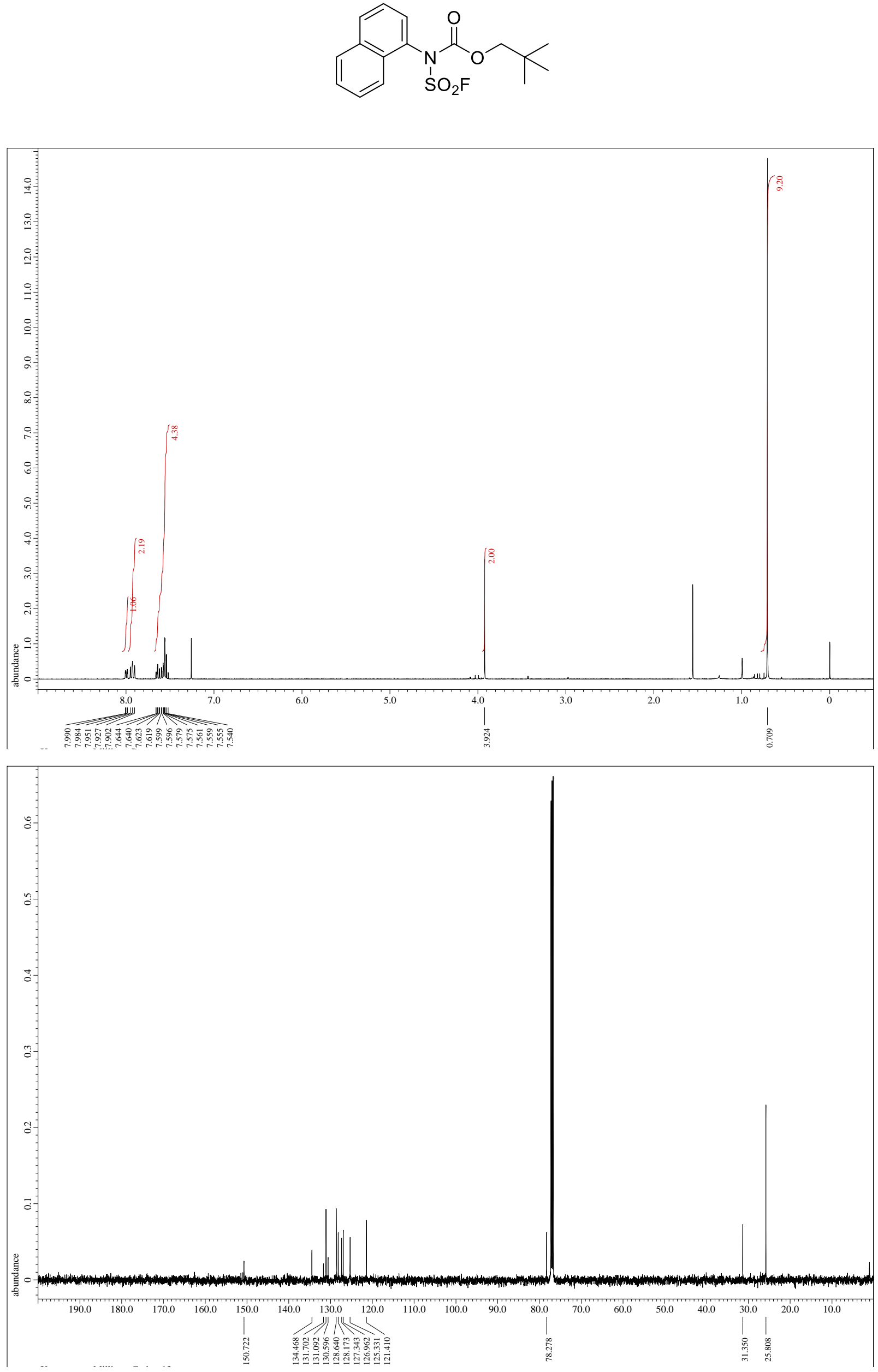


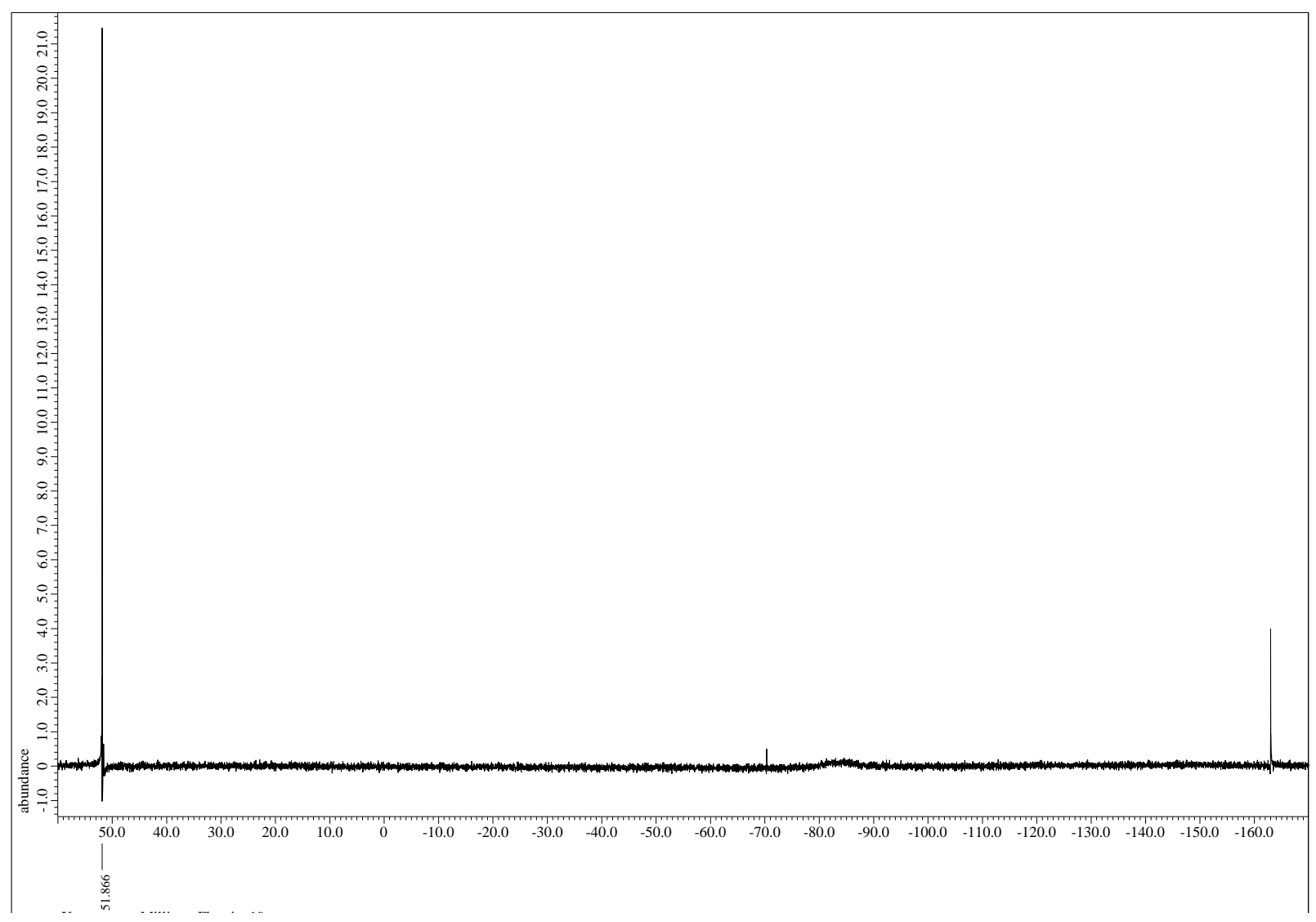


neopentyl (3,5-dimethylbenzyl)(fluorosulfonyl)carbamate (26)
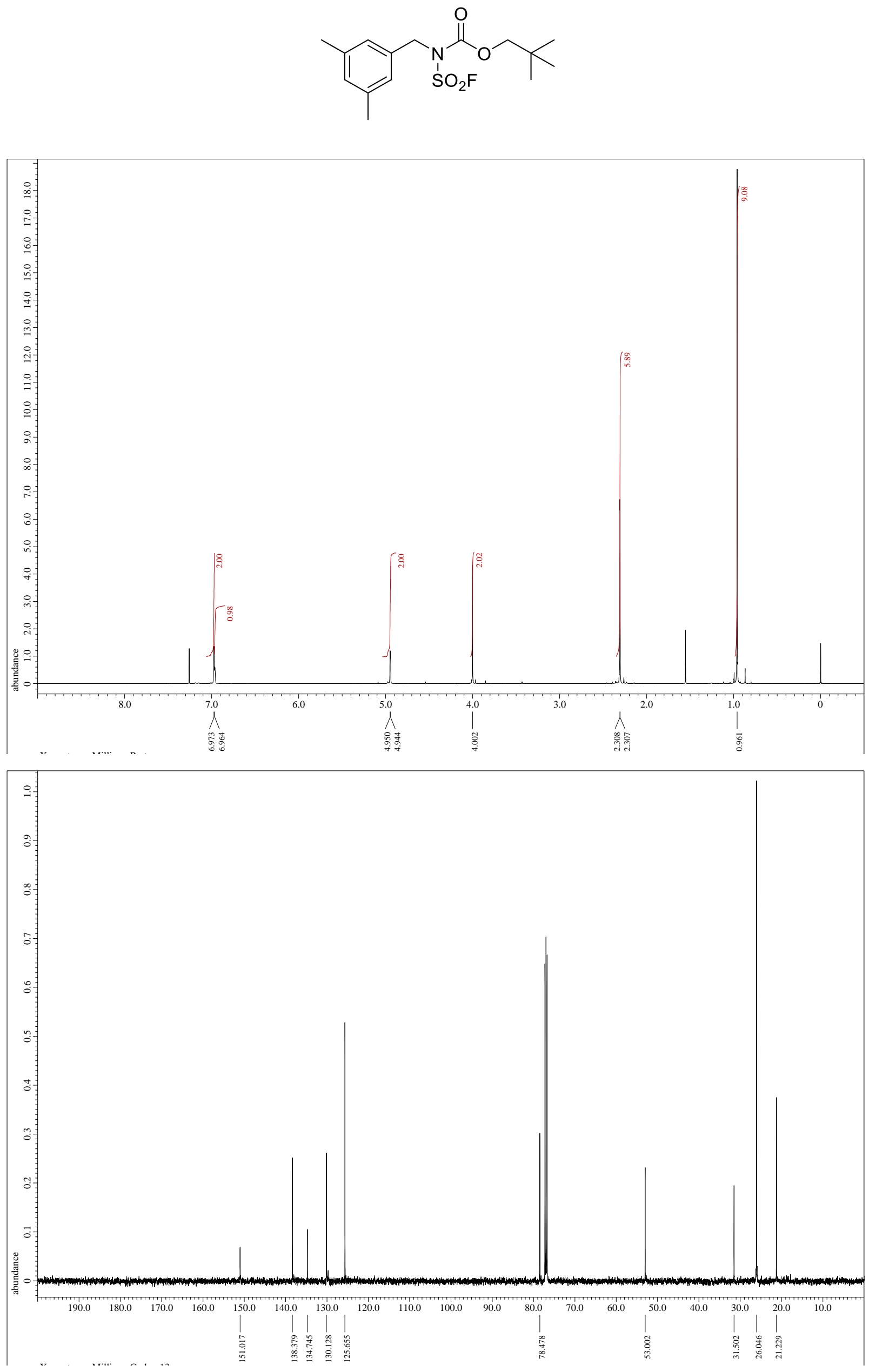


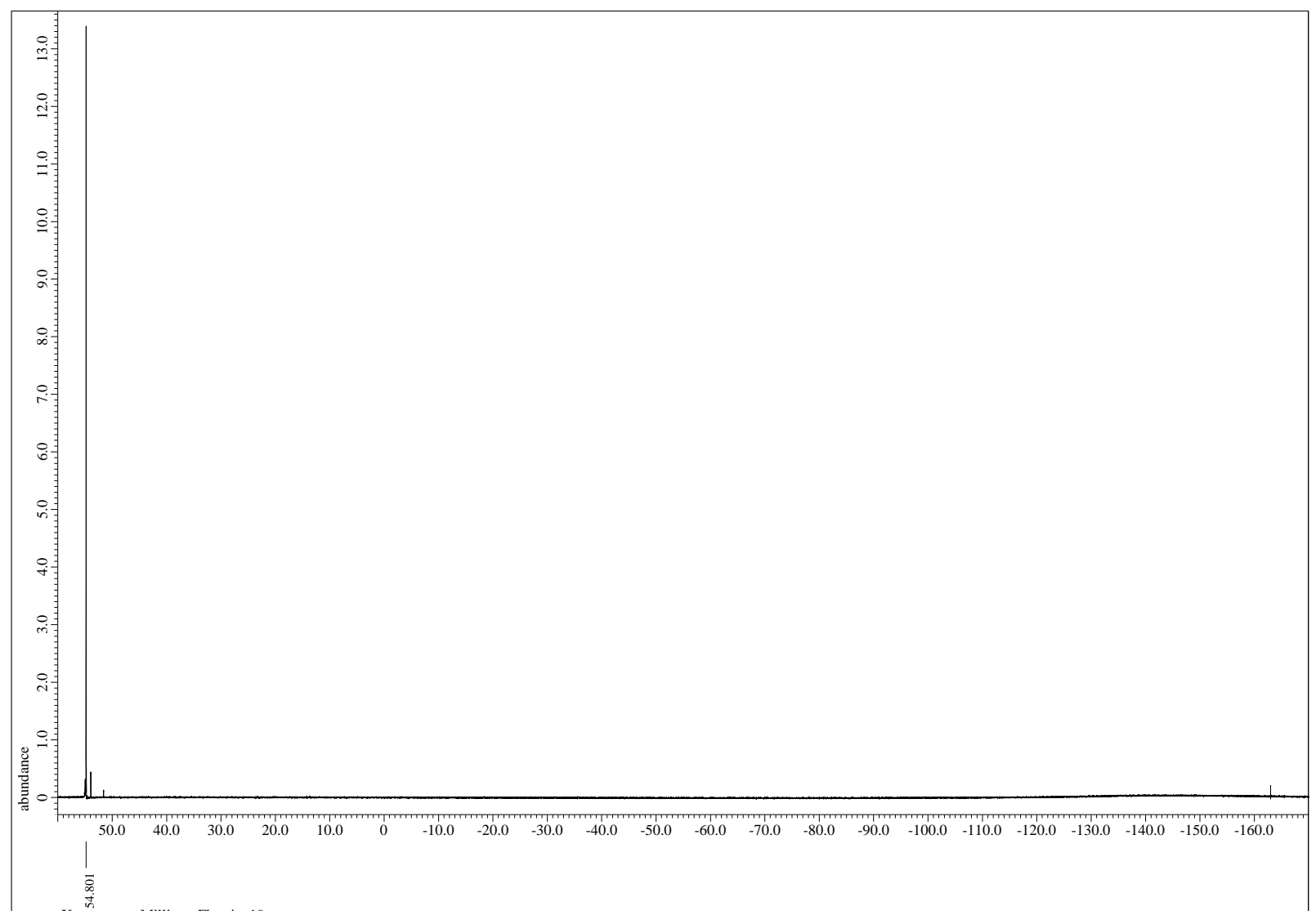


neopentyl (5-acetylthiophen-2-yl)(fluorosulfonyl)carbamate (S1)
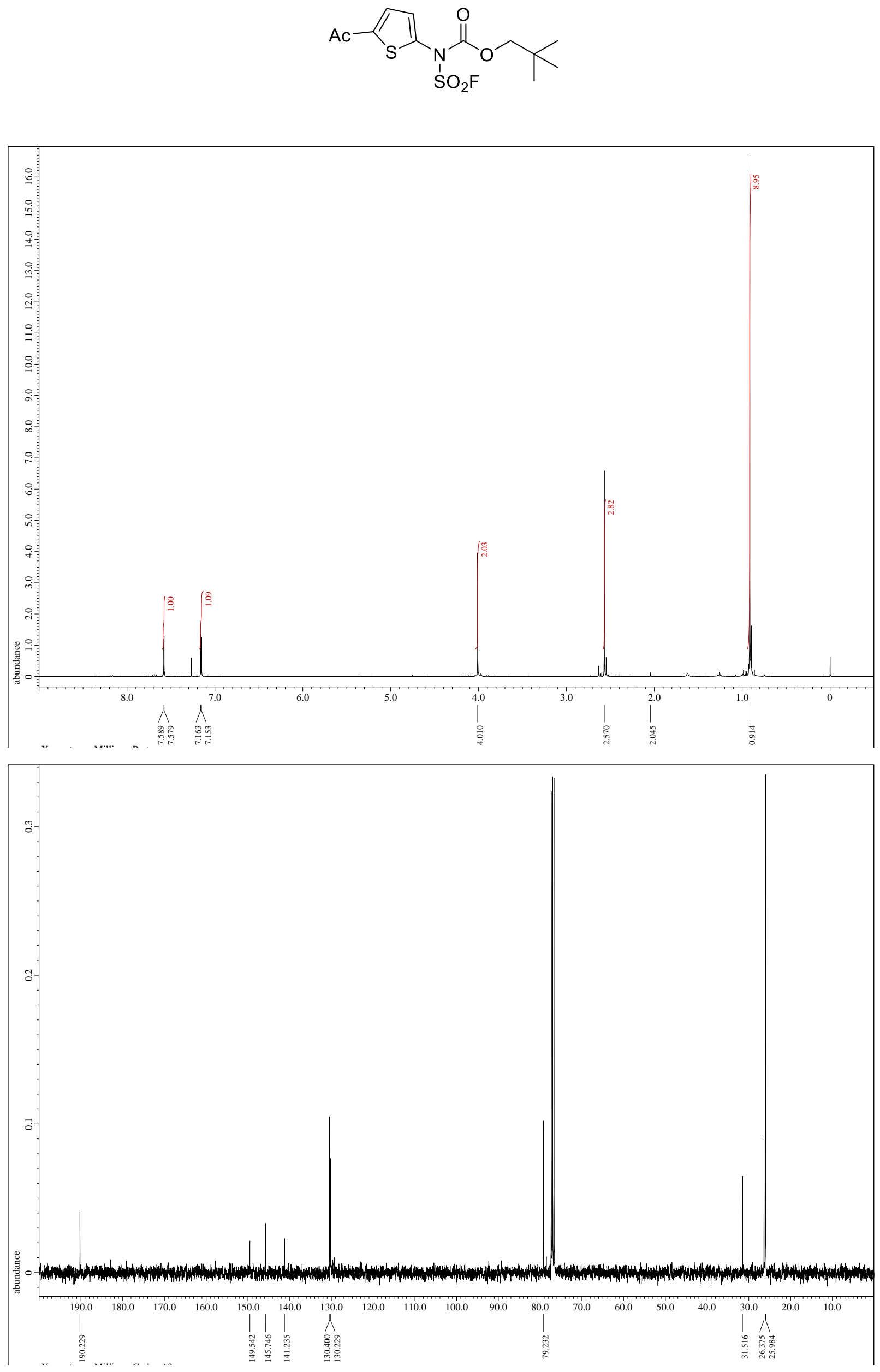


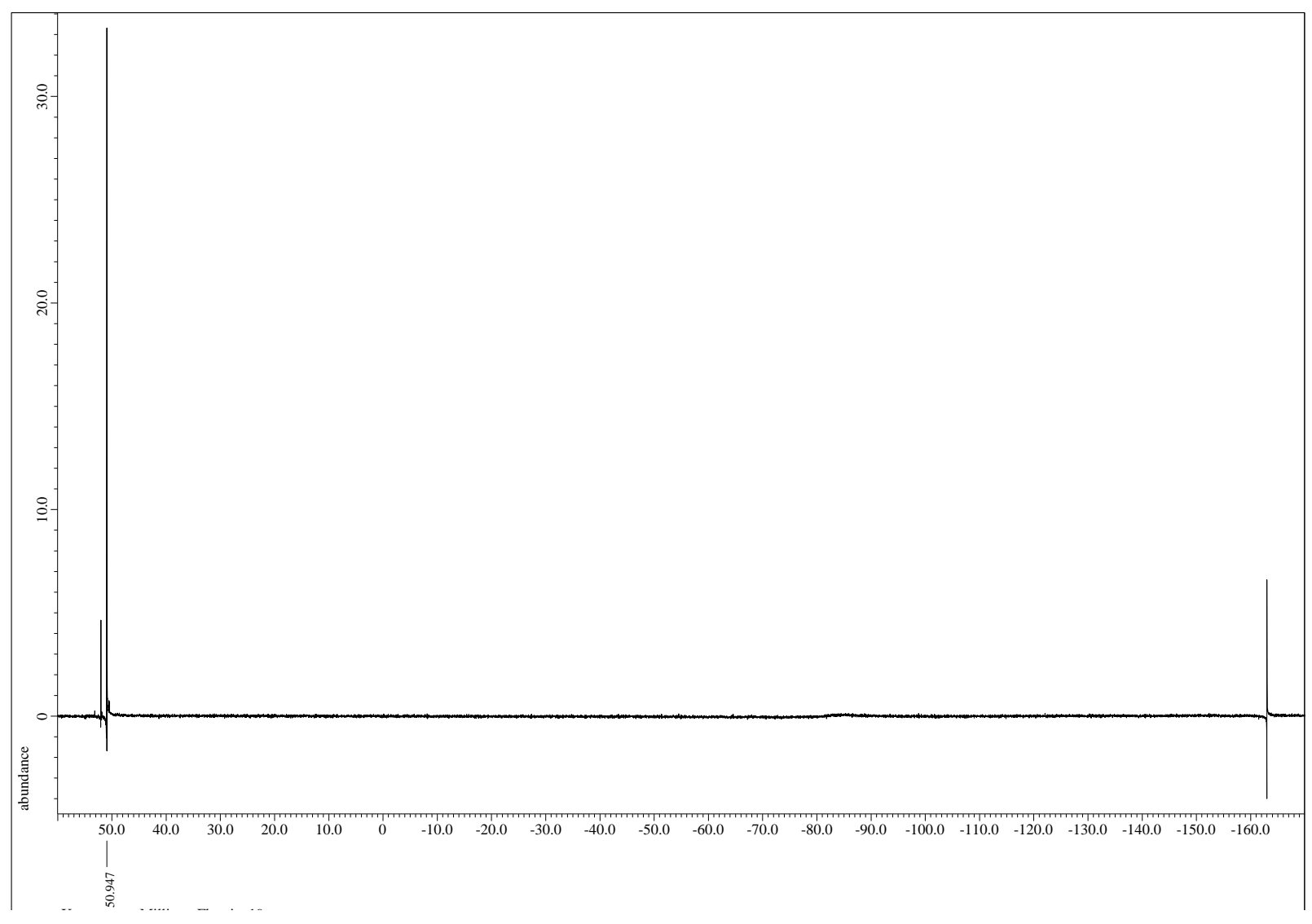


neopentyl (fluorosulfonyl)(furan-2-yl)carbamate (S2)
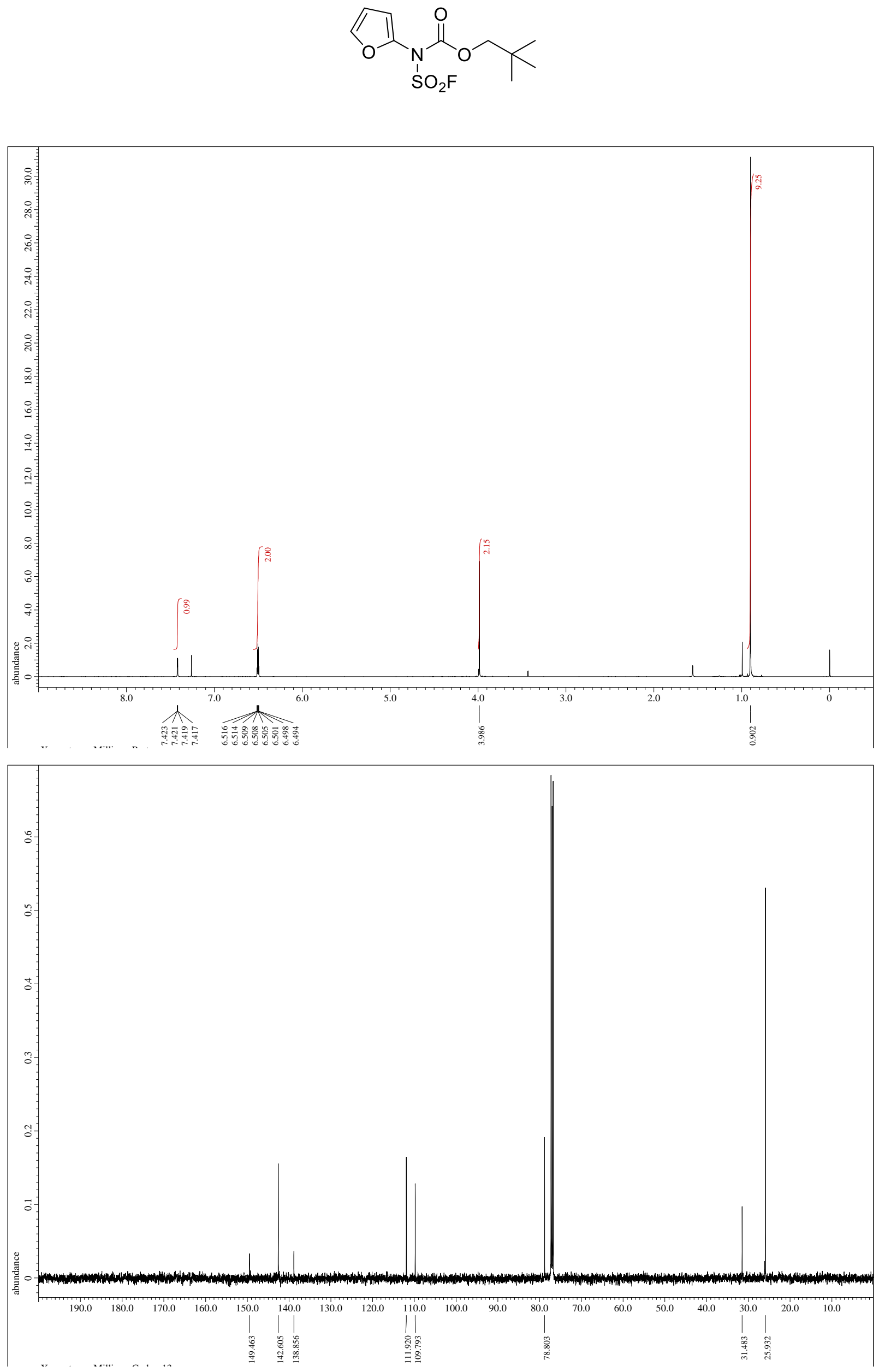


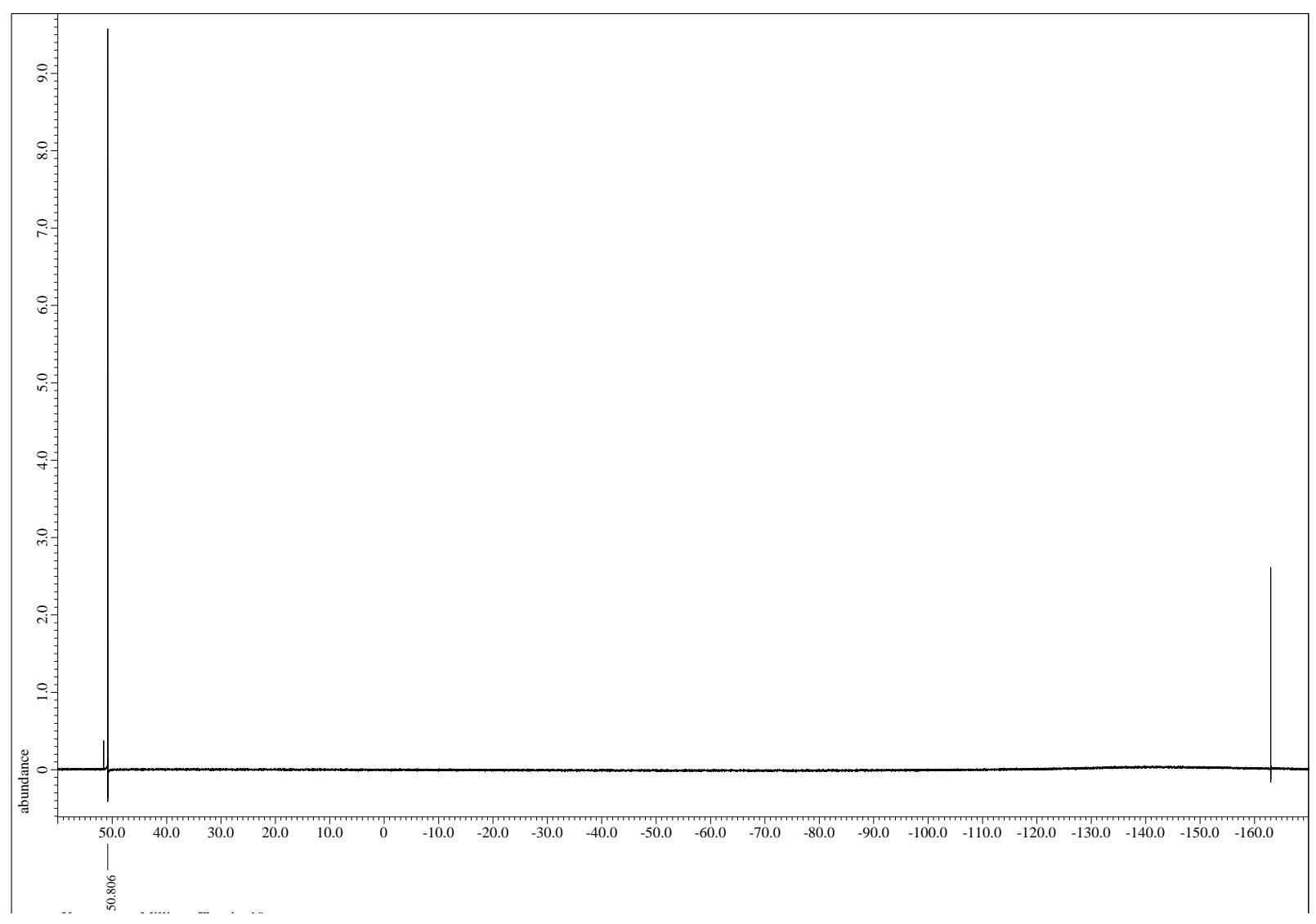


neopentyl (fluorosulfonyl)(5-methylfuran-2-yl)carbamate (S3) and

neopentyl (fluorosulfonyl)(furan-2-ylmethyl)carbamate (S3')

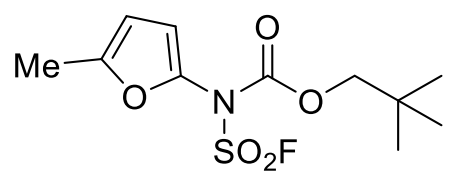

S3<smiles>CC(C)(C)COC(=O)N(Cc1ccco1)S(=O)(=O)F</smiles>

S3'
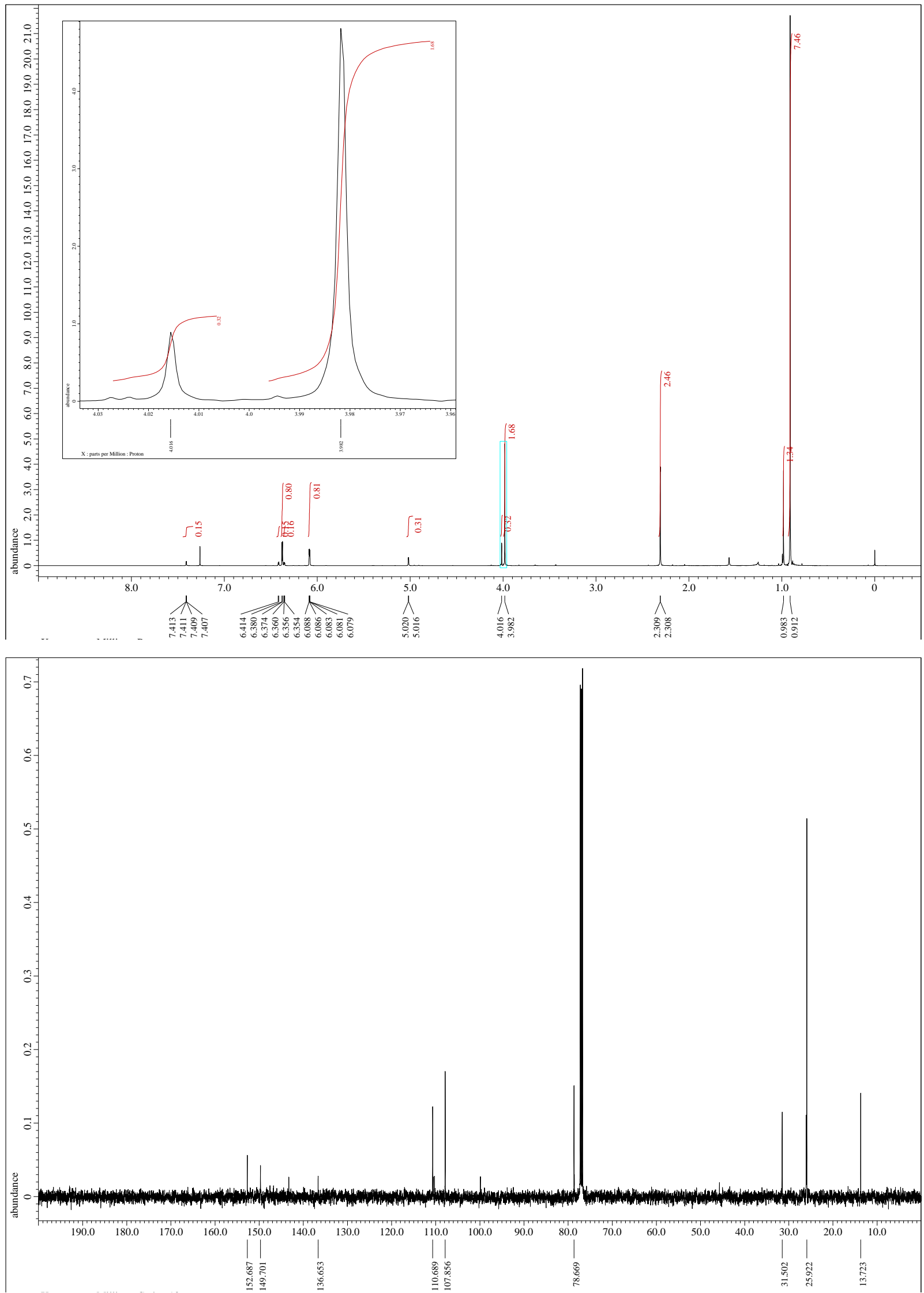


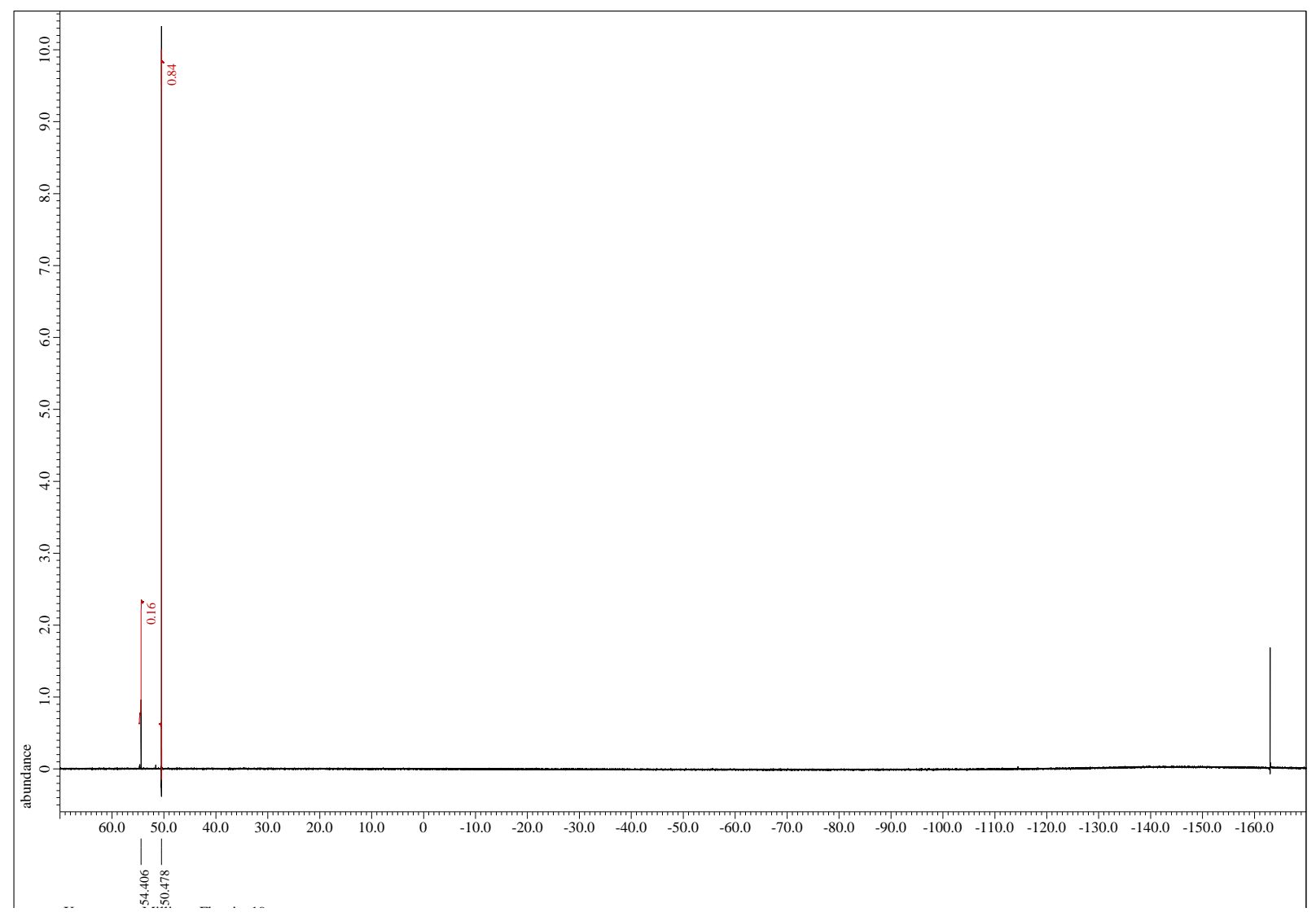


neopentyl (5-acetylfuran-2-yl)(fluorosulfonyl)carbamate (S4)
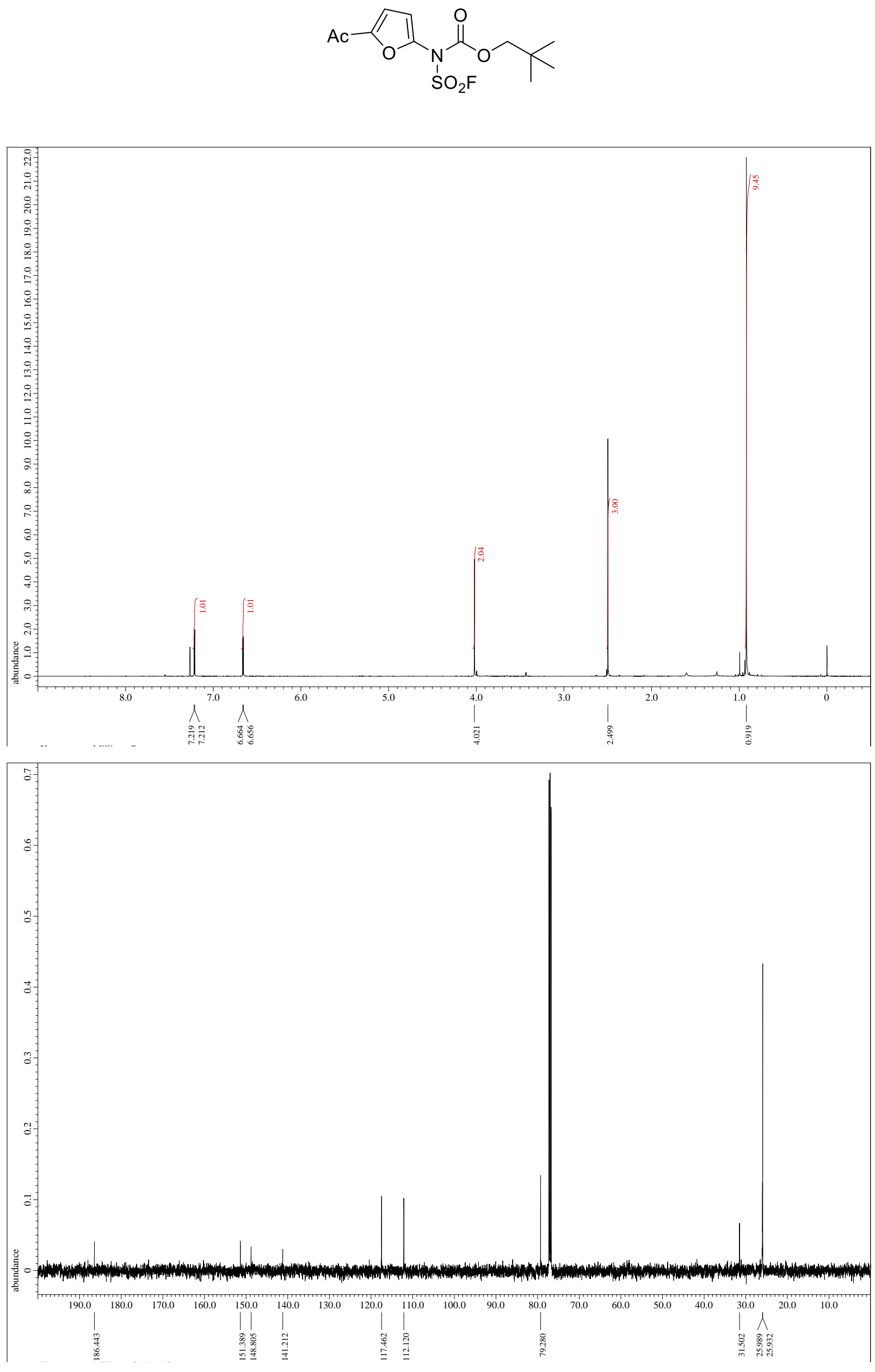


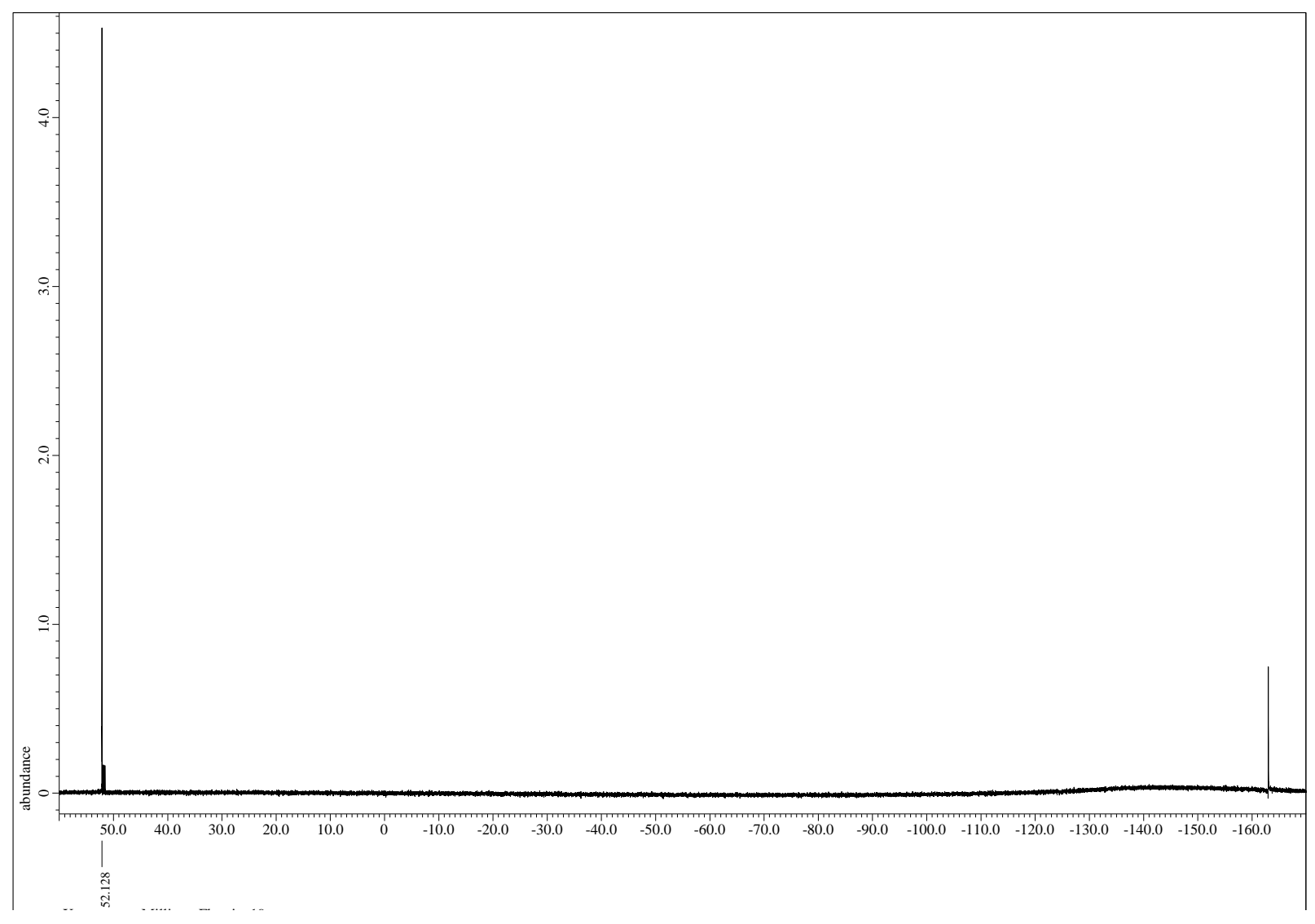


methyl 5-((fluorosulfonyl)((neopentyloxy)carbonyl)amino)furan-2-carboxylate (S5)
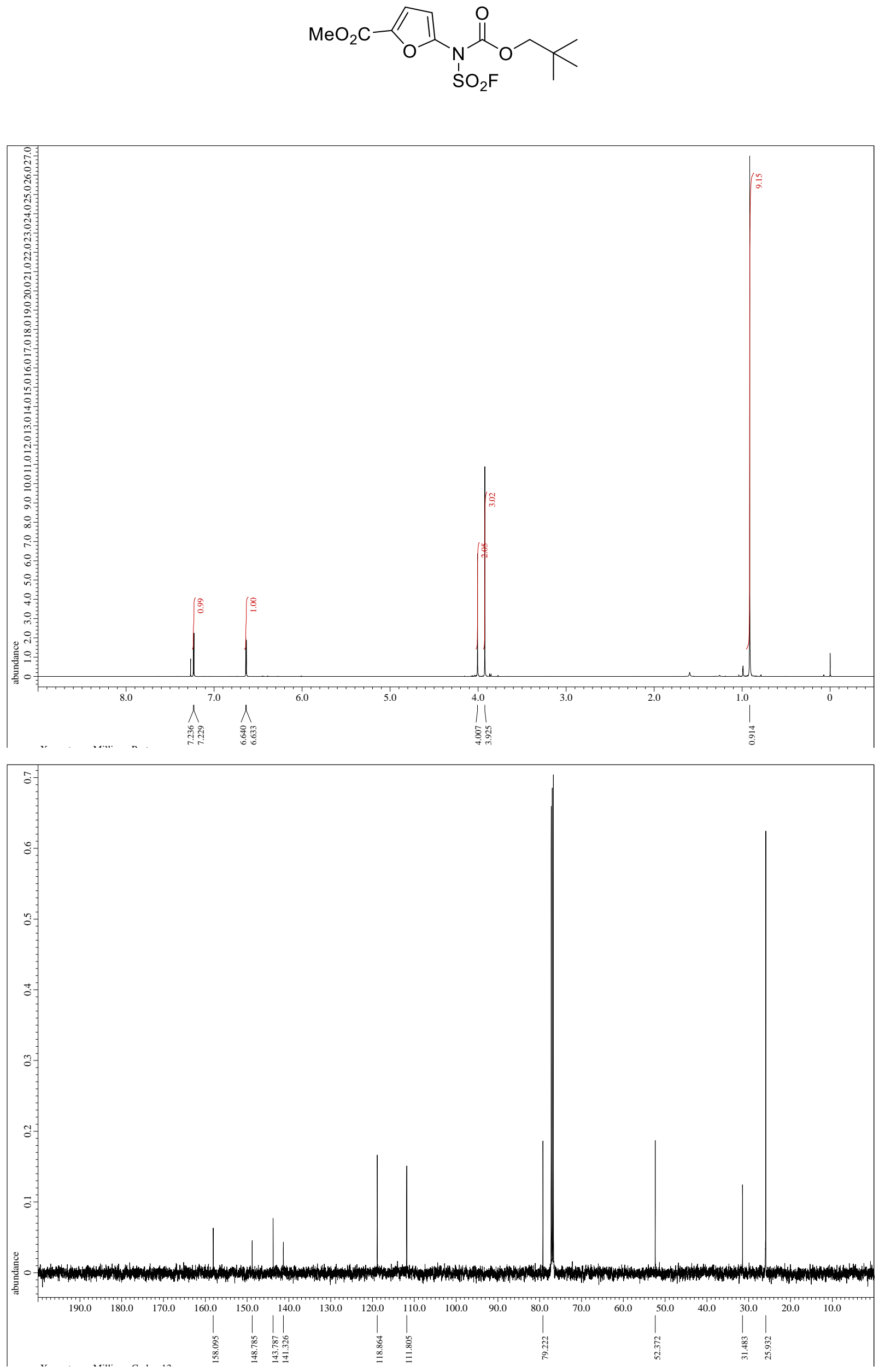


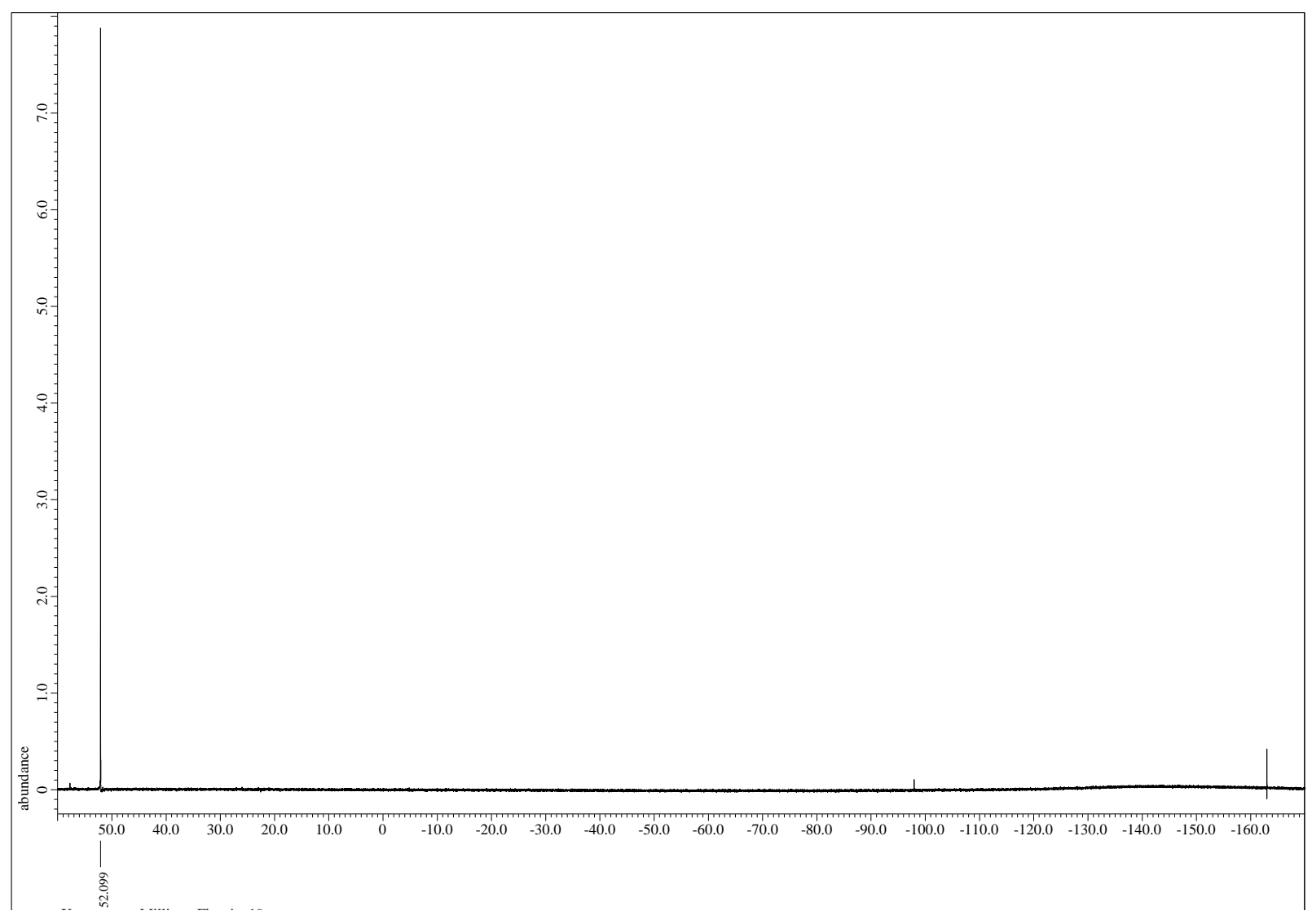


neopentyl (5-bromofuran-2-yl)(fluorosulfonyl)carbamate (S6)
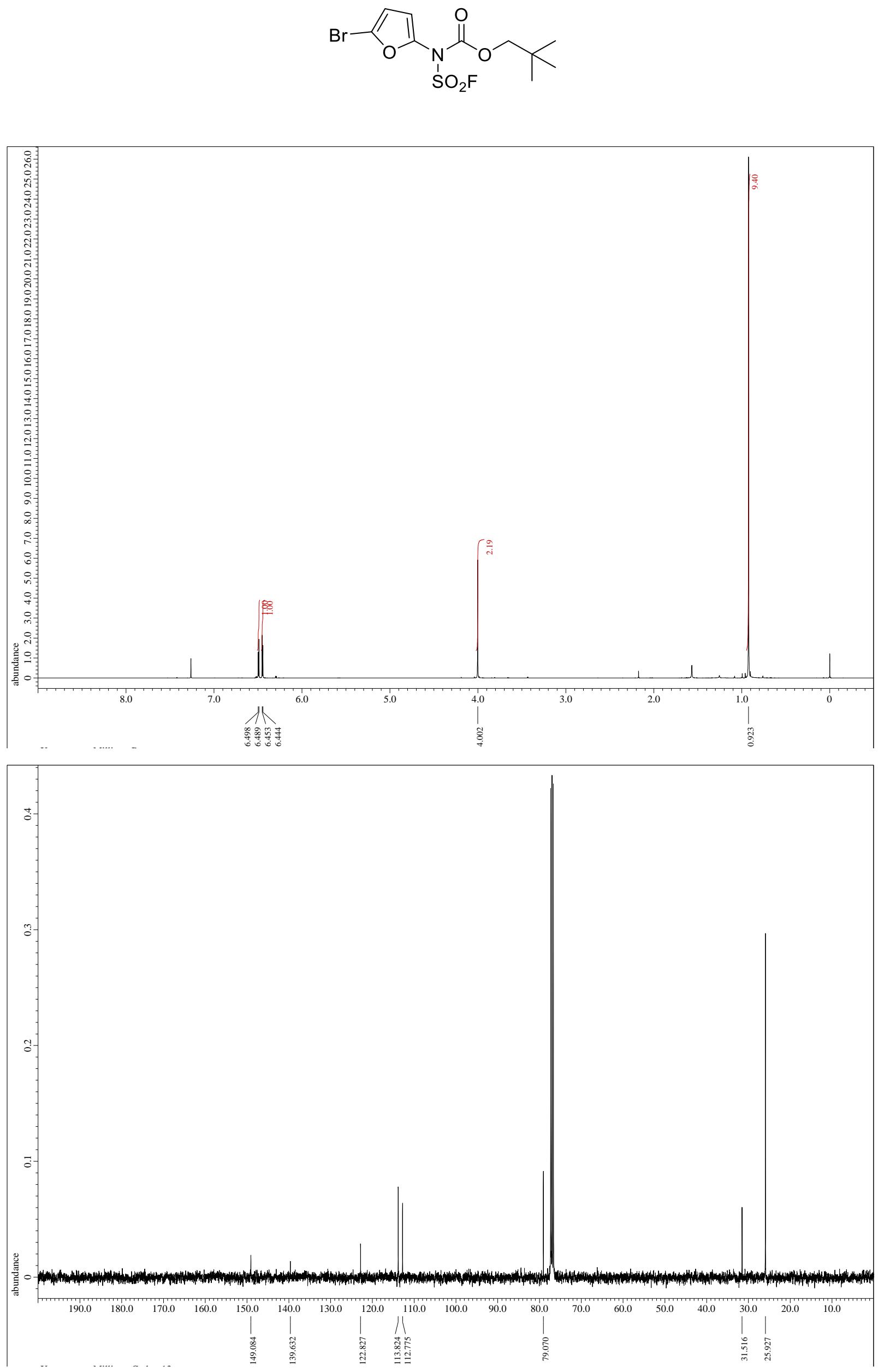


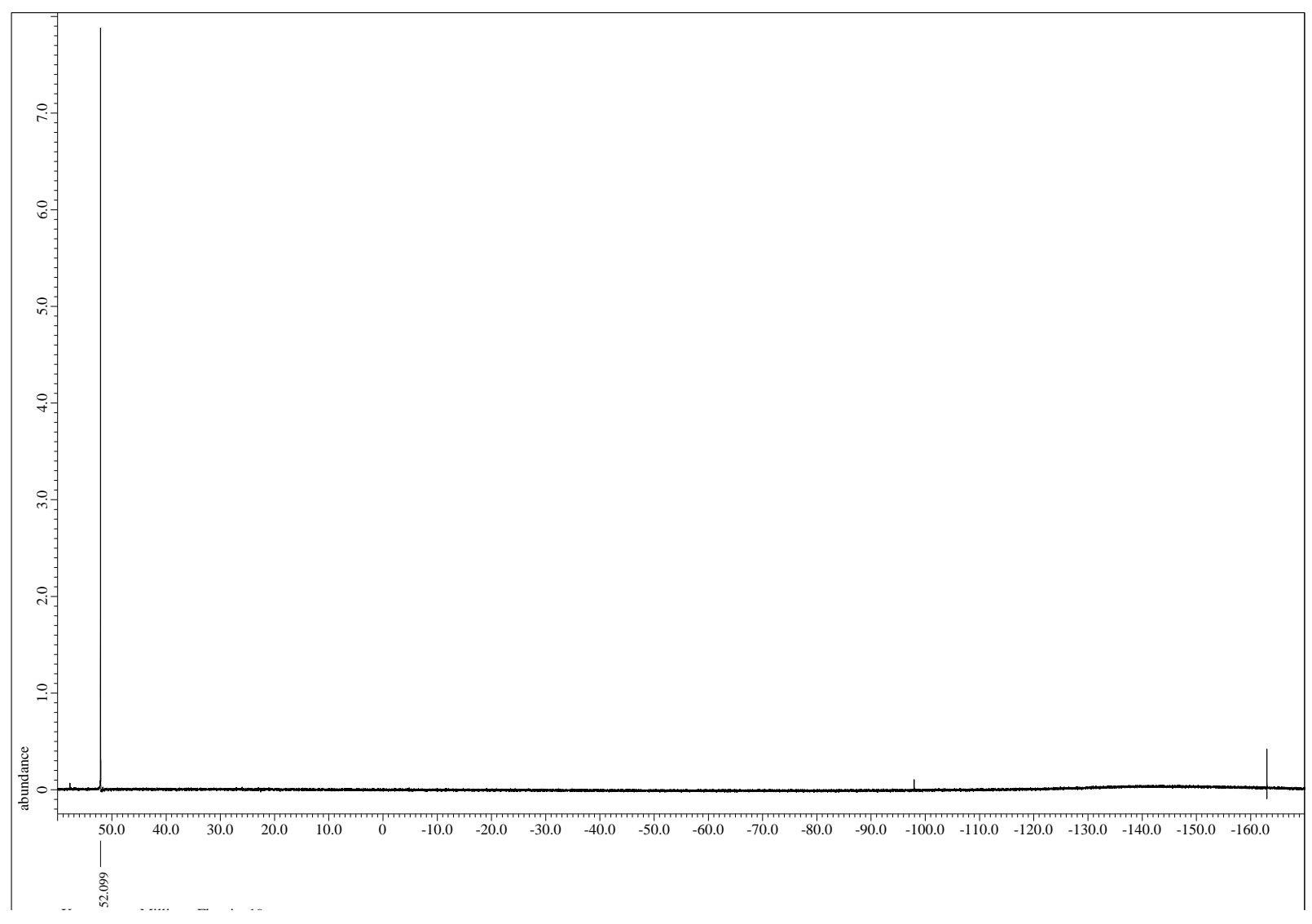



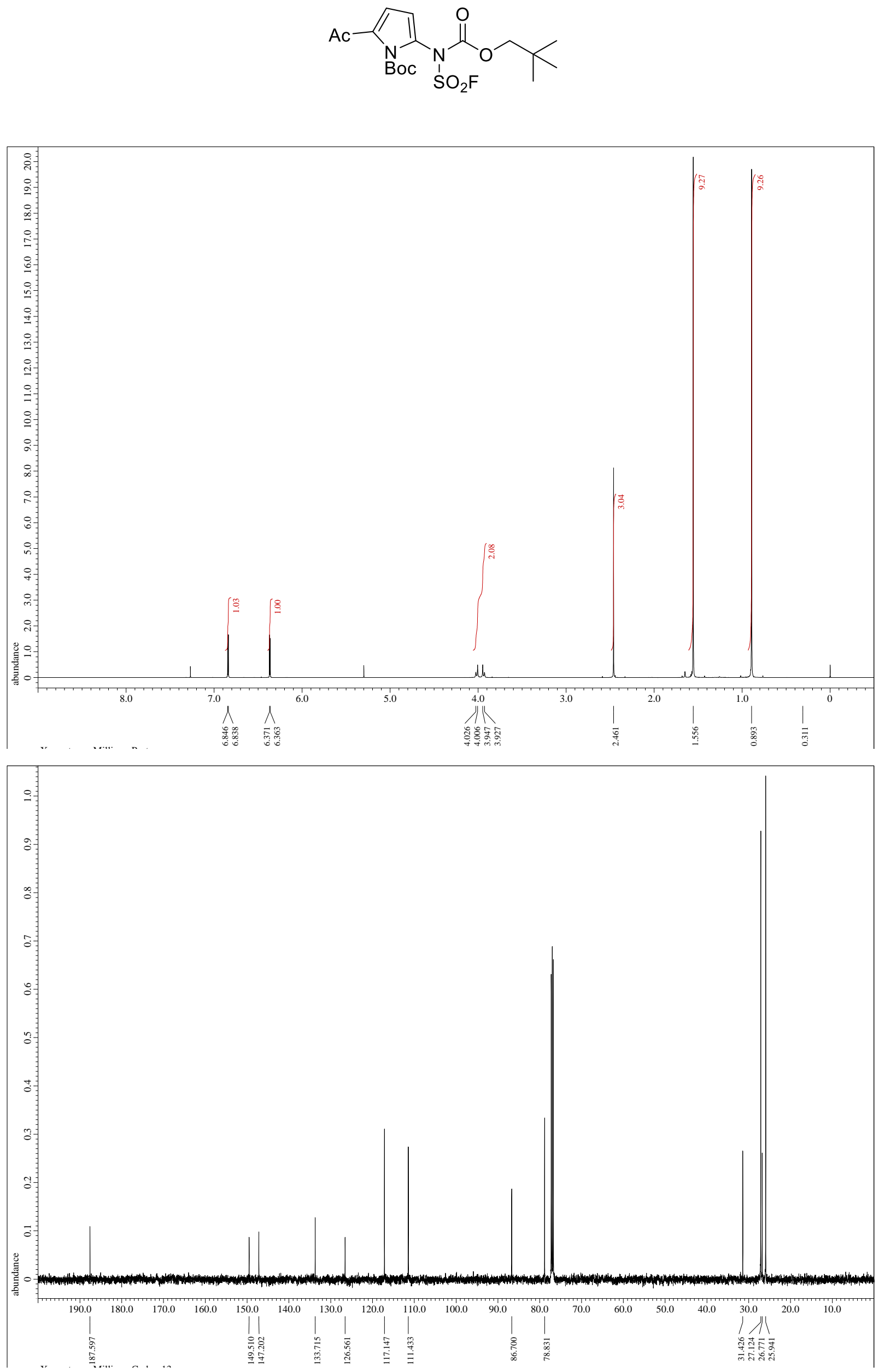


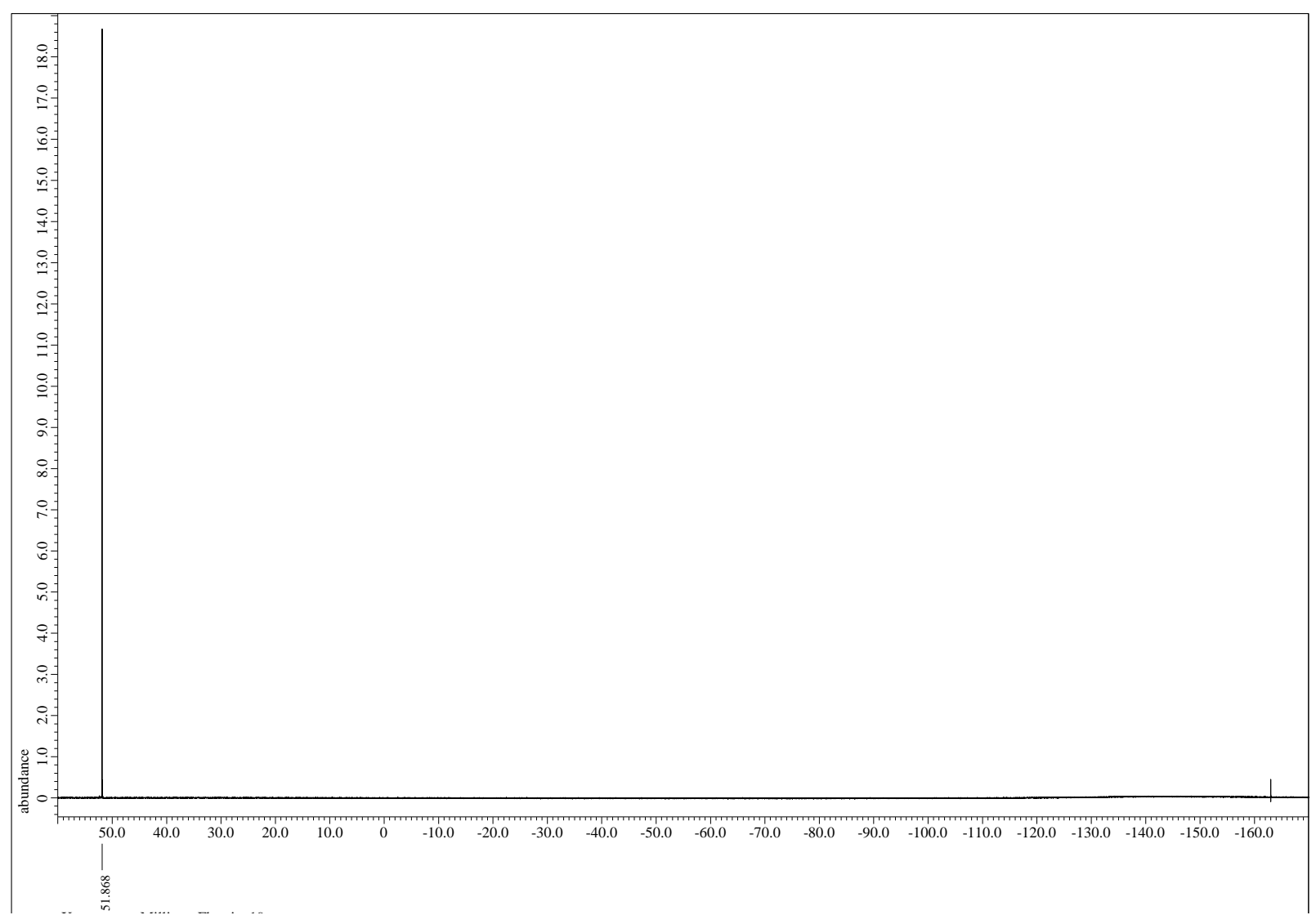


dicarboxylate (S8)
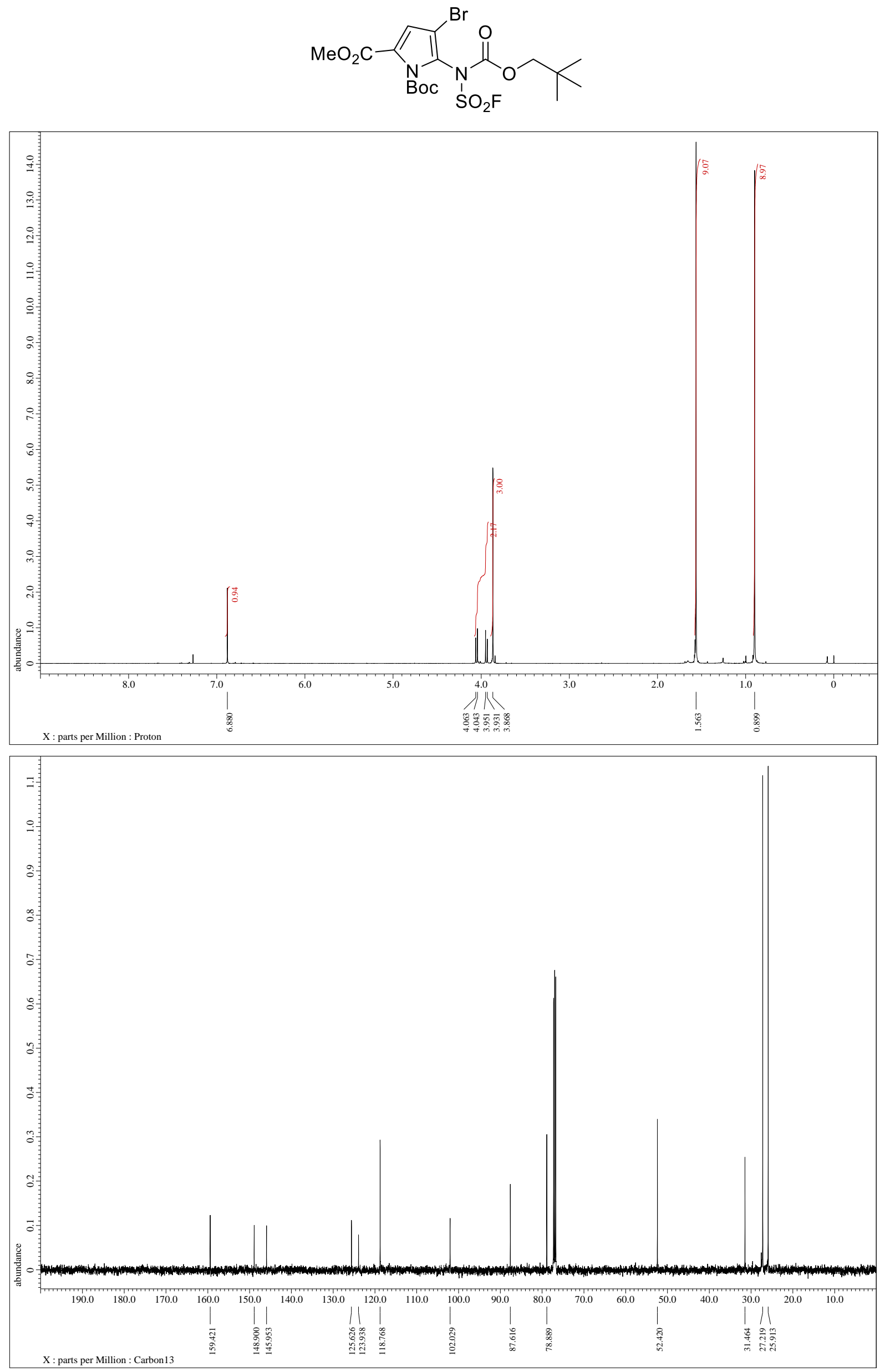


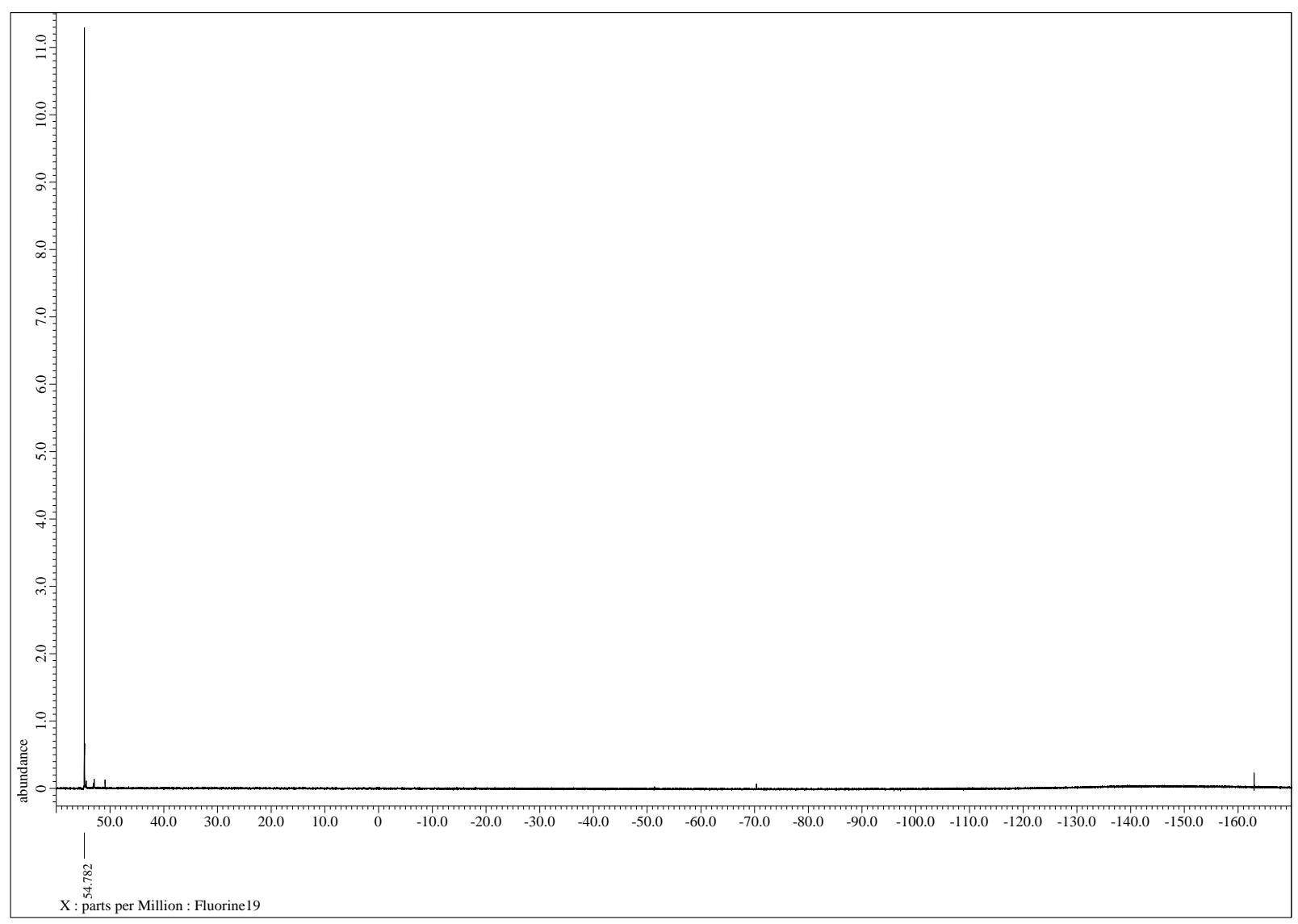


neopentyl (2,5-diphenyloxazol-4-yl)(fluorosulfonyl)carbamate (S9)
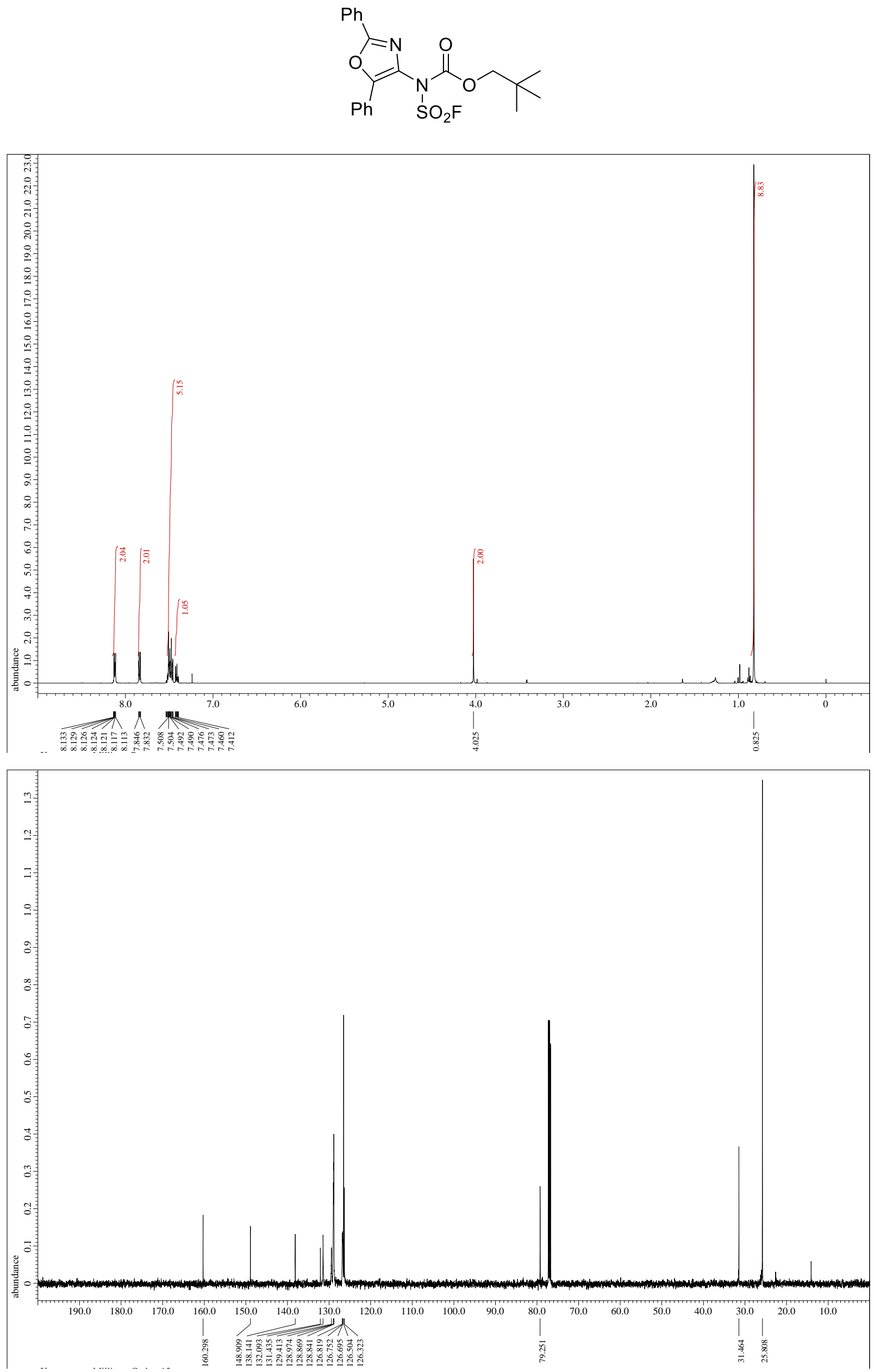


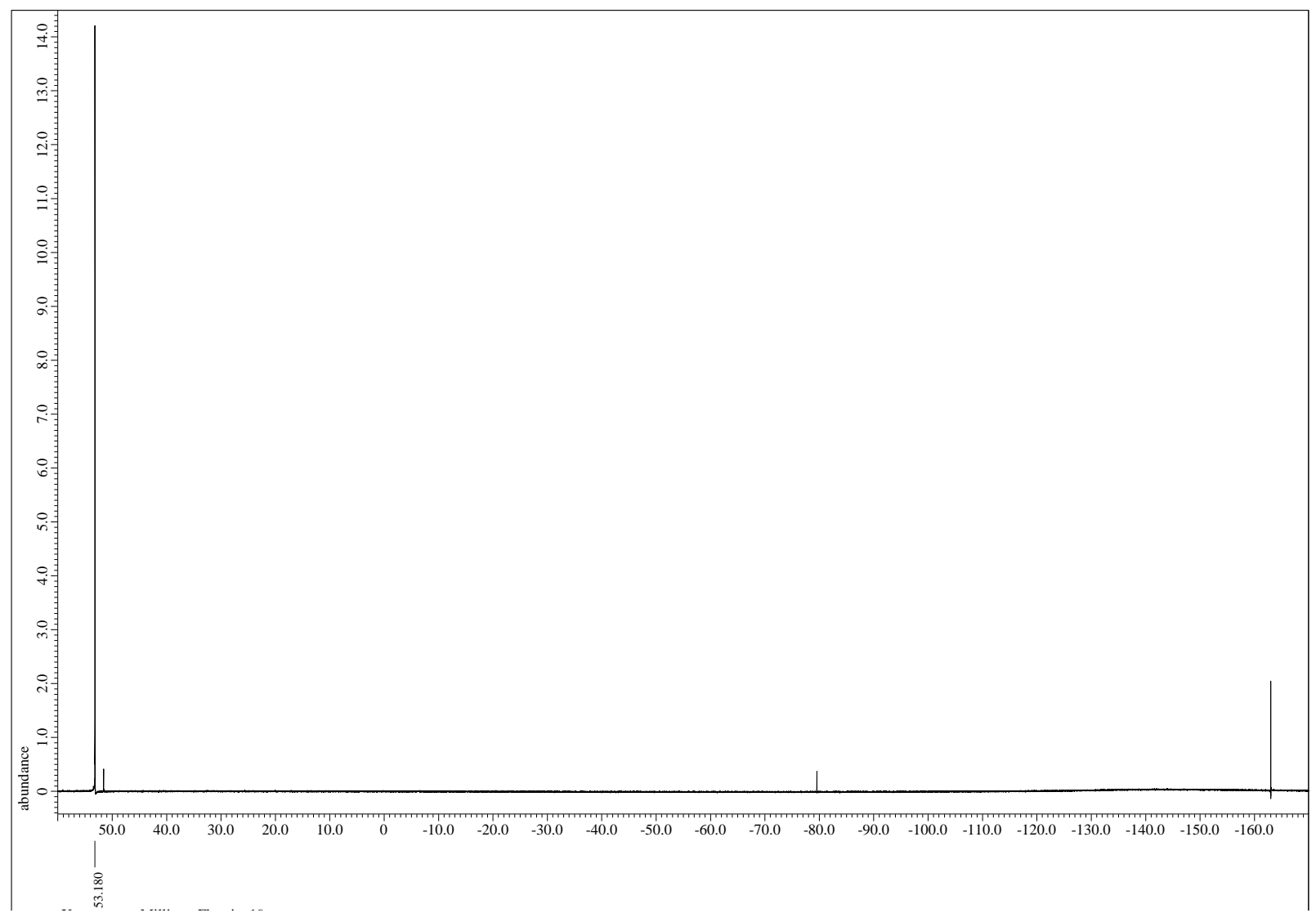


neopentyl (3-bromobenzofuran-2-yl)(fluorosulfonyl)carbamate (S10)
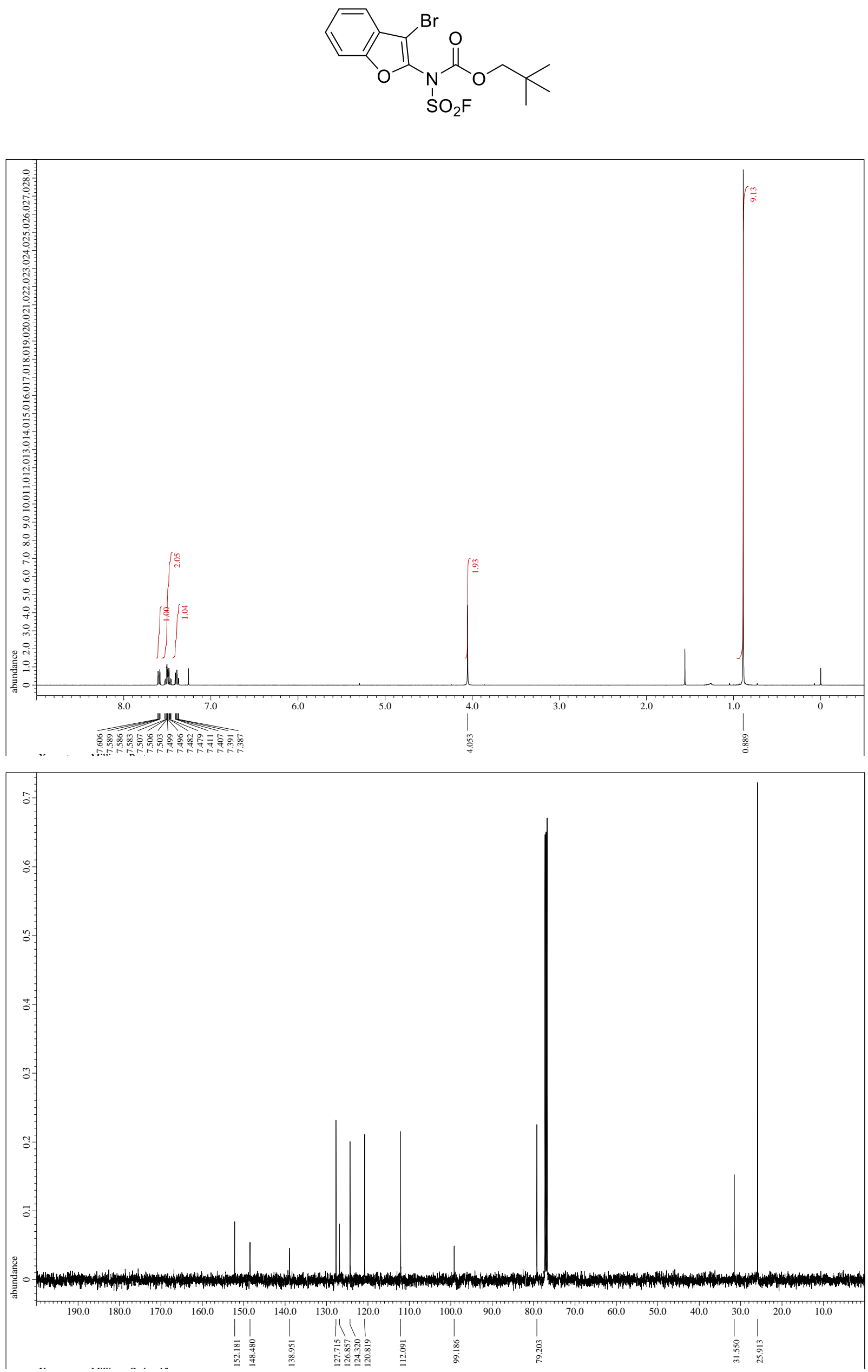


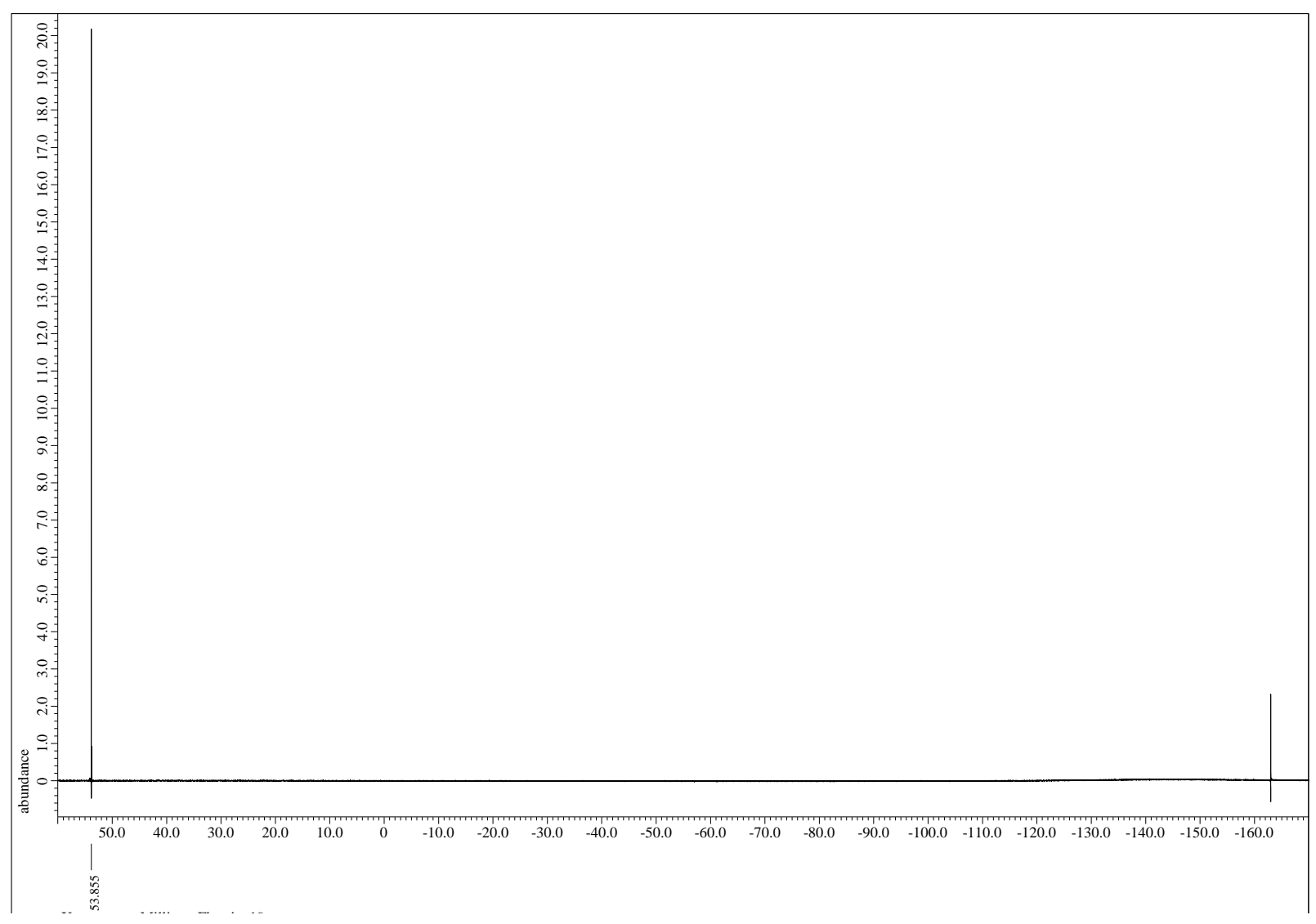



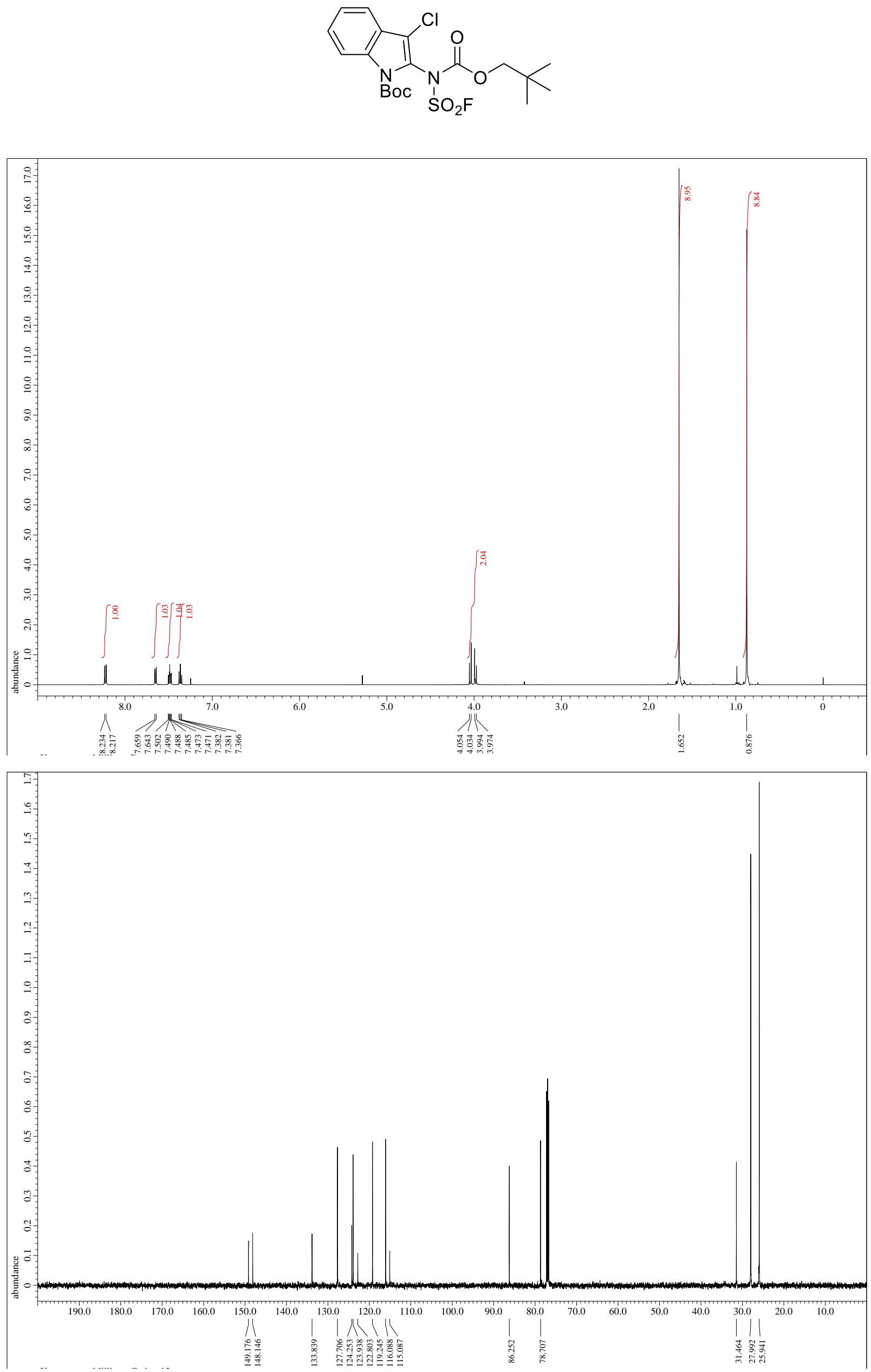


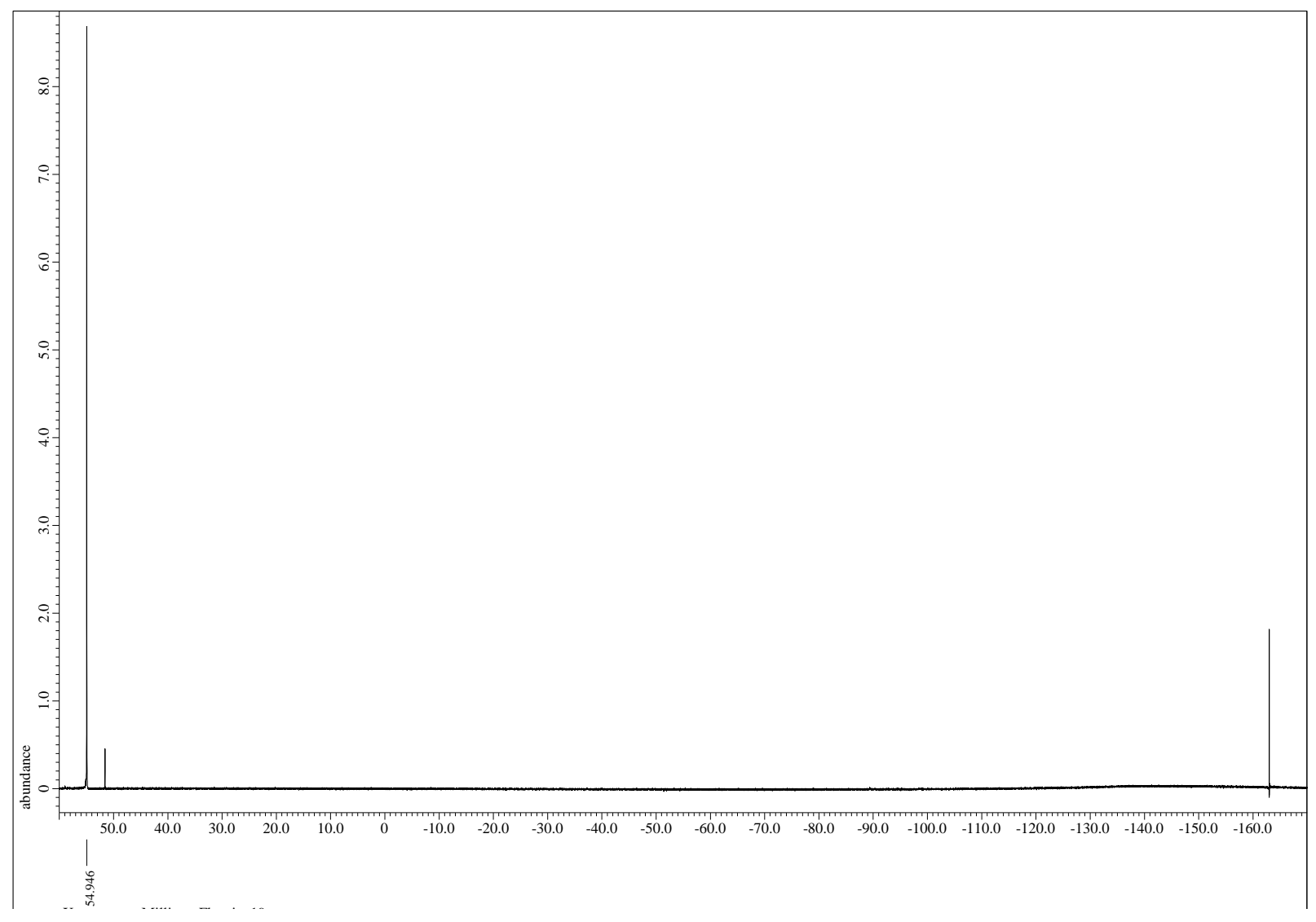


neopentyl (fluorosulfonyl)(perylen-3-yl)carbamate (S12)<smiles></smiles>
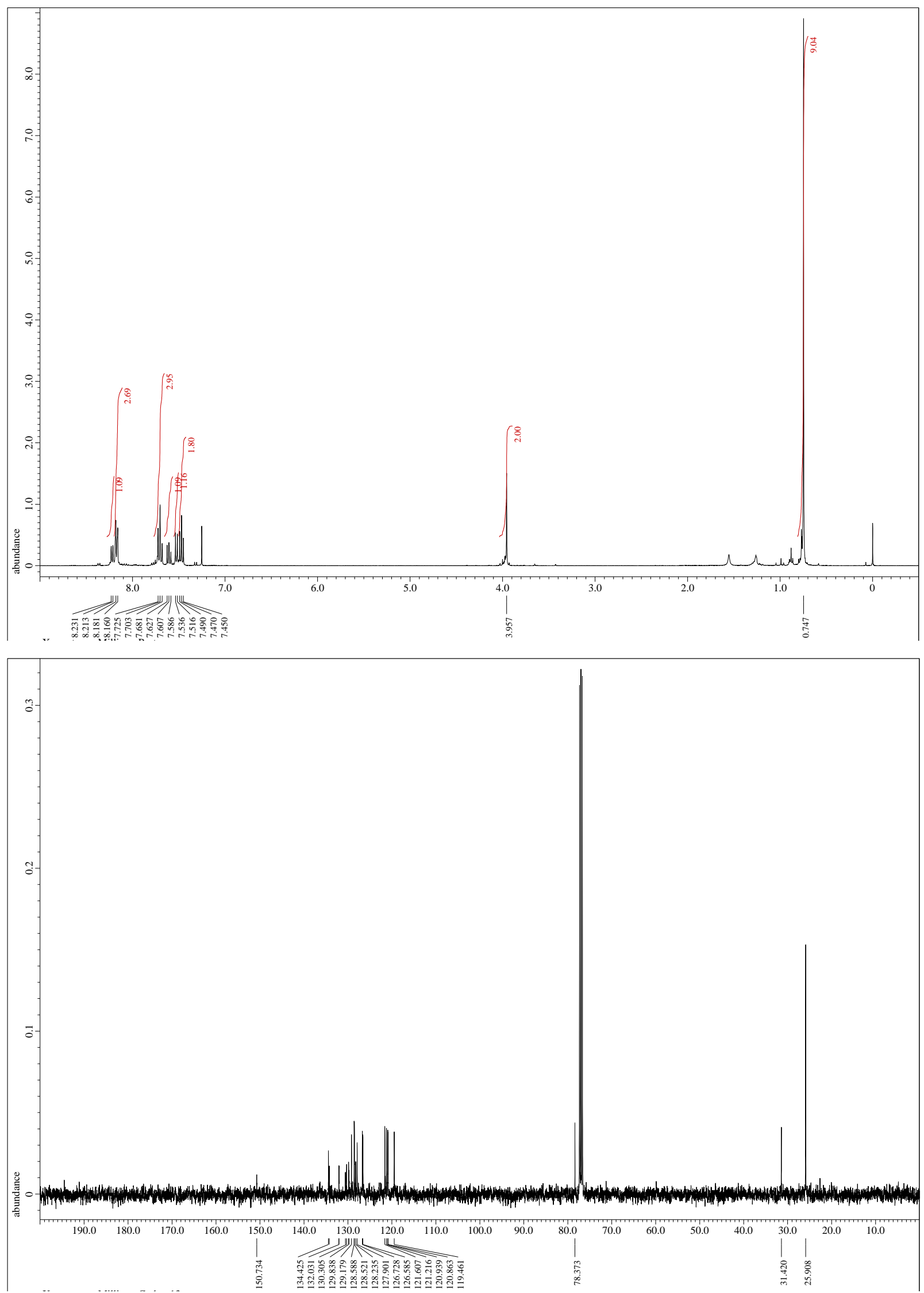


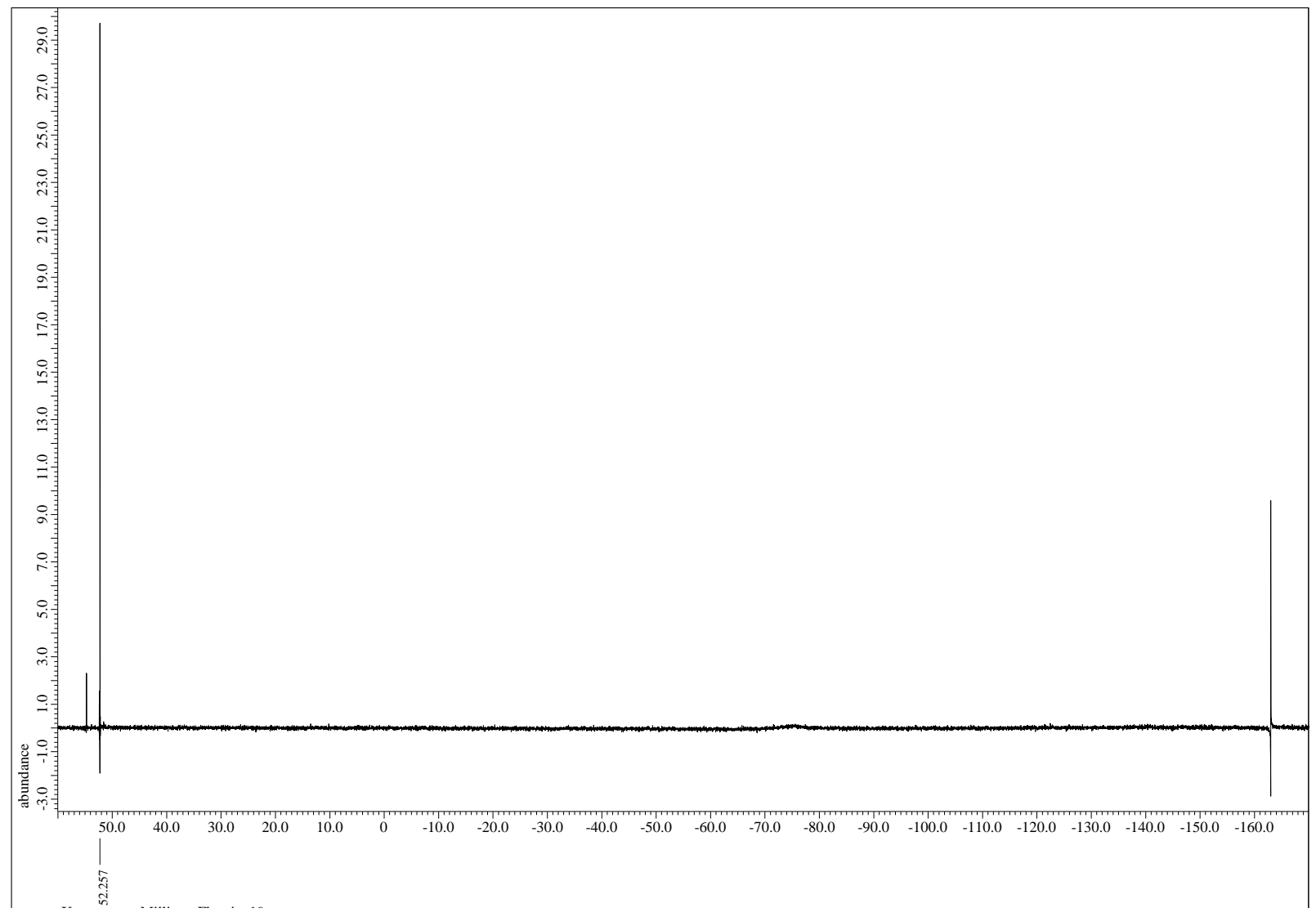


neopentyl (fluorosulfonyl)(phenyl)carbamate (25)<smiles>CC(C)(C)COC(=O)N(c1ccccc1)S(=O)(=O)O[Na]</smiles>
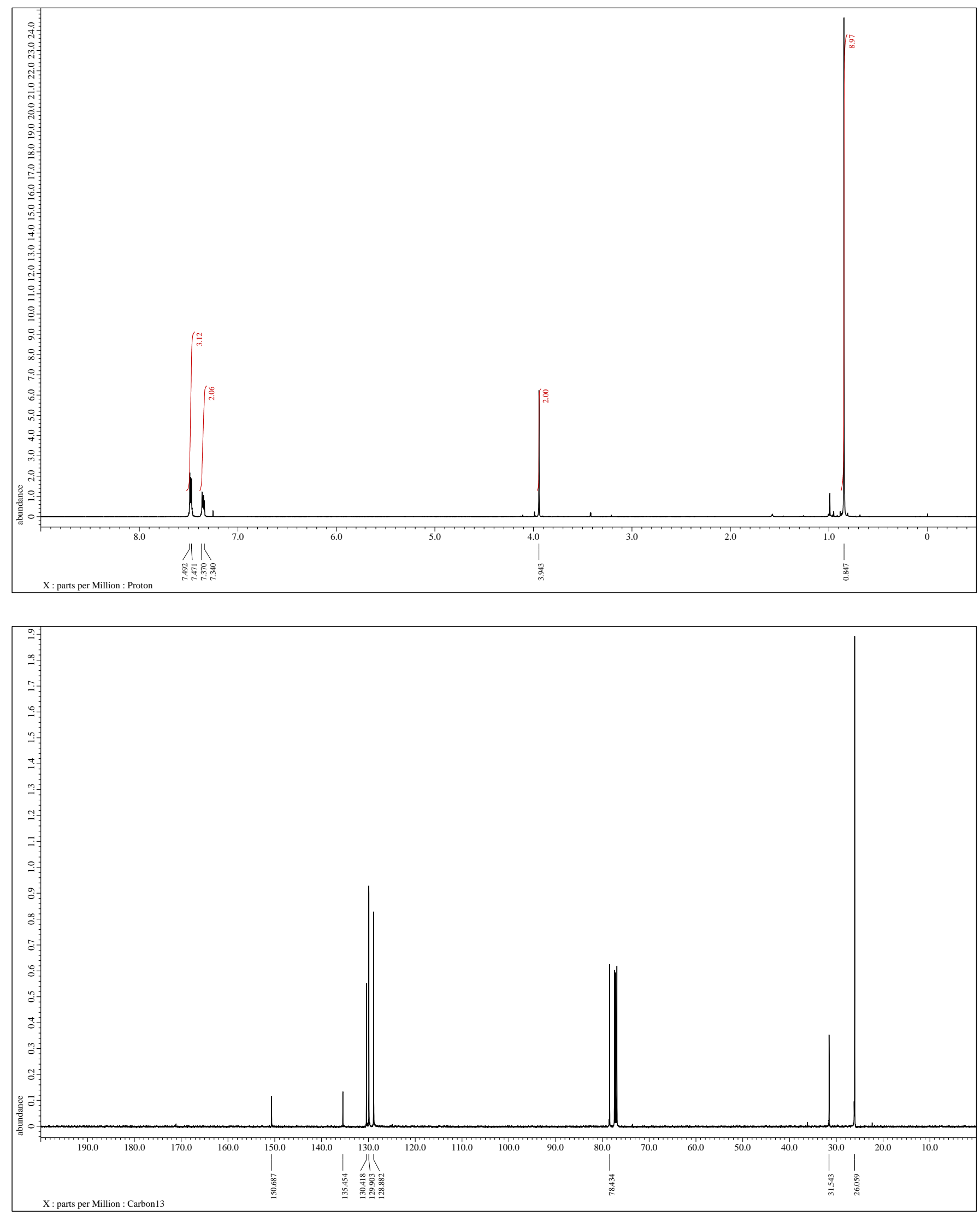

S134 


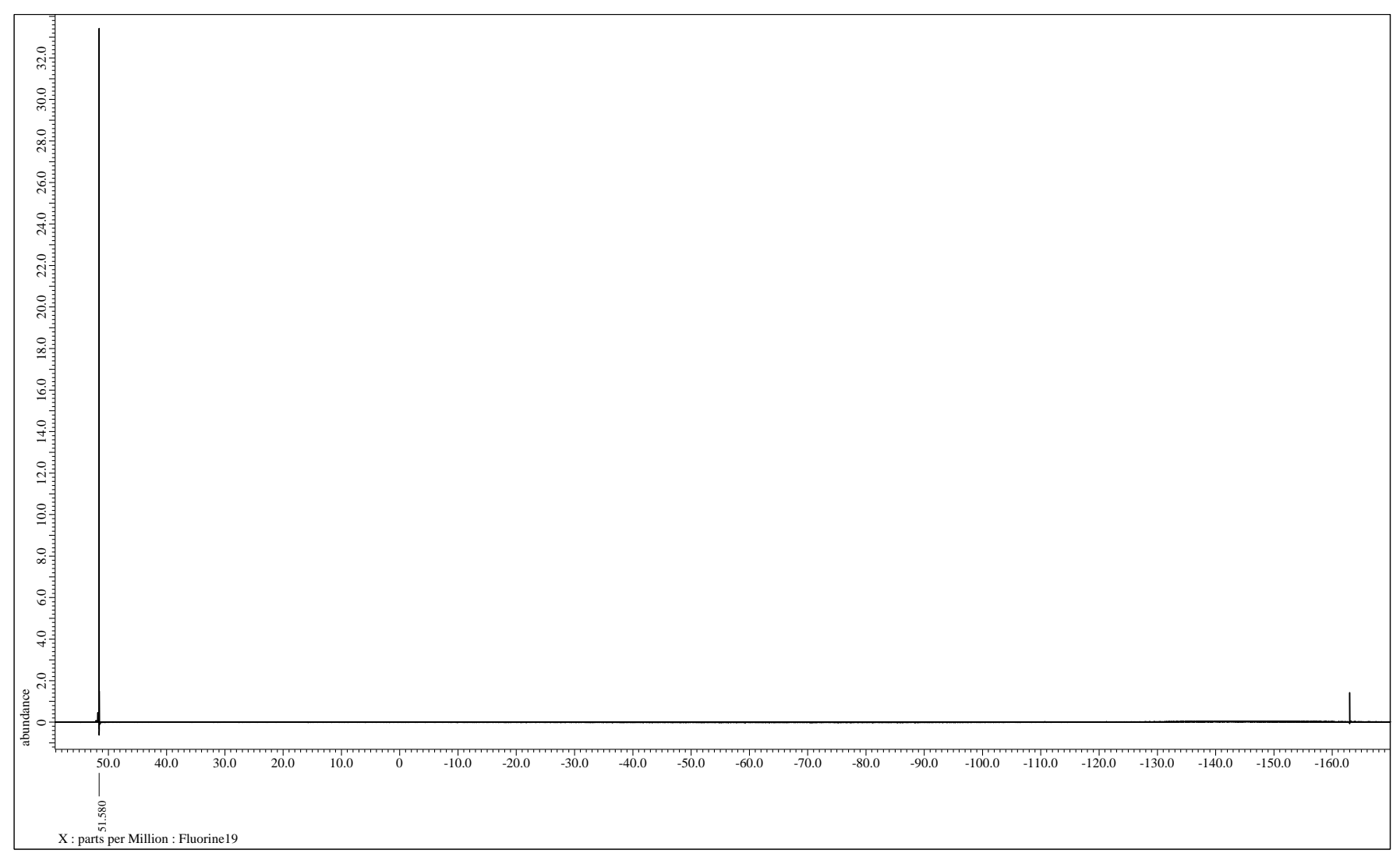




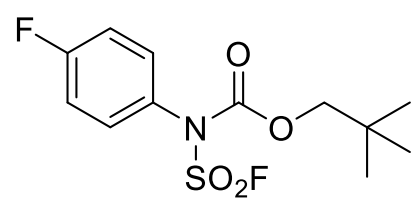

$0: m: p=37: 14: 49$
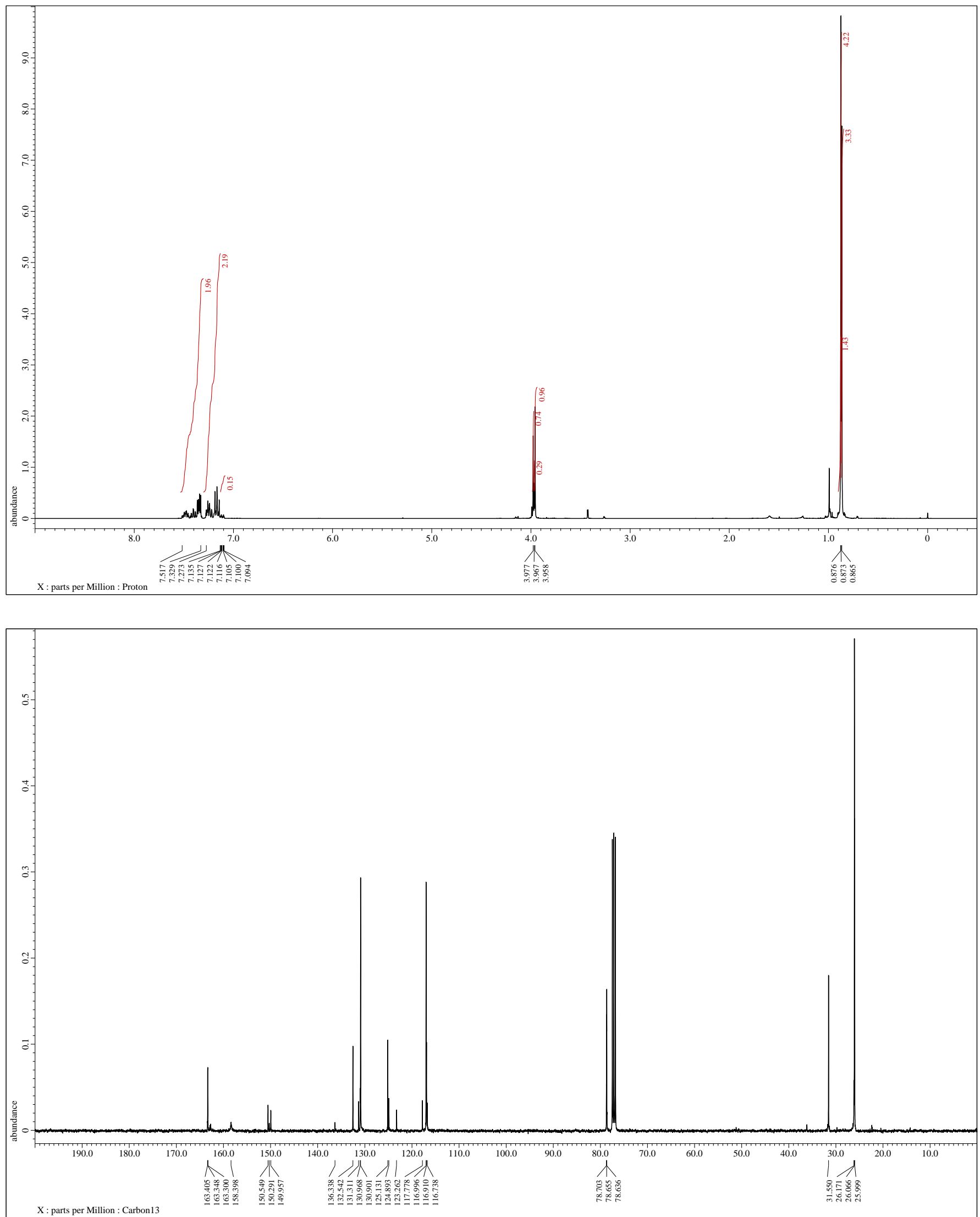

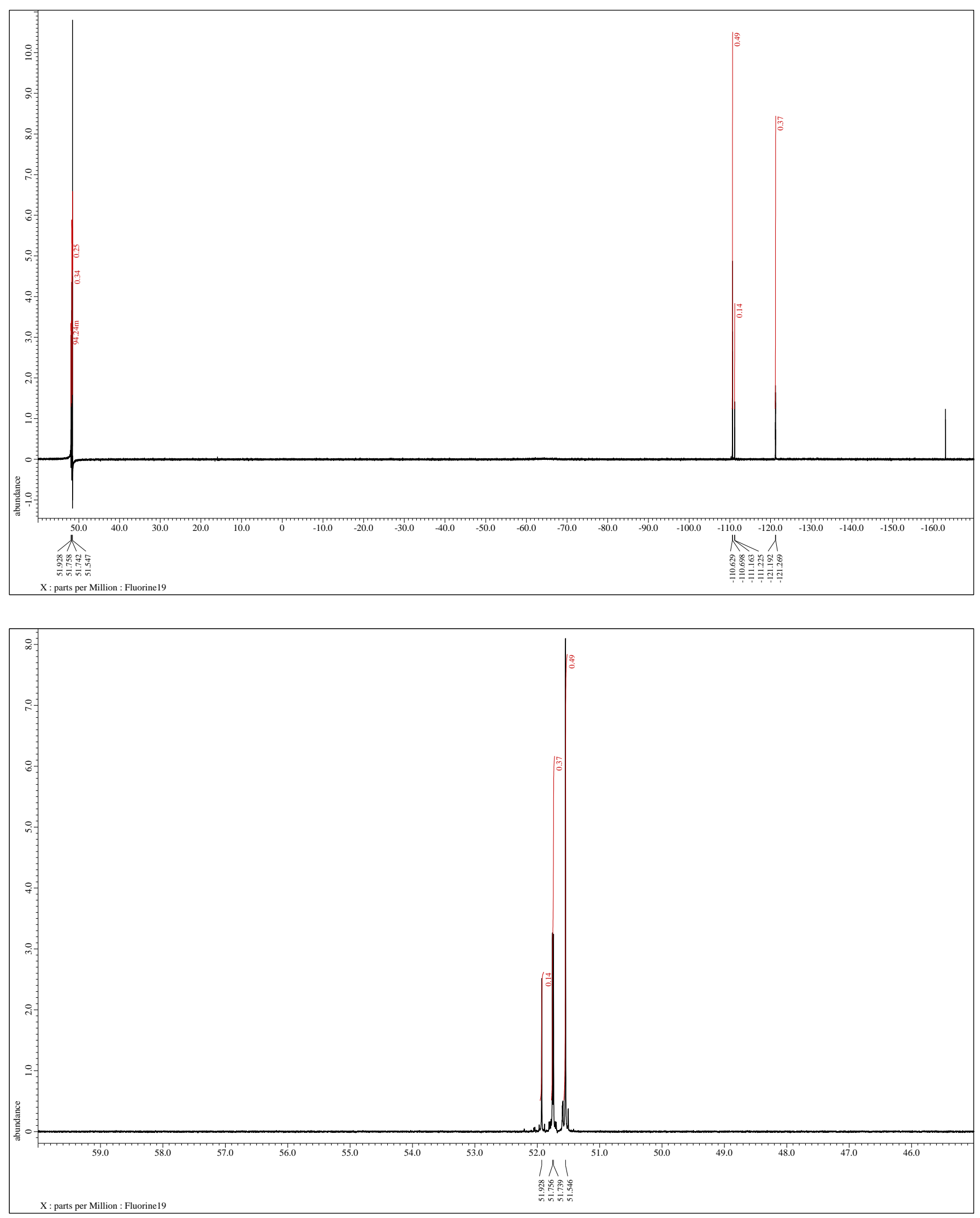
neopentyl (chlorophenyl)(fluorosulfonyl)carbamate (28)

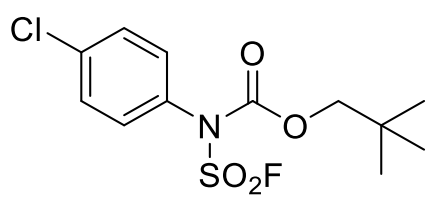

$o: m: p=42: 3: 55$
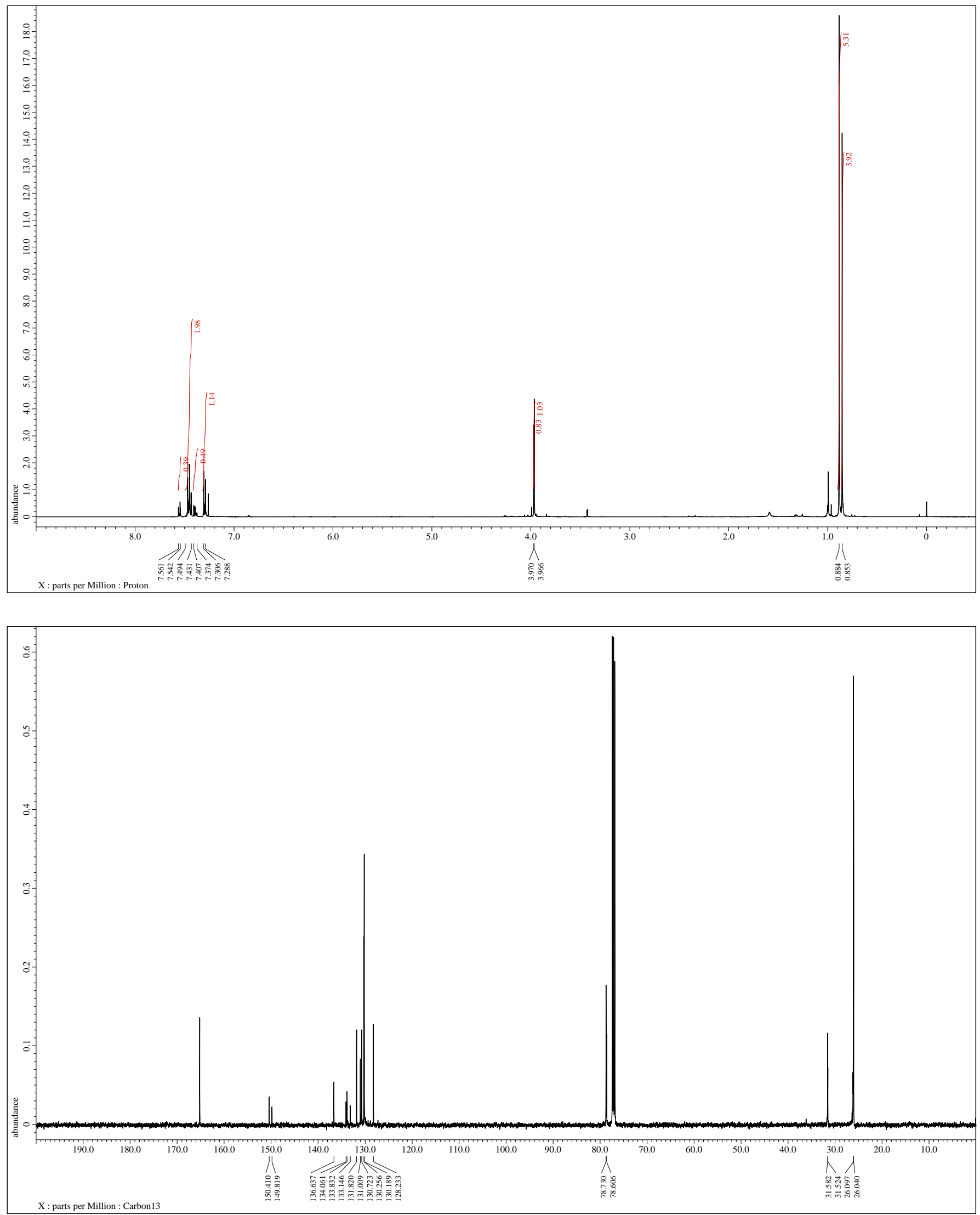

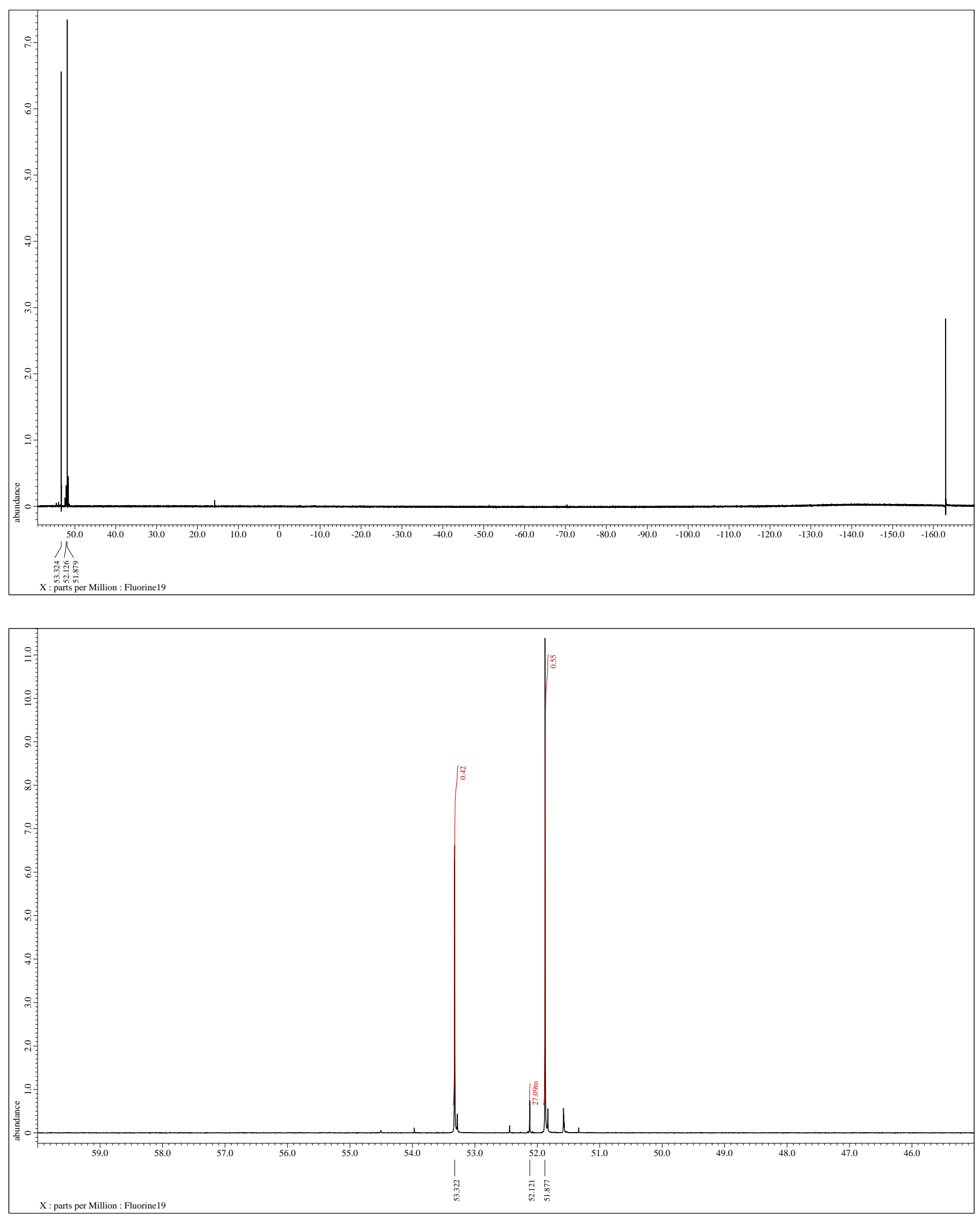
neopentyl (bromophenyl)(fluorosulfonyl)carbamate (29)<smiles>CC(C)(C)COC(=O)N(c1ccc(Br)cc1)S(=O)(=O)OF</smiles>

$$
o: m: p=44: 6: 50
$$
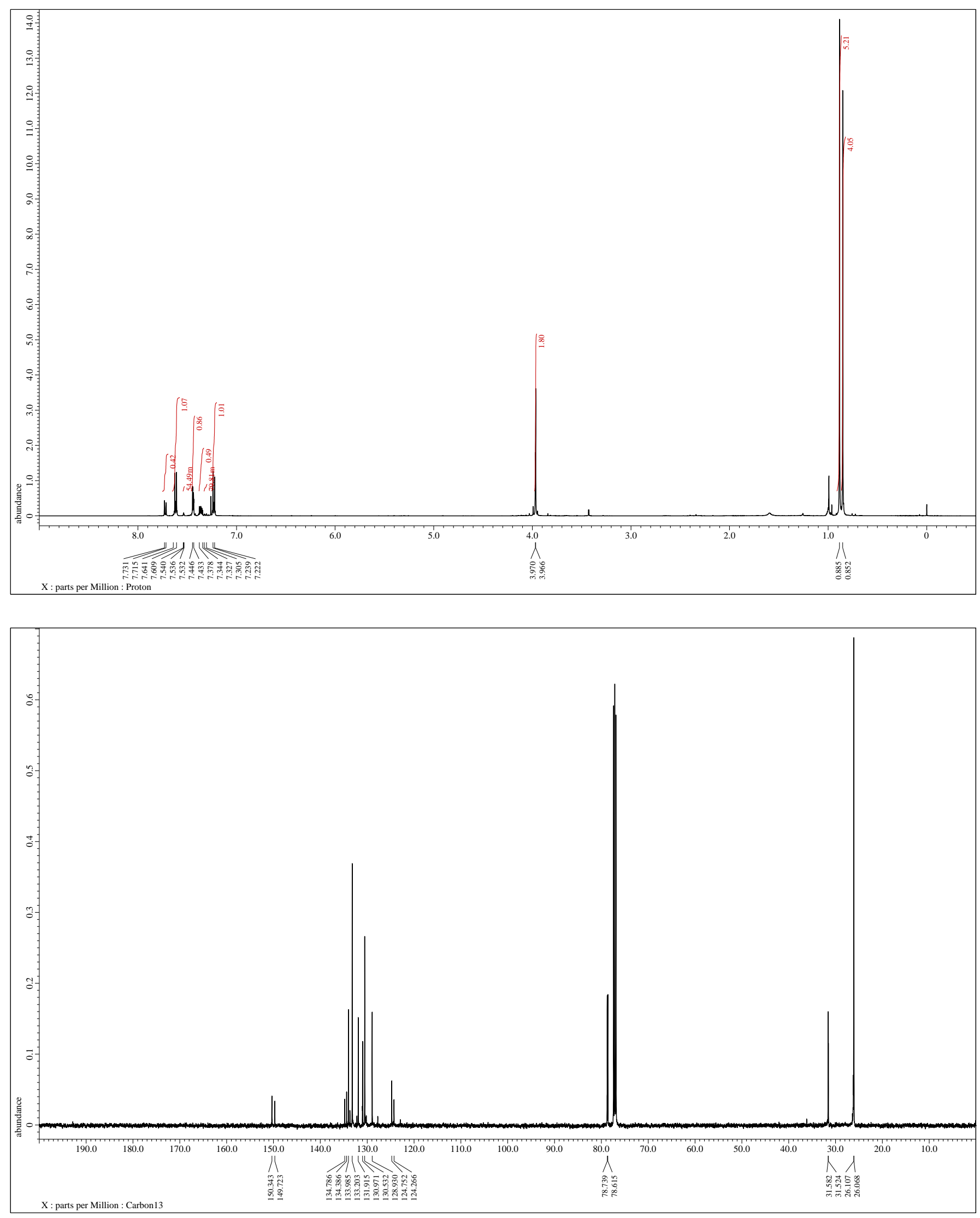

$\mathrm{S} 140$ 

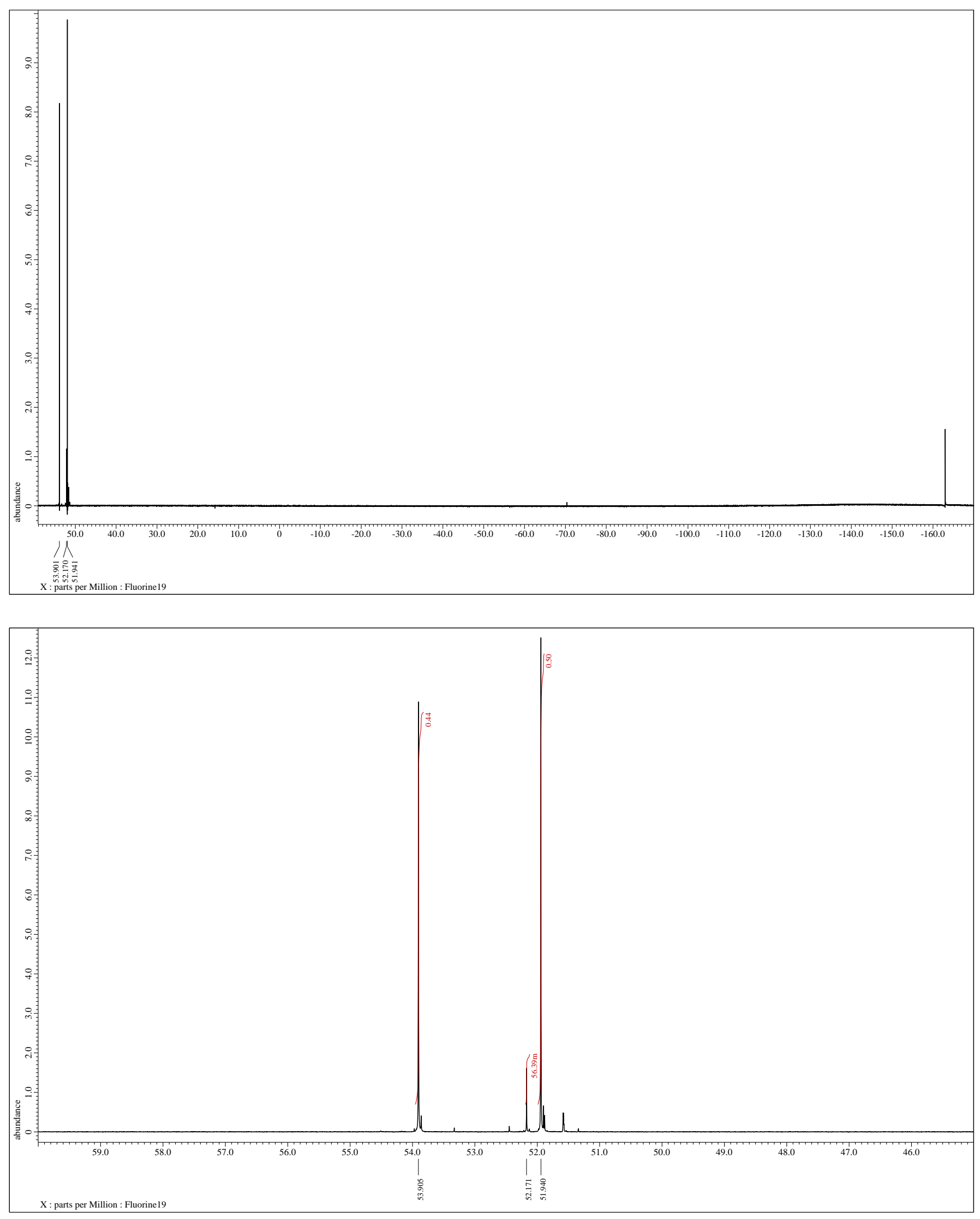


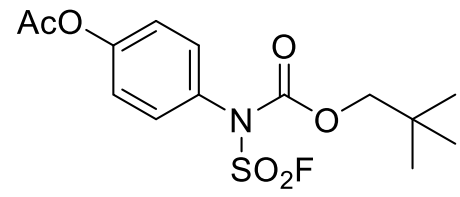

$o: m: p=38: 1: 61$
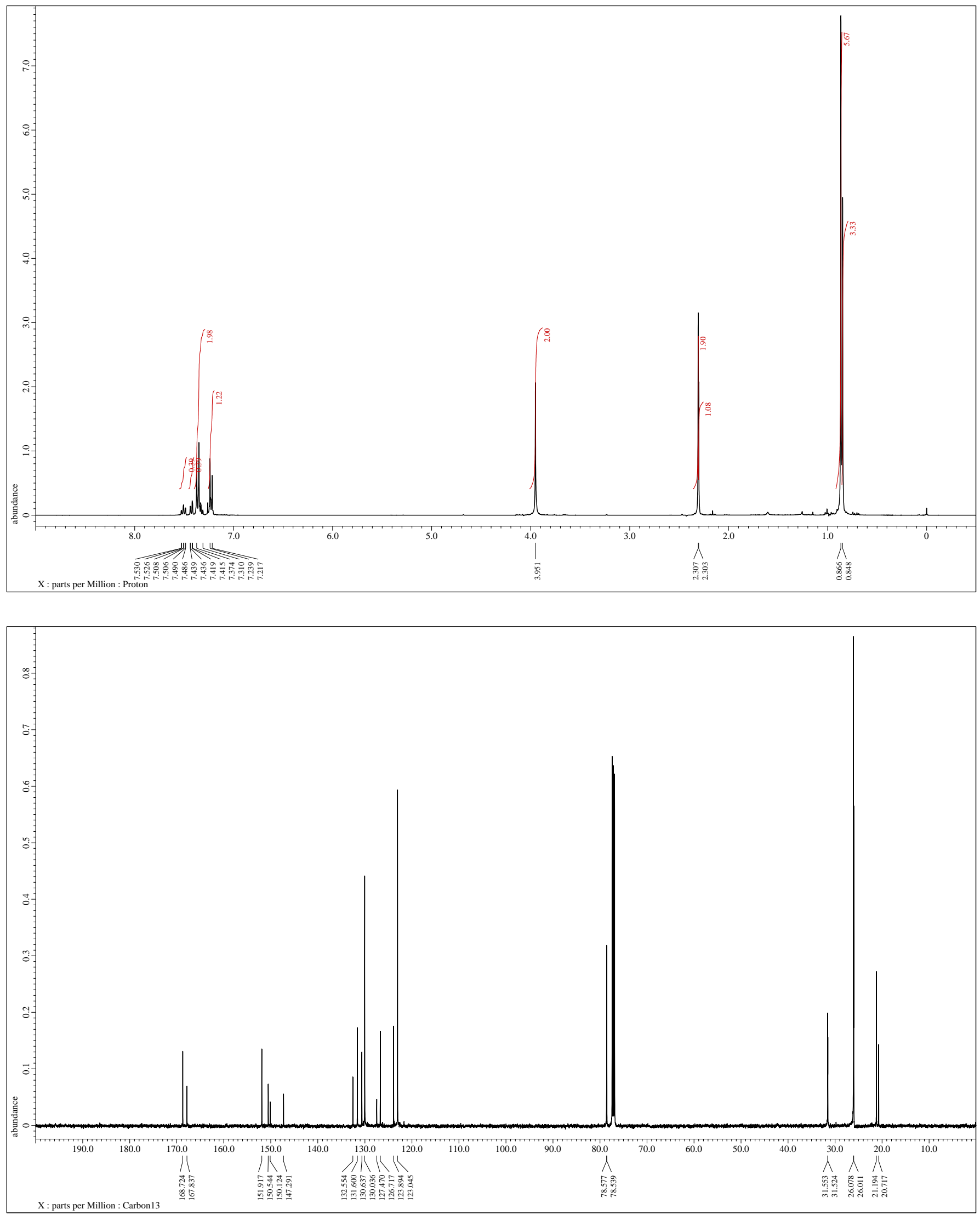

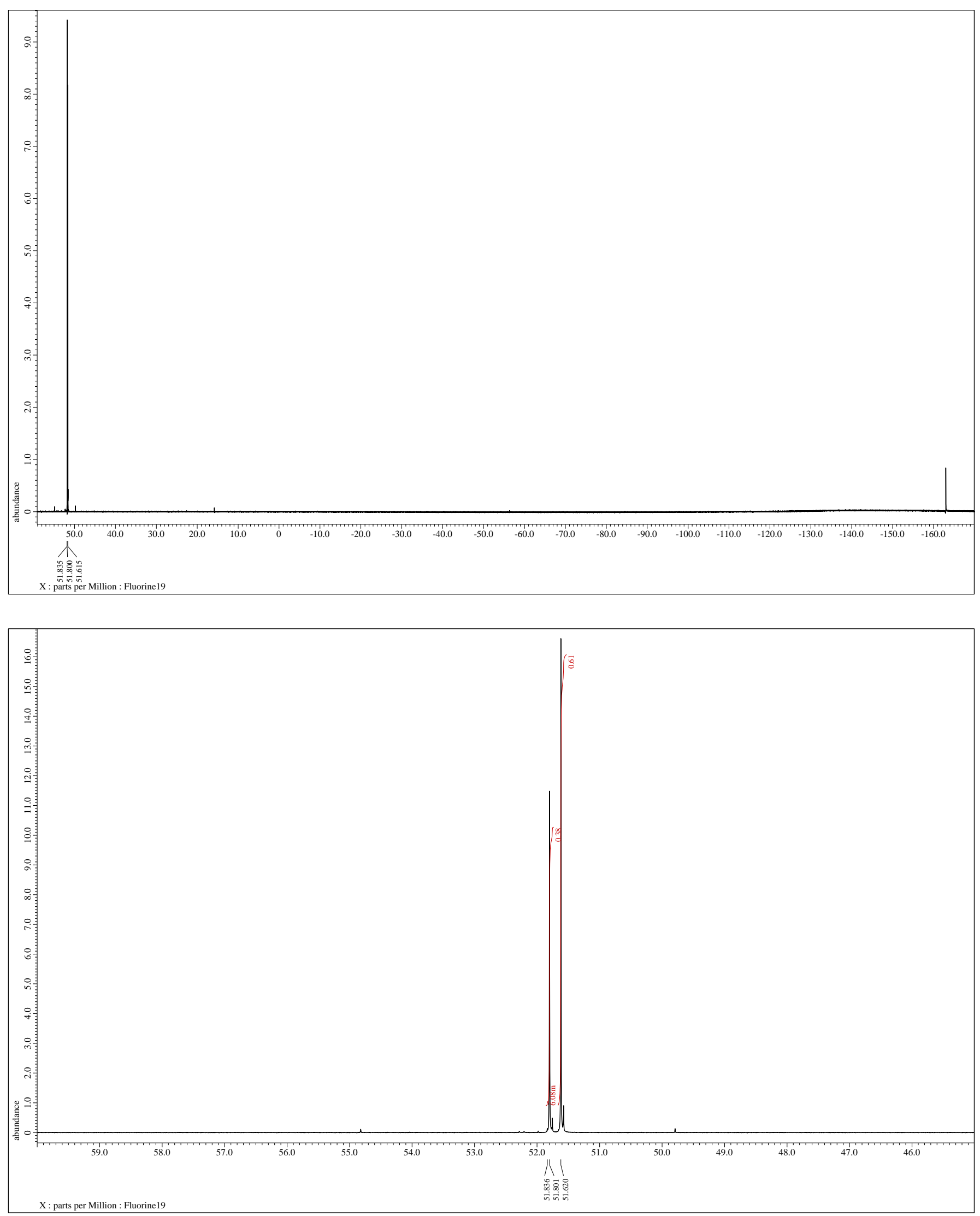


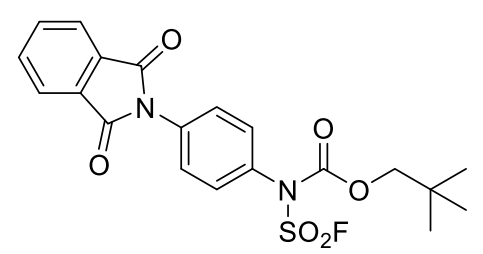

$o: m: p=5: 16: 79$
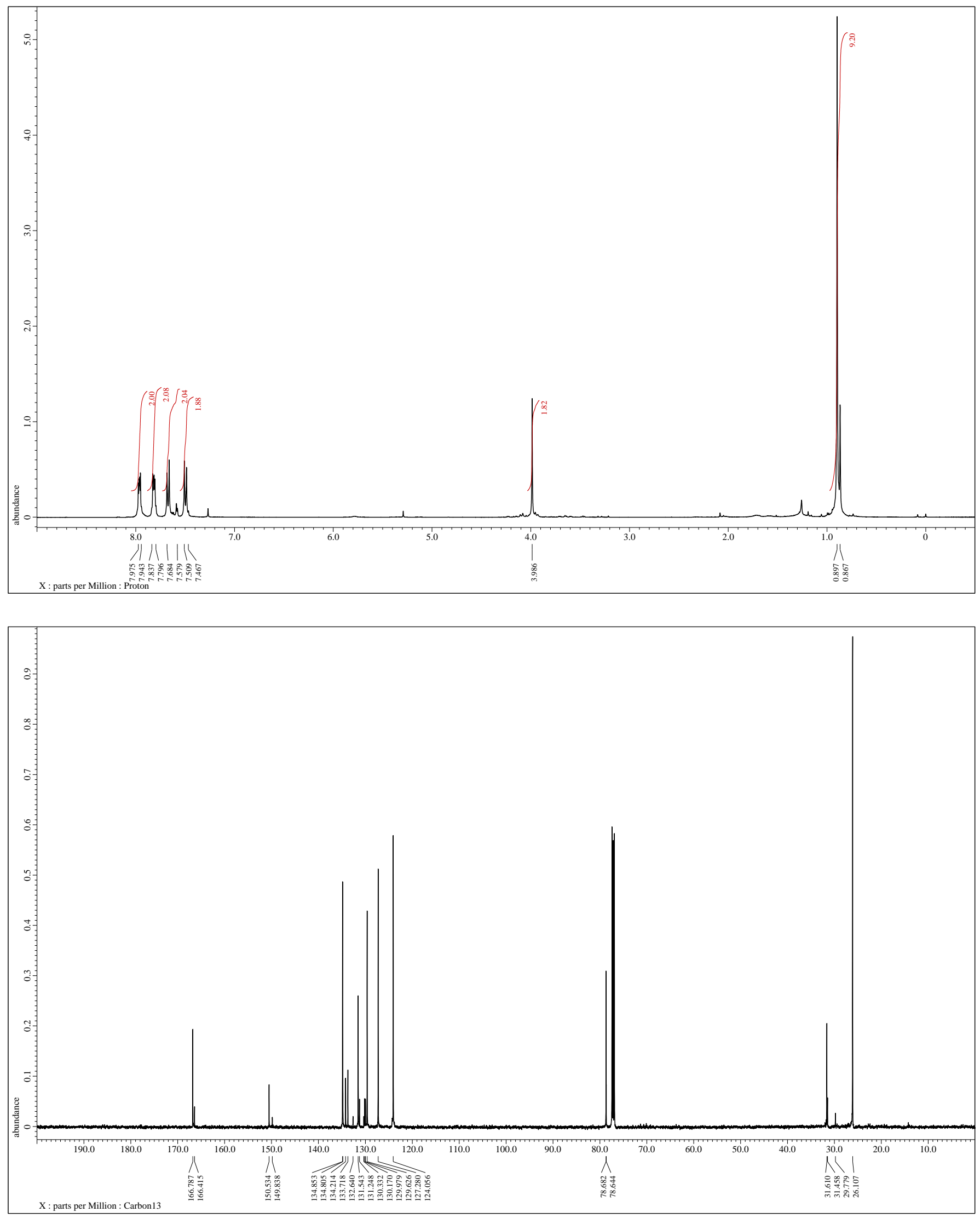

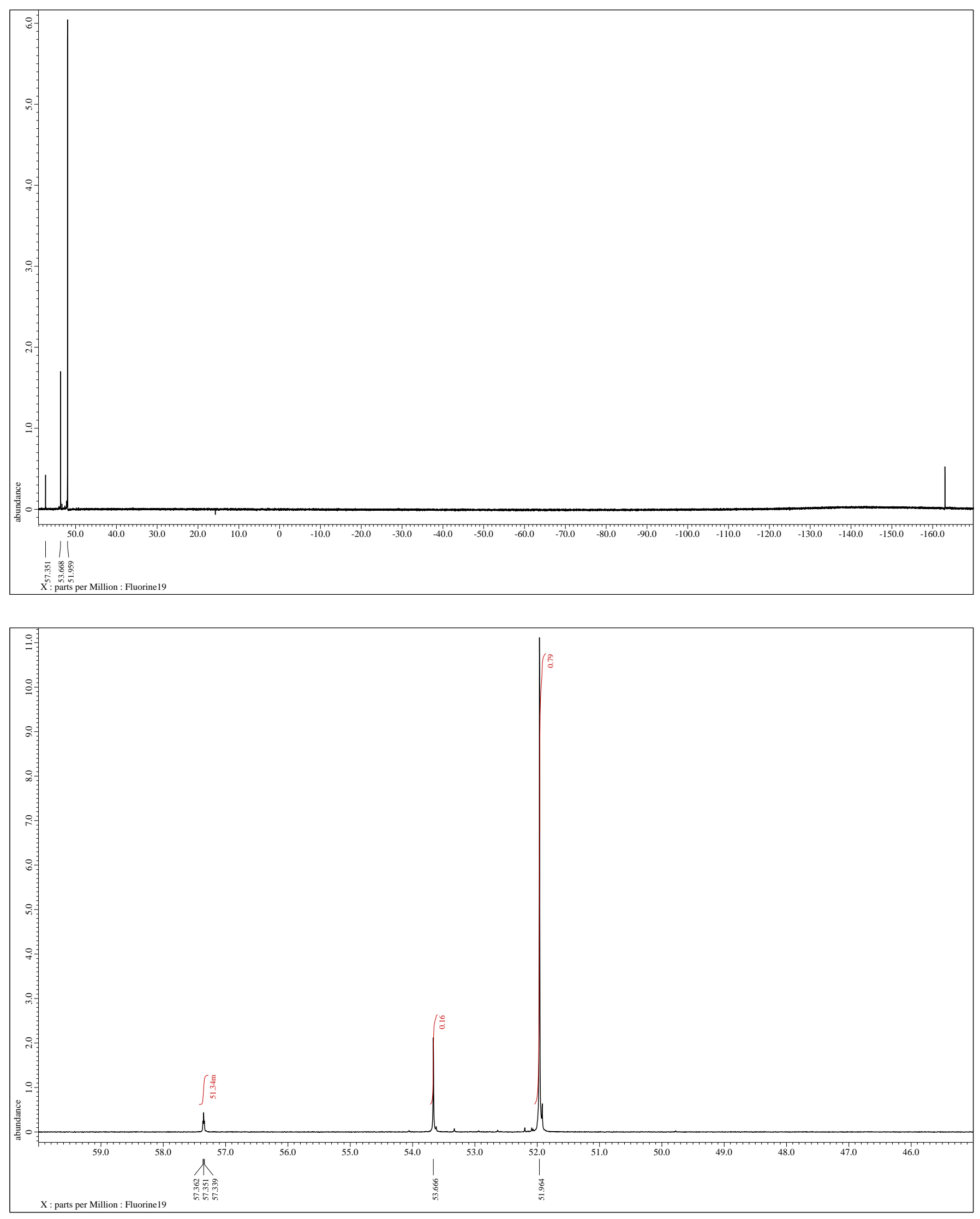


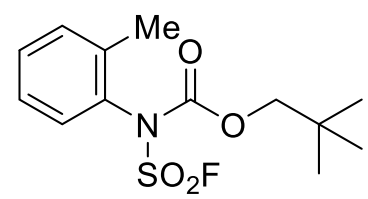

$o: m: p=63: 4: 33$
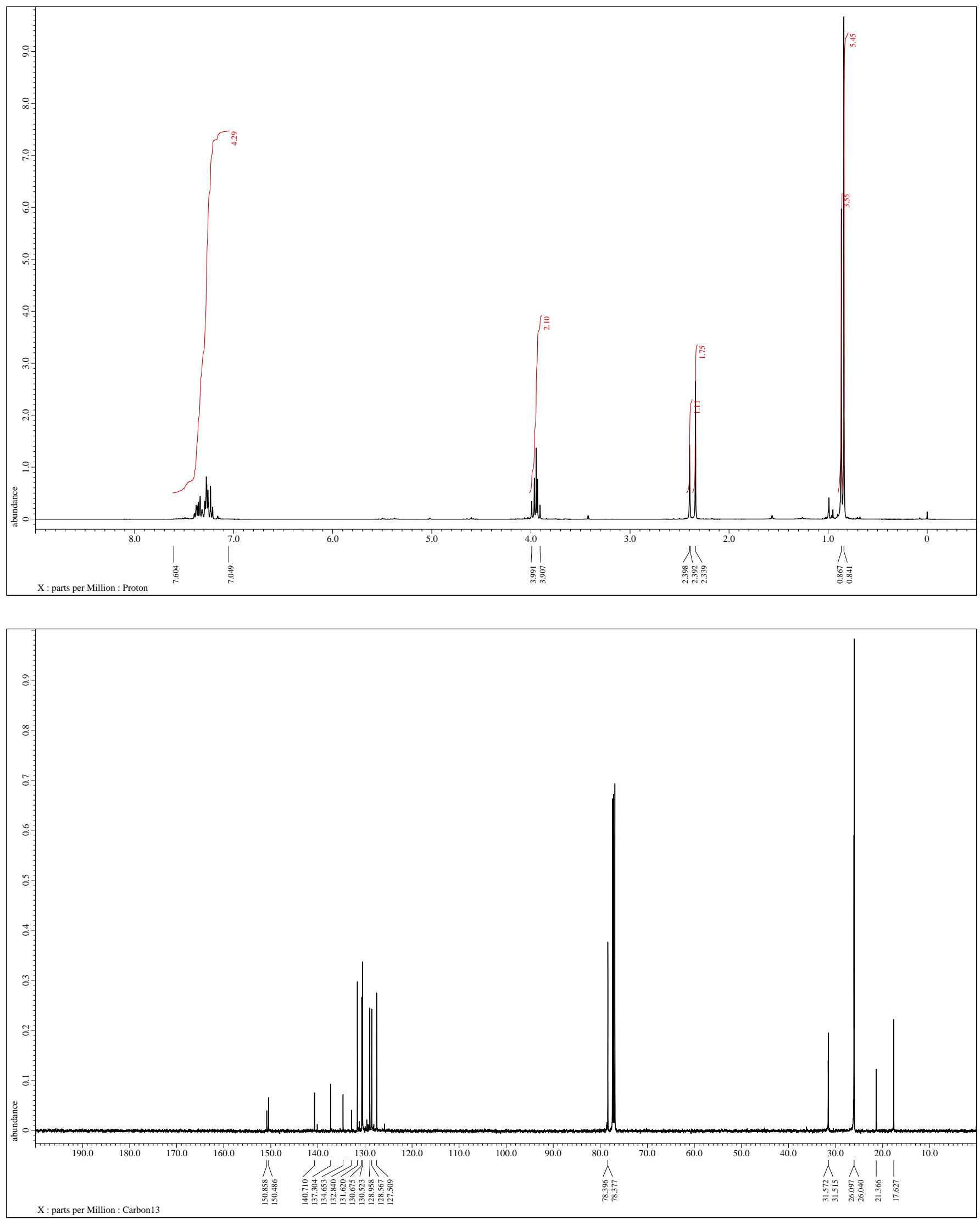

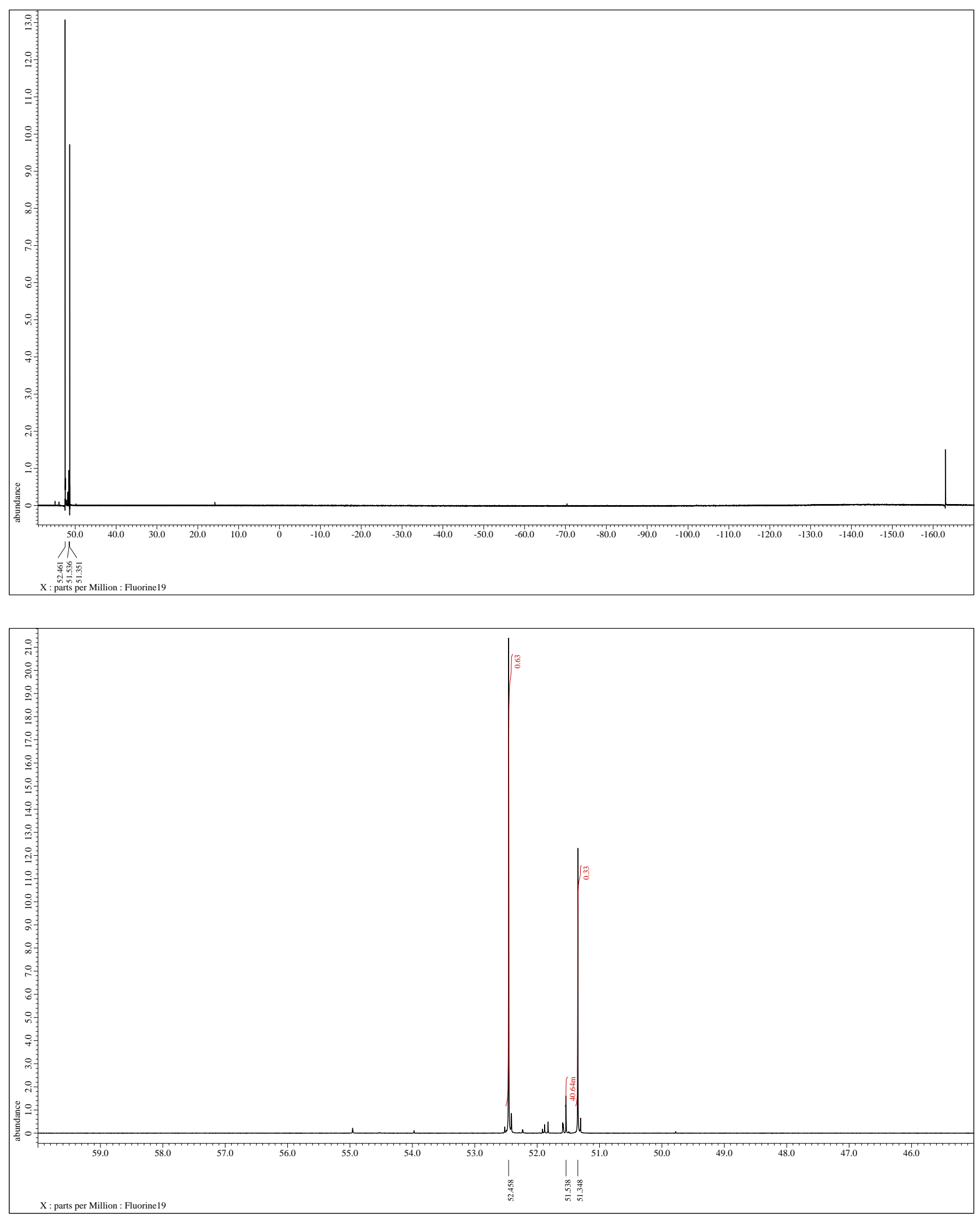
neopentyl (2,4-dibromophenyl)(fluorosulfonyl)carbamate (33) and

neopentyl (2,6-dibromophenyl)(fluorosulfonyl)carbamate (33')
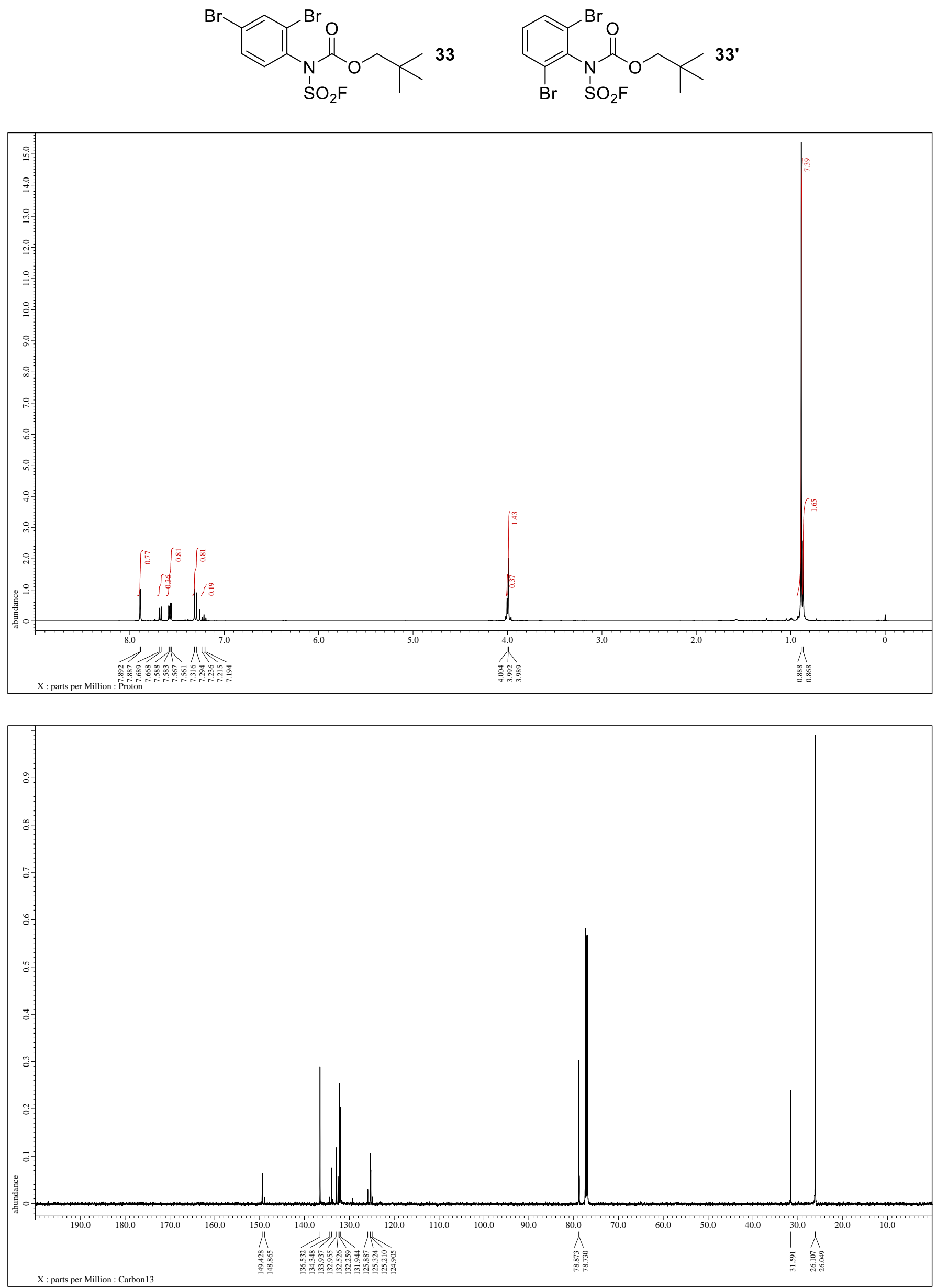

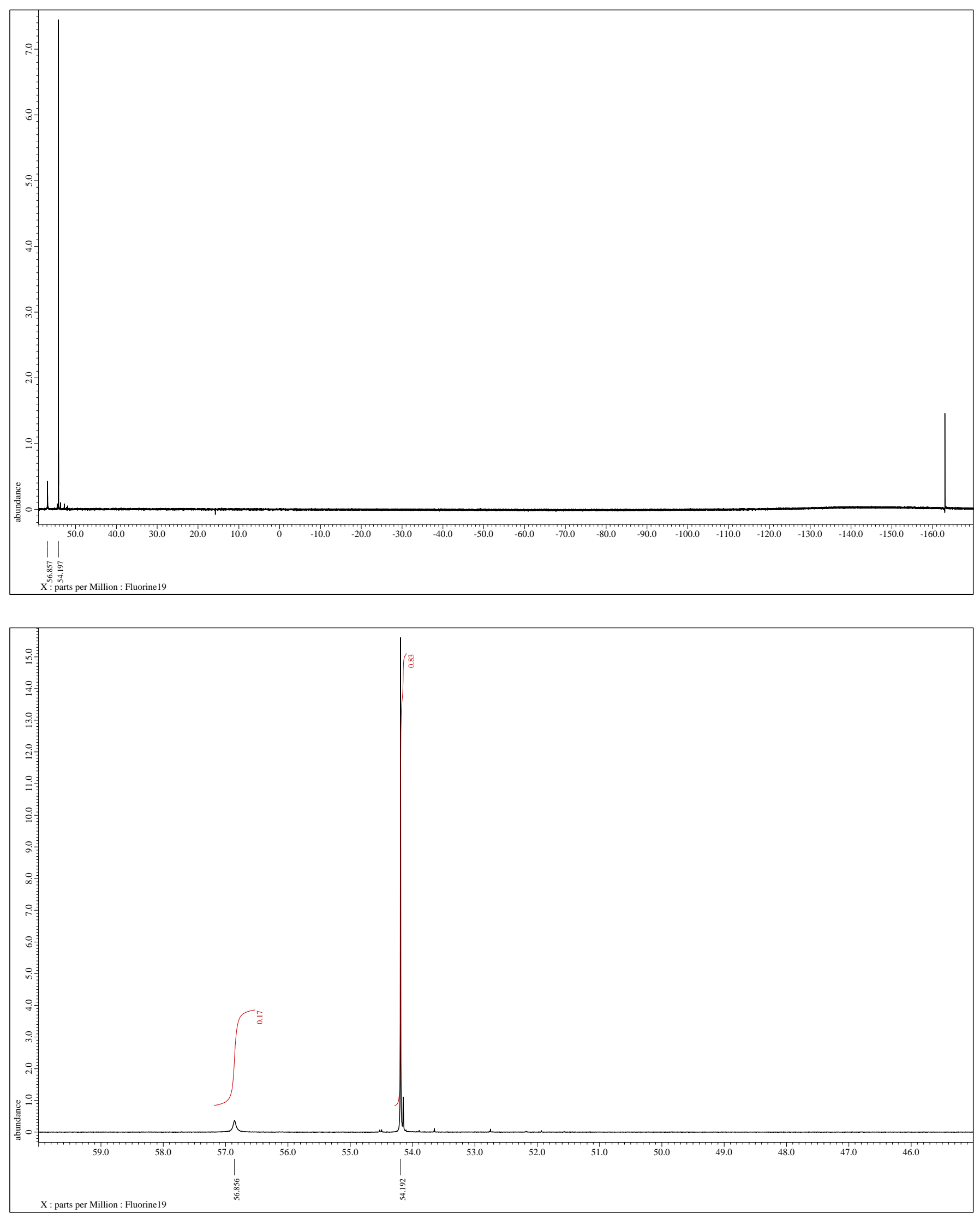

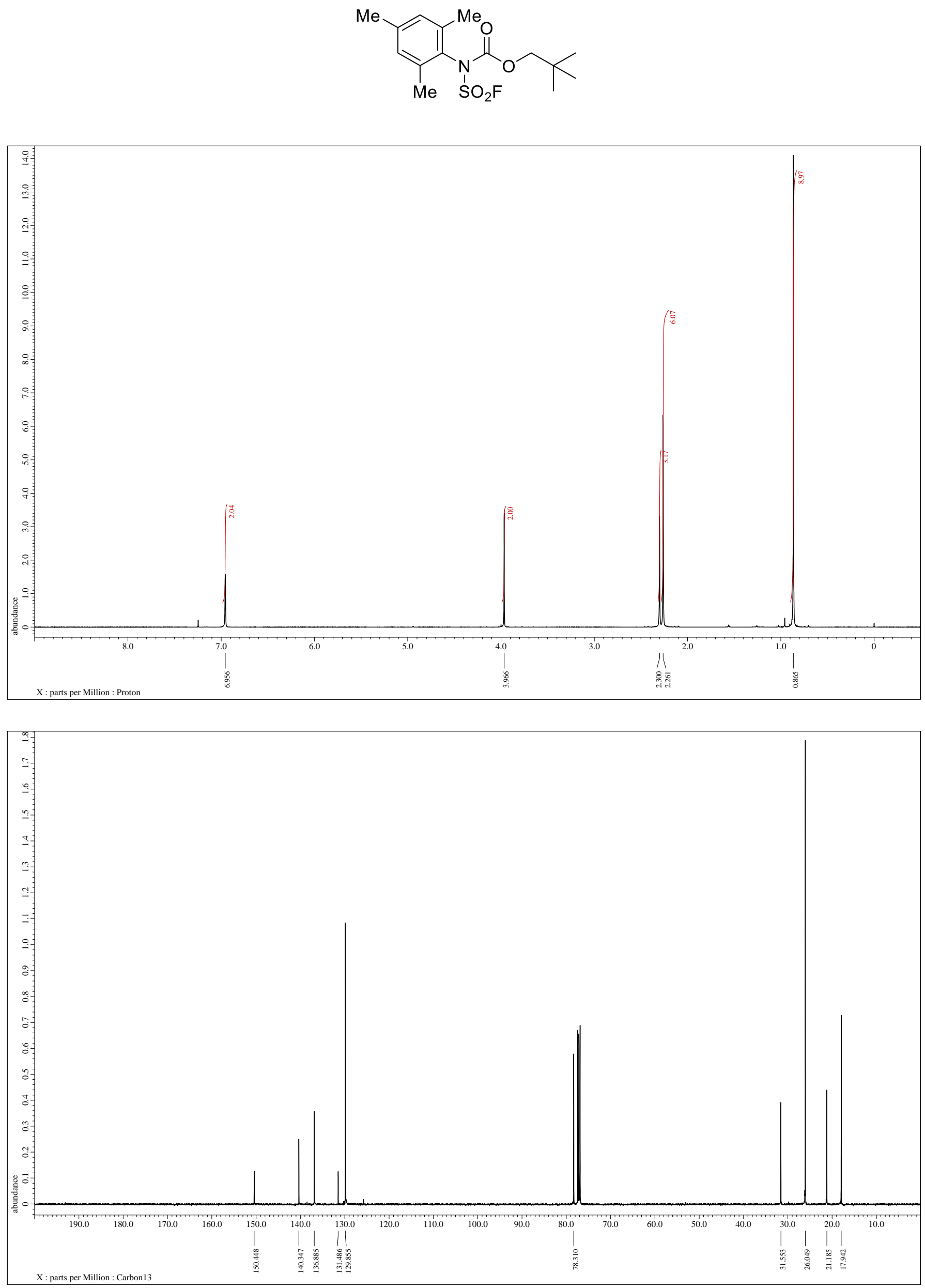


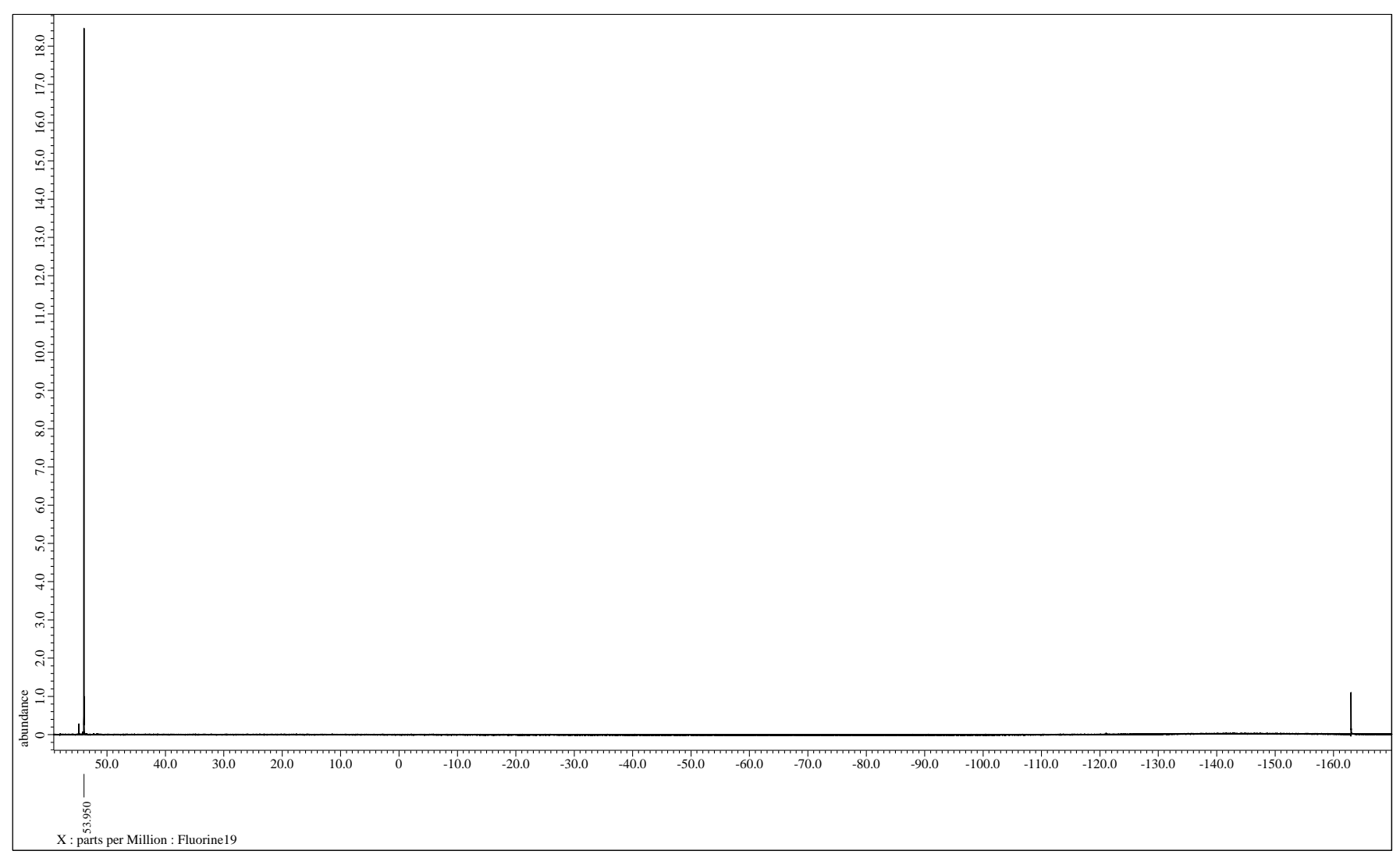



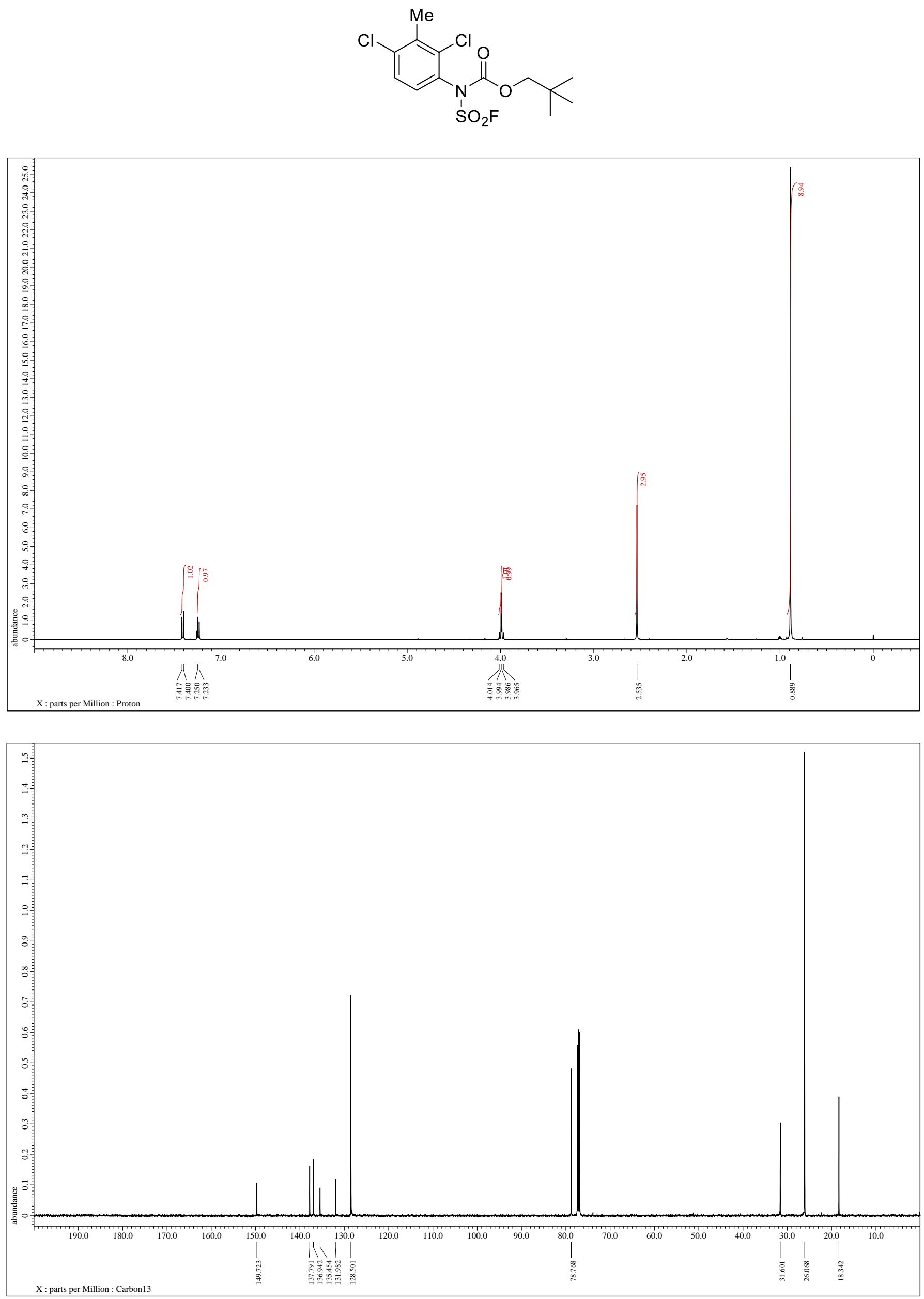


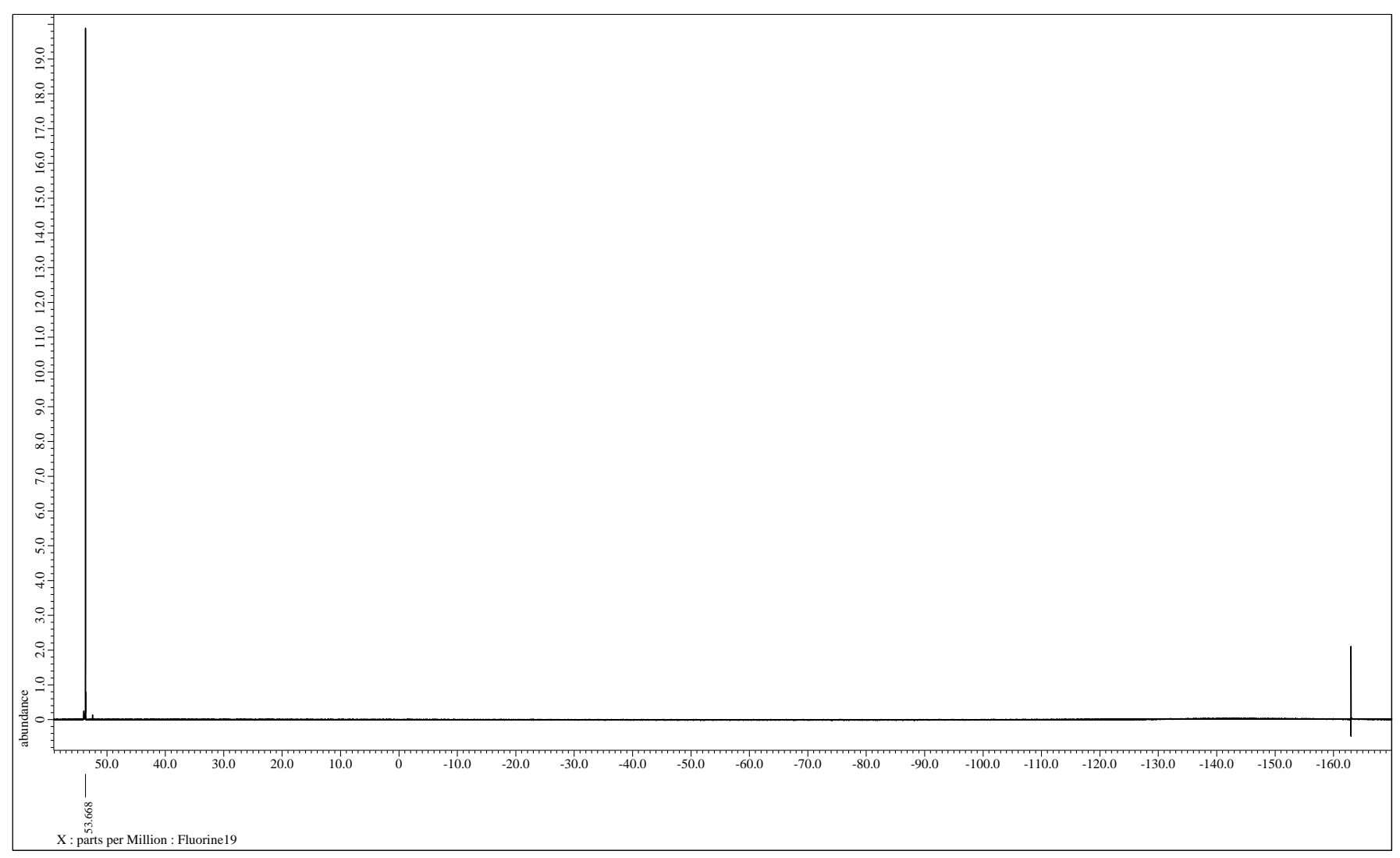


neopentyl (3-bromo-2,4-dimethylphenyl)(fluorosulfonyl)carbamate (36) and neopentyl (4-bromo-3,5-dimethylphenyl)(fluorosulfonyl)carbamate (36')
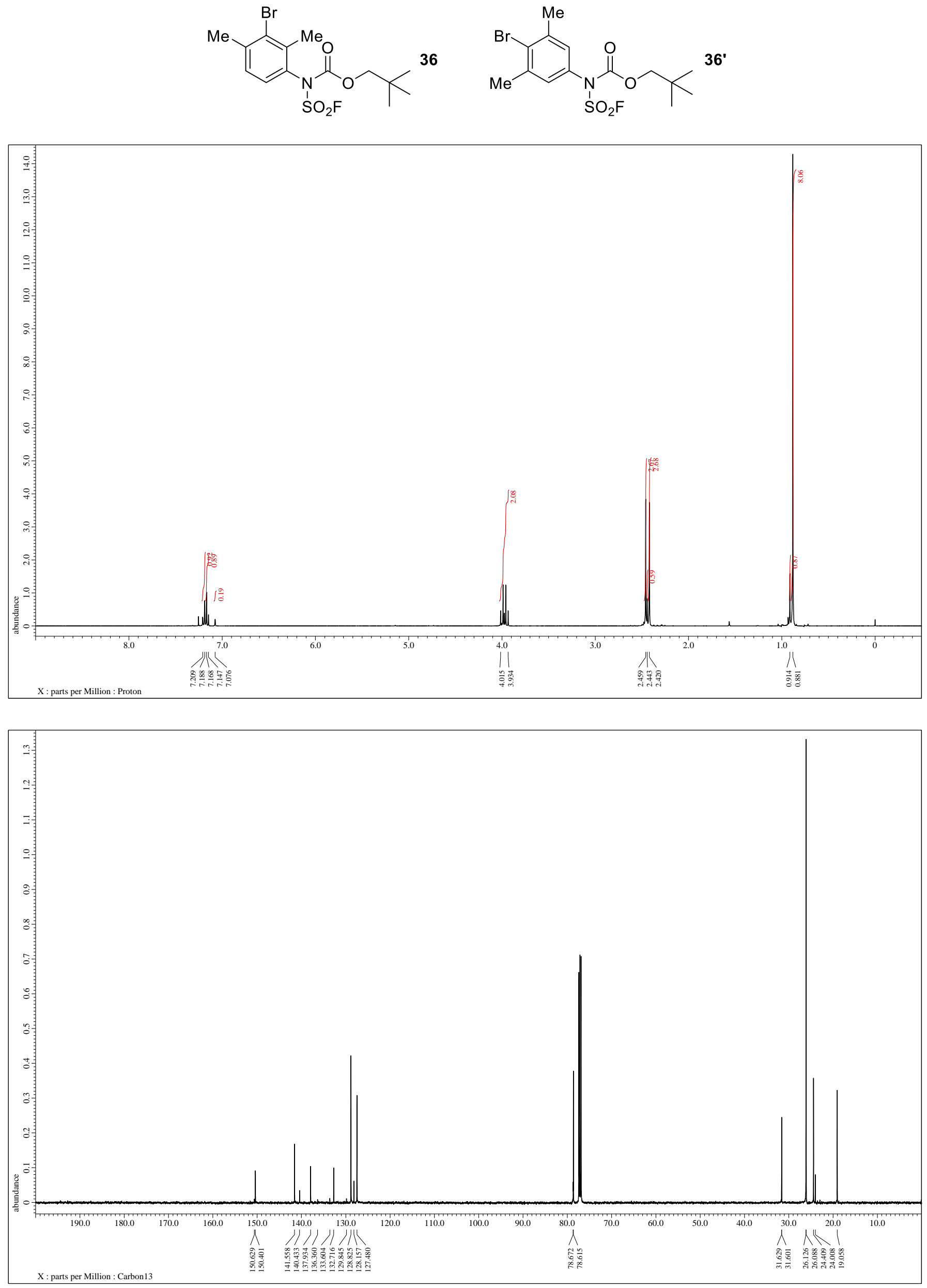

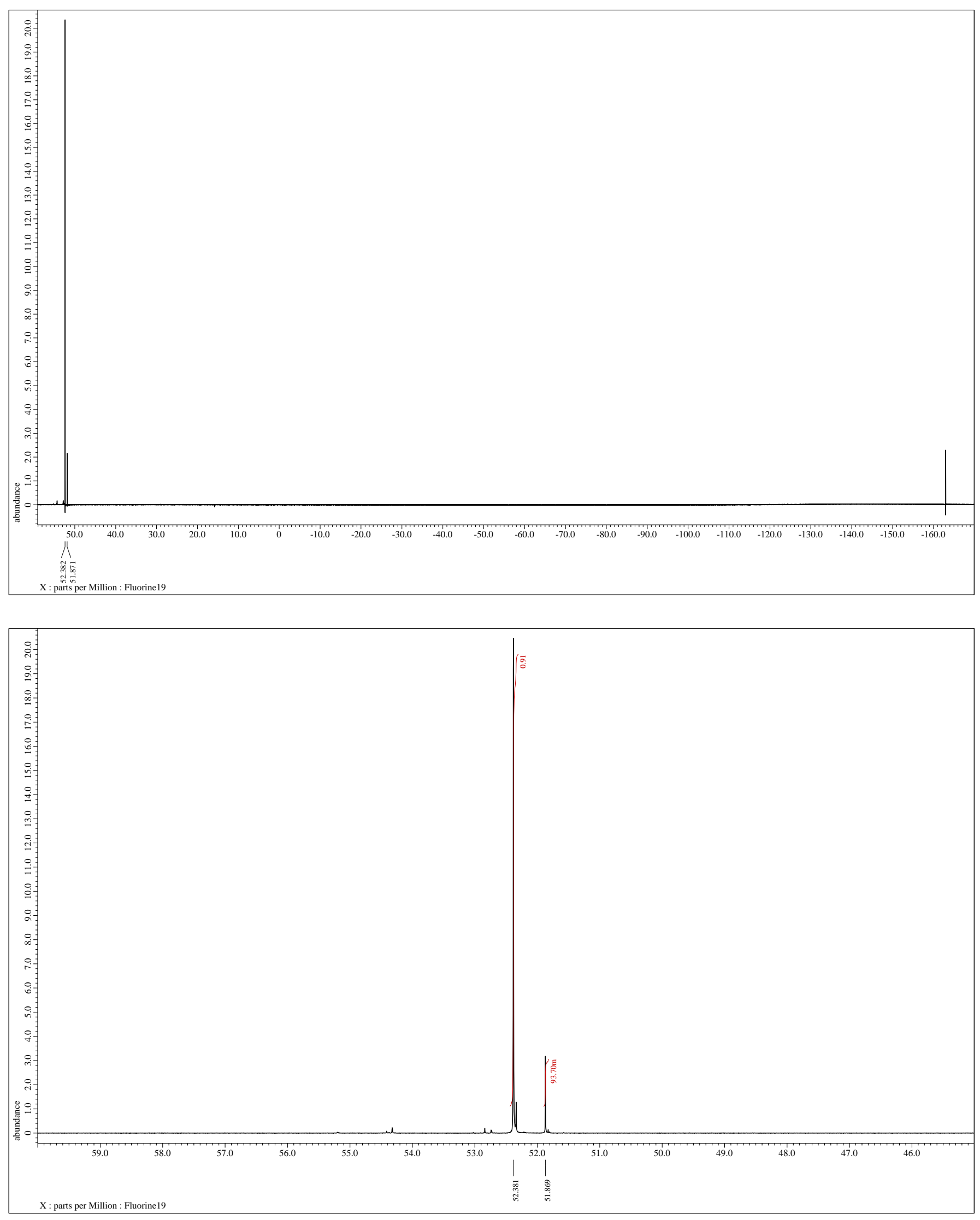
4-((fluorosulfonyl)((neopentyloxy)carbonyl)amino)-5-methyl-1,3-phenylene diacetate (37) and 2-((fluorosulfonyl)((neopentyloxy)carbonyl)amino)-5-methyl-1,3-phenylene diacetate (37')<smiles>CC(=O)Oc1cc(OC(C)=O)c(N(C(=O)O[Na])C(=O)OCC(C)(C)C)c([N+](=O)[O-])c1</smiles>

37<smiles>COc1cc(C)cc(OC(C)=O)c1N(C(=O)O[Na])C(=O)OCC(C)(C)C</smiles>
$37^{\prime}$
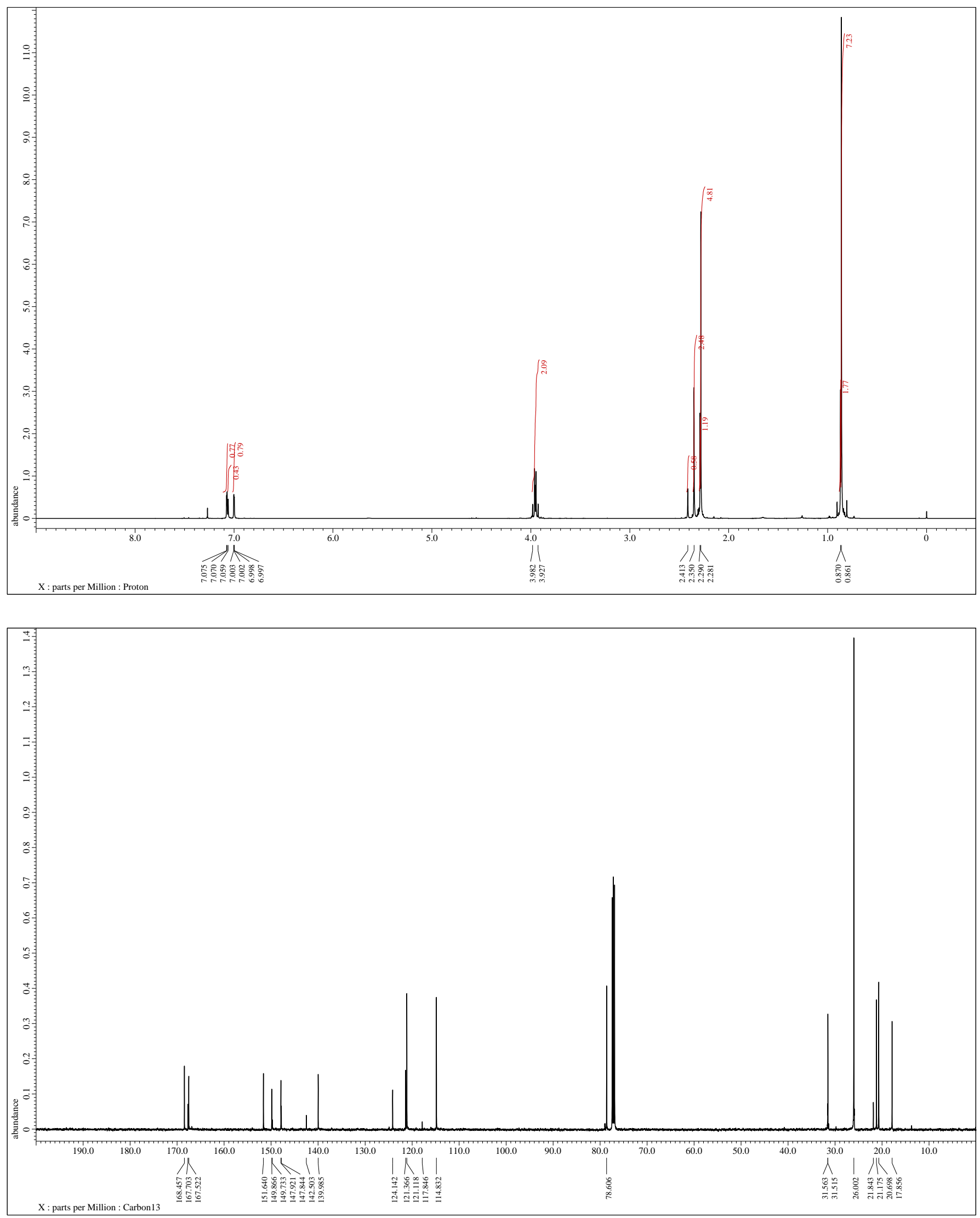

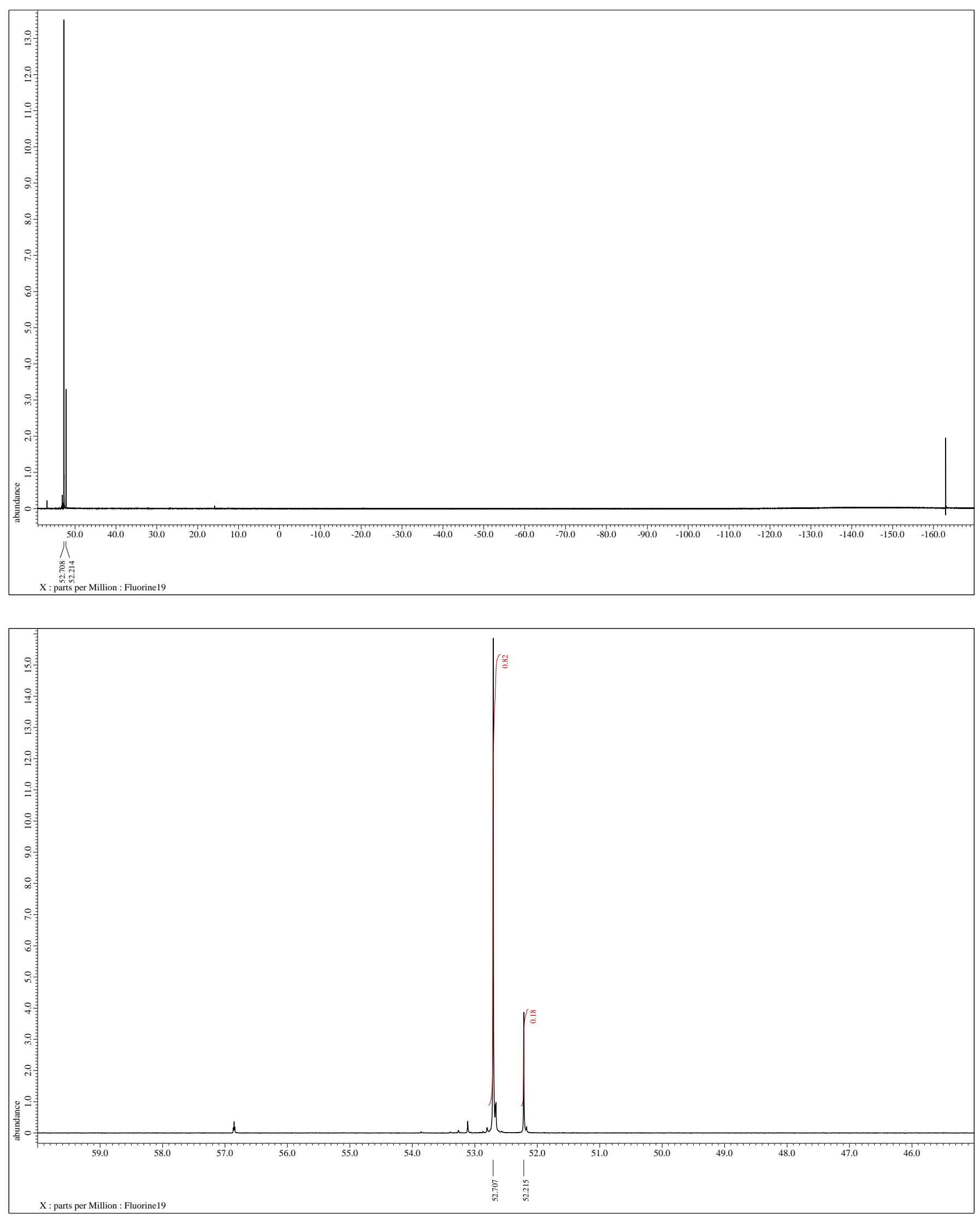

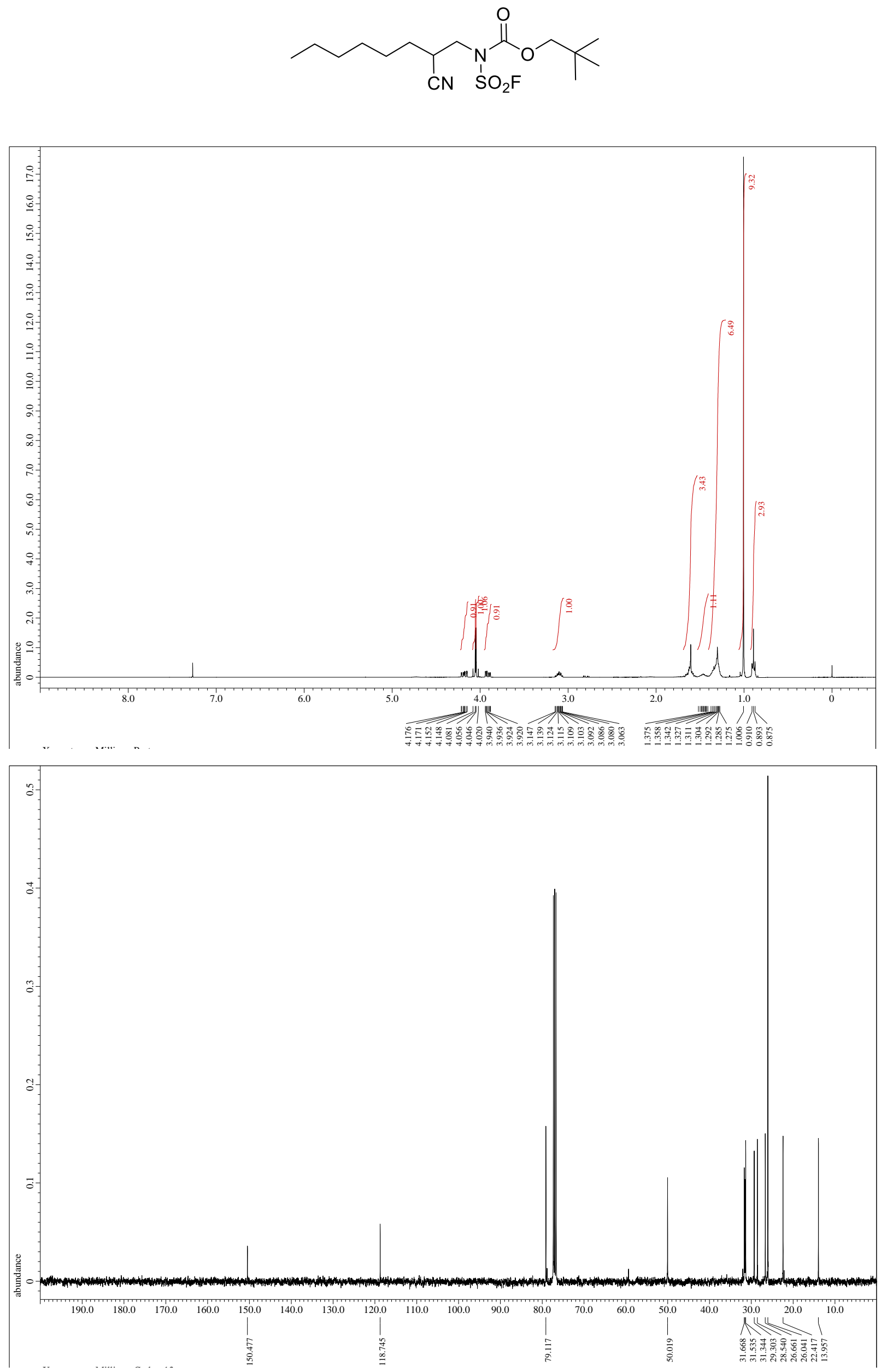


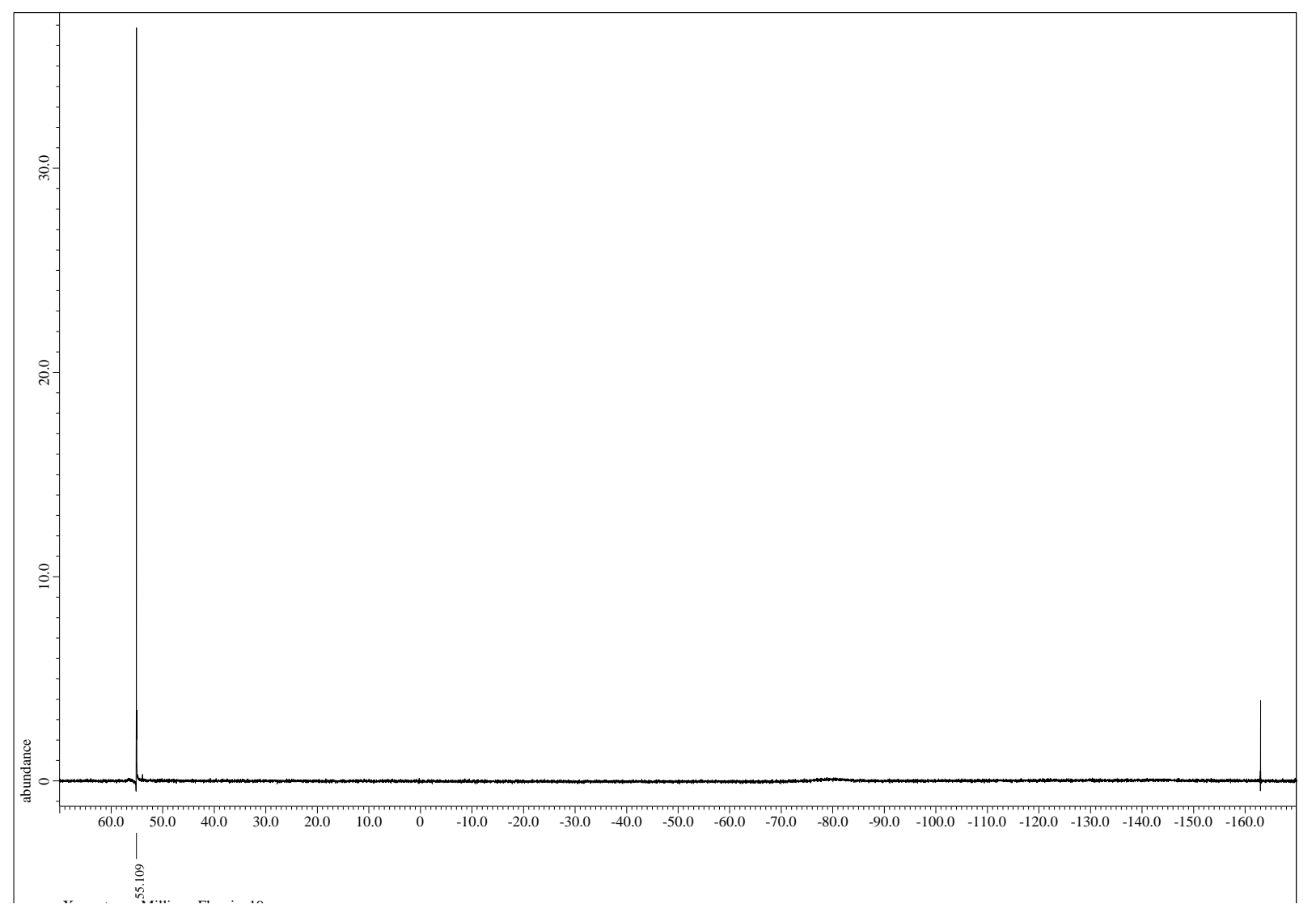



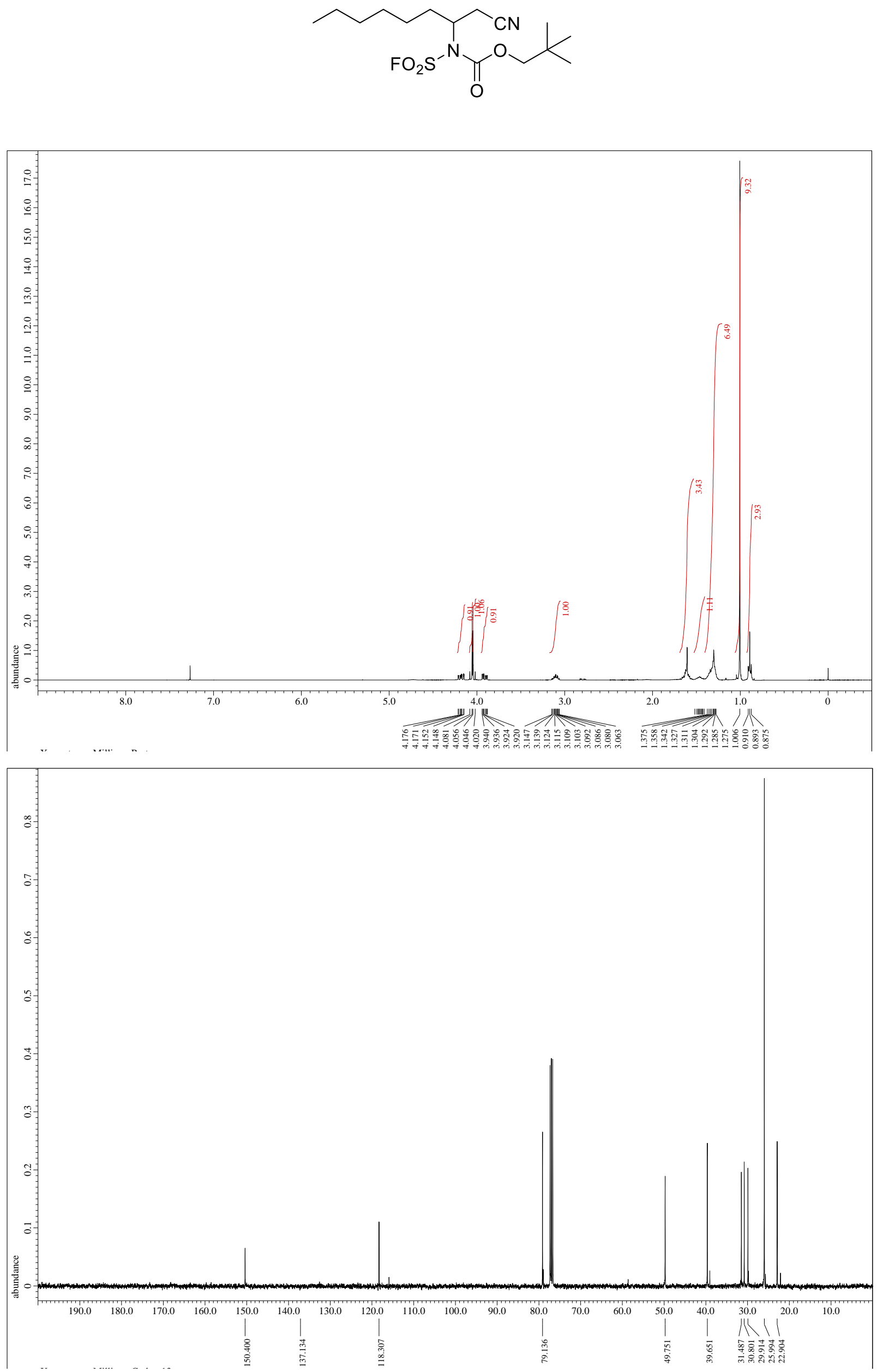


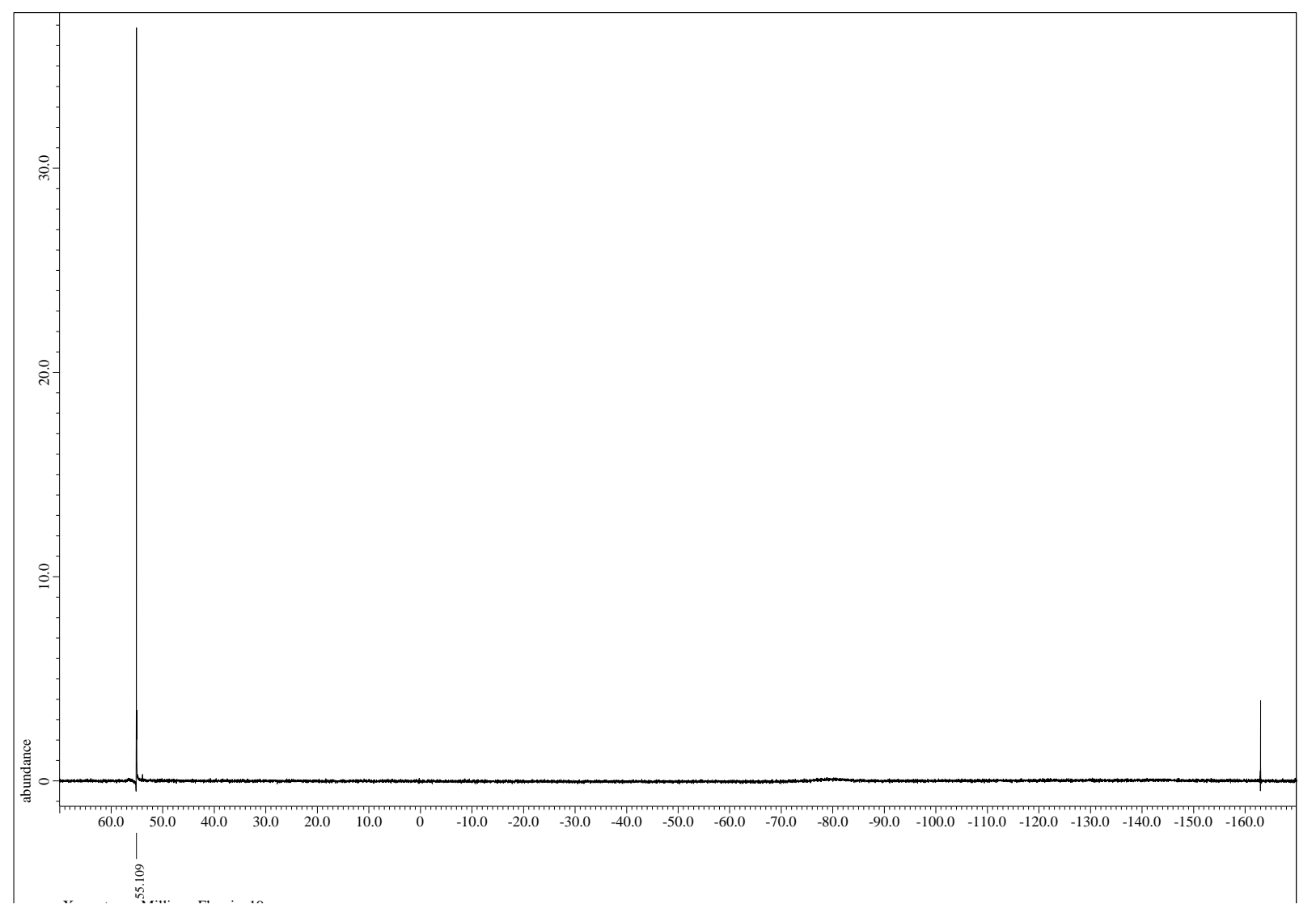



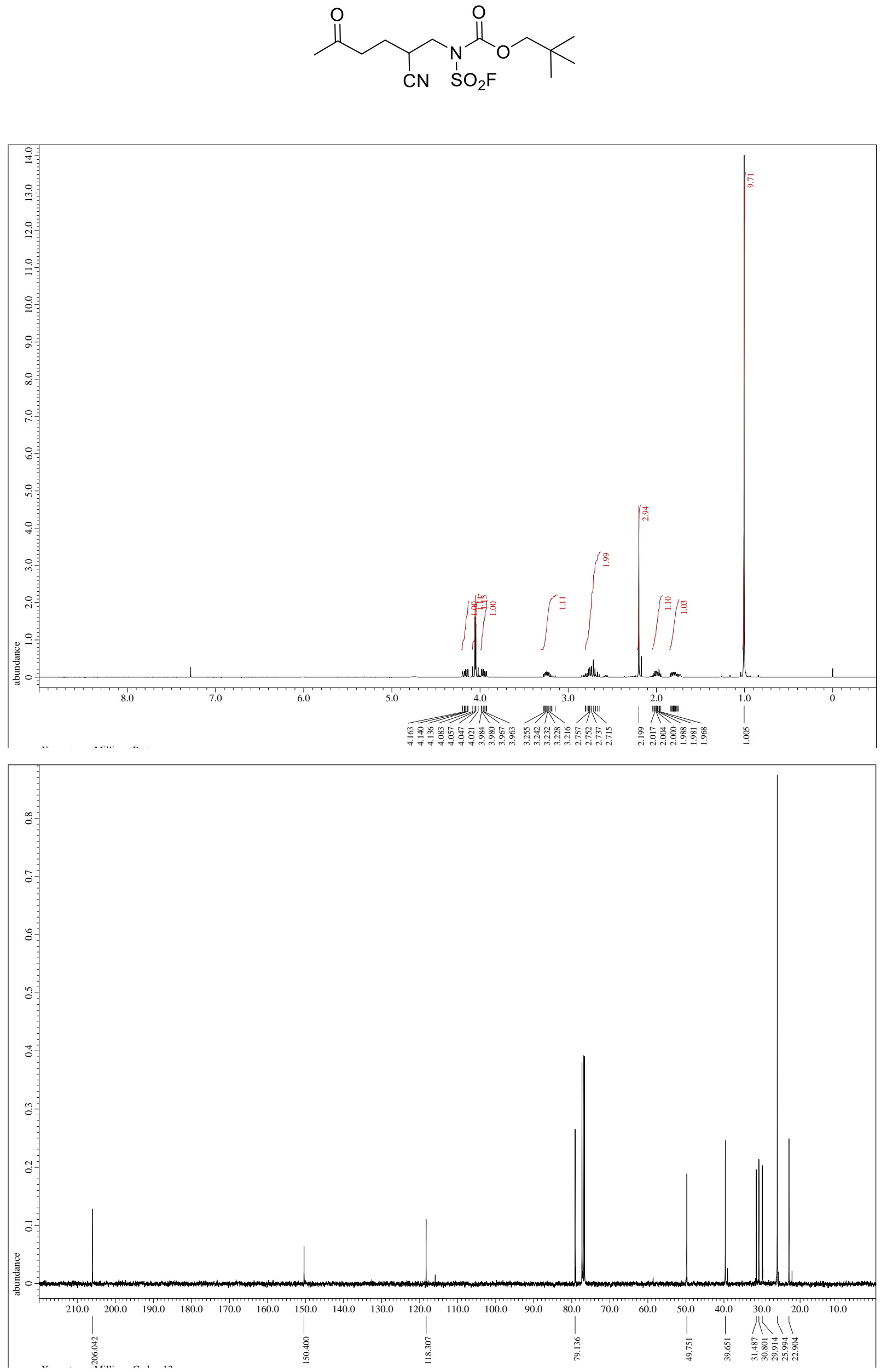


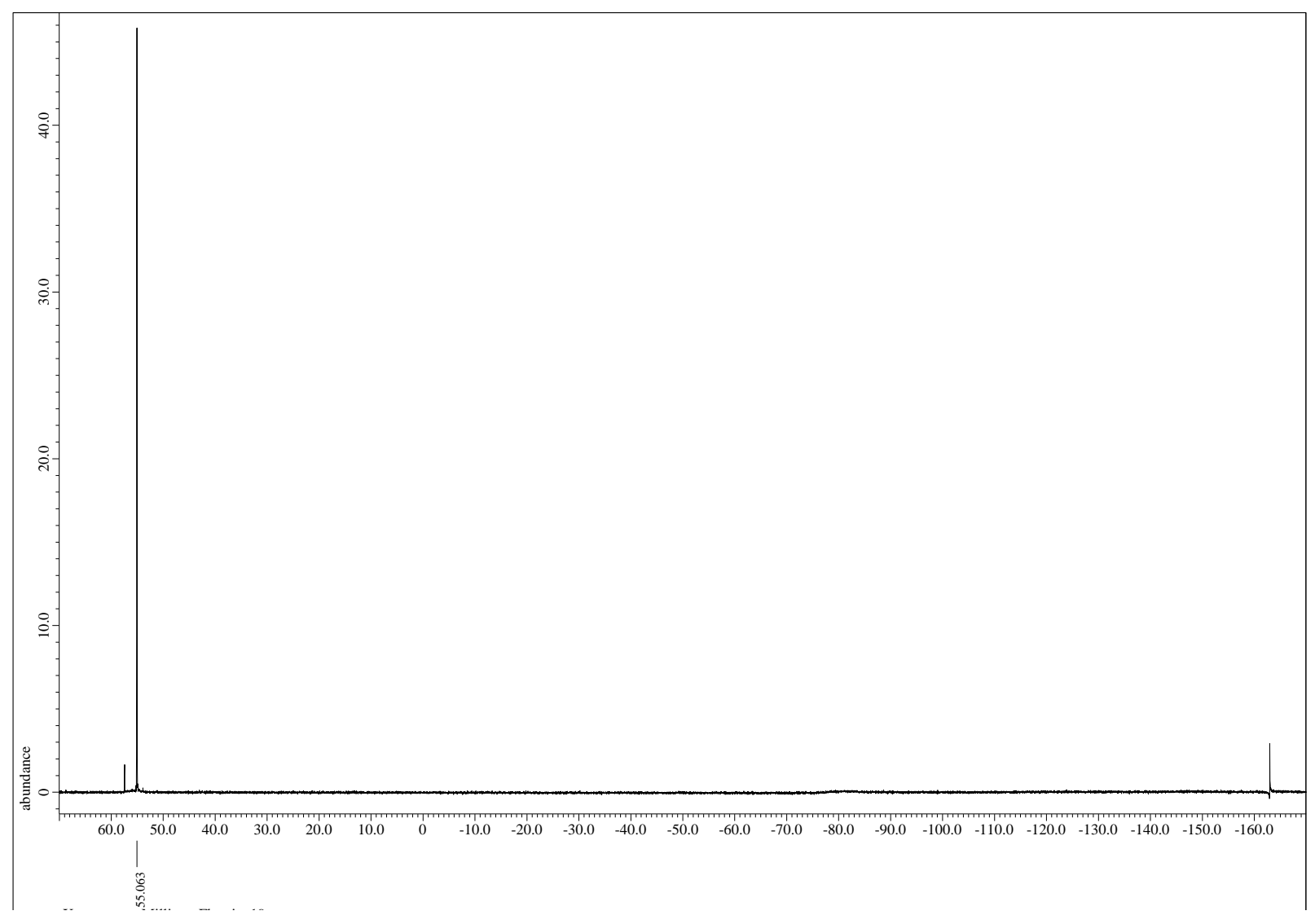


methyl 5-cyano-6-((fluorosulfonyl)((neopentyloxy)carbonyl)amino)hexanoate (40)
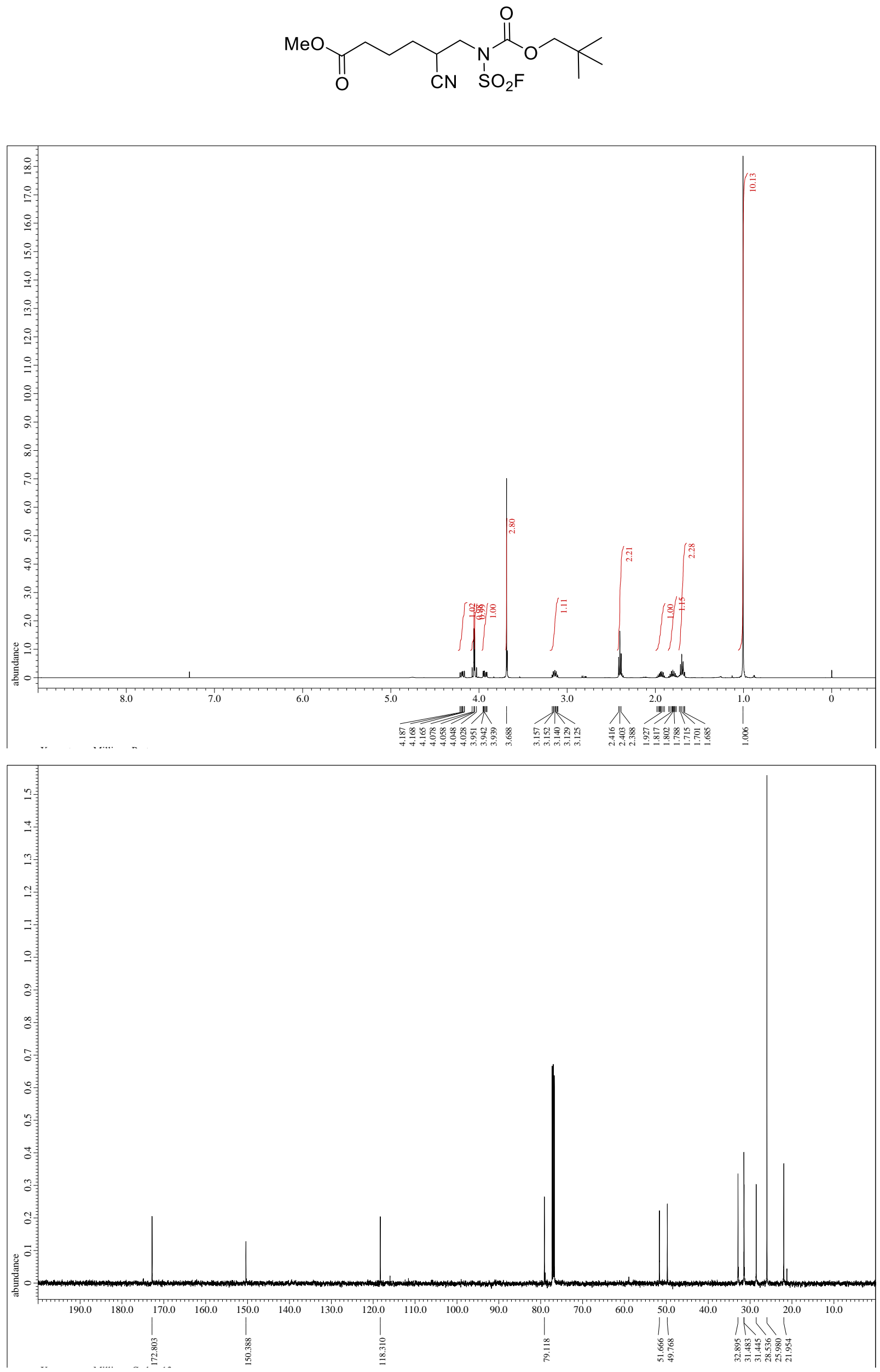


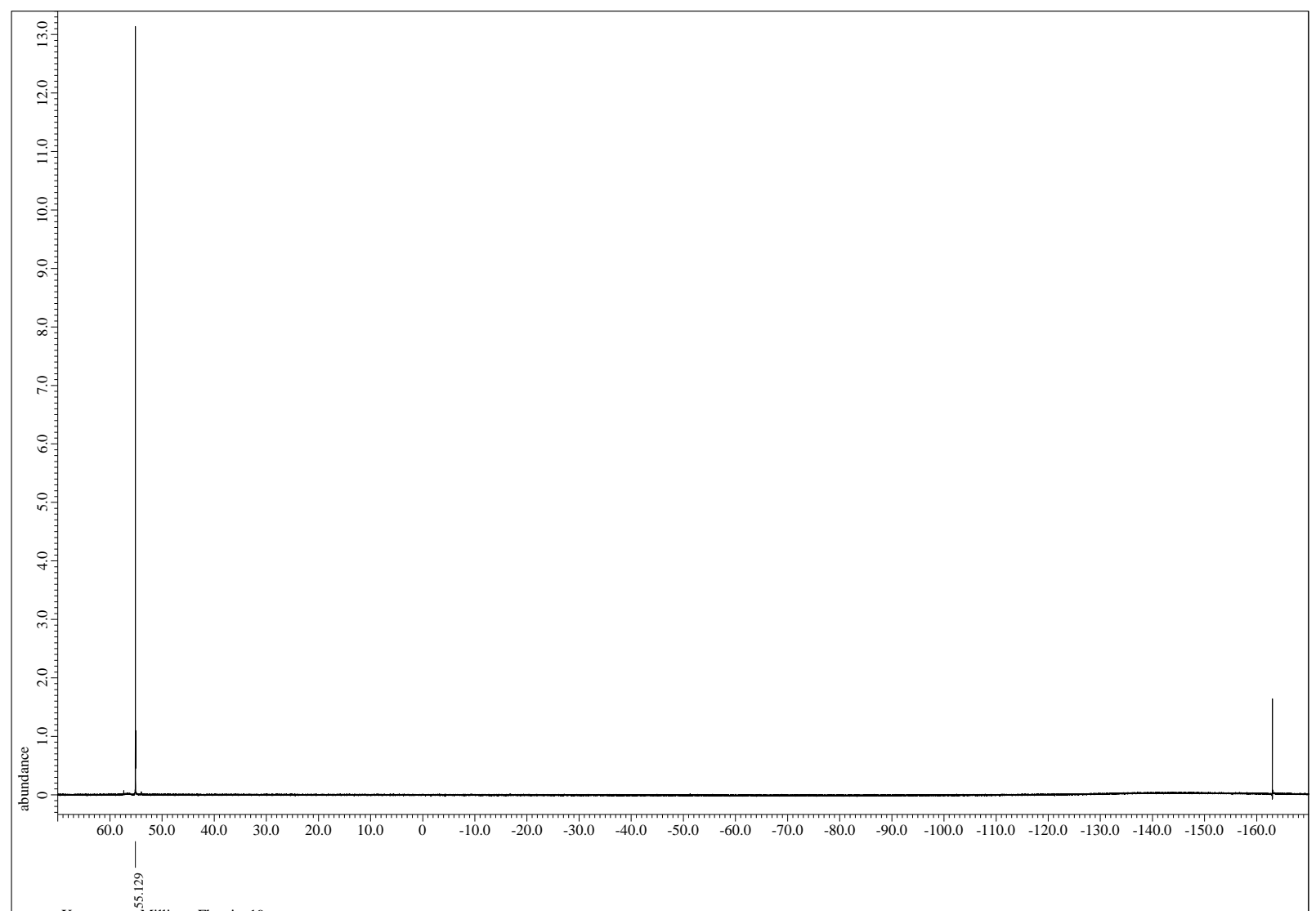



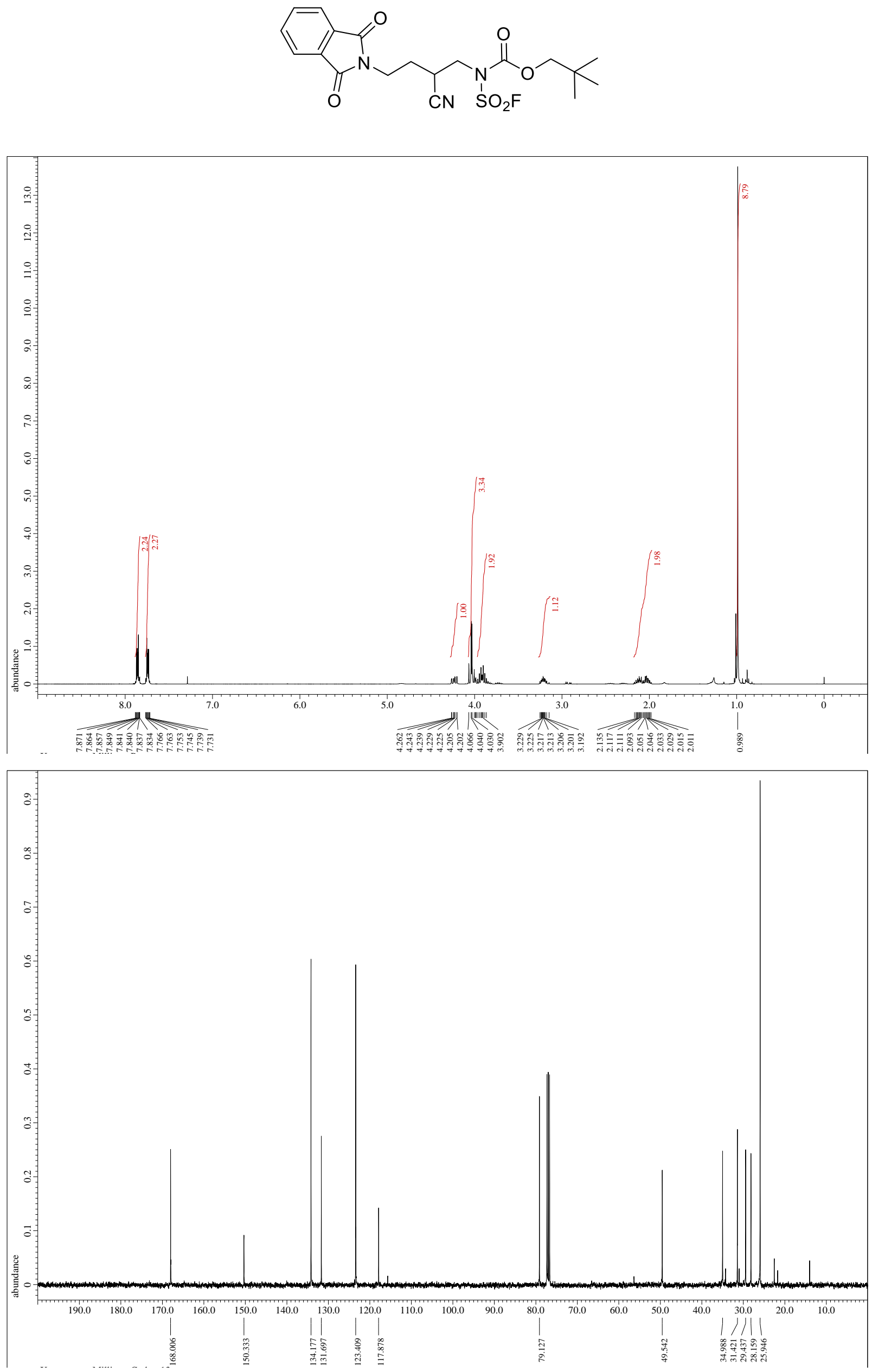


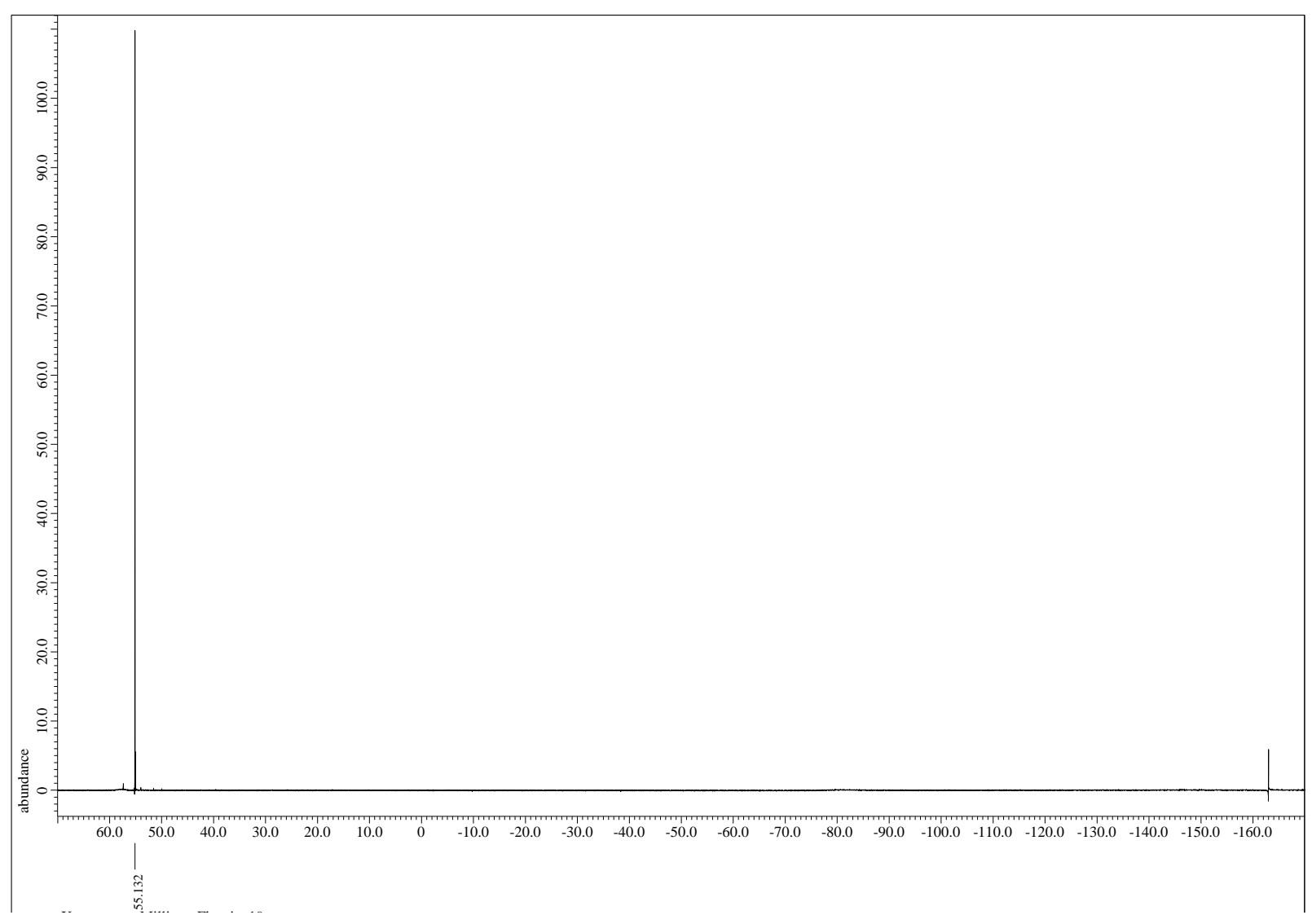



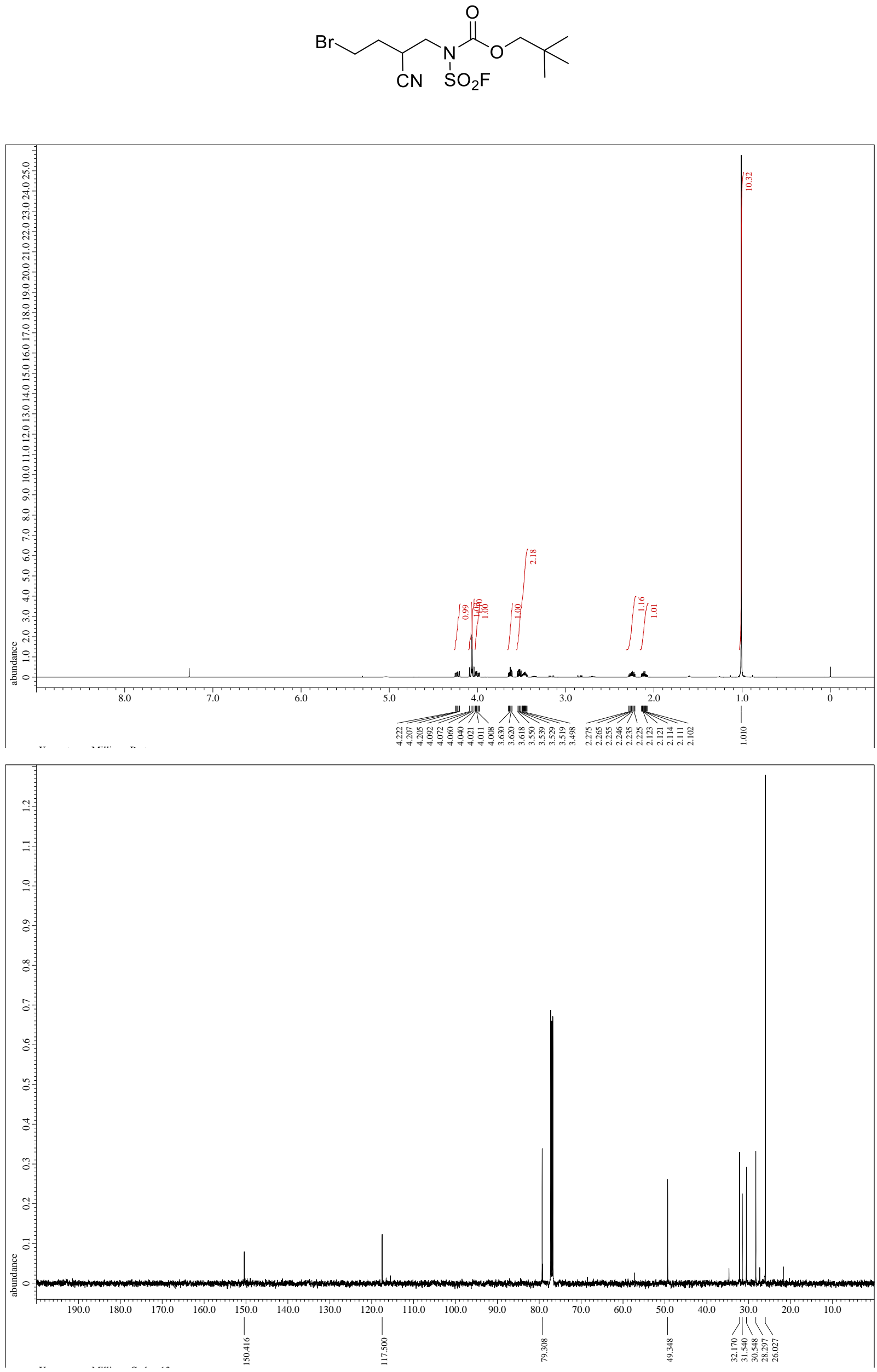


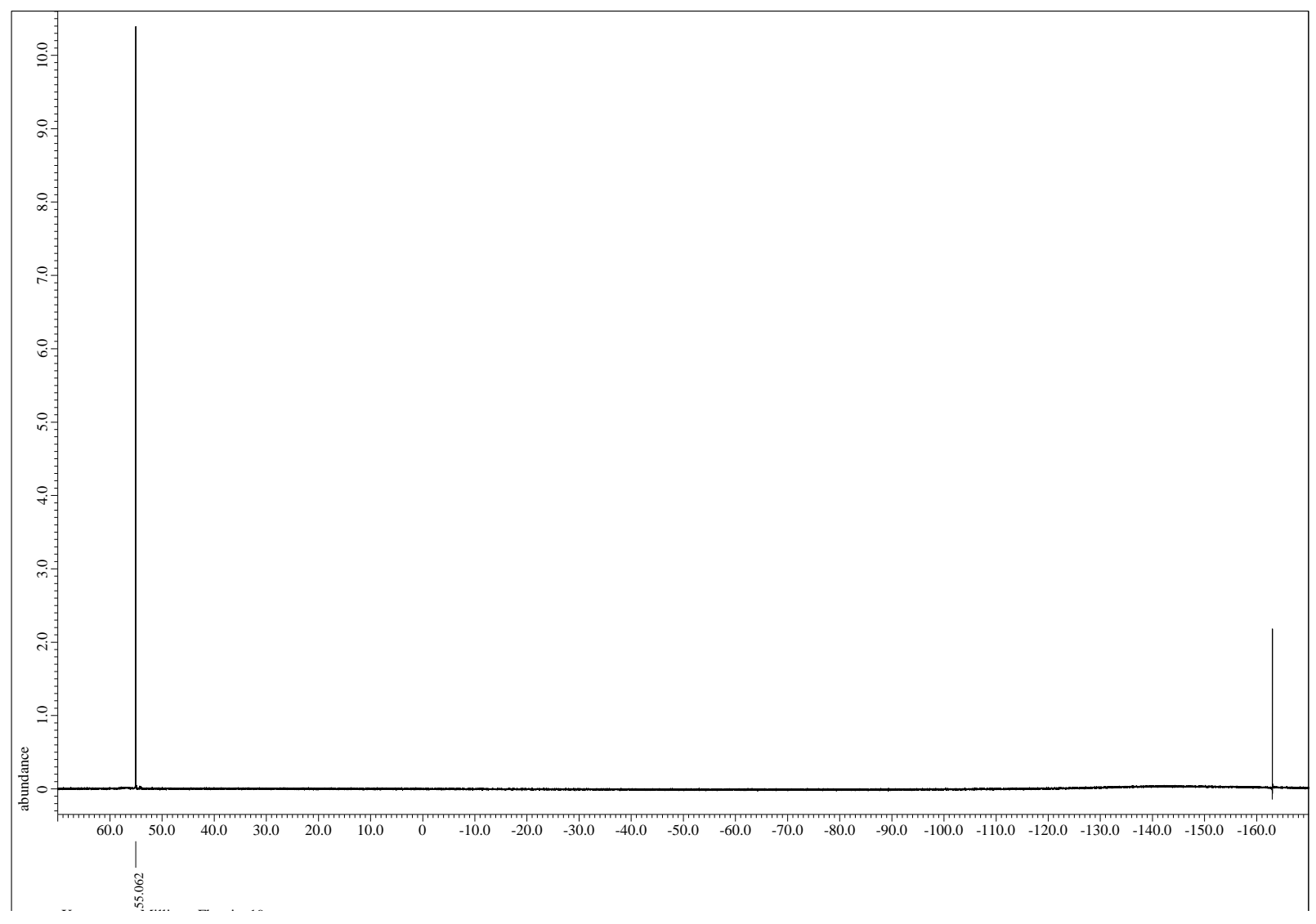



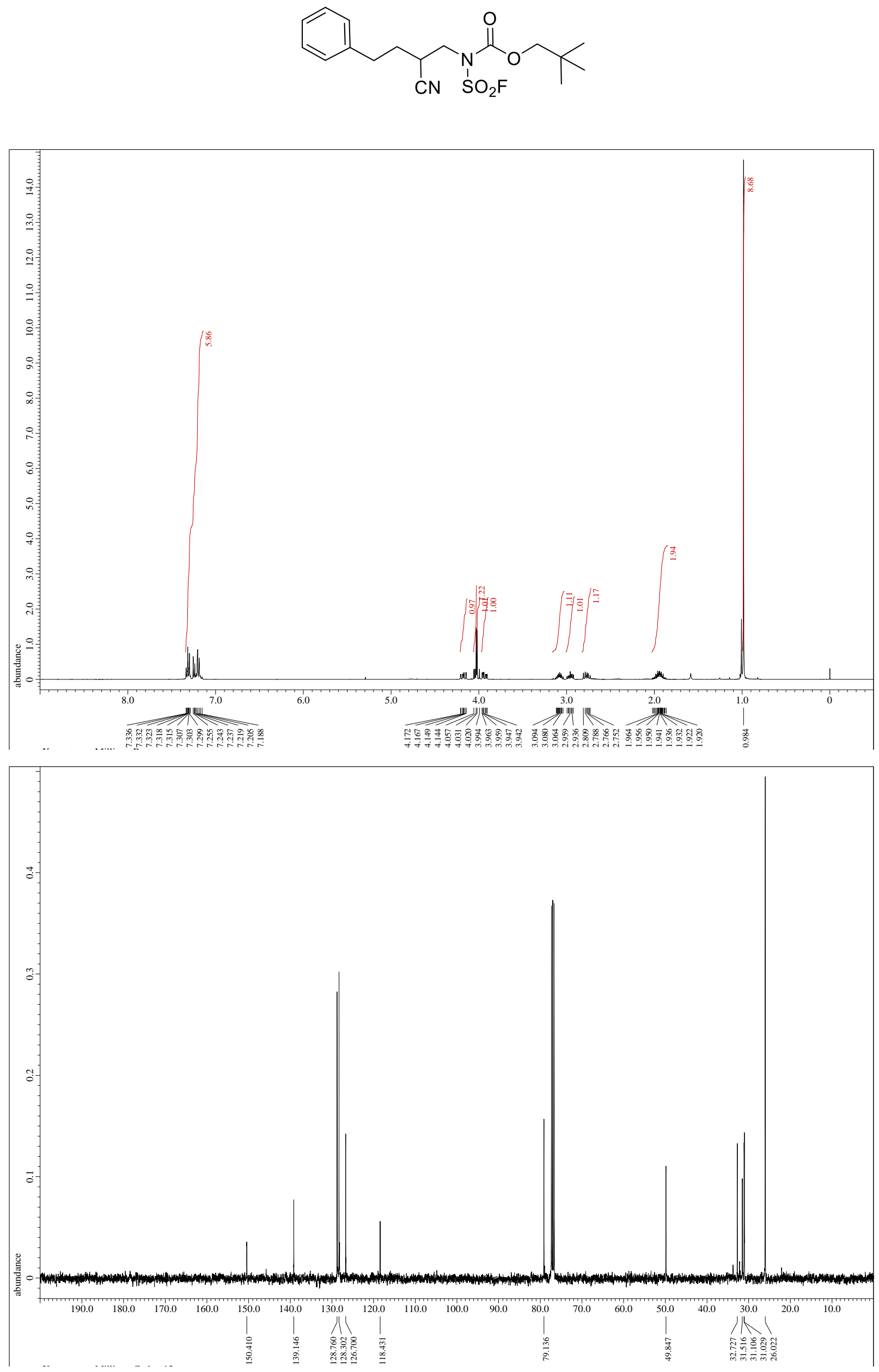


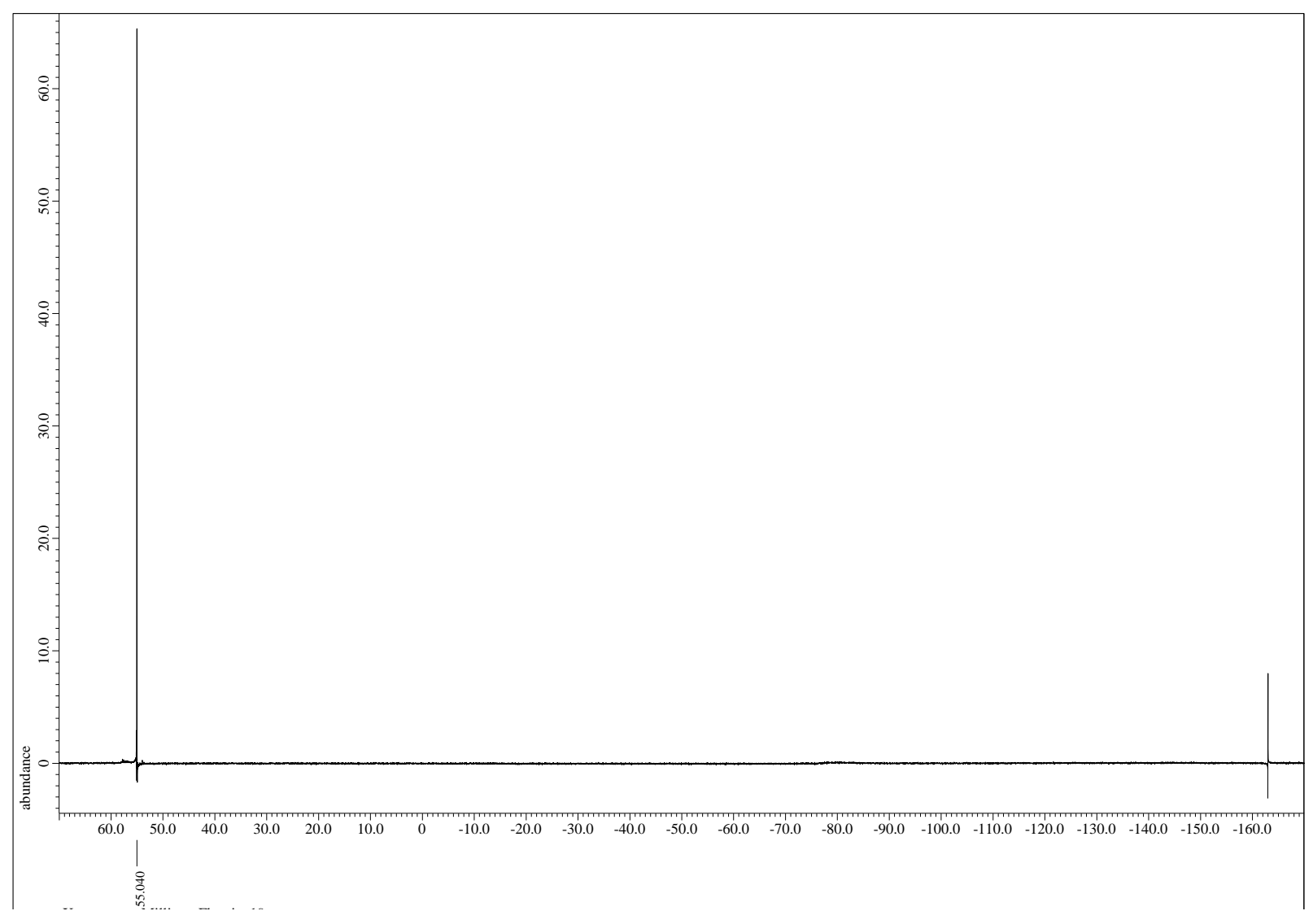



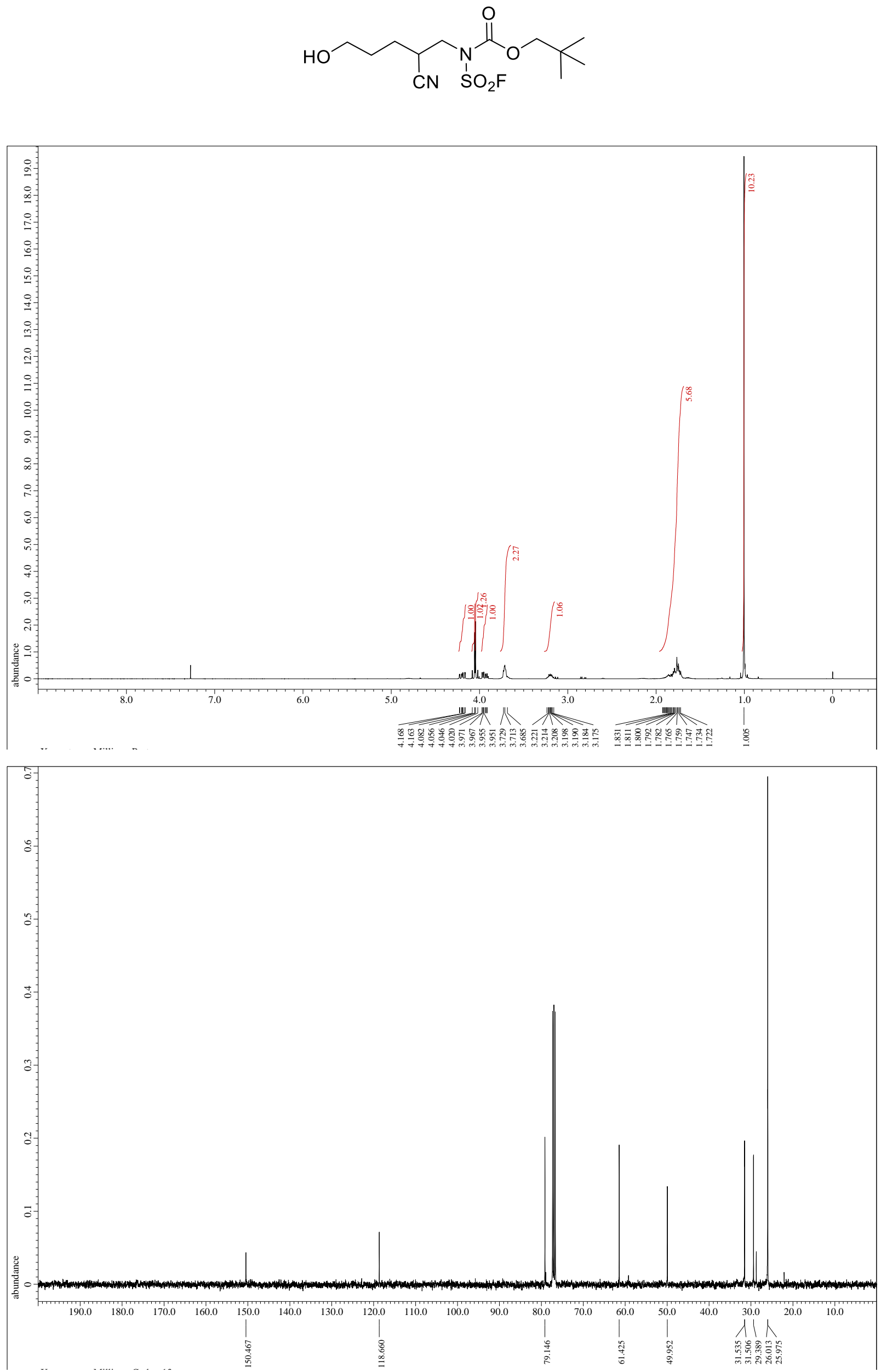


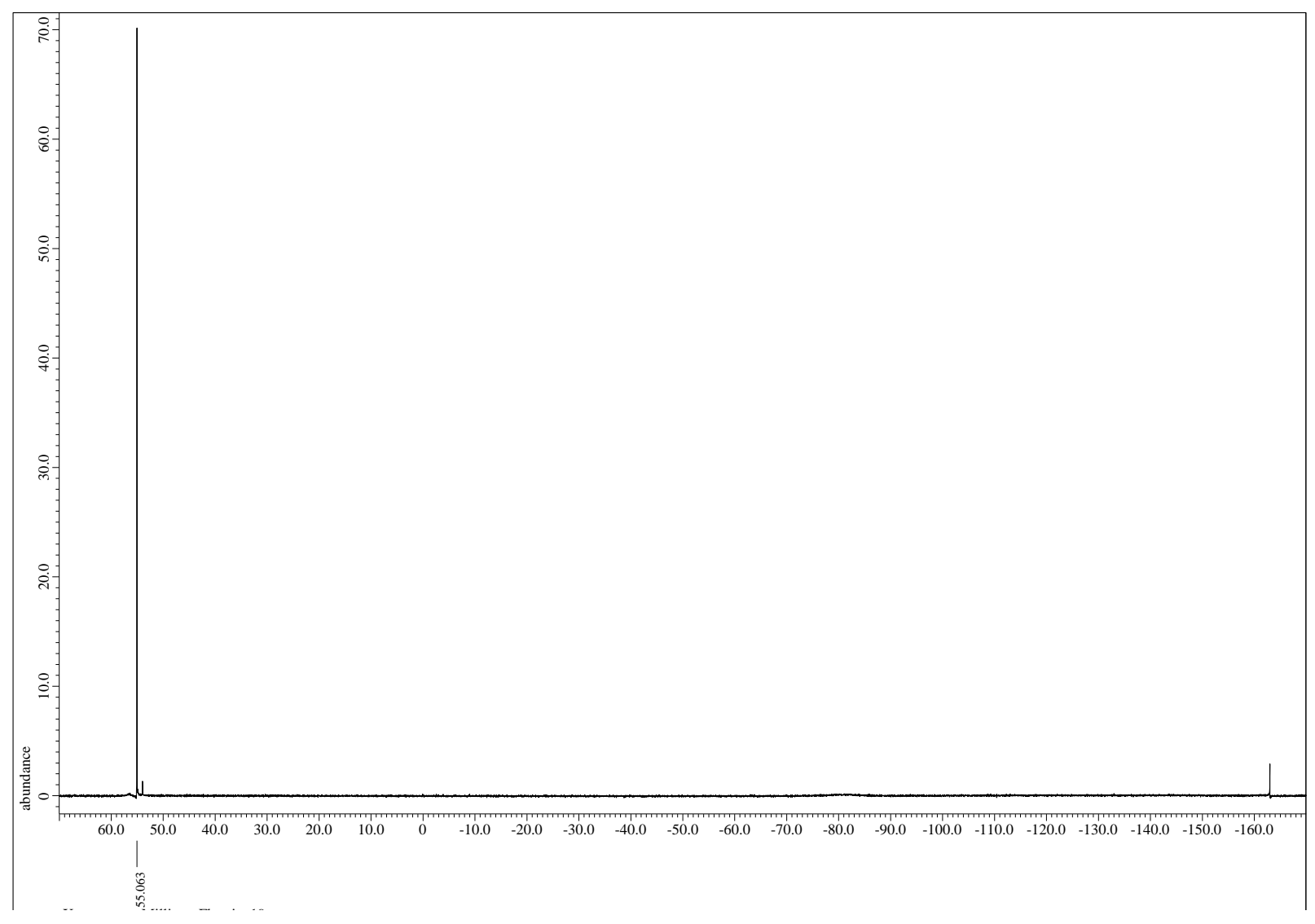



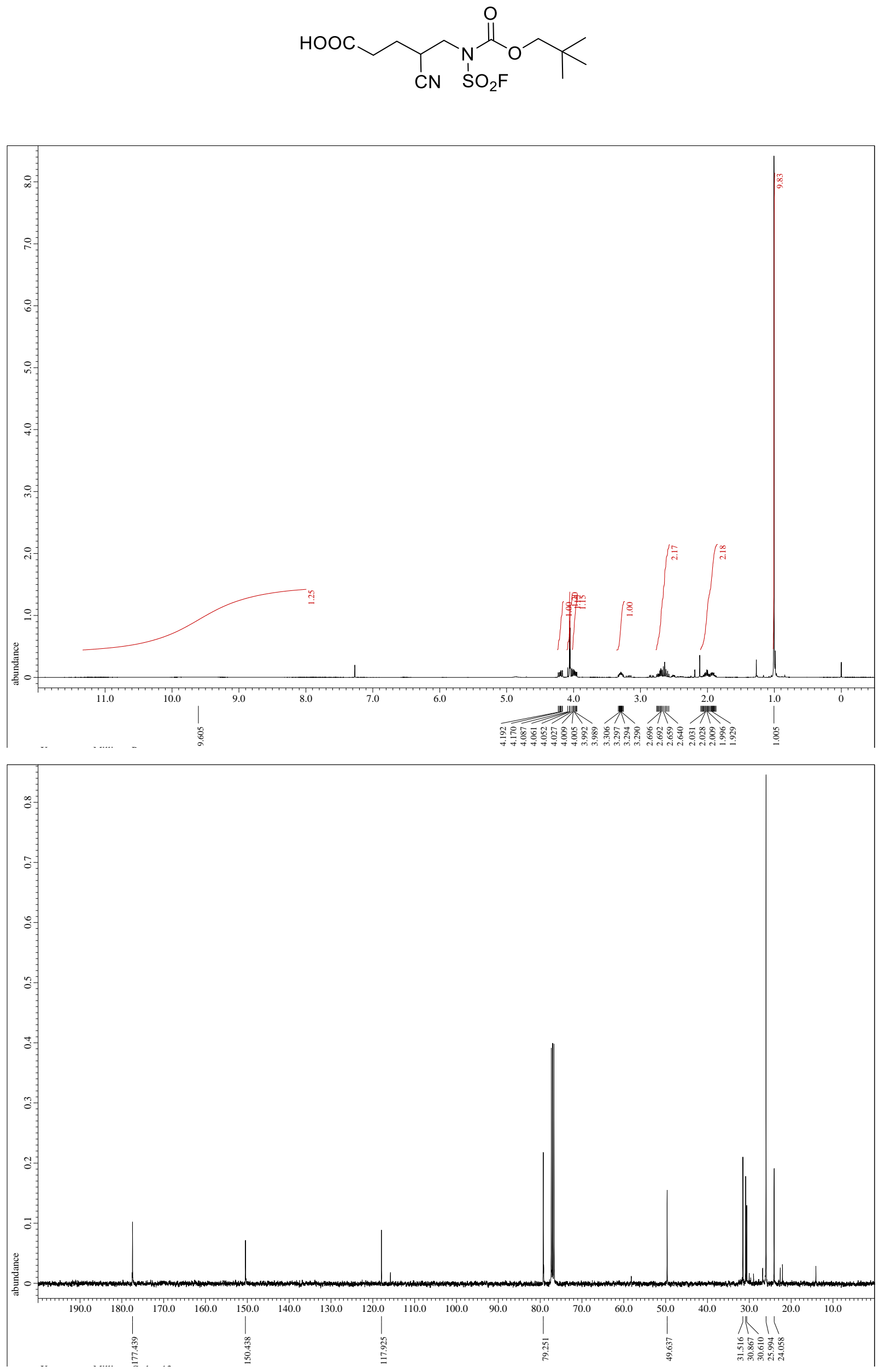


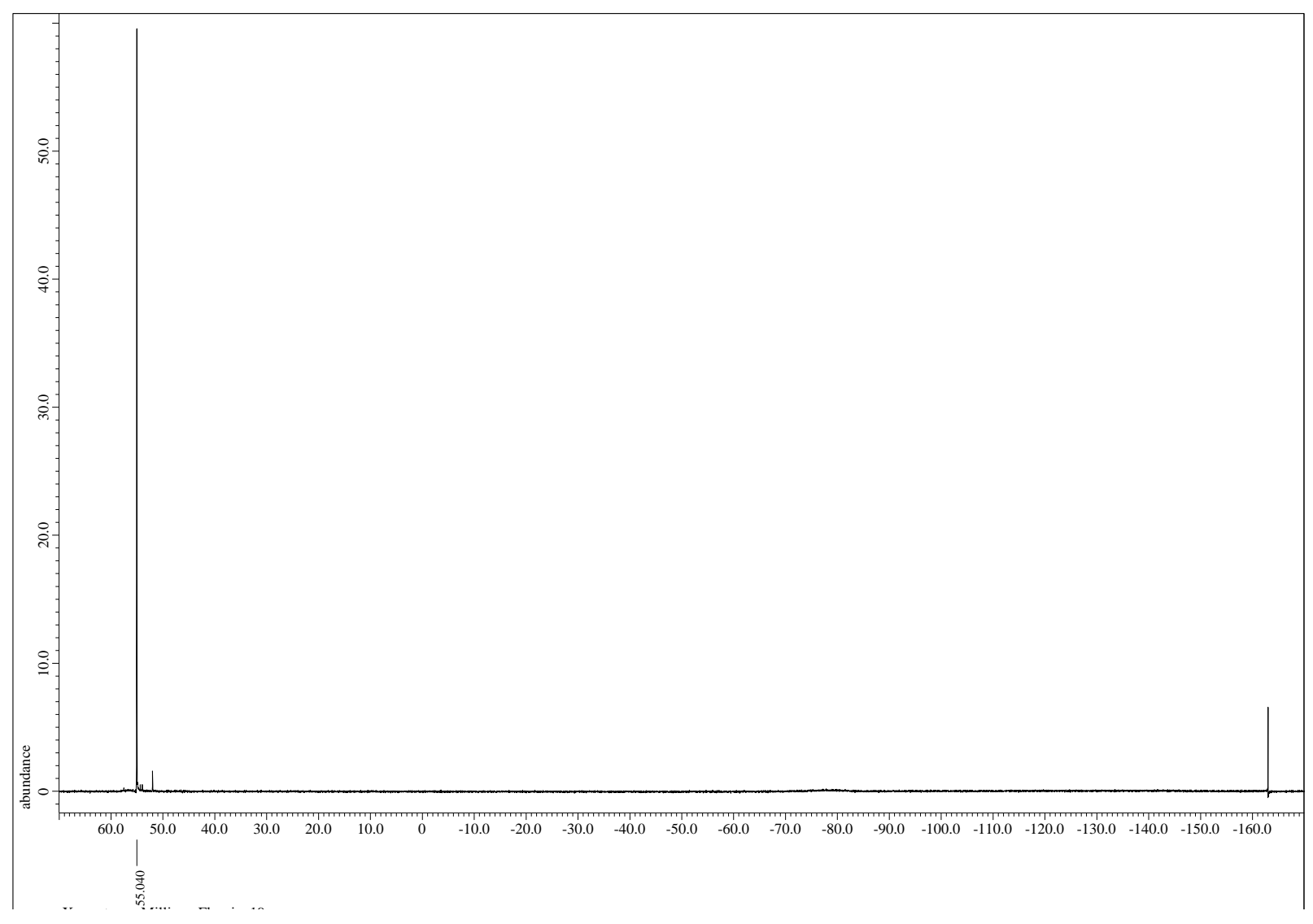



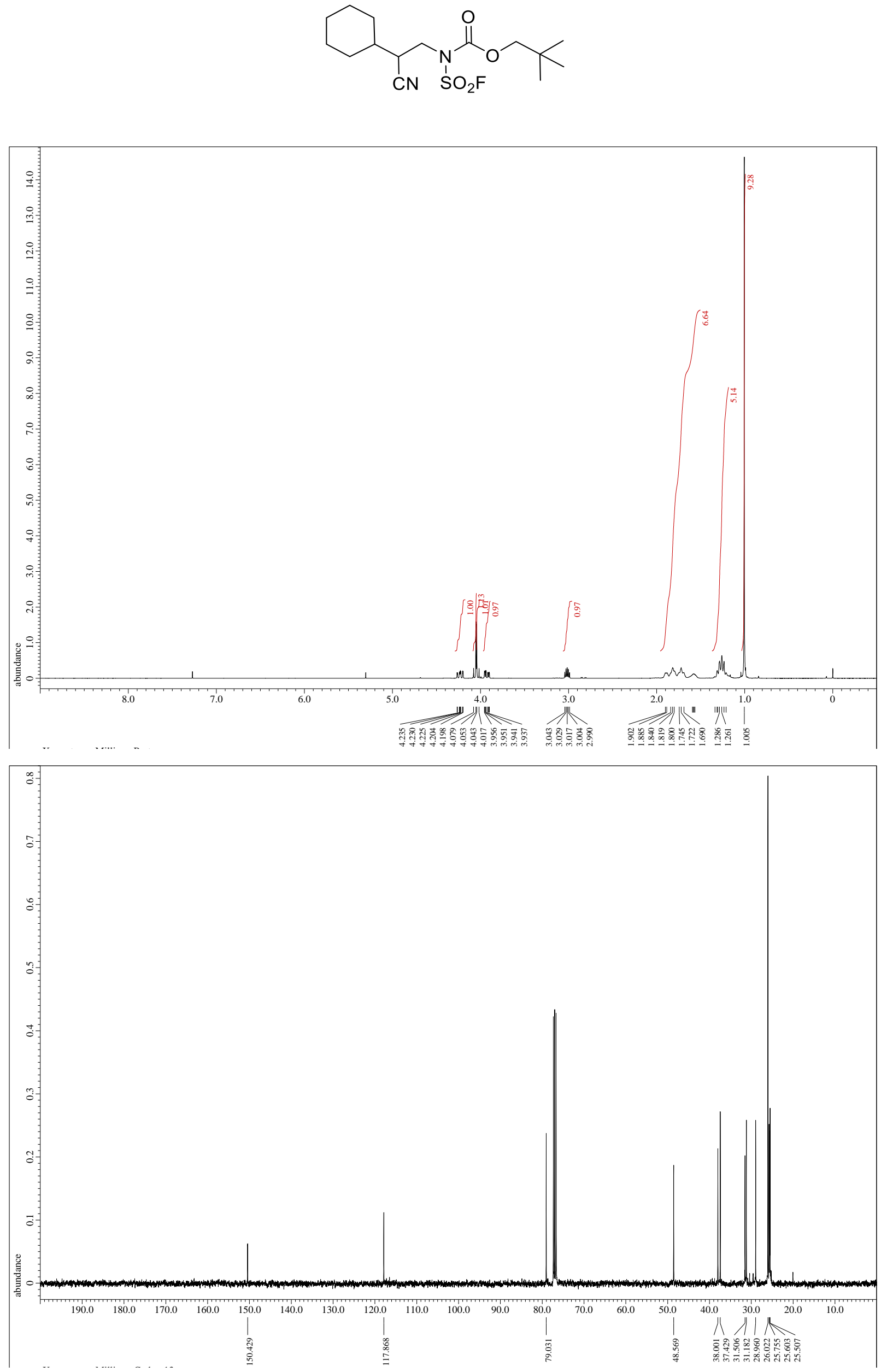


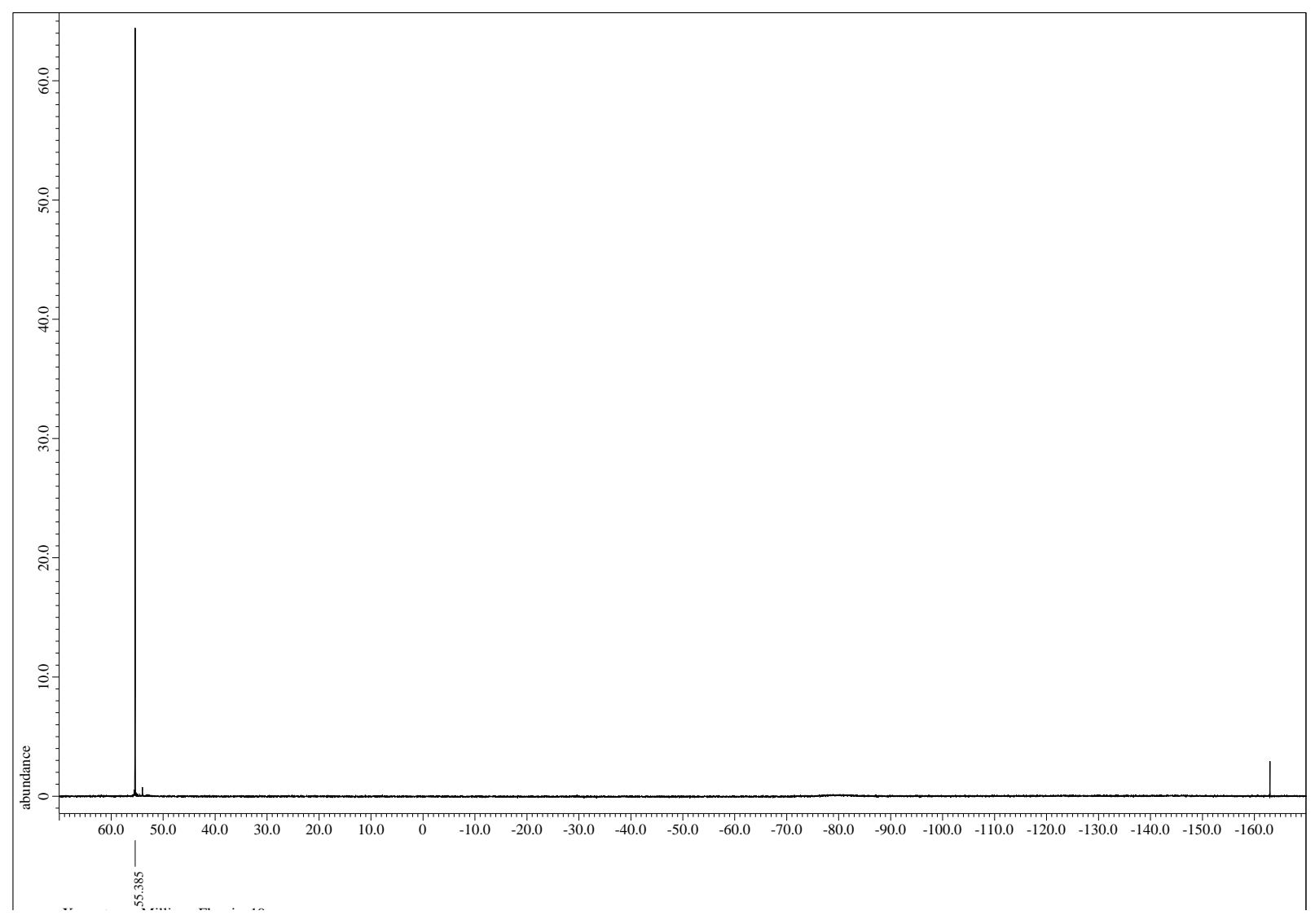



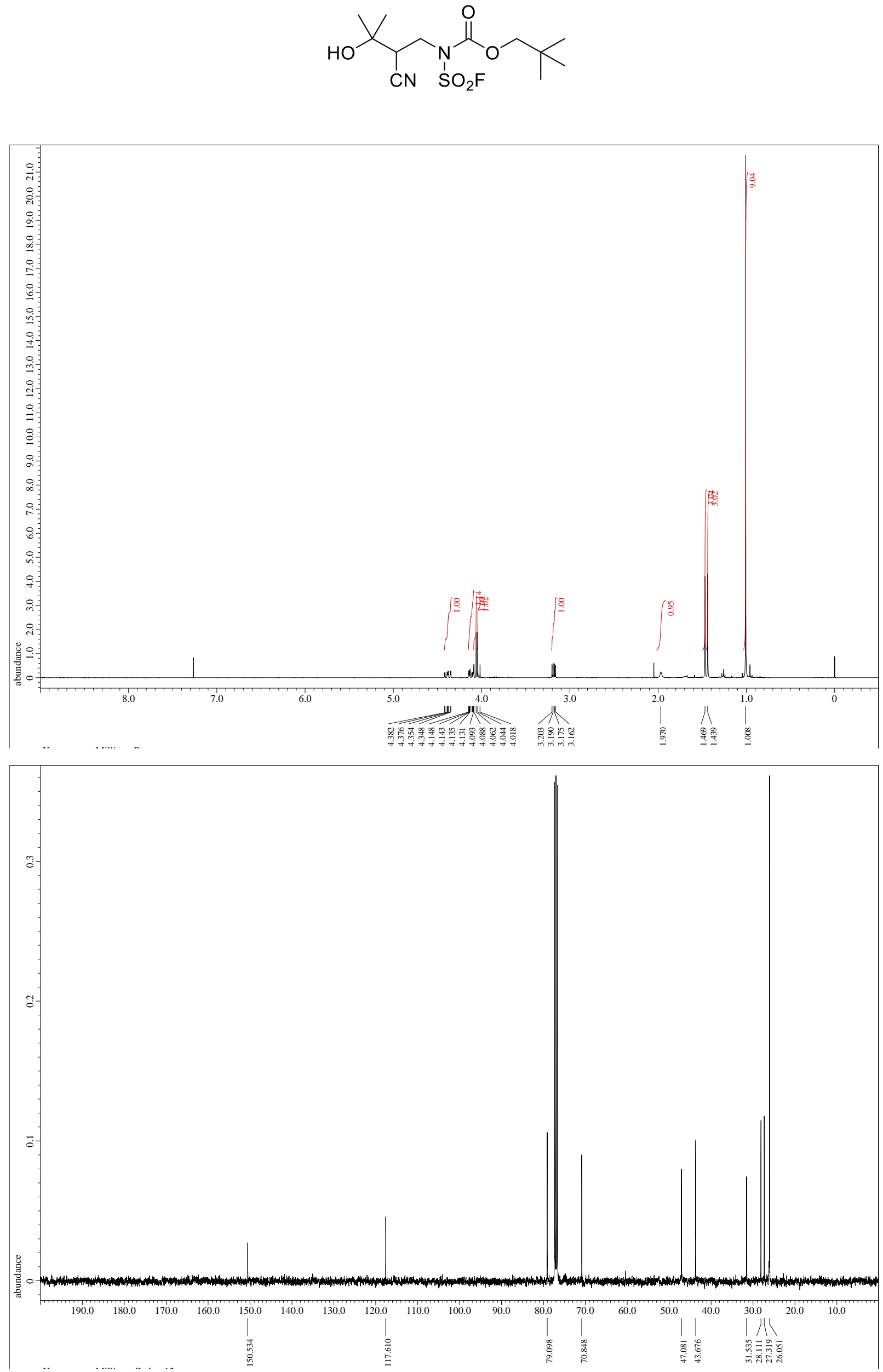


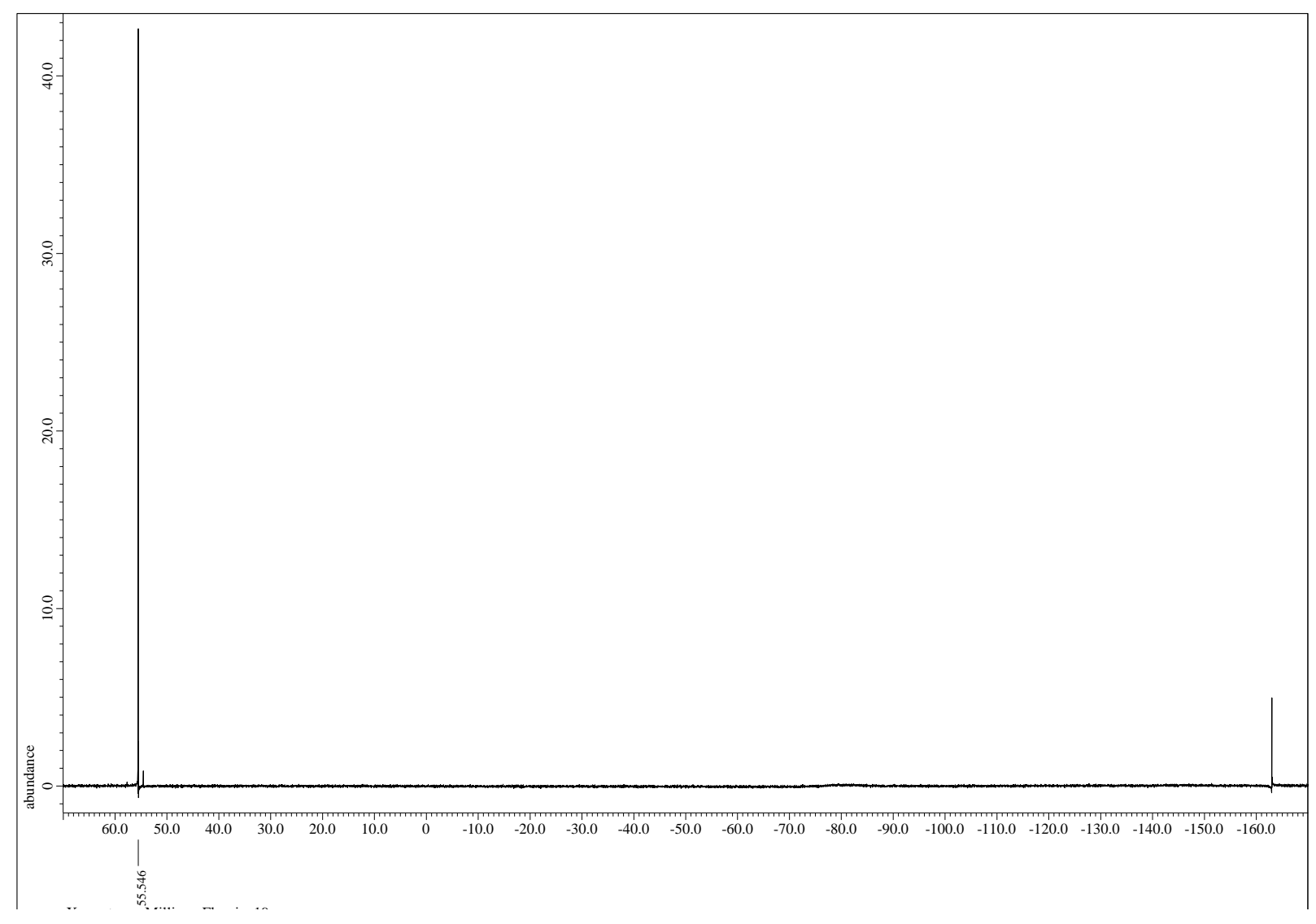


neopentyl rel-(4R,5S)-(5-cyanooctan-4-yl)(fluorosulfonyl)carbamate (48) and neopentyl rel-(4S,5S)-(5cyanooctan-4-yl)(fluorosulfonyl)carbamate (48')

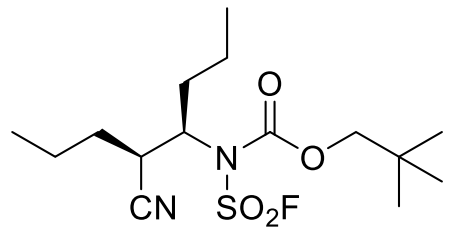

48
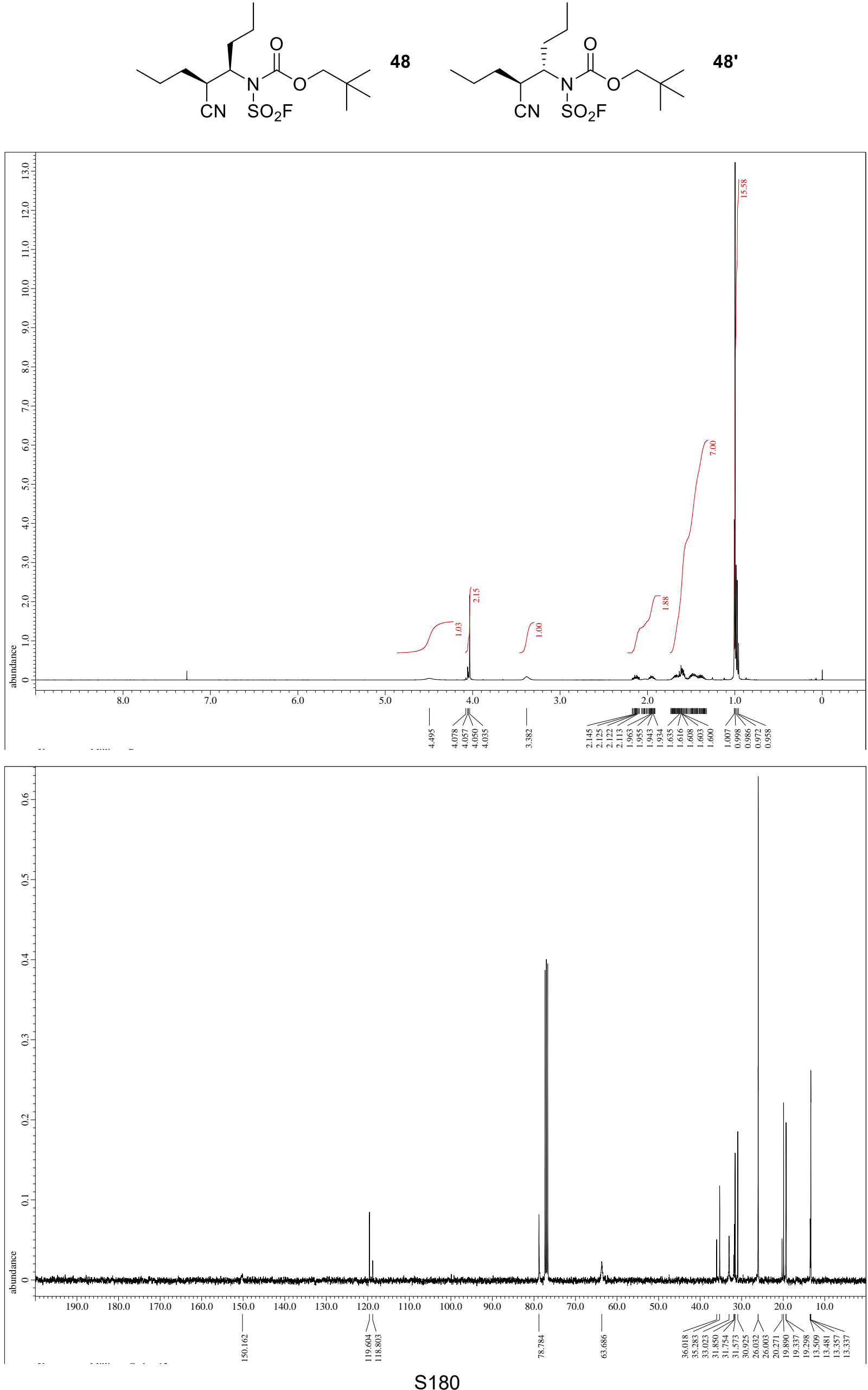


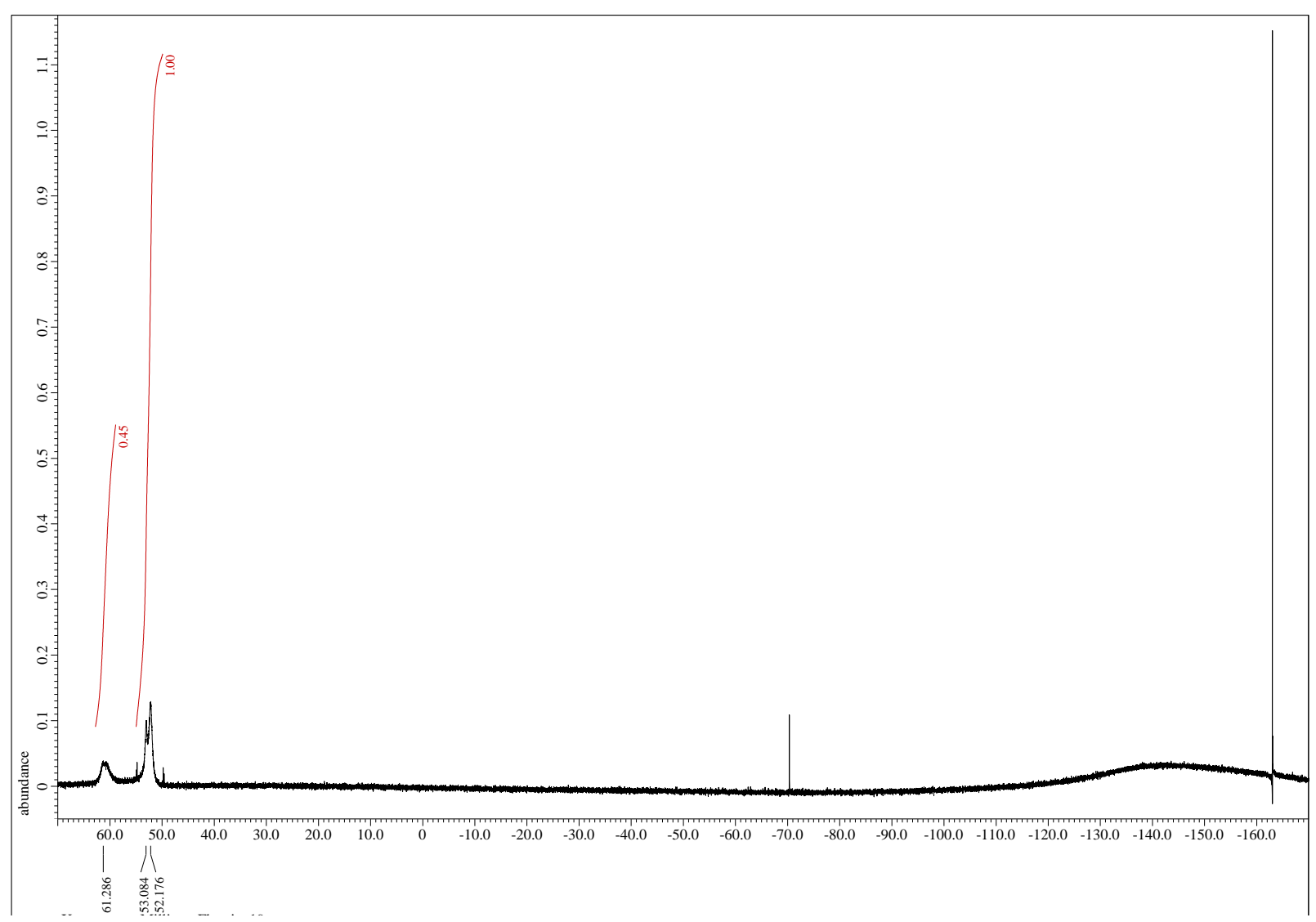



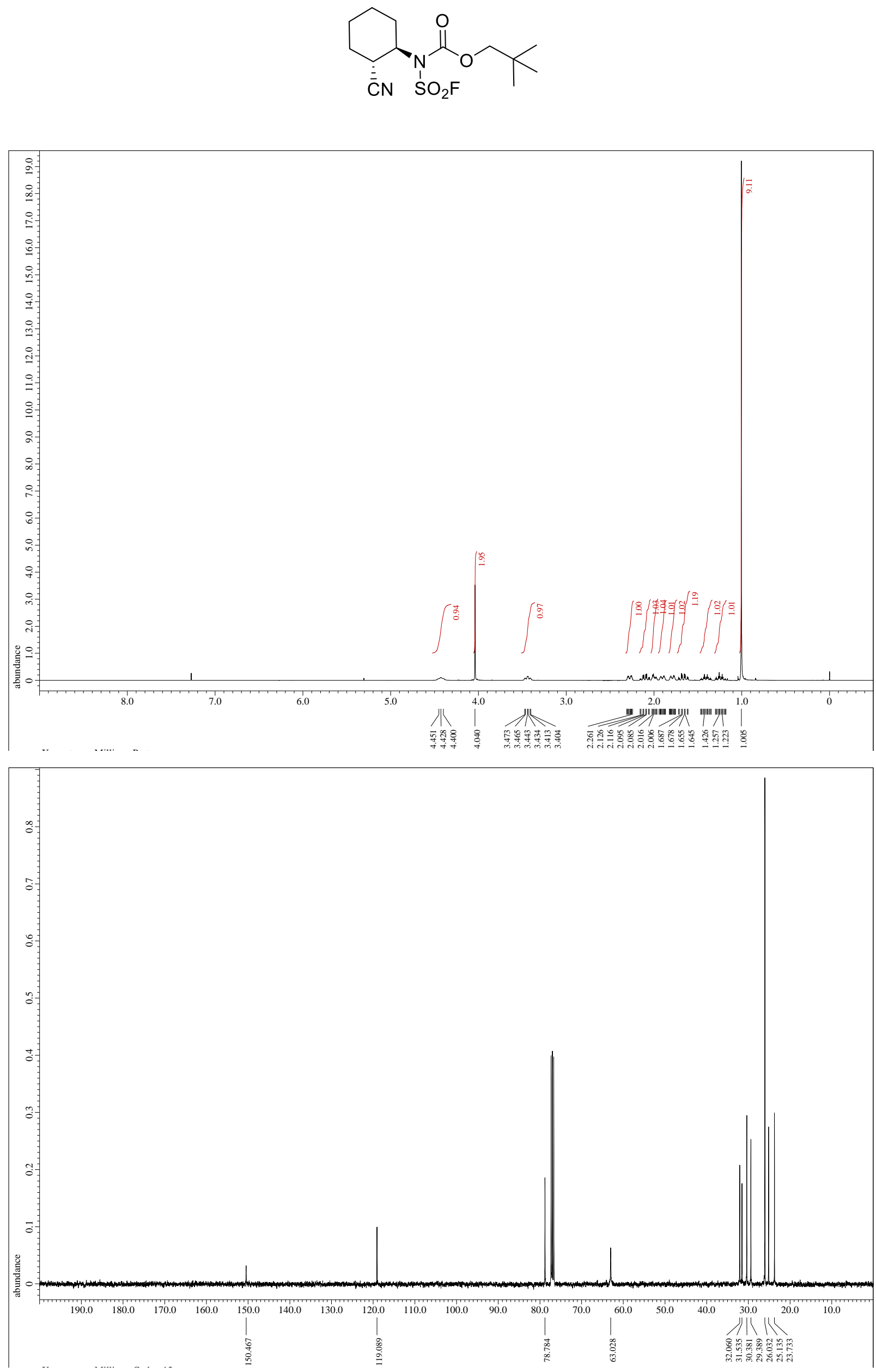


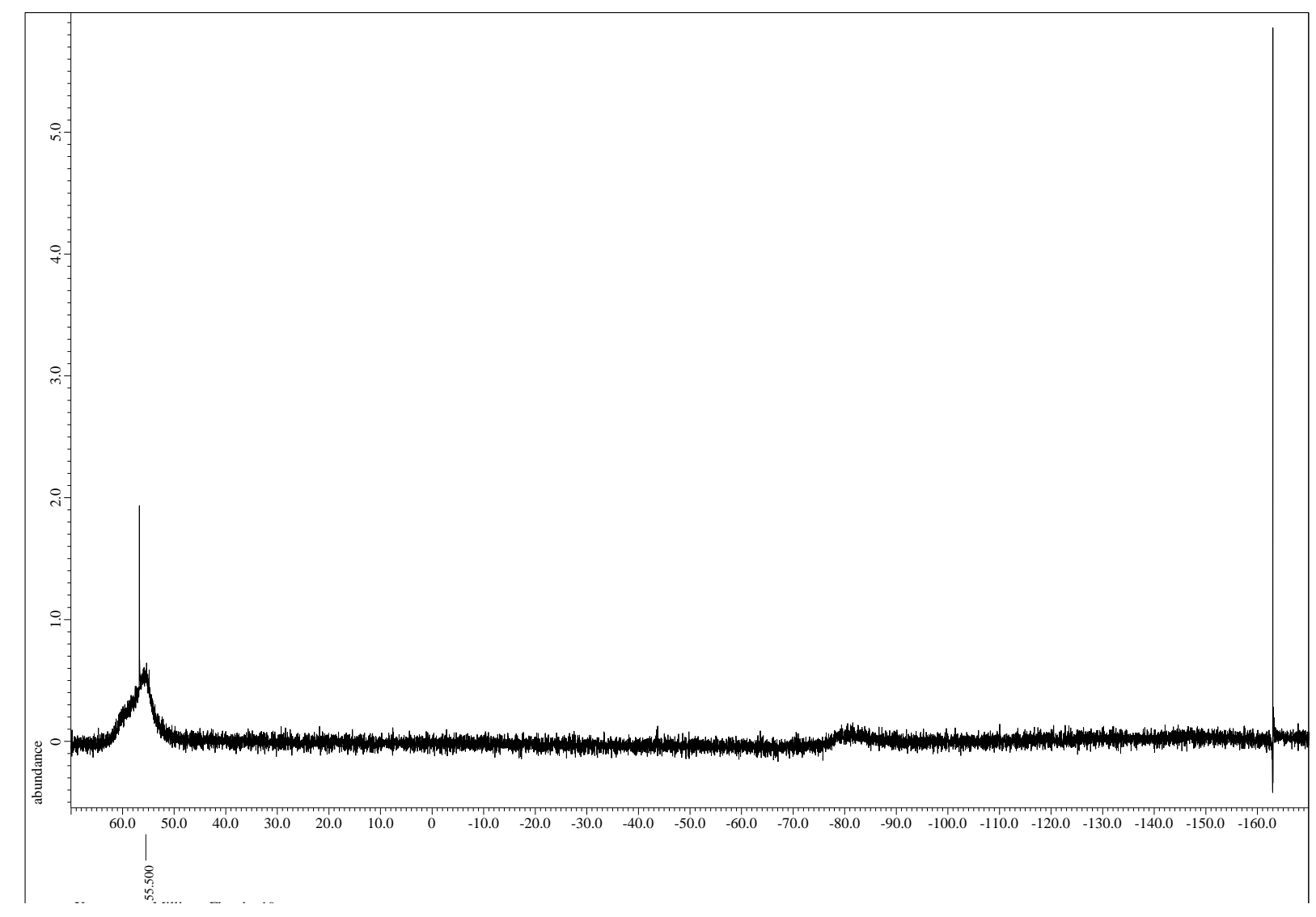



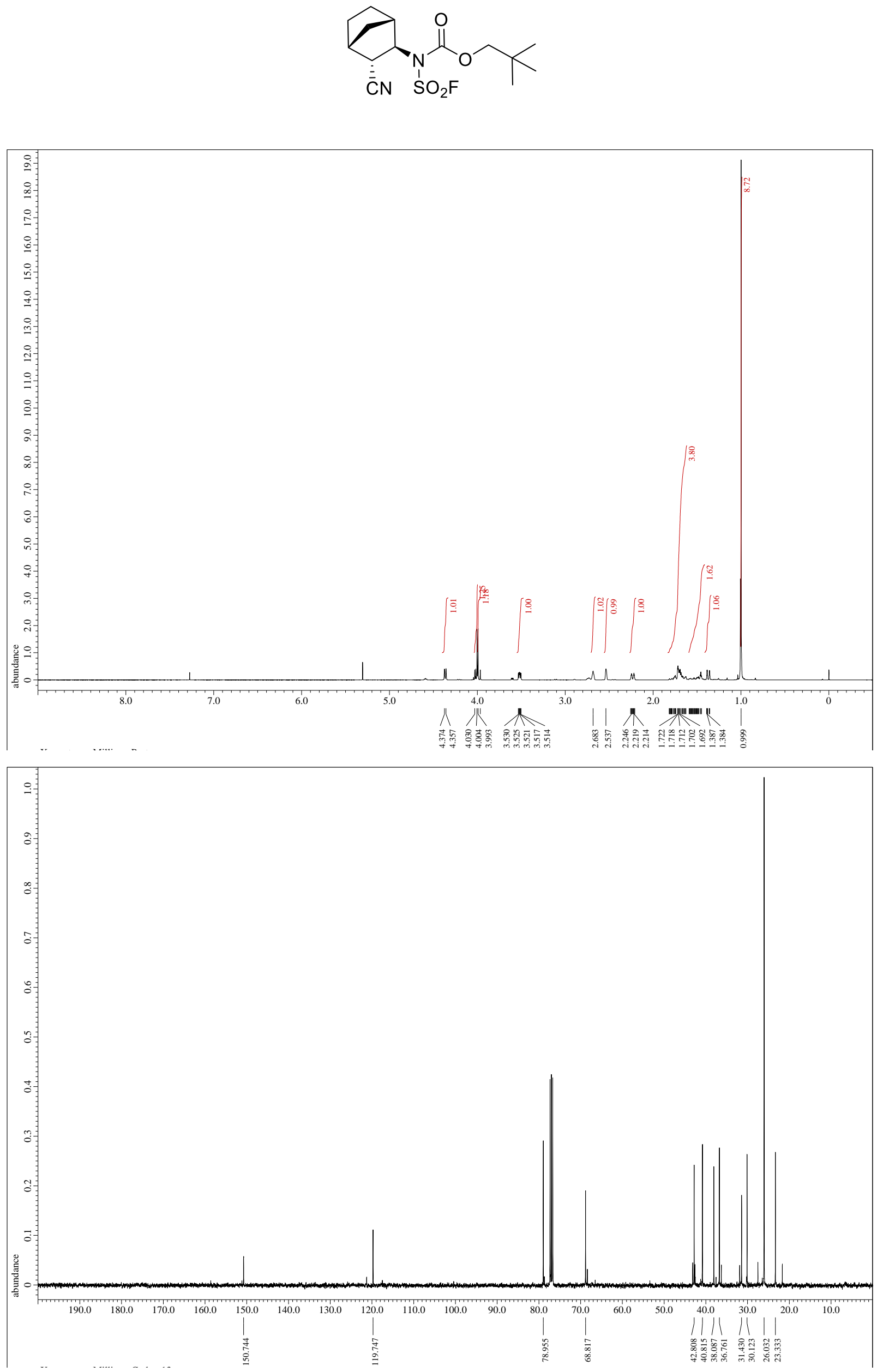


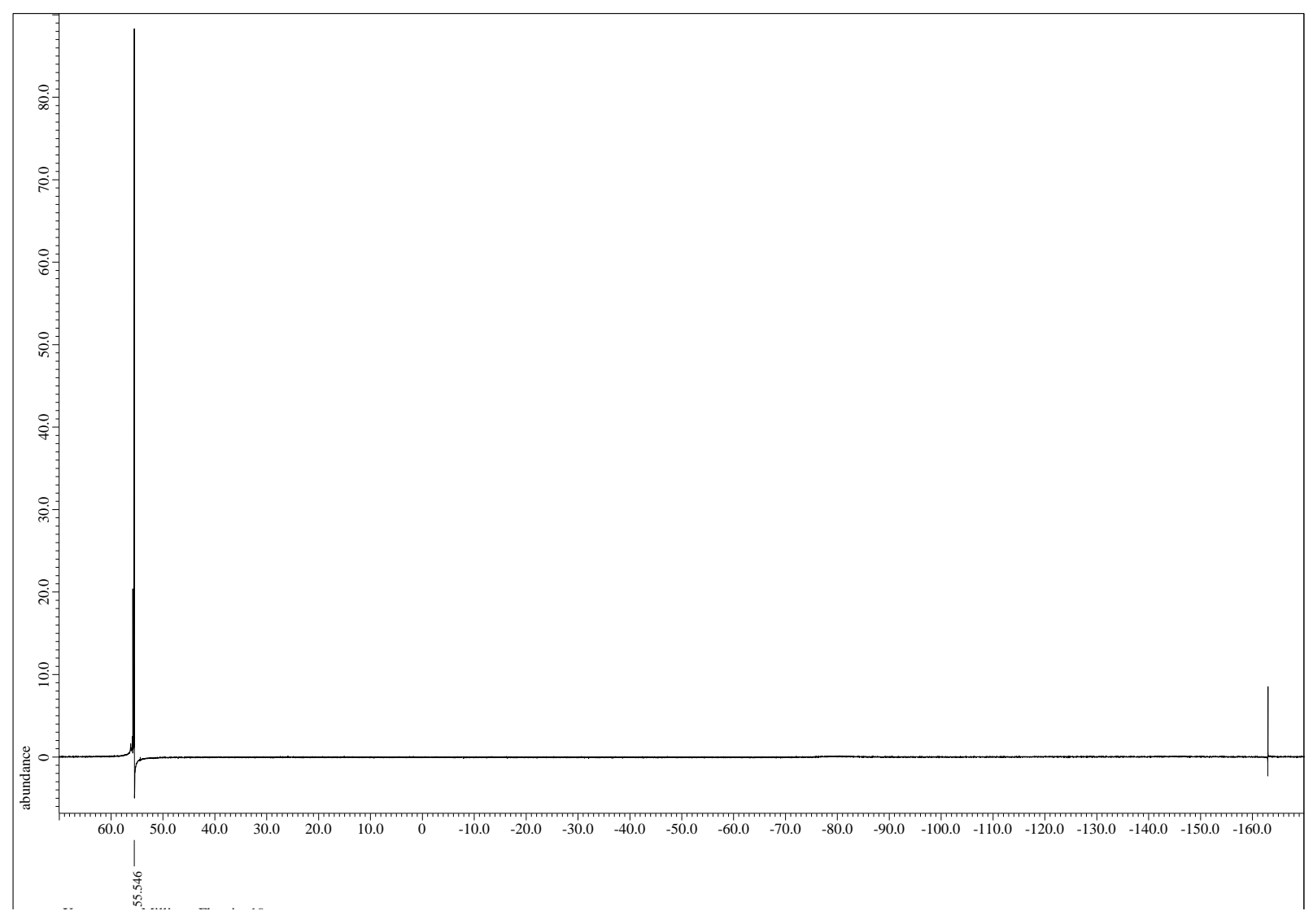



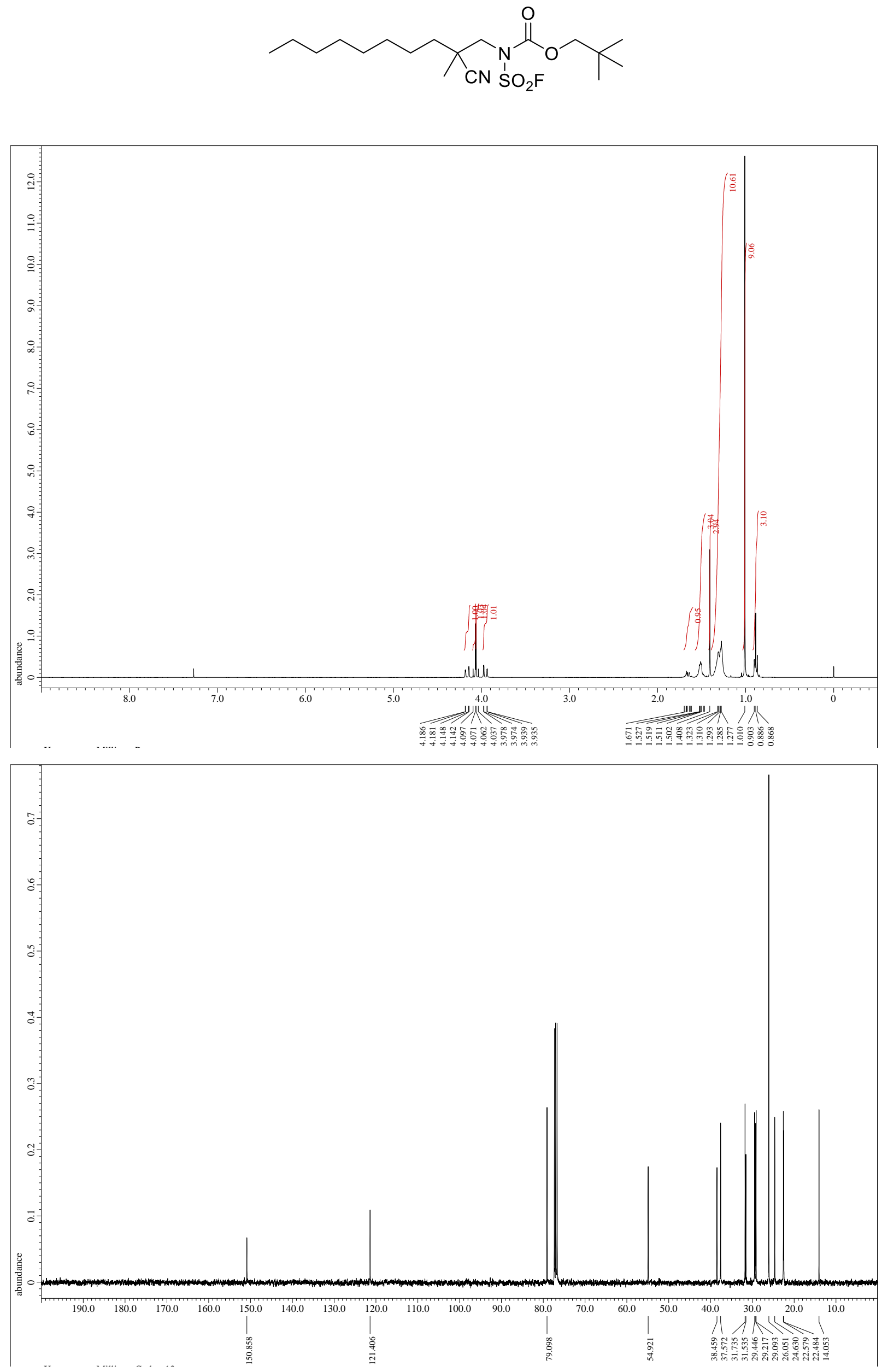


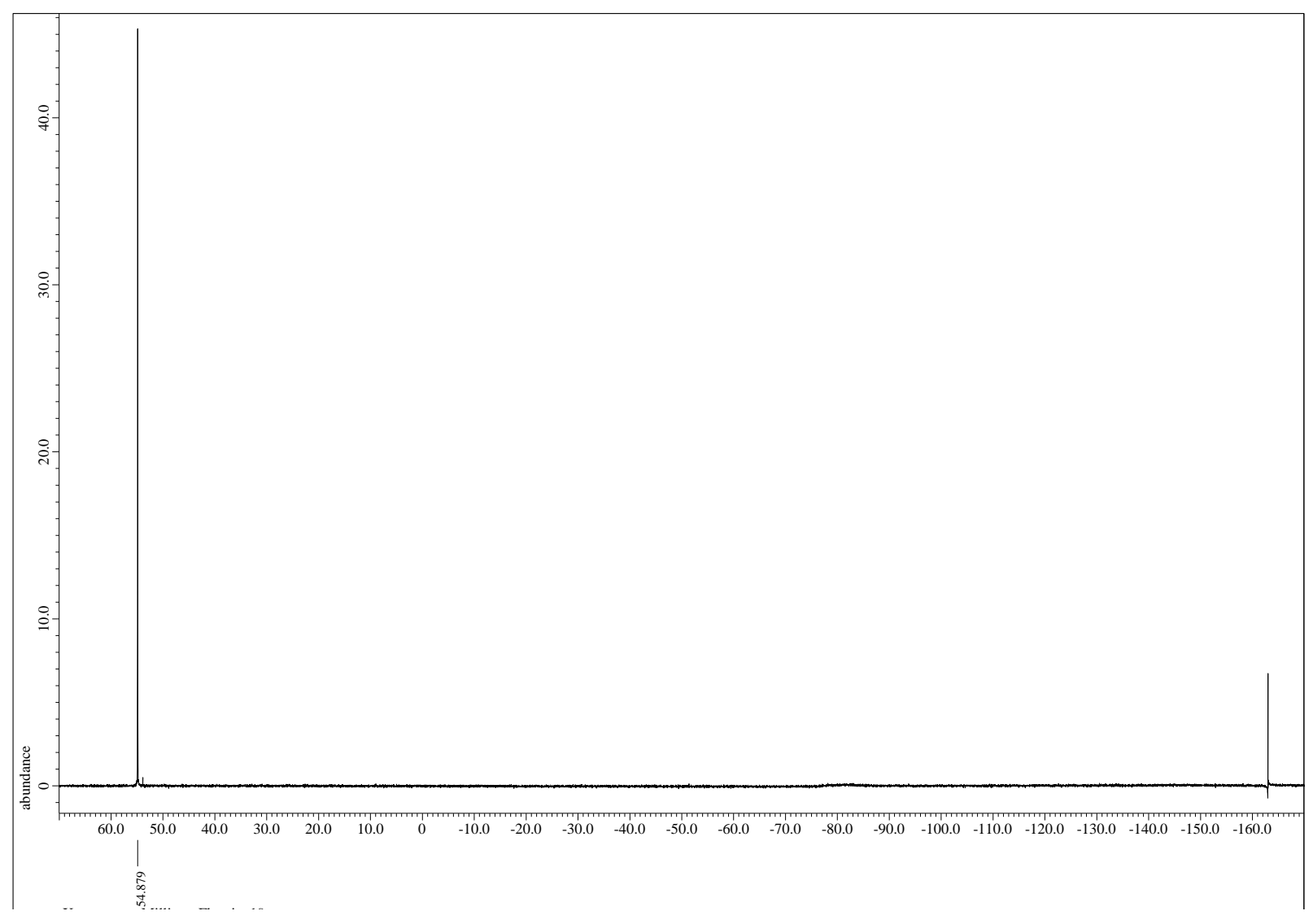


neopentyl (2-cyano-4-hydroxy-2-methylbutyl)(fluorosulfonyl)carbamate (52)
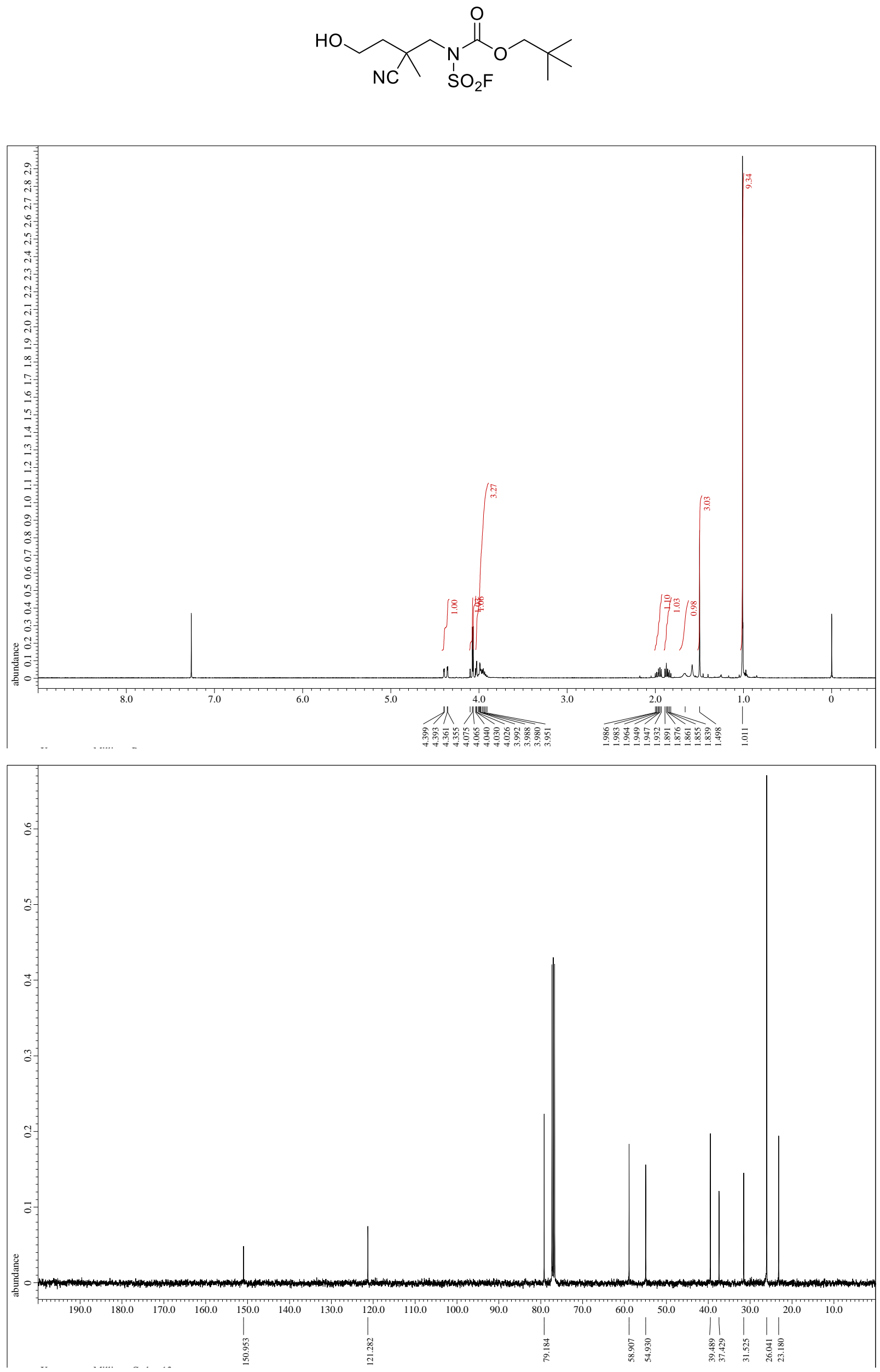


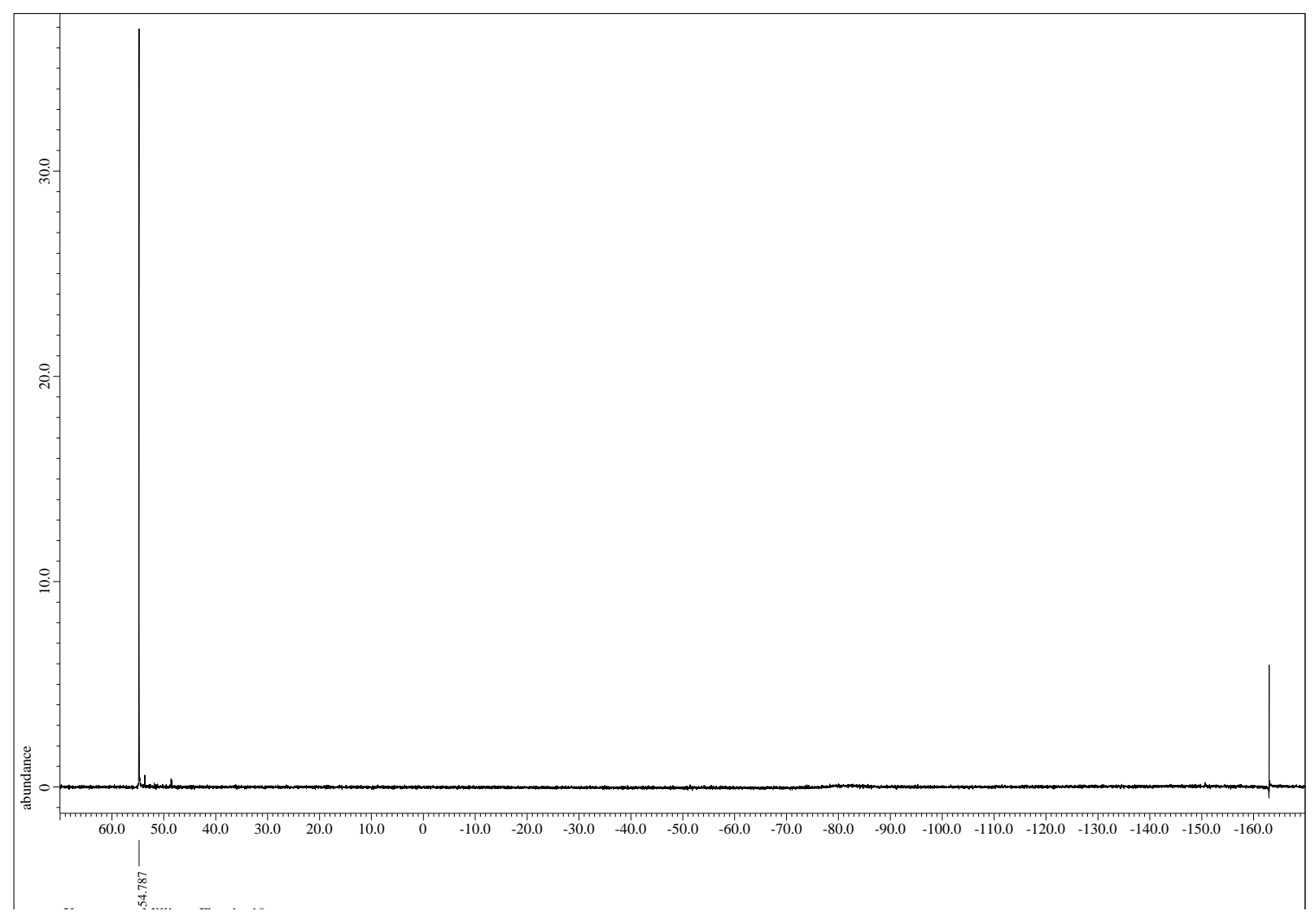



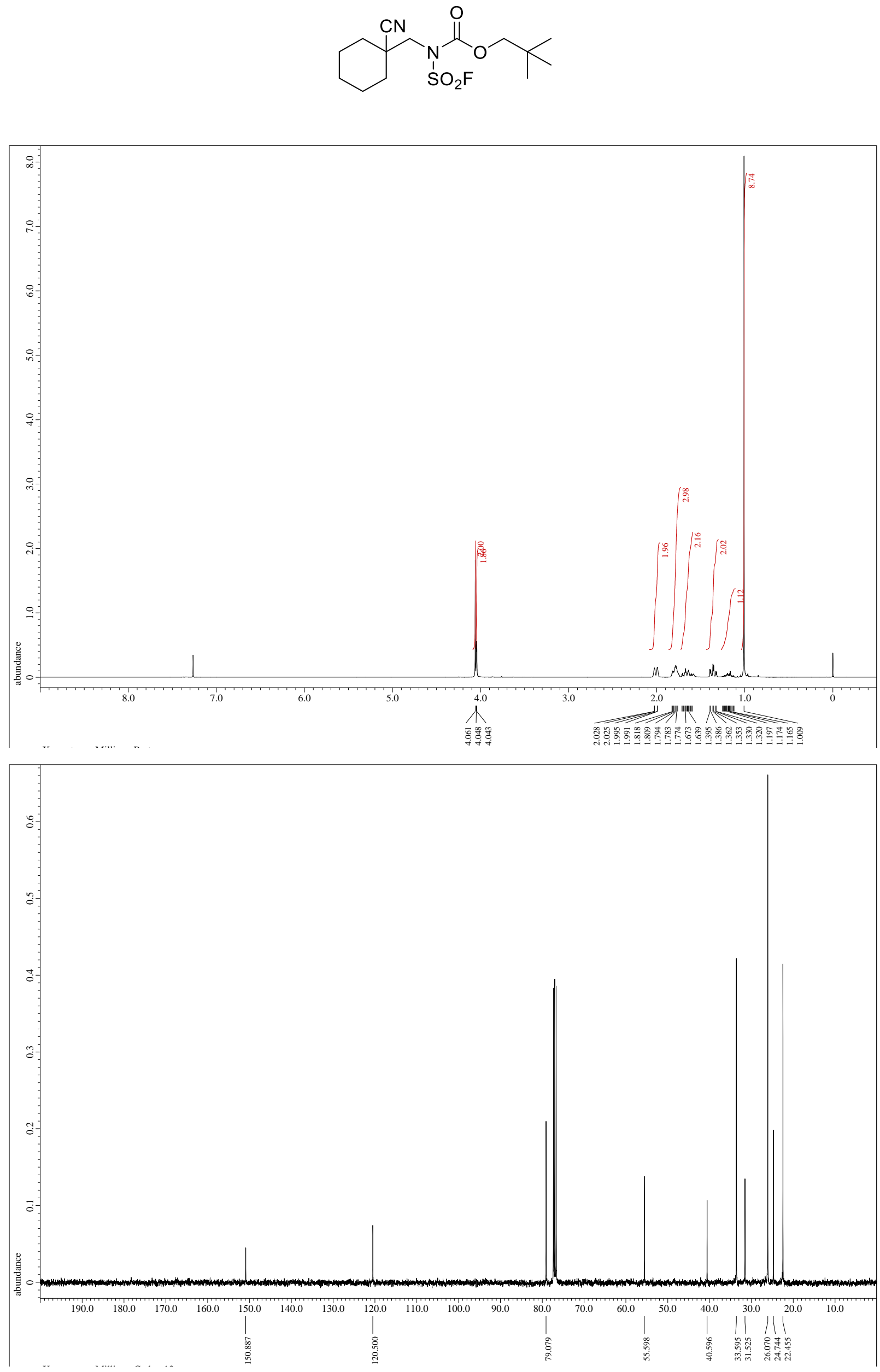


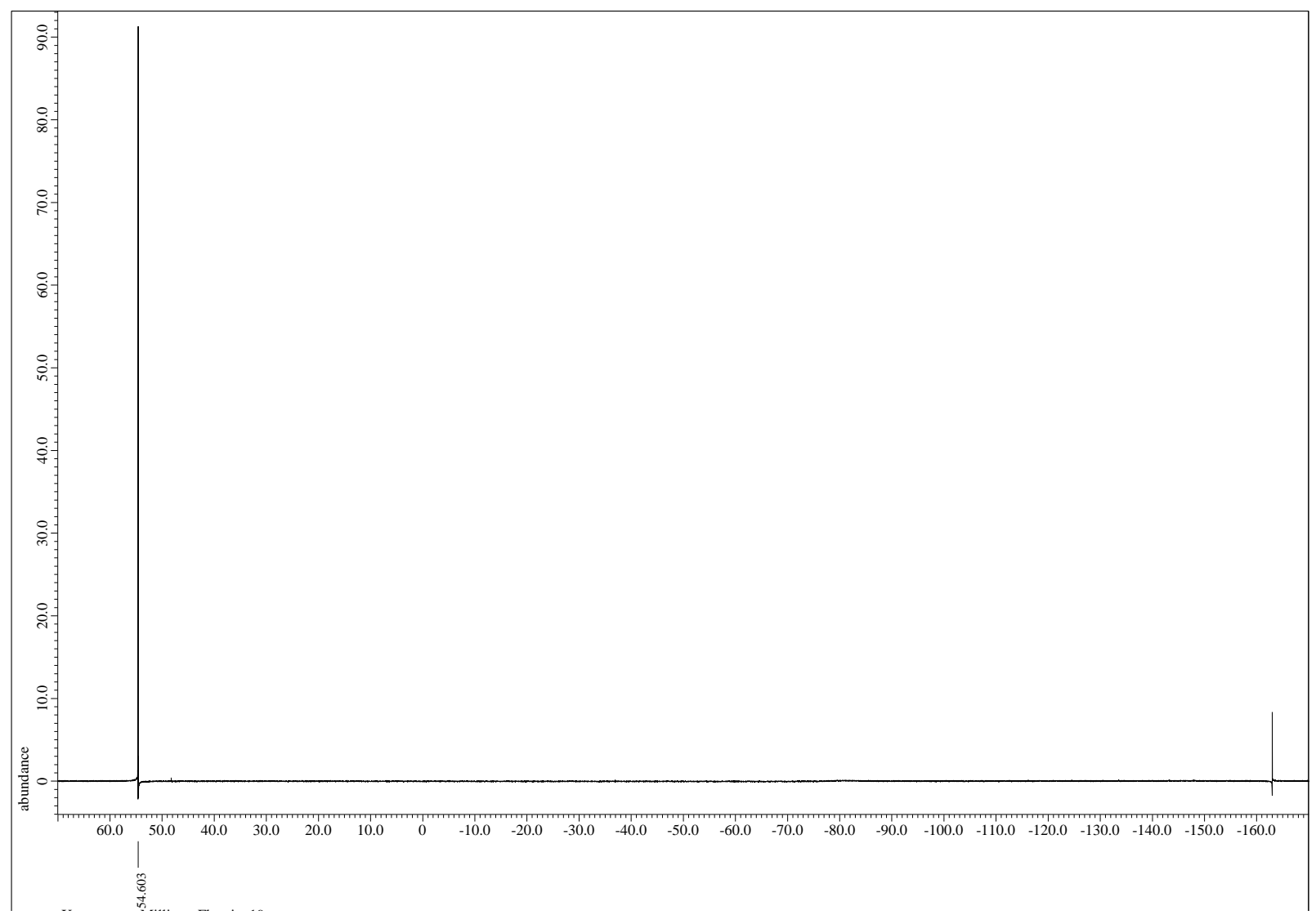



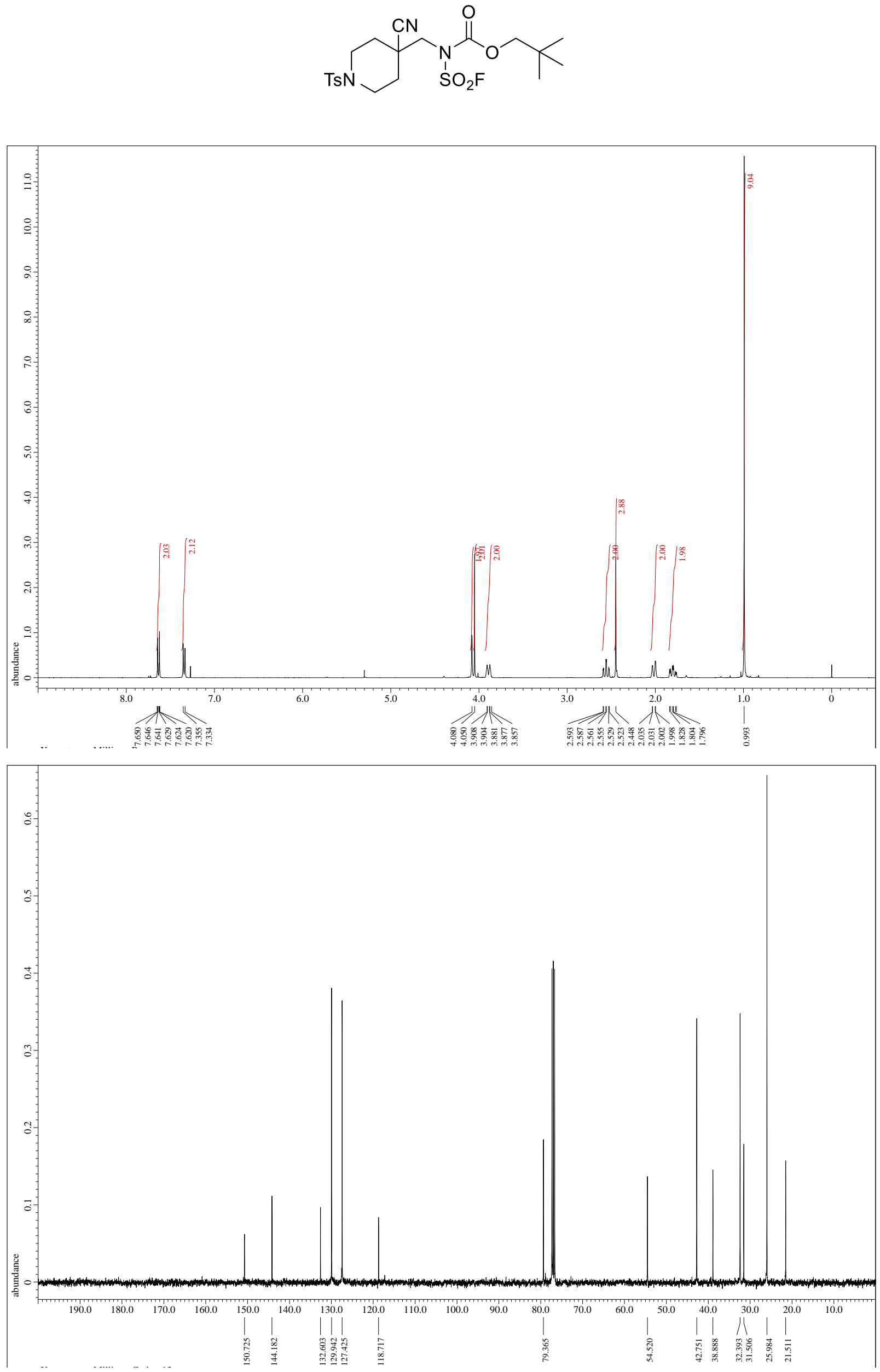


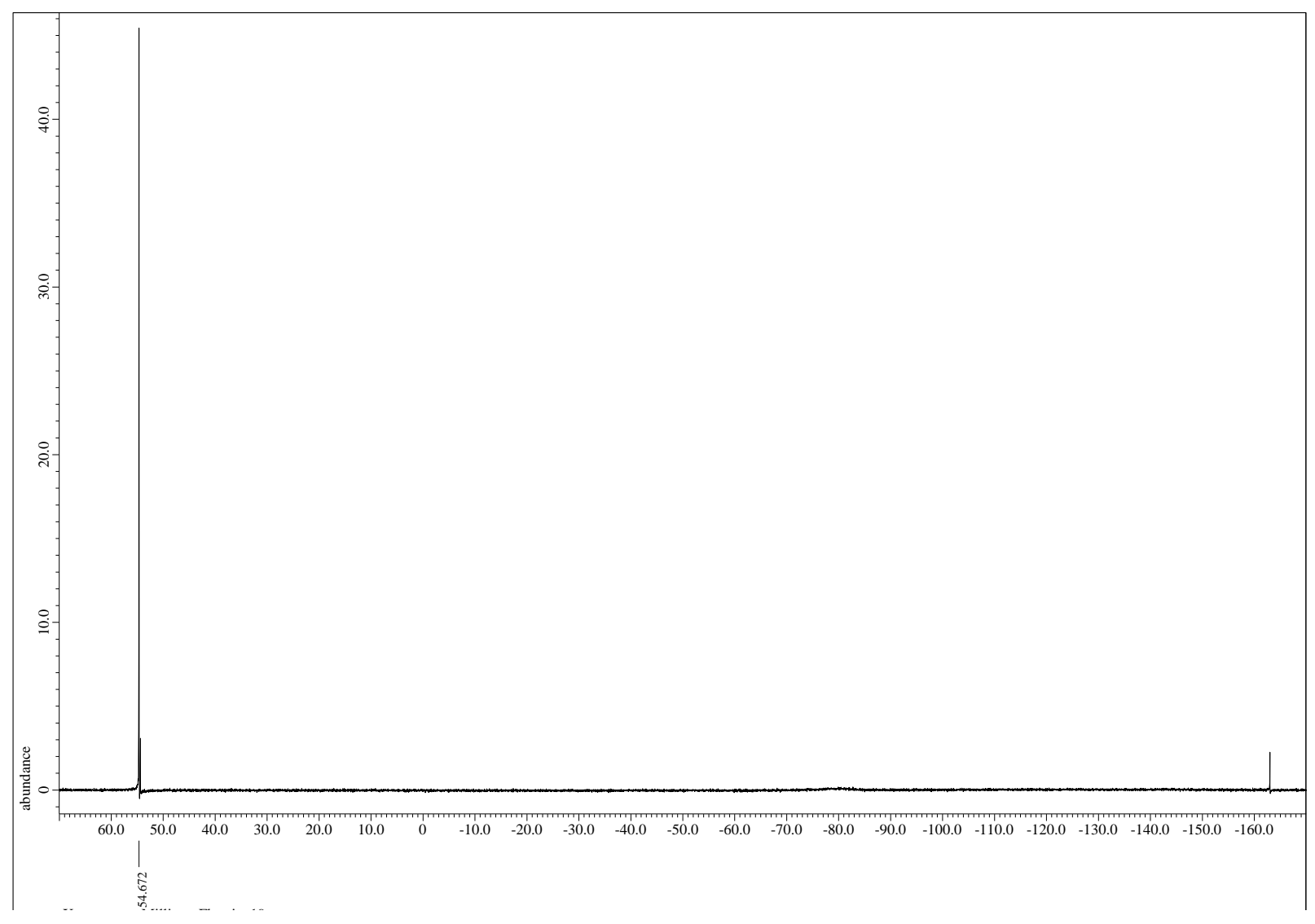


neopentyl (2-cyano-2-methylundecan-3-yl)(fluorosulfonyl)carbamate (55)
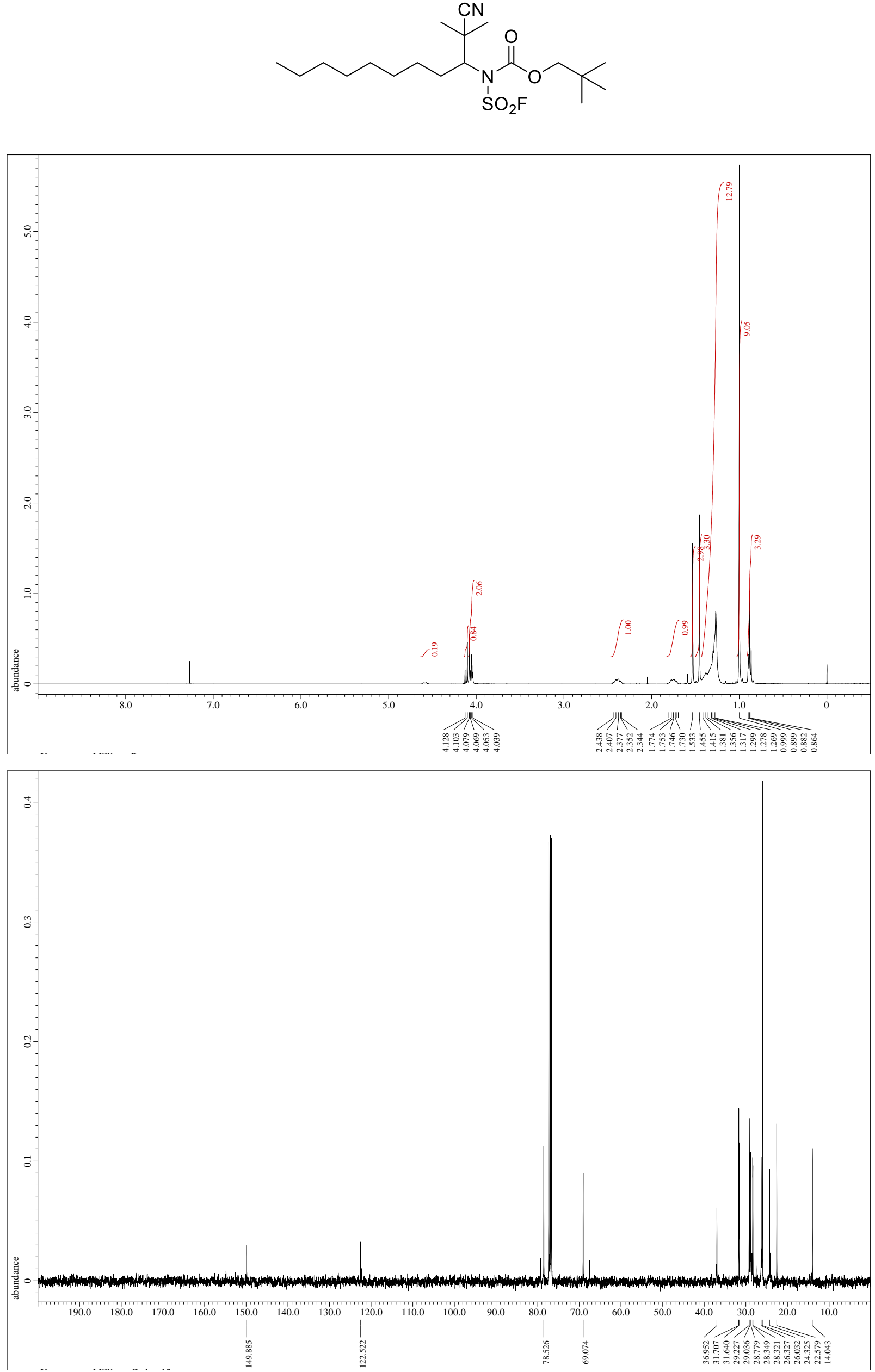


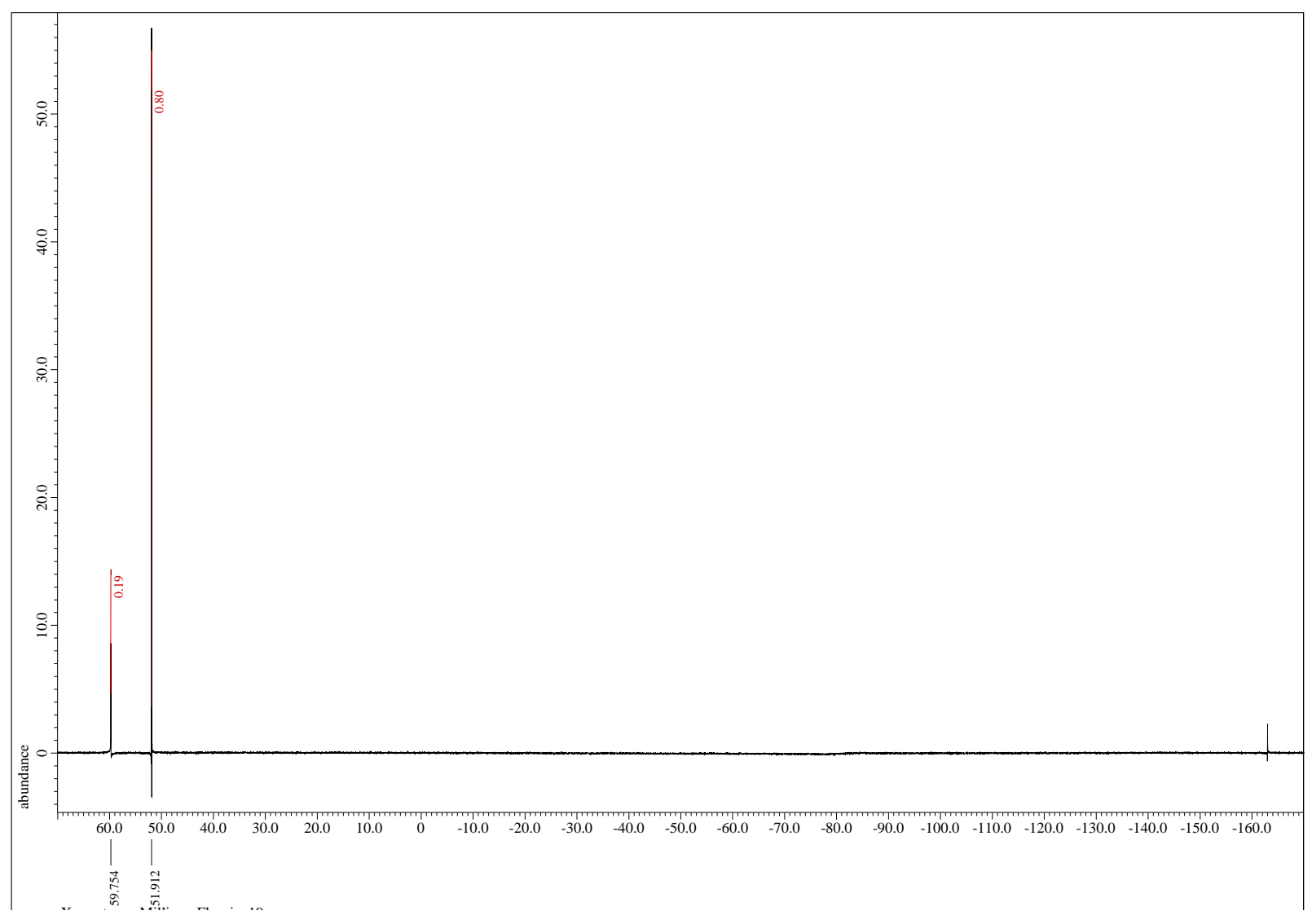



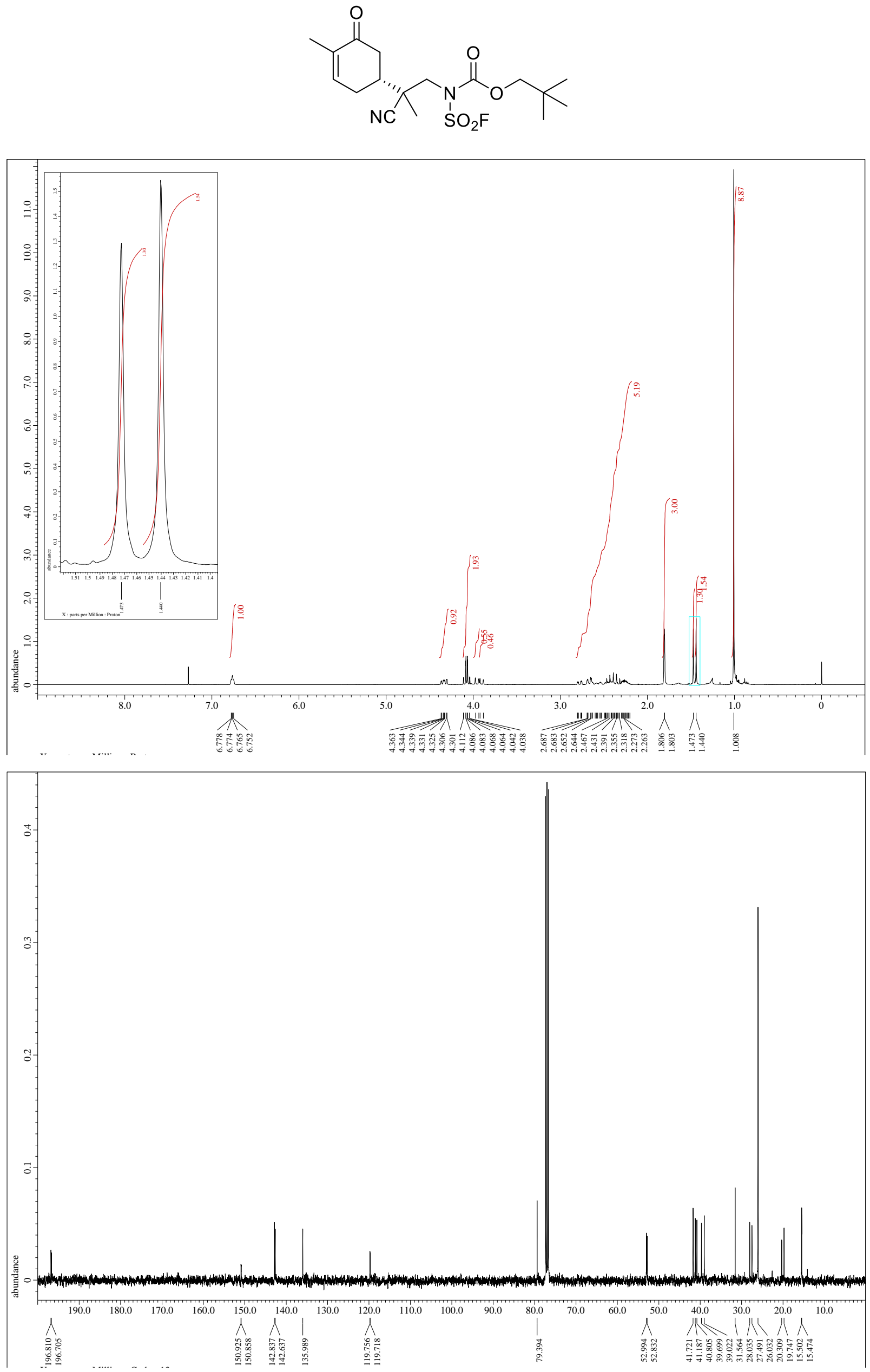


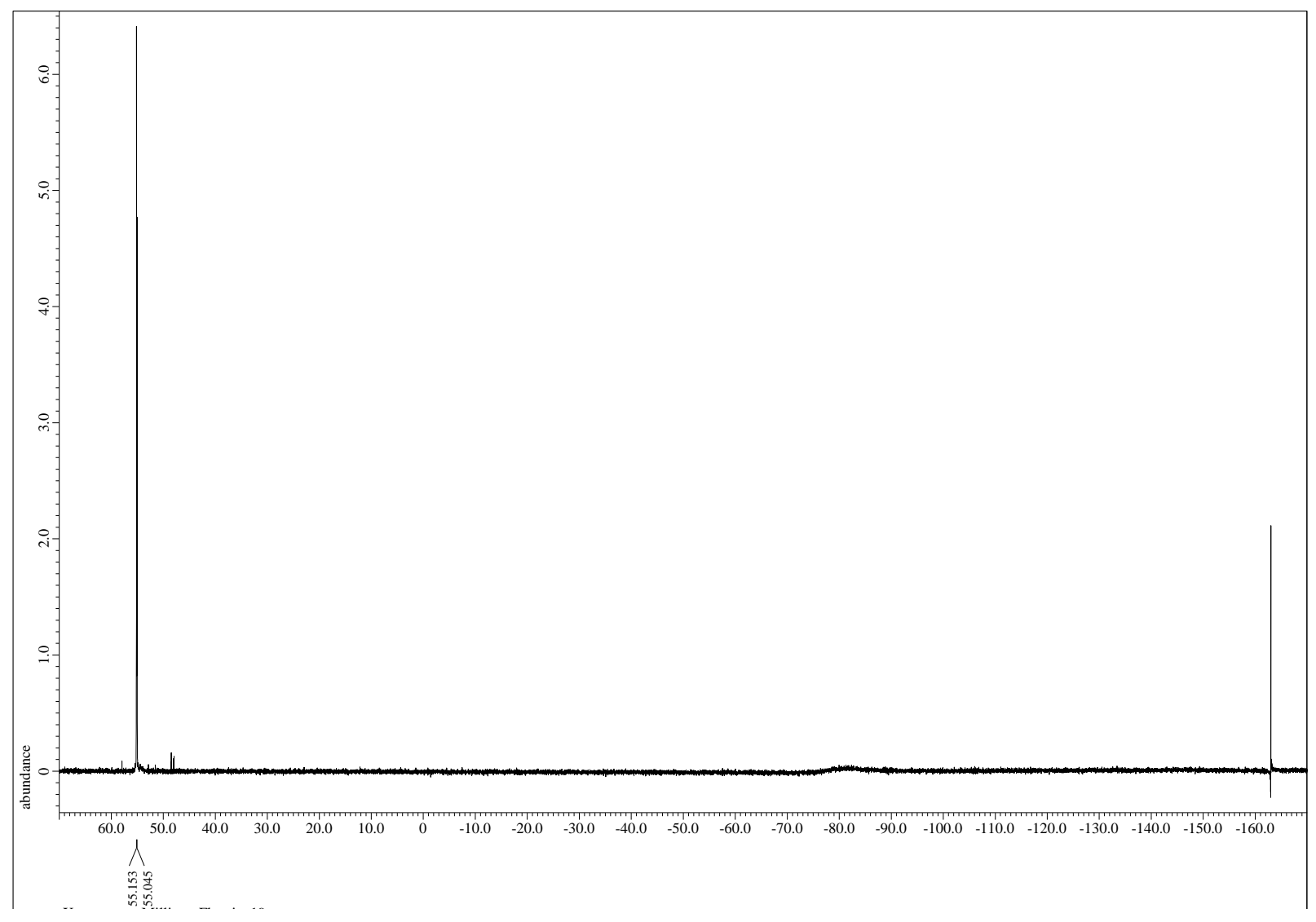



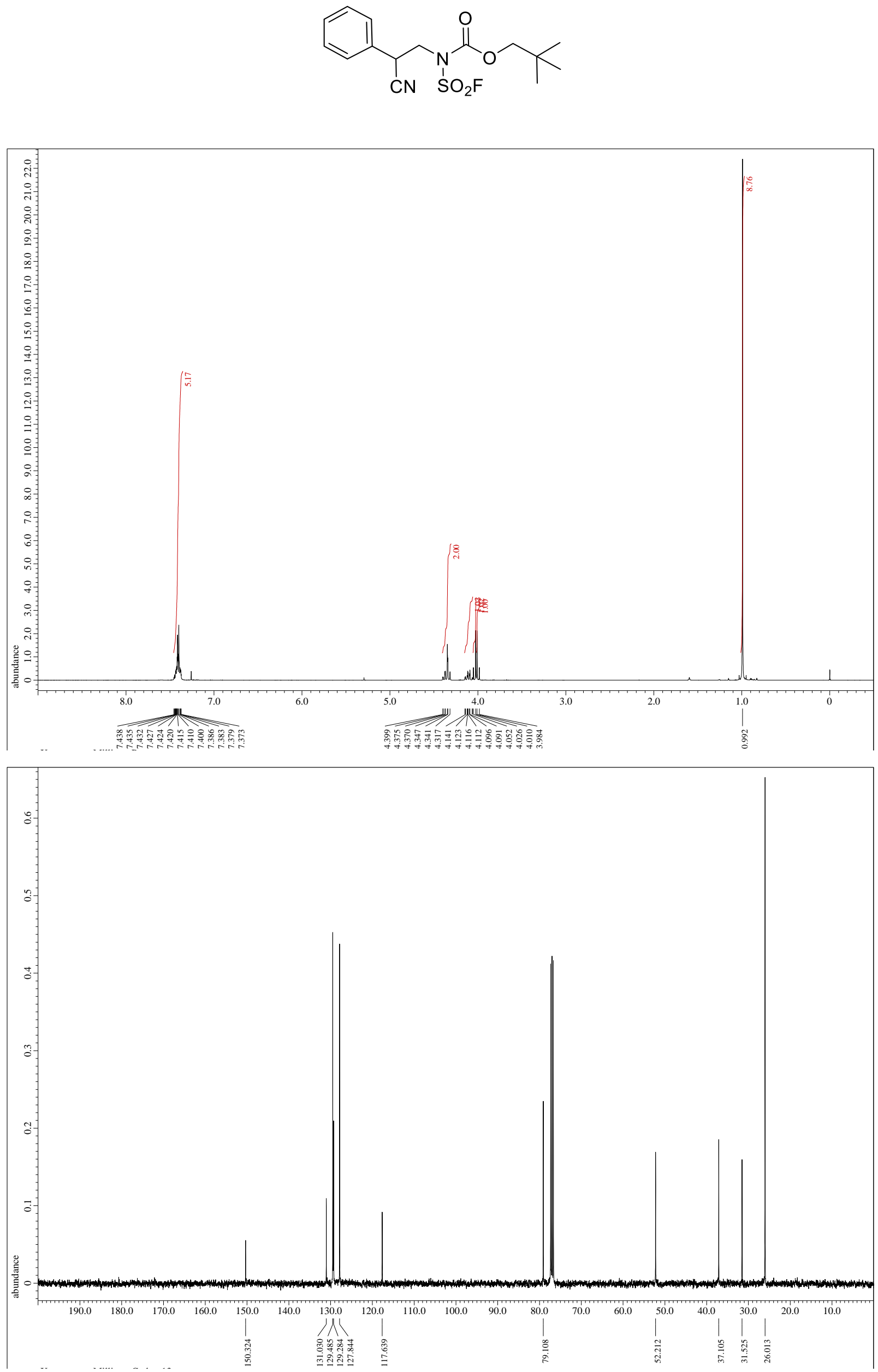


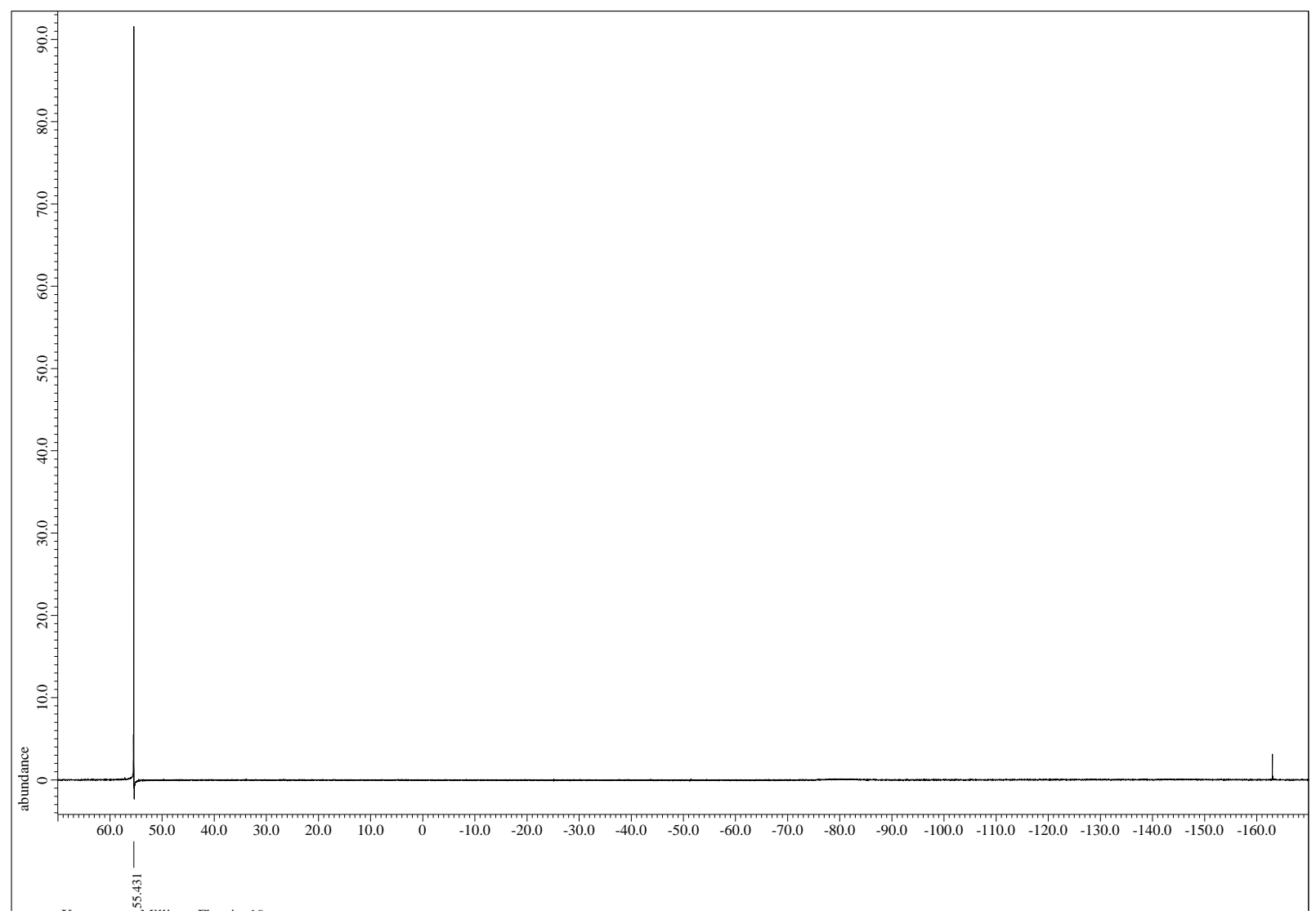



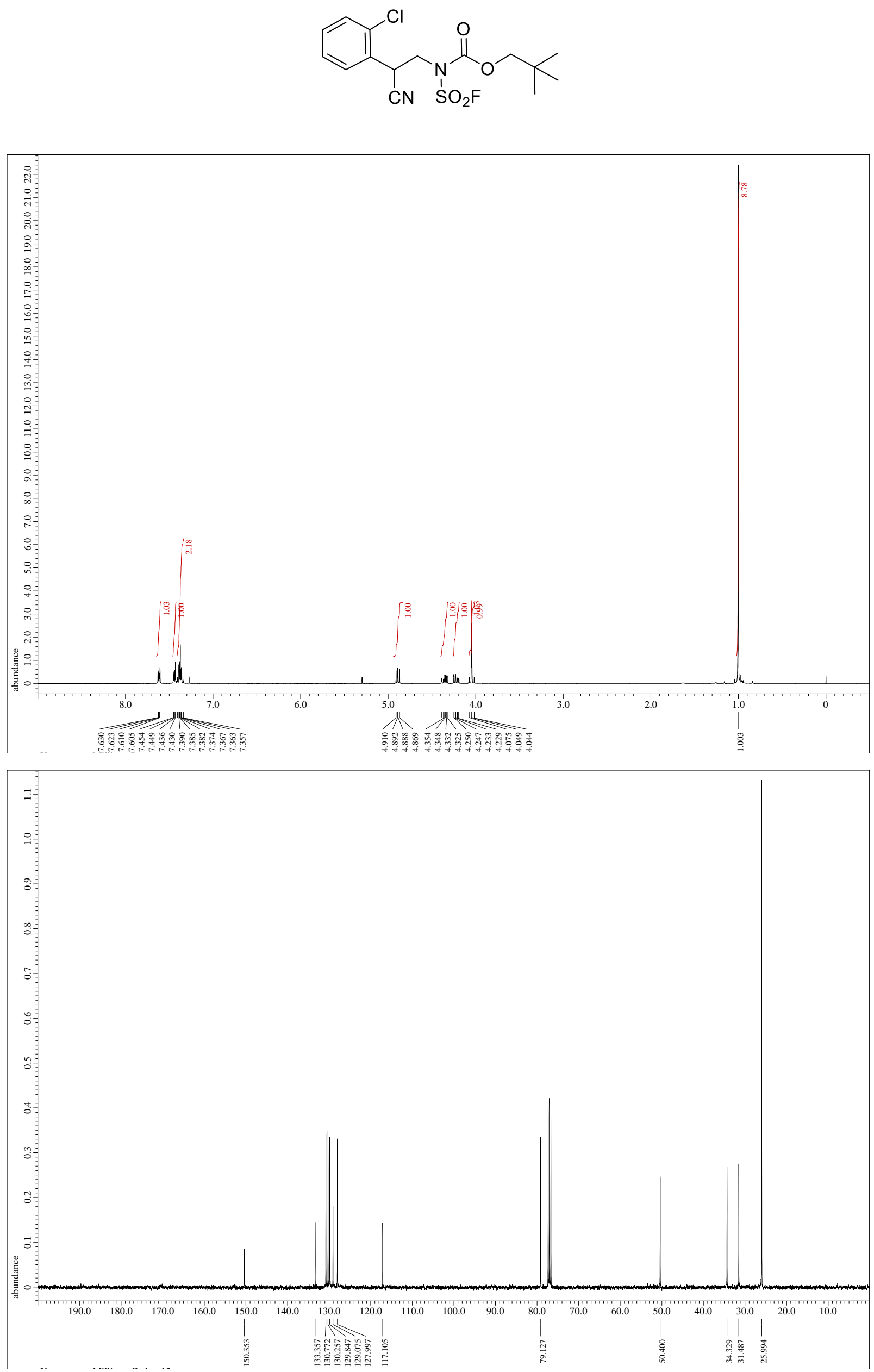


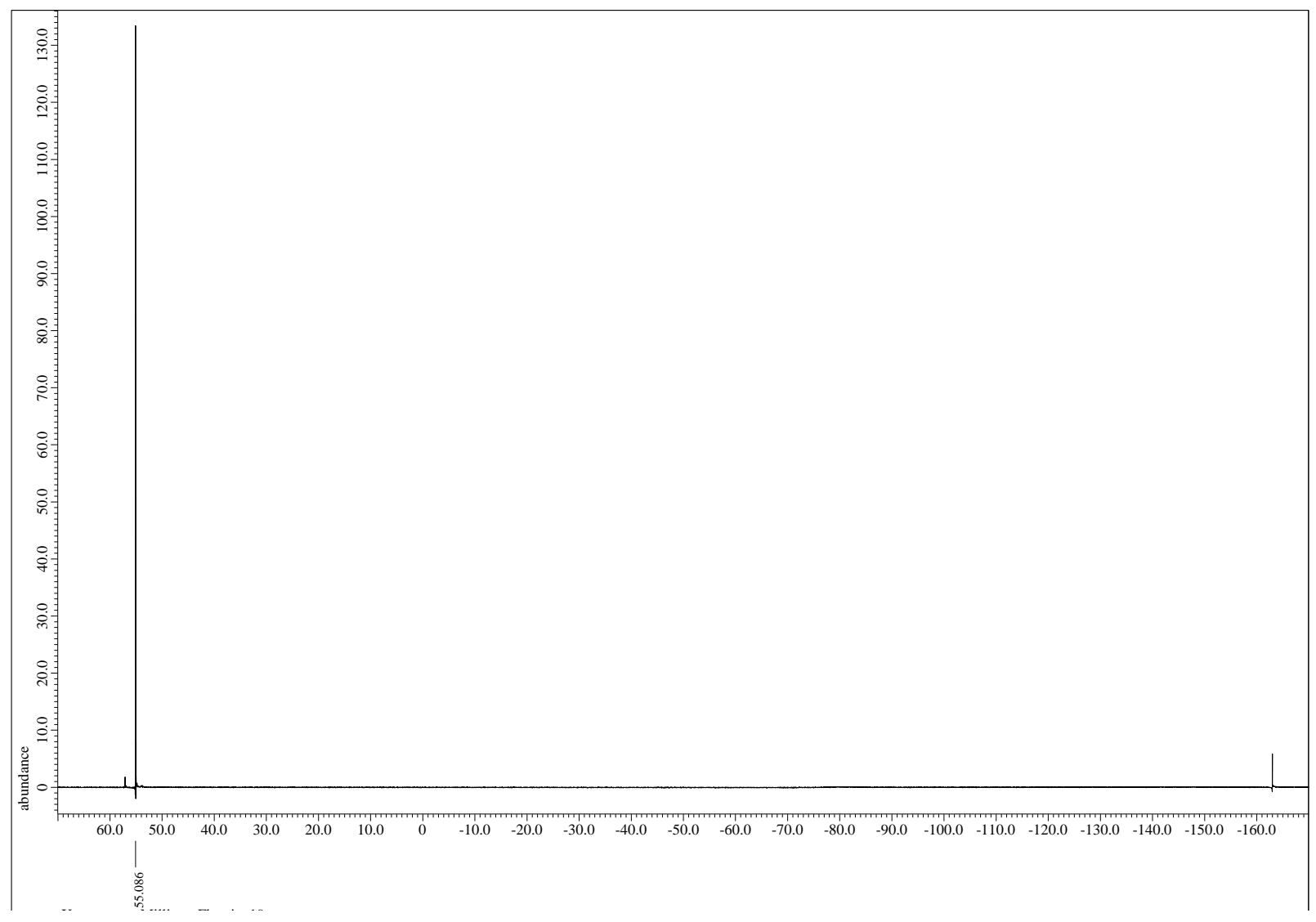


neopentyl (2-(3-chlorophenyl)-2-cyanoethyl)(fluorosulfonyl)carbamate (59)
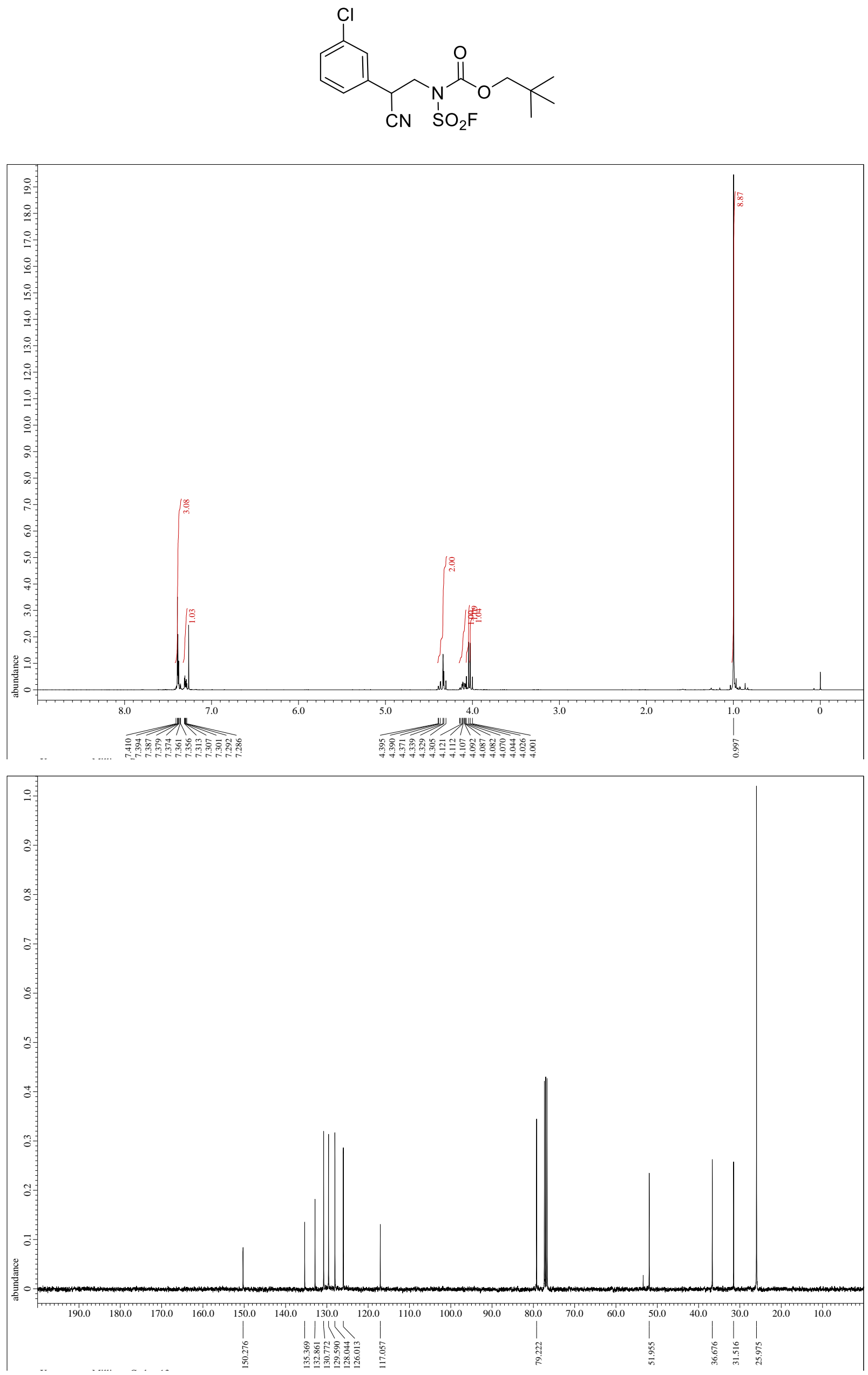


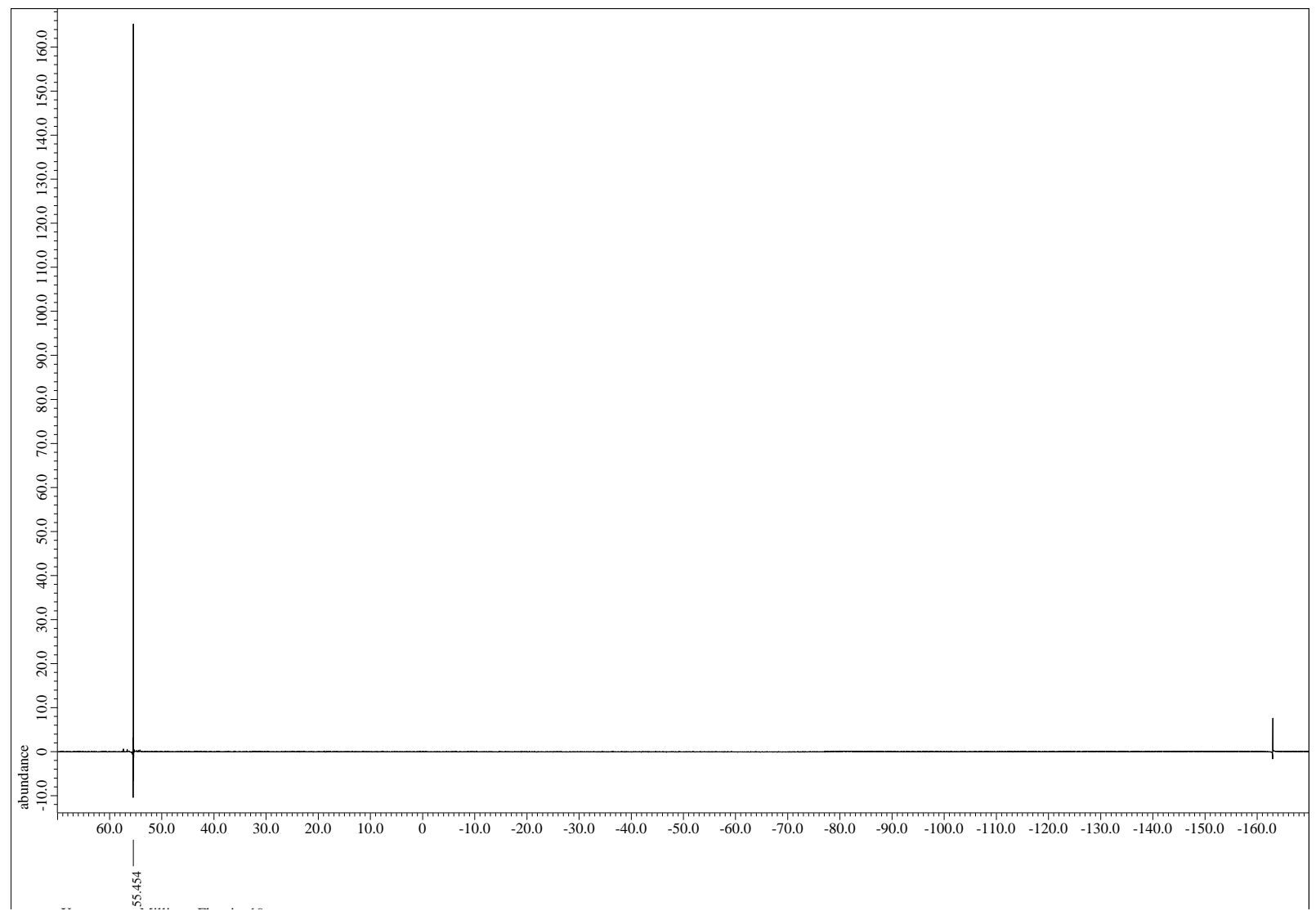



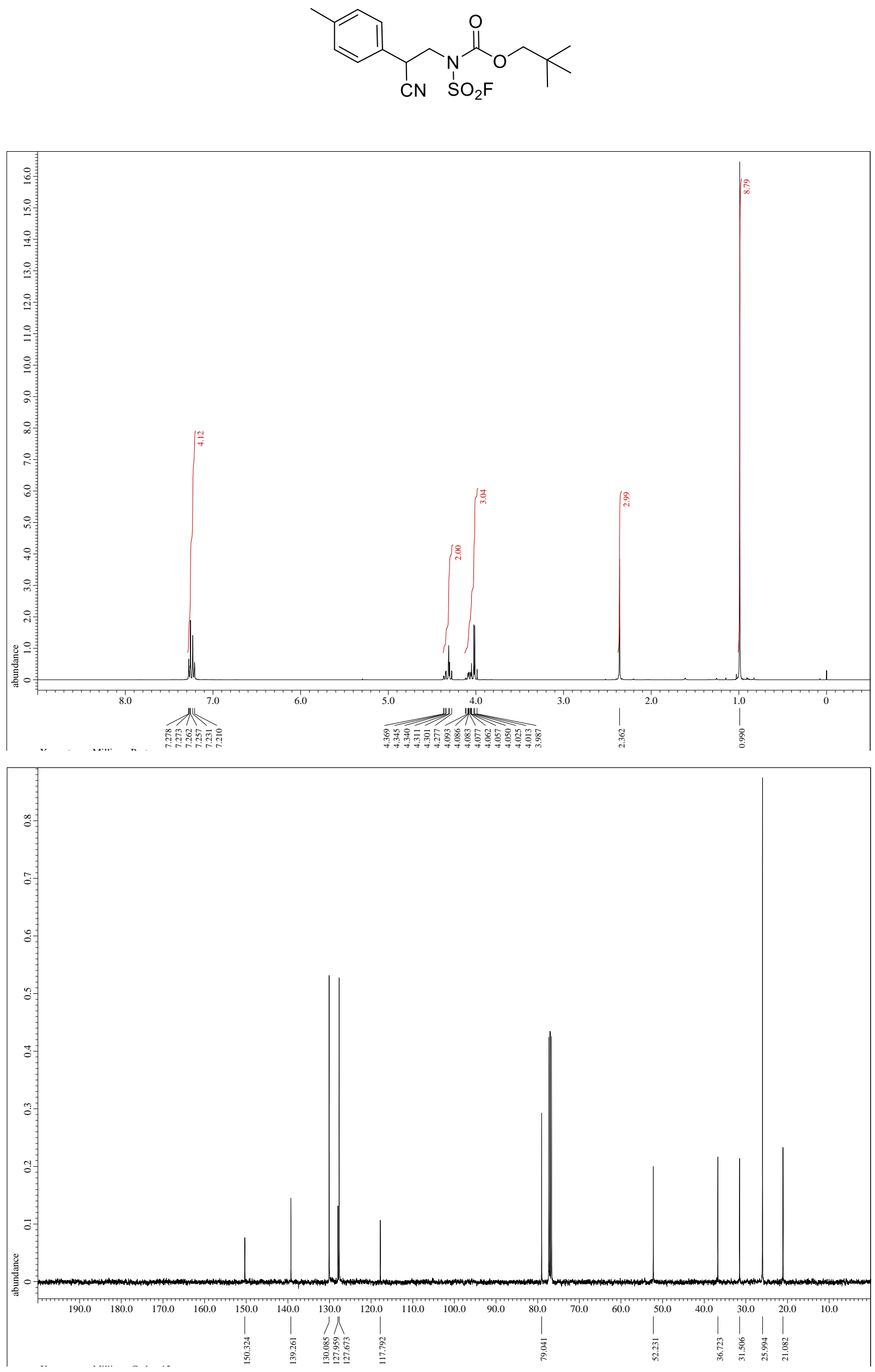


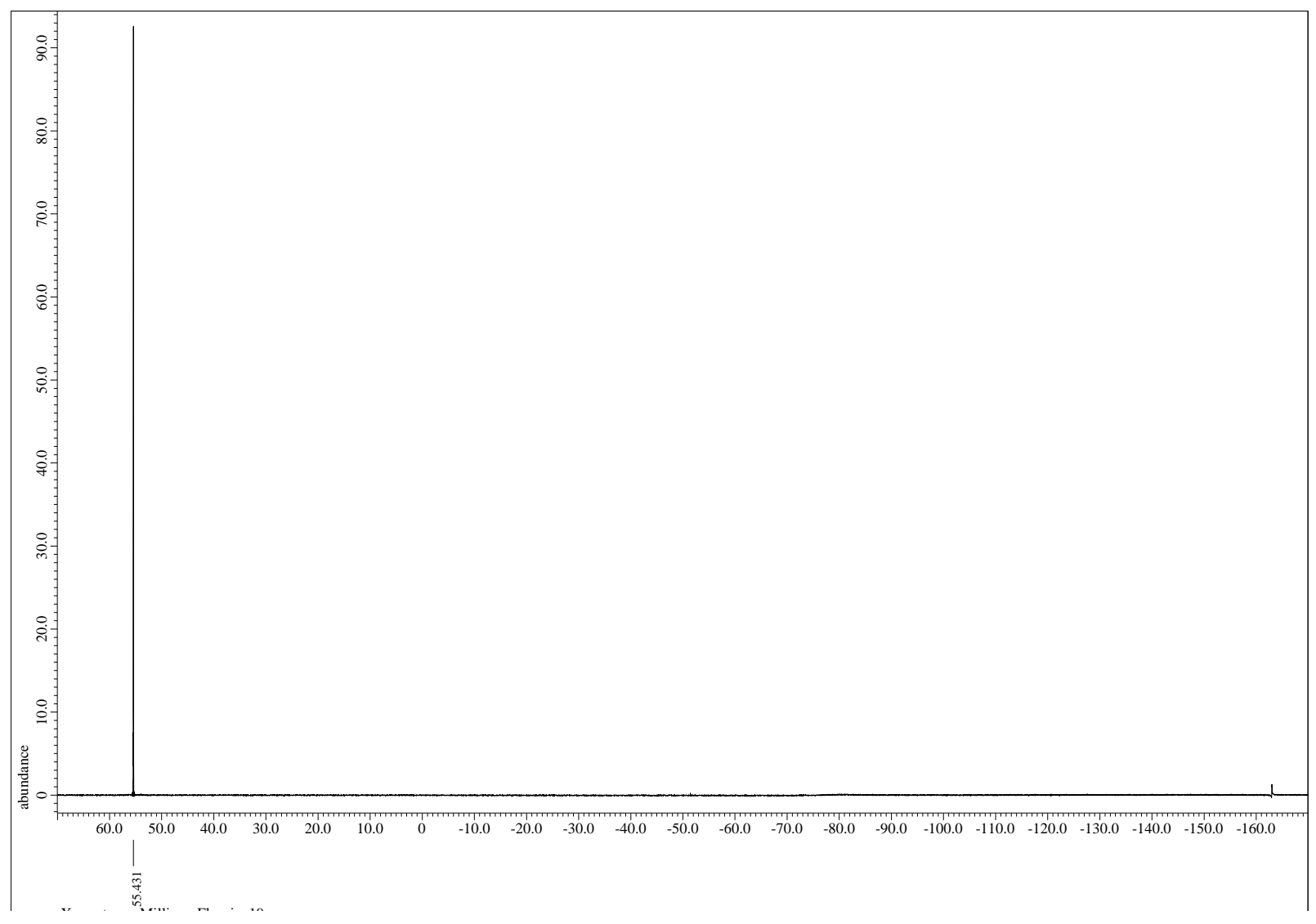



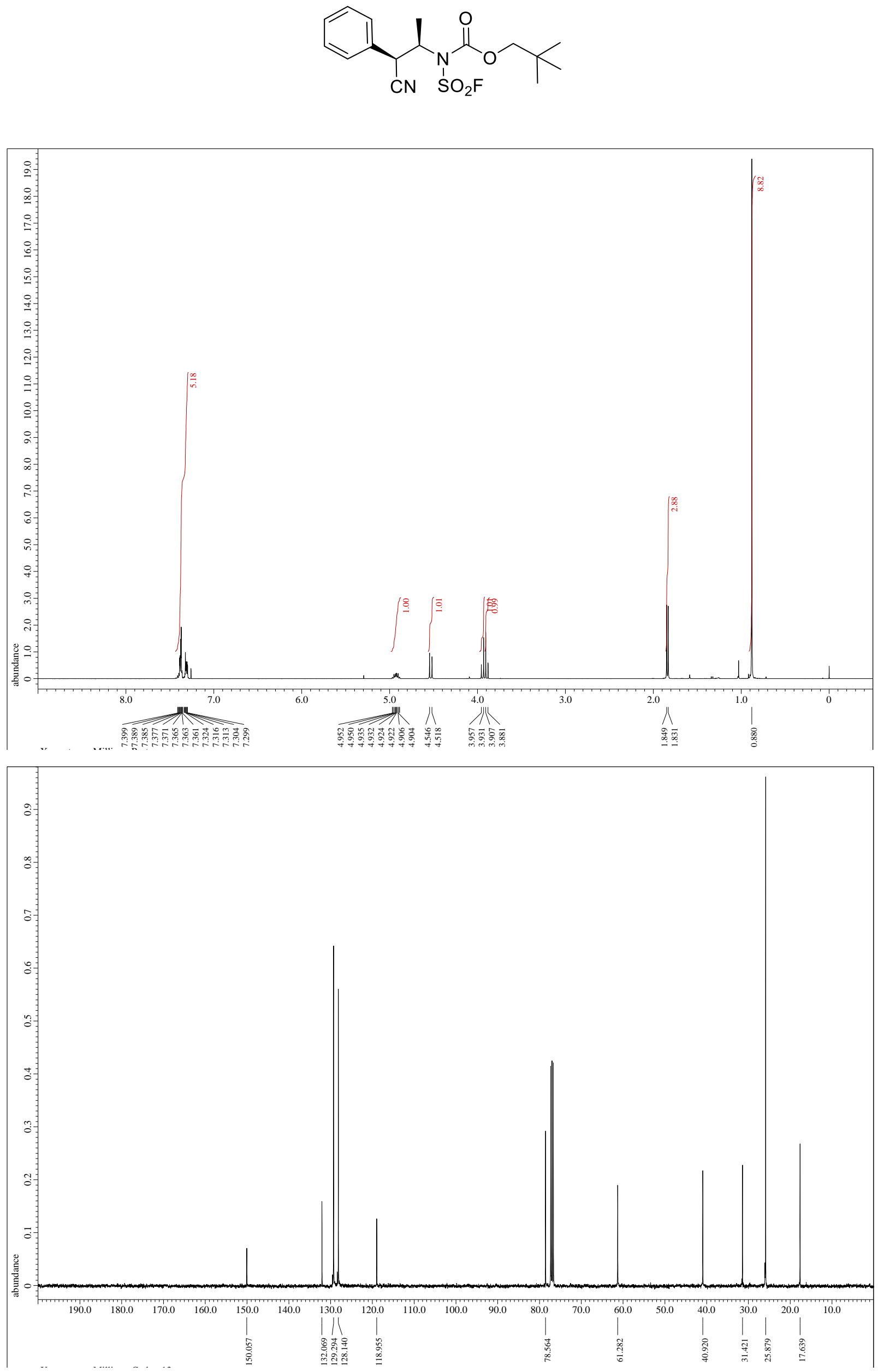


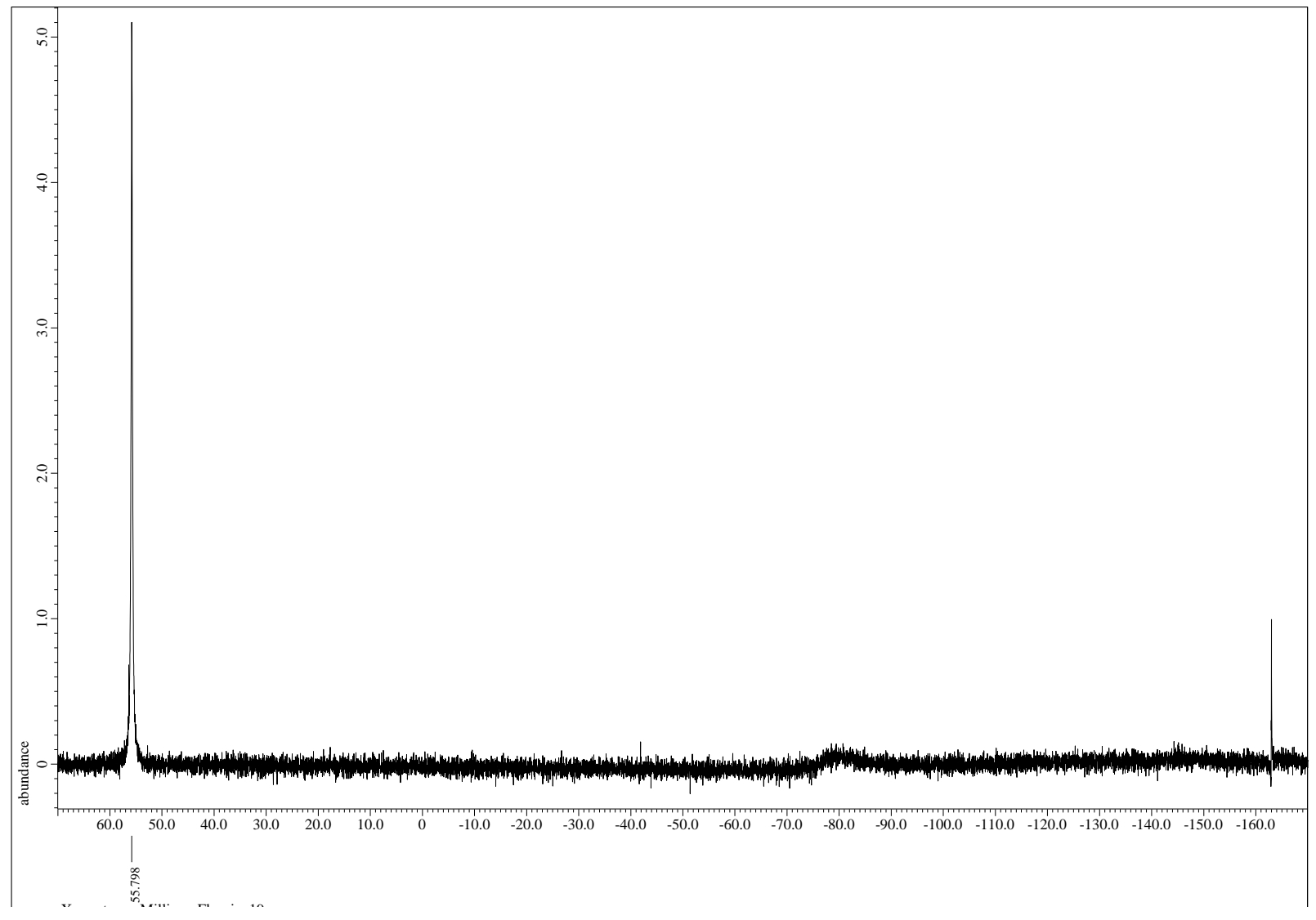



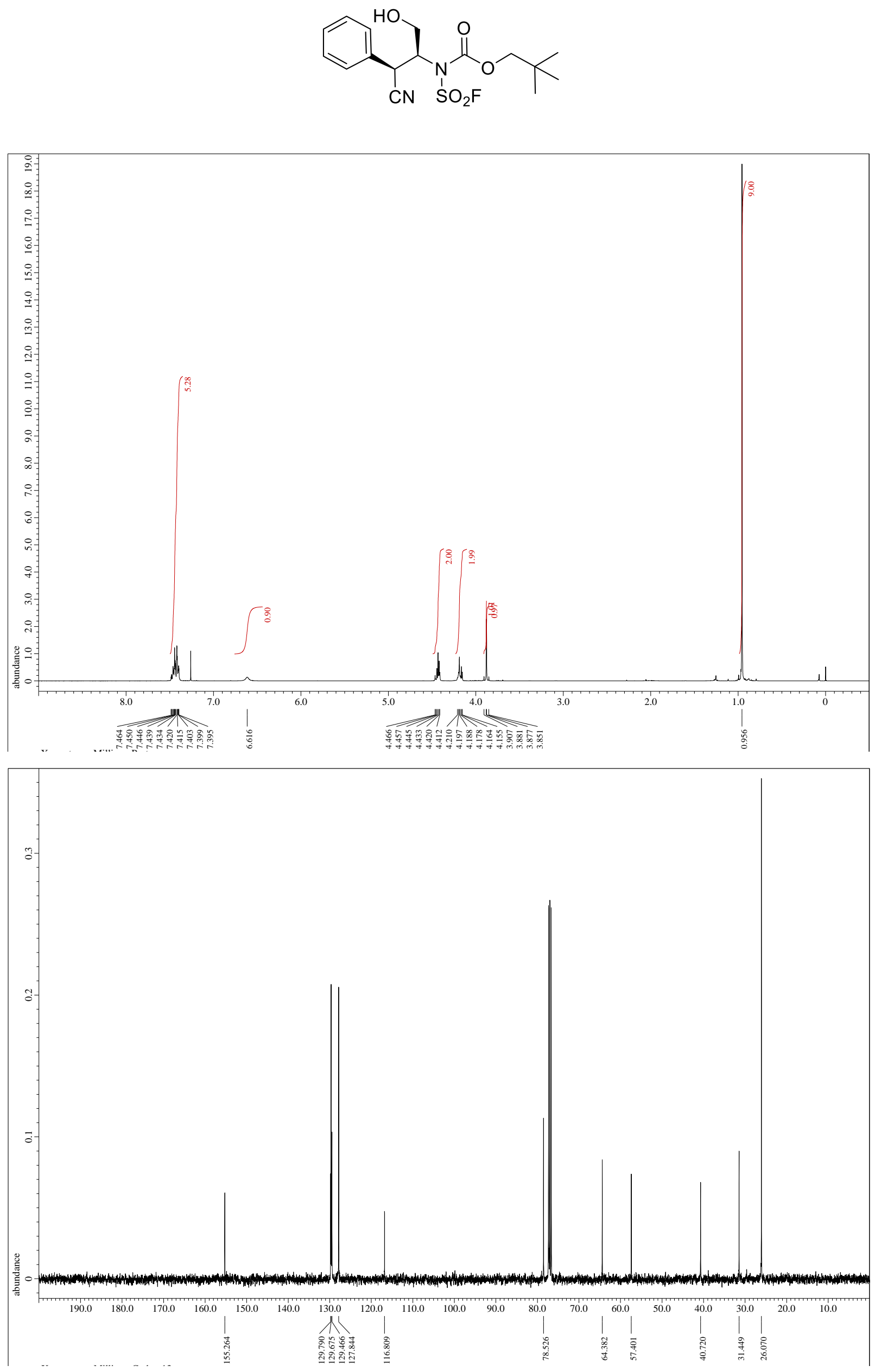


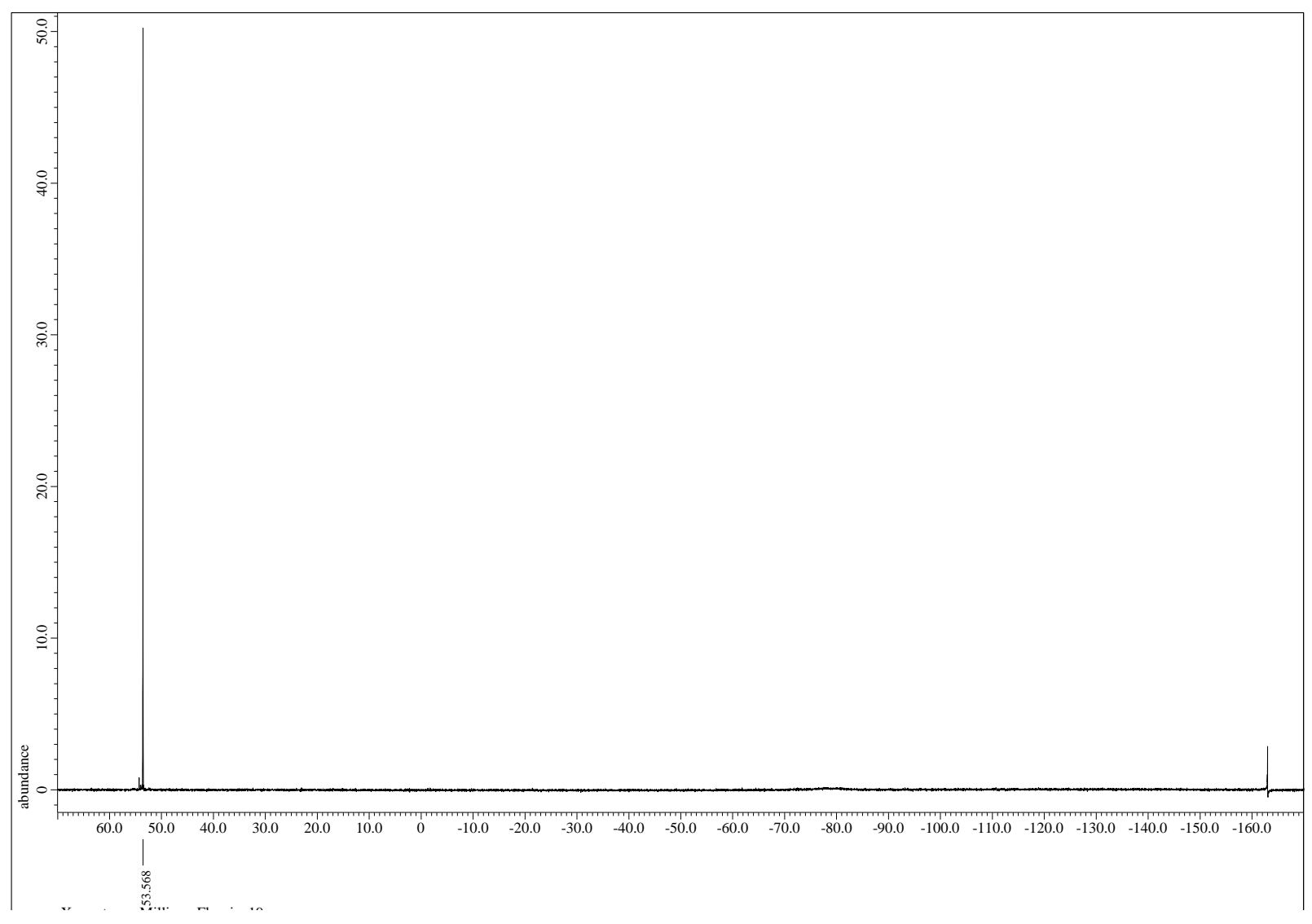




$$
\overbrace{n \mathrm{Hex}}^{\mathrm{CN}} \mathrm{NHBOC}
$$
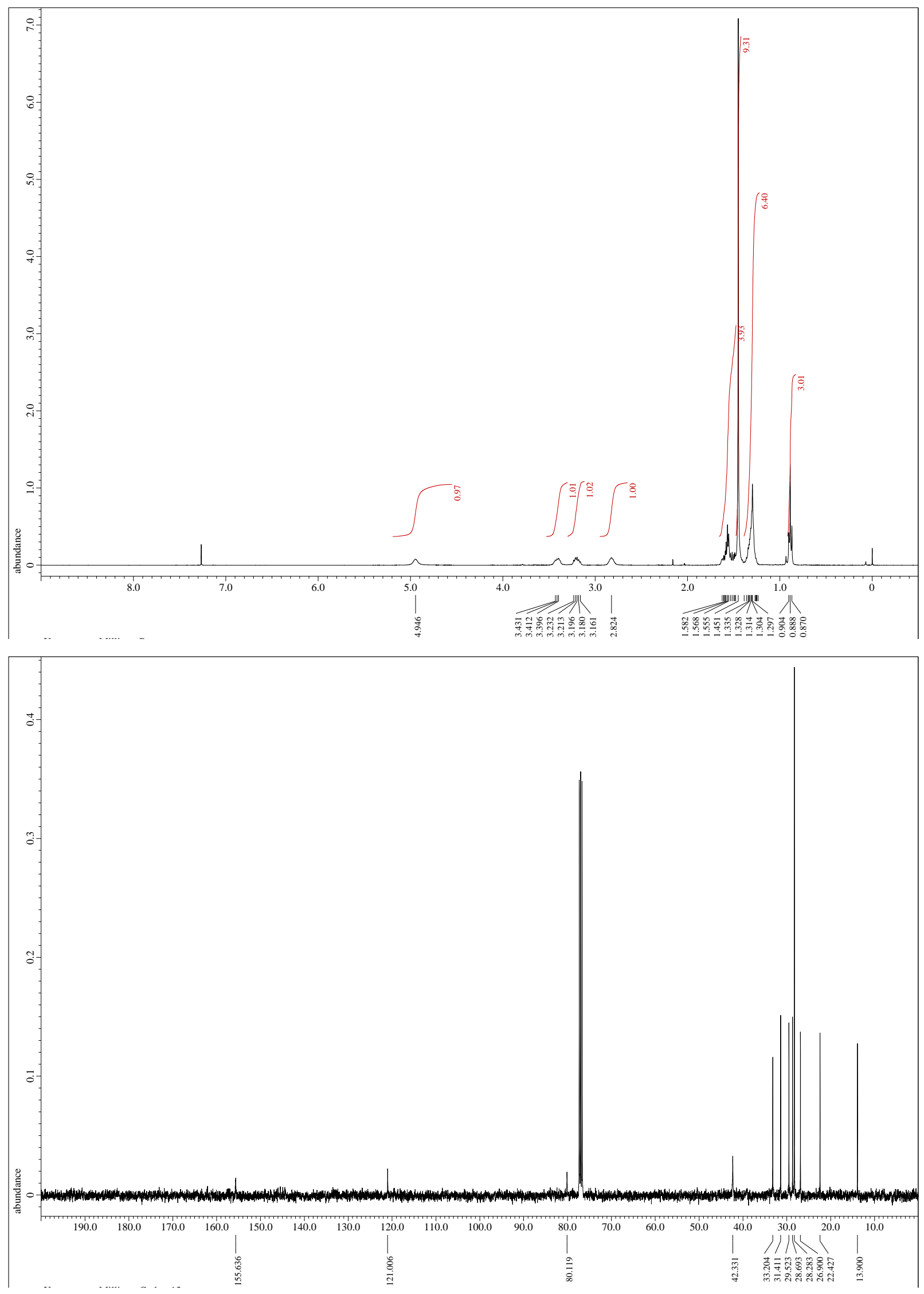

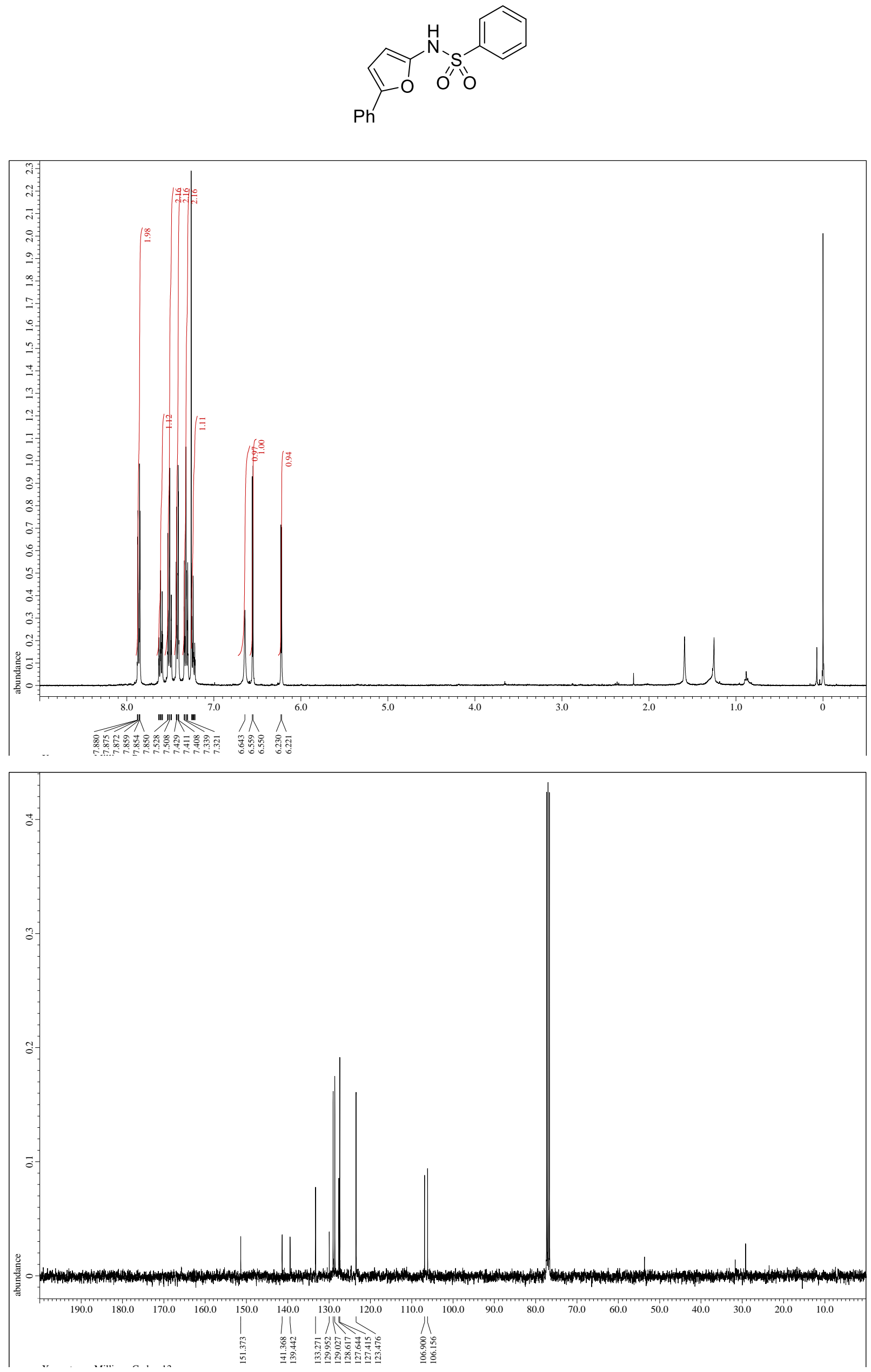


$$
\overbrace{O O O N O}^{N}
$$
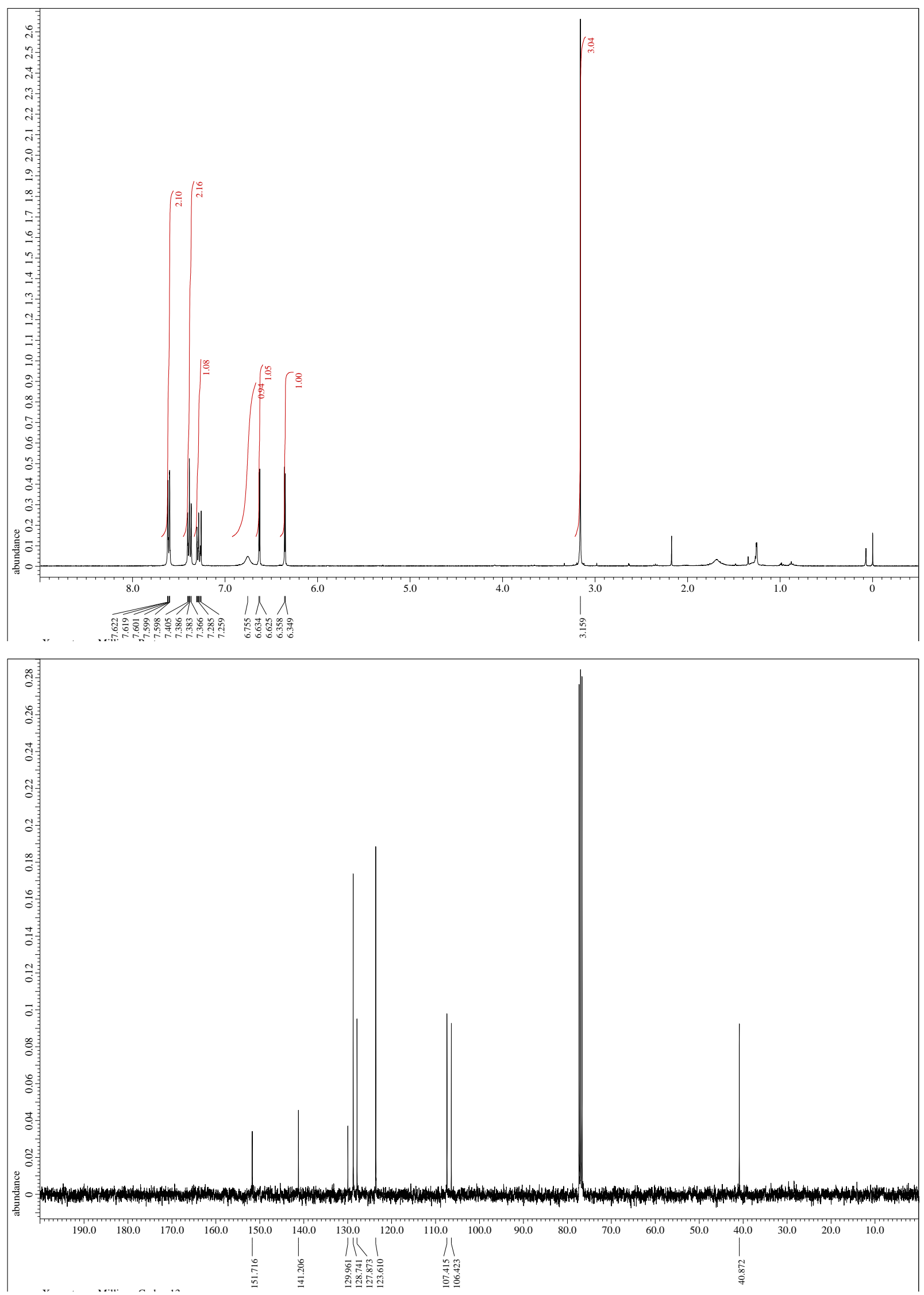

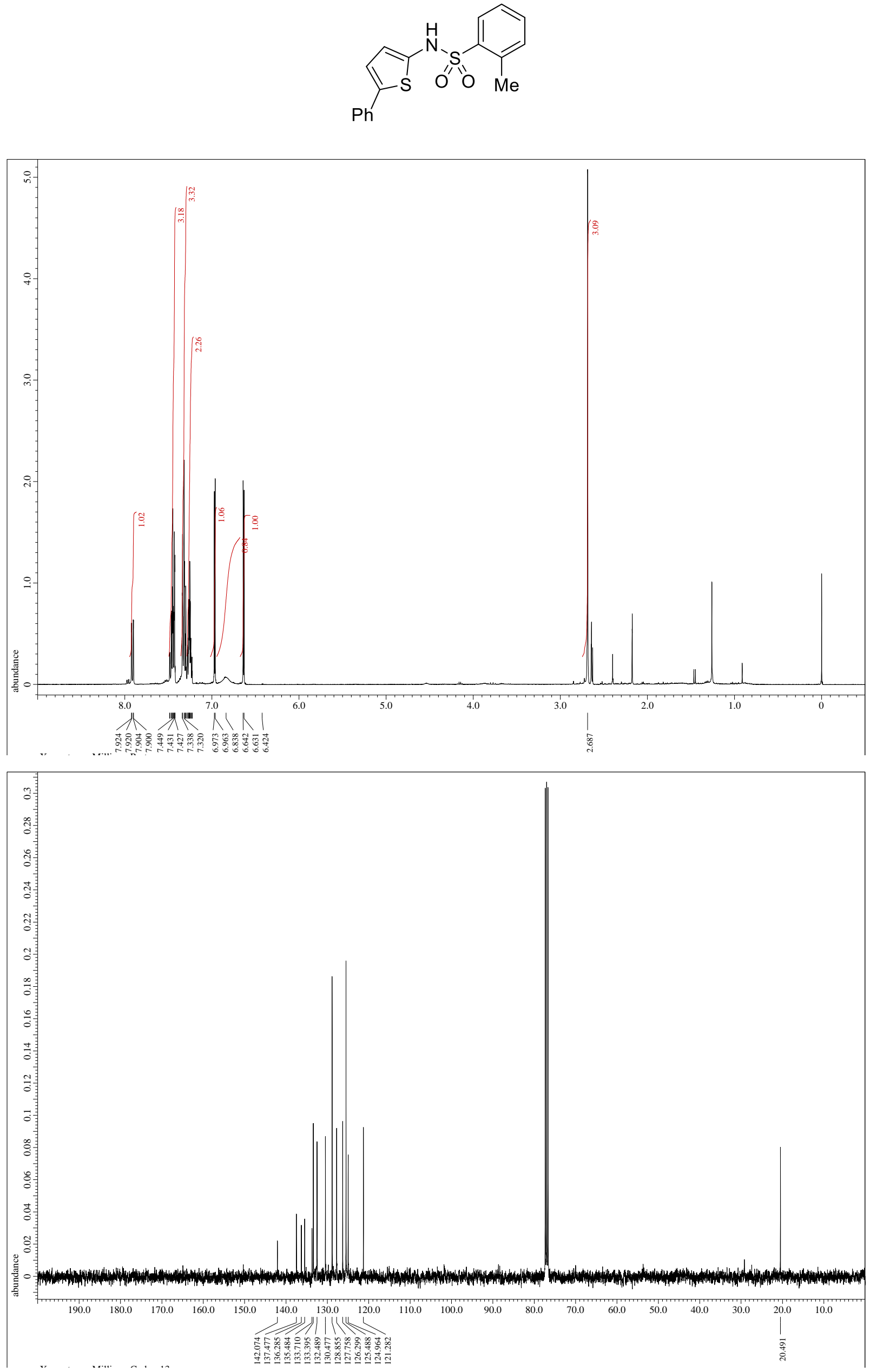

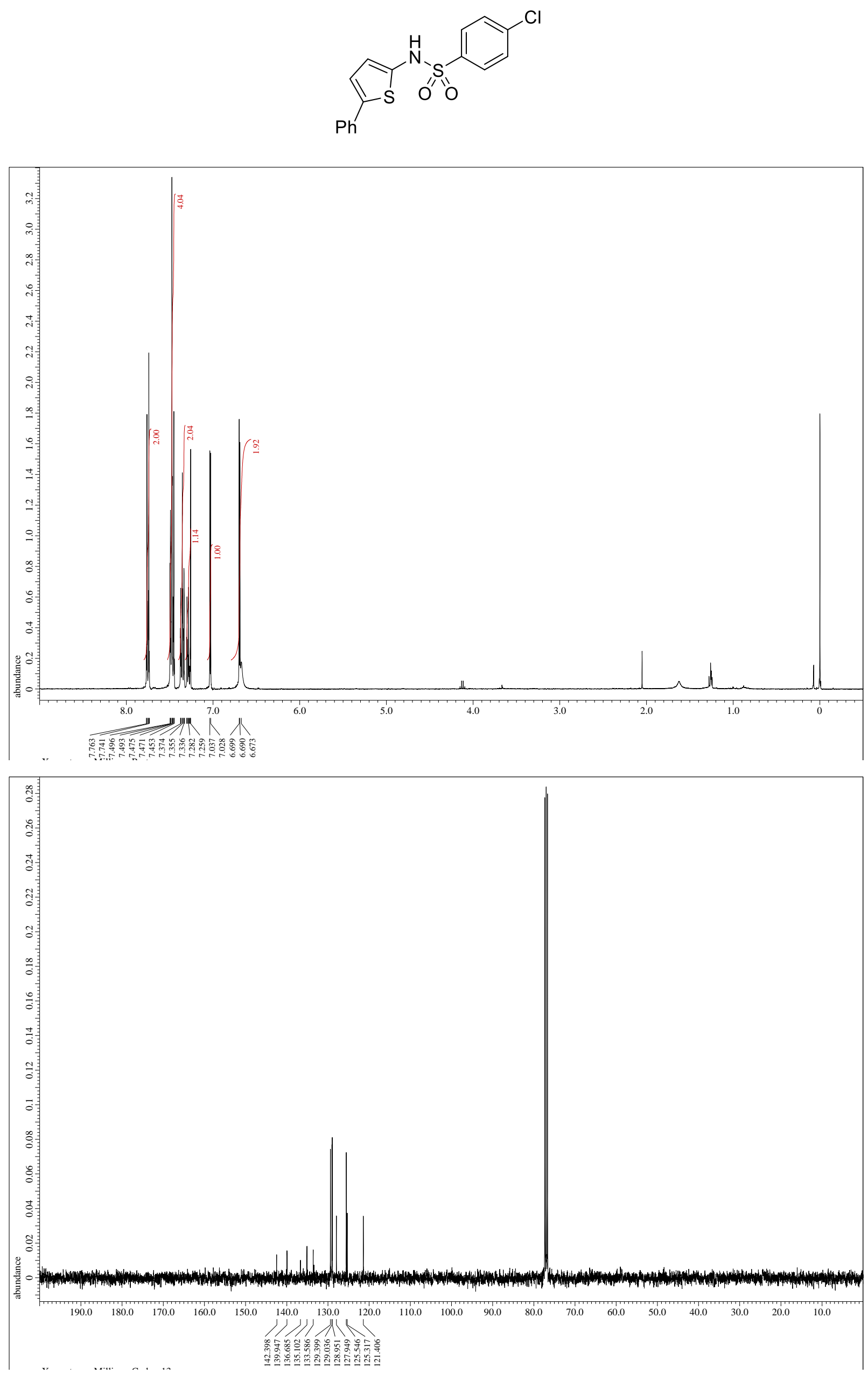

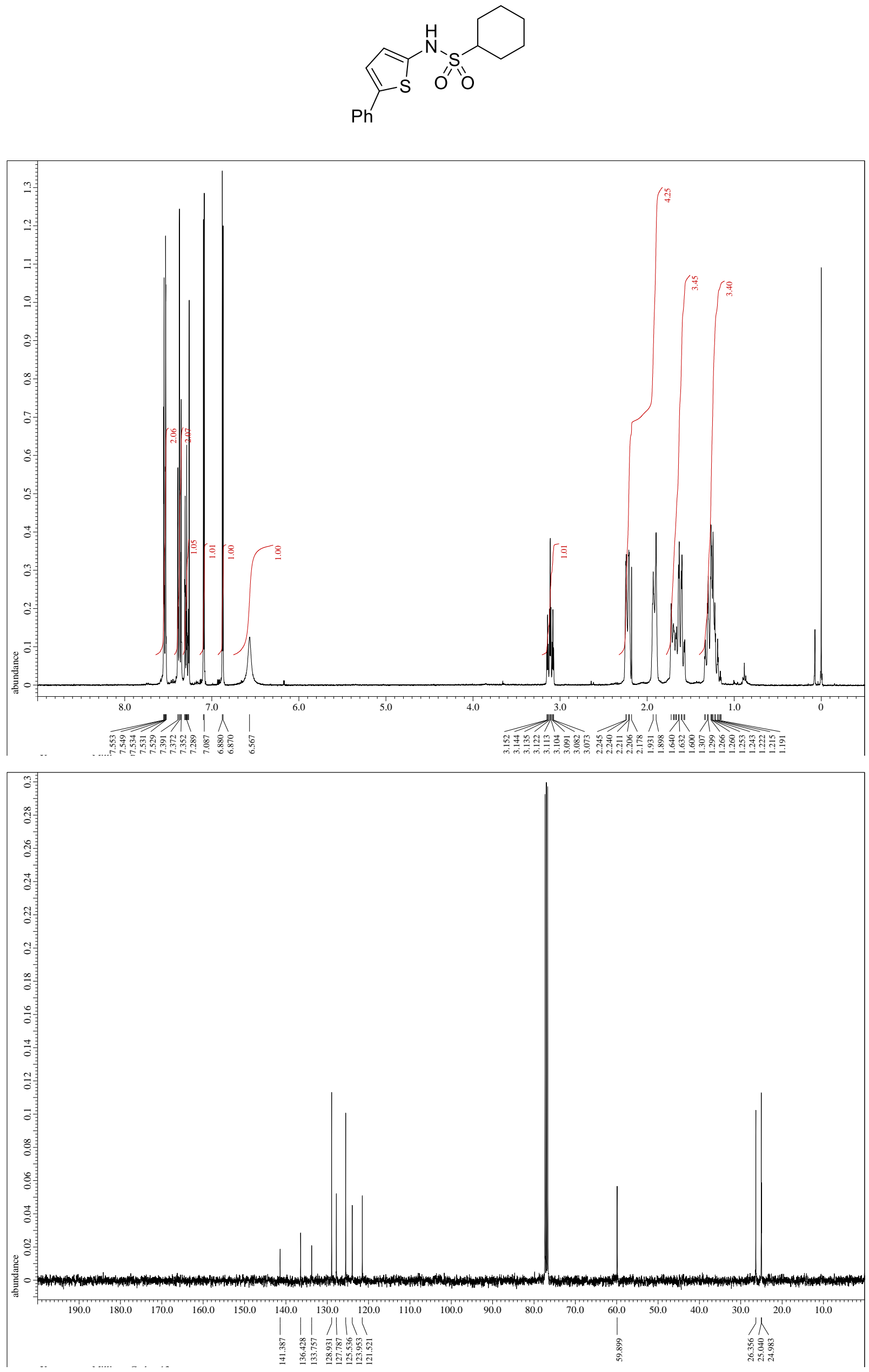

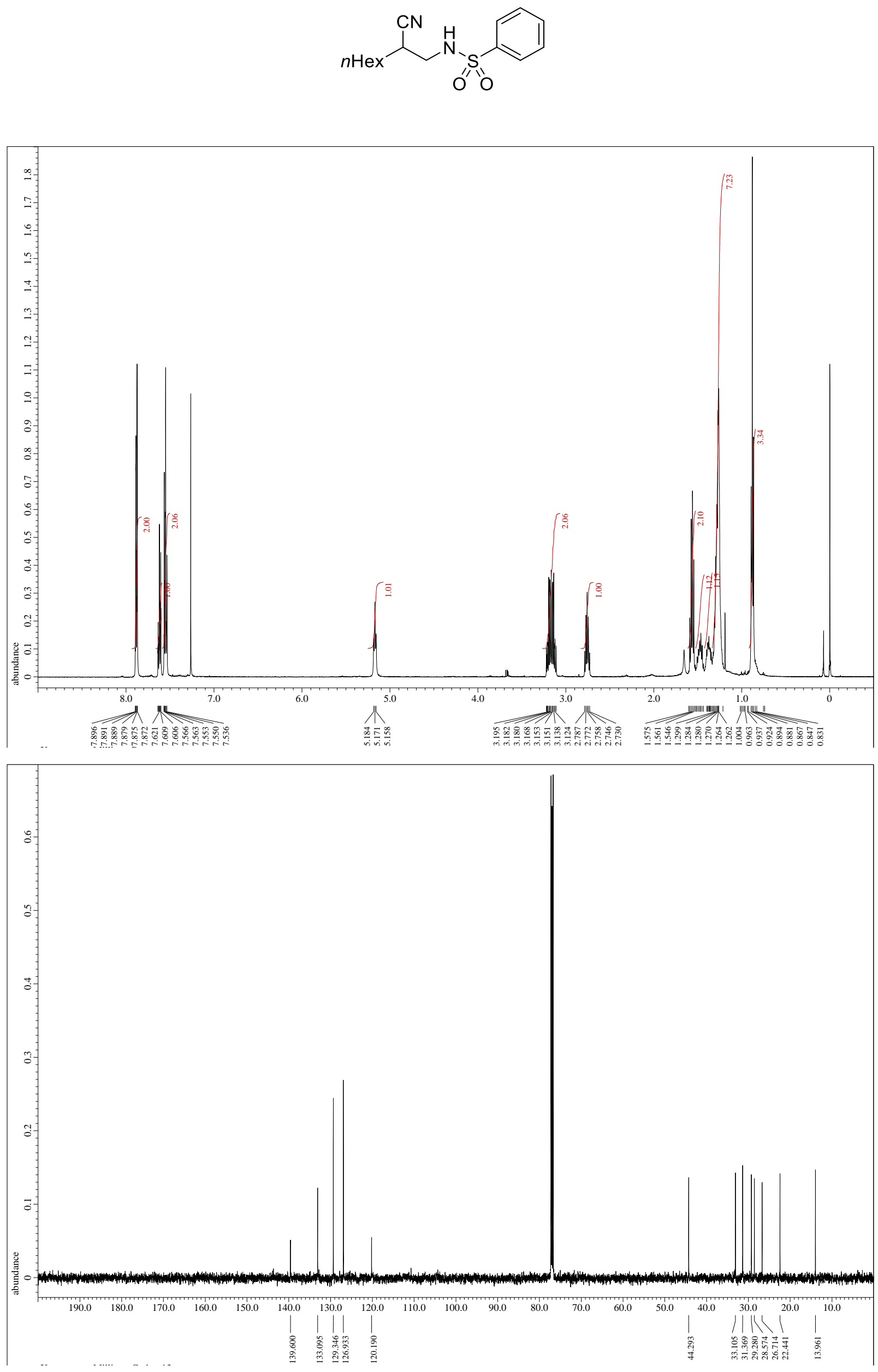
<smiles>CS(=O)(=O)NCC(C#N)C#N</smiles>
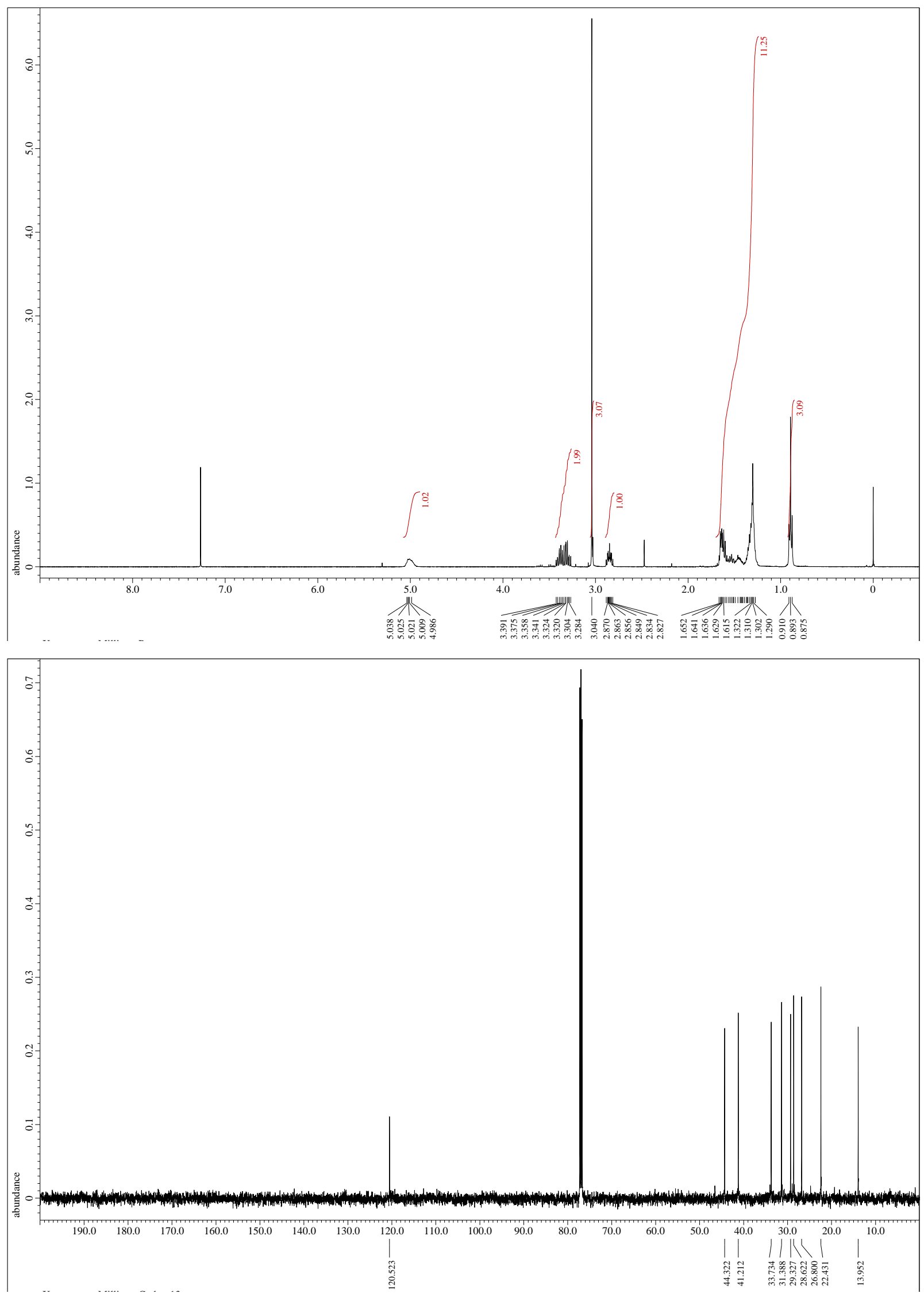

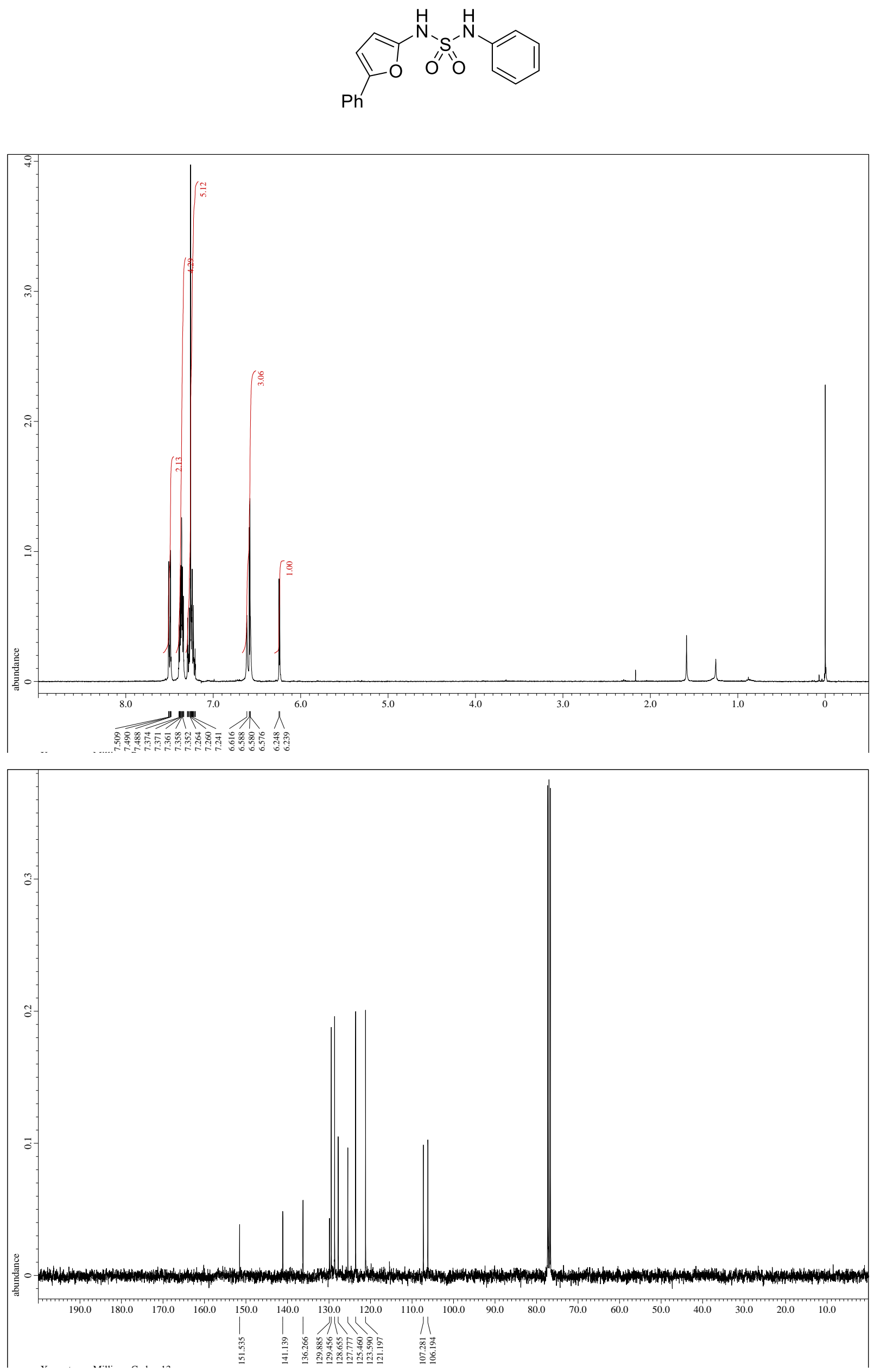

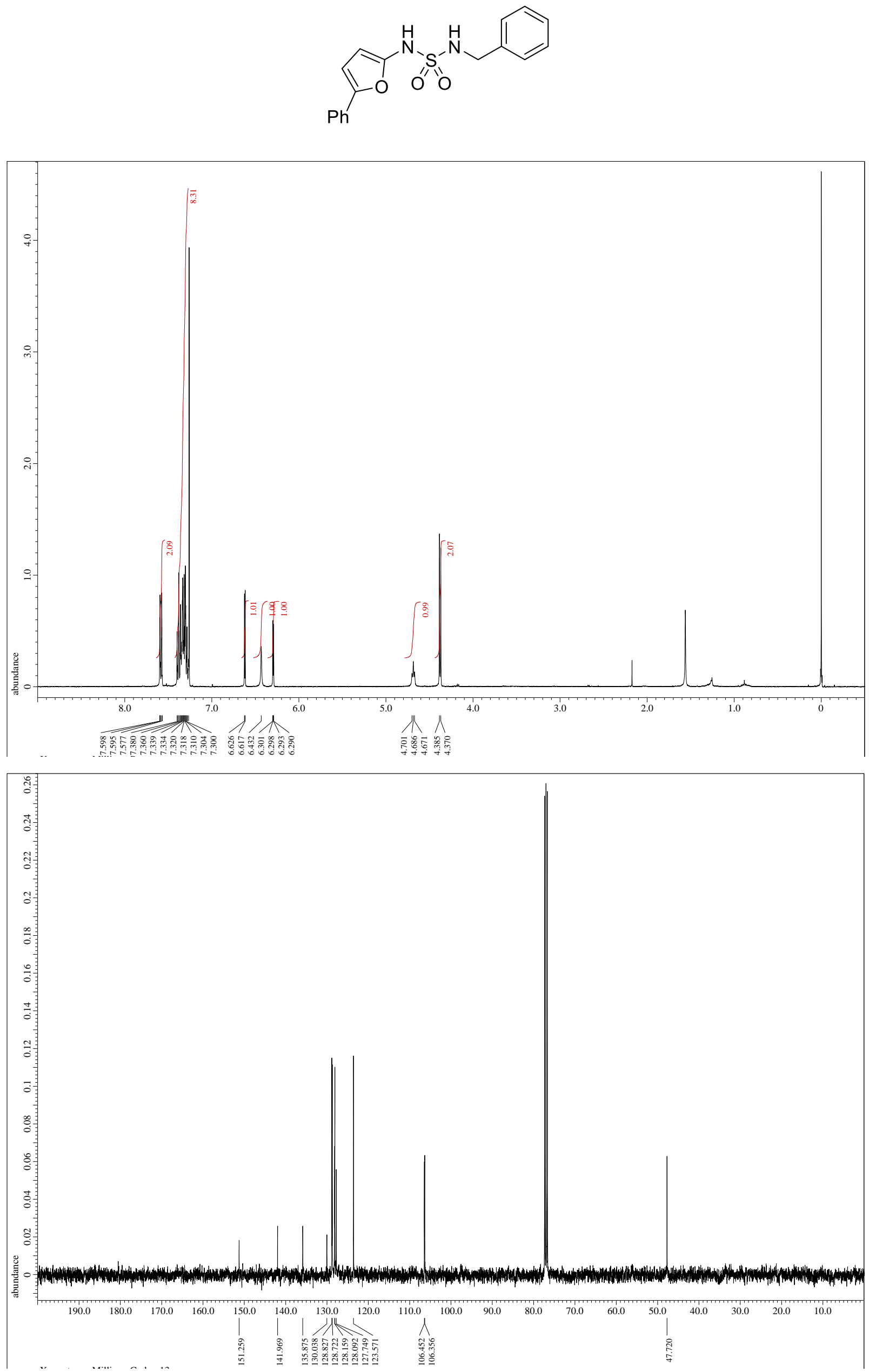

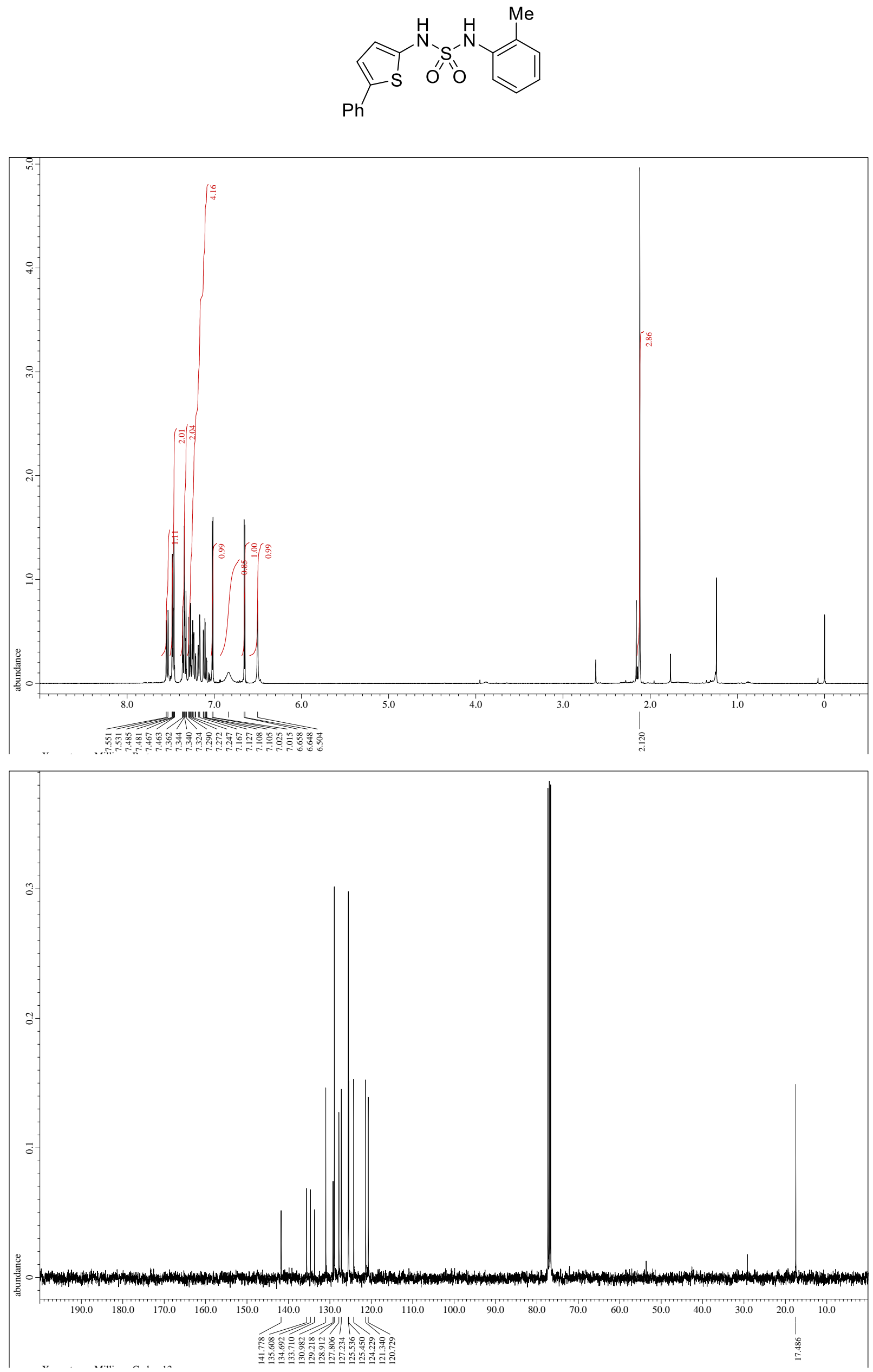

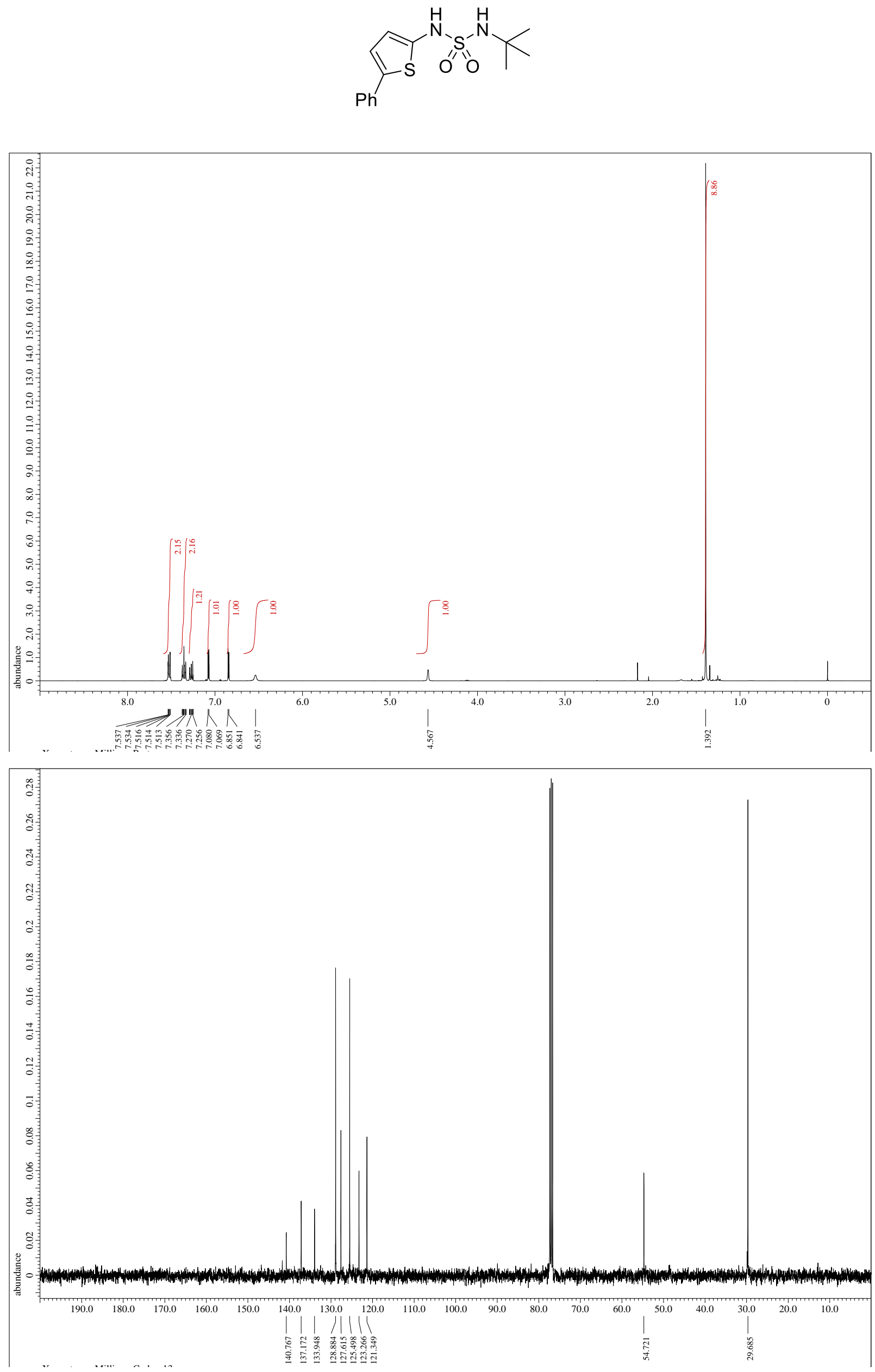

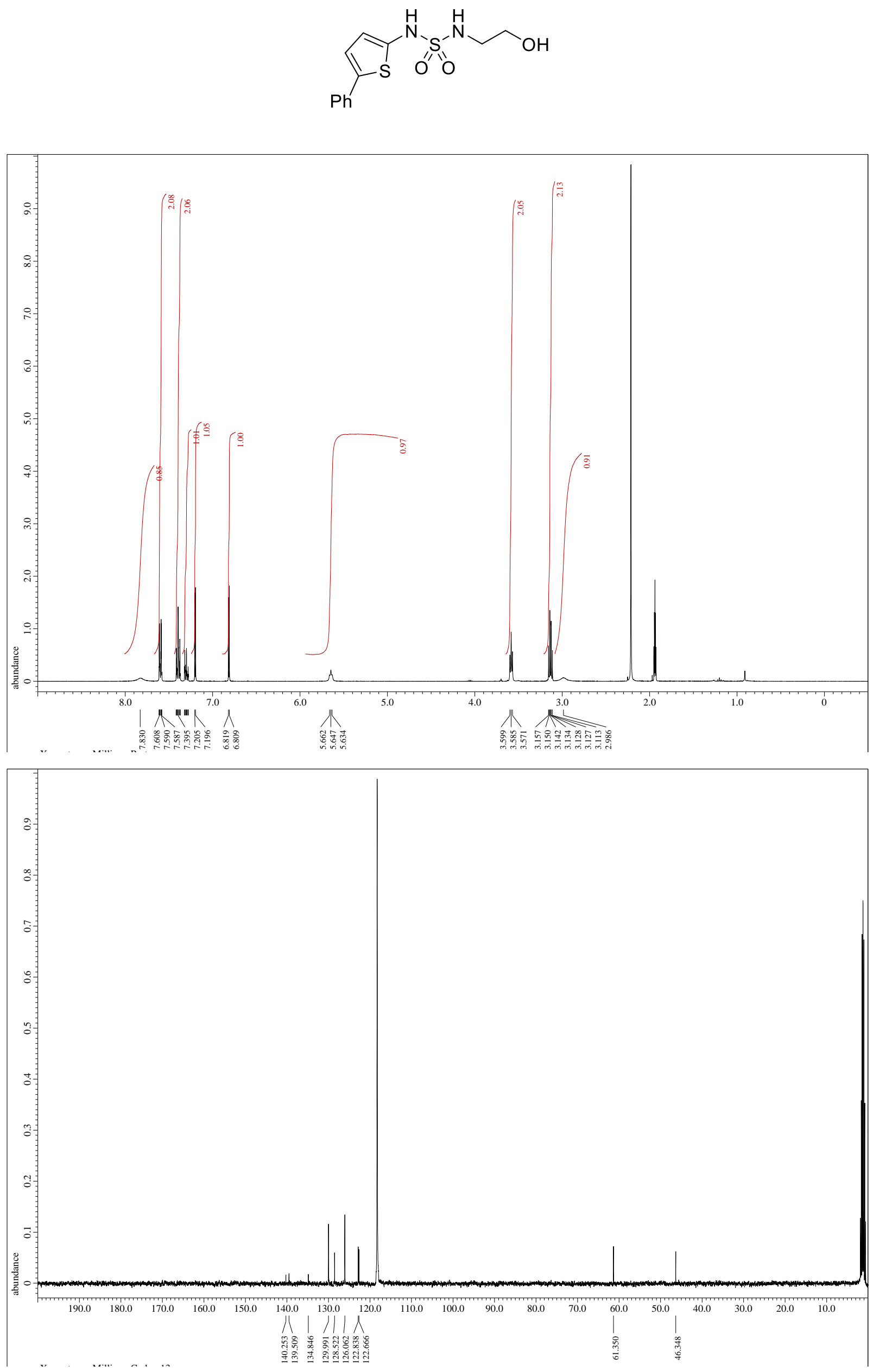

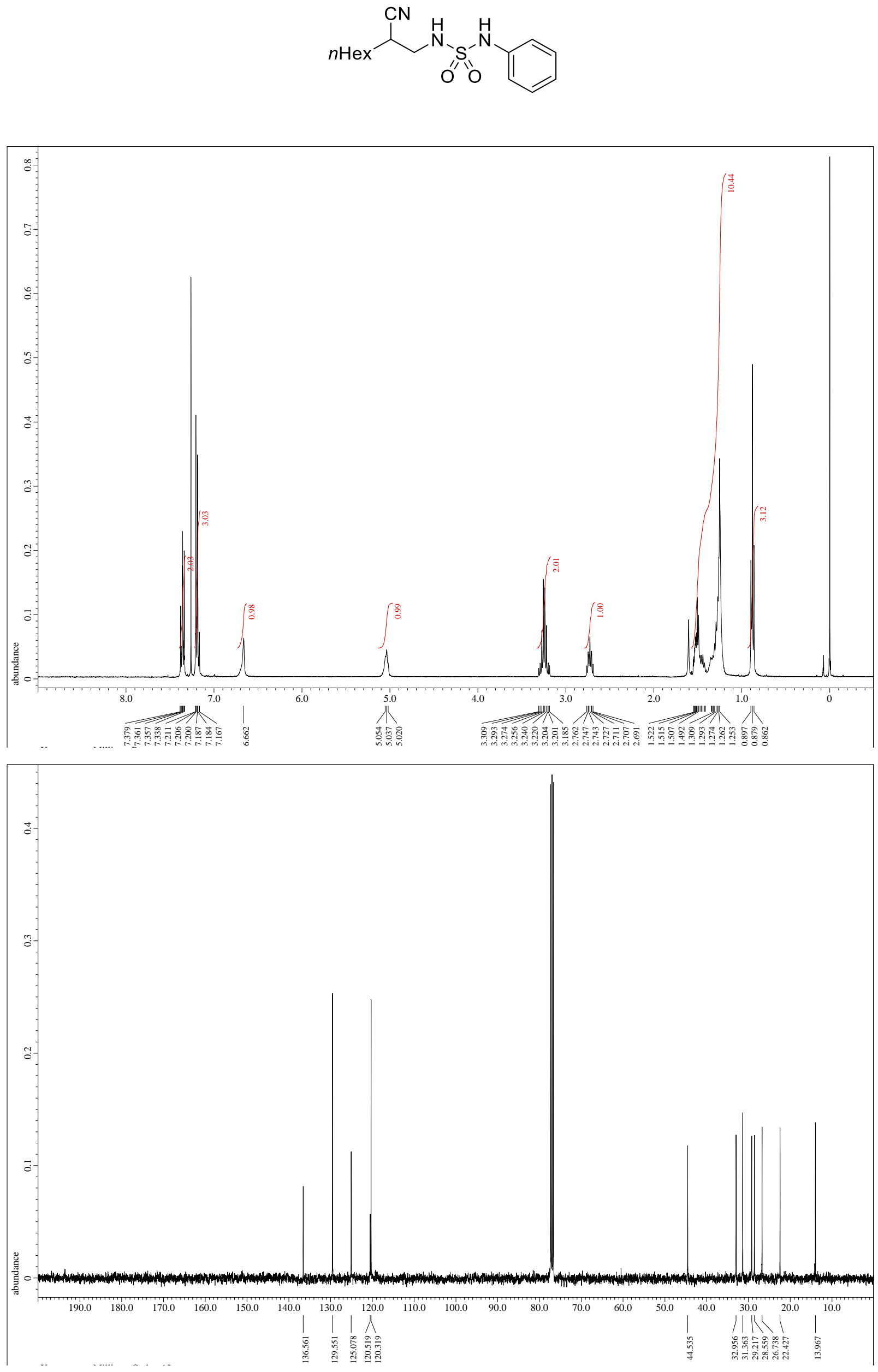

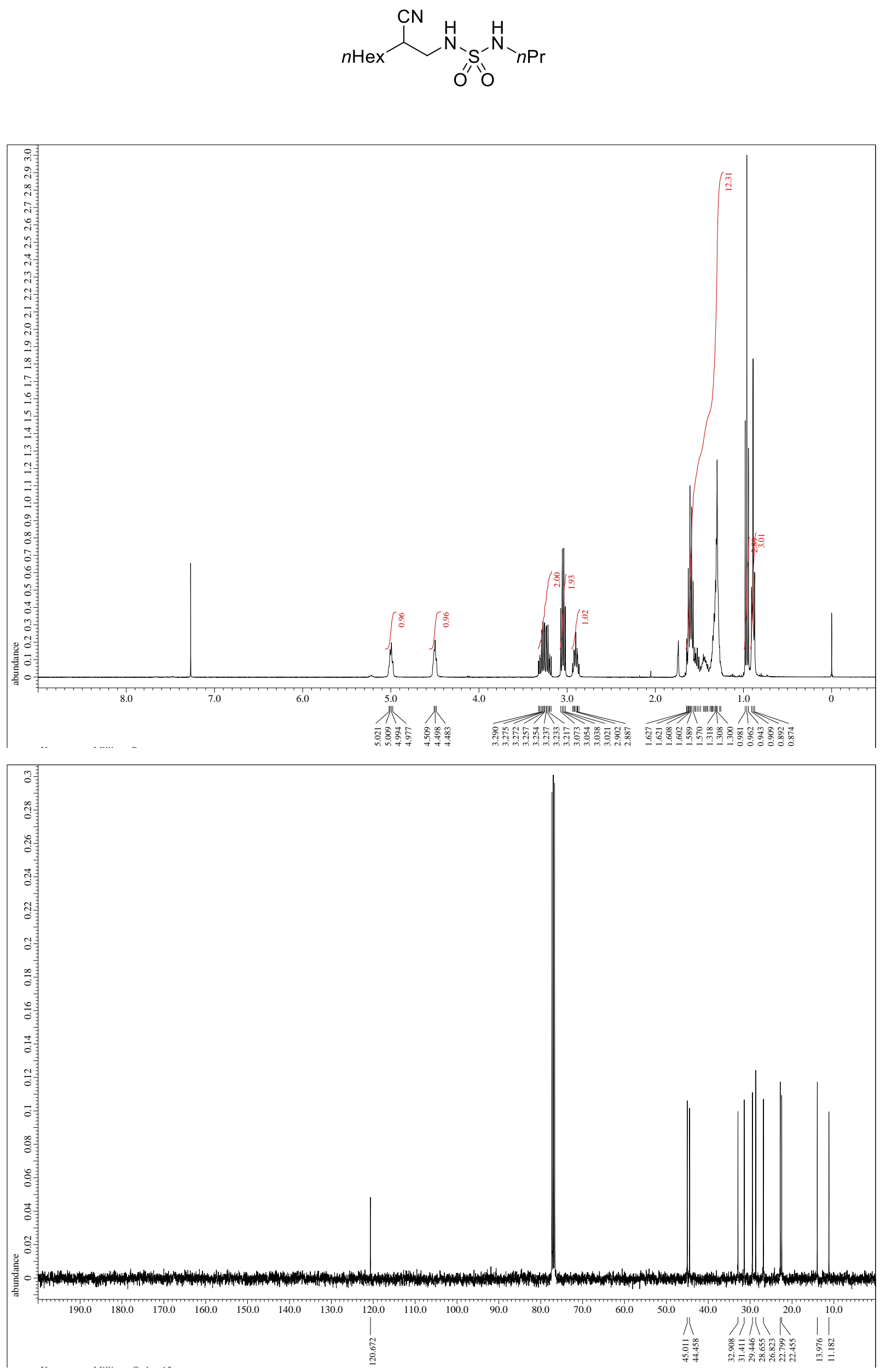